\title{
Emission Cross Sections for Rotational Transitions of Molecules of Astrophysical Interest
}

by

Susan Ames

Walter F. Huebner

\section{notres X}

PORTIONS OF THIS REPORT ARE ILLEGIRLE. It has been reproduced fro the best avallable copy to permit the broadest possible avallab1l1ty.

An Allirmative Action; Equal Opportunity Employer 
Printed in the United States of America. Available from National Technical Information Service U.S. Department of Commerce

$$
5285 \text { Port Royal Road }
$$

$$
\text { Springfield, VA } 2216
$$

Price: Printed Copy $\$ 6.00$ Microfiche $\$ 3.00$

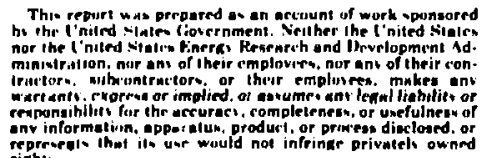

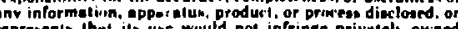

represte. 
EMISSION CROSS SECTIONS FOR ROTATIONAL

TRENSITIONS OF MOLECULES OF ASTROPHYSICAL INTEREST

by

Susan Ames and Walter $F$. Huebner

ABSTRACT

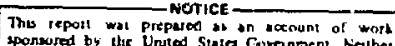

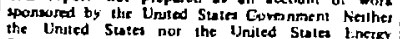

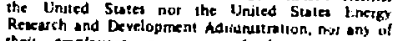
thei emgloyees. not any of they contractors. $\rightarrow i$ renty. expjes ot inpled, of atsumes ony ingal unblusy of retponsibilily for the sccurscy, somple senes of usefulnee of any informu tion. appesalid, piod uct or

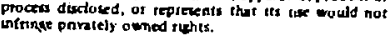

The emission cross sections and Einstein A coefficients of the microwave transitions of numerous molecules of astrophysical interest are presented.

\section{INTRODUCTION}

In recent years, radio observations of the interstellar medium and of comets have revealed the presence of numerous molecules by detecting their rotational and some vibrational transitions, which generally lie in the microwave frequency range. For recent reviews on interstellar molecules, see Zuckerman and Palmer ${ }^{1}$ and Turner, ${ }^{2}$ and for a review of radio observations on comets, see Snyder. ${ }^{3}$ We have computed emission cross sections and Einstein $A$ coefficients for transitions whose frequencies are known securely for molecules of astrophysical interest. We hope that these cross sections will prove useful both in identifying observed lines and in suggesting lines for which to search, because for comparable column densities of the molecular species the observed line intensities would be proportional to the cross sections. The Einstein coefficients for spontaneous emission are useful to compare with collisional excitation rates in assessing the departure from thermal equilibrium.

Only molecules whose rotational constants have been measured are considered. For these molecules, we have chosen only those transitions for which $J \leqslant 20$ and whose frequencies have been measured in the laboratory or calculated with confidence. That is, the calculated frequencies must lie near other observed transitions that agree well with similarly calculated values. We have not caiculated any frequencies; all frequencies given are taken from the references. Hyperfine

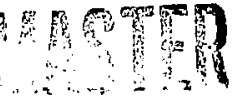


splitting is treated as unresolved; the total line strength is assumed to be in the theoretically strongest component when the line was resolved in the laboratory; ctherwise, the blended value of the frequency is used as reported in the reference. All calculations are made using the approximation that the molecules are rigid rotors, but we emphasize that this affects only the cross sections, not. the 1 ine frequencies, because the frequency values are obtairied from observations. Nevertheless, because many niolecules differ significantly from rigid rotors, our calculations should be regarded as only a first approximation, and the original literature should be checked for comments and for correction procedures for the particular molecule, when more precise values are desired. Linear molecules are listed in Table I, symmetric rotors in Table II, and asymmetric rotors in Table III. Within each table, the molecules are ordered as follows: First, those containing hydrogen and second, by increasing total number of atoms of the next higher atomic number. For detail see the contents listed for each table.

When the data were available, we included rotational transitions within excited vibrational states. Each vibrational mode is identified using the notation of the reference from which we took the data.

All frequencies, $\mathrm{NU}$, in the tables are in gigahertz, as are the rotation constants. The dipole moment, MU, is in debyes $\left(10^{-18}\right.$ esu $\left.\mathrm{cm}\right)$; the Einstein coefficient, A, (spontaneous transitions integrated over the normalized line shape) is in inverse seconds; and the cross sections, SIGMA, are averaged over the line width in square centimeters.

\section{CALCULATIONS}

Townes and Schawlow ${ }^{4}$ (TS) give the general expression for optical depth as

$$
\tau\left(\nu^{\prime}\right)=\frac{8 \pi^{3} N f|\mu|^{2} v^{2}}{3 c k T^{\top}} \varphi\left(v^{\prime}\right)
$$

where $N$ is the column density of molecules, $f$ is the fraction of molecules in the initial state, $|\mu|^{2}$ is the electric dipole moment matrix element, and $\varphi$ is the normalized line profile function. The component of $\mu$ appropriate to the particular transition was used when it was known; otherwise it was assumed that the total value reported in the reference applies to the strongest moment. The mean optical depth in the line is then 


$$
\tau=\frac{1}{\Delta \nu} \int_{-\infty}^{\infty} \tau d \nu^{\prime}=\frac{8 \pi^{3} N f|\mu|^{2} v^{2}}{3 c k T \Delta v}
$$

where $\Delta v$ is the observed FWHM. The cross section is defined as $\sigma \equiv \bar{\tau} / N$. These cross sections are computed for four temperature valles, 20, 50, 100, and $200 \mathrm{~K}$. A value of $L V=1 \mathrm{kllz}$ has been used in calculating tre cross sections, $\sigma_{\text {calc. }}$ Hence, $N=\bar{\tau} / \sigma_{\text {calc }}\left(\frac{\Delta v}{1 M H z}\right)$. The general expression ior the Einstein $A$ coefficient is

$$
A=\frac{64 \pi^{4} \nu^{3}}{3 h c^{3}}|\mu|^{2}
$$

The fractional occupation of the upper level is

$$
f=\frac{(2 J+1) e^{-W / k T}}{Q_{r} Q_{v}}
$$

where $Q_{r}$ and $Q_{v}$ are the rotational and vibrational partition functions, respectively,

$$
\begin{aligned}
& Q_{r}=\sum_{J}(2 J+1) e^{-W_{J} / k T}, \\
& Q_{v}=\prod_{v}\left(1-e^{-W_{v} / k T}\right)^{-g_{v}},
\end{aligned}
$$

$g_{v}$ is the degeneracy of the vibrational level $v, W$ is the energy of the upper level as measured from the absolute ground state, $W_{J}$ is the energy of this level as measured from the ground rotational level of the particular vibrational state, and $W_{v}$ is the energy of the ground rotational level in the particular vibrational state involved (in the vibrational ground state, $W_{v}=0$ ). 
A. Linear bolecules

Fur emissinn, $\mathrm{J} \rightarrow \mathrm{J}-1$, the dipole moment elenent is (TS)

$$
|\mu|^{2}=i^{2} \frac{J}{2 J+T} .
$$

Using the relation for the rotation constant $B$,

$$
B=\frac{v}{2 J},
$$

we $o b$ ๖ain

$$
\sigma=\frac{4 \pi^{3} v^{3} \mu^{2}}{3 c k T B \nu Q_{r} Q_{v}} e^{-W / k T}
$$

The energy of the upper level is given by

$$
W=w_{J}+w_{v}=h B J(J+1)+w_{v}
$$

$Q_{v}$ is set equal to $l$ in our calculations, but $Q_{r}$ is evaluated by performing Summation (5a).

Transitions associated with $\ell$ doublet bending in linear molecules are listed in Table Ib. For these transitions, the dipole moment matrix element is given by

$$
|\mu|^{2}= \begin{cases}\mu^{2} \frac{\ell^{2}}{(J+1) J}, & \Delta J=0, \\ \mu^{2} \frac{J^{2}-\ell^{2}}{J(\angle J+1)}, & J \rightarrow J-1,\end{cases}
$$

"In our notation, $\mathrm{J}$ always refers to the upper leveí. 
ard the energy of the upper level is given by

$$
W=W_{v}+B h\left[u(u+1)-\ell^{2}\right] \text {, }
$$

where $w_{v}$ is the bending mode energy.

Transitions between ${ }^{3} \Sigma$ states for low $\mathrm{J}$ rekresent a case intermediate between Hund's Case a and Case b. The only molecule we have included to which this applies is SO. We have used the line strength factors that were computed taking this into account, and $W$ values from the appropriate reference listed in Table Ic. B. Sylmietric Rotars

For these riolecules, is gives the dipole matrix eleilent as

$$
|\mu|^{2}= \begin{cases}\frac{\mu^{2} k^{2}}{J(J+1)^{F},} & \Delta u=0 \\ \mu^{2} \frac{J^{2}-k^{2}}{\pi(2 J=1)^{F},} & \jmath \rightarrow J-1,\end{cases}
$$

with the selection rule $\Delta K=0$. Here $F$ is the factor that accounts for nuclear spin degeneracy.

$$
F=\frac{S(I, K)}{4 I^{2}+4 I+1}
$$

where $I$ is the nuclear spin and $S(I, K)$ is given by

$$
S(I, K)= \begin{cases}2\left(4 I^{2}+4 I+3\right), & K \text { a multiple of } 3 \\ 4 I^{2}+4 I+3 & , K=0 \\ 2\left(4 I^{2}+4 I\right) & , K \text { not a multiple of } 3 .\end{cases}
$$


The population factor $f$ is given as in $E q$. (4) with $Q_{v}$ as given by $E q$. (5b). In this case (assuming $f^{\prime}=B$ ).

$$
W=B h J(J+1) i(C-B) h k^{2}+b
$$

and

$$
a_{r}=\sum_{J=0}^{\infty} \sum_{k=-J}^{+J}(2 J+1) e^{-W_{J} / k T} .
$$

The cross section is given by

$$
\sigma=\frac{8 \pi^{3} \mu^{2} v^{2}}{3 c k T \Delta v Q_{r} Q_{v}} F\left\{\begin{array}{l}
\frac{k^{2}(2 J+1)}{\left.J_{1} J+1\right)}, \Delta J=0 \\
\frac{J^{2}-k^{2}}{J}, J+J-1 .
\end{array}\right.
$$

As above, $Q_{v}$ was set equal to 1 and $Q_{r}$ was evaluated by using Summation (14). These cross sections are given in Table II.

C. Asymmetric Rotors

For asymmetric rotors, the dipole matrix element for emission $\mathrm{J} \rightarrow \mathrm{J}-1$ is given by

$$
|\mu|^{2}=\frac{\mu^{2} s}{2 J+1}
$$

where $S$ is the line strength factor. This transition strength factcr is computed for each transition, for each molecule, using the program by Wacker and Pratto ${ }^{5}$ which R. Beebe of New Mexico State University kindly furnished us. The population factor is given by Eq. (4), and the energy of the upper level, assuming $A>B>C$, 
is given by

$$
W=\frac{h}{2}\left[(A+C) J(J+1)+(A-C) E_{\tau}(K)\right]+W_{v}=W_{J}+H_{v},
$$

Where $E_{\tau}(k)$ is the energy parameter for level $J_{\tau}$ of a molecule with asymmetry parameter

$$
K=\frac{2 B-A-C}{A-C}
$$

For an energy level $J_{K_{-1}} K_{+1}, \tau$ is defined as $\tau \equiv K_{-1}-K_{+1} \cdot E_{\tau}(K)$ is computed for each $J$ level, for each rioleclite, by diagonalizing the energy matrix, as describec by Turmer: Hicks, and Reitwiesner. ${ }^{6}$ Hence, the cross section is given ty

$$
\sigma=\frac{8 \pi^{3} \mu^{2} s v^{2}}{3 c k T \Delta v Q_{r} Q_{v}} e^{-W / k T} .
$$

As above, $Q_{v}$ was set equal to 1 . For the calculations at $20 \mathrm{~K}, \eta_{r}$ was evaiuated using the summation

$$
\eta_{r}=\sum_{J=0}^{\infty} \sum_{\tau=-J}^{+J}(2 J+J) e^{-w_{j} / k T} .
$$

At higher temperatures, we used the approximation given by Herzberg: 7

$$
\mathrm{O}_{r}=\mathrm{e}^{\sqrt{B C} h /(4 k T)} \sqrt{\frac{\pi}{A B C}\left(\frac{k T}{h}\right)^{3}}\left[1+\frac{1}{12}\left(1-\frac{\sqrt{B C}}{A}\right) \frac{\sqrt{B C} h}{k T}+\ldots\right] .
$$

This expression assumes that $B$ and $C$ are the two most nearly equal rotation constants. Otherwise, $B C$ is replaced by $A B$ and $A$ by $C$. These cross sections are given in Table III. 
III. FINDING LIST OF ALL LINES

AII transitions contained in Tables Id-C, II, and III have been sorted together by frequency. This frequency-ordered list appears in Table IV, which lists the chemical formula of the molecule and the Table (I - III) in which it appears, for each transition frequericy.

\section{REFERENCES}

1. B. Zuckerman and P. Palmer, "Radio Radiation from Interstellar Molecules," Ann. Rev. As. AD. 12, 279 (1974).

2. B. E. Turner, "Interstellar Molecules," in Galactic and Extra-Galactic Radio Astronomy, G. L. Verschuur and K. I. Kellernann, Eds. (Springer-Verlag, NY, HeideTberg, Berl in. 1974).

3. L. E. Snyder, "Radio Detections of Molecular Transitions from Comet:: A Review," in IAU Colloquium Ho. 25, A Study of Comets, MASA SP-393 (1976).

4. C. H. Townes and A. L. Schawlow, Microwave Spectroscopy (McGraw Hill, Nlew York, Toronto, Lendon, 1955).

5. P. F. Wacker and M. R. Pratto, Microwave Spectral Tables, IBBC Monograph 70 , Vo?. II, U.S. Government Printing Office, Washington, D. C. (1964).

6. T. E. Turner, B. L. Hicks, and G. Reitwiesner, Ballistics Research Laboratories report No. 878, Ballistics Research Laboratory, Aberdeen, MD (1953).

7. G. Herzberg, Molecular Spectra and Molecular Structure, Vol. II, Infrarec and Ramen Spectra of Polyatomic Molecules (Van Nostrand Co., Inc., Princeton, N.J., 1964). 


\section{TABLE I}

\section{LINEAR 11OLECULES}
(A) rotational transitions
(B) 2-doubling transitions
(C) transitions between ${ }^{3} \sum$ states

\section{Table IA - Contents}

1. $\mathrm{HCN}$

2. $\mathrm{HCNO}$

3. $\mathrm{C}_{2} \mathrm{H}$

4. $\mathrm{HC}_{3} \mathrm{H}$

5. $\mathrm{OH}$

6. ClO

7. $\mathrm{CN}$

8. NCO

9. $\mathrm{FCN}$

10. $\mathrm{CO}$

11. OCS

12. CS

13. FCCH

14. $\quad 10$

15. SiN

16. NS

17. $\mathrm{N} O \mathrm{O}$

18. SiOt

19. $\$ 10$

20. Sis

Table IB - Cortents

1. $\mathrm{HCN}$

2. OCS

3. $\mathrm{HCNO}$

Table IC - Contents

1. SO hydrogen cyanide fulminic acid ethynyl radical cyanoacety? ene hydroxyl radical chiorine ronoxije cyano-radica?

fluorine cyanide carbor monoxide carbonyl sulfide carbon monosulfide fluoroacetylene nitric oxide

nitrogen sulfide nitrous oxide

silicon monoxide silicon monosulfide

hydrogen cyanide carbonyl sulfide fulminic acid

sulfur monoxide

\section{Notes to Table IA}

a. Value of $\mu$ is assumed to be 1 .

b. See also Table IB. 


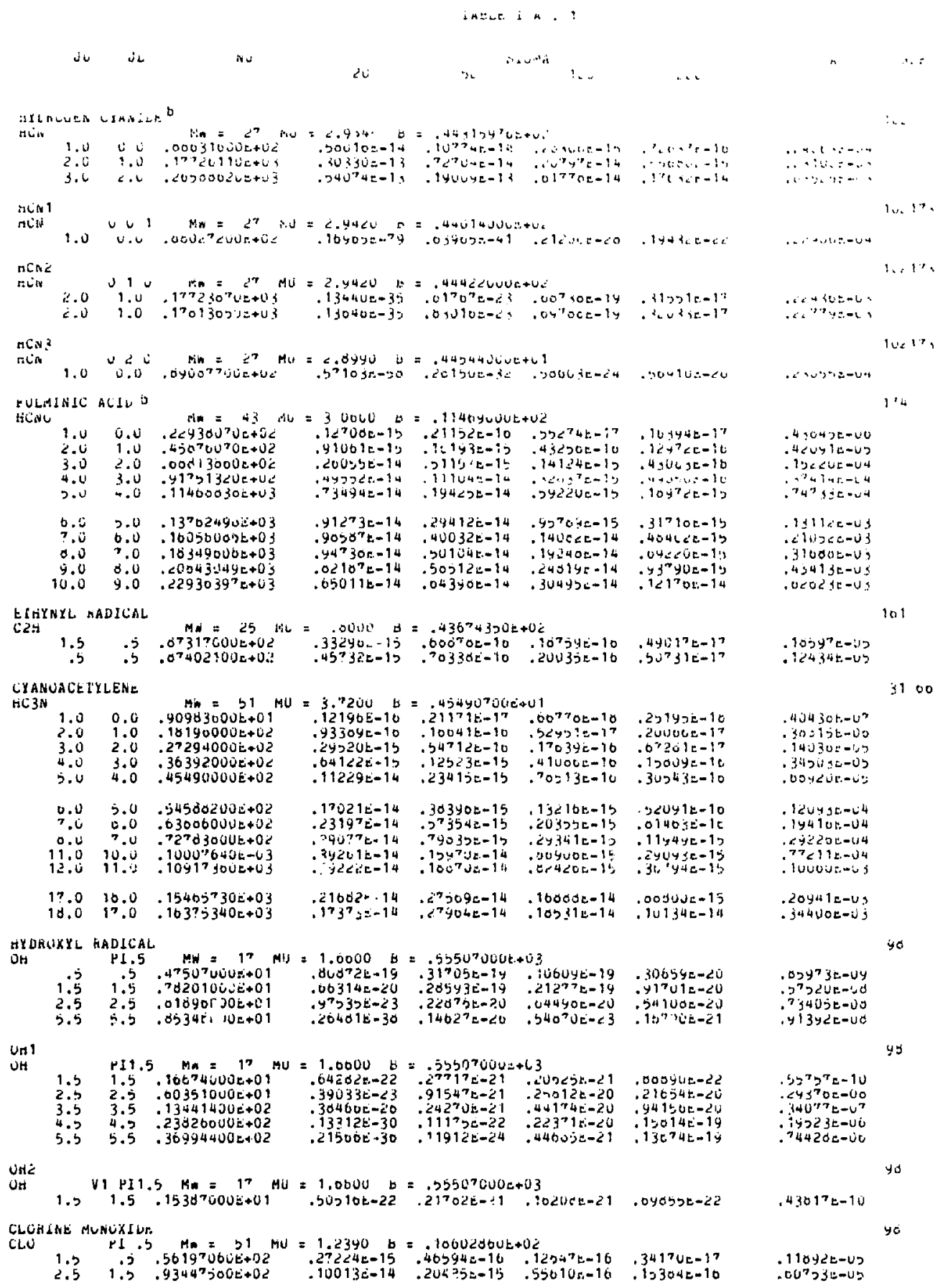




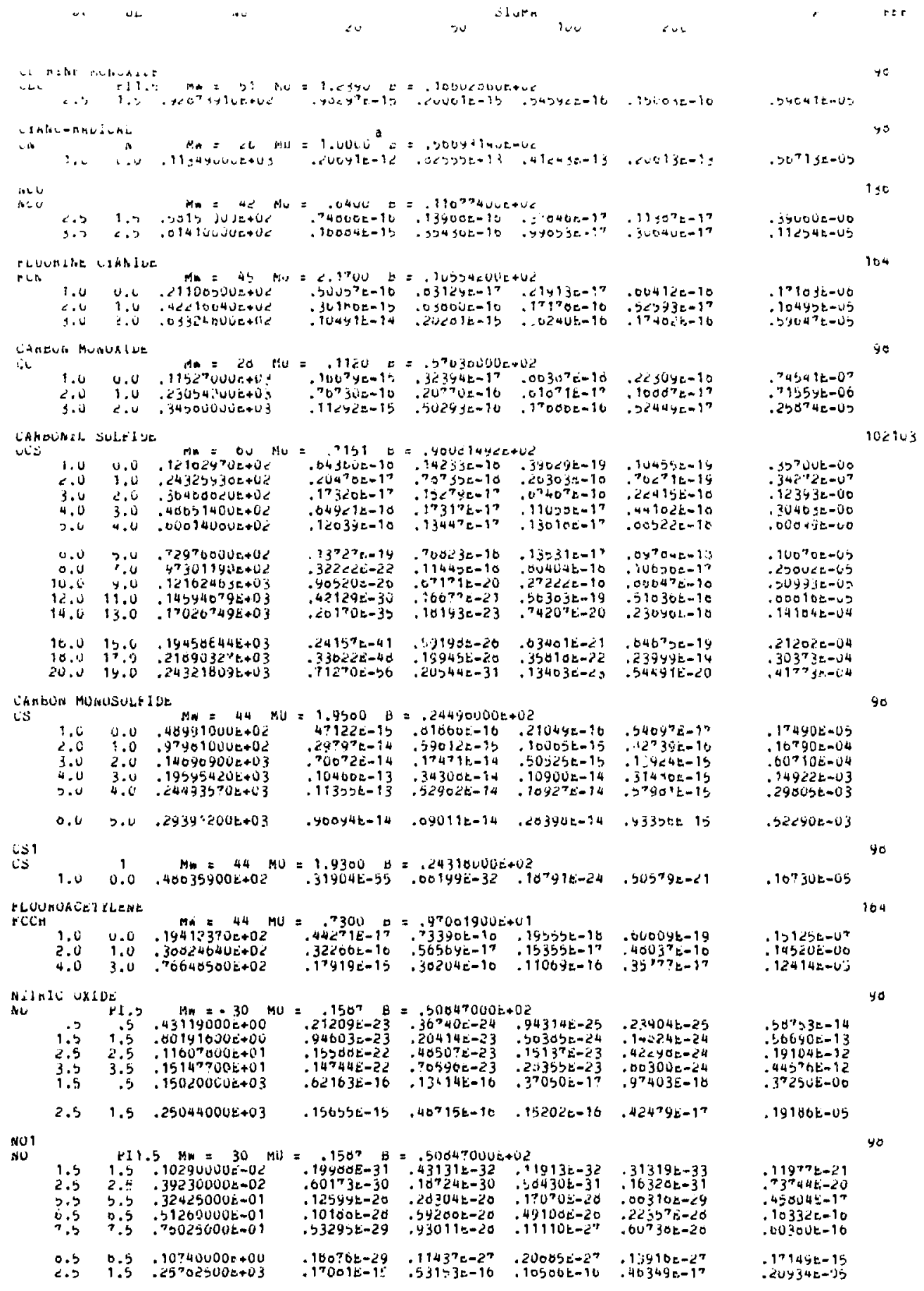


fABic $1 \mathrm{n}, 3$

Jis JL Bu

20

20

SIUAA

10.

¿Uง

ner

$\operatorname{Sin}$

$1.0 \quad M m=4 z M L=3.0000 \quad B=2.031000 e+i 2$

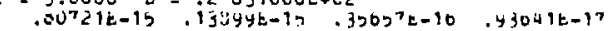

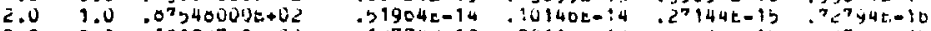

$3.0 \quad 2.0 .13120^{7} v 0 E+C j \quad .12^{774 E-13} .30110 e-14.03004 E-15.2<7428-16$

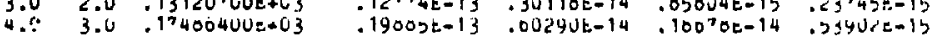

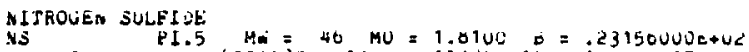

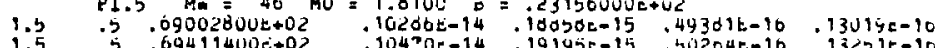

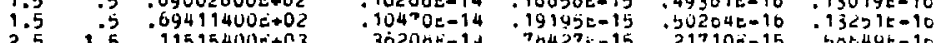

$\begin{array}{llll}.30206 E-14 & 70427 i-15 & .21710 E-15 & .30049 E-10 \\ .365008-14 & .79252 i-15 & .21930 E-15 & .5440^{7}=-10\end{array}$

NS 1

P11.5 Mm $=40 \mathrm{MU}=1.8100 \mathrm{~b}=.23 ! 50000 \mathrm{E}+02$

MitfuUs OXIOE

$\mathrm{N} 2 \mathrm{O}$

$\begin{array}{rrr}1.0 & 0.0 & .25120000 \mathrm{~mL}=4 \\ 2.0 & 1.0 & .5 \mathrm{C} \times 30000 \mathrm{E}+0 \\ 3.0 & 2.0 & .75370000 \mathrm{E}+0 \\ 4.0 & 3.0 & .10049174 \mathrm{E}+0\end{array}$

$\begin{array}{lll}4.0 & 3.0 & 10049174 E+03 \\ 5.0 & 4.0 & 13561370 E+03\end{array}$

$0.0 \quad 5.0 \quad .15073505 E+03$

$1.0 \quad 6.0 \quad 07565562 \mathrm{e}+05$

$0.0 \quad 7.0 \quad .200475206+03$

$\begin{array}{lll}10.0 & 9.0 & .25121150 E+03 \\ 12.0 & 11.0 & .301442^{7} 0 \mathrm{E}+03\end{array}$

1201

,2

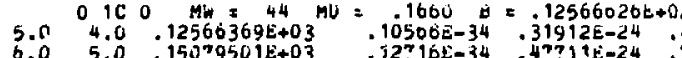

6.0 $5.0+15079501 E+03$

$\begin{array}{rrr}10.0 & 6.0 & .17592559 E+03 \\ 10.0 & 9.0 & .2513 ! 134 \mathrm{E}+03\end{array}$

$.37100 t-14 \quad .00546 E-15 \quad .22297 E-15$

$.60439=-10$

117145

$104^{17}=-00$
$102^{n} 30-07$

$5085:=-C^{7}$

$1440 t, 2-U$

icestise-do

$.50099 E-00$

.814 CUe-Ua

$1225,2 \mathrm{c}=u \mathrm{~s}$

. $24 c ? 3 x-0 n$

$.4 \gtrless 1$ CUR $-6 \dot{5}$

$2013 \mathrm{~V}=-00$

. $54 ? 590-06$

$0149^{7} \varepsilon-00$

$.24,242 \varepsilon-0 j$

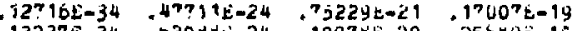

$.1323^{7} E-34 \quad .039 d d E-24 \quad 10978 E-20 \quad .25689 E-1$.

$.0412 ? E-19$

$\mathrm{N2O}^{\mathrm{202}}$

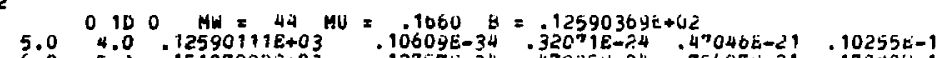

$6.0 \quad 5.0 \quad: 1510^{7992 E+03} \quad .12^{7} 57 E-34 \quad .47935 E-24 \quad .7560^{7} E-21: 170096-19$

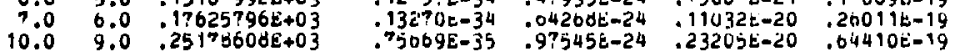

$\mathrm{N} 203$
$\mathrm{~N} 20$

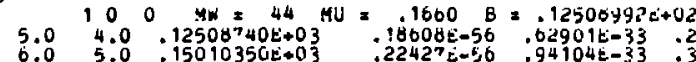

$\begin{array}{rll}0.0 & 5.0 & 150103506+03 \\ 10.0 & 0.0 & .1751196 ! \mathrm{H}+03 \\ 10.0 & 9.0 & .25015904 \mathrm{E}+03\end{array}$

$\begin{array}{rll}.18608 E-56 & .62901 \mathrm{E}-33 & .20045 E-25 \\ .22427 E-56 & .94104 \mathrm{E}-33 & .33194 \mathrm{E}-25\end{array}$

. $12420201123 j 5-2$

$.13481 \mathrm{E}-56 \quad: 19252 \mathrm{E}-32 \quad .10215 \mathrm{E}-24$

$17105 \dot{E}-21$
$.424 \mathrm{U} 2 E-21$

204

NEO

$\begin{array}{rrrr} & 02 & 0 \quad M W=44 M \\ 5.0 & 4.0 & .12508494 E+03 \\ 6.0 & 5.0 & .15106020 E+03 \\ 7.0 & 6.0 & .17623417 E+03 \\ 10.0 & 9.0 & .25174812 E+03\end{array}$

$10608=1258 d d ? 4 E+02$

$.03938 c-53$. 10421E-31 .11273E-24

$.10093 E-52.2^{7} 534 \mathrm{E}-31.1011^{3} \mathrm{E}-24$

$\begin{array}{rrr}.10500 \mathrm{E}-52 & .36 \mathrm{Y} 16 \mathrm{E}-31 & .20434 \mathrm{E}-24 \\ .59000 \mathrm{E}-53 & .56032 \mathrm{E}-31 & .55002 \mathrm{E}-24\end{array}$

$150^{71} 1 \mathrm{k}-21$

$.204480-21$

$.402504-21$

$.99004 t-21$

N205

N2O

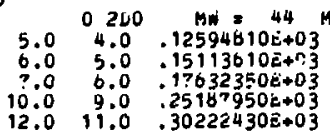

$=.1560 \quad B=.12595000 \varepsilon+02$

$.42062 \mathrm{E}-53=13900 \mathrm{E}-31 \quad .90296 \mathrm{E}-25 \quad .14029 \mathrm{E}-21$

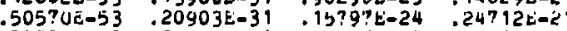

$.52594 E-53$.20023E-31.23048E-24.9614E-2

$\begin{array}{llll}.29973 E-53 & .42522 E-31 & .404 ? 4 E-24 & .93135 E-21 \\ .12691 E-53 & .117120 E-31 & .03409 E-24 & .14000 E-20\end{array}$

N206

$\mathrm{N} 206$
$\mathrm{~N} 2 \mathrm{O}$

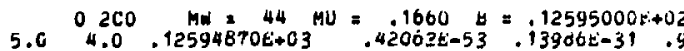

$5.0 \quad 4.0 \quad .12594870 E+C 3 \quad .42002 t-53 \quad .13906 t-31.9029 d E-25$

$6.0 \quad 5.0 \quad .15113760 E+03 \quad .50571 E-53 \quad .20903 E-31 \quad .1{ }^{7} 97 E-24$

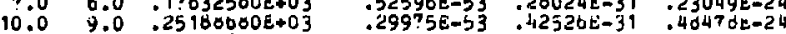

$12.0 \quad 11.0 \quad: 30225620 \mathrm{E}+03$ $.12095 \varepsilon-53 \quad .42133 k-31 \quad .63429 \mathrm{E}-24$

$140305-2$

$24712 \dot{10}-21$

. 3 ? $610 \mathrm{E}-21$ $.93143 \dot{1}-21$ SIO+

N MH $=44 M U=3.0000 \quad \mathrm{H}=.21520000 \mathrm{t}+02$

$1.0 \quad 0.0 .43080000 E+02 \quad .76483 \mathrm{E}-15.13155 \mathrm{E}-15.33743 \mathrm{E}-10.68751 \mathrm{E}-17$

$2.0 \quad 1.0 \quad .86117000 t+02 \quad .49692 t-14 \quad .96750 t-15 \quad .25003 E-15 \quad .04445 E-16$

$.12290 \mathrm{E}-13 \quad: 20832 \mathrm{E}-14 \quad: 01999 \mathrm{E}-15 \quad$.22711E-15

$29095=-0 n$

$.21042 E-U 0$

$.01360 \mathrm{E}-06$

$.243^{79} 9=-05$

$.265342-00$ $.50004 E-U 0$ $.23910 \mathrm{t}-05$

$.29003 \mathrm{i}-00$

$.51020 \mathrm{e}-0 \mathrm{~b}$ $.8192 \%+110$ $.24308 t=0$ ?

. $2 y 12^{2} r-00$ $.51105 \mathrm{k}=\mathrm{COO}$ $.02051 \mathrm{t}-00$ $.2440 ? 5-05$ $.42499 k-05$

$.2912 a z-06$ $.51106 \mathrm{~b}-00$ $.02054 \mathrm{E}-06$ $.24409 \mathrm{E}-05$ . $45120-45$

- 27910t-0s $.20^{7} 01 \mathrm{k}-04$ $.96719 t-04$ 
TABLE $1 \mathrm{~h}, 4$

Ju JL

$\angle U$

30

ILMA

100

200

SILILUR MUIUXILE
SIU

$$
\begin{array}{lll}
1.0 & 0.0 & .43423600=+42 \\
2.0 & 1.0 & .06046000 E+42 \\
5.0 & 2.0 & .130260000+03 \\
4.0 & 3.0 & .173000302+03 \\
5.0 & 4.0 & .217104000+03 \\
0.0 & 7.0 & .200517001+03 \\
7.0 & 0.0 & .303920102+03
\end{array}
$$

i $=3.0900$

$.03404=-15$. $14354 E=15$. $30030 E-10.90^{7} D^{7} E-17$

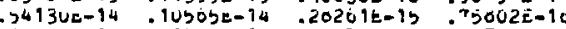

$.13349 \mathrm{E}-13 \quad .31450 \mathrm{E}=14 \quad .095^{\circ} \mathrm{OL}=15 \quad .24792 t-15$

$.20624 \mathrm{E}-13.03050 \mathrm{E}-14.9520 \mathrm{0}-14.50355=-15$

$.24110 \mathrm{E}-13$.99510t-14.34361E-14 .1U445E-14

$.22243 E-13 \quad .13431 i-1 j \quad .52353 z-14 \quad .16 y 50=-14$

$\sin 1$

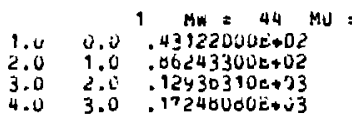

$M J=3.1180 \quad b=.27^{n} 8^{7} 500 E+02$

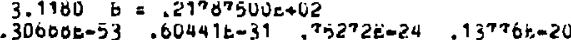

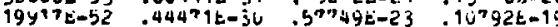

$49117 t-52 \quad 13230 t-29 \quad 10304 t-22$.35297t-19

$.76022 \mathrm{e}-52 \quad 20543 \mathrm{c}-29 \quad 39903 t-\varepsilon 2$. $40235 t-19$

sive?

$1.2 \mathrm{Mh}=44 \mathrm{MU}=3.1370 \mathrm{D}=.21707500 \mathrm{C}+02$

$1.0 \quad 6.0 \quad .42020>00 \mathrm{~L}+02 \quad .23294 t-91.39972 t-40.17760 E-31 \quad .21070 E+24$

$2.0 \quad 1.0 \quad .05040300 \mathrm{c}+02 \quad .15118 \mathrm{E}-40 \quad .249 y \mathrm{CL}-45 \quad .13030 \mathrm{E}-3 \mathrm{C} \quad .10507 \mathrm{~L}-23$

$3.0 \quad 2.0 \quad .120450^{7} 0 \mathrm{~L}+03 \quad .37203 \mathrm{E}-90 \quad .74409 E-45, .43203 \mathrm{E}-30 \quad .5390^{7} \mathrm{E}-2 \mathrm{C}$

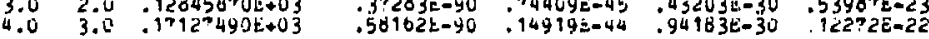

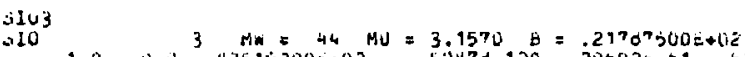

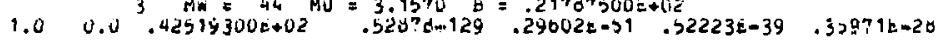

SJLJCUN NUNOSULFIDE

IJ $\quad M n=60 M U=1.7500 \quad B=.90774030 \mathrm{z}+01$

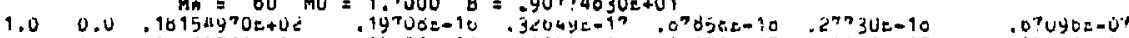

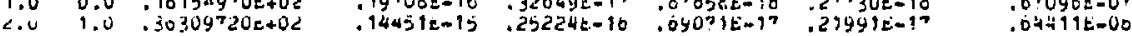

Sisi
sis

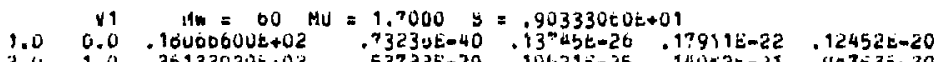

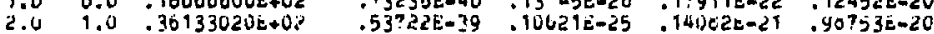

S1s2

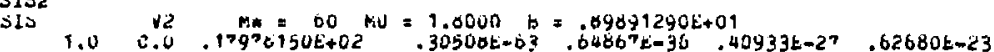

$9712^{7}$

$30490 \mathrm{em} 05$

$292^{7} 05-14$

$10504 \mathrm{c}-03$

$20015=-03$

51 to $1 \mathrm{t}-0$ 's

$.911025-03$
$.14 t 35 t-02$

$\$ 512^{7}$

$30245 \mathrm{k}=0$

$29035 t=04$

$.10449 E-03$

$.250005-03$

.24Y77t-

$.124 L U E-13$
$.255^{2}$ OE-U 3

45127

$.29^{\prime} \div 5 E-05$

2750

$.06 ? 21 \varepsilon-07$

$.03475=06$

b7

$.73045 \mathrm{E}-0 ?$ 
jable d D. 1

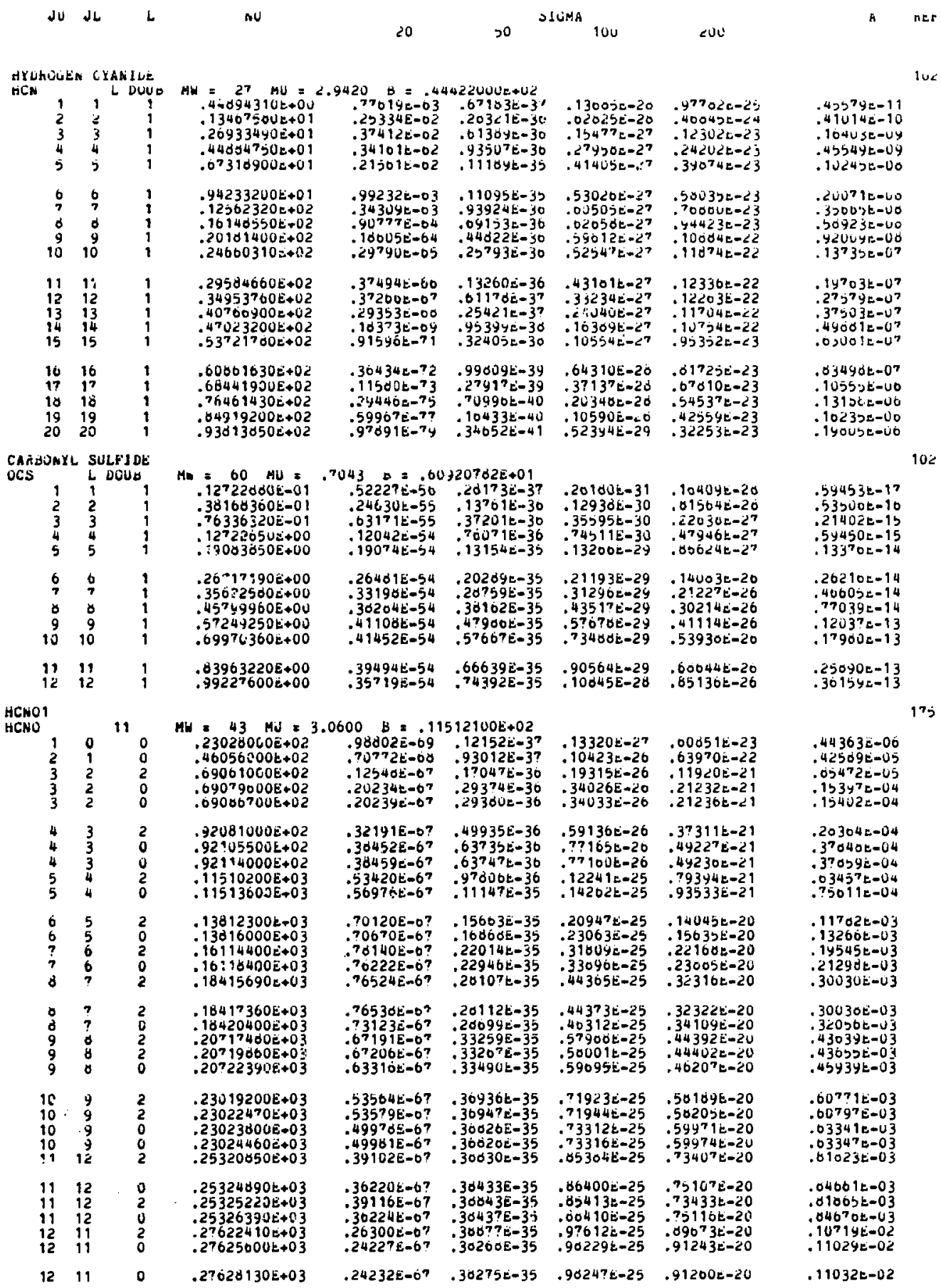


JABLE I D. 2

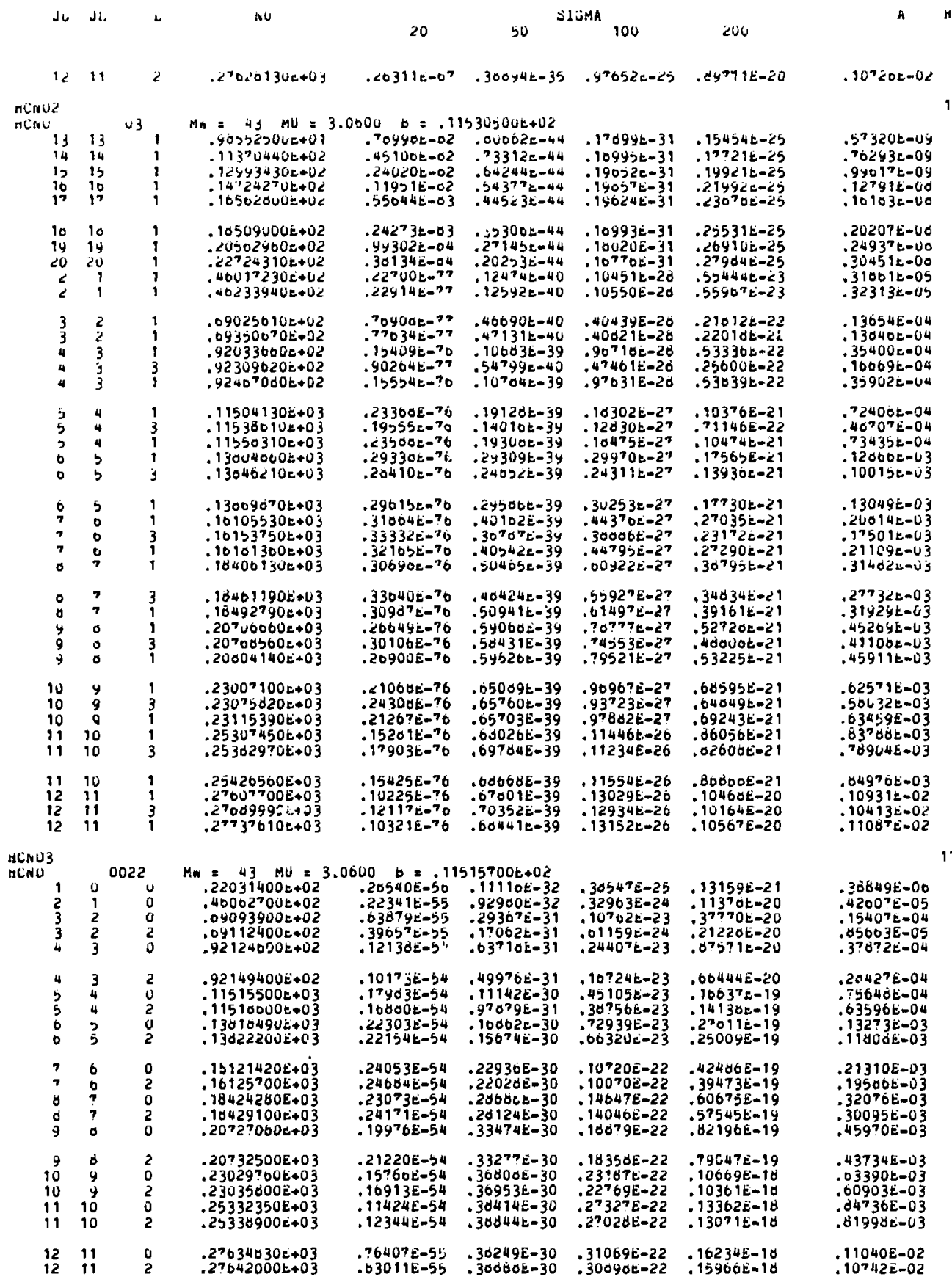


TADLE I D , 3

Nu

20

50

SILPAA 100

200

net

$\mathrm{HCNO} 4$

HCNO

$\begin{array}{rrr} & & 2200 \\ 1 & 0 & 0 \\ 2 & 1 & 0 \\ 3 & 2 & 2 \\ 3 & 2 & 0 \\ 4 & 3 & 2 \\ 4 & 3 & 0 \\ 5 & 4 & 2 \\ 5 & 4 & 0 \\ 6 & 5 & 2 \\ 0 & 5 & 0 \\ 7 & 0 & 2 \\ 7 & 6 & 0 \\ 0 & ? & 2 \\ 8 & 7 & 0 \\ 9 & 0 & 0 \\ 0 & 9 & 0 \\ 1 & 10 & 0 \\ 2 & 11 & 0\end{array}$

$\mathrm{MW}=43 \mathrm{MU}=3.0000 \quad \forall=.11490200 \mathrm{E}+02$ $.22990000 \mathrm{e}+02 \quad .023524 \mathrm{E}-64 \quad .00205 \mathrm{t}-44$ $459920045+125$ $.00460700 \mathrm{c}+02$ $.009806400 \mathrm{E}+02$ $.10052 \mathrm{E}-\mathrm{d} 3 \quad .5220^{7} \mathrm{E}-4 \mathrm{~T}$ $97004 t-31 \quad 22001 E-24$
$77909 t-30 \quad 17470 E-23$ $.29095 E-03.920302-43.144 j 9 t-2 y$.32509E-23 $.48198 z-03 \quad .105102-42.25404 t-29 . b^{7} 9935-23$ $.06968400 E+0$ $707130-d 3 \quad 05102-42$ ? $10194 \mathrm{E}-24$

$.91943500 \mathrm{e}+02$

$.91983500 \mathrm{E}+02$
$.1149600 \mathrm{E}+93$ 7o?13E-d3 .20070t-42

$.99614 t=03$

$.11497700 \mathrm{E}+03$

$.35020 E-42$
$.54990 t-42$

$.57750 \mathrm{e}-29$

$13440 \mathrm{t}-22$ $.13797110 \mathrm{~b}+0$

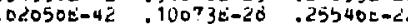

- 10073E-20 .25540L-L'

$\begin{array}{ll}15003 E-20 & -36375 E-22 \\ 17201 E-20 & .42705 E-22\end{array}$

$.94030=-42$

$.10095300 E+03$

-10040L-02

$.12383 \mathrm{E}-41.23 d 17 \mathrm{~L}-20.0 \cup\langle 730-22$

18101E-d2 12903E-41 25371E-20 .0524<E-2

$10260 \mathrm{k}-02$ 15015E-41.33225E-20 .0039bki-?

$18394600 \mathrm{E}+03$

$10200 E-02$
$17451 E-d 2$

$10142 E-410340095-20$. $031735-2$

$.15120 \mathrm{E}-62$

$.168416-41.44693 t-20$

$.12 \mathrm{~b} 23 \mathrm{c}-21$

$.11943 \mathrm{E}-02 \quad .20^{2} 24 \mathrm{E}-41 \quad .5469^{7} \mathrm{E}-20 \quad .1030 \mathrm{BE}-\angle 1$

$.22992900 \mathrm{~s}+03$

$.25291400 E+03$

$.06620 E-03 \quad .29636 t-41.64710 E-20$

$.57900 \mathrm{~L}-03 \quad .21551 E-41 \quad .735 d 4 E-20 \quad .24935 E-21$

$.44902 c-00$ $.42413 \mathrm{t}-\mathrm{U} 2$ $.03174 \mathrm{k}-0$ ? i $230 \mathrm{u}-\mathrm{u} 4$ 10,0

3'OYOL-U4 $.03<34 \mathrm{E}-04$ $73300=-04$ $117415-05$ $.132110-03$

$144700-09$ $.21291 \mathrm{E}-\mathrm{U} 3$ $.29427 \dot{-1}-43$ - 31y<0 -03 $.45^{7} 52=03$

$.03 \quad 7 E-03$ $.04: \angle 0 E=03$ . 1UyOUe-UL 
IAELE 1 ᄂ 1
JU AU UL OL
106
20
slutin
100
zUu
her SLLTUR mubudL.t
SU

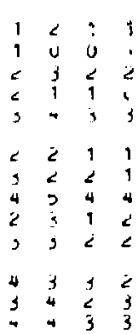

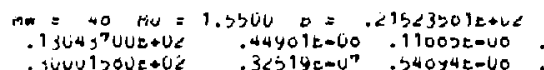
IUUu $15002+02$ $30<U 10<U E+U \angle$ $.0<y j$ icuUe+UC

$.0005444 L=+02$ $.23144=-40$ $.14 y+9=-60$

$34334 c-69$
$13971 k-40$

$. C^{7} y E-U Q$. ${ }^{7} O Z^{7} O E-U Y$

.

cos

$.10314 E-00 \quad .<g^{T} U A E-U^{T}$

$.33^{n} 34 E-40$

$1<7$ by $=00$

$.32243 \varepsilon-04$

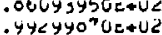

ívulyute+03

$.10 \times 25210 x+63$

- YUST Je-00

$.6900^{7} E=05 \quad 3543^{7} t=3 d$

$\begin{array}{ll}.10010 E-U^{7} & .40021 E-C O \\ .15200 E-00 & .402^{2} 2 E-114\end{array}$

$.24904 t-c 0$

.

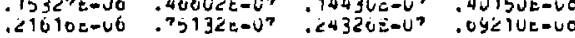

$.40062 k-4 b .11903 \mathrm{e}-00.35220 \mathrm{k}-U^{7} .4530<2-40$

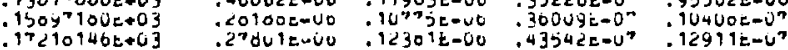

Sul

$\begin{array}{llll}1 & 2 & 1 & 1 \\ 1 & 0 & 0 & 1 \\ 2 & 1 & 2 & 2 \\ 2 & 1 & 1 & 0 \\ 3 & 4 & 9 & 3\end{array}$

$M_{*}=40 \mathrm{MU}=1.5500 E \geqslant .21351010 \mathrm{E}+U 2$

$12^{7} 529 v 0 \mathrm{~d}+\mathrm{J}$

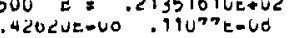

$<y+4930 v e+U$

$32154 \mathrm{E}-U^{7} \quad .74 L^{7} \mathrm{~J}=-40$

$3255<-00$

$154014 u k E+02$

. 20 y $05 u e+02$

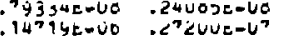

$130110-00.00234 \mathrm{e}=10$

. $0404447 v E+U 2$

.04544E-vo

$\begin{array}{ll}754 j E-U Y & .20730 E-04 \\ 1340 E-U 0 & .102^{7} 0 E-U 0\end{array}$

$102^{7} 0 E-U O$
$.31140 E-U Y$

$2 y 004 z-u^{3}$ 240C4E-U6 $.1 y^{7} 9 j e-u b$

.26y06c-05 $.50130=-40$

$.53535 E-L \overline{5}$

- 11040t-ive

$.11040 \mathrm{E}-\mathrm{LS}$

- $11013 \mathrm{E}-0 \mathrm{a}$

$.229452-14$

32201t-04 $.431578-04$
$.594700=04$

$27743 z-07$ $23930 \mathrm{~b}-\mathrm{vo}$ 10605 e-vo . 20000 e-U5 $.53050 t-V O$ 
TABLE II

TRANSITIONS OF SYMMETRIC ROTORS

\section{Table II - Contents}
1. $\mathrm{CH}_{3} \mathrm{NO}_{2}$
2. $\mathrm{CH}_{3} \mathrm{OH}$
3. $\mathrm{CH}_{3} \mathrm{CN}$
4. $\mathrm{CH}_{3} \mathrm{NC}$
5. $\mathrm{CH}_{3} \mathrm{NCO}$
6. $\mathrm{CH}_{3} \mathrm{NCS}$
7. $\mathrm{CH}_{3} \mathrm{C}_{2} \mathrm{H}$
8. $\mathrm{NH}_{3}$

\author{
nitromethane \\ methanol \\ methyl cyanide \\ methyl isocyanide \\ methyl i socyanate \\ methyl isothiocyanate \\ methylacetylene \\ armonia
}

Note to Table II

a. Hindered internal motions affect this molecule severely, so the cross sections are not very accurate. 
$\pi 0-3 \zeta \varepsilon L L 2$. $\pitchfork 0-36 \exists \varepsilon ! 8$. TO-gटाLit.

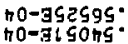
$n 0-2<+600^{\circ}$ $\pi 0-3<206 \varepsilon^{\circ}$

$\rightarrow 0-3 \angle 026 E^{\circ}$

$\pi 0-39 \pi 9 \pi \varepsilon^{\circ}$ $70-36622 \varepsilon^{\circ}$ in-3056E2 TO- 5 ? $8 \varepsilon$ L ד0-970691.

$\pi 0-3589 \pi 1^{\circ}$ SO- $3 \varepsilon L \varepsilon 6 Z^{\circ}$ $50-76$ Sth! $90-3 L+\pi t 8$ 90-320+ह8.

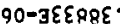
$90-\Xi ! \varepsilon \subseteq 9 \varepsilon$ LO-3G18ZZ $10-301095$
$10-305258$

L-JOSSटE.

LO-3t501"

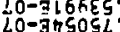
$90-301+12$. 90-उहटट।

90-356991 $90-3+6512$ $90-30510 ?$ LO-729त9. LO-JLZEHG

98

$\rightarrow 0-\exists \varepsilon \pitchfork \varepsilon z h$ की $0-7+2210$ $\rightarrow O-\exists$ RIOEE.

त०-वह। TO-3! I SRE : $\pi 0-7100 \pi 7$ $70-\mathrm{g} \pi \angle 6 E \mathrm{n}$ hO-3ROZOH

त)-JSEहดl : $0-32 E 566^{\circ}$ tก-эSट65! $50-3 L 1966$ 与०- $₹$ เ $566^{\circ}$

HO-7506Lt. $70-3126 L$ L no-3ilssi. SO-70?10E. 与०-उดदा LE.

40-วุด $56 \pi$ S०-3£ ट56t $50-78519 \varepsilon$ $00-7200: 5$

$90-7 \angle L O L E$ 90-360nLL

$60-319268$

60-390658.

$11-300 \angle 5 \varepsilon$.

Q

$50-7991179$ รว-วRSQย己 Q5 1

$f 94$
$21-35628$

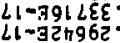

$\angle 1-3+09 \varepsilon 6^{\circ}$. $91-3+99 L$.

Li:-graSty Ll $-70<9 \pi 6^{\circ}$ $9 l-3+10 \pi 1$. $L L-3 L+B+L$

$9 l-39 \pi 6 \pitchfork \varepsilon$ $91-390562$. $9 i-3 \varepsilon \varepsilon z \varepsilon 5:$ $1-051<2$

ᄂ - $998019 \circ$ Lᄂ - $3 \varepsilon \varepsilon 909^{\circ}$ $L$ L -319662 L-3Tत9LR.

91-39tzदz

9l-3iLOSE.

$91-3 h 6<\pi l$.

Q

$\angle L-39056 \varepsilon$. LI$81-39 R h l R$

$81=301 \varepsilon i 5$

$9 l-39<9 \varepsilon L^{\circ}$

Li-3z80ls.

¿ᄂ.जEโOLE.

LL-386LLC.

81-3LPROS

८ا-з६ट०Lट.

$81-3682 \pi \varepsilon$.

$81-326 L E 2$

$61-3 \pi n z 22$

$61-05 \angle 692$

LL-3098LI.

$61-3 L 02 I I$.
$61-35 E+19$.

$61-35 E \pi 19^{\circ}$

$61-3699 \mathrm{~g}_{\mathrm{C}}$

$61-30050$

.

$9 l-3 t 269 n$.

91-396296. 9l-3ॄgट6L. Gl-8JE5L.

SI-36061!. Sl-9t6LG2. 91-39h0LS: 91-329S2z. SI-3SEESt. SI-3h9002. 9l-319819. 9l-30t19t.

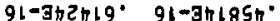

9l-3L2658. $51-36098^{\circ}$ 9l-328559. 5l-32E98l. 9l-3hLOzL:

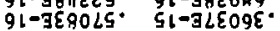

$61-322 \pi 05:$

$81-3 E$ OnSL

9l-3TटLटE.

$9 l-35 \varepsilon 5 \pi 2$

$\angle 1=3 \pi \angle \varepsilon q 6^{\circ}$

LL $-368968^{\circ}$

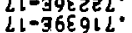

gl- $5 \xi 5 \pi c^{\circ}$

gi-jELati.

gl-3irgs!:

$\angle 1-36 z \varepsilon 9 C^{\circ}$

$\angle L-3 \varepsilon \pi 96 \pi^{\circ}$

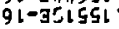

$01-3<0101$.

Lᄂ-362!Lट"

$9 l-3919 \varepsilon 2$

$61-3 \angle 8081$.

$61-3 \angle 89 E 9^{\circ}$

$81-356 \mathrm{~L}<\mathrm{C}^{\circ}$

i=st8c0c

$1=36099$.

$81-319585$.

al-3ios

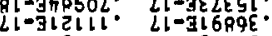

$1-3 g \varepsilon 5 \pi \pi^{\circ}$

$91-\exists \xi 99 \pi \varepsilon^{\circ}$

$81-3 \pi E 06 E^{\circ}$

$\iota 1-3\left\llcorner 92 \measuredangle \iota^{\circ}\right.$

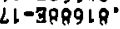

$81-36921 l^{\circ} \cdot 91-310955$.

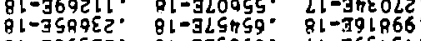

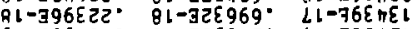

$61-70619 \pi^{\circ} 81-30592^{\circ}$

$6 !-3 L L 292^{\circ} 81-352 E 2 l^{\circ}$ BL-3EgET5.

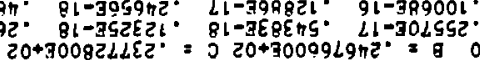

$91-300021:$

$81-9 i L E 6 !$

$81-392925$.
$16-71000 \pi^{\circ}$

$L L-3 L 5+E^{\circ} 9 L-3 L O Q \pi L$

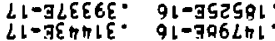

$9 L-312 n \varepsilon 8$.

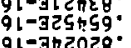

$91-3 \pitchfork 202 \beta^{\circ}$

$\xi 1-3 \pi \varepsilon 96 \varepsilon^{\circ}$

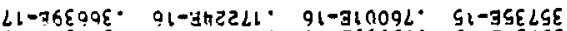

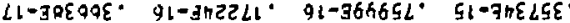

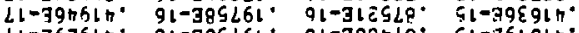

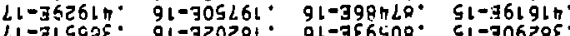
(1-71590द. 91-32028:

Gl-706?:QE.

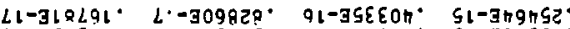

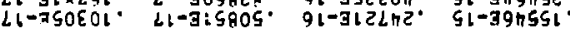

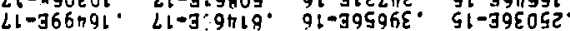

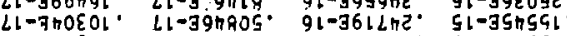

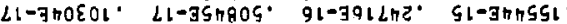

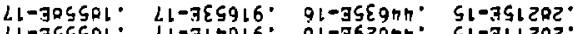

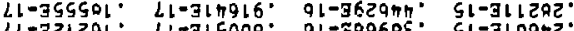

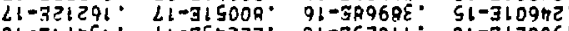

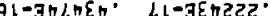

$01-36 \sum_{12}$

$\left.\angle 1-3 \angle 9)^{\circ}\right)^{\circ}$

$01-302 \pi 11$

Q1-3हि $6268^{\circ}$

$91-359699^{\circ}$

Pl-? 0.0695

ol-70005\%:

$61-345622$
$61-30+6 ? 2$

$\angle 1-329152$

$21-709162$

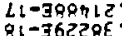

$91-39+25 l^{\circ}$

$51-362611$.

$91-3 \angle \pi 25 l: 51-38561 !$

$91-3 \operatorname{le} 2 \mathrm{~L}$

$21-7856 \pi 5^{\circ} 91-310691$.

$91-3252 \circ \varepsilon=21-3+56 \cap 2.91-3 \angle 6891$.

$02-3040 \varepsilon \varepsilon^{\circ} 61-79 L 5 \geq 1^{\circ} 61-3 h 5 \varepsilon 9 \varepsilon^{\circ} 61-35909 \pi^{\circ}$

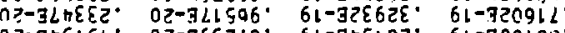

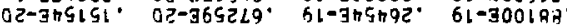
$12 z-361<10^{\circ} 0 z-360510^{\circ} 51-316291.61-310690^{\circ}$

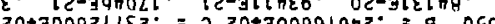

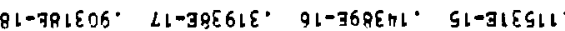

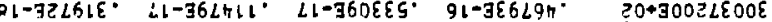

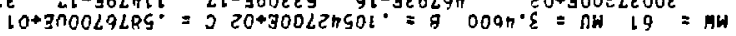

$00 ?$

001

พพอIS

05

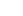

$50+3929+1561^{\circ}$

है।atzl LSE6

है0+309905ह 61

$\varepsilon 0+3 z \xi L L \pi \varepsilon 6 L$

$0+36 E \pi S H E 6 L^{\circ}$

$0+3 L E S L T E 6 L$

\& O+30\&89E9HL.

O+39HEEL STL.

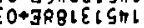

$\varepsilon 0+3 L \varepsilon 9215 \pi 1$

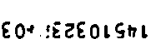

E

$20+30$ ER $85 L 6$

2043015 SS $196^{\circ}$

$20+3095 \pi h \angle 96^{\circ}$

$20+302 h 1+1.96$

$20+3062+1656$

20* 70 ES InEE?

ट0* उ0दहE।दLL.

Z0+3000LLदL.

$=$

กง $\varepsilon 0+3 \hbar 1190591^{\circ}$ E0+361050591:

E0+30LL2! gSt. $80+3288199 \mathrm{gl}:$ EO+GQLOLECE: EO+39696RhE :

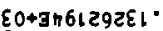

E0+3L669ST2!

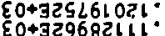

EO+JSBELOLOL.

$20+301769 L^{\circ} 6^{\circ}$ $20+309 \varepsilon E \pi 85 \pi^{\circ}$

$20+3008890 \pi h^{\circ}$

$20+3069 z 5 \pitchfork 8 E$

$20+300669692^{\circ}$

20+30E 09IERE.

20+3081BL85?"

$20+30 E+1+552:$

$20+30884215 z^{\circ}$

$20+30 \pi 18.052:$

$20+3000656 \pi 2^{\circ}$

$20+30 \theta \varepsilon+\varepsilon 6 \pi 2^{\circ}$

$20+30 \angle \hbar E E G \leftrightarrow Z^{\circ}$

$20+300<826 \pi 2^{\circ}$

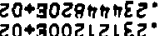

$20+300 \& 2966 \mathrm{~L}$

$20+300$ g50OL.

$\cdot I=n K Z \varepsilon=$

$\varepsilon 0+\pi \varepsilon \varepsilon \sqcap L H E 6 L$

ZD+3050LLERT
IDOUQLLAT

0
0

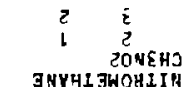

GNYHI JWOHIIA

y 
ABLE : : .

\begin{tabular}{|c|c|c|c|c|c|c|c|c|}
\hline \multirow{2}{*}{$\dot{U U}$} & \multirow[t]{2}{*}{$J L$} & \multirow[t]{2}{*}{ A } & \multirow[t]{2}{*}{$\mathrm{NL}$} & \multicolumn{4}{|c|}{$\therefore \triangle M A$} & \multirow[t]{2}{*}{$\dot{A}$} \\
\hline & & & & +4 & $\because$ & 1.6 & $\therefore$ & \\
\hline $\begin{array}{l}3 \\
4 \\
5 \\
0 \\
?\end{array}$ & $\begin{array}{l}3 \\
4 \\
5 \\
0 \\
7\end{array}$ & $\begin{array}{l}1 \\
1 \\
\vdots \\
i\end{array}$ & 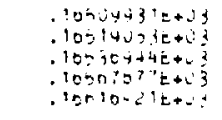 & 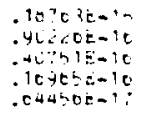 & 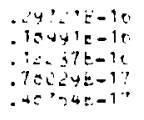 & 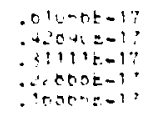 & 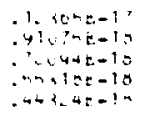 & 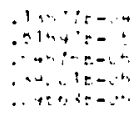 \\
\hline $\begin{array}{l}3 \\
7 \\
9 \\
3 \\
4\end{array}$ & $\begin{array}{l}2 \\
0 \\
0 \\
0 \\
0\end{array}$ & $\begin{array}{l}3 \\
\vdots \\
i \\
2 \\
2 \\
2 \\
2\end{array}$ & 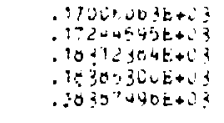 & $\begin{array}{l}.50511=-13 \\
.12101 E+15 \\
.33100 E-10 \\
.57322 E=15 \\
.334+3 E-10\end{array}$ & $\begin{array}{l}.34004 E-10 \\
.133=-10 \\
.04501=-10 \\
.1 ? 200 t=19 \\
.04440 t-10\end{array}$ & $\begin{array}{l}.804005-10 \\
.474005-10 \\
.4500 u t-10 \\
.43401 \leq-15 \\
.440+15-10\end{array}$ & 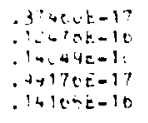 & 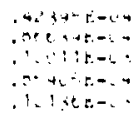 \\
\hline$\stackrel{6}{10}$ & $\begin{array}{l}7 \\
y\end{array}$ & $\frac{3}{c}$ & $\begin{array}{l}1917 j 3 u b t+u a \\
-190403<<t+L 3\end{array}$ & $\begin{array}{l}.0+925=19 \\
.140+j=-10\end{array}$ & $\begin{array}{l}3: 17 n=-17 \\
-1<2005=-10\end{array}$ & $\begin{array}{l}* x+40-18 \\
-7 \leq 1 \leq-10\end{array}$ & $\because\left\{\begin{array}{l}1,-5-10 \\
1: i \leq-10\end{array}\right.$ & 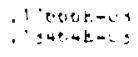 \\
\hline $\begin{array}{r}\text { METHYL } \\
\text { Cri } 3 \text { CN } \\
2 \\
2 \\
5 \\
5 \\
5\end{array}$ & $\begin{array}{l}1 \\
1 \\
4 \\
4 \\
4\end{array}$ & $\begin{array}{l}1 \\
0 \\
4 \\
3 \\
2\end{array}$ & $\begin{aligned} & M W=49 M U=3 . \\
& . 30794000 E+02 \\
& .39795300 E+02 \\
& .91929200 E+02 \\
& .91971350 E+0 E \\
& .91900000 E+02\end{aligned}$ & $\begin{array}{l}00 \quad 8=.914 \\
.430942-15 \\
.44950 E-12 \\
.41004 E-12 \\
.11992 E-12 \\
.13200 E-13\end{array}$ & $\begin{array}{l}00306 t+i 1 \\
.34450 t-10 \\
.45503 t-10 \\
.20375 t-14 \\
.20003 t=14 \\
.05000 t-15\end{array}$ & 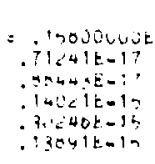 & 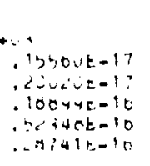 & 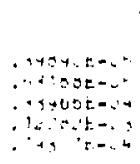 \\
\hline $\begin{array}{l}5 \\
5 \\
0 \\
6 \\
0\end{array}$ & $\begin{array}{l}4 \\
4 \\
5 \\
5 \\
5\end{array}$ & $\begin{array}{l}1 \\
0 \\
5 \\
4 \\
3\end{array}$ & $\begin{array}{l}.91905350 E+02 \\
.91907070 E+02 \\
.11033079 E+03 \\
.11034900 E+03 \\
.11030452 E+03\end{array}$ & $\begin{array}{l}.51080 E=14 \\
.37673 E=14 \\
.11491 E=10 \\
.84015 E=12 \\
.10031 E=12\end{array}$ & $\begin{array}{l}.03002 t-i 5 \\
.27014 t-12 \\
.97192 t-14 \\
.40077 t-14 \\
.40507 t-14\end{array}$ & $\begin{array}{l}1<015 t-14 \\
.124<9 t=12 \\
.37075 t=15 \\
.3545 E E=12 \\
.5000 t-12\end{array}$ & 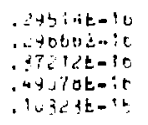 & 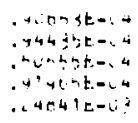 \\
\hline $\begin{array}{l}0 \\
6 \\
6 \\
7 \\
7\end{array}$ & $\begin{array}{l}5 \\
5 \\
5 \\
0 \\
0\end{array}$ & $\begin{array}{l}2 \\
1 \\
0 \\
0 \\
5\end{array}$ & $\begin{array}{l}.11037501 E+03 \\
.11030139 E+03 \\
.11038347 E+03 \\
.12009057 E+03 \\
.12871769 E+03\end{array}$ & $\begin{array}{l}.10520 E-13 \\
.09390 E-14 \\
.44947 E-16 \\
.11815 E-00 \\
.21473 E-16\end{array}$ & $\begin{array}{l}.14043 E-14 \\
.16043 E-16 \\
.09554 E-19 \\
.11344 E-12 \\
.41002 E-13\end{array}$ & 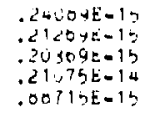 & 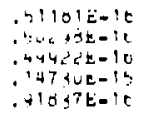 & 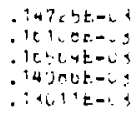 \\
\hline $\begin{array}{l}7 \\
7 \\
7 \\
7 \\
7\end{array}$ & $\begin{array}{l}0 \\
0 \\
0 \\
6 \\
0\end{array}$ & $\begin{array}{l}4 \\
3 \\
2 \\
1 \\
0\end{array}$ & $\begin{array}{l}.12873991 E+03 \\
.12875703 E+03 \\
12876952 E+03 \\
.12877702 E+03 \\
.12077943 E+03\end{array}$ & $\begin{array}{l}.11013 E-11 \\
.23040 E-12 \\
.22306 E-13 \\
.61515 E-14 \\
.50226 E-14\end{array}$ & $\begin{array}{l}.83145 E-14 \\
.7417 y E-14 \\
.20432 E-14 \\
.142 U U E=14 \\
.12507 E=14\end{array}$ & $\begin{array}{l}.04104 z-15 \\
.94370 t-15 \\
.37152 t=15 \\
.31989 E=15 \\
.30400 t=15\end{array}$ & $\begin{array}{l}.41549 t-10 \\
.17294 E-15 \\
.01411 E-10 \\
.70025 E-10 \\
.7000 U t-10\end{array}$ & 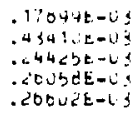 \\
\hline $\begin{array}{l}0 \\
8 \\
6 \\
0 \\
0\end{array}$ & $\begin{array}{l}7 \\
7 \\
7 \\
7 \\
7\end{array}$ & $\begin{array}{l}7 \\
0 \\
5 \\
4 \\
3\end{array}$ & $\begin{array}{l}.14703509 E+03 \\
.14707304 E+03 \\
.14710407 E+03 \\
.14712941 E+03 \\
.14714928 E+03\end{array}$ & $\begin{array}{l}.50761 E=07 \\
.20420 E-00 \\
.20011 E=10 \\
.13004 E=11 \\
.26094 E-12\end{array}$ & $\begin{array}{l}.41740 E-12 \\
.24350 \mathrm{E}-12 \\
.35251 \mathrm{E}-13 \\
.12000 \mathrm{E}-13 \\
.10121 \mathrm{E}-13\end{array}$ & $\begin{array}{r}.32 ? 44 t=14 \\
.40337 t-14 \\
.15351 E=14 \\
.99305 E-15 \\
.13019 E-14\end{array}$ & $\begin{array}{l}.14405 E-15 \\
.34990 E-15 \\
.10403 E=15 \\
.14690 E-15 \\
.20240 E=15\end{array}$ & $\begin{array}{l}.43503 E-C 4 \\
.34+04 t-0 j \\
.24300 E-U 3 \\
.30404 E-03 \\
.60767 E-C 3\end{array}$ \\
\hline $\begin{array}{l}8 \\
0 \\
0 \\
9 \\
9\end{array}$ & $\begin{array}{l}7 \\
7 \\
7 \\
0 \\
6\end{array}$ & $\begin{array}{l}2 \\
1 \\
0 \\
6 \\
7\end{array}$ & $\begin{array}{l}.14710346 E+03 \\
.14717195 E+03 \\
.14717472 E+03 \\
.10536543 E+03 \\
.10541293 E+03\end{array}$ & $\begin{array}{l}.23675 E-13 \\
.85665 E-14 \\
.01050 E-14 \\
.10305 E=04 \\
.91553 E=07\end{array}$ & $\begin{array}{l}.27032 \mathrm{E}-14 \\
.10493 \mathrm{E}-14 \\
.10267 \mathrm{E}-14 \\
.30070 \mathrm{E}-11 \\
.85407 \mathrm{E}-12\end{array}$ & $\begin{array}{l}.52750 E=15 \\
.44710 E=15 \\
.42291 E=15 \\
.11251 E=13 \\
.72590 E=14\end{array}$ & $\begin{array}{l}.11975 E-15 \\
.11297 E-15 \\
.11075 E-15 \\
.31106 E-15 \\
.3437 \text { \&E=15 }\end{array}$ & $\begin{array}{l}.37531 E-03 \\
.39414 t-03 \\
.40042 \mathrm{t}-03 \\
.11999 \mathrm{t}-03 \\
.02607 \mathrm{E}-03\end{array}$ \\
\hline $\begin{array}{l}9 \\
9 \\
9 \\
9 \\
9\end{array}$ & $\begin{array}{l}0 \\
0 \\
0 \\
0 \\
0 \\
0\end{array}$ & $\begin{array}{l}0 \\
5 \\
4 \\
3 \\
2\end{array}$ & $\begin{array}{l}.16545434 E+03 \\
.16546939 \mathrm{E}+03 \\
.16551793 \mathrm{E}+03 \\
.16554031 \mathrm{0}+03 \\
.16555618 \mathrm{E}+03\end{array}$ & $\begin{array}{l}.24 \mathrm{CJ} J E-L O \\
.30410 \mathrm{E}-10 \\
.14194 \mathrm{E}-11 \\
.25427 \mathrm{E}-12 \\
.23165 \mathrm{E}-13\end{array}$ & $\begin{array}{l}.37=54 E-12 \\
.40574 E-13 \\
.15594 E-13 \\
.12714 \mathrm{E}-13 \\
.33291 \mathrm{E}-14\end{array}$ & $\begin{array}{l}.00710 \mathrm{E}-14 \\
.22903 \mathrm{E}-14 \\
.13983 \mathrm{E}-14 \\
.10796 \mathrm{E}-14 \\
.70337 \mathrm{E}-15\end{array}$ & $\begin{array}{l}.00011 \mathrm{E}-15 \\
.25557 \mathrm{E}-15 \\
.21510 \mathrm{E}-15 \\
.37130 \mathrm{E}-15 \\
.16014 \mathrm{E}-15\end{array}$ & $\begin{array}{l}.43624 E-63 \\
.39017 E-U 3 \\
.46007 E-0 \\
.10197 E-U 2 \\
.54539 E-43\end{array}$ \\
\hline $\begin{array}{r}9 \\
9 \\
10 \\
10 \\
10\end{array}$ & $\begin{array}{l}8 \\
6 \\
9 \\
9 \\
9\end{array}$ & $\begin{array}{l}1 \\
0 \\
0 \\
7 \\
6\end{array}$ & $\begin{array}{l}16550571 E+03 \\
.16550095 E+03 \\
118373628 \mathrm{E}+03 \\
.10370931 \mathrm{0}+03 \\
.18383534 \mathrm{E}+03\end{array}$ & $\begin{array}{l}.82457 E-14 \\
.58419 E-14 \\
.15591 E-04 \\
.10425 E-06 \\
.2 \zeta 224 E-08\end{array}$ & $\begin{array}{l}.22535 E-14 \\
.19781 E-14 \\
.76255 E-11 \\
.12064 E-11 \\
.49730 E-12\end{array}$ & $\begin{array}{l}.58990 E-15 \\
.55613 E-15 \\
.24234 E-13 \\
.11760 E-13 \\
.11677 E=13\end{array}$ & $\begin{array}{l}.15510 \mathrm{E}-15 \\
.15153 \mathrm{E}-15 \\
.70200 \mathrm{E}-15 \\
.50247 \mathrm{E}-15 \\
.91943 \mathrm{E}-15\end{array}$ & $\begin{array}{l}.50074 E-03 \\
.57365 E-03 \\
.2630<E=03 \\
.40243 E-03 \\
.10100 \mathrm{E}-02\end{array}$ \\
\hline $\begin{array}{l}10 \\
10 \\
10 \\
10 \\
10\end{array}$ & $\begin{array}{l}9 \\
9 \\
9 \\
9 \\
9\end{array}$ & $\begin{array}{l}5 \\
4 \\
3 \\
2 \\
1\end{array}$ & $\begin{array}{l}.18387421 k+03 \\
.18390605 E+03 \\
.18393079 E+03 \\
.1839484 E+03 \\
.18395900 E+03\end{array}$ & $\begin{array}{l}.29100 E-10 \\
.13106 E-11 \\
.23322 E-12 \\
.20630 E-13 \\
.72907 E-14\end{array}$ & $\begin{array}{l}.60579 E-13 \\
.18706 E-13 \\
.14964 E-13 \\
.30050 E-14 \\
.25909 E-14\end{array}$ & $\begin{array}{l}.31201 E-14 \\
.16381 E-14 \\
.24964 E-14 \\
.69199 E-15 \\
.74254 E-15\end{array}$ & $\begin{array}{l}.30388 E-15 \\
.29563 E-15 \\
.49049 E-15 \\
.22021 \varepsilon-15 \\
.20404 E-15\end{array}$ & $\begin{array}{l}.59263 E-03 \\
.06409 E-03 \\
.14394 E-02 \\
.75940 E-03 \\
.78335 E-03\end{array}$ \\
\hline $\begin{array}{l}10 \\
11 \\
11 \\
11 \\
11\end{array}$ & $\begin{array}{r}9 \\
10 \\
10 \\
10 \\
90\end{array}$ & $\begin{array}{r}0 \\
10 \\
9 \\
8 \\
7\end{array}$ & $\begin{array}{l}.18390262 E+03 \\
.20190702 E+03 \\
.20204076 E+03 \\
.20210600 E+03 \\
.20216493 E+03\end{array}$ & $\begin{array}{l}.51530 E-14 \\
.23539 E+U 1 \\
.10147 E=01 \\
.10707 E=04 \\
.99610 E-07\end{array}$ & $\begin{array}{l}.22747 E-14 \\
.60842 E-09 \\
.17393 E-09 \\
.10936 E-10 \\
.16219 E-11\end{array}$ & $\begin{array}{l}.69837 \mathrm{E}-15 \\
.18432 \mathrm{E}-12 \\
.18090 \mathrm{E}-12 \\
.38 j 00 \mathrm{E}-13 \\
.16502 \mathrm{E}-13\end{array}$ & $\begin{array}{l}.19000 E-15 \\
.15499 E-14 \\
.29900 E-14 \\
.11640 E-14 \\
.06103 E-15\end{array}$ & $\begin{array}{l}.74131 \mathrm{E}-03 \\
.10252 \mathrm{E}-03 \\
.09609 \mathrm{E}-03 \\
.49645 \mathrm{E}-03 \\
.02703 \mathrm{E}-03\end{array}$ \\
\hline $\begin{array}{l}11 \\
11 \\
11 \\
11 \\
11\end{array}$ & $\begin{array}{l}10 \\
10 \\
10 \\
10 \\
10\end{array}$ & $\begin{array}{l}6 \\
5 \\
4 \\
3 \\
2\end{array}$ & $\begin{array}{l}.20221567 E+03 \\
.20225787 E+03 \\
.20229370 E+03 \\
.20232154 E+03 \\
.20234010 E+03\end{array}$ & $\begin{array}{l}.22673 \mathrm{E}-08 \\
.25209 \mathrm{E}-10 \\
.11088 \mathrm{E}-11 \\
.19427 \mathrm{E}-12 \\
.17021 \mathrm{E}-13\end{array}$ & $\begin{array}{l}.59630 \mathrm{E}=12 \\
.70232 \mathrm{E}-13 \\
.21247 \mathrm{E}=13 \\
.16681 \mathrm{E}=13 \\
.42665 \mathrm{E}-14\end{array}$ & $\begin{array}{l}.15479 E-13 \\
.39863 \mathrm{E}-14 \\
.22934 \mathrm{E}-14 \\
.29645 \mathrm{E}-14 \\
.10851 \mathrm{E}-14\end{array}$ & $\begin{array}{l}.12795 E-14 \\
.40803 E-15 \\
.30721 E-15 \\
.04352 E-15 \\
.28121 E-15\end{array}$ & $\begin{array}{l}.14630 \mathrm{E}-02 \\
.03000 \mathrm{E}-03 \\
.91705 \mathrm{E}-03 \\
.14572 \mathrm{E}-02 \\
.10220 \mathrm{E}-02\end{array}$ \\
\hline
\end{tabular}




\begin{tabular}{|c|c|c|c|c|c|c|c|c|c|c|}
\hline is & $\because:$ & in. & & s. & $\Omega$ & $\therefore: 4$ & 1U & 200 & $\dot{A}$ & HER \\
\hline $\begin{array}{l}11 \\
11 \\
1< \\
1< \\
1<\end{array}$ & $\begin{array}{l}1.2 \\
13 \\
11 \\
11 \\
11\end{array}$ & $\begin{array}{l}i \\
\imath \\
y \\
i\end{array}$ & & 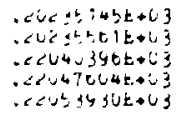 & $\begin{array}{l}.34 t<y t-14 \\
.4<949 t-14 \\
.14<09 t-69 \\
.15 U 54 t-64 \\
.04405 t-67\end{array}$ & $\begin{array}{l}.205118-14 \\
.<4963 E-14 \\
.<470 E-194 \\
.19540 \mathrm{~F}=10 \\
.10007 \mathrm{E}-11\end{array}$ & $\begin{array}{l}.04030 \mathrm{t}-15 \\
.04346 \mathrm{t}-15 \\
.27955 \mathrm{t}-12 \\
.32743 \mathrm{t}-13 \\
.01460 \mathrm{t}-13\end{array}$ & $\begin{array}{l}.25 y 15 t-15 \\
.25215 t-15 \\
.4005 d t-14 \\
.16413 E-14 \\
.11762 t-14\end{array}$ & 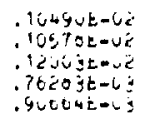 & \\
\hline $\begin{array}{l}1 \% \\
12 \\
12 \\
12 \\
12\end{array}$ & $\begin{array}{l}11 \\
11 \\
11 \\
11 \\
11\end{array}$ & $\begin{array}{l}i \\
\vdots \\
5\end{array}$ & & 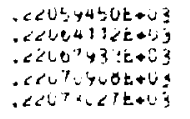 & 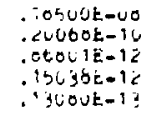 & $\begin{array}{l}070045-12 \\
.70032 E=13 \\
.20050 k-19 \\
.17745 E-13 \\
.6505 E-14\end{array}$ & $\begin{array}{l}14640 E-13 \\
.40404 E-14 \\
.41412 E-14 \\
.95167 E-14 \\
.12740 E=14\end{array}$ & $\begin{array}{l}.10019 \mathrm{t}-14 \\
.02500 \mathrm{t}-15 \\
.40034 \mathrm{E}-15 \\
.00240 \mathrm{t}-15 \\
.34013 \mathrm{E}-15\end{array}$ & 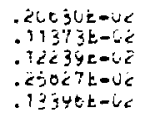 & \\
\hline 16 & 11 & $\vdots$ & & 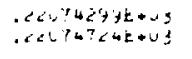 & $\begin{array}{l}\alpha=77 T E-14 \\
. \alpha<5<k-14\end{array}$ & $\begin{array}{l}.<y C t z-14 \\
. \angle t i T C z-14\end{array}$ & $\begin{array}{l}16504 k-14 \\
.404962-1=\end{array}$ & $\begin{array}{l}\text {.313bik-15 } \\
.3104 \mathrm{~L}-15\end{array}$ & 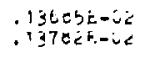 & \\
\hline $\begin{array}{c}\text { METHE: } \\
\text { citjor } \\
\qquad\end{array}$ & $!$ & $\vdots$ & Min & $\begin{array}{l}=49 \quad M U=3.0 \\
.40 t: C l t t+J C \\
.46+1 t(i t+j<\end{array}$ & 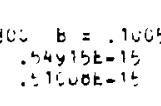 & 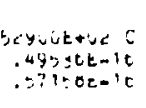 & 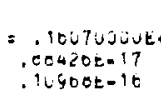 & $\begin{array}{l}+\dot{0} \\
.10 \cos 4 t=17 \\
.<4<4 ? 5-17\end{array}$ & $\begin{array}{l}.4 \$ 45 \dot{C} t-6\rangle \\
.00007 t=-5\end{array}$ & $\Rightarrow$ \\
\hline 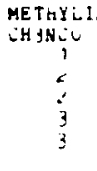 & $\begin{array}{c}u \\
\vdots \\
1 \\
\vdots \\
\vdots\end{array}$ & $\begin{array}{l}t E \\
0 \\
t \\
1 \\
j \\
1\end{array}$ & Me & 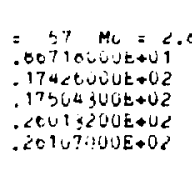 & 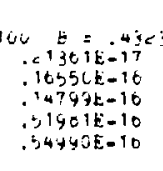 & 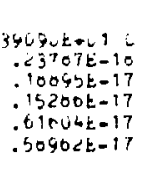 & $\begin{array}{l}=730+5206 k \\
.54325 E-19 \\
.435: 1 E-10 \\
.34045 E-10 \\
.14304 E-17 \\
.13207 E-17\end{array}$ & 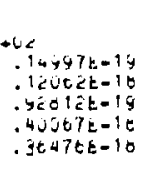 & 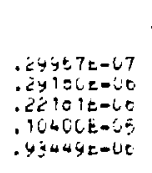 & $2 t$ \\
\hline $\begin{array}{c}\text { METHYLI: } \\
\text { LH BNE:- } \\
\vdots \\
\vdots \\
\vdots\end{array}$ & $\begin{array}{l}\text { SUTH: } \\
\vdots\end{array}$ & $\begin{array}{l}\text { YANAT } \\
u \\
\dot{v} \\
v\end{array}$ & & 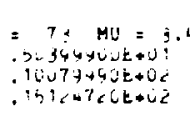 & $\begin{array}{l}b=.2517 \\
.07 \cup 4 u t-1 c \\
.7351 k-17 \\
.770 b u t-1 c\end{array}$ & $\begin{array}{l}77000 k+j 1: \\
.00<07 E=19 \\
.00+50 k=10 \\
.02 b u E-17\end{array}$ & 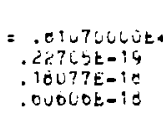 & 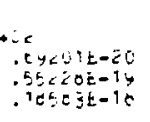 & $\begin{array}{l}.00036 E-L 0 \\
.03179 E=-27 \\
.30163 t-60\end{array}$ & $x i$ \\
\hline $\begin{array}{c}\text { METHYLA } \\
\text { EH } 3 \text { C H } \\
4 \\
2 \\
3 \\
3 \\
3\end{array}$ & $\begin{array}{c}\text { CETY } \\
\vdots \\
1 \\
2 \\
c \\
2\end{array}$ & $\begin{array}{l}1 \\
0 \\
2 \\
1 \\
0\end{array}$ & mb & $\begin{array}{l}=4 L \quad M L= \\
.34102710 t+02 \\
.3410 .37 \cup E+02 \\
.51270000 E+02 \\
.51273700 E+02 \\
.51274750 E+02\end{array}$ & $\begin{array}{l}E=.854 \\
.21475 E-17 \\
.20035 E-17 \\
.47400 E-17 \\
.75954 E-17 \\
.05451 \mathrm{E}-17\end{array}$ & $\begin{array}{l}.070 r_{t}+616 \\
.23910 E-10 \\
.31892 E-18 \\
.50910 E-10 \\
.91070 \mathrm{E}-10 \\
.10240 \mathrm{E}-17\end{array}$ & $\begin{array}{l}=.05450700 \mathrm{E} \\
.50900 \mathrm{E}-19 \\
.67959 \mathrm{E}-19 \\
.12431 \mathrm{E}-18 \\
.19691 \mathrm{k}-10 \\
.22370 \mathrm{k}-16\end{array}$ & $\begin{array}{l}.00 \\
.14255 E-19 \\
.19007 \mathrm{t}=1 \mathrm{y} \\
.25947 \mathrm{t}-14 \\
.50362 \mathrm{E}-19 \\
.03364 \mathrm{E}-19\end{array}$ & 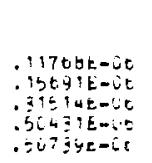 & $94 \quad 2$ \\
\hline $\begin{array}{l}5 \\
5 \\
5 \\
5 \\
5\end{array}$ & $\begin{array}{l}4 \\
4 \\
4 \\
4 \\
4\end{array}$ & $\begin{array}{l}4 \\
3 \\
5 \\
1 \\
0\end{array}$ & & $\begin{array}{l}.85431340 E+02 \\
.85442610 E+02 \\
.05450760 E+02 \\
.0545567 \mathrm{JE} \rightarrow 02 \\
.05457290 \mathrm{0}+02\end{array}$ & $\begin{array}{l}.94395 k-17 \\
.34994 k-10 \\
.22909 k-10 \\
.20254 k-16 \\
.01340 k-10\end{array}$ & $\begin{array}{l}.14724 \mathrm{E}-17 \\
.52300 \mathrm{E}-17 \\
.34371 \mathrm{E}=17 \\
.39280 \mathrm{E}-17 \\
.40925 \mathrm{E}-17\end{array}$ & $\begin{array}{l}.34621 E-10 \\
.12313 E-17 \\
00620 E-10 \\
.42370 E-16 \\
.96229 E-16\end{array}$ & $\begin{array}{l}.10172 E-10 \\
.20175 E-18 \\
.23745 E-10 \\
.27140 E-1 c \\
.20272 E-10\end{array}$ & $\begin{array}{l}.12 C 20 E=05 \\
.350+1 E-05 \\
.23390 E=65 \\
.20743 E-05 \\
.27059 E=05\end{array}$ & \\
\hline $\begin{array}{l}7 \\
7 \\
7 \\
7 \\
7\end{array}$ & $\begin{array}{l}0 \\
0 \\
0 \\
0 \\
0\end{array}$ & $\begin{array}{l}0 \\
5 \\
4 \\
3 \\
2\end{array}$ & & $\begin{array}{l}.11955000 \mathrm{t}+03 \\
.11956112 \mathrm{0}+03 \\
.11900102 \mathrm{0}+03 \\
.11901707 t+03 \\
.11902913 \mathrm{E}+03\end{array}$ & $\begin{array}{l}.23324 E-10 \\
.21544 E-10 \\
.29633 E-10 \\
.71857 \mathrm{E}-10 \\
.40427 E-10\end{array}$ & $\begin{array}{l}.40073 E-17 \\
.44394 E-17 \\
.01003 E-17 \\
.14007 E-10 \\
.83305 E-17\end{array}$ & $\begin{array}{l}.12576 \mathrm{E}-17 \\
.11013 \mathrm{E}-17 \\
.15974 \mathrm{E}-17 \\
.38795 \mathrm{E}-17 \\
.21793 \mathrm{E}-17\end{array}$ & $\begin{array}{l}.30971 E-10 \\
.35909 k-10 \\
.49501 k-10 \\
.12004 E-17 \\
.07533 k-10\end{array}$ & 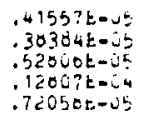 & \\
\hline $\begin{array}{l}i \\
7 \\
0 \\
0 \\
0\end{array}$ & $\begin{array}{l}7 \\
6 \\
7 \\
7 \\
7\end{array}$ & $\begin{array}{l}1 \\
0 \\
7 \\
0 \\
5\end{array}$ & & $\begin{array}{l}.11903547 E+03 \\
.11903622 E+03 \\
.13660015 E+C 3 \\
.13003403 E+03 \\
.13000274 E+03\end{array}$ & $\begin{array}{l}.43127 E-16 \\
.44027 E-10 \\
.110735-16 \\
.41301 E-10 \\
.20017 E-16\end{array}$ & $\begin{array}{l}.6 \text { J69E-17 } \\
.90724 E-17 \\
.27783 E-17 \\
.10378 \mathrm{E}-16 \\
.72303 E-17\end{array}$ & $\begin{array}{l}.23240 E-17 \\
.23733 E-17 \\
.77017 E-16 \\
.28989 E-17 \\
.20197 E-17\end{array}$ & $\begin{array}{l}.72043 t-18 \\
.73547 t-10 \\
.24053 t-18 \\
.92830 E-1 t \\
.64077 t-18\end{array}$ & $\begin{array}{l}.70075 E-c 5 \\
.704615-05 \\
.27609 E-05 \\
.10315 E-04 \\
.71006 E-05\end{array}$ & \\
\hline $\begin{array}{l}8 \\
0 \\
0 \\
0 \\
0\end{array}$ & $\begin{array}{l}7 \\
7 \\
7 \\
7 \\
?\end{array}$ & $\begin{array}{l}4 \\
3 \\
2 \\
1 \\
0\end{array}$ & & $\begin{array}{l}.13608019 E+03 \\
.13670448 E+03 \\
.13071760 E+03 \\
.13672536 E+03 \\
.13672793 E+05\end{array}$ & $\begin{array}{l}.35479 E-16 \\
.41329 E-10 \\
.44370 E-10 \\
.46593 E=16 \\
.47335 E-16\end{array}$ & $\begin{array}{l}.09019 E-17 \\
.20406 E-16 \\
.11132 E=16 \\
.11690 E-16 \\
.11876 E-10\end{array}$ & $\begin{array}{l}.24867 E-17 \\
.57002 E=17 \\
.31098 \mathrm{E}=17 \\
.32650 \mathrm{E}-17 \\
.33170 \mathrm{E}-17\end{array}$ & $\begin{array}{l}.79630 E-18 \\
.16253 \mathrm{E}-17 \\
.99563 E-18 \\
.10457 \mathrm{E}-17 \\
.10624 \mathrm{E}-17\end{array}$ & $\begin{array}{l}.00510 E-05 \\
.20293 E-04 \\
.11072 z-04 \\
.11020 E-14 \\
.11813 t-04\end{array}$ & \\
\hline $\begin{array}{l}9 \\
9 \\
9 \\
9 \\
9\end{array}$ & $\begin{array}{l}8 \\
0 \\
0 \\
0 \\
8\end{array}$ & $\begin{array}{l}0 \\
5 \\
4 \\
3 \\
2\end{array}$ & & $\begin{array}{l}.15371155 E+03 \\
15374372 \mathrm{E}+03 \\
.15377015 \mathrm{E}+03 \\
.15379000 \mathrm{E}+03 \\
.15360537 \mathrm{E}+03\end{array}$ & $\begin{array}{l}.51697 E-16 \\
.32180 E-16 \\
.37305 E-16 \\
.82800 E-16 \\
.44284 E-16\end{array}$ & $\begin{array}{l}.16167 \mathrm{E}-16 \\
.10070 \mathrm{E}-16 \\
.11699 \mathrm{E}-16 \\
.25920 \mathrm{E}-16 \\
.13000 \mathrm{E}-10\end{array}$ & $\begin{array}{l}.46082 \mathrm{E}-17 \\
.30304 \mathrm{E}-17 \\
.35185 \mathrm{E}-17 \\
.77971 \mathrm{E}-17 \\
.41701 \mathrm{E}-17\end{array}$ & $\begin{array}{l}.10175 \mathrm{E}-17 \\
.10009 \mathrm{E}-17 \\
.1109 \mathrm{IE}-17 \\
.25007 \mathrm{E}-17 \\
.13056 \mathrm{E}-17\end{array}$ & $\begin{array}{l}10772 \mathrm{E}=04 \\
11608 \mathrm{E}=04 \\
13573 \mathrm{E}=\mathrm{U} \\
3000 \mathrm{~L}=\mathrm{U} \\
30090 \mathrm{E}-04\end{array}$ & \\
\hline $\begin{array}{r}9 \\
9 \\
10 \\
10 \\
10\end{array}$ & $\begin{array}{l}8 \\
6 \\
9 \\
9 \\
9\end{array}$ & $\begin{array}{l}1 \\
0 \\
7 \\
0 \\
5\end{array}$ & & $\begin{array}{l}.15301427 E+03 \\
.15301710 E+03 \\
.17074605 E+03 \\
.17078029 E+03 \\
.17062413 E+03\end{array}$ & $\begin{array}{l}.46014 E=10 \\
.40591 E=16 \\
.27587 E=16 \\
.54205 E=10 \\
.31774 E-16\end{array}$ & $\begin{array}{l}.14400 E-16 \\
.14588 E-16 \\
.80450 E-17 \\
.21700 E-16 \\
.12725 E-16\end{array}$ & $\begin{array}{l}.43331 E-17 \\
.43674 \mathrm{E}-17 \\
.28223 \mathrm{E}-17 \\
.7080 \mathrm{EE}=17 \\
.41542 \mathrm{E}-17\end{array}$ & $\begin{array}{r}.14397 \mathrm{E}-17 \\
.1457 \mathrm{BE}-17 \\
.97701 \mathrm{E}-18 \\
.24533 \mathrm{E}-17 \\
.14301 \mathrm{E}-17\end{array}$ & $\begin{array}{l}10720 \mathrm{E}=04 \\
.10930 \mathrm{E}=04 \\
.11873 \mathrm{t}=04 \\
.290<0 \mathrm{E}=04 \\
.174 \mathrm{E}=04\end{array}$ & \\
\hline $\begin{array}{l}10 \\
10 \\
10 \\
10\end{array}$ & $\begin{array}{l}9 \\
9 \\
9 \\
9\end{array}$ & $\begin{array}{l}4 \\
3 \\
2 \\
1\end{array}$ & & $\begin{array}{l}.17085350 E+03 \\
.17087627 E+03 \\
.17039259 E+03 \\
.17090237 E+03\end{array}$ & $\begin{array}{l}.35599 \mathrm{E}-10 \\
.77152 \mathrm{E}-16 \\
.40703 \mathrm{E}-10 \\
.41980 \mathrm{E}-16\end{array}$ & $\begin{array}{l}.14257 E-16 \\
.30098 E-16 \\
.10301 E-16 \\
.10812 E-16\end{array}$ & $\begin{array}{l}.46543 E-17 \\
.10087 E-16 \\
.53216 \mathrm{E}-17 \\
.54866 E-17\end{array}$ & $\begin{array}{l}.10112 \mathrm{E}-17 \\
.34919 \mathrm{E}-17 \\
.18422 \mathrm{E}-17 \\
.19000 \mathrm{E}-17\end{array}$ & $\begin{array}{l}19292 E-04 \\
.42460 E-04 \\
.22406 t-04 \\
.23110 E-04\end{array}$ & \\
\hline
\end{tabular}


$.17040500 t+03$

. $10770390 \mathrm{c}+03$

$.10700442 E+0.3$
$.10790340 E+03$

$.107+3034 \varepsilon+03$

$107 \rightarrow$ i30yeitus
20

$.42400=-10 \quad, 10403 E-10$
$.160928=10 \quad .000745=17$

$.50432=-10 \quad .60470 E-10 \quad .94590 E-17 \quad .34254 E-17$

$20491 E-10 \quad .14950 E-10 \quad .5344<E-17$.1y J5be-17

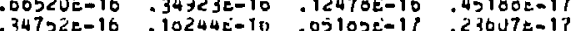

347

$.32047 E-10 \quad 10 ? 14 E-10$
$32945 E-10 \quad .00715-10 \quad .07424 E-17 \quad .24410 E=1 ?$
$.31173 \mathrm{k}-10 \quad .10300 \mathrm{E}-10 \quad .50473 \mathrm{e}-17 \quad .<1170 \mathrm{E}-17$
UL a N N

$\begin{array}{ll}10 & 9 \\ 11 & 10 \\ 11 & 10 \\ 11 & 10 \\ 11 & 10 \\ 11 & 10 \\ 11 & 10 \\ 11 & 10 \\ 11 & 10 \\ 11 & 10\end{array}$

0
0
7
0
5
4
3
2
1
0

$\begin{array}{cc}\operatorname{maII} & \\ \operatorname{Cr} 302 \pi & \\ 2 & 1 \\ 2 & 1 \\ 3 & 2 \\ \frac{3}{3} & 2\end{array}$

10
1
1
0
1
$.18701795 \mathrm{t}+03$

$107979346+03$

21349E-10 112U0E-10 , NUU4SE-17 .14503E-17

$.23345=-04$

$.10,170=04$

$.4375 j 5-v 4$

$.247<3 c-04$

$.2705 \%=-04$

-301000 -04

30405004

. $312002=04$

$11005=-40$

$.150238-00$

- SUOSOE-00

.$\$ 721$ UE-va

. $31700 \mathrm{e}-00$

AMHON 1 A

$.5141275 \mathrm{JE} \rightarrow 02$

$50102 \mathrm{E}-22$

- +0000E-21 .1200\% 120

$.159505-20$.5054ye-20

$.50 y+3 x-20$

$.9 y 730 e-21$.31020e-20

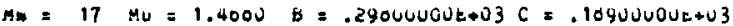

$.14047 a-04$

$n \in \boldsymbol{r}$

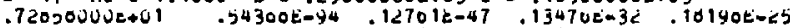

$.73700 j 00 \mathrm{e} \rightarrow 0$

.7617 Gouvt +01

$.70943700 e+01$

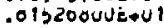

$51394 \mathrm{e}-03 \div 13410 \mathrm{E}-43$

$.37003-107$

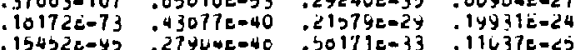

$.001200-31.037400-25$

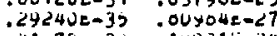

$.07020700 \mathrm{~L}+01$

$.07009000 \mathrm{E}+\mathrm{N} 1$

-

$.0023 y 00 u t+01$

$.92721000 t+01$

$.30003 E-74$

$.01330=040$

(11037e=c

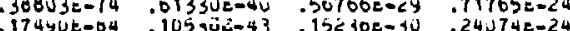

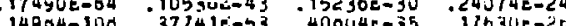

$14904-100$ - 3774le-53 .400U4E-35 .1163Ue-20

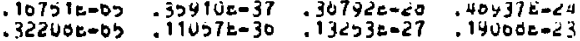

$.51730 k-97 \quad .90309 t-49 \quad .44948 k-33 \quad .126<0 t-2 j$

$.07330=-75 \quad .43099 t-40 \quad .04771 E-2 y \quad .10302 t-23$

$.02127 \varepsilon-02 \quad .5405 J E-43 \quad .19033 \mathrm{E}-30 \quad .14041 \mathrm{E}-24$

$.49752 \mathrm{E}=00 \quad .23001 t-44 \quad .65024 \mathrm{t}-31 \quad .14013 \mathrm{E}-24$

$.65792 \varepsilon=00 \quad: 15529 E-36 \quad: 34074 E-27 \quad: 00074 E-23$

$.9 d 143000 \mathrm{~s}+01$

$.103971208+02$

$10426760 \mathrm{E}+02$

$.10426760 e+02$

$10402020 \varepsilon+02$
$.1053030 v E+02$

$.107545002+02$

$.443018-54$

$.91917 \mathrm{E}-27$

$13213 \mathrm{E}-75$ 4020UE-40 $12702 \mathrm{E}-20.27097 \mathrm{t}-23$

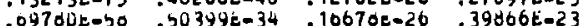

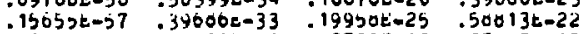

$.205050-50$

$396060-33$
$.50419 t-49$

$.02739 \mathrm{E}-33$

$.50013 \mathrm{t}-22$
$.27442 \mathrm{E}-25$

$-10759020 t+02$

- 10030100e+02

- I1 $32700 e+02$

. $11073100 e+U 2$

$.13410 t-57$
$.53524 t-65$

. 1ว519t-33

$.00003 E-26$

$.15335 c-22$

11940e-06 . du<73e-30

$.02709 \mathrm{~L}-27$. IU202E-L2

$.39003 t-27 \quad$.yy100t-23

$.54005 \mathrm{E}-29 \quad$ :164jut-23

$.100005-01$

$113 \mathrm{dut}-40$

10550e-40

$.15015 E-\angle 5$

$.16450=-23$

$.20009 k-50 .+1205 \mathrm{e}-33$

$.604 \mathrm{USE}-51$. 34204t-31

$.23437 t=67 \quad, 12212 k-36$
$.93727-120 \quad 10077 t-59$

$.93727-120 \quad .10077 t-59$
$.13155 t-50 \quad .12092 \mathrm{t}-30$

$.55002 \mathrm{c}-25$

$.70002 \mathrm{t}=2$

$.30075 E-30$
$.20127 E-24$

$.27600 \mathrm{e}-22$
$.25945 \mathrm{E}-22$

$.25945 E-22$

$.97253 E-20$
$.10790 \mathrm{E}-21$

. 12330400 s'02

$.124 \mathrm{e} 1040 \mathrm{i}+02$

$.1267+1208+02$

$.03752-113.055408=55$

$.74970 \mathrm{E}-36$

$.92235 E-27$

$.1990 E=-33 \quad .14330 E-25$

$.10050 \dot{0}-25 \quad .00000 \mathrm{t}-22$

$.49774 \mathrm{E}-59 \quad .15545 \mathrm{t}-33$

$.60320 t-09 \quad .40002 E-45$

$.105945-50$

$.37462 \mathrm{E}-30$

$71047 E-31$

$.30429 \mathrm{E}-24$

$.494925-21$

$.125940^{\circ}-7$

$.37219 \dot{e-41}$

$.10033 E-23$

$.13297490 t+02$

$13612300 \mathrm{C}+02$

$13700960 \mathrm{0}+02$

$.13719510 E+02$

$.13974540 \mathrm{e}+02$

$.20121 \pm-51$

$.00713 \pm-6$

- 170 Joed20

$10997 \mathrm{E}-30$

$.20742 \mathrm{e}-37$

$.42441 E-29$

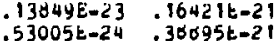

$.53005 t-24 \quad, 36095 t-21$

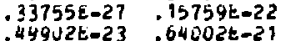

$.97746 E-00$

$.35435 \mathrm{E}-25$

$143705005+02$

$14022700 E+02$

1519554 Ueto

$.15193540+02$
$.151959000^{\circ}+02$

$.15233360 E+02$

$15200240 \varepsilon+02$

$154125 \angle 02+02$

$.15523900 \mathrm{t}+02$
$.15632000 \mathrm{t}+02$

$.97740 E-00$
$.39140 \mathrm{E}=44$

. $17159 \mathrm{e}-33$

10296E-2

$.21160 E-21$

. 403 JE- 52

-12 122 E- 30

$.60534 \mathrm{E}-24$

$.20745 \mathrm{E}-20$

$.55005-115$

. $272016-55$

$.00534 \mathrm{E}-24$

$0=11 \mathrm{t}-21$

$.87600-103$

$12002 \varepsilon=50$

$.11541 E-33$

$.170024-20$

$.06240 t-39$

- 3950 jej-41

$.41041 E-20$

$.254605-22$

$.22557 \mathrm{t}-3$

$.59303 E-29$

$033308-21$

$.19012 \mathrm{E}-24$

$.41052 \mathrm{e}-23$

$.23001 \mathrm{~b}-20$
.1005 1E-22

436 SE $=00$

- $11470 \mathrm{O}-\mathrm{O}$

- $\$ 137=-v 0$

- 4 Uuose-0y

$.416208-00$

$.22257 \mathrm{~L}-00$

- $00 y<3=-40$

$.12390=-0$

$17777 E-U y$
$70403 t-0 y$

.0027 yE-UO

$.43977 \mathrm{t}-\mathrm{U}$

2 2y $160 \mathrm{E}-\mathrm{U}$

$.02941 \mathrm{e}-00$

$.42460 t-40$

$.15547 \mathrm{t}=07$

$.10073 E-07$

$32027 \dot{E-0}$

$60012 \mathrm{E}-00$

- $31590 \mathrm{~K}=07$

$.14203 \mathrm{e}-00$

. SDE 30t-00

$.03194=00$

$.10113 \mathrm{k}-07$

$77770=00$

$59301 E-0 y$

$31933 \mathrm{E}=07$

.25007E=00

. 32306t-07

. $31917 E-07$

15379E-U7

O J054E-UT

$.14473 E=07$

$.311096-07$

. IU545t-00

.14075t-07

$.30510 E=07$

$.45630 E-00$

$.59070 \mathrm{k}-07$

-22300ع-07

13973.00

$.139730=06$

$.10473 \varepsilon=00$

$.63771 \mathrm{t}-0$ ?

.12201 e-Ub

$.25024 \mathrm{k}-07$

$.50972 E=07$ 
Ihele I1. 3

\begin{tabular}{|c|c|c|c|c|c|c|c|c|}
\hline נل J & $J L$ & n. & Mu & نL & نان & 100 & cus & $n$ \\
\hline $\begin{array}{c}0 \\
11 \\
10 \\
0 \\
y\end{array}$ & $\begin{array}{c}0 \\
11 \\
0 \\
y\end{array}$ & $\begin{array}{l}\dot{c} \\
l \\
\vdots \\
2 \\
\vdots \\
b\end{array}$ & 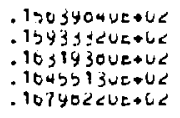 & 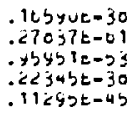 & 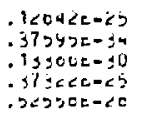 & 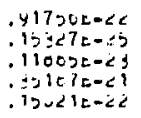 & 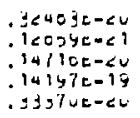 & 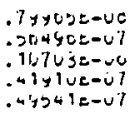 \\
\hline $\begin{array}{l}1 \\
7 \\
0 \\
7 \\
12\end{array}$ & $\begin{array}{r}1 \\
7 \\
0 \\
7 \\
12\end{array}$ & $\begin{array}{l}1 \\
2 \\
4 \\
3 \\
3\end{array}$ & 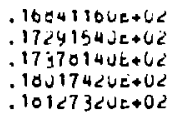 & $\begin{array}{l}.11140 t-33 \\
.21442 \varepsilon-33 \\
.35440 c-3 y \\
.20320 z-33 \\
.0 y 100 c-72\end{array}$ & $\begin{array}{l}.323<0 E-24 \\
11042 t-\angle j \\
17100 x-25 \\
.449202-2 j \\
.54<40=-0\end{array}$ & 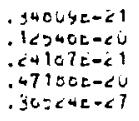 & $\begin{array}{l}.30201 E-<0 \\
14144 t-1 y \\
117<2 c=1 y \\
04033 c-1 y \\
.9 y 03 c-2 c\end{array}$ & 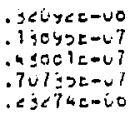 \\
\hline $\begin{array}{l}11 \\
13 \\
10 \\
0 \\
4\end{array}$ & $\begin{array}{r}11 \\
13 \\
10 \\
0 \\
y\end{array}$ & $\begin{array}{r}0 \\
10 \\
1 \\
1\end{array}$ & $\begin{array}{l}.10162540 \mathrm{U}+02 \\
.10177990 \mathrm{U} 2 \\
.10205500 \mathrm{U} 2 \\
.10391050 c+02 \\
.10499200 \mathrm{U} 202\end{array}$ & 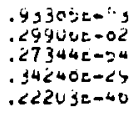 & $\begin{array}{l}.19201 \varepsilon-54 \\
.25132 E-204 \\
.29540=-51 \\
.41059 c-<c \\
.50071 c-20\end{array}$ & 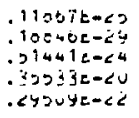 & $\begin{array}{l}14141 L-<1 \\
.21444 E-<3 \\
0+4 y+c-21 \\
13 e 20 E-1 y \\
.07411 L-<0\end{array}$ & 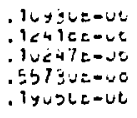 \\
\hline $\begin{array}{r}15 \\
0 \\
10 \\
0 \\
7\end{array}$ & $\begin{array}{r}15 \\
0 \\
10 \\
b \\
t\end{array}$ & $\begin{array}{r}12 \\
3 \\
13 \\
2 \\
4\end{array}$ & 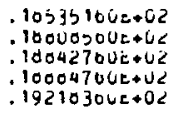 & $\begin{array}{l}.75700-1 u 5 \\
.01700 E=40 \\
.71750-110 \\
.050 y 7 c-2 y \\
.45 y 0 j t-34\end{array}$ & $\begin{array}{l}.40050=-51 \\
1<0+0 E-\angle 5 \\
10004 E-50 \\
1<00 y=-21 \\
.21414 E-<3\end{array}$ & $\begin{array}{l}1<<0+c-33 \\
.210+2 c-21 \\
1 y+j c e-32 \\
1200 y c-19 \\
330 y t-<0\end{array}$ & 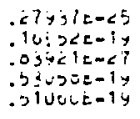 & 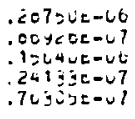 \\
\hline $\begin{array}{l}0 \\
b \\
b \\
0 \\
9\end{array}$ & $\begin{array}{l}b \\
b \\
b \\
0 \\
y\end{array}$ & $\begin{array}{l}3 \\
1 \\
2 \\
0 \\
7\end{array}$ & $\begin{array}{l}.1975740 u t+0 z \\
.19030410 E+0< \\
.2037140 \mathrm{~J} t 02 \\
.2071 \text { y210e+02 } \\
.20735440 E+02\end{array}$ & $\begin{array}{l}.077 \supset y c-E y \\
.2510 \cup E-c z \\
.40454 c=-5 \\
.12130 c=40 \\
.03319 E-40\end{array}$ & 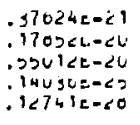 & $\begin{array}{l}.40200=-1 y \\
.<7251=-1 y \\
.90<47 E-1 y \\
.34340=-1 \\
.1<701 E-2<\end{array}$ & 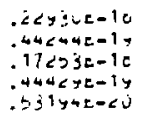 & 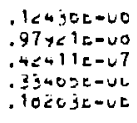 \\
\hline $\begin{array}{r}7 \\
10 \\
6 \\
11 \\
4\end{array}$ & $\begin{array}{r}7 \\
10 \\
0 \\
11 \\
4\end{array}$ & $\begin{array}{l}2 \\
0 \\
4 \\
9 \\
1\end{array}$ & $\begin{array}{l}.20004030 t+02 \\
.20052510 E+02 \\
.20494610 t+02 \\
.21070700 E+02 \\
.21134290 E+02\end{array}$ & $\begin{array}{l}.74020 c-30 \\
.41000 E-20 \\
.14114 E-24 \\
.37200 E-04 \\
.44700 E-\angle C\end{array}$ & $\begin{array}{l}.171 \text { j4E- : } \\
.10370 E=51 \\
.10154 E-\angle 1 \\
.70392 E-35 \\
.43440 E-19\end{array}$ & $\begin{array}{l}.970 j t 5-\angle U \\
. j y 064 t-24 \\
3374+E-14 \\
.10013 t-\angle 2 \\
.3000 t-10\end{array}$ & 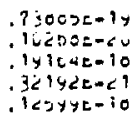 & 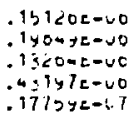 \\
\hline $\begin{array}{r}5 \\
12 \\
4 \\
3 \\
5\end{array}$ & $\begin{array}{r}3 \\
12 \\
4 \\
3 \\
3\end{array}$ & $\begin{array}{r}3 \\
10 \\
2 \\
1 \\
4\end{array}$ & $\begin{array}{l}.21205270 E+02 \\
.21391550 \mathrm{~L}+02 \\
.21703360 E+0 z \\
.22234530 \mathrm{E}+02 \\
.22053000 \mathrm{E}+02\end{array}$ & $\begin{array}{l}.04363 E-25 \\
.53209 t-74 \\
.00134 E-22 \\
.14604 t-19 \\
.10303 t-25\end{array}$ & $\begin{array}{l}.10017 E-19 \\
.03000 E-39 \\
.13304 E-10 \\
.01404 E-10 \\
.77521 E-20\end{array}$ & $\begin{array}{l}.37157 t-10 \\
.11019 E-27 \\
.57207 t-10 \\
.71462 c=10 \\
.25430 t=10\end{array}$ & 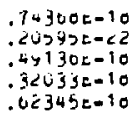 & 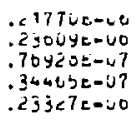 \\
\hline $\begin{array}{r}4 \\
0 \\
3 \\
7 \\
15\end{array}$ & $\begin{array}{r}4 \\
0 \\
3 \\
7 \\
15\end{array}$ & $\begin{array}{r}3 \\
5 \\
2 \\
6 \\
13\end{array}$ & $\begin{array}{l}.22600290 E+02 \\
.22732430 E+02 \\
.22834170 E+02 \\
.22924940 E+02 \\
.23004000 E+02\end{array}$ & $\begin{array}{l}.11453 E-27 \\
.24554 E-30 \\
.37732 t-19 \\
.75709 E-35 \\
.90083-100\end{array}$ & $\begin{array}{l}.34023 \mathrm{E}-10 \\
12904 \mathrm{E}-21 \\
.10944 \mathrm{E}-17 \\
.10453 \mathrm{E}-23 \\
.20990 \mathrm{E}-52\end{array}$ & $\begin{array}{l}.21047 \mathrm{t}-17 \\
.30005 \mathrm{E}-19 \\
.2577 \mathrm{UE}-17 \\
.744) 5 \mathrm{E}-20 \\
.25304 \mathrm{E}-34\end{array}$ & $\begin{array}{l}.21201 \mathrm{E}-17 \\
.2774<2-10 \\
.12444 \mathrm{E}-17 \\
.14370 \mathrm{E}-10 \\
.13130 \mathrm{E}-25\end{array}$ & $\begin{array}{l}.34540 t-U t \\
.009 U y a-U b \\
.14 y 32 E-00 \\
.50203 E-U b \\
.3223 \angle E-U t\end{array}$ \\
\hline $\begin{array}{l}2 \\
8 \\
9 \\
1 \\
2\end{array}$ & $\begin{array}{l}2 \\
0 \\
9 \\
1 \\
2\end{array}$ & $\begin{array}{l}1 \\
7 \\
6 \\
1 \\
2\end{array}$ & $\begin{array}{l}.23096790 \mathrm{t}+02 \\
.23232240 \mathrm{0}+02 \\
.23657480 \mathrm{0}+02 \\
.23694490 \mathrm{0}+02 \\
.23722630 \mathrm{0}+02\end{array}$ & $\begin{array}{l}.22075 t-17 \\
.34634 t-42 \\
.21279 c-49 \\
.73055 L-10 \\
.42491 E-17\end{array}$ & $\begin{array}{l}.52730 \mathrm{t}-17 \\
.30017 \mathrm{t}-20 \\
.45000 \mathrm{t}-29 \\
.31301 \mathrm{t}-10 \\
.10253 \mathrm{t}-10\end{array}$ & $\begin{array}{l}.25997 c-17 \\
.23553 \mathrm{E}-21 \\
.9913 j \mathrm{E}-23 \\
.8725 \mathrm{E}-17 \\
.937 \vdots U \mathrm{E}-17\end{array}$ & $\begin{array}{l}72024 t=10 \\
.270,27 t=19 \\
.01007 E=20 \\
.141<5 E-17 \\
.29507 \varepsilon=17\end{array}$ & $\begin{array}{l}.77204 c-07 \\
.321608-00 \\
.35420 c-10 \\
.25020=-00 \\
.33407 \varepsilon-10\end{array}$ \\
\hline $\begin{array}{r}16 \\
3 \\
4 \\
10 \\
5\end{array}$ & $\begin{array}{r}16 \\
3 \\
4 \\
10 \\
5\end{array}$ & $\begin{array}{r}14 \\
3 \\
4 \\
9 \\
5\end{array}$ & $\begin{array}{l}.23777400 E+02 \\
.23070130 E+02 \\
.24139410 E+02 \\
.24205297 \mathrm{E}+02 \\
.24532900 \mathrm{0}+02\end{array}$ & $\begin{array}{l}11340-120 \\
.50109 \mathrm{~L}-19 \\
.16406 \mathrm{t}-22 \\
.36060 \mathrm{E}=57 \\
.10070 \mathrm{E}-20\end{array}$ & $\begin{array}{l}.19713 \mathrm{E}-57 \\
.55209 \mathrm{E}-17 \\
.10070 \mathrm{E}-10 \\
.59754 \mathrm{c}-32 \\
.55400 \mathrm{E}-20\end{array}$ & $\begin{array}{l}.07400 E-37 \\
.9757 \text { yE-17 } \\
.15170 \leq-17 \\
.25000 E-24 \\
.29003 E-10\end{array}$ & $\begin{array}{l}.70400 \mathrm{E}-27 \\
.53907 \mathrm{E}-17 \\
.17704 \mathrm{E}-17 \\
.22440 \mathrm{E}-20 \\
.40207 \mathrm{E}-10\end{array}$ & $\begin{array}{l}.30440 E-110 \\
.70759=-20 \\
.4239 y 2-00 \\
.705092-00 \\
.402900-00\end{array}$ \\
\hline $\begin{array}{r}17 \\
11 \\
6 \\
12 \\
7\end{array}$ & $\begin{array}{r}17 \\
11 \\
6 \\
12 \\
7\end{array}$ & $\begin{array}{r}15 \\
10 \\
0 \\
11 \\
7\end{array}$ & $\begin{array}{l}.24000100 E+02 \\
.24 d d 1900 E+02 \\
.25050020 E+02 \\
.25695230 E+02 \\
.25715170 E+02\end{array}$ & $\begin{array}{l}.30902-134 \\
.22271 E-06 \\
.46349 E-31 \\
.30220 E-70 \\
.44665 E-37\end{array}$ & $\begin{array}{l}.13202 t-62 \\
.90063 t-36 \\
.14354 t-21 \\
.12346 t-39 \\
.41644 t-24\end{array}$ & $\begin{array}{l}.34542 E-39 \\
.52952 E-20 \\
.70007 t-19 \\
.07617 t-20 \\
.32279 t-20\end{array}$ & $\begin{array}{l}.73420 \mathrm{E}-2 \mathrm{a} \\
.16057 \mathrm{E}-21 \\
.72794 \mathrm{E}-10 \\
.20750 \mathrm{E}-22 \\
.11009 \mathrm{E}-10\end{array}$ & $\begin{array}{l}.03170=-00 \\
.43 y 0 y=000 \\
.16445=05 \\
.45510=-00 \\
.55102 E=00\end{array}$ \\
\hline $\begin{array}{r}0 \\
13 \\
9 \\
14 \\
10\end{array}$ & $\begin{array}{r}13 \\
13 \\
14 \\
10\end{array}$ & $\begin{array}{r}0 \\
12 \\
9 \\
13 \\
10\end{array}$ & $\begin{array}{l}.26518910 E+02 \\
.26055000 E+02 \\
.27476000 E+02 \\
.27772520 E+02 \\
.28004730 E+02\end{array}$ & $\begin{array}{l}.11650 E-43 \\
.16570 E-00 \\
.05123 E-51 \\
.32022 E-90 \\
.21952 E-59\end{array}$ & $\begin{array}{l}.10916 E-26 \\
.15383 E-43 \\
.25997 E-29 \\
.22442 E-40 \\
.70553 E-33\end{array}$ & $\begin{array}{l}.10287 t-21 \\
.11077 t-29 \\
.13911 t-22 \\
.34125 E-32 \\
.17 t 24 E-24\end{array}$ & $\begin{array}{l}.311025=14 \\
.42000 E=-23 \\
.13372 E=19 \\
.17400 E-24 \\
.11772 E-20\end{array}$ & $\begin{array}{l}.02372 t-60 \\
.11275 E-05 \\
.14059 t-05 \\
.04130<E=00 \\
.041320 E-W 0\end{array}$ \\
\hline $\begin{array}{l}15 \\
11 \\
12 \\
13 \\
14\end{array}$ & $\begin{array}{l}15 \\
11 \\
12 \\
13 \\
14\end{array}$ & $\begin{array}{l}14 \\
11 \\
12 \\
13 \\
14\end{array}$ & $\begin{array}{r}.29061140 \mathrm{E}+02 \\
.29914660 \mathrm{E}+02 \\
.31424970 \mathrm{E}+02 \\
.33156950 \mathrm{E}+02 \\
.35134440 \mathrm{E}+02\end{array}$ & $\begin{array}{l}.15674-110 \\
.1602 \mathrm{bE}-65 \\
.33177 \mathrm{E}-76 \\
.24377 \mathrm{E}-89 \\
.50901-101\end{array}$ & $\begin{array}{l}.29634 E-53 \\
.17501 E-30 \\
.39745 E-40 \\
.10344 E-44 \\
.24702 E-49\end{array}$ & $\begin{array}{l}.13515 E-34 \\
.30052 t-2 j \\
.72204 t-2 d \\
.22009 E-30 \\
.15425 t-32\end{array}$ & $\begin{array}{l}.11993 E-25 \\
.17022 E-21 \\
.40491 E-22 \\
19033 E-23 \\
160+7 E-K 4\end{array}$ & $\begin{array}{l}.754 i 5 t-00 \\
.923290-00 \\
.21350-45 \\
.12135 t-05 \\
.15<30 e-05\end{array}$ \\
\hline $\begin{array}{l}15 \\
16\end{array}$ & $\begin{array}{l}15 \\
16\end{array}$ & $\begin{array}{l}15 \\
10\end{array}$ & $\begin{array}{r}37305180 E+02 \\
.39941540 E+02\end{array}$ & $\begin{array}{l}.30220-113 \\
.63034-127\end{array}$ & $\begin{array}{l}.54163 \mathrm{E}-54 \\
.13638 E-59\end{array}$ & $\begin{array}{l}11263 E-34 \\
.13957 E-37\end{array}$ & $\begin{array}{l}.23341 \mathrm{E}-25 \\
.20071 \mathrm{t}-27\end{array}$ & $\begin{array}{l}.30002 E-0 S \\
.22004 E-U S\end{array}$ \\
\hline
\end{tabular}


Table III - Contents

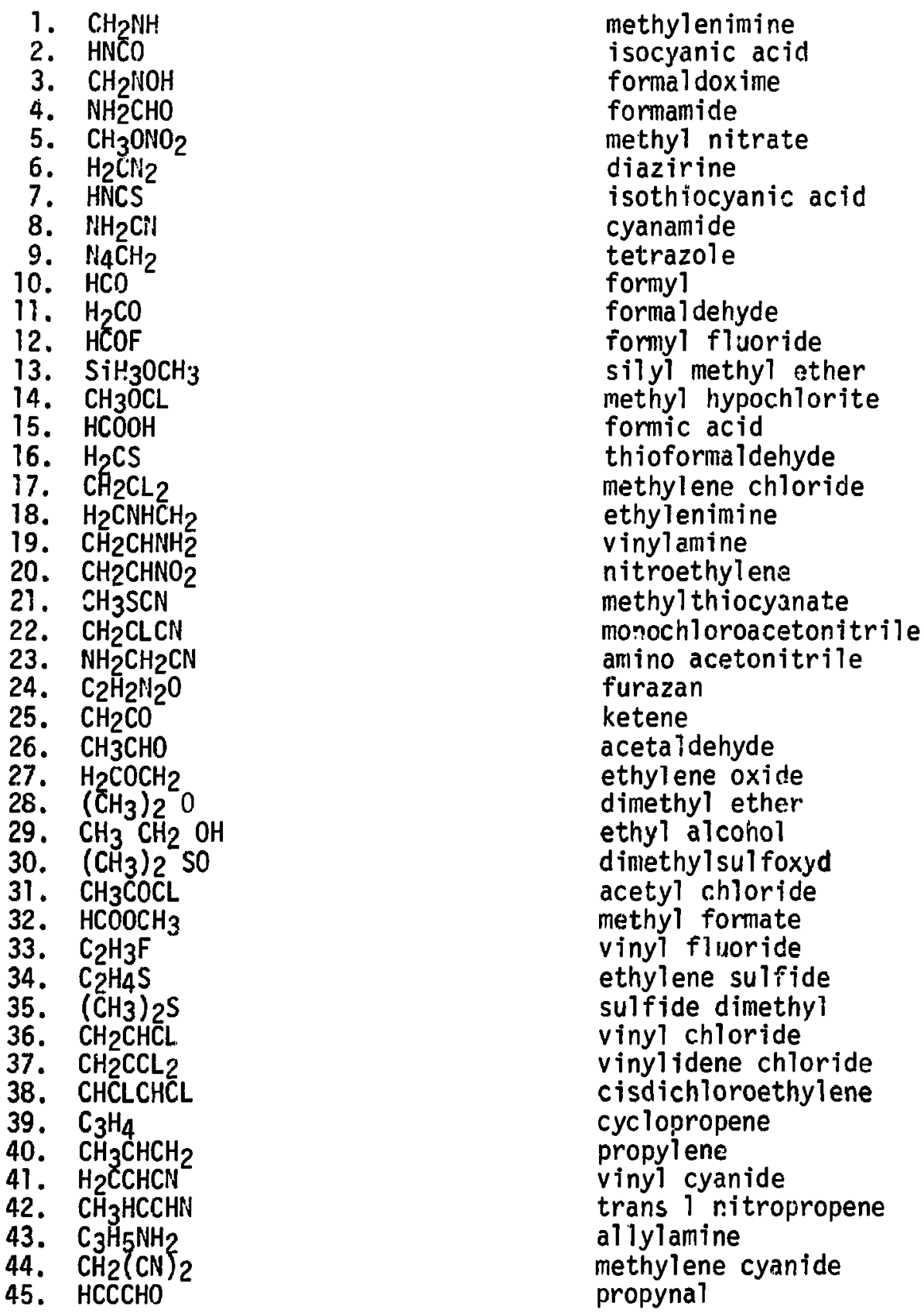


46. $\mathrm{HCCCH}_{2} \mathrm{OH}$

47. $\mathrm{CH}_{2} \mathrm{CHC}+\mathrm{HO}$

48. $\left(\mathrm{CH}_{2}\right)_{3} \mathrm{O}$

49. $\left(\mathrm{CH}_{3}\right)_{2} \mathrm{CHOH}$

50. $\mathrm{CH}_{2}(\mathrm{CHO})_{2}$

51. $\mathrm{C}_{3} \mathrm{H}_{2} \mathrm{O}_{3}$

52. $\mathrm{HCCSCH}_{3}$

53. $\mathrm{CH}_{2} \mathrm{CHCH}_{2} \mathrm{SH}$

54. $\mathrm{CH}_{3} \mathrm{SC}_{2} \mathrm{H}_{3}$

55. $\mathrm{C}_{4} \mathrm{H}_{4}$

56. $\mathrm{CH}_{3} \mathrm{CHCCH}_{2}$

57. $\left(\mathrm{CH}_{3}\right)_{2} \mathrm{CCH}_{2}$

58. $\mathrm{HCCCH}_{2} \mathrm{CH}_{2} \mathrm{OH}$

59. $\mathrm{CH}_{2} \mathrm{CHCHCN}$

60. $\mathrm{C}_{4} \mathrm{~N}_{2} \mathrm{H}_{4}$

61. $\mathrm{NC}_{4} \mathrm{NH}_{2} \mathrm{H}_{4}$

62. $\mathrm{C}_{2} \mathrm{H}_{2} \mathrm{OC}_{2} \mathrm{H}_{2}$

63. $\mathrm{CH}_{2} \mathrm{CCH}_{3} \mathrm{CHO}$

64. $\left(\mathrm{CH}_{3}\right)_{2} \mathrm{CCO}$

65. $\left(\mathrm{H}_{2} \mathrm{CCH}\right)_{2} \mathrm{O}$

66. $\mathrm{C}_{4} \mathrm{SH}_{6}$

67. $\mathrm{C}_{5} \mathrm{H}_{6}$

68. $\mathrm{CH}_{3} \mathrm{C}_{3} \mathrm{HCH}_{2}$

69. $\left(\mathrm{CH}_{3}\right)_{2} \mathrm{CCCH}_{2}$

70. $\mathrm{C}_{5} \mathrm{H}_{5} \mathrm{~N}$

71. $\mathrm{C}_{5} \mathrm{OH}_{8}$

72. $\mathrm{C}_{6} \mathrm{H}_{6}$

73. $\mathrm{C}_{6} \mathrm{OH}_{4}$

74. HNO

75. $\mathrm{HNO}_{3}$

76. $\mathrm{NH}_{2} \mathrm{NO}_{2}$

77. $\mathrm{HN}_{3}$

78. $\mathrm{H}_{2} \mathrm{O}$

79. $\mathrm{H}_{2} \mathrm{~S}$

80. $\mathrm{COCL}_{2}$

81. NOF

82. NOCL

83. $\mathrm{NO}_{2}$

84. $\mathrm{NO}_{2} \mathrm{CL}$

85. NSF

86. $\mathrm{OF}_{2}$

87. $\mathrm{S}_{2} \mathrm{O}$

88. $\mathrm{CL}_{2} \mathrm{O}$

89. $\mathrm{SO}_{2}$

90. $\left(\mathrm{SO}_{2}\right.$

91. $\mathrm{O}_{3}$

92. SiF $_{2}$ propargyl alcohoi

$s$ trans acrolein

oxetane

isopropyl alcohcl

vinyl formate

vinylene carbonate

methyl thioethyne

allyl mercaptan

methyl vinyl sulfide

vinylace tylene

methylallene

isobutylene

butynol

crotononitile

pyrimidene

2 aminopyridine

furan

methacrolein

dimethyl ketene

divinyl ether

3 methyl thiophene

trans 3 penten 1 yne

penten

dimethylallene

pyridine

cyclopentene oxide

fulvene

cyclohexene oxide

nitroxyl

nitric acid

nitramide

hydrazoic acid

water

hydrogen sulfide

phosgene

nitrosyl fluoride

nitrosyl chloride

nitrogen dioxide

nitryl chloride

thionitrosyl fluoride

oxygen difluoride

disulfurmonoxide

chlorine monoxide

sulfur dioxide

polymer sulfur monoxide ozone

silicon difluoride 


\section{TABLE III (cont)}

Notes to Table III

a. Value of $\mu$ is assumed to be 1 .

b. Cross sections are uncorrected for fraction of molecules in the vibration state $v_{i}$. 
50-7!EดnE SO-76L209.

CO-3505E!

SO-3PQIEI:

s0-7900 LI:

on-3605il:

$00-790250^{\circ}$

L0-7i 2966 .

90-7ह0 0 Q

op-zहo

०0-30960ह

90-3E0902:

90-7Lद6I!

90-32+rial.

90-ahE291:

10-36co6n"

¿ी-3iटा।

LO-7000LI.

20-30igEn.

LOFELLS

99\&6.

9

5o-7t119t

E-21<091.

ถO-Fn?

$70-7 C 6<09$

E0-700hL

E0-76R6hL

EO-3KESZ!

no-zonเza.

$\rightarrow 0-3069 \pi E^{\circ}$

nO-3GL hHE.

n-zSEh!9.

$50-30<961$.

70-7LSTAR.

So-zglali.

ho-aitsht.

$\rightarrow 0-3190 L 1$.

$a 0-31+<09^{\circ}$

$40-7665 \varepsilon 9^{\circ}$

on-3g Loi

on- गदE०ก

an-3E66t।

COC-305R

Qn-72EQ $Q$ L :

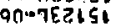

20-7.706ns

LO- सहाOSL

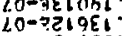

On-aSOLSS.

pn-जnzzह1.

คด-สEटLSL

6O-R9L LEट

01-310995

$01-321702$

LI-3⿻5nLL

$11-72 \angle 9 L 1$

ZІ-चESEL

ह1-ESGEQL

EI-30LE Q?

QवE5०
QL.

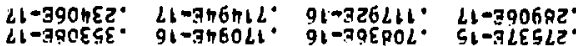

Q1-3inth6: L1-300562:

Qt $-3 \zeta L E O L$

$61-32 L \pi 59$

Q1-3985टt.

$96-30+001$.

$L 1=32 h L 4$.

L1-39160s.

$81-35 f \varepsilon 9 \varepsilon^{2}:$

$02-35596 \varepsilon^{\circ}$

LI-3LiLG!

Lt-3n62ht

$12-3066 \varepsilon 6^{\circ}$

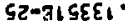

gi-30E 602 .

$61-3026 E$.

$12-3050 \pi 1$

62-3699LE .

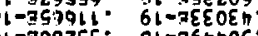

Q1-3992EE.

L - -

$02-\operatorname{scz} 26$

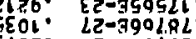

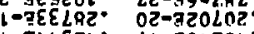

$81-39 L 2 \pi+$

$91-3 n+69 l$

Ri-360.92.

PI-JaEEZ1

Pl- $-7600 t !$

$81-71 \angle \varphi_{0}$

$21-3 h n 9 \varepsilon 1$.

Q

$61-7 L L S_{L}$.

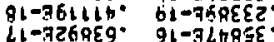

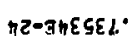

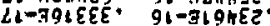

Q1-390101. 02-3605z2.

$61-36 \angle 0<P^{\circ}$

$6.35918 L^{\circ}$

$6 t-39590 E^{\circ}$

$81-79612 !$

Pl-35PE21.

$61-3<8240$

12-3E9016.

R1-380102 $81-3 E Z 5 E 2^{\circ}$ 61-3ELSOZ

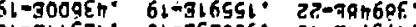

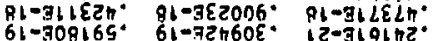

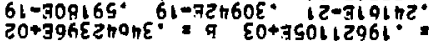

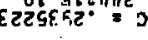

$\angle 1-7 E Z R O L^{\circ}$

$9 L-3 G_{L} L 5$. $^{\circ}$

91 - 301 ดह?

LL-a!shthe

St-32t $\varphi_{9 !}$

$9 L-3 L L E Q \varepsilon^{\circ}$

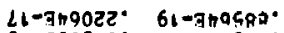
$9 t-392 E t t^{\circ}$

Ol- $3<\varepsilon+8 \varepsilon^{\circ}$

Sl-36? है:

Gi -36094

hi $\rightarrow$ a LQEQ1.

$91-\pi 5 z \varepsilon ! t$

91 -

$9 L-7 \pi+20 L^{\circ}$

St-7PSS.tht:

$91-35$ SEE

Ql-

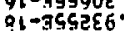

$9 \mathrm{l}-96 \mathrm{~A} h \mathrm{L6}$

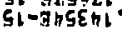

Li-3069h?

Gt-3hh+62:

St-3LalkL:
St-76?asc.

Li-งE $690 \varepsilon$

or-a00098.

$9 i-36 \pi z 51$

91-TEG।GL

G1-3602zह.

Gl-3ic5n

hL-3250.

ol-366LLE.

Li-3entrol.

$9 i-30 h \pi 9 i$.

Oा -जा G9HE:

$91-\rightarrow 0 L \pitchfork \rightarrow E^{*}$

$8 i-3 \pi 599 l^{\circ}$

Ql-35lang.

द)-30ERI:

S1-7द厶Ll!

ci-3rious.

SI-702LSE:

G1-ais?

Mi-aiहzez.

$91-3220 \varepsilon 1$.

L1-3161L: $91-7 L 95 L 2$.

G1-366!R2.

LI-JGESEn

a1-sokn L6.

nI-TGOLZt.

LI-36ILE

Sl-392 ros.

hi-70LTOL:

$9 \iota-\pi<8 เ n$

RL-JSSIER:

$\angle L=36 \varepsilon \angle 9 a$

L1-3915nt०

$11-30 \mathrm{pct}$.

9l-30E! เ

Q $1-3+99 p 2$

$61-36 h t 2 \pi$

LI-สsहz2i.

QI-FPOLLi:

$\angle-3 P \pi L T^{\circ}$.

t?-25hLद

PI-7दL161

$\angle 1-900 \pi 68$

$\angle i-3+261 L$

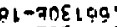

Qi $-7054+1$ :

GL-7E OO6P.

$61-322551$.

เこ-7ZเこLE

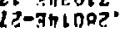

LITQ⿱一𫝀口LQQE:

PL-TQPQL?

$\zeta L=62 Q L L$

$0:-16 \varepsilon 20 L$.

$l_{1}=329562$

OZ-7GETAR

LI- $\varepsilon_{21}<0^{\circ}$

61-99gटzi:

ดा-3แtहlट

Q1-7565l?

$L-9 \varepsilon \varepsilon O L L$

61-020500

$61-3 \circ \varepsilon \varsigma_{1}$.

61 -7695ह2:

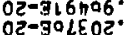

$Q_{1}-300$ CEI

QL-7iglPI:

GL-DELONE.

政

$61-310 L 52$

$61-7010 \varepsilon \varepsilon$
$02-7 \rightarrow \pi 162$

9i $-39 t L 2 !$

$61-7 ? 0<56$.

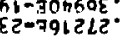

oz-70! เSg.

6L-3द $9 L \pi E^{\circ}$

$F L-3 L L G L^{\circ} \quad L L-3 \geq n Z Z L^{\circ}$

$61-79 L c_{15} \cdot 61-306162$

12-75ट00L. हट-702दा5.

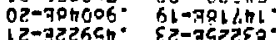

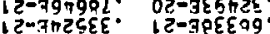

(a)

เट-7เ6Qกเ

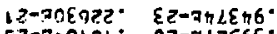

टर-700 $65^{\circ}$

$\begin{array}{lllllll}\varepsilon 0+\pi 0026060 l^{\circ} & 9 & 2 & L & L & ! & 9 \\ \varepsilon 0+30146 L S O l^{\circ} & \varepsilon & l & \varepsilon & h & c & h\end{array}$

20+3OSPQUES6

$20+30 S \pi \angle 25 \angle 0^{\circ}$

20+7009501เ:

$20+301019100^{\circ}$

$20+3009+5699^{\circ}$

$20+302116 \pi 00^{\circ}$

$20+30 H S 2 T O A S:$

20+302+262LS.

20+3076L

$20+30620556 \pi^{\circ}$

$20+30$ HPESSSH:

20+305SS90SE:

20+30pRhOLE:

$20+30222568 ? ?^{\circ}$

$20+301490002$.

$20+706052$ ig:

$20+3005$ EST92.

ZO+ M090EQLSE.

$00 E S \cdot L=N K 62$

$\varepsilon 0+30 \angle O L T \angle A C$

E0+30101.2992:

En+z0.211L52.

EO+300LL\&9G2.

80+30त591 0द2.

$0+30+5+9 G 92$.

0+302509h52:

$0+300021$ sit?

EOमJOSPEIOE?

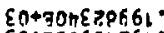

E0+50069n261.

$80+305656161:$

$80+30529161$.

$50+2009 ; 6 E Q_{1}$.

E0+3000902hL

$D+30 i$ - L $>\varepsilon \varepsilon L$

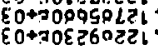

E0+70606901:

OO+7DEQEOOL:

$20+300061610^{\circ}$

$20+702 \angle 08262$

Z0+70ZE $00259^{\circ}$

$20+309626650^{\circ}$

$20+700 \angle 05405$

$20+30 E 009025$

20+702L $0156 E$

?०+3กEGSELLE

$20+3 n 2 T S L P S ?:$

$20+206060051$.

$20+501 \pi O E L G$.

$0+30610<200^{\circ}$

$10+3020$ है $\$ 9 द$.

10+anool6pas.

$10+706011$ tht

$10+700$ icgtak.

$10+702$ LE $0161^{\circ}$ 


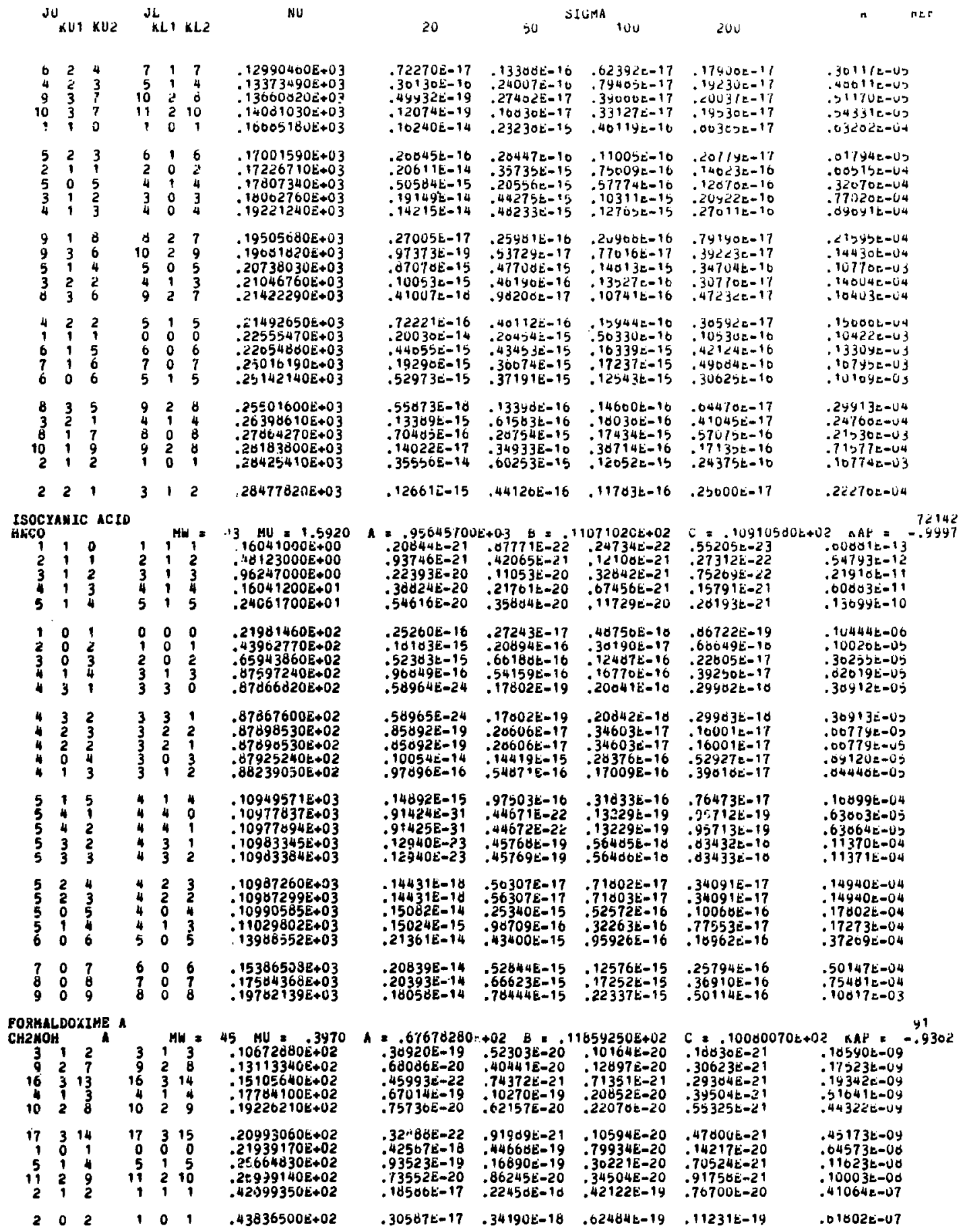


TABLE III. 3
Ji
id
20
50
Slomh
100
200
A $\mathrm{HER}$

\begin{tabular}{|c|c|c|c|}
\hline ENUn & & & $M_{*}=$ \\
\hline 34 & 3 & 5 & 12 \\
\hline 12 & 9 & 13 & 212 \\
\hline y & 7 & 10 & 70 \\
\hline & & & \\
\hline י & 0 & 11 & $2 y$ \\
\hline 3 & 0 & 12 & $2+1$ \\
\hline 2 & ; & 0 & 10 \\
\hline 0 & 4 & 3 & 13 \\
\hline 1 & 7 & 7 & 20 \\
\hline 9 & 1 & 2 & 02 \\
\hline t & & & \\
\hline & & & \\
\hline $\begin{array}{ll}10 \\
1 \\
1\end{array}$ & $\begin{array}{l}0 \\
1\end{array}$ & $\begin{array}{c}10 \\
0\end{array}$ & $\begin{array}{ll}2 & 9 \\
0 & 0\end{array}$ \\
\hline & 2 & $i$ & 1 \\
\hline 0 & $\overline{2}$ & 1 & 01 \\
\hline
\end{tabular}

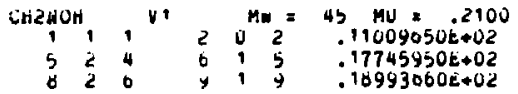

FUhmAMIDE

MHECHO

$\begin{array}{rrr}10 & 3 & 17 \\ 17 & 4 & 13 \\ 11 & 3 & \\ 10 & 4 & 1 \\ 5 & 2 & \\ 19 & 4 & 15 \\ 1 & 1 & 0 \\ 12 & 3 & 9 \\ 6 & 8 & \\ 20 & 4 & 16\end{array}$ 7 A

A $\quad \mathrm{Mm}=45$

$45 \mathrm{MJ}=3.6160$

$.56439100<+00$

$.63247000 \mathrm{E}+00$

$.90332700 t+00$

$.90704500 E+00$

$.99755000 \mathrm{E}+0 \mathrm{C}$

$\begin{array}{rrr}10 & 4 & 15 \\ 5 & 2 & 4\end{array}$

ig 410

1? 310

$\begin{array}{rrr}0 & 2 & 5 \\ 20 & 4 & 17\end{array}$

$13 \quad 310$

$\begin{array}{lll}7 & 2 & 5\end{array}$

$14 ; 11$

13311

$\begin{array}{lll}7 & 2 & 6\end{array}$

$\begin{array}{rrr}7 & 2 & 6 \\ 14 & 3 & 12 \\ 2 & 1 & 2\end{array}$

$\begin{array}{lll}15 & 1 & 2 \\ 15 & 13\end{array}$

$\begin{array}{rrr}15 & 2 & 0 \\ 10 & 3 & 13 \\ 9 & 2 & 7 \\ 3 & 1 & ?\end{array}$

$\begin{array}{rrr}0 & 2 & 7 \\ 10 & 3 & 14\end{array}$

$\begin{array}{rrr}9 & 2 & 0 \\ 3 & 1 & 3\end{array}$

1025

10315

$\begin{array}{rrr}10 & 3 & 15 \\ 1 & 2 & 9\end{array}$

$\begin{array}{lll}10 & 2 & 9\end{array}$

$143 \quad 16$

$\begin{array}{rrr}11 & 3 & 16 \\ 0 & 0 & 10\end{array}$

19316

$\begin{array}{rrr}5 & 1 & 4 \\ 12 & 2 & 10 \\ 20 & 3 & 17 \\ 0 & 1 & 5\end{array}$

$19 \quad 317$

$\begin{array}{rrr}5 & 1 & 5 \\ 12 & 2 & 11\end{array}$

2003 1e

$13 \quad 211$

$\begin{array}{lll}2 & 1 \\ 2 & 0 \\ 7 & 1 \\ 2 & 9 & 0\end{array}$

$13 \quad 212$

$\begin{array}{lll}1 & 1 & 1 \\ 7 & 1 & 1 \\ 1 & 1 & 0\end{array}$

14
13

$\begin{array}{ccc}15 & 2 & 1 \\ 3 & 1 & 3\end{array}$

$\begin{array}{lll}3 & 1 & 3 \\ 3 & 0 & 3\end{array}$

322

$.15012500 E+01$

$.15390500 E=01$

$.1629 b 150 E \rightarrow 0$

$.19076600 E+01$

.250024005401

.35571000 k40

-39634490E+01

. $461 \triangle 9700 E+01$

$.58777010 E+01$

$.50737130 k+01$

$.84712010 \mathrm{D}+01$

$.91313090 k+01$

.923656000401

$.13409370 \dot{0}+02$

$.15392700 E+02$

$.16322070 \mathrm{E}+02$

$19112420 E+02$

$.21207330 E+02$

$.219002605+02$

.230d1760t +02

$.26135730 E \div 02$

$.20616030 \mathrm{0}+02$

. 32290190t+02

.34663900E +02

$.40874910 t+02$

.42306070E+02

$.43020130 E+02$

$.43954470 \varepsilon+02$

$.447681908+02$

$.55254690 E+02$

$.56487150 E+02$

.63507 100E+02

$.63023200 E+D 2$
$.21501 E-17$

$.20<79 k-18$

$.49410 E=19$

$.90097 t-20$

$.52300 \mathrm{~b}-07$

$A=67676200 \mathrm{c}+02 \mathrm{~B}=11854250 \mathrm{E}+02 \mathrm{C}=1006 \mathrm{CO} 070 \mathrm{E}+02$ KAt $=-430$

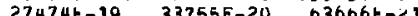

$10274 E-19$. 10507E-20 .34010E-2

$45520 \mathrm{E}-21.11400 \mathrm{E}-20 \quad .59270 \mathrm{E}-21$

$\begin{array}{rrr}.2 C \delta 43 E-20 & .17132 E-20 & .54637 E-21 \\ .27242 E-19 & .61600 E-20 & .14263 E-20\end{array}$

$.61320 \mathrm{k}-22$

$.17910 E-21$

$.12973 E-21$
$26837 E-21$

$12251 \mathrm{k}-0 \mathrm{~g}$

$.15417 \mathrm{E}=09$

$100043=0 y$

$.06416 \mathrm{E}-10$

$.37033 \dot{E}-0 y$

$.33337 E-20 \quad .40350 x-20 \quad .16314 E-20 \quad .43616 E-21$

$10000 k-19 \quad, 47400 \mathrm{k}-20 \quad .13725 \mathrm{k}-20$

.31051E-2

$.42700 \mathrm{E}-0 Y$

$.3317 \mathrm{dE}-U \mathrm{U}$

$10723 E=00$

$32490 E-19: 11109 t-19: 29662 t-20$

$33120 E-10.40149 E-19$.907t-5E-20

$.04337 \bar{E}-2$ ?

$430165-00$

$60075 \mathrm{E}-19 \quad .23957 \mathrm{E}-19 \quad .64616 \mathrm{E}-20$

$.10925 \mathrm{E}-20$

$.22705 E-00$

$.10770 \mathrm{E}-17$.2U420E-10 .30413t=19

$A=.00033020 t+02 \quad b=.11029500 k+02$ $.72504 \mathrm{E}-20 \quad .59001 \mathrm{E}-20 \quad .20420 \mathrm{E}-20$ $.42119 \mathrm{E}-10 \quad .44197 \mathrm{t}-19 \quad .79009 \mathrm{E}-20$ $: 30270 E-17$ :33032E-10 :0102UE-19

$529 y 0 \mathrm{E}-07$

$C=.10087990 \mathrm{E} * 02$ KAH $=-.9304$

$.52371 \mathrm{E}-29 \quad .41261 \mathrm{~b}-09$

$.14067 t-20 \quad .043 d 1 t-40$

$.70030 E-20 \quad .41047 \pm-07$

$.61622 E=07$

$A=.60633020 \mathrm{E}+02 \quad \mathrm{~d}=.11429560 \mathrm{E}+02$ $=13652 \mathrm{E}-19 \quad 15502 \mathrm{E}-20^{11029560 \mathrm{C}+02}$ $.20704 \mathrm{E}-19 \quad .46757 \mathrm{E}-20 \quad .10769 \mathrm{E}-20$

$C=.10007990 E+02 \quad$ XAP $=-\$ 9304$

$.51326 \mathrm{E}-22 \quad 11956 \mathrm{E}=09$

$.217 d 9 t-21 \quad . .5006 t-09$

$.73103 \mathrm{E}-20 \quad .32200 \mathrm{t}-20 \quad .93254 \mathrm{E}-21$

$A=.72716950 E+0 \mathrm{E} \quad \mathrm{E}=113 \% 3454 E+U 2$

$.60559 \mathrm{E}-27.01514 \mathrm{E}-2$ ? $33007 \mathrm{EAC1}$

$.20030 E-23$.95144E-22 .13003E-21

$.1047 t \mathrm{E}-20 \quad .17973 \mathrm{E}-20 \quad .81640 \mathrm{E}-21$

$.19019 t-23 \quad 1525 b E-21 \quad .24956 k-21$

$.20073 E-19 \quad .06477 E-20 \quad .15457 E-20$

$.21071 \mathrm{E}-2$

$.10737 \mathrm{E}-0 \mathrm{Y}$

$=.90339040 \mathrm{E}+01$

$.80629 \mathrm{E}-22$

$.64163 E-22$

$.23134 \mathrm{E}-21$

$13423 E-21$

$15000 E-23.22563 E-21.44852 E-21$

$.22541 E-10 \quad .25007 E-19 \quad .47609 E-20$

$.14244 \mathrm{E}-20 \quad .35348 \mathrm{EE}-20 \quad .18156 \mathrm{E}-20$

$.70054 E-19 \quad .19630 E-19 \quad .48540 E-20$

$.26568 \mathrm{E}-21$

$.86092 E-2$

$.54709 E-2$

$.10149 E-20$

$.169 d \Delta t-20 \quad .62879 t-20 \quad .30902 E-20$

$.13637 b-16 \quad .40555 E-19: .12450 E-19$

$.17945 E-20 \quad .10223 E-19$

$.10139 \mathrm{E}-17 \quad .12390 \mathrm{t}-10$

$.109195-20$

. $12390 \mathrm{t}-10 \mathrm{2} 260 \mathrm{E}-20$

$.23340 E-19$
$.12103 E-19$

$.110068-20$

$26904 E-20$

$239725-20$

$42583 E-20$

$.21573 E-16 \quad .94910 \mathrm{E}-19 \quad .27306 E-19 \quad .61850 \mathrm{E}-20$

$.14321 \mathrm{E}-20 \quad .21245 \mathrm{E}-19 \quad .19804 \mathrm{E}-19 \quad .00394 \mathrm{E}-20$

$.20927 E-18$. $10020 E=18$.53200E-19 .12b01E-19

$\begin{array}{llll}.24231 E-17 & .32574 E-10 & : 53300 E-19 & 11734 E-19 \\ .10731 E-20 & .37446 \mathrm{E}-19 & .30493 E-19 & .13513 \mathrm{E}-19\end{array}$

$.10731 E-20$

$.33550 \mathrm{~b}-18 \quad-26617 \mathrm{E}-18 \quad .93402 \mathrm{~b}=19$

$.75500 \mathrm{E}-21.33137 \mathrm{E}=19$ : $44344 \mathrm{E}=19$

$.23293 .18-19$

$.24627 \mathrm{E}-19$

$215,00 E=19$

$.39649 \mathrm{E}-19$

$339495=16$

$.38171 E-16$
$.34745 E-17$

$.02152 \mathrm{E}-16$

1 $1052 k=10$

$204707 \quad 00132$

$.47331 E-29 \quad .37495 k-19 \quad .61093 E-19$

$.59272 E-17$.1059LA+N $.22634 \mathrm{E}=16$

$.30055 \mathrm{E}-1 \mathrm{O} \quad .50170 . .19$

$26909 E-21$

$.50170 . .-10$

$.22302 \mathrm{E}-18$

. 327 38E-19

$.43991 E-19$

$.71556 \mathrm{E}-17$

$15457 E=17$

$.35164 E-10$

$47004 \mathrm{t}-19$

$.70579 E-19$

$\begin{array}{ll}.24905 E-18 & .60909 t-18 \\ .14452 E-19 & .17551 E-16\end{array}$

$.14452 E-19$

$.31163 \mathrm{E}=10$

$.9365 d \varepsilon \times 19$

$23910 E-15$.26640E-16

$.70264 \mathrm{E}-17$

$.20648 E-16$

$32974 \mathrm{E}-17$

$.16527 E-15$

$.20205 E-16$

. 5035 1L-10

$.873936-10$

$.09411 \mathrm{E}-1 \mathrm{~d}$

$.10232 \mathrm{E}-10$.09109E-16 .400U1E-16 .13220E-16

$.73725 E=17.25359 E-17$.07592E-10 .14672E-10

$.12099 k=18 \quad .73492 t-18 \quad .50873 k-10 \quad .17796 k-16$

$.49007 \mathrm{E}=15$.00151E-16 .12799E-16 .23672E-17

$.09130 t-15$. $84410 E-16$.15067E-10 .20470E-T7

$.2123 \mathrm{kE}-15 \quad .37052 \mathrm{E}-16 \quad .70639 \mathrm{E}-17 \quad .15220 \mathrm{E}-17$

$.22222 \mathrm{E}-1$

$.19005 \mathrm{E}-1$

$.97615 t-11$

$.67009 \mathrm{e}-11$

$.200602=10$

$.21130 k-10$

$.27704 \mathrm{E}-09$

- $37423 t=10$

. 11299E-09

$.62337 \mathrm{k}-10$

+ $12701 \mathrm{c}=09$

$.40301 E-09$

- $39492 \mathrm{~L}-09$

$.24950 E=00$
$.11169 E=00$

$.106246=00$

$.291708-00$

$.49902 \mathrm{t}-00$

$.99954 \mathrm{E}=0 \mathrm{C}$

$.70902 E=0 d$

$.12904 E-07$

.27709E-07

$.10117 \mathrm{e}-07$

$.30273 E-67$

. $4 d 3 \partial 7 t=06$

. 3442ठE-07

$.62485 E-07$

$.64269 t-07$

$.693 d 2 t=07$

. $12251 E-00$

. 12506E-06

. $31100 \mathrm{E}-05$

$.40352 \mathrm{E}-05$

$21790 \mathrm{t}-00$

. 30772E-05

$.22904 E-00$

$36053 E-06$

-34506E-00

$.13351 \mathrm{t}=04$

.10701 to-04

$.93322 \mathrm{t}=03$ 


\begin{tabular}{|c|c|c|c|c|c|c|c|c|c|c|c|}
\hline \multirow{2}{*}{\multicolumn{2}{|c|}{${ }_{K U}^{J U}$}} & \multirow{2}{*}{\multicolumn{3}{|c|}{$\mathrm{JL}_{\text {KL }}$}} & \multirow{2}{*}{ KLL } & \multirow[t]{2}{*}{ NU } & \multicolumn{4}{|c|}{ SI UKA } & \multirow[t]{2}{*}{$n$} \\
\hline & & & & & & & 20 & 50 & 100 & $\angle O U$ & \\
\hline $\begin{array}{r}3 \\
3 \\
9 \\
16 \\
4\end{array}$ & $\begin{array}{l}2 \\
1 \\
1 \\
2 \\
1\end{array}$ & $\begin{array}{r}1 \\
2 \\
8 \\
14 \\
4\end{array}$ & $\begin{array}{r}2 \\
2 \\
9 \\
16 \\
3\end{array}$ & $\begin{array}{l}2 \\
1 \\
1 \\
2 \\
1\end{array}$ & $\begin{array}{r}0 \\
1 \\
9 \\
15 \\
3\end{array}$ & $\begin{array}{l}.63737000 E+02 \\
.05913100 E+02 \\
.00949630 \mathrm{0}+02 \\
.69829620 \mathrm{0}+02 \\
.81693540 \mathrm{t}+02\end{array}$ & $\begin{array}{l}.21300=-15 \\
.50399 \varepsilon-15 \\
.63873 \varepsilon-17 \\
.73412 E-19 \\
.10230 E-14\end{array}$ & $\begin{array}{l}.37210 c=10 \\
.756100-10 \\
.29414 E-17 \\
.73100=-16 \\
.15277 c-15\end{array}$ & $\begin{array}{l}.70 y 17 c-17 \\
14737 E-10 \\
.00174 E-10 \\
.00412 c-10 \\
.30741 E-10\end{array}$ & $\begin{array}{l}.15<0 v e=17 \\
.27312=17 \\
17411=-10 \\
.229032=10 \\
.2747+2=17\end{array}$ & 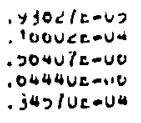 \\
\hline $\begin{array}{r}10 \\
4 \\
17 \\
4\end{array}$ & $\begin{array}{l}1 \\
0 \\
2 \\
2 \\
3\end{array}$ & $\begin{array}{r}9 \\
4 \\
15 \\
3 \\
2\end{array}$ & $\begin{array}{r}10 \\
3 \\
17 \\
3 \\
3\end{array}$ & $\begin{array}{l}1 \\
0 \\
2 \\
2 \\
3\end{array}$ & $\begin{array}{r}10 \\
3 \\
16 \\
2 \\
1\end{array}$ & $\begin{array}{l}.04073760 c+02 \\
.04542400 \mathrm{0}+02 \\
.04779000 c+02 \\
.84007940 E+02 \\
.04009150 E+02\end{array}$ & $\begin{array}{l}.51279 E-97 \\
.13332 E-114 \\
.40940 E-19 \\
.55391 E-15 \\
.15357 E-15\end{array}$ & $\begin{array}{l}.32349 E-17 \\
.10305 E-15 \\
.70313 E-16 \\
.10932 t-15 \\
.47413 E-16\end{array}$ & $\begin{array}{l}.10520 E-17 \\
.300350-10 \\
.000296-10 \\
.24140 E-10 \\
.12150 \mathrm{E}-10\end{array}$ & $\begin{array}{l}.292448-10 \\
.670772-17 \\
.200272-10 \\
.47114 E-17 \\
.25004=-17\end{array}$ & 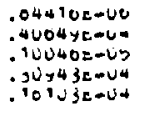 \\
\hline $\begin{array}{r}4 \\
4 \\
11 \\
11\end{array}$ & $\begin{array}{l}3 \\
2 \\
1 \\
1 \\
2\end{array}$ & $\begin{array}{r}1 \\
2 \\
3 \\
10 \\
16\end{array}$ & $\begin{array}{r}3 \\
3 \\
3 \\
11 \\
18\end{array}$ & $\begin{array}{l}3 \\
2 \\
1 \\
1 \\
2\end{array}$ & $\begin{array}{r}0 \\
1 \\
2 \\
11 \\
17\end{array}$ & $\begin{array}{l}.64891150 \mathrm{t}+02 \\
.05093360 E+02 \\
.07840930 E+02 \\
.10057240 E+03 \\
.10129930 t+03\end{array}$ & $\begin{array}{l}.15350 E-15 \\
.55700 E-15 \\
.11409 E-14 \\
.30005 E-17 \\
.21094 E-19\end{array}$ & $\begin{array}{l}.47415 t-16 \\
.11001 t-15 \\
.17400 E-15 \\
.33940 t-17 \\
.63090 z-10\end{array}$ & $\begin{array}{l}.12156 \mathrm{E}-10 \\
.24304 \mathrm{t}-10 \\
.35206 \mathrm{t}-1 \mathrm{t} \\
.1239 j \mathrm{t}-17 \\
.75557 \mathrm{E}-10\end{array}$ & $\begin{array}{l}.25003 E-17 \\
.40031 \mathrm{E}-17 \\
.00790 \mathrm{c}-17 \\
.31443 \mathrm{~J}-15 \\
.34347 \mathrm{e}-10\end{array}$ & $\begin{array}{l}.10104 E-44 \\
.31257 E-64 \\
.4<400 E-44 \\
.12104 E-67 \\
.15002 E-U 5\end{array}$ \\
\hline $\begin{array}{l}5 \\
5 \\
5 \\
5 \\
5\end{array}$ & $\begin{array}{l}1 \\
0 \\
2 \\
4 \\
4\end{array}$ & $\begin{array}{l}5 \\
5 \\
4 \\
2 \\
1\end{array}$ & $\begin{array}{l}4 \\
4 \\
4 \\
4 \\
4\end{array}$ & $\begin{array}{l}1 \\
0 \\
2 \\
4 \\
4\end{array}$ & $\begin{array}{l}4 \\
4 \\
3 \\
1 \\
0\end{array}$ & $\begin{array}{l}10200440 E * 03 \\
.10546430 E * 03 \\
105972702+03 \\
106106106+03 \\
.10010820 E+03\end{array}$ & $\begin{array}{l}.10000 E-14 \\
.20126 E-14 \\
.938 d 1 E-15 \\
.07303 E-16 \\
.67303 E-16\end{array}$ & $\begin{array}{l}.27071 t-15 \\
.32307 t=15 \\
.21503 E-15 \\
.45320 t-16 \\
.45320 E-10\end{array}$ & $\begin{array}{l}.50470 \mathrm{~b}-10 \\
.00013 \mathrm{c}-10 \\
.50163 \mathrm{c}-10 \\
.15009 \mathrm{e}-16 \\
.15003 \mathrm{t}-10\end{array}$ & $\begin{array}{l}.113032-10 \\
127102-10 \\
.10400=-10 \\
.305322-17 \\
.30532 e-17\end{array}$ & 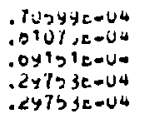 \\
\hline $\begin{array}{r}5 \\
5 \\
5 \\
5 \\
12\end{array}$ & $\begin{array}{l}3 \\
3 \\
2 \\
1 \\
1\end{array}$ & $\begin{array}{r}3 \\
2 \\
3 \\
4 \\
11\end{array}$ & $\begin{array}{r}4 \\
4 \\
4 \\
12\end{array}$ & $\begin{array}{l}3 \\
3 \\
2 \\
1 \\
1 \\
1\end{array}$ & $\begin{array}{r}2 \\
1 \\
2 \\
3 \\
12\end{array}$ & $\begin{array}{l}10613460 E+03 \\
10614160 t+03 \\
10054100 t+03 \\
.10975360 E+03 \\
110370 d 0 t+03\end{array}$ & $\begin{array}{l}.34022 t-15 \\
.34020=-15 \\
.94065 E-15 \\
.17513 t-14 \\
.20300=-17\end{array}$ & $\begin{array}{l}.12239 \mathrm{E}-15 \\
.12241 \mathrm{E}-15 \\
.2174 \mathrm{E}=15 \\
.3229 \mathrm{E}-15 \\
.34102 \mathrm{E}-17\end{array}$ & $\begin{array}{l}.33024 t-10 \\
.33020 L-16 \\
.51679 t-16 \\
.66074 E-16 \\
.14141 t-17\end{array}$ & $\begin{array}{l}.72125 E-17 \\
.72134 \mathrm{E}-17 \\
.102756-10 \\
.12990 E-70 \\
.30253 \mathrm{i}=10\end{array}$ & 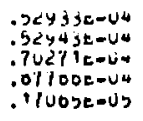 \\
\hline $\begin{array}{r}19 \\
6 \\
6 \\
6 \\
6\end{array}$ & $\begin{array}{l}2 \\
1 \\
0 \\
2 \\
5\end{array}$ & $\begin{array}{r}17 \\
6 \\
6 \\
5 \\
2\end{array}$ & $\begin{array}{r}19 \\
5 \\
5 \\
5 \\
5\end{array}$ & $\begin{array}{l}2 \\
1 \\
0 \\
2 \\
5\end{array}$ & $\begin{array}{r}18 \\
5 \\
5 \\
4 \\
1\end{array}$ & $\begin{array}{l}11933230 E+03 \\
.12240200 E+03 \\
.12624700 E+03 \\
.12711290 E+03 \\
.12733040 E+03\end{array}$ & $\begin{array}{l}10000 E-19 \\
.20054 E-14 \\
.25540 E=14 \\
.12040 E-14 \\
.19020 E-16\end{array}$ & $\begin{array}{l}.55201 E-16 \\
.42997 t-15 \\
.49192 E-15 \\
.34900 E-15 \\
.34394 E-16\end{array}$ & $\begin{array}{l}.00200 t-10 \\
.90304 E-16 \\
.10777 E=1 b \\
.00217 E-10 \\
.15097 t-10\end{array}$ & $\begin{array}{l}.40551 E-10 \\
.19102 E-10 \\
.21207 E-10 \\
.14017 E-10 \\
.45449 E-17\end{array}$ & 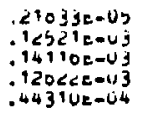 \\
\hline $\begin{array}{l}6 \\
6 \\
6 \\
6 \\
6\end{array}$ & $\begin{array}{l}5 \\
4 \\
4 \\
3 \\
3\end{array}$ & $\begin{array}{l}1 \\
3 \\
2 \\
4 \\
3\end{array}$ & $\begin{array}{l}\mathbf{5} \\
\mathbf{5} \\
5 \\
5 \\
5\end{array}$ & $\begin{array}{l}5 \\
4 \\
4 \\
3 \\
3\end{array}$ & $\begin{array}{l}0 \\
2 \\
1 \\
3 \\
2\end{array}$ & $\begin{array}{l}.12733040 E * 03 \\
.12734060 E+03 \\
.12734040 E * 03 \\
.12739380 E+03 \\
.12741240 E \bullet 03\end{array}$ & $\begin{array}{l}19020 E-16 \\
13230 E-15 \\
.13236 E=15 \\
.50707 E-15 \\
.50770 E=15\end{array}$ & $\begin{array}{l}.34394 \mathrm{E}-16 \\
.10699 \mathrm{E}-15 \\
.10699 \mathrm{E}=15 \\
.21942 \mathrm{E}=15 \\
.21947 \mathrm{E}=15\end{array}$ & $\begin{array}{l}.15097 \mathrm{t}-10 \\
.37012 E=10 \\
.37013 E=16 \\
.0293 d E-16 \\
.62955 t-16\end{array}$ & $\begin{array}{l}.45441 E-17 \\
.94512 E-17 \\
.94513 E-17 \\
.14172 E-16 \\
.14176 E-16\end{array}$ & $\begin{array}{l}.44310 t-U 4 \\
.00597 E-04 \\
.00590 \mathrm{E}-04 \\
.10092 \mathrm{E}-63 \\
.10097 \mathrm{E}-03\end{array}$ \\
\hline $\begin{array}{r}6 \\
6 \\
13 \\
20 \\
7\end{array}$ & $\begin{array}{l}2 \\
1 \\
1 \\
2 \\
1\end{array}$ & $\begin{array}{r}4 \\
5 \\
12 \\
18 \\
7\end{array}$ & $\begin{array}{r}5 \\
5 \\
13 \\
20 \\
6\end{array}$ & $\begin{array}{l}2 \\
1 \\
1 \\
2 \\
1\end{array}$ & $\begin{array}{r}3 \\
4 \\
13 \\
19 \\
6\end{array}$ & 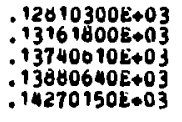 & $\begin{array}{l}.12777 E-14 \\
.22312 E-14 \\
.16953 t-17 \\
.44640 E-20 \\
.23652 E=14\end{array}$ & $\begin{array}{l}.35379 E-15 \\
.40195 E-15 \\
.33087 E-17 \\
.40454 E-18 \\
.59595 E-15\end{array}$ & $\begin{array}{l}.67464 \mathrm{E}-16 \\
.1097 \mathrm{E}-16 \\
.15087 \mathrm{t}-17 \\
.0277 \mathrm{EE}-18 \\
.14376 \mathrm{E}-15\end{array}$ & $\begin{array}{l}.16291 E-16 \\
.22007 t=16 \\
.45413 E-10 \\
.46455 E-10 \\
.29614 t-16\end{array}$ & $\begin{array}{l}.13124 t=03 \\
.15507 \mathrm{E}=03 \\
.23303 \mathrm{t}-05 \\
.30742 \mathrm{E}=05 \\
.20212 \mathrm{E}-03\end{array}$ \\
\hline $\begin{array}{l}7 \\
7 \\
7 \\
7 \\
7\end{array}$ & $\begin{array}{l}0 \\
2 \\
0 \\
6 \\
5\end{array}$ & $\begin{array}{l}7 \\
6 \\
2 \\
1 \\
3\end{array}$ & $\begin{array}{l}6 \\
6 \\
6 \\
6 \\
6\end{array}$ & $\begin{array}{l}0 \\
2 \\
6 \\
6 \\
5 \\
1\end{array}$ & $\begin{array}{l}0 \\
5 \\
1 \\
0 \\
2\end{array}$ & $\begin{array}{l}14607100 E+03 \\
114022340 E+03 \\
11455440 E+03 \\
.14855640 E+03 \\
.14656730 E+03\end{array}$ & $\begin{array}{l}.26340 \mathrm{E}-14 \\
.14513 \mathrm{E}-14 \\
.35608 \mathrm{E}-17 \\
.35008 \mathrm{E}-17 \\
.33690 \mathrm{E}-16\end{array}$ & $\begin{array}{l}.67424 E-15 \\
.49600 E-15 \\
.21337 E-16 \\
.21337 \mathrm{E}-16 \\
.75924 E-16\end{array}$ & $\begin{array}{l}.15050 \mathrm{E}-15 \\
.13159 \mathrm{E}-15 \\
14703 \mathrm{E}-16 \\
.14703 \mathrm{E}-10 \\
.37007 \mathrm{E}-16\end{array}$ & $\begin{array}{l}.32311 E-16 \\
.20496 \mathrm{t}-10 \\
.51340 \mathrm{t}=17 \\
.51316 \mathrm{t}-17 \\
.11164 \mathrm{E}-17\end{array}$ & $\begin{array}{l}.22402 \mathrm{E}-U 3 \\
.21237 \mathrm{t}-03 \\
.04770 \mathrm{E}-04 \\
.01778 \mathrm{t}-04 \\
.11400 \mathrm{U}-0 \mathrm{~s}\end{array}$ \\
\hline $\begin{array}{l}7 \\
7 \\
7 \\
7\end{array}$ & $\begin{array}{l}5 \\
4 \\
4 \\
3 \\
3\end{array}$ & $\begin{array}{l}2 \\
4 \\
3 \\
5 \\
4\end{array}$ & $\begin{array}{l}6 \\
6 \\
6 \\
6 \\
6\end{array}$ & $\begin{array}{l}5 \\
5 \\
4 \\
3 \\
3\end{array}$ & $\begin{array}{l}1 \\
3 \\
2 \\
4 \\
3\end{array}$ & $\begin{array}{l}14856730 E * 03 \\
114859940 E * 03 \\
114859580 E * 03 \\
14866760 E * 03 \\
114870930 E * 03\end{array}$ & $\begin{array}{l}.33690 E-16 \\
.17640 \mathrm{E}-15 \\
.17840 \mathrm{E}-15 \\
.61440 \mathrm{E}-15 \\
.61464 \mathrm{E}-15\end{array}$ & $\begin{array}{l}.75924 E-16 \\
.17803 E-15 \\
.17863 E-15 \\
.32896 E-15 \\
.32912 E-15\end{array}$ & $\begin{array}{l}.37607 k-16 \\
.67000 E-16 \\
.07800 E-16 \\
.70734 E-15 \\
.10140 E-15\end{array}$ & $\begin{array}{l}11164 t-10 \\
.17562 E=10 \\
.17562 t=16 \\
.23050 t=-36 \\
.23662 t=10\end{array}$ & $\begin{array}{l}.11400 E-03 \\
.15090 \mathrm{E}-03 \\
15090 \mathrm{E}-03 \\
.14051 \mathrm{E}-03 \\
.19067 \mathrm{t}-03\end{array}$ \\
\hline $\begin{array}{c}7 \\
7 \\
14 \\
8 \\
8\end{array}$ & $\begin{array}{l}2 \\
1 \\
1 \\
1 \\
0\end{array}$ & $\begin{array}{r}5 \\
6 \\
13 \\
8 \\
8\end{array}$ & $\begin{array}{r}6 \\
6 \\
14 \\
7\end{array}$ & $\begin{array}{l}2 \\
1 \\
1 \\
1 \\
0\end{array}$ & $\begin{array}{r}4 \\
5 \\
7 \\
7\end{array}$ & $\begin{array}{l}149792806+03 \\
.15343240 \mathrm{0}+03 \\
.15754800 \mathrm{E}+03 \\
.16295690 \mathrm{0}+03 \\
.16732090 \mathrm{E}+03\end{array}$ & $\begin{array}{l}.140968-14 \\
.24657 E-14 \\
.10232 E-17 \\
.23950 E-14 \\
.28116 E-14\end{array}$ & $\begin{array}{l}.50493 E-15 \\
.66434 E-15 \\
.30931 E=17 \\
.76700 E-15 \\
.85122 E-15\end{array}$ & $\begin{array}{l}13417 E-15 \\
.16279 E-15 \\
.16960 E-17 \\
19908 E-15 \\
.21665 E-15\end{array}$ & $\begin{array}{l}.29079 t-16 \\
.330 d 1 E-16 \\
.52030 E-10 \\
.42645 t-16 \\
.40010 E-10\end{array}$ & $\begin{array}{l}.2191 j e-03 \\
.25121 t=03 \\
.31132 t=05 \\
.30497 \varepsilon=03 \\
.33475 t-03\end{array}$ \\
\hline $\begin{array}{l}8 \\
8 \\
0 \\
8 \\
8\end{array}$ & $\begin{array}{l}2 \\
2 \\
7 \\
7 \\
6\end{array}$ & $\begin{array}{l}7 \\
7 \\
2 \\
1 \\
3\end{array}$ & $\begin{array}{l}7 \\
7 \\
7 \\
7\end{array}$ & $\begin{array}{l}2 \\
2 \\
7 \\
7 \\
6\end{array}$ & $\begin{array}{l}6 \\
6 \\
1 \\
0 \\
2\end{array}$ & $\begin{array}{l}16929950 E+03 \\
.16929950 E+03 \\
16976650 E+03 \\
.16978650 E+03 \\
16979130 E+03\end{array}$ & $\begin{array}{l}.14710 E-14 \\
.14710 E-14 \\
.44968 E-18 \\
.44968 E-18 \\
.50319 E-17\end{array}$ & $\begin{array}{r}.64171 E-15 \\
.64171 E-15 \\
.11010 E-16 \\
.110105-16 \\
.44627 E-16\end{array}$ & $\begin{array}{l}.16464 E-15 \\
.18464 E-15 \\
.12129 E-16 \\
.12129 E-16 \\
.33364 E-16\end{array}$ & $\begin{array}{l}.41641 \mathrm{k}-16 \\
.41641 \mathrm{E}-16 \\
.53524 \mathrm{E}=17 \\
.53524 \mathrm{E}-17 \\
.12129 E=16\end{array}$ & $\begin{array}{l}.32574 \mathrm{E}-03 \\
.32574 \mathrm{E}-03 \\
.02161 \mathrm{E}=04 \\
.02101 \mathrm{E}-04 \\
.15330 \mathrm{E}=03\end{array}$ \\
\hline $\begin{array}{l}8 \\
8 \\
8 \\
8 \\
8 \\
0\end{array}$ & $\begin{array}{l}6 \\
5 \\
5 \\
4 \\
4\end{array}$ & $\begin{array}{l}2 \\
4 \\
3 \\
5 \\
4\end{array}$ & $\begin{array}{l}7 \\
7 \\
7 \\
7 \\
7\end{array}$ & $\begin{array}{l}6 \\
5 \\
5 \\
4 \\
4\end{array}$ & $\begin{array}{l}1 \\
3 \\
2 \\
4 \\
3\end{array}$ & $\begin{array}{r}169791308+03 \\
.16981130 E+03 \\
.169811300 * 03 \\
.169062008+03 \\
.16906300 E+03\end{array}$ & $\begin{array}{r}.54379 E-17 \\
.41867 E-16 \\
.418 d 7 E-16 \\
.19733 t-15 \\
.19733 E-15\end{array}$ & $\begin{array}{l}.44627 E-16 \\
.11902 E-15 \\
.11982 E-15 \\
.25236 E-15 \\
.25236 E=15\end{array}$ & $\begin{array}{l}.33364 E-16 \\
.64526 E=10 \\
.64526 E-16 \\
.10392 E-15 \\
.10392 E=15\end{array}$ & $\begin{array}{l}12129 E-16 \\
.19909 t-16 \\
.19944 t-16 \\
.28039 t-16 \\
.20039 t-16\end{array}$ & $\begin{array}{l}.15330 t-03 \\
.21371 \mathrm{t}-03 \\
.21371 \mathrm{E}-03 \\
.20326 \mathrm{t}=03 \\
.20327 t=03\end{array}$ \\
\hline $\begin{array}{r}9 \\
8 \\
8 \\
8 \\
15\end{array}$ & $\begin{array}{l}3 \\
3 \\
2 \\
1 \\
1 \\
1\end{array}$ & $\begin{array}{r}6 \\
5 \\
6 \\
7 \\
14\end{array}$ & $\begin{array}{r}7 \\
7 \\
7 \\
7 \\
15\end{array}$ & $\begin{array}{l}3 \\
3 \\
2 \\
1 \\
1\end{array}$ & $\begin{array}{r}5 \\
4 \\
5 \\
6 \\
15\end{array}$ & $\begin{array}{l}16995620 \mathrm{1}+03 \\
: 17003950 \mathrm{E}+03 \\
117162110 \mathrm{~L}+03 \\
17518570 \mathrm{E}+03 \\
: 17868290 \mathrm{0}+03\end{array}$ & $\begin{array}{l}.6423 d E-15 \\
.64277 E-15 \\
.14966 E-14 \\
.24237 E-14 \\
.57971 E-16\end{array}$ & $\begin{array}{l}.43935 E-15 \\
.43971 E-15 \\
.65577 \mathrm{E}-15 \\
.84050 \mathrm{E}-15 \\
.27974 \mathrm{E}-17\end{array}$ & $\begin{array}{l}.14686 \mathrm{E}-15 \\
.14699 \mathrm{E}-15 \\
.10922 \mathrm{E}=15 \\
.22403 \mathrm{E}-15 \\
.17934 \mathrm{E}-17\end{array}$ & $\begin{array}{l}.35699 \mathrm{E}-16 \\
.35732 \mathrm{E}-16 \\
.42735 \mathrm{E}-16 \\
.48629 \mathrm{E}-16 \\
.60370 \mathrm{E}-10\end{array}$ & $\begin{array}{l}.30215 t-03 \\
.30259 t-03 \\
.33935 t-03 \\
.3705 t-03 \\
.40803 t-05\end{array}$ \\
\hline
\end{tabular}


JU WL ALE ALTALE

nu

ن

50

SILIMA

tou

200

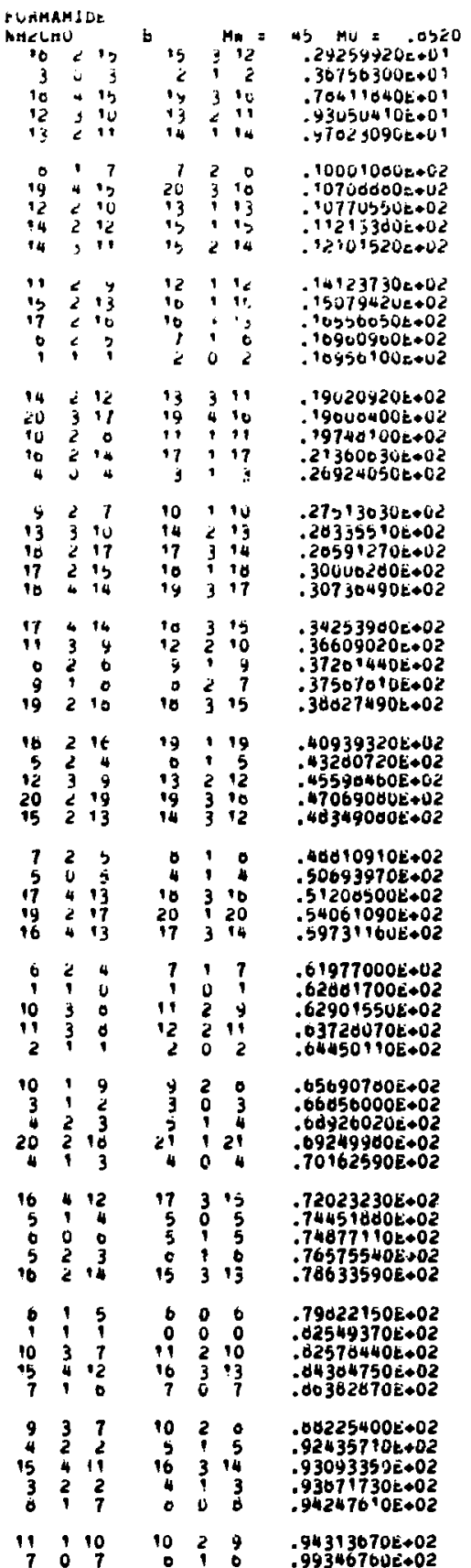

$A=.72710950 E+U \angle E=.11373454 E+U L$ $.4044 ! k-22=4 \angle 202 k-2 !$ $45542 c=19$ $.1007 \mathrm{e}-22$ $.30202 c-20$ $.1030+6-20$

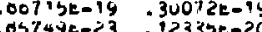
$.47344 c-20 \quad .70902 t-20$ $100 \mathrm{cos}-20$.jto $.10020 E-20,94003 k-20$

$.1011 \mathrm{gte}-19,10011 \mathrm{t}-19$ $.77030 \mathrm{c}-21,47994 \mathrm{cos}$ $.04050 \mathrm{~L}-\mathrm{Z}^{\prime} .90240 \mathrm{E}=20$ $.20055 E-10 \quad .79000 \mathrm{k}-19$ $.05022 \mathrm{t}-10.75300 \mathrm{t}-\mathrm{tg}$

$.80019 t-20$. $33365 t-19$ $.20041 \mathrm{t}-22.42307 \mathrm{t}-20$ $.57043 \mathrm{E}-19 \quad+45732 \mathrm{E}-19$

$.55702 \mathrm{c}-21.06067 \mathrm{E}-20$ - 3u7y15-17 $.42460 \mathrm{E}-10$

$.10049 k-10$ $17007 \mathrm{E}-10$ $.70544 \mathrm{E}-21$ $42032 t-2 !$

$52855 \mathrm{E}-21$ $90795 t-19$ $.90795 t-19$ $.5330,3 t-1 d$ $.55290 \mathrm{t}-21$

$.2 / 123 t-21$ $19201 \mathrm{E}-17$ . $2757 E-19$ $29070 \varepsilon-2$ .20015t-19

$12095 \mathrm{E}-17$ .11677L-16 $.1561 k-20$ $15329 t-21$ $.35404 \mathrm{t}-20$

$.20457 \mathrm{~L}-17$ $.20859 E=16$ $.43701 E-1 d$ $.27247 \mathrm{Ee} 1 \mathrm{~d}$ $.32409 \mathrm{t}-10$

10900e-1d $.053450-19$ . $20552 \mathrm{c}-19$ $.73200 \mathrm{x}-20$

$24304 t=19$ $.16591 \mathrm{E}-16$ . ¿3405E-10 $.43002 \mathrm{t}-18$ $.2560^{\circ} \mathrm{E}-19$

$.82153 z-20$ $.44329 E-10$ .20537 ílo $.20537 E-18$ $.24644 E-19$
$.7503 E-18$

$.44303 E-10$ 19745t-1? $.533405-19$ $.8452 ? t-20$ $.90075 \mathrm{t}-19$

\section{$.73257 \mathrm{E}-1 \mathrm{H}$} $.239478-17$ $.53551 \mathrm{k}-1 \mathrm{~d}$ $.46766 \mathrm{E}-18$ -38694t-17

20330E-17 $.40903 E-16$ .44297E-17 $.77500 e-22$ $.45766 \mathrm{E}-16$

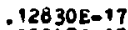
550 U ह-17 $.67422 \mathrm{t}-16$ Buzote-zo $.696265-17$

5072 1E-20 - 460095-16 $24309 t-16$ $45817 \mathrm{k}-17$ $.30444 E-19$

$.1380456=10$ $.03647 \mathrm{E}-17$ . $46959 \mathrm{t}-17$ -10549E-1?

$44490 \mathrm{E}-16$ 24065E- 16 .717 16E-18 $.140716-19$

$091145-17$ $.27554 E-17$ $.07032 E-10$ $.24577 \mathrm{E}-10$

$.06732=16$

$12296 \mathrm{bi}-17$ $656825-17$ $176798-19$ $.61599 \mathrm{E}-17$ 11079E-17 1397 1E-!7 $29026 E-10$ 10759E-17 $33147 E_{-16} .11495 E-10$

$.27003 k-17.24654 E-17$ $.37041 \mathrm{t}-16 \quad .095515-17$
$.33411 E-C^{\prime}$

$10405 \mathrm{c}-20$

$.15543 \mathrm{t}-2 \mathrm{O}$

$.40042 \mathrm{e}-2$

$.22055 t-20$

$.8 u ̈ 154 \mathrm{k}-20$ $24520 E-2 U$

$.34344 t-2 \downarrow$ $.23503 t-20$ $64155 \mathrm{E}-20$

$70914 E-20$ . 32044t-20 . $423116-20$ $19001 E-19$ $.13064 k=19$

$14737 \mathrm{E}-19$ $.04845 E-20$ $.10002 \mathrm{c}-19$ $.49179 \mathrm{E}-20$ $.03225 k-14$

$34704 t-19$ - jo37ok-19 $231468-19$ $716552=20$ $.23197 \mathrm{k}-19$

$33143 E-19$ $.75327 \mathrm{t}-19$ . 12622E-10 .34900E-19

$.97155 t-20$ $.10303 E-1$ .1054ठE-1O $.41073 E-1$ .12116E-18

$11772 t-10$ . 36649E-10 .72500 t-19 $.12200 E-19$ 110?5t-10

. $16115 E-10$ . $44150 E-10$ .21730t- 10 $.21244 k-18$ $.74742 E-10$

$.41745 t-18$ $.10702 \mathrm{~L}-17$ $.193108-18$ $.14306 \mathrm{E}-19$ $.14155 E ̈-17$

15070E- 10 .17675E-17 - 0287 - 17 $.24528 E-18$ $.29990 t-18$

. 1 1079E-17 $.50762 E-10$ .3505 IE-10 $.2305: 2=10$

. 405960-? .20656E-18 $.20763 E-10$ . $220165-10$ $.30639 E-17$

$.90747 t-40$ .21US2 $E-17$ $.07760 \mathrm{E}-19$
$.12499 E-29$

19000k-Ź?

$03571 E-21$

$.14652=-20$

.60ty

$.17399 t-20$

$14535 t-20$

$.90446 t-a 1$

$.70196 \mathrm{E}-2$ ?

. $22203 E-20$

$.10700 \mathrm{t}-20$

$.11419 r-20$

- $37623 E-20$

प1 $29 E-20$

$.63623 \mathrm{t}-20$

. $505108-20$

$.40021 E-20$

TUT OE-2O

$.15492=-19$

$.2109 E=20$

. $12301 \mathrm{E}=19$

$.10329 E-15$

? o0ob-20

$.6253 \mathrm{t}-19$

$21340 E-19$

15305 k-19

$.207246-19$

$.171760-19$

$44422 E-20$

20003E- 19

31765E-19

$.22294 E-19$

$.42382 E-19$

$.25514 k-19$

$.73780 t-19$

355 Y7E-19

$616705-20$

$.497006-19$

- $37873 E-19$

79703t-1Y

5230 TE 19

$.60199 \mathrm{t}-19$

. $3636 \mathrm{k}-10$

. M0T2E-18

$158375-12$

d020 3 - 20

$.26797 E-18$

.7130 UE- 19

$.34741 E-10$

$.20245 E-18$

$.49729 \mathrm{E}-19$

$43089 t i-10$

$.91606 E-19$

$.95499 E-10$

$90033 E-19$

$10332 E-16$

$.56631 \mathrm{t}-19$

.19916E-18

$.44177 E-19$

$.66500 \mathrm{c}-10$

$23055 \mathrm{E}-10$

$.42915 \mathrm{E}-10$

20470700832 $.14440 \mathrm{E}-10$ $.63072 k-10$ $.33105 E-05$ $.37209 t-0 y$ $.260 \mathrm{OOt}=0 \mathrm{y}$

$.77302 t-69$ $.02400 e-19$ 3310 ź .11160 e-00

$.10556 t-v o$ $.07674 \mathrm{t}-0 \mathrm{y}$ . $25203 E-00$ -39U55t-00 $.99507 t-00$

$.47146 E-00$ $.50102 \dot{c}-60$ $.32000 \mathrm{t}-00$ $.10047 t=0 a$ - 3004JE-U?

$.97975 t-00$ 14553k-07 . 12404k-07 .37020 E

$27100 \mathrm{~L}-\mathrm{U} 7$ .33516 te 07 $8.0307 \mathrm{t}-07$ $.45015 E-0$ ? $.29230 \mathrm{~b}-07$

.70270E-00 $50005 E-0 ?$ 


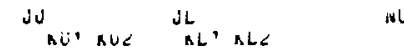

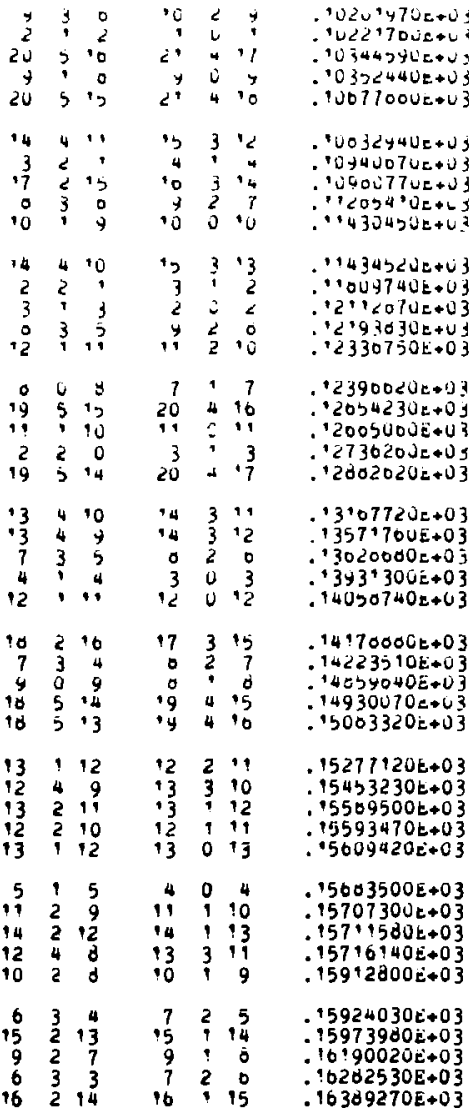

$\begin{array}{rrrrrr}8 & 2 & 6 & 0 & 1 \\ 7 & 2 & 5 & 7 & 1 & 6 \\ 7 & 2 & 5 & 7 & 1 & 6 \\ 17 & 2 & 5 & 17 & 1 & 16 \\ 17 & 5 & 3 & 18 & 4 & 14\end{array}$

$\begin{array}{llllll}7 & 5 & 3 & & 9 & 9 \\ 6 & 2 & 4 & 6 & 1\end{array}$

$\begin{array}{rrrrrr}6 & 2 & 4 & 6 & 1 & 5 \\ 17 & 5 & 12 & 18 & 4 & 15 \\ 14 & 1 & 13 & 14 & 0 & 14 \\ 10 & 0 & 10 & 9 & 1 & 9 \\ 6 & 1 & 6 & 5 & 0 & 5\end{array}$

$\begin{array}{rrrrrr}19 & 2 & 17 & 18 & 3 & 10 \\ 5 & 2 & 3 & 5 & 1 & 4 \\ 11 & 4 & 8 & 12 & 3 & 9 \\ 10 & 2 & 16 & 10 & 1 & 17 \\ 11 & 4 & 7 & 12 & 3 & 10\end{array}$

$4 \quad 2 \quad 2$

4

$10960300 \mathrm{E}+0$

$.17177060 \dot{E}+03$

$.17238120 k+03$

$.7270 .40 \mathrm{k}+03$

$.17310330 \mathrm{E}+03$

$.17310400 E+U 3$
$.17377260 \mathrm{E}+03$

$.17448220 E+03$

$17509630 \mathrm{e}+03$

$.17640700 E+03$

$17720040 \mathrm{E}+03$

$17803780 E+03$

$.179 \cdot 0090 E+03$ co

$x^{i}$

$\therefore$ U.A

us.

rat

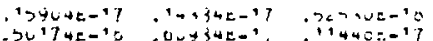

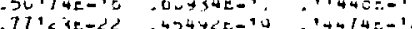

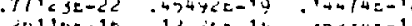

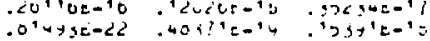

$-412400-1 y \quad .4 y+i 46-10$

$.73 i 2<x-17 \quad-1<074 x-1$

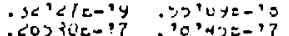

$.43045=0$

$.5209 / \mathrm{ten} y$

4y>soz-1?

$0.007 \varepsilon-10$

$304250-17$
.$<40050-17$

$12<02-10$

$.203<45=10$

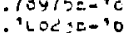

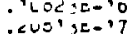

$.307,3 e-1$,

4 10102-10

$21,142=10$

axydse-10

$.341002=1$

$40010 \mathrm{k}-10$

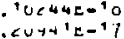

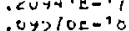

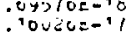

- 4iveve-to

$.29034 E-20$

$.14475 E^{-10}$

$.907<0 E-17$

$.45454 E-1 y$

$.241350-10$

44 U1L-?

b3409e-17 .032'4L-10

. $3 \dot{3}+20 \mathrm{e}-\mathrm{C}^{\prime}$

golgote-y

$44000-17$
$230220=90$

$\therefore<0^{1} 40-10$

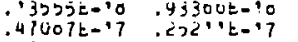

$00254=-10$

$.03704 \mathrm{E}-10$

:1224t-1' :0744t-10

$.03704=-10$
$.07347=-10$
$.710075=-10$

$33703=-17$

. $2<9<7 \mathrm{e}-1 y$

. JU0. DE-1?

- $9014=-10$

$.0300=20$

0 OSALEE-10

$.20570=-10$

47y YOE-'?

$2009 y=-17$

$\because 0$ yyc=0

ne $23=-10$

$37,504=-77$

$30730=-10$

उybi $3 x=-0$

$30352 k-10$

- $537012-17$

$.524 Y 7 E-17$

$.524 Y 7 E-17$
$.14034 E-17$

$\because 30246-10$

$.24009 \mathrm{e}-17$

$00743 \mathrm{e}-10$

$.040 .3 E=17$

$.53397 \mathrm{E}-17$

.00 12k-7o

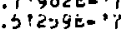

? 3001e-15

$.6794 \mathrm{x}-10$

$.235^{\prime} \mathrm{Je}-10$

. 4y0a4e-1?

$24734 \mathrm{E}-17$

$\because 12716=10$

$.7355 \mathrm{UL}-17$

$.00074=-17$

.9155 'E-'。

$.74002 c-17$

$.20500 \mathrm{E}-16$.21000-10

$.70222 \mathrm{E}-17$

$30350 \mathrm{U}=17$

$.07057 e-10$

$75069 \mathrm{E}-17$
$.39797 \mathrm{E}-10$

$39797 \mathrm{E}-10$
$.7205 \mathrm{te}-17$ $.91529 \mathrm{E}-17$ $.23140 E-10$ $.314902-17$

$.070370-19$
$.03350 \mathrm{O}-17$
$.73<74 \mathrm{E}-17$

$.73<74 \mathrm{E}-17$
$.90324 \mathrm{E}-10$

.540 10E-17

$.7305 ! e=17$

$.55912 \mathrm{~L}-16$

$.73403 E-10$

$.24590 \mathrm{~d}-16$

$.25220 \mathrm{E}-10$

$.70970 \mathrm{E}-17$

$.07094 \mathrm{E}-17$

$.07014 \mathrm{E}-17$

So $125=-17$

$33392 \mathrm{e}-10$

$.25220 \mathrm{E}=10$

$04525=16$ .3000E-10

$.50020 t-10$

$.24749 \dot{0}=16$

$.32236 \mathrm{E}-10$

$.97951 \mathrm{e}-17$

$.61297 \mathrm{E}-17$

$.572022-10$

$.53731 \mathrm{t}=17$

$.02406 E-? ?$
$.00602 \mathrm{E}=?$
$.30635 \mathrm{k}-16$

of6re?

$.14650 \mathrm{E}-15$

$.14000 \mathrm{E}-19$. 77 (C) $3 \mathrm{E}-10$

$.10048 E-15$

$.63907 t-16$

.231 J4E-16

112 $105-17$

$.53792 E=17$

$.52359 \mathrm{E}=17$

$.14018 \mathrm{E}-16$

$20480 \mathrm{E}=17$

$.442750-17$
$.20836 E=17$

$.52359 \mathrm{E}=17$
$.11653 \mathrm{E}=17$

$.10195 \mathrm{E}-? 5$

$.20 ! 33 t=16$

$.44479 \mathrm{t}-17$ nov $x=10$

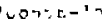

ON'OHE-16

$11=4 \cdot \ldots-1=$

$1 \cdot+8025-10$

$32030=-1$,

is $4: 1 x-1,0$

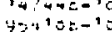

$170 \mathrm{sec}-1 \mathrm{e}$

suytorast

$20 i<+0-10$

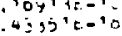

$11+4, c-10$

. 070 endo

'Luer-17

is $42 x<-1 y$

$221305-10$

$.440730-15$

$.03000=10$

1<yo:-17

- 3TSOUE-10

$-1 y 00=-10$

$\therefore 132=-1$

-3yource-'o

$.7<0>3 e-10$

$.2 y 00>t-10$

$.20422 \mathrm{E}-?$

$.24<050-17$
$40405-17$

$.90033 \mathrm{E}=10$

$.9404=-17$

$.2150^{\prime} \mathrm{E}-17$

$.30003 \mathrm{c}^{-10}$

? $0454 \mathrm{E}=?$

$.94004 \mathrm{t}=9 \mathrm{C}$

$.22103 \ddot{E}-17$

173 J0e-?

$.2 U 34^{\circ} \mathrm{t}-10$
$.227 j \mathrm{EE}=77$

$.0003 u k-17$

$.145<4 \mathrm{t}-17$

$.4524 k-17$

$.233408-17$

$.3920=-10$

$120^{1} 0 \mathrm{CE}-17$

. $32105 E-10$

$.10732 \mathrm{E}-17$

$: 14224 c-17$
$13007 \mathrm{E}=17$

$.50070 \mathrm{t}-10$

$.0900 \mathrm{t}-17$

$30024 \mathrm{E}-10$

$.2394 u t=17$

$30040=10$

$.07904 \mathrm{t}-10$
MA2CHO $, V, D_{0}^{M H}=45, M U=3.6100$

ME IHYL NITAATE

CH $30 \mathrm{NO2}$

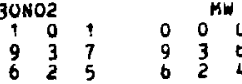

$77 \mathrm{HU}=3.0000$ $.8: 459000 E+0$. $.63000400 \mathrm{E}+0$
$A=$ $=\begin{array}{r}7271706: 0 E+02 \quad B=.19373450 E+02 \\ .32381 E-16 \quad .34309 k-17^{16} .69703 E-90\end{array}$

\section{A}

$.50504=5060 E$

$.39705 \mathrm{t}-10$

$.42042 \mathrm{E}-10$ a

i.j yes

$140,5=02$

losice-v

$i+1,1=-x$

$1,14+5-6$

as lica-ca

कारतe-s.

- TUUE-S

- nojacta

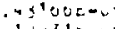

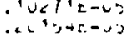

$4<x<=-4$

' $\angle 0<00-2$

=4 3 tor-u

gasuck $=0$

$130: 16-v 3$

'jujue-u

1984te-US

c4039e-co

. outaient

. $2 y^{2} y^{1}+5=-2$

IUターYEーU

<UPग E-U.

$.41900 \mathrm{E}-\mathrm{J}$

-c $3+0 \mathrm{c}-\mathrm{L}=$

- $20445-U 4$

0
$003015=u$

$-92^{2} 7<x-u b$

$121700-c 4$

.

ic jjent

$10033 c-0$

$19 y 14=-64$

व?73E-U4

.9900t-J

$.1497 j \mathrm{E}-U_{4}$

$.1225^{\prime} \mathrm{t}=\mathrm{u} 4$

$1233 j \mathrm{c}-04$

1233je-04

tojyle-ua

1ट374t-0 4

- $30937 \mathrm{e}-05$

1jojze-ua

- 1 coose-ia

. $440^{1}$ OE-US

$123 ! 4=-04$

$30350 e-$ is

. 1025OE-U4

$31903 r-05$ 
JU KUL JL KL KLE

$\begin{array}{llllll}4 & 1 & 4 & 1 & 1 \\ 2 & 1 & 2 & 1 & 1 \\ 2 & 4 & 2 & 1 & 4 \\ 2 & 1 & 1 & 1 & 0 \\ 3 & 1 & 3 & 2\end{array}$

$\begin{array}{llllll}3 & 0 & 3 & 2 & 0 & 2 \\ 3 & 2 & 1 & 2 & 2 & 0 \\ 3 & 1 & 2 & 4 & 1 & 1 \\ 4 & 1 & 4 & 3 & 1 & 3 \\ 4 & 0 & 4 & 3 & 0 & 3\end{array}$

322303

$\begin{array}{llllll}4 & 2 & 3 & 3 & 2 \\ 4 & 3 & 1 & 3 & 3 & 0\end{array}$

$\begin{array}{lll}4 & 2 & 3 \\ 4 & 2 & 2\end{array}$

$4 \div 3$

D $1 A \angle 2 N 2 H E$

$\$ 2 \mathrm{Cr}^{\circ}$

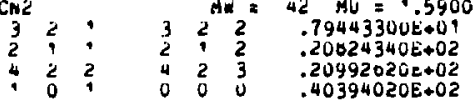

ISUTHLOLZAMIC ACIL

\begin{tabular}{|c|c|c|c|c|c|c|}
\hline $\begin{array}{l}6 \\
2 \\
2 \\
2 \\
0 \\
0\end{array}$ & $\begin{array}{l}1 \\
0 \\
1 \\
1 \\
0\end{array}$ & $\begin{array}{l}2 \\
\vdots \\
1 \\
0 \\
0\end{array}$ & $\begin{array}{l}? \\
? \\
? \\
?\end{array}$ & $!^{4}$ & $\begin{array}{l}1 \\
1 \\
0 \\
7 \\
7\end{array}$ & $\begin{array}{l}5 y \quad: 1 U=1.7200 \\
.23424500 E+02 \\
.23450000 E+02 \\
.23499500 E+02 \\
.93692700 E+02 \\
.930299 ? 0 t+02\end{array}$ \\
\hline $\begin{array}{l}0 \\
0 \\
0 \\
0 \\
0\end{array}$ & $\begin{array}{l}2 \\
2 \\
3 \\
3 \\
4\end{array}$ & $\begin{array}{l}6 \\
7 \\
5 \\
6 \\
7\end{array}$ & $\begin{array}{l}7 \\
7 \\
7 \\
7 \\
7\end{array}$ & $\begin{array}{l}2 \\
2 \\
3 \\
3 \\
1\end{array}$ & $\begin{array}{l}5 \\
6 \\
4 \\
5 \\
6\end{array}$ & $\begin{array}{l}.93863200 \mathrm{E}+02 \\
.93003200 \mathrm{~L}+02 \\
.93875280 \mathrm{~L}+02 \\
.93075280 \mathrm{E}+02 \\
.93994960 \mathrm{0}+02\end{array}$ \\
\hline $\begin{array}{l}9 \\
9 \\
9 \\
9 \\
9\end{array}$ & $\begin{array}{l}1 \\
0 \\
2 \\
2 \\
3\end{array}$ & $\begin{array}{l}9 \\
9 \\
7 \\
0 \\
0\end{array}$ & $\begin{array}{l}0 \\
0 \\
0 \\
0 \\
0\end{array}$ & $\begin{array}{l}1 \\
0 \\
2 \\
2 \\
3\end{array}$ & $\begin{array}{l}0 \\
6 \\
6 \\
7 \\
5\end{array}$ & $\begin{array}{l}.10540363 E+03 \\
.1055580 \mathrm{E}+03 \\
.10559561 t+03 \\
.10559561 \mathrm{0}+03 \\
.10560918 \mathrm{0}+03\end{array}$ \\
\hline $\begin{array}{r}9 \\
9 \\
10 \\
10 \\
10\end{array}$ & $\begin{array}{l}3 \\
1 \\
\vdots \\
0 \\
2\end{array}$ & $\begin{array}{r}7 \\
10 \\
10 \\
0\end{array}$ & $\begin{array}{l}0 \\
0 \\
9 \\
9 \\
9\end{array}$ & $\begin{array}{l}3 \\
\vdots \\
1 \\
0 \\
2\end{array}$ & $\begin{array}{l}0 \\
7 \\
9 \\
9 \\
7\end{array}$ & $\begin{array}{r}10500918 E+03 \\
: 10574377 E+03 \\
177+1404 E+03 \\
11720545 E+03 \\
11732747 E+03\end{array}$ \\
\hline $\begin{array}{l}10 \\
10 \\
10 \\
10 \\
16\end{array}$ & $\begin{array}{l}2 \\
3 \\
3 \\
1 \\
1\end{array}$ & $\begin{array}{r}9 \\
7 \\
0 \\
9 \\
10\end{array}$ & $\begin{array}{r}9 \\
9 \\
9 \\
9 \\
15\end{array}$ & $\begin{array}{l}2 \\
3 \\
3 \\
1 \\
1\end{array}$ & $\begin{array}{r}0 \\
0 \\
7 \\
0 \\
15\end{array}$ & $\begin{array}{l}11732747 E+03 \\
.11734263 E+03 \\
.11734263 E+03 \\
.17749195 E+03 \\
.16737057 E+03\end{array}$ \\
\hline $\begin{array}{l}10 \\
16 \\
16 \\
16 \\
10\end{array}$ & $\begin{array}{l}0 \\
2 \\
4 \\
3 \\
3\end{array}$ & $\begin{array}{l}16 \\
15 \\
14 \\
13 \\
14\end{array}$ & $\begin{array}{l}15 \\
15 \\
15 \\
? 5 \\
15\end{array}$ & $\begin{array}{l}0 \\
2 \\
2 \\
3 \\
3\end{array}$ & $\begin{array}{l}15 \\
14 \\
13 \\
12 \\
13\end{array}$ & $\begin{array}{l}.18764515 E+03 \\
.18771173 \mathrm{E}+03 \\
.18771222 \mathrm{E}+03 \\
.18773656 \mathrm{E}+03 \\
.18773668 \mathrm{0}+03\end{array}$ \\
\hline 16 & 1 & 15 & 15 & 1 & 14 & $.1879761 j E+03$ \\
\hline
\end{tabular}

$12009400 \mathrm{e}+02$ . 150<<300e+02 $.10135050 t+02$ $.17500040 \mathrm{E}+02$ $.2244 \div 530 E+02$

$.2302 \$ 450 t+02$ $.25044000 t+02$ $.26237300 \mathrm{c}+02$ . $2 y 765970 \varepsilon+02$ $.31449420 \mathrm{e}+02$

$.31052050 \mathrm{e}+02$ $.32461300 E+02$ - $324327 j 0 t+02$ $.32904200 \mathrm{E}+02$
$.33093000 \mathrm{U}+02$

$.3477 \div 440=+02$

$3+2$

$2 \mathrm{MU}=-.5900$ $20424340 \mathrm{0}+02$ $20492020 \mathrm{E}+02$ $5 y$ 的 $=1.7200$ $.23450000 t+02$ $23499500 \mathrm{E}+02$ $.93692700 t+02$
$936299 \% 0 t+02$

$.53863200 \mathrm{E}+02$ $93003200 \mathrm{x}+02$ $.93875200 \mathrm{i}+02$ $.93994960 \mathrm{t}+02$

$10540363 E+03$

$10559561 t+03$

$10560918 \mathrm{E}+03$

$.10560918 E+03$

$\because 0574377 E+03$

$11732747 E+03$

$.19732747 \mathrm{E}+03$

$635+03$

$.11749195 \mathrm{E}+03$

$16737057 \mathrm{E}+03$

$.18771736+03$

$67712222+03$

$.18797615 \mathrm{E}+03$

\section{CYANAMIDE}

\begin{tabular}{|c|c|c|c|c|c|}
\hline $\begin{array}{c}12 \mathrm{CN} \\
1 \\
15 \\
16 \\
2 \\
2\end{array}$ & $\begin{array}{l}0 \\
1 \\
1 \\
1 \\
0\end{array}$ & $\begin{array}{r}1 \\
14 \\
15 \\
2 \\
2\end{array}$ & $\begin{array}{r}0 \\
15 \\
16 \\
!\end{array}$ & $\begin{array}{cc}\mathrm{HW} \\
0 & 0 \\
1 & 15 \\
1 & 16 \\
1 & 1 \\
0 & 1\end{array}$ & $\begin{array}{r}42 \text { HU }=4.3200 \\
.19995100 E+02 \\
.3162 d 600 E+02 \\
.35642800 E+02 \\
.39725600 E+02 \\
.39990800 E+02\end{array}$ \\
\hline $\begin{array}{r}2 \\
17 \\
3 \\
3 \\
3\end{array}$ & $\begin{array}{l}1 \\
1 \\
1 \\
2 \\
2\end{array}$ & $\begin{array}{r}1 \\
16 \\
3 \\
2 \\
1\end{array}$ & $\begin{array}{r}1 \\
17 \\
2 \\
2 \\
2\end{array}$ & $\begin{array}{rr}1 & 0 \\
1 & 17 \\
1 & 2 \\
2 & 1 \\
2 & 0\end{array}$ & $\begin{array}{l}.40253000 E+02 \\
.40320800 E+02 \\
.59587700 E+02 \\
.59972800 E+02 \\
.59972800 E+02\end{array}$ \\
\hline $\begin{array}{l}3 \\
3\end{array}$ & 0 & $\begin{array}{l}3 \\
2\end{array}$ & $\begin{array}{l}2 \\
2\end{array}$ & 0 & $\begin{array}{l}.59985700 E+0 \hat{E} \\
.60378500 E+02\end{array}$ \\
\hline
\end{tabular}

20

SIGMA

jo

:0u
200
ME

$\begin{array}{ll}.5230^{1} \mathrm{E}-10 & .59575 \mathrm{E}-19 \\ .2737 \mathrm{j}=17 & .20077 \mathrm{E}-10\end{array}$

$.42504 \mathrm{E}=17$

.4445 CE.

$.39317 \mathrm{TE}-10$

$.10903 E-19$

.5 ! $20 \mathrm{E}-19$

$.79555 \mathrm{E}-19$

$.70092 \mathrm{E}-19$

$.20313 E-10$

$13033 \mathrm{t}=10$

.4'06E-17

$.25537 E-10$

.74 ode-17 d5017t-10

$13707 \mathrm{E}$

$.15100 \mathrm{c}-17$

$23031 \mathrm{E}-10$ 15
.$<0076 \mathrm{t}-17$

.2007 bE- 17
$.30959 t-17$

$.27053 \mathrm{E}-10$

.2705 3E-10

$.49459 E-10$
$.50004 E-10$

$395318-10$

$.44700 t-? 9$

$.62310 \mathrm{E}-20$

$209690-10 \quad .24044 \mathrm{E}-17$

$1909 E-10$

$01305 \mathrm{t}-10$

$.1443 ! \mathrm{t}=17$

$.90522 \mathrm{t}-19$

$.46470 \varepsilon-10$

$.27451 \mathrm{c}-10$

$.10090-19$

$.50716 E=18$

. $31234 t-16$

$301750=? 7$

$.66975 \mathrm{c}-10$

$A=.40956720 \mathrm{E}+02 \quad \mathrm{E}=.23007760 \mathrm{E}+02$

$.12766 \mathrm{E}-10$

52700E-17

$.21 ! 16 t-10$

$87904 \mathrm{E}-10$

.98d8E-10

(1500-17

$.33705 \mathrm{E}-17$

$.60920 E-16$

$A=.14030000 \bar{E}+04 \quad \overline{\mathrm{B}}=.50834240 \mathrm{E}+0$ !

$.05409 E-10 \quad .06173 \mathrm{E}-18 \quad .3159 \mathrm{dE-?b}$

$.39634 \mathrm{E}-10 \quad .47597 \mathrm{E}-17 \quad .05655 \mathrm{E}-18$

$.05994 \mathrm{E}-18$.80710E-10 .39718E-18

$.20307 \mathrm{t}-10 \quad .49930 \mathrm{E}-16 \quad .29909 t=16$

$.45520 t-16$

$.64921 E-2$

$.64921 E-21$

2 $1005 E=15$

25025E-17

$.198505-28$

.67 bo6t-18

$.67666 \mathrm{E}-18$

$.51572 \mathrm{E}-2 ?$

$.25025 \mathrm{E}-17$
$.66153 \mathrm{E}-19$

$.66153 E-19$

.50195E-16

$.22116 \mathrm{E}-16$

$.284706-10$

$.64473 E-16$

31467E-16

.1068E-14

$.27024 E-15$

$.29062 E-16$

.72746E-2

- BO274E-18

$.61600 E=16$

- $00291-21$

$34343 E-17$

$.13554 \mathrm{E}-20$

$.92609 E-19$

$.13554 \mathrm{E}-28$

-31562E-16

$.32684 E-16$

$.70041 \mathrm{E}-21$

$.66629 \mathrm{E}-21$

$.92609 E-19$

.647 d3E-16 . $30031 E-16$

.79?19E-16 -38675E-i6

$: .0926 \mathrm{E}-17.44969 \mathrm{E}-17$

$.76041 \mathrm{E-2}$

. 109260-17

$.14363 \mathrm{E}-28$

$.86109 \mathrm{E}-21$

$.44969 \mathrm{E}-17$

.12293E- 18

$.66109 \mathrm{E}-21$

$.12293 \mathrm{E}-18$

$.32739 E-16$

$.795725-16$

$39027 \mathrm{E}-16$

$.13614 \mathrm{E}-16$

$.13128 E-15$

$.10443 \mathrm{E}-15$

$.47946 t-15$

.5447 1E-15

.20732E-15

.32634E-'?

$.18425 \mathrm{E}-17$

$.11967 \mathrm{E}-16$

$.11967 E-16$

.184

63735E-2। $150165-20$

- $33827 \mathrm{E}-18$

$.33827 E-18$
$.33827 \mathrm{E}-18$

$.15016 \mathrm{E}-20$

$.10184 \mathrm{E}-15$

$.93733 E-16$

$.13148 \mathrm{E}-15$

$.99774 E-20$

.11359E-07

. $11230 \mathrm{E}=00$

$.10403 E=00$

$.17939 \mathrm{t}-06$

$.47474 t=00$

45682E-19

$.63236 \mathrm{t}-06$

$.41310 \mathrm{E}-00$

$.75037 \mathrm{e}=0 \mathrm{0}$

$.12037 \mathrm{E}=0 \mathrm{0}$

.14537 E-05

$09207 E-19$

10252t-io

$.27487 \mathrm{E}-07$

$.12573 t-05$

$.70762 x-06$

$.49506 \mathrm{E}-U 7$

$14342 E-05$

$32731 \mathrm{c}-20$

$.12916 E-10 \quad .19251 t-05$

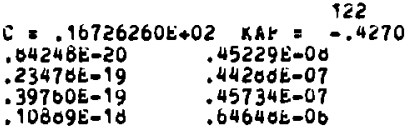

472

$C=.58456220 E+01$ KAr $=-.9999$

$.80142 \mathrm{t}-19 \quad .13270 \mathrm{E}-06$

$.15277 E-10 \quad .17700 E=06$

$.80654 \mathrm{E}-19$ 13400E-00

$.01348 \mathrm{t}-17 \quad .13119 \mathrm{t}-04$

$.09095 E-17 \quad .13306 \mathrm{E}-04$

$.20234 \mathrm{E}-17$

$.20234 \mathrm{E}-17$

-31501E-16

31501E-10

$12563 \mathrm{Em} 04$

$12563 E-04$

$.11520 \mathrm{E}=04$

$.11520 E=04$

$.13247 E=04$

$.85449 \mathrm{E}-17$

$.123665-16$
$.28481 \mathrm{E}-17$

$.28481 E-17$

$.45232 E-18$

$.188650-04$

$.19905 E-04$

$.18257 \mathrm{E}-04$

$.182578=0$ i

$.1707 \partial E-04$

$.45232 \mathrm{E}-10$

$.85966 \mathrm{E}-17$

$11423 E-16$

$16494 \mathrm{E}-16$

$.17078 E-04$

.19040 E-04

$.26075 E-04$

$.26455 E-04$

$.25424 E-04$

. $38356 \mathrm{E}-17$

$.25424 \mathrm{E}-04$

$.241095=04$

$.24109 E=04$

$.26329 \mathrm{E}-04$

$.10940 \mathrm{E}-03$

$114915 \times 16$

$.37486 \mathrm{E}-16$

$.11031 E-03$

. 10070E-03

$.10070 \mathrm{E}-03$

$12323 E-16$

$: 10659 \mathrm{E}-03$

$\because 106595-03$

$.19046 \mathrm{E}-03$

$.37682 E-16$

165

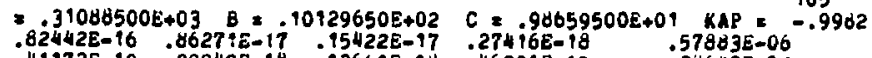
$.41172 E-19$.20940E-16

$.22925 E-19$. $18541 E-18$

21554E-15. $36834 E-16$

$.13661 \mathrm{E}-10$

.14196-18

$.57883 \mathrm{E}-06$

$.46391 E-19$

$.51798 \mathrm{E}-19$

$.94958 \mathrm{E}-17$

. $6404-0$

$.1,08548-05$
$.55570 E-05$

$.22060 \mathrm{E}-15.37790 \mathrm{~L}-16$

$.12104 E=16$

217:4E-17

$.42503 E-05$

. 46546E-07

$.17508 \mathrm{E}-04$

.14293E-1d .56928E-17?

. $301415-16$. 5890

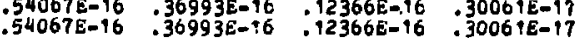

$11156 E-04$

$.17510 \mathrm{E}-14$.21161E-15 .39689E-16 .72268E-17

$.00536 E-17$

$.20094 \mathrm{E}-04$ 
JU AU2 NL KL KLC

in

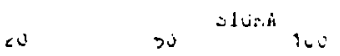

$\therefore$ ¿

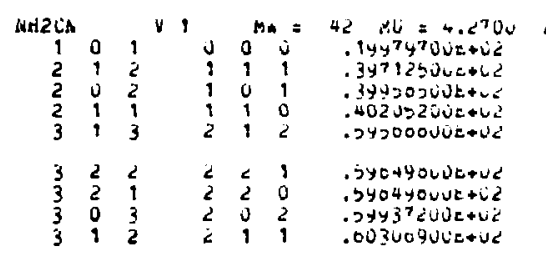

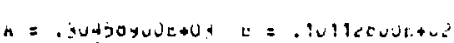

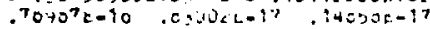

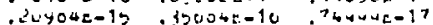

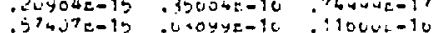

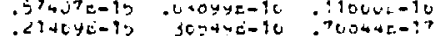

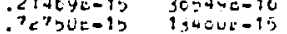
. $29120=-10$

$.240 Y 25=10 \quad .30,71 E-10$

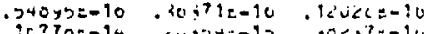

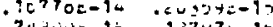

$1<j<2=-10$
$0<, 7=-10$

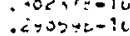

TETARZULE

त4 $4 \mathrm{CH}$

$\begin{array}{lll}3 & 2 & 1 \\ 1 & 0 & 1 \\ 2 & 2 & 1 \\ 2 & 1 & 2 \\ 4 & 2 & 2\end{array}$

A

$32^{\mathrm{M} m}=$

TU AL $=1.910 \mathrm{U}$

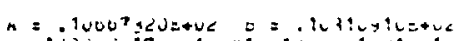

$.9400 y=-17 \quad .10001=-10$

$.13473 \leq-17 \quad 13 y^{7} \supset E=90$

. 1u० 1بs-17

. 5j 1 vue-17

11Ujet-10

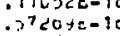

alcris-1y

$1525150 u r+c 2$

$.20030^{7} 0=-02$

423

$\begin{array}{lll}3 & 2 & 2 \\ 2 & 0 & 2 \\ 2 & 1 & 1\end{array}$

$\begin{array}{lll}3 & 0 & 3 \\ 1 & 0 & 1\end{array}$

202502 dUt +02

.2037051 vetul

.30172430 etu2

- $972540-17$

(4) ${ }^{7}$ YL-1:

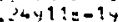

- ¿L Iu $T_{c}=1$

$103 x-10$

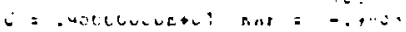

$.25 y+2 E-17 \quad$-3UOUSE -10

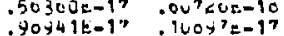

$.3 U \dot{i+4 L-1,}$

$30 j+4 t-1$

1) $16=-1$.

, cus ic $\therefore=$ ?

$\therefore 44: 3:-?^{7}$

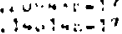

D'vons.:?

- it 4 Z:

$+4+41=$

$0,0,7,7=0$

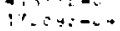

$\therefore u=x-17$

in: $25=0$

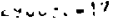

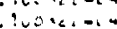

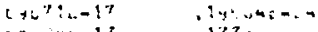

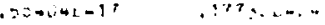

JeTRAZuLE

$14 \mathrm{CH}$

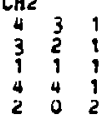

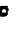

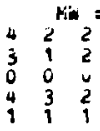

$\begin{array}{lll}4 & 2 & 2 \\ 3 & 2 & 2 \\ 4 & 3 & 2 \\ 2 & 1 & 2 \\ 2 & 2 & 1\end{array}$

$\begin{array}{lll}4 & 1 & 3 \\ 3 & 1 & 3 \\ 4 & 2 & 3 \\ 1 & 0 & 1 \\ 1 & 1 & 0\end{array}$

FORHYL

HCO

$\begin{array}{lll}2 & 1 & 1\end{array}$

PORMALDEHYDE

H2LO

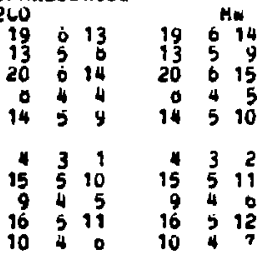

$\begin{array}{rrrrrr}5 & 3 & 2 & 5 & 3 & 3 \\ 17 & 5 & 12 & 17 & 5 & 13 \\ 10 & 4 & 7 & 11 & 4 & 8 \\ 6 & 3 & 3 & 10 & 5 & 14\end{array}$

1240124

$\begin{array}{rrrrrr}19 & 5 & 14 & 19 & 5 & 15 \\ 2 & 2 & 0 & 2 & 2 & 1\end{array}$

$\begin{array}{rrrrrr}2 & 2 & 0 & 2 & 2 & 1 \\ 20 & 5 & 15 & 20 & 5 & 10 \\ 13 & 4 & 9 & 13 & 4 & 10\end{array}$

$\begin{array}{rrrrrr}7 & 3 & 4 & 7 & 3 & 5\end{array}$

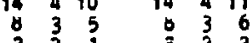

$\begin{array}{rrrrrr}3 & 2 & 1 & 5 & 2 & 2 \\ 15 & 4 & 11 & 15 & 4 & 12\end{array}$

$\begin{array}{rrr}9 & 3 & 6 \\ 16 & 4 & 12 \\ 17 & 4 & 13 \\ 4 & 2 & 2 \\ 10 & 3 & 7\end{array}$
7. MU $=1.07 !$ $.1420020 u t+U L$

$.14770050=+u<$

isyj7570couz

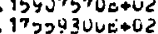

$. \angle 0 V 1+j^{7} v e+U 2$
$.20030740 \mathrm{E}+02$

$20254020 x+02$

$.26303000 \mathrm{E} * 02$

$.26300340 \mathrm{E}+02$

$.37242150 \mathrm{~L}+02$

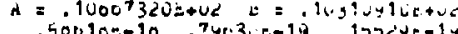
$.50010=-10 \quad .7 y 03=2=10$ DIVYE-10.01033e-1t $.442 U \angle E=10 \quad .42074 \mathrm{t}-19$ $.05122 E-10$.0OY2JE-14 .100040-17 .:75:aUL=10

$.150<<2-17 \quad .17,500-10$ $.006230-10 \quad 144002-10$ .191958-17.17i DOe-10 $.17140 \mathrm{E}=1 \mathrm{r} .10 \cdot 00 \mathrm{c}-10$ $.32830 t-17 \quad 30<14 t-10$ $12524=-1 y$
$1124 y=-y$ $.017000-c$ $12 \leq 2 c c-1 y$
.1200

$.3 j>0^{7} x-1 y$ $.1 y 435-1 y$ - 340:9e-1y $.33339 c-14$ - $050000-1 y$

$29 \mathrm{MU}=1.0000$ $.06070550 \mathrm{E}+0 \mathrm{Z}$

$30 \mathrm{MU}=2.3310$ - I1100000t-0? 14750000 Ẽ 02 $20500000 E-02$ $245400005=02$ - 31100000 e-02

$45730000 \mathrm{E}-\mathrm{UL}$ $.02120000 t-02$ $.03740000 t=02$ 11 400juei=01 . $14645000 \mathrm{~L}-01$

$18204000 \mathrm{c}-61$ .21050 UOUE-01 .317900uve-01 $36200000 e-01$ $.54037000 \mathrm{E}=01$

$.03440000 t-01$ $.05300000=001$ 71900000 E-U1 $.10040900 \mathrm{e}+00$ $11902000 \mathrm{E}+00$

$.13090000 \mathrm{E}+00$ $.21400000 \mathrm{~L}+00$ $35550000 E+00$ - j70uUuJoe+0u

$.00107400 E+00$ $.014700 \mathrm{CDE}+0 \mathrm{C}$ .9d915UUUe+UO $100>0>00 t+01$ $.11132000 E+01$
$A=.07033000 \mathrm{~L}+63 E=.4470400 \mathrm{Ut}+\mathrm{UL}$

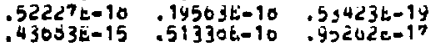

$h=.20210000 e+03 E=.3003000 u=+i c$

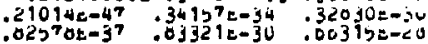
$.25010 \mathrm{~L}-40 \quad .27424 \dot{2}-34 \quad .5312 j e-30$ $.71314 E-30 \quad .92354 \varepsilon-27 \quad .30212 \varepsilon-20$ $.23430 \mathrm{c}=37 \quad: 129 e^{7} \mathrm{E}=29 \quad: 17300 \mathrm{E}-27$

$.15 j 91 \mathrm{E}-25$.11009e-z4 ov721te-2b $.74337 E-30: 10057 E-29$.30105E-C7 $.00995 E-50 \quad .2400^{7} E-20 \quad .1001 j E-2 j$ $.104025=30$ :10732e-<9:74293E-27 75772t-90 :72212e-2b . .50uylt-25

.

.

$32900=40$ $13902 \mathrm{E}-25$

$.2<405 E=<4$

$.500000-23$

$.20100=-\angle C$

$.20604 \mathrm{E}-3 \mathrm{U}$.22077E-2j

$.32030 E-41$

$.22077 E-2 j \quad .3374 y=-24$

$.20145 e-21$

11092

?7ग00E-2द

$2 j 054 t-42$ r024er-a

$.64043 z-33$

$.702 y 0 E= \pm 0$
$.2 y 10<E-25$

$3^{7} 320 E-20$

-33590E-24.1072yt-2a - 1795 CE-j1 $.32^{7} 8^{7} \mathrm{E}-<5$

3735>t-24

jo

. $4404 t^{-2}$

. $35004=-32$

1 15b07L-20

$.27470 E-? 4$.77000i-22

Sod

$.71057 \mathrm{~L}-20$

$-207200-25$

$19150 \mathrm{k}-21$

- $307000-23$

147 UUE-24

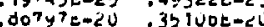

.11041tezl itjtode-21

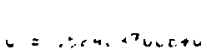

$\therefore 7,8=60$

intecuitito

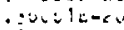

$96404:-?: 4$

. $14 x^{2}=-14$

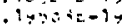

$0,202 \times r-1 y$

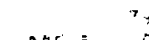

. $42, \because-2$

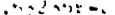

. $7 y=1: \ldots$

. $1400=0$

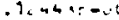

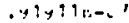

.

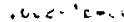

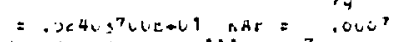

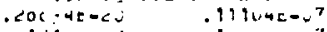

- inviyeas

inglyeco

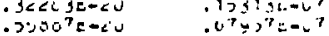

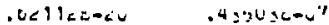

$.32<0 u=-c j \quad \because 13 y<z-v^{7}$

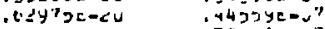

. Pyjocies .734010-u?

.2.BEvUE-60

1e 30135

$c=.41$ yrugoutatuc ant $=-$. ybiu

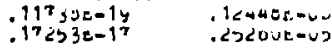

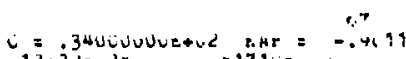

$.13,320-20$

$31000-20$

$.31000 E-20$
$.3<070 \mathrm{E}-20$

$.04111=-27$

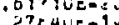

.

$.2 \dot{1}^{7}$

$.2 U^{7}>O E-18$
$.2<U L E=16$

$.20 y 53 z-25$

$.241450-20$

$.100320-<5$

$.0<\angle 07<-<0$

$.0 \ngtr<7 y k=<$ ?

- $27 \dot{1} 13 k-17$

$.1>^{7} \partial^{2}=-17$

. $\angle \rightarrow 0 y 1 c-17$

$.94105 \mathrm{E}-$ ?

- suvsoc-10

$.04050 E-25.10159 E-23 \quad .00250 E-64 \quad .34>03 E-24$

$.202^{72} 25-39 \quad .17929 t-29 \quad .12040 E-20 \quad .14343 t-23$

$.230710-24$

$.31212=05$

$.70+13 t-24$

.01 पi 30E-<5

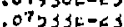

$.11430 t-<4$

$.1050 \leq t-23$

$.115 y 9 x-12$ $.222<0=-16$ $.<4>90<-1$, $<=0442-13$ $.22333=-14$

$.11<71_{c-<c}$

. $43>4 i<t=<3$

$41740=-<2$

140370

$147370-<1$
$.92761 \mathrm{E}-<3$

$.1052 x=-14$

$.113120-14$

. $1515.2=-1$.

4771<e-14

$.4+0 c^{2} \mathrm{t}-14$

$.1<0 y U_{C}=<1$

$-10131 \mathrm{E}=<2$

$.43075 c i-21$

- jétueres?

- COU $40 E-1:$

$215=10$

$4700-1$ 


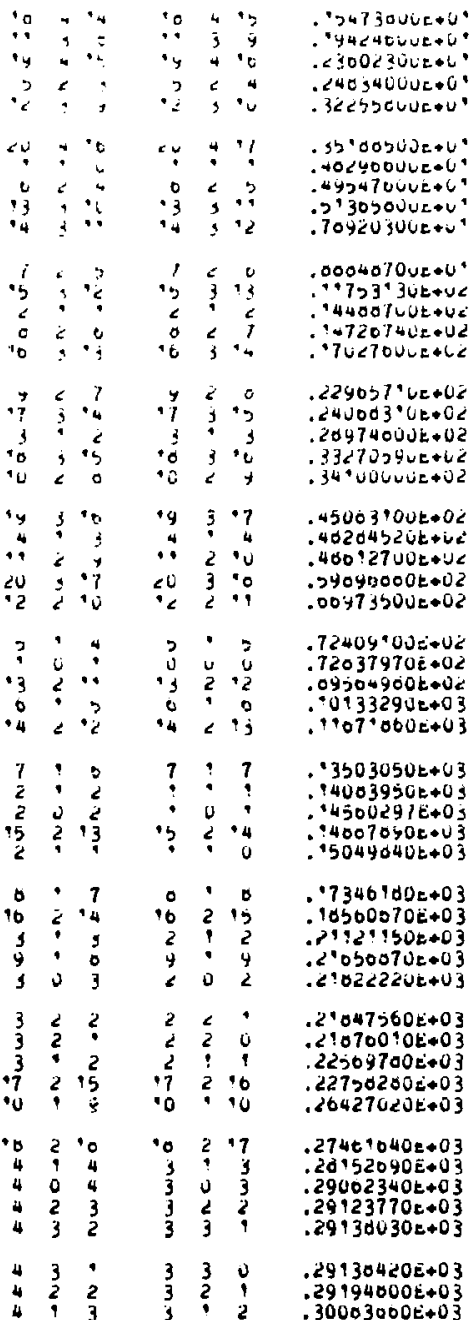

rOHAYL FLUUHILE A acur

$\begin{array}{llllll}2 & 0 & 2 & 1 & 1 & 1 \\ 2 & 0 & 2 & 1 & 0 & 1 \\ 7 & 1 & 7 & 0 & 1 & 0 \\ 7 & 0 & 7 & 0 & 0 & 0 \\ 7 & 2 & 0 & 0 & 2 & 5 \\ 7 & 3 & 9 & 0 & 3 & 4 \\ 7 & 3 & 4 & 0 & 3 & 3 \\ 7 & 2 & 5 & 0 & 2 & 4 \\ 7 & 1 & 0 & 0 & 1 & 5\end{array}$

48 $\mathrm{ML}=\quad .5950$
$.42950700 \mathrm{E}+02$ $.44296000 \mathrm{t}+02$

$.15004073 E+03$

$15412002 E+03$

$.15495555 E \div 03$

$.15523477 \mathrm{t+03}$

$.15525193 E+03$

$.15592397 k+03$

$.159010496+03$
$.010045-35$ $.2 Y 300 E-23$ $.33032 \mathrm{e}-30$

isooe- 9

$.027<E-25$

.j96万人e-37

. $340<00-7$

$\because 30 u=-9$

$.4930^{4}=-20$

(c) $2442-<T$

'cutuen?

$\because 907 t-27$

¿cuobe-io

$.7393 t-20$

$2477 e-<0$

- 977 e-<0

$.507 \mathrm{e}-<9$
$.4040 \mathrm{t}-90$

- $90703 x-34$

- vo!ze-co

$.57004 c-3 \angle$ $.20440 k-10$

$.27003 c-33$

$.573 \cup 4 E-22$

. DzUAE-0 $.45259 \mathrm{E-1}$

$.5904 k-23$

$.0525^{\circ} \mathrm{e}-1$ ?

$.111910-23$

$.37+47 \mathrm{e}-17$

$.21<44 c=-14$

$.53070 c-14$

$.23429 t-14$

$.2019 \mathrm{k}-17$ $.97007 t-20$

$.51955 e-14$

.3ว400t-?

¿0700e-13

$.50299 z-15$

$.5039 d E-05$

$.54407 E-14$

$.63734 \dot{\varepsilon}-27$

$.74513 E-19$

$.33902 \mathrm{E}-20$ $.05010 \mathrm{e}=14$

- $12602 t-13$

. $54464 E-\div 5$

$.27350 t-10$

$.27357 \mathrm{~L}-16$

. egoyot-15

$.00192 \mathrm{E}-14$

\section{¿couve-25 \\ $.74590=-20$ \\ .20yyst-1y \\ 't5 3' $t-2$}

- $50=27 z-20$

- SES4Ut-?0

- Sy $50>2-1 y$

35
$\because 267 y-24$

Tusor-10 $.40 y 0<$ enaz $.24400 \mathrm{E}=7$ - jinyte-co

.5700ie-22

$.37+7 k=10$ ? $2440 ! e-22$ . $5{ }^{2} 5{ }^{2}=-17$

- vé79t-22

D $3 ! 7 e=18$

$.72957 k-23$ $.005305=17$
$.000 ! t=10$ 205207s-2i $.94205 t-19$

-335E- 6 - $000 \%$ each $.04774 \mathrm{E}-9$ ?240ट-?6 $.94700 E-? 9$

$.11207 \varepsilon-16$ $.42 ? 96 t-15$

$.75000 t-15$ $.17056 E \ldots$ $.47519 E-? 5$

$.92300 k-17$ $.00450 t=20$ :3774t-14 . OT7UTE-??

$.20057 E+44$

$.44750 t-15$ $.440595-15$ $.15297 E-14$ $32905 E-20$ $.44044 E=? 7$

$122904-20$ . $2020 \mathrm{t}=-4$ - 30548tra-14 $? 0023 \mathrm{~L}=14$ 1942 IE-15

$.14422 \varepsilon-? 5$

$.200<0=-14$
$.00520 c-23$ $.70740 E-\bar{x}$.

$.60327 \mathrm{t}-23$

$\therefore 90: 3=-19$

- yupue-zó

$.7 ?<076-23$

$9727 t-90$

$35333 \mathrm{c}-9$

$.9300=-20$

$.220 y 5 x=06$

$70570 \mathrm{e}=19$

$31507 \mathrm{c}-<\mathrm{C}$

$.54340 E-? 0$

- 3द? 3e-10

.2'0jst-"0 $354950-<0$ $13703 E-17$ $.340946-20$

.30293 to- 0

$.29937 \mathrm{E}-20$ $25445 k-17$
$37587 k-90$ $24490-30$ $422226-10$

-30904E- 7 .20U32k-?6 $+3274 E-10$ 510500-?? $.40752 E=16$

$.620 .37 L-17$ $.954 z 2 c-10$ T492te-1s 3547 UE- 10 . $90594 \mathrm{E}=15$

.67022 E- 7 .20003t-:0 .3374 YE- 5 .0004 ?E-?

$.15732 t-15$

$.15779 E-15$

.36005t-15

$.21649 E-10$

$.64064 E-17$

$.15315 t-90$ $.73650 \mathrm{t}-15$ $.93134 t-15$ $.43753 \mathrm{E}-1$

$.14: 03 t-95$ $.43944 t-? 5$ $.021700-15$ j5.07t=$754 \cdot 7 t-2$

$.00944 E-42$

. 37900e-íu

.5452c=2u

-c007e-c"

. 2<4jut-? 9

.<O700E-zU

$.442045-20$

izuyor-19

$.7745 ? t .-20$

$.0020 x-10$

. 5070z=-19

- Jozbr-'o

.543 Ut-?

.207 OE-10

$.20744 E=19$

$.99 \cdot 2 t-10$

$.25497 \mathrm{t}-?$

.3725 !k-90

$.27097-10$

$.29065 \mathrm{t}-49$

$.375 S^{-i} c=10$

.959'6t-?0

- $30130 \mathrm{t}-17$

.40550 E-?O

$14247 \mathrm{E}-17$

$.507430=+6$

$.94333 \mathrm{E}-17$

$.18481 E-10$

$.27007 t-10$

$.00041 \mathrm{c}-10$

$.21030 \mathrm{e}=16$

$.24417 t-17$

$.72000 E-10$

$.70238 \mathrm{E}-10$

$.29059 \mathrm{E}-17$

$.09950 t-10$

. $39290 \mathrm{E}-10$

$39395 \mathrm{E}-10$

.79 . $47 E=? 6$

.73 ? $42 E-10$

$.3 z \forall 73 t-17$

$.12090 \mathrm{E}-10$

$.0409 E-15$

.19600E-15

$.50851 k-16$

$.50053 \mathrm{E}-16$

. $1750 \mathrm{E}-15$

$.10512 t-15$
$.10023 e-10$ $.31394 t-10$

$.3442 y<-10$

$12004 x-04$

. $21312-6 y$

- Tozye-0y .35023 ë-LO $.72925 t-U y$ . IOA4t-iy - juybe-io

$.31425 c=00$ $37057 t=00$ - jeusye-vi 9107 je-ci

. OOS 3 ite-u?

$.33350 e-0$ ? $.645710=0$ ? . $2021 \mathrm{c}=00$ $.20433 e-47$ $.06545 \mathrm{c}-07$

$.22914 \mathrm{E}=00$ . $350: 0 t=00$ . $9124 \mathrm{E}=00$ $.2700 ! E-v 0$ $.40095 \mathrm{e}-\mathrm{CO}$

$.00 ! 15 E-00$ $.09404 k=05$ $.929 y 4 L-00$ $15702 i-i s$ $17522=05$

$.27990 E-05$ $.53004 E-04$ $.70063 E-U 4$ -30g1t-oj $.6407+E-C 4$

$.46104 E-05$ $.5 .2327 \mathrm{~L}-05$

$22700 \mathrm{E}=03$ $.72171 \mathrm{E}-05$ $.20101 E-03$

15703E-U3 $.15764 E-03$ $.27099 E-03$ . B4060E-05 $.10790 \mathrm{E}=04$

$12962 \mathrm{E}=04$ $.50795 E-03$ .08971 t-03

$.52075 \mathrm{E}-03$ $.30423 t-03$

$.30424 \mathrm{E}-03$ $.52457 E-03$

$.71742 E=03$

$A=y ! 1536000+02 \quad E=.11700000 E+02$ $.48050 \mathrm{E}-17 \quad .00100 \mathrm{k}-10$. $11422 \mathrm{E}-16$ $.82990 E-17$.91903E-10 $.74040 E-1 \mathrm{t}$.20U70E=?6 $.92527 \varepsilon-16$.22925E-:0 .39000t-10 :10030t-10 $.96010 \mathrm{E}-10$ $.49203 \mathrm{E}=17$ $.54025 \mathrm{E}-17$ 
JU KUT KU2 JLL! KL2

$\begin{array}{rrrrrr}5 & 0 & 5 & 4 & 1 & 4 \\ 7 & 2 & 6 & 0 & 1 & 7 \\ 5 & 1 & 4 & 5 & 0 & 5 \\ 5 & 2 & 4 & 0 & 1 & 5 \\ 6 & 1 & 5 & 6 & 0 & 6 \\ 7 & 1 & 6 & 7 & 0 & 7 \\ 1 & 1 & 1 & 0 & 0 & 0 \\ 8 & 1 & 7 & 0 & 0 & 0 \\ 9 & 1 & 8 & 9 & 0 & 9 \\ 4 & 2 & 3 & 5 & 1 & 4 \\ 2 & 1 & 2 & 1 & 0 & 1 \\ 10 & 1 & 9 & 10 & 0 & 10 \\ 9 & 0 & 9 & 0 & 1 & 0 \\ 3 & 1 & 3 & 2 & 0 & 2 \\ 3 & 1 & 3 & 2 & 0 & 2 \\ 4 & 1 & 4 & 3 & 0 & 3 \\ 4 & 1 & 4 & 3 & 0 & 3\end{array}$

SILYL METHYL ETtiSR

\begin{tabular}{|c|c|c|c|}
\hline $\begin{array}{l}5 \\
1 \\
2 \\
3 \\
4\end{array}$ & $\begin{array}{l}0 \\
1 \\
1 \\
1 \\
1\end{array}$ & $\begin{array}{l}5 \\
0 \\
1 \\
2 \\
3\end{array}$ & $\begin{array}{l}4 \\
1 \\
2 \\
3 \\
4\end{array}$ \\
\hline $\begin{array}{l}5 \\
6 \\
7 \\
8 \\
9\end{array}$ & $\begin{array}{l}1 \\
1 \\
1 \\
1\end{array}$ & $\begin{array}{l}4 \\
5 \\
6 \\
7 \\
6\end{array}$ & $\begin{array}{l}5 \\
6 \\
7 \\
6 \\
9\end{array}$ \\
\hline${ }_{10}^{1}$ & 1 & 1 & $\begin{array}{r}0 \\
10\end{array}$ \\
\hline
\end{tabular}

\section{aE PARITY}

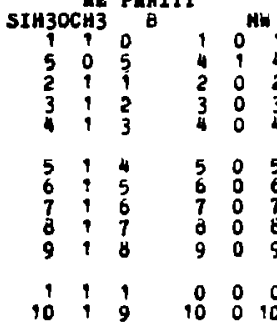

EA PAF TIY

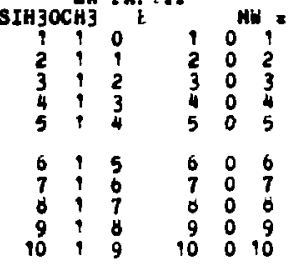

AA PAKITY

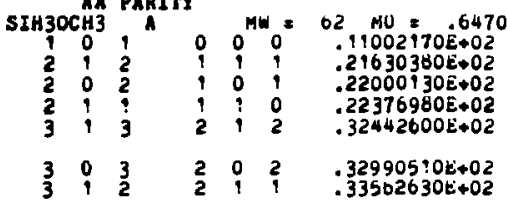

. 30950000E-02 $39044300=02$ $.90700040 E+U C$ $.939041340 \mathrm{e} * 02$ $.95339460 E * 0 z$

$.10003005 E+03$ $.10155030 E+03$ $11499300 \mathrm{E} * 03$ $.11929055 \mathrm{E}+03$

$.12234395 \dot{E}+03$ $.1230 ! 235 E+03$ $130900300+03$ $.14246244 \mathrm{E}+03$

$.109927>0 E+03$

$.16192756 F+03$

$62 \mathrm{HU}=.9500$ $.28461330 E+02$ $20050020 E+02$ $29430 d 50 E=02$ $.30200340 E+02$

. 31995900c+02 $32413970 E+02$ $33873300 c+02$ . $35594130 x+02$ $.37594300 E+02$

$39110530 E+02$ $.39093700 E+02$

$62 \mathrm{MU}=.9500$ $.20457700 \mathrm{E}+02$ $.26467650 E+02$ $20 d 32910 E+02$ $.29404390 \mathrm{E}+02$ $30179690 \varepsilon+02$

$.31909150 E \rightarrow 02$ $32306230 E+02$
$.33846320 E+02$ $.35562060 E+02$ $.37567250 \mathrm{E}+02$

. 39041290E+02

$62 \mathrm{HU}=.9500$ $.29670060 \mathrm{E}+02$ $209708506+02$ $30431500 E+02$ .34?70100E+02

. $32217040 \mathrm{E}+02$ $.33546750 E+02$ $.35164640 \mathrm{E}+02$ . $37077 \triangle 20 E+02$ .30297010E+02
20

30

SIGMA - vo

$2 \mathrm{su}$
nEP

\begin{tabular}{|c|c|c|c|c|}
\hline $\begin{array}{l}.34007 t-16 \\
.00020 E-97 \\
.34 y 53 E-92 \\
.42030 E-16 \\
.304 ? \cdot E=15\end{array}$ & $\begin{array}{l}.50003 E-17 \\
.29404 E-17 \\
.74073 E-10 \\
.04008-16 \\
.0302<5-10\end{array}$ & 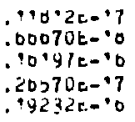 & 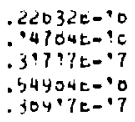 & 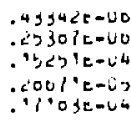 \\
\hline $\begin{array}{l}.31050 E=15 \\
.20091 E-15 \\
.24790 E=! 5 \\
.10000=-15 \\
.03792=-10\end{array}$ & $\begin{array}{r}.00974 E-10 \\
.2459, E=-10 \\
.92300 E-10 \\
.93902 E-10 \\
.9439 E=-10\end{array}$ & $\begin{array}{l}.222 \supset 2 \mathrm{E}-10 \\
.45722 E-17 \\
.25212 \mathrm{Y}-10 \\
.20045 E-10 \\
.32323 E-17\end{array}$ & 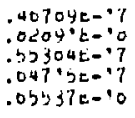 & 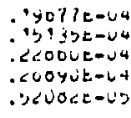 \\
\hline $\begin{array}{l}.410278-15 \\
.13437 E=! 5 \\
.22074 E-15 \\
.030108-15 \\
.03010 E-95\end{array}$ & $\begin{array}{l}.5,370 \mathrm{E}-90 \\
.91274 \mathrm{E}-10 \\
.90+07 \mathrm{E}-10 \\
.07002 \mathrm{E}-16 \\
.07002 \mathrm{E}-10\end{array}$ & 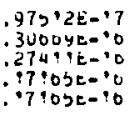 & 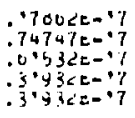 & 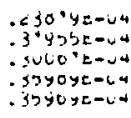 \\
\hline $\begin{array}{l}04500 E-15 \\
04560 E-15\end{array}$ & $\begin{array}{l}13: 4 y t-12 \\
: 1314 y E-15\end{array}$ & $\begin{array}{l}.200235-10 \\
.20 Y 250-10\end{array}$ & $\begin{array}{l}.2094<E=: 7 \\
.5094<c=-7\end{array}$ & $\begin{array}{l}\Rightarrow 1005-64 \\
.5 \cdot 102=-44\end{array}$ \\
\hline
\end{tabular}

$A=.335600000 t+02 \quad B=.57090020 e+0^{4}$ $.22154 \mathrm{E}=17.2053 \mathrm{IE}-10$. $540 \mathrm{OB} 3 \mathrm{E}-19$ $.21135 E-17$.22603E-10 .40097E-'Y $.34045 E-17 \quad .3774^{4} E-10 \quad .00703 E=19$ $.45275 \mathrm{E}-17$.52609k-10 $97544 \mathrm{E}=19$ $.54331 t=17$.07457t-10 :12700E-?

$.00072 \mathrm{E}-17$.6995!E-18 . 5935E-1C $.64730 \mathrm{E}=17$.90042E-10 .99209c-10 $05942 \mathrm{E}-17.90950 \mathrm{0}-17.32052 \mathrm{E}-10$ $.64077 E-97 \quad .1223^{\prime}=-17$

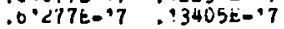
$.32052 \mathrm{E}=90$ $.20030 E=10$
$.30042 t=10$

26j91E-17 .28524t-16 .594908-9y $.56106 \mathrm{E}-17 \quad .14440 \mathrm{~d}=17$

$.34057 \mathrm{E}-10$

$\dot{c}=.3353$ sovitat $^{2}$

Cuidue-iy

$.730 \cdot 1 \mathrm{E}-2 \mathrm{C}$

$2140 \mathrm{a}=9$

-7tsut-iy

. $23354 \mathrm{t}-19$

$.29543 t-19$

$.303460-19$

$.4300 \mathrm{de}=9 \mathrm{y}$

$.5<<0 . t-1, y$

.6 . $590 \mathrm{CE}=19$

rat $=-.9747$

$.40 Y 70 \mathrm{C}-\mathrm{U} 7$

. 2ijot-lo

$.2243 t=v 0$

$139700-00$

1400t-vo

$.91701=-20$

. $\supset<33 c-v 0$

.1074 比-Ut

. OOSYE-vo

$.21054 E-30$

.$<4027 \mathrm{e}-40$

$. \angle 7095 \mathrm{~L}=\llcorner 0$

$A=.33560000 E+02 \quad E=.57090020 E+0 !$ 21099E-17.22644E-10 .4U027 E-? $.22195 E-17$.20SOLE-10 .54764E-19 $\begin{array}{rrr}.22195 E-17 & .20304 E-10 & .54764 E-19 \\ .33904 E-17 & .37673 E-16 & .00660 \mathrm{E}-19\end{array}$ $.45194 \mathrm{E}-17 \quad .52594 \mathrm{E}=10 \quad .97410 \mathrm{~L}=19$ $.54235 E-17$.67330E-!8 :127454-10

$.5070 d E-17$. $61010 \mathrm{E}=18.15907 \mathrm{c}-16$ $.04029 k-17$.95802t-1A :.9257t-10 $.05036 \mathrm{E}-17$. $10939 \mathrm{E}-17$.22010E-10 $.64579 E-17 \quad .12213 E-17$.26595E-10 $.61949 E-17 \quad .13305 k-17 \quad .3059 d t-10$ $\begin{array}{lll}.26551 E-17 & .20401 E-16 & .51341 E-19 \\ .56109 E-17 & : 14429 E-17 & .34010 E-16\end{array}$

$c=.53532000 k+u$

$.72007=-20 \quad, 2 ! G 5 E-U 0$

$.0070 c=19$.479ULE=U?

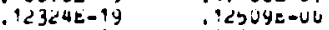

$.17020 E-19$.3?4UE=-UO

$.233^{\prime} 3 t-19 \quad .4023=-00$

$.29493 \mathrm{E}-19$

$.30205 \mathrm{E}-19$

$.43010 \mathrm{E}-19$

$.52902 e-19$

$.6: 510 E-19$

1 $594=00$

$.10704 t-06$

$.10014 \mathrm{E}=00$

$.21000 \mathrm{U}-\mathrm{U}$

$.23975 e-C a$

$\begin{array}{ll}.99050 \mathrm{E}-20 & .20901 \mathrm{k}-06 \\ .71000 \mathrm{E}-19 & .27039 \mathrm{t}-00\end{array}$

$A=.33560800 E+02 \quad B=.57098020 E+09$ $.22949 \mathrm{k}-17 \quad .24630 \mathrm{E}-1.0 \mathrm{~d} .44406 \mathrm{E}-19$ $.36179 k-17 \quad .40106 \dot{0}=10 \quad .73094: 5-19$ $.40952 \mathrm{E}-17 \quad .54640 \mathrm{E}-10$ $.55944 E-17 \quad .68467 E-10$ $.60003 \mathrm{E}-17$.8907E-98 .15997E-10

$.63959 \mathrm{E}-17$

$.94006 \mathrm{E}-10$

. $19057 \mathrm{E}-10$ $.64676 \mathrm{E}-17 \quad .10746 \mathrm{E}-17$ $.03126 \mathrm{E}-17$ $11936 \mathrm{E}-17$ $.596055-17$ $13039 \mathrm{E}-17$ $.22413 \mathrm{E}-10$ $.25997 E-18$ $.54519 \mathrm{E}-17$ $.14020 \mathrm{E}-17$

$.29006 \mathrm{E}-18$ $.33023 k=10$

$C=.53532000 \mathrm{E}+01$ BAF $=-0.9747$ $.792700=20 \quad .13731 t-00$ $.13120 t=? \quad .13741 \mathrm{t}=00$ $.16314 \mathrm{E}-19 \quad .13914 \mathrm{E}-00$ $.23704 \mathrm{E}-99 \quad .9377 \mathrm{E}=0 \mathrm{O}$ $.29509 \mathrm{E}-19$.\$5207E-00

.37909t:-?9 $.430305-19$

$.16444 E-50$ $5900 \mathrm{t}-14$ $.0124 \mathrm{E}=04$ $.20301 k-00$ $\begin{array}{ll}.59417 t-19 & .23050 e-00 \\ .69050 t-19 & .20472 t=00\end{array}$

$A=.33500800 E+02 \quad B=.57090020 E+U 1 \quad C=.53532000 t+U 1 \quad$ KAP $=-\$ 5747$ $.10435 \mathrm{E}-18$. $10754 \mathrm{E}-19$. $.19127 \mathrm{E}=20$ .53707E-18 :59440E-19: :10029E-19 $.79131 \mathrm{E}-18: 84185 \mathrm{E}-19: ? 5134 \mathrm{E}-19$ $.57331 E-10 \quad .63555 E-19 \quad .11503 E-19$ $.19059 \mathrm{E}-17$

$.23040 \mathrm{E}=? \mathrm{O} \quad .42632 \mathrm{E}=? \mathrm{3}$

$.33917 \mathrm{E}-21$

$.19431=-20$ $.210302-40$ $.14793=-07$ $.20752 \mathrm{E}-01$ $.10370 E-07$ $.207916-20$ .6 3300e-07

$.24647 E-17$.27504E-16 .50236E-ig .90273E-20 .7497 : -07 $.21945 E-17 \quad .24607 E-18 \quad .45580 E-19 \quad .02477 E-20 \quad$.70174t-U7 
Ju, net nL. KL

ly

\begin{tabular}{|c|c|c|c|c|c|c|}
\hline \multirow{2}{*}{\multicolumn{2}{|c|}{-justrar }} & \multicolumn{2}{|l|}{ rAT:II } & \multicolumn{2}{|c|}{$H_{n}=$} & t2 KL \& .647C \\
\hline & & , & 0 & $v$ & 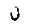 & $.190 \mathrm{UE}+7 \mathrm{UE}+\mathrm{UL}$ \\
\hline $2^{2}$ & ’ & $\epsilon$ & ? & 1 & s & $.2^{\prime} 03$ 'BdUE+U2 \\
\hline$<$ & $\dot{v}$ & $<$ & • & $u$ & ' & $.22000190=+02$ \\
\hline 4 & 4 & ' & 4 & 4 & $c$ & $.2237-504 x+02$ \\
\hline 4 & $v$ & 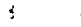 & $<$ & $v$ & $<$ & $.3299 \mathrm{JJ}+U \mathrm{UL}+\mathrm{UL}$ \\
\hline 9 & 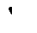 & $\alpha^{\prime}$ & $<$ & • & י & . $33>0<04 u t+0<$ \\
\hline & $E \dot{A}$ & rhnIII & & & & \\
\hline 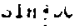 & ns & A & & & $=$ & to $K_{16}=.0470$ \\
\hline ' & U & , & $u$ & c & $\approx$ & $.00 y y 2 y 42 x+42$ \\
\hline$<$ & - & $<$ & ; & + & ; & $.299303762+02$ \\
\hline$<$ & 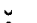 & $c$ & ? & $u$ & ' & $.219 j^{+}+97 i c+L<$ \\
\hline 2 & 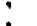 & ' & ' & 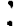 & $u$ & $.22052: 362+02$ \\
\hline 1 & • & 3 & 2 & ' & $<$ & $.327022500+42$ \\
\hline 3 & ? & 3 & $<$ & ? & $\vdots$ & $\begin{array}{l}.329033 y 0 t+c 2 \\
.33^{\circ} 05330 \mathrm{c}+02\end{array}$ \\
\hline
\end{tabular}

MeInXL nXruinLCndIE

C:3üCl

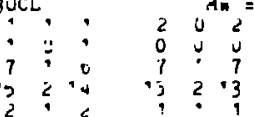

$$
\begin{aligned}
& \begin{array}{llllll}
2 & 0 & 5 & \vdots & 0 & 1 \\
2 & 0 & 4 & 0 & 9 & 0 \\
3 & 1 & 1 & 2 & 1 & 2
\end{array} \\
& \begin{array}{lll}
3 & 2 & 2 \\
3 & 2 & 1 \\
1 & 1 & 4 \\
2 & 1 & 1
\end{array} \\
& \begin{array}{lll}
2 & 5 & 1 \\
2 & 2 & 0 \\
2 & 0 & 1
\end{array}
\end{aligned}
$$

ot $\mathrm{MU}=1.0000$

$.11033340 \mathrm{c}+12$

$.197 \div 700 t+02$

$.155 ? 4000 \mathrm{E}+\mathrm{C} ?$

. $7469240 \mathrm{E}+6 \mathrm{C}$ ?

$.233 \cdot 427 U E+0$ ?

$.23 y 20400 \mathrm{E}+02$ $.24500040 E+02$ $.2995 Y 050 E+1 j 2$

$.349002102+02$

$.35070400 t+02$

.3590355 UE+OL

$.35941250 \mathrm{i}+02$

$36300 j 2 \mathrm{Uk}+\mathrm{UL}$

$.37023770 \mathrm{t}+02$

\begin{tabular}{|c|c|c|c|c|c|}
\hline $\begin{array}{ll}1 & 1 \\
0 & 3 \\
9 & 2 \\
3 & 1\end{array}$ & $\begin{array}{r}0 \\
13 \\
7 \\
2 \\
2\end{array}$ & $\begin{array}{r}1 \\
16 \\
y \\
3 \\
3\end{array}$ & $\begin{array}{l}1 \\
3 \\
2 \\
1 \\
1\end{array}$ & $\begin{array}{r}1 \\
14 \\
0 \\
3 \\
3\end{array}$ & $\begin{array}{l}.10300000 z+09 \\
.097 \div: 000 \mathrm{0}+09 \\
.96960700 z+0 ? \\
.90320000 \mathrm{0}+09 \\
.96320100 \mathrm{0}+01\end{array}$ \\
\hline $\begin{array}{l}3 \\
2 \\
3 \\
2\end{array}$ & $\begin{array}{r}4 \\
0 \\
3 \\
13 \\
9\end{array}$ & $\begin{array}{r}7 \\
10 \\
4 \\
10\end{array}$ & $\begin{array}{l}3 \\
2 \\
1 \\
3 \\
2\end{array}$ & $\begin{array}{r}13 \\
9 \\
4 \\
10 \\
10\end{array}$ & $\begin{array}{l}.12600560 t+02 \\
.14325370 z+02 \\
.16304190 \mathrm{0}+02 \\
.17206100 \mathrm{0}+02 \\
.20297090 \mathrm{E}+02\end{array}$ \\
\hline $\begin{array}{l}0 \\
3 \\
9 \\
2 \\
3\end{array}$ & $\begin{array}{c}10 \\
10 \\
17\end{array}$ & $\begin{array}{r}0 \\
19 \\
5 \\
12 \\
20\end{array}$ & $\begin{array}{l}0 \\
3 \\
1 \\
2 \\
3\end{array}$ & $\begin{array}{r}0 \\
17 \\
5 \\
19 \\
10\end{array}$ & $\begin{array}{l}.22471180 E+02 \\
.23203400 E+02 \\
.24560900 E+02 \\
.27758400 E+02 \\
.30521650 E+02\end{array}$ \\
\hline $\begin{array}{ll}0 & 3 \\
0 & 1 \\
3 & 2 \\
2 & 1 \\
2 & 0\end{array}$ & $\begin{array}{r}17 \\
j \\
11 \\
2 \\
2\end{array}$ & $\begin{array}{r}20 \\
6 \\
13 \\
1 \\
1\end{array}$ & $\begin{array}{l}3 \\
? \\
2 \\
1 \\
0\end{array}$ & $\begin{array}{r}10 \\
0 \\
12 \\
1 \\
1\end{array}$ & $\begin{array}{l}30527+00 E+02 \\
.34370830 E+02 \\
.3609630 E+02 \\
.43303710 E+02 \\
.44917750 E+02\end{array}$ \\
\hline $\begin{array}{l}1 \\
1 \\
2 \\
1 \\
2\end{array}$ & $\begin{array}{r}0 \\
1 \\
12 \\
7 \\
13\end{array}$ & $\begin{array}{r}7 \\
1 \\
4 \\
0 \\
15\end{array}$ & $\begin{array}{l}1 \\
1 \\
2 \\
\vdots \\
2\end{array}$ & $\begin{array}{r}7 \\
0 \\
13 \\
0 \\
14\end{array}$ & $\begin{array}{l}45001010 E+02 \\
.46501400 E+02 \\
.47554530 E+02 \\
.58615410 E+02 \\
.00007010 E+02\end{array}$ \\
\hline $\begin{array}{l}1 \\
0 \\
2 \\
2 \\
1\end{array}$ & $\begin{array}{l}3 \\
3 \\
2 \\
1 \\
2\end{array}$ & $\begin{array}{l}5 \\
2 \\
2 \\
2 \\
2\end{array}$ & $\begin{array}{l}1 \\
0 \\
2 \\
2 \\
1\end{array}$ & $\begin{array}{l}2 \\
2 \\
1 \\
0 \\
1\end{array}$ & $\begin{array}{l}.04936300 E+02 \\
.67251050 \mathrm{E}+02 \\
.67494740 E+02 \\
.67536090 \mathrm{0}+02 \\
.69052030 \mathrm{E}+02\end{array}$ \\
\hline $\begin{array}{ll}4 & 1 \\
4 & 0 \\
4 & 2 \\
4 & 3 \\
4 & 3\end{array}$ & $\begin{array}{l}4 \\
4 \\
3 \\
2 \\
1\end{array}$ & $\begin{array}{l}3 \\
3 \\
3 \\
3 \\
3\end{array}$ & $\begin{array}{l}1 \\
0 \\
2 \\
3 \\
3\end{array}$ & $\begin{array}{l}3 \\
3 \\
2 \\
1 \\
0\end{array}$ & $\begin{array}{l}.86545900 E+02 \\
.89579200 E+02 \\
.89861500 E+02 \\
.69948300 E+02 \\
.69950300 E+02\end{array}$ \\
\hline $\begin{array}{l}2 \\
1\end{array}$ & $\frac{2}{3}$ & 3 & 2 & $\begin{array}{l}1 \\
2\end{array}$ & $\begin{array}{l}.90 \div 64500 E+02 \\
.93048500 E+02\end{array}$ \\
\hline
\end{tabular}

FoHAL ACID nCOUn

A

A $=$ $10307 \mathrm{E}-10 \quad .17800 \mathrm{k}-19 \quad .32240 \mathrm{k}-20$ $33622 t-10 \quad 30707 t-19$ 65 $431 t-20$ $.00403 \dot{E}-14.11655 \mathrm{E}-9 \mathrm{~g} \quad .24746 \mathrm{E}-20$ $.03510 \mathrm{E}-20.02989 \mathrm{E}-20.31015 \mathrm{~L}-20$ $.175 d>t-17,1970<t-18,3018 \cdot z=19$

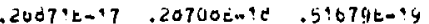

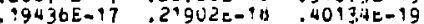
.95009k-1E . 1537?E-id .39745E-19 $.04035 \mathrm{t}-17 \quad .7039$ Ee-? $.14223 \mathrm{E}+10$ $.03097 t-17 \quad .93404 t=8 \quad .17121 E-10$

$.32700 \mathrm{E}-17 \quad .45302 \mathrm{E}-10 \quad .00923 \mathrm{E}-19$ $.32774 \mathrm{E}-17$. $45396 \mathrm{E}-10$. $09100 \mathrm{E}=19$ $.45230 E-17$.49!97E-4ه .69094k-19 $.72937 \mathrm{E}-17.02491 \mathrm{E}-10$, $15062 \mathrm{E}-18$

$A=.77512200 E+0 E \quad B=.120 j 5110 E+02$ $.40771 \mathrm{E}-19 \quad .47170 \mathrm{E}-20 \quad .67201 \mathrm{E}-21$ $15687 t-29 \quad .31516 E-20 \quad .32505 t-20$ $.44194 t-19 \quad .20590 t-19.93036 \mathrm{E}-20$ $.43 ? 546=i d \quad .54051 E-19 \quad .11544 E-19$ $.43159 \mathrm{E}-10 \quad .5905: 19.19 \quad .11544 \mathrm{E}-19$

$+1305 E-29.39079 k-20$ $49705 E-19 \quad 44703 E-19$.96379t-10 $.7397: E=16 \quad 19573 E-16 \quad .23659 E-19$ $.7-277 E-22 \quad .47095 E-20 \quad .71246 \mathrm{E}-20$ $.46909 E=19 \quad .63251 E-19$

$.20144 E-19$

$.60037 c-11$ . $439: 3 \mathrm{E}-2 \mathrm{i}$ $10261 E-7$.

$4257 \mathrm{~T} E-11 \mathrm{~F}$ .2354 É-22

$63001 \mathrm{E}-18$

$52069 \mathrm{E}-20$

१ $10903 E=90$

$91286 \mathrm{E}=90$ $.97028 \mathrm{E}-20$ $.97028 \mathrm{E}-20$
$.41036 \mathrm{E}-19$ $.36653 E-19$ $.23544: \div-22$ !2 $150 \mathrm{E}-77$ . $330895=-9$ . $25750 \mathrm{E}-16$ $43057 t-16$

$.12674 \mathrm{E}-17.30230 \mathrm{~L}-10$ $.29435 t-16.36443 t-17$ $23135 t-19,10 y 94-18$ $11850=-17 \quad 44203 E-18$ .14652E-19 . 11392E-18

$.80103 E-16 \quad .11805 k-16$ $.12333 z-15 \quad .15227 t=16$ $30308 t-16$.65832E-17 $36506 \mathrm{E}-16$. boObOt-17 $.99560 \mathrm{E}=16 \quad .13023 \mathrm{E}-16$

$.17 \Delta 7 \Delta E-15$.2731日E-16 23496t-15 , 33005E-10 $93792 \mathrm{E}-16,19313 \mathrm{E}=16$ 24736E-16 .82?09E-?7 $.24737 \mathrm{E}-16$ $.82112 \mathrm{E}-17$ $.12544 \mathrm{E}-19$

$.12546 \mathrm{t}-19$ $.63548 \mathrm{E}-19$ $.53393 \mathrm{E}-19$ $.59000 \mathrm{E}-10$ $.80246 \mathrm{E}-98$

$.90545 \mathrm{E}-19$ $.60 \mathrm{b94t-16}$ $.09440 \mathrm{~b}-19$ $12094 \mathrm{E}-10$ $.05028 \mathrm{E}-19$

$.23125 \mathrm{e}-17$ $.28766 \mathrm{E}-17$ $.14126 E-17$ $.14176 \mathrm{E}-17$ $.20632 \mathrm{E}-17$

. 554:0E- 17 . 65090E-17 $.43269 \mathrm{E}-17$ . $2 \div 509 \mathrm{E}-17$ $.21570 \mathrm{E}-17$

$.94323 \mathrm{E}-16$ 19809E- 15
$19435 t-15$

$.43559 \mathrm{E}-17$ $.630+5 E-17$
200

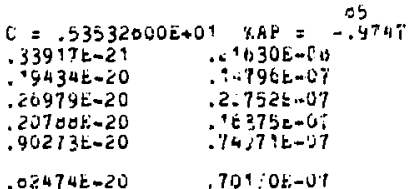

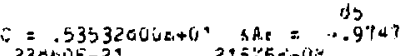

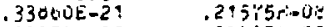

$.9974 E-20 \quad .9975-0 j$

$.20734 \mathrm{t}-20 \quad$. $20701 \mathrm{~L}+\mathrm{C}$ ?

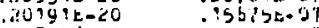

$.70727 E-20 \quad$.053प2kn

$. \$ 0120 t-20 \quad .747 d d z-07$

$.0 C 033 i-20 \quad .070 \$ 4 E-i$ ?

$C=56700200 \mathrm{~b}+0 ! \quad 64 P=\frac{1120}{1.965 \mathrm{t}}$

$.57677 \mathrm{~L}-29 \quad .320195 \mathrm{~L}-00$

$.9005 t-20 \quad$.66574E-00

$.47943 \mathrm{e}-2.9 \quad .77<50 \mathrm{t}-6 \mathrm{9}$

$.00107 \mathrm{E}-21 \quad .944 \exists 3 \mathrm{E}-09$

$.650902-20 \quad .4425 \mathrm{JE}-0 \mathrm{i}$

$. \exists 2: 095-20$

$.72235 \mathrm{E}-20$

. OOT5OE-T:

$.25609 \mathrm{E}+99$

$637 a d t-1, ?$

. 7760 :-vi

. $2030302-17$

. $99465-18$

$10564 \varepsilon_{i}-19$

$.16599 \mathrm{E}-19$

.1594 'E- 19

$.27107 \mathrm{E}-19$

$12 \delta 26 E-2 \dot{0}$

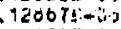

. $20036 \mathrm{Em}=\mathrm{b}$

. $2 \$ 27 \mathrm{CE}=0 \mathrm{~b}$

f 357509159

$C=.104: 6120 \mathrm{z}+0 \% \mathrm{KAF}=-.9511$

$.15764 \mathrm{E}-21 \quad$.4956.

$.13079 E-20 \quad, 51252 E-54$

$.220005-20 \quad .06444 E=159$

$.21460 E-20 \quad .17040 F+00$

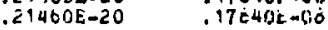

$.232103-20 \quad .124065-04$

$49653 \mathrm{t}-20 \quad .37097$ zi-jo

$.44973 \mathrm{E}-20 \quad .49555 \mathrm{E}=0 \mathrm{H}$

$.36844 E-20 \quad$.21 34.JE $=1,3$

$.70670 \mathrm{E}-20$

$.556731-1: 0$

$.20080 \mathrm{E}-19$

$.556935-20$

$.80220 \mathrm{E}-20$

$111648-19$

$.00300 E-20$

$.803075-20$

$.12846 \mathrm{E}-19$

? $6570 \mathrm{E}-19$

$10897 \mathrm{E}-18$

$.15860 \mathrm{E}-18$

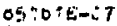

$.60552 E^{\circ}=610$

. $11919-117$

.$: 13935.007$

$.12227 \varepsilon-3 ?$

$.1226 .8 E-07$

$.2 \div 84_{1} 26-07$

- $223264-07$

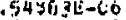

-31597 $=06$

. 19032E-19

$.12594 \mathrm{t}-1 \mathrm{~d}$

$.23291 \mathrm{E}-19$

.2057 $8 \mathrm{E}=19$

. $31212 \mathrm{E}-19$

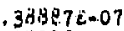

- 585 5月E-UD

. $40787 E=07$

. $14539 \mathrm{E}-\mathrm{U} 7$

?०194E-0?

$42 b 07 \vec{E}-18$

$.23499 k=05$

. $2940010-05$

. $164294-05$

. 16,$17 ;=05$

.2924 LE=05

$27610 E-18$

$.49509 E-10$

$.6082+45-05$

$.7190 .54=05$

. $\$ 472 \mathrm{t}=05$

. $3106 \mathrm{ct}=05$

$.31071 \mathrm{E}-0 \mathrm{~S}$

. 55c25t-05

$.757 ? 2 E=05$ 
JU

KU1 KU2 JL KLT KL2

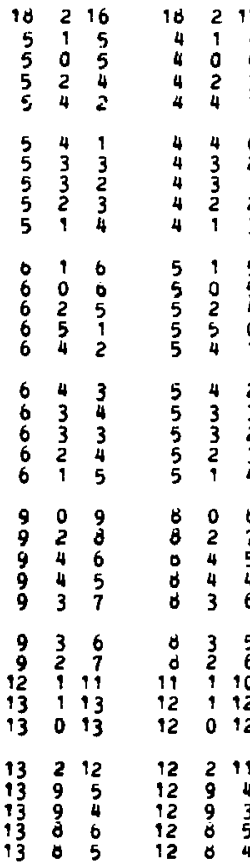
$\begin{array}{rrrrrr}13 & 10 & 4 & 12 & 10 & 3 \\ 13 & 10 & 3 & 12 & 10 & 2 \\ 13 & 7 & 7 & 12 & 7 & 6 \\ 13 & 7 & 0 & 12 & 7 & 5 \\ 13 & 11 & 3 & 12 & 11 & 2\end{array}$

13112212111 $\begin{array}{rrrrrr}13 & 11 & 2 & 12 & 11 & 1 \\ 13 & 12 & 2 & 12 & 12 & 1 \\ 13 & 12 & 1 & 12 & 12 & 0 \\ 13 & 6 & 0 & 12 & 0 & 7 \\ 13 & 0 & 7 & 12 & 0 & 6\end{array}$

$\begin{array}{rrr}13 & 5 & 9 \\ 13 & 5 & 6 \\ 13 & 4 & 10 \\ 13 & 4 & 9\end{array}$ 13311

13310

FOAMIC ACIO $\mathrm{HCOOH}$

$\begin{array}{rrr}6 & 2 \\ 1 & 1 \\ 2 & 0 \\ 11 & 1 \\ 16 & 2 & 1 \\ 18 & 2 & 17 \\ 4 & 0 & 4 \\ 9 & 2 & 7 \\ 18 & 4 & 14 \\ 9 & 1 & 10 \\ 19 & 2 & 10 \\ 18 & 3 & 1 \\ 11 & 3 & 9 \\ 9 & 1 & 9 \\ 2 & ?\end{array}$

is $2 \quad 13$
$.10763010 \mathrm{E}+03$ $10812670 E+03$ $.11174600 E+03$

- $11228710 \mathrm{E}+03$ $.11243230 \mathrm{E}+03$

$.11243230 \mathrm{E}+03$ $.11245900 \mathrm{E}+03$ $.11246700 \mathrm{E}+03$

. $1289440 E+03$

$.11631150 E+03$

$12907160 \mathrm{t}+03$

$.13376710 \mathrm{E}+03$

$.13468040 \mathrm{E}+03$

$13492850 \mathrm{k}+03$

$.13493 d 50 E+03$

$.13498560 E+U 3$

$.13500490 E+03$

13940 $370 \mathrm{E}+03$

$.19875520 \mathrm{E}+03$

$.20167370 E+03$

$.20252730 \mathrm{E}+03$

$.20253000 E+03$

$.20205450 \mathrm{E}+03$

$.20281520 E+03$

$.205127 \triangle 0 E+03$

$.27692920 \mathrm{E}+03$

$.27918210 E+03$

$.28293980 E+03$

$.290314105+03$

$.292429 C 0 E+03$

$.29242190 \mathrm{E}+03$

$.29242990 E+03$

$.29242990 \mathrm{E}+03$

$.29243610 k+03$

$.29243610 E+03$

$.24244440 E+03$

$.29244440 E+03$

$.29246170 \mathrm{E}+0$.

$.292461706+03$

$.29249610 \mathrm{E}+03$

$.29249610 \mathrm{t}+03$

$.29250120 k+C$

$.29250120 \mathrm{e}+03$

$.29262300 \mathrm{E}+03$

$.29202400 \mathrm{E}+0$

$.29206450 \mathrm{E}+03$

$.29290260 \mathrm{E}+03$

$.29302770 \mathrm{t} \rightarrow 03$

$.29404320 c+03$
$.34939 \mathrm{E}-15 \quad .49495 \mathrm{E}-10 \quad .10510 \mathrm{E}=?$

156:7t-15

$.99649 \mathrm{i}=17$

$37901 E-10$

$.75870 \mathrm{ki}=17$

$.99049 \mathrm{E}-17$

$.53974 \mathrm{E}-16$

. 539 dOt- 6

- $5706 \mathrm{E}-1$

$.75070 \mathrm{E}-1 \mathrm{~T}$

$.2906 / E-10$

.30271E-?6

$55590 \mathrm{k}-10$

. 35269t-15

$.43557 \mathrm{E}-15$

$.20709 E-15$

$.19223 \mathrm{~s}-10$

$.19220 \mathrm{~B}-16$

$.79084 \mathrm{E}-10$

$.79101 \mathrm{E}-16$

$.20920 \mathrm{t}-15$

$.37572 \mathrm{E}-15$

$.40190 \mathrm{E}-15$

$.20450 \mathrm{E}-15$

$.25667 \mathrm{E}-16$

b67E-16

.00607 É-16

$.86677 t-16$

. $20673 \mathrm{E}-15$

- $12712 \mathrm{E}-15$

$97053 E-16$

$.10486 E-15$

$.53164 E-16$

-13520E-2

$.13520 \mathrm{E}-21$

$24112 E-20$

$.24112 t-20$

$.51595 E-23$

$.51595 \mathrm{E}-23$

.29962E- 19

.29962 e-19

. $12706 \mathrm{E}-24$

$.1270 B E-24$

$.17046 \mathrm{E}-26$

$.17040 \mathrm{~s}-26$

$.20204 \mathrm{Em}$

$.20264 \mathrm{E}-10$

$16339 \mathrm{E}-17$
$.16359 \mathrm{E}-17$

$.72716 \mathrm{E}-17$

$.72720 \mathrm{E}-17$

$.23120 \mathrm{k}-16$

$.23128 \dot{0}-16$

75911 E-16

$.87144 E-16$

.00841E-9h

$.55932 E=17$

$17770 \mathrm{E}-10$

. $37493 \mathrm{E}=16$

$.37503 \mathrm{E}-16$

0 !670k-10

$.44975=10$

. $17295 \mathrm{E}-15$

$.13049 \mathrm{~b}-15$

$.51663 E-16$

$51664 \mathrm{E}-1 \mathrm{~b}$

$.89417 \bar{E}-16$

$.89531 E-16$

. $13377 \mathrm{E}-15$

. $10319 \mathrm{E}-15$

$19236 E-15$

$.14931 \mathrm{E}-15$

59755E-18

$59755 \mathrm{E}-16$

$.21033 E-17$

$.21033 E-? 7$

. $3905 t-10$

$13905 \mathrm{E}=18$

$.6244 \div \mathrm{t}-17$

$.02441 \mathrm{E}-17$

. 25570 E- 19

.25570t-19

. $30021 \mathrm{E}-20$

$30821 \mathrm{t}-20$

. $15630 \mathrm{E}-16$

.15030E-16

. $34528 E-16$

- 34つ2४ E-10

$.65094 \mathrm{E}=10$

$65100 E=10$

$10627 \mathrm{E}-15$

99015E-17

20284E-??

. 202d4ti-i7

.50411 É-11

$.50416 \mathrm{e}-17$

.90537 E- $?$

$.92010 \mathrm{t}-16$

$.17259 \mathrm{t}-10$

$.19330 c-16$

$.15347 E-16$

. $734 \mathrm{E}=17$

$.05712 \mathrm{t}-17$

$.11091 \mathrm{E}-1 \mathrm{t}$

$.11094 \mathrm{t}-16$

$.15572 E-16$

$.19042 t-16$

$.49538 t-16$

$.420 \div 9 E-16$

$.24747 \mathrm{E}-16$

$.2474 \sigma E-16$

$.34286 \mathrm{E}=16$

$.34330 \ddot{-16}$

$.43 d 94 E-16$

$.05094 \mathrm{E}-10$

$.69330 \mathrm{E}-16$

$.79919 \mathrm{E}-16$

$.37204 \mathrm{E}-17$

.37204E-17

$.76244 E-17$

$.70244 E^{2}-17$

$.1593 \mathrm{dE}=17$

$.15930 \mathrm{E}-17$

$.94045 E-16$

$.14045 \mathrm{e}-10$

$.56847 \mathrm{t}-18$

$.50047 t-18$

$.14245 t-10$

$.14245 t=18$

$.23540 \mathrm{E}-16$

$.23546 \mathrm{E}-16$

- 36201 E- 16

- $36201 \mathrm{E}-10$

$51278 \mathrm{EE}-16$

.51269t- 16

$.67035 \mathrm{E}-16$

$.67412 \mathrm{t}-16$

$.10673 \mathrm{E}-15$

$$
\text { 1.05 }
$$

$=.77512200 t+02 \quad B=.12055110 t+02$ $\begin{array}{lll}.34129 E-19 & .10031 \mathrm{E}-19 & .25301 \mathrm{E}-20 \\ .77707 \mathrm{E}-19 & .09691 \mathrm{E}-20 & .16560 \mathrm{E}-20\end{array}$ $\begin{array}{rr}.77707 E-19 & .09691 \mathrm{~s}-20 \\ .81630 \mathrm{~b}-19 & .91470 \mathrm{~d}-20\end{array}$ $.00004 \mathrm{E}-20 \quad .57460 \mathrm{e}-20$ $.02041 \mathrm{t}-22$. $09000 \mathrm{ki}=\alpha 1$

$10732 \mathrm{E}-20$

$.20 d 15 \mathrm{E}-20$

$.61958 \mathrm{E}-21$

$.43701 \mathrm{E}-22 \quad .15904 \mathrm{e}-20$

$.3309 b z-16 \quad .46407 \mathrm{~s}-19$

$12372 E-22 \div 443 U E-20$

$.86295 t-19 \quad .43534 t-19$

$.20095 \mathrm{~m}-20$

$.91079 E-20$

$274915-20$

$.139400-19$

$35003300 \mathrm{t}+0$

. उ05/Yy50E+02

$.30706090 k+02$

. $39537900 z+02$

$.49034940 E+02$

$.405 a^{4} 2 \angle 0 E+02$

, 3037 ○ं-22

$.20300 t-20$

$.31309 k-20$

$.40452 t-20$

$92004 \mathrm{c}-20 \quad .26739 \mathrm{k}-20$

b2750t-19

$.10291 \mathrm{k}-17$

$.20479 t-1 y$

$91043 \mathrm{E}-20$

$.24070 \mathrm{e}-19$

$.19990 \mathrm{t}-20 \quad .15543 \mathrm{e}-19$

$.596555-19$

$.264246-17$

$23007 c-17$

$10321 \mathrm{E}-11$

$.05044 t-16$

$.05044 \dot{z}-18$

$.12932 \varepsilon-17$

$12933 t-17$

$.23494 \mathrm{E}-17$

-3400टt-17

$.30240 \mathrm{t}-17$

.3240 ÚE-17

0037 t- ?

$16794 t-17$

$10797 \mathrm{E}-17$

$.25304 \mathrm{E}-17$

$32690 c-17$

.39705k-17

11147t-16

$.10241 \mathrm{E}-10$

$72014 \mathrm{E}=17$

$.89260 E=17$

.0940:E-17

$10572 E-10$

$23205 t-10$

.25595k-10

.24504 E- 16

. $39031 E-17$

$39031 E-17$

$.61034 \mathrm{E}-17$

$22022 \mathrm{e}-17$

$.60504=-17$

$.06504 \mathrm{E}-17$

$.11270 \leq-17$

$.11270 \mathrm{E}-17$

$.40710 \mathrm{E}=18$

$.40710 \ddot{E}-18$

$.12075 \mathrm{t}-10$

$.12075 \mathrm{E}=16$

.15505t-10

-155d5E-15 


\begin{tabular}{|c|c|c|c|c|c|c|}
\hline $\begin{array}{r}13 \\
0 \\
5 \\
10 \\
17\end{array}$ & $\begin{array}{l}2 \\
1 \\
0 \\
3 \\
3\end{array}$ & $\begin{array}{r}12 \\
0 \\
5 \\
? 6 \\
? 4\end{array}$ & $\begin{array}{r}12 \\
7 \\
4 \\
17 \\
10\end{array}$ & $\begin{array}{l}3 \\
2 \\
1 \\
4 \\
4\end{array}$ & $\begin{array}{r}9 \\
5 \\
4 \\
13 \\
13\end{array}$ & $\begin{array}{l}.50653020 t+02 \\
.53251920 e+02 \\
.53206950 t+02 \\
.57491650 t+02 \\
.605209702+02\end{array}$ \\
\hline $\begin{array}{r}1 \\
7 \\
10 \\
2 \\
1 !\end{array}$ & $\begin{array}{l}1 \\
1 \\
1 \\
\vdots \\
2\end{array}$ & $\begin{array}{l}0 \\
7 \\
y \\
1 \\
y\end{array}$ & $\begin{array}{r}1 \\
6 \\
4 \\
2 \\
1 n\end{array}$ & $\begin{array}{l}0 \\
2 \\
2 \\
0 \\
3\end{array}$ & $\begin{array}{l}1 \\
4 \\
0 \\
2 \\
0\end{array}$ & $\begin{array}{l}.67094560 E+02 \\
.67967130 \mathrm{E}+02 \\
.68492010 \mathrm{E}+02 \\
.60704140 \mathrm{0}+02 \\
.66924510 \mathrm{E}+02\end{array}$ \\
\hline $\begin{array}{l}3 \\
9 \\
17 \\
10\end{array}$ & $\begin{array}{l}3 \\
1 \\
1 \\
2 \\
1\end{array}$ & $\begin{array}{r}5 \\
2 \\
0 \\
15 \\
9\end{array}$ & $\begin{array}{r}12 \\
3 \\
9 \\
16 \\
10\end{array}$ & $\begin{array}{l}2 \\
0 \\
0 \\
3 \\
0\end{array}$ & $\begin{array}{r}11 \\
3 \\
9 \\
14 \\
10\end{array}$ & $\begin{array}{l}.09654370 E+02 \\
.71324890 E+02 \\
.11034010 \mathrm{~L}+03 \\
11419060 \mathrm{0}+03 \\
.12180770 \mathrm{0}+03\end{array}$ \\
\hline 19 & $\begin{array}{l}0 \\
5\end{array}$ & $\begin{array}{r}0 \\
15\end{array}$ & $\begin{array}{r}7 \\
20\end{array}$ & 1 & $\begin{array}{r}7 \\
10\end{array}$ & $\begin{array}{l}13098020 \mathrm{t}+03 \\
.13624040 \mathrm{0}+03\end{array}$ \\
\hline
\end{tabular}

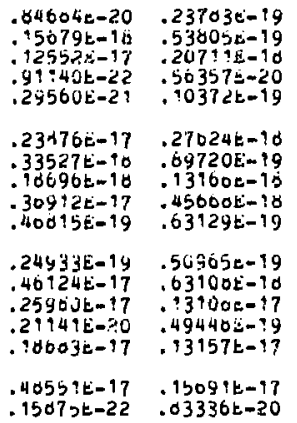

$.12730 E-19$ $.14291 \mathrm{E}-? 9$ $.04550 \mathrm{E}-20$ $.12003 \mathrm{E}-19$

$.51066 \mathrm{E}-19$ $.21935 E-19$ $.44436 \varepsilon-19$ $.06334 E-19$ $.20094 E=19$

$.24539 \mathrm{E}-\div 9$ -39564E-1D . $336 \mathrm{bOL}-19$ $.444096=16$ .25 505t-19 $.43096 \mathrm{k}-19$ $.12337 \mathrm{E}-10$

$.4005 j E-10$
$.39 \cdot 59 t-20$ -30У00 $.02656 E-20$ $43542 t-20$ $.00360 \mathrm{c}-20$

$.92345 E-20$ $.4560^{\circ} \mathrm{E}-20$ $.10055 \mathrm{t}-19$ $15783 E-49$ $.70535 \mathrm{E}-20$

. 7 1592 $\mathrm{b}^{-20}$ . $22935 \mathrm{E}=19$

\begin{tabular}{|c|c|c|c|c|c|}
\hline $\begin{array}{l}2 \\
3 \\
3 \\
2 \\
3\end{array}$ & $\begin{array}{r}3 \\
: 3 \\
14 \\
4 \\
15\end{array}$ & $\begin{array}{r}5 \\
16 \\
17 \\
6 \\
10\end{array}$ & $\begin{array}{l}c^{n} \\
3 \\
3 \\
2 \\
3\end{array}$ & $\begin{array}{r}16 \\
4 \\
14 \\
25 \\
5 \\
26\end{array}$ & $\begin{array}{r}\text { UU }=1.647 \\
.10445700 E+00 \\
.14324300 E+00 \\
.20449400 E+00 \\
.20867700 E+00 \\
.20607100 E+00\end{array}$ \\
\hline $\begin{array}{l}2 \\
3 \\
3 \\
2 \\
2\end{array}$ & $\begin{array}{r}5 \\
10 \\
17 \\
0 \\
7\end{array}$ & $\begin{array}{r}7 \\
19 \\
20 \\
0 \\
9\end{array}$ & $\begin{array}{l}2 \\
3 \\
3 \\
2 \\
2\end{array}$ & $\begin{array}{r}6 \\
17 \\
10 \\
7 \\
0\end{array}$ & $\begin{array}{l}.37500000 E+00 \\
.39300000 E+00 \\
.53120400 E+00 \\
.02627000 E+00 \\
.98373200 E+00\end{array}$ \\
\hline $\begin{array}{l}1 \\
2 \\
2 \\
2 \\
1\end{array}$ & $\begin{array}{r}0 \\
0 \\
y \\
10 \\
1\end{array}$ & $\begin{array}{r}1 \\
10 \\
11 \\
12 \\
2\end{array}$ & $\begin{array}{l}1 \\
2 \\
2 \\
2 \\
1\end{array}$ & $\begin{array}{r}1 \\
9 \\
10 \\
11 \\
2\end{array}$ & $\begin{array}{l}.10404880 E+01 \\
.14747070 E+01 \\
.21267060 E+0 ! \\
.29776030 \dot{0}+0 ? \\
.31393000 E+09\end{array}$ \\
\hline $\begin{array}{l}2 \\
2 \\
1 \\
2 \\
2\end{array}$ & $\begin{array}{r}11 \\
12 \\
2 \\
13 \\
14\end{array}$ & $\begin{array}{r}13 \\
14 \\
3 \\
15 \\
10\end{array}$ & $\begin{array}{l}2 \\
2 \\
1 \\
2 \\
2\end{array}$ & $\begin{array}{r}12 \\
13 \\
3 \\
14 \\
15\end{array}$ & $\begin{array}{l}.40561370 E+01 \\
.5401910 E+01 \\
.62700500 E+01 \\
.70520709 E+0 ? \\
.90501000 t+01\end{array}$ \\
\hline $\begin{array}{l}1 \\
2 \\
2 \\
1 \\
2\end{array}$ & $\begin{array}{r}3 \\
15 \\
10 \\
4 \\
17\end{array}$ & $\begin{array}{r}4 \\
17 \\
10 \\
5 \\
19\end{array}$ & $\begin{array}{l}i \\
2 \\
2 \\
i \\
2\end{array}$ & $\begin{array}{r}4 \\
16 \\
17 \\
5 \\
10\end{array}$ & $\begin{array}{l}.10463970 \mathrm{c}+02 \\
.11430530 \mathrm{0}+02 \\
.14201900 \mathrm{0}+02 \\
.15695120 \mathrm{0}+02 \\
.17505920 \mathrm{0}+02\end{array}$ \\
\hline $\begin{array}{l}2 \\
1 \\
1 \\
0 \\
1\end{array}$ & $\begin{array}{r}10 \\
5 \\
0 \\
7 \\
7\end{array}$ & $\begin{array}{r}20 \\
0 \\
7 \\
0 \\
0\end{array}$ & $\begin{array}{l}2 \\
1 \\
1 \\
0 \\
1\end{array}$ & $\begin{array}{r}19 \\
6 \\
7 \\
0 \\
0\end{array}$ & $\begin{array}{l}.21397440 \mathrm{t}+02 \\
.21971700 \mathrm{r}+02 \\
.29293290 \mathrm{0}+02 \\
.34351430 \mathrm{t}+02 \\
.37050830 \mathrm{E}+02\end{array}$ \\
\hline $\begin{array}{c}1 \\
1 \\
4 \\
0 \\
1\end{array}$ & $\begin{array}{r}6 \\
9 \\
2 \\
2 \\
10\end{array}$ & $\begin{array}{r}9 \\
10 \\
1 \\
11\end{array}$ & $\begin{array}{l}1 \\
\vdots \\
i \\
i\end{array}$ & $\begin{array}{r}y \\
10 \\
1 \\
1\end{array}$ & $\begin{array}{l}.47007730 E+02 \\
.57518000 E+02 \\
.67653620 t+02 \\
.68699410 E+02 \\
.69090610 \mathrm{~b}+02\end{array}$ \\
\hline $\begin{array}{l}\vdots \\
1 \\
1 \\
\vdots \\
2\end{array}$ & $\begin{array}{l}9 \\
19 \\
12 \\
3 \\
2\end{array}$ & $\begin{array}{r}1 \\
12 \\
13 \\
2 \\
2\end{array}$ & $\begin{array}{l}1 \\
\vdots \\
1 \\
\vdots \\
4\end{array}$ & $\begin{array}{r}0 \\
12 \\
13 \\
2 \\
1\end{array}$ & $\begin{array}{l}.09740720 \mathrm{0}+02 \\
.01541000 \mathrm{0}+02 \\
.95100200 \mathrm{0}+02 \\
.10147760 \mathrm{0}+03 \\
.10303000 k+03\end{array}$ \\
\hline $\begin{array}{l}0 \\
2 \\
1 \\
\vdots \\
?\end{array}$ & $\begin{array}{r}3 \\
1 \\
2 \\
13 \\
14\end{array}$ & $\begin{array}{r}2 \\
2 \\
2 \\
14 \\
15\end{array}$ & $\begin{array}{l}j \\
2 \\
1 \\
1 \\
1\end{array}$ & $\begin{array}{r}2 \\
0 \\
1 \\
14 \\
15\end{array}$ & $\begin{array}{l}.90304020 E+03 \\
.10305100 E+03 \\
.10401700 E+03 \\
.10970940 E+03 \\
.12534 ? 30 \mathrm{x}+03\end{array}$ \\
\hline $\begin{array}{l}1 \\
3 \\
3 \\
0 \\
2\end{array}$ & $\begin{array}{l}4 \\
\vdots \\
4 \\
3\end{array}$ & $\begin{array}{l}3 \\
3 \\
3 \\
3 \\
3\end{array}$ & $\begin{array}{l}1 \\
3 \\
3 \\
0 \\
2\end{array}$ & $\begin{array}{l}3 \\
1 \\
5 \\
3\end{array}$ & $\begin{array}{l}13529700 \mathrm{e}+\mathrm{U} 3 \\
: 13730920 \mathrm{e}+03 \\
.13730 y 20 \mathrm{v}+03 \\
.13737110 \mathrm{c}+03 \\
.13730 \mathrm{v}=\mathrm{t}+03\end{array}$ \\
\hline
\end{tabular}
$.23496 t-19$ $.10840 \dot{E}-10$

$.07642 \mathrm{t}-19$ $.16700 \mathrm{E}=19$ $.91463 \mathrm{E}-19$

$.71945 k-00$ $.60922 \mathrm{E}-40$ $.24449 \mathrm{t}-07$ $.11053 E-07$ $.17118 E=07$

$.11803 E-00$ $.13515 E-07$ $.27501 E \backsim C 7$ $.12633 \mathrm{E}-06$ .10191607

$.20196 c-07$ . $3531 \mathrm{E}-06$ $.39074 E-06$

10689E-06 $.46932 \mathrm{E}-06$

$.40121 E-00$

$. \$ 5572 \mathrm{t}-00$

THIOFUKMALDEHYYE Hicis
$A=.29100000 E+03 \quad b=.97099555 E+02$ $.17307 E-22$. $18035 E-22$.09306E-23 $.41474 E-20,12450 t-24$ $.19500 \mathrm{E}-20 \quad .1365: E-24$ $356051 \mathrm{i}-22$ 49935t-22 $.02057 \dot{E}-29.13943 \mathrm{E}-24$

$55991 \mathrm{E}-22.91100 \mathrm{E}-2$ ? $.30609 E-29 \quad .13327 \varepsilon-24$ $10202 \mathrm{t}-29 \quad 11900 \mathrm{t}-24$ 7077 oE-22 $.74251 \varepsilon-22$ $.66060 \mathrm{E}-22$ $.50612 \mathrm{E}-22$ $33772 E-22$

9794E-22 . 39090E-21 $10250 E-22.92457 E-21$ $37452 E-16 \quad 70004 E-14$ $.47251 \mathrm{t}-23 \quad .89042 \mathrm{E}-21$ $.19425 \mathrm{t}-23 \quad .01537 \mathrm{t}-21$

.5740yE-10 .143716-10 $.71511 \mathrm{E}-24 \quad .09010 \mathrm{E}-21$ $2304 i t=24 \quad .50430 \mathrm{E}-2$ $.69245 \mathrm{E}-9 \mathrm{O} \quad .22203 \mathrm{E}-? \mathrm{~b}$ $.70340 \leq-25$

$16874 \mathrm{E}-25$

$.6 y 322 E-18$ $.59349 t=10$ $.50526 \mathrm{E}-16$ $.44262 t-10$

$29909 \mathrm{E}-10$ $.17026 E-10$ $.14483 \mathrm{e}-15$ . $30342 e-15$ . $84113 \mathrm{e}-19$

$15277 t-15$ $.41933 \mathrm{E}-19$ $17003 E-19$ $45402 t=15$ $40206 E-? 0$

$.10102 t-14$ $.44222 \mathrm{E}-16$ $.47533 t-15$ oc $30=2-20$ $.23021 \mathrm{t}-20$

$.02015 k-15$ $.20003 t-17$ .20003b-17 $.17215 \mathrm{~s}-14$ $.92910 \mathrm{c}=10$ $.64395 \mathrm{E}-20$ $.17 \div 95 \mathrm{E}=18$
$.60175 \mathrm{E}-24$ $.90915 E-24$ $.21205 \mathrm{k}-22$ $.13599 k-23$

$.52935 \mathrm{E}-22$ $.17780 \mathrm{E}-23$ $.22206 \mathrm{E}-23$ $11346 \mathrm{EE} 2$ ? $.21572 \mathrm{E}-29$

. 33 90t-20 . $37154 \mathrm{t}-21$ $.50602 E=21$ $.86043 E-21$

$.12022 E-20$ $.15726 E-20$ $17147 E-19$ . $1954: E-2 D$ $.23161 t-20$

$.34300 E=19$ $.20276 \mathrm{E}-20$ $.200 \div 5 \mathrm{E}-20$ $.57929 \mathrm{E}-19$ .43 to $40-2$

$.31307 \mathrm{E}-21$ $.30154 \mathrm{E}=10$ - 30094 E- 10 . $00505=-17$ $.40900 E-10$

$.42269 e-10$ . 400s $1 \mathrm{t}-10$ $.25394 \mathrm{E}-10$ .4536 पE-:0 . 379 4CE-10

$.20905 t-16$ . 3:992 - $2003 y=10$ . $92126 \mathrm{t}-16$ $.208360=16$

$.13007 \mathrm{e}-15$ $.20047 t-1 t$ $.97320 \mathrm{c}-10$ $.20199 t-10$ $.14940 \mathrm{E}-10$

$.20223=-15$ :17j1 t-16 - 11751t-10 .20000e-15 $.752000-16$
$30290 \mathrm{E}-20$ $.80678 \mathrm{E}-19$ $.11859 \mathrm{~L}-18$ $10918 E-17$ . 151:3E-18

$18159 \mathrm{e}-10$ .20740E-10 $.5391 ? \mathrm{~b}-17$ $84499 \mathrm{E}-17$ $.22001 \mathrm{E}-10$

$.57212 \mathrm{E}-17$ $.230338-10$ $24759 k=16$ $20>30 k-10$ $.00974 E-17$

$.27137 t=\div 6$ .89008t=17 $21702 \mathrm{E}-1 \mathrm{~b}$ $23000 \mathrm{x}-10$ 22b?de-10

$.40110 t-10$ $0 J 434 \mathrm{t}=? 7$ .00434 E- 97 $60204 \mathrm{E}-10$ . $20050 E-96$
$C=.1005306 \mathrm{~J} i+02$ $.10007 \mathrm{E}=23$ $.67060 \mathrm{E}-24$ $.11795 E-23$ . $50090 \mathrm{E}-23$ $.7056 \mathrm{E}-23$

$.15360 \mathrm{E}-22$ $.27319 E-23$ $.40230 \mathrm{E}-23$

$35190 \mathrm{E}-22$ $.72051 E-22$

$.25393 \mathrm{E}-2$ ?

$.13478 E-21$ $23392 E-21$ 30095E-21 $.12485 \mathrm{E}-20$

$.50719 E-29$ $80234 E-2$ ? . $34090 \mathrm{E}-20$ $.12930 \mathrm{E}-20$ $.16412 t-20$

$70639 E-20$ $.21432 E-20$ $.27091 \mathrm{k}-20$ $.12419 k-19$

. $39614 k-20$ 19539E-19 30625E-19

$.50041 E-19$ $.62167 E-19$ $.10445 E-17$ $.12332 \mathrm{E}-17$ $.74515 E-19$

$.11092 \mathrm{E}-17$ $.00572 E=19$ .9763 dE-?9 .4 477 Ut-17 $.215390-17$

$.50473 \mathrm{E}-17$ $.29540 \mathrm{t}-17$ $.43207 \mathrm{E}-77$ $.10705 E-10$ . 110<jE-10

$.90059 \mathrm{k}-17$ $.27900 \varepsilon=17$ $.11573 \mathrm{~s}-10$ $20340 t-19$ .27 y00z- 7 $.00097 \mathrm{~s}-17$

KAP $=$
$.47977 E=i 4$ $.30073 \mathrm{t}-14$ $.79305 \mathrm{E}-14$ $.27398 \mathrm{E}-13$ $.19449 \mathrm{E}-13$

$.11974 t-12$ $.45299 \mathrm{k}-13$ $.10116 E-12$ $.43065 \mathrm{~L}-1 \mathrm{C}$ $.73348 \mathrm{E}=11$

$.18092 E-\$ 0$ $.36703 k-11$ $92136 \mathrm{E}-11$ $.21322 \varepsilon=10$ 

sU
dt

NU

$\begin{array}{rrr}4 & 2 & 2 \\ 4 & 1 & 3 \\ 16 & 1 & 15 \\ 17 & 1 & 16 \\ 5 & 1 & 5\end{array}$

$\begin{array}{rrr}3 & 2 & 1 \\ 3 & 1 & 2 \\ 96 & 1 & 96 \\ 17 & 9 & 17\end{array}$

$\begin{array}{lll}5 & 4 & \\ 5 & 4 \\ 5 & 0 & 1 \\ 5 & 3 & 5\end{array}$

$\begin{array}{lll}5 & 3 & 2\end{array}$

441

$\begin{array}{lll}4 & 4 & 0 \\ 4 & 0 & 4\end{array}$

$\begin{array}{lll}4 & 3 & 2 \\ 4 & 3 & 1\end{array}$

$\begin{array}{rrr}5 & 2 & 4 \\ 5 & 2 & 3 \\ 5 & 1 & 4 \\ 10 & 1 & 17 \\ 19 & 1 & 14\end{array}$

423

$\begin{array}{rrr}4 & 2 & 2 \\ 4 & 9 & 3 \\ 18 & 9 & 18\end{array}$

16

$\begin{array}{lll}6 & 1 & 6 \\ 6 & 5 \\ 6 & 5 \\ 6 & 0 & 6 \\ 6 & 4 & 3\end{array}$

$\begin{array}{lll}6 & 0 & 6 \\ 6 & 4 & 3\end{array}$

$\begin{array}{lll}6 & 4 & 2 \\ 6 & 3 & 4 \\ 6 & 3 & 3 \\ 6 & 2 & 5\end{array}$

62

$\begin{array}{rl}6 & 9 \\ 70 & 9 \\ 7 & 1 \\ 7 & 6 \\ 7 & 6\end{array}$

$\begin{array}{lll}7 & 5 & 3 \\ 7 & 5 & 2 \\ 7 & 0 & 7 \\ 7 & 4 & 4 \\ 7 & 4 & 3\end{array}$

$\begin{array}{lll}5 & ? & 5 \\ 5 & 5 & ? \\ 5 & 5 & 0 \\ 5 & 0 & 5 \\ 5 & 4 & 2\end{array}$

$\begin{array}{lll}5 & 4 & 9\end{array}$

$\begin{array}{lll}3 & 3 & 3 \\ 5 & 3 & 2\end{array}$

$\begin{array}{lll}5 & 3 & 4 \\ 5 & 2 & 3\end{array}$

$\begin{array}{rrr}5 & 1 & 4 \\ 20 & 1 & 20 \\ 6 & 9 & 6 \\ 6 & 6 & 9 \\ 6 & 6 & 0\end{array}$

$\begin{array}{lll}0 & 5 & 2 \\ 6 & 5 & 1 \\ 6 & 0 & 0 \\ 6 & 4 & 3\end{array}$

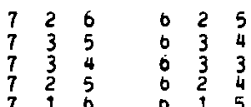

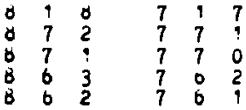

$\begin{array}{lll}0 & 0 & 0 \\ 0 & 5 & 4 \\ 0 & 5 & 3 \\ 0 & 4 & 5 \\ 0 & 4 & 4\end{array}$

$\begin{array}{lll}7 & 0 & 7 \\ 7 & 5 & 3 \\ 7 & 5 & 2 \\ 7 & 4 & 4 \\ 7 & 4 & 3\end{array}$

$\begin{array}{lll}8 & 2 & 7 \\ 0 & 3 & 0 \\ 0 & 3 & 5 \\ 0 & 2 & 6 \\ 0 & 1 & 7\end{array}$

$\begin{array}{lll}7 & 2 & 6 \\ 7 & 3 & 5 \\ 7 & 3 & 4 \\ 7 & 2 & 5 \\ 7 & 1 & 0\end{array}$
$.13741180 \mathrm{E}+03$ $13946340 e+03$ $14200020 \varepsilon+03$ $.96991350 E+03$

$17167070 E+03$ $17167070 E+03$ 17 $.17171100 \mathrm{E}+03$

$.17172020 E+03$ - $97177940 \mathrm{E}+03$ $17030010 k+03$ . 1900d9o0k+uz

$20292350 \mathrm{E}+\mathrm{U}$

$20594280 \dot{0}+03$

$.20594200 \mathrm{E}+0$

$.20590740 E+0$

$.20600990 \mathrm{~L}-03$

$.20000190 \mathrm{E}+03$

$.20005 \cdot 90 \mathrm{E}+0$

$2005220 \mathrm{E}+03$

.2001589 UE +03

$.20920010 E+03$

$21000350 t+0$

$23672000 \mathrm{E}+0$

$.24017060 \mathrm{E}+0$

$.24017000 E+03$

$.24026140 \mathrm{E}+03$

$24026940 t+03$

$24020630 \mathrm{E}+03$

$24033940 \mathrm{E}+03$

$24033940 E+03$

$24036 \div 80 \dot{2}+03$

$.24039230 k+03$

$24039300 t+0$

$244041 d 0 E+03$

$.27052000 \mathrm{E}+03$

$.27437150 \mathrm{E}+03$

2'7437150E +03

$27446220 \mathrm{E}+03$
$27440220 \mathrm{E}+03$

$.27452000 \mathrm{E}+03$

$.27457750 \mathrm{t}+03$

$.27405910 \mathrm{E}+03$

$.27465910 \mathrm{t}+\mathrm{C} 3$

$.27470220 \mathrm{E}+03$

$27473100 \mathrm{E}+03$

$27473320 \mathrm{E}+03$

$27000050 E+U 3$
¿ง

so

$\pm 164$

10

200

os0U5e-15 .2127yt-1j. .5UO7Oc-1b. $1040 U E-10$

73303E-21.10554E-10 .20790E-10.1220te-10

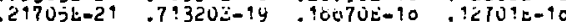

$.10920 \mathrm{k}-14$

$.7 \div 3202-14$

$21111 \mathrm{E}-19$

$.211 \div 1 \mathrm{E}-19$

$.22257 \mathrm{E}=14$

$.37030 \mathrm{E}-17$

. $2 b 289 \mathrm{k}-17$

$25209 \mathrm{E}-17$

$.47655 \mathrm{~s}-95$

$.26407 E-10$

$.93394 \mathrm{t}-15$

$13404 \dot{E}-15$

$1396 u=-15$

$.1390 \mathrm{dL}-15$

$50930 \mathrm{~b}-22$

$.13021 \mathrm{E}-2 \mathrm{z}$

. $35993 \mathrm{E}=15$

$40 ! 621,-9$

$197 \triangle 0 \mathrm{OE}-14$

5011 Uย́-22

$.49504 E-15 \quad .14000 E-15$

$0070=$

$472 y 4 y-? 7$

$.472948-i 7$

$21902 \mathrm{e}-16$

$.21102 \mathrm{E}=16$

lag 5 De- 10

.536yOt- 10

$.53724 E-10$

.10330z-10

$\therefore 139250-10$

$.27 ! 93 e-17$

$.27145 \mathrm{k}-17$

.767 i

$.707 \div 2 \mathrm{i}-17$

.$: 3999 \mathrm{~b}-90$

.4009t-:0

$.200590-10$

i. ?900r-?c

$50114 \mathrm{~s}-22$

. 31573E-46

$.23445 \mathrm{E}-14$

$20303 \mathrm{t}-10 \quad: 19175 \mathrm{E}=17$

$.67503=-15 \quad .10937 \varepsilon-15$

$343218-19$

$.34321 \mathrm{k}-19$

$.4079 \mathrm{E}-17$

$.55323 t=? 7$

.20 y $3 \mathrm{t}-17$

$.20963 \mathrm{E}=17$

$.35040 \mathrm{E}-10$

$.09010 \mathrm{~L}=17$

$14930 \mathrm{0}-15$

$.472931-16$

$20919 k-15$

$14943 \mathrm{E}-15$

$200565-15$

$11043 z-14$

$.29749 E-23$

$10044 \mathrm{E}=\mathrm{I}$

$515150-15$

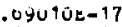

. $30094-16 \quad .14704 \mathrm{~L}-10$

$40910 \mathrm{t}-10$

$009470-10 \quad .24300 \mathrm{e}-10$

$17002 c-19$ 19507E-10 .12095t-10

$.63000 \mathrm{t}-15$. $20705 \mathrm{E}-15$.47710t-10

$.27502 E-25$
$27502 F-25$

$.55220 E-10 \quad .13205 E-17$
$.55220 E-10 \quad: 13205 E-17$

$.71609 E-22$

$71609 E-22$

$37000 E-19$

$370000-19$

$57307 \mathrm{E}-10$

$8596 b \mathrm{~b}-15$

$84570 \mathrm{t}-17$

$.4340 \mathrm{bt}-17$

$.50 j 65 \mathrm{E}-17$

$.434 \mathrm{dot}-17.50305 \mathrm{E}-1 \%$

$.23953 \mathrm{t}-15 \quad .53411 \mathrm{t}-10$

$.12539 \mathrm{c}-10$

$.13756 \mathrm{E}-15$

$.04530 \mathrm{E}-17$

$19590 \mathrm{E}=16$

$.19590 \mathrm{U}-16$

$.12539 \mathrm{t}-10$

$.45322 \ddot{E}-17$

$.27269 \mathrm{t}-15$

$.12980 \mathrm{t}-15$

$.37720 \mathrm{E}=10$

$.24919 \mathrm{k}-16$

$.24199 \mathrm{t}-10$

$.37709 \mathrm{e}=10$

$13702 t-15$

$.04004 \mathrm{E}-16$

$.27297 \varepsilon-15$

. $59769 \mathrm{E}-16$

$.50359 k-10$

$.03306 \mathrm{E}-15$

$.72965=-15$

$.0703 \mathrm{t}-16$

.35731 E-29

$.35731 \mathrm{E}-29$

$.35070 \mathrm{E}-25$

$.50150 \mathrm{E}-21$

$.26470 \mathrm{k}-15$

$.09 ! 54 k-10$

$.56150 \mathrm{~b}-21$

. 32202E-19

$.19495 \mathrm{E}-10$

$.11495 \mathrm{t}-10$

$.119 ! 3 E-11$

$69154 k-10$

उUस2OE=17

. 30420E-17

$.10129 \mathrm{E}-14 \quad .97692 \mathrm{c}-15$

$.74003 \mathrm{t}-16$

$.31323 \mathrm{E}-15$

$\mathrm{k}-16$
$\mathrm{t}-17$

$.60744 E-22$

$.01734 E-10$

$.707790-17$

$.87550 \mathrm{E}-17$

$19512 \varepsilon=10$

$.20539 \mathrm{E}-10$

$.10792 \mathrm{e}-10$

.31809E-19

. $31919 \mathrm{E}-15$

$.19512 \mathrm{E}-10$

$.10836 \mathrm{t}-15$

$17353 k-15$

$.53001 \mathrm{t}-10$

$.02309 t-10 \quad .35400 \mathrm{~L}-16$
$.17379 t-15$

$.70300 \mathrm{E}-? 6$

. 3:950E-15

$.5309: \leq-16$

$.90839 E-? 5$ $.74742 E-1$

METHYLENE CHLOHTUE CH2Cle

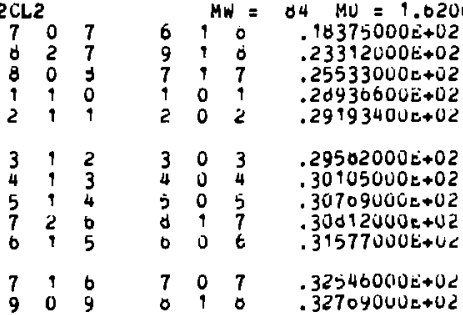

$A=$

$=.32001800 \mathrm{t}+0$
$.22505 \mathrm{t}-17$
$.12552 \mathrm{t}-17$

$02 \quad b=$

$c=.30652030 \varepsilon+0$

$19 \mathrm{C}$

$\begin{array}{ll}.29237 \mathrm{E}-10 & .50340 \mathrm{E}-19 \\ 20727 \mathrm{E}-10 & .10399 \mathrm{E}-19\end{array}$

$.45074 \mathrm{bi}-17$

$.36461 \mathrm{E}-17$

$20727 \mathrm{E}-10$

$03050 E-10$

$.13205 E-19$

$.12090 \mathrm{E}-10$

$.09322 \mathrm{E}=19$

$.03110 \mathrm{U}-20$
$2330 \mathrm{j}-14$

$.23700 \mathrm{e}-19$

$.12302 \mathrm{c}-9$

$.09394 t-11$

$.90069 \mathrm{t}-10$

$.10000 \mathrm{t}-10$. $11542 \mathrm{E}-17$

$.11767 \mathrm{E}=10$. $14102 \mathrm{E}-17$

$.164505-10$

. $295790-4 y$

$.21440 E=10.3070 y t-9 y$

$.20053 E-10.40501 \mathrm{e}-1$

$.04559 E-19$
$.32111 t-10$

$.20000 \mathrm{c}=17 \quad$.31690E-10

$.142 ? 5 t-10 \quad .99324 t-17$

$.37003 E-10 \quad .70407 E-19$
$.235400-90 \quad .44001=-14$

1 AAF $=-.40<4$

$.40917 \mathrm{E}-07$

: $150 \mathrm{ck}-00$

-37006e-vo

$370314=00$

. j̧U⿺gk-نo

$.4005 y 5-40$

$.43900 \mathrm{E}-40$

.03 10UE-U?

. 
TABLE III . 15

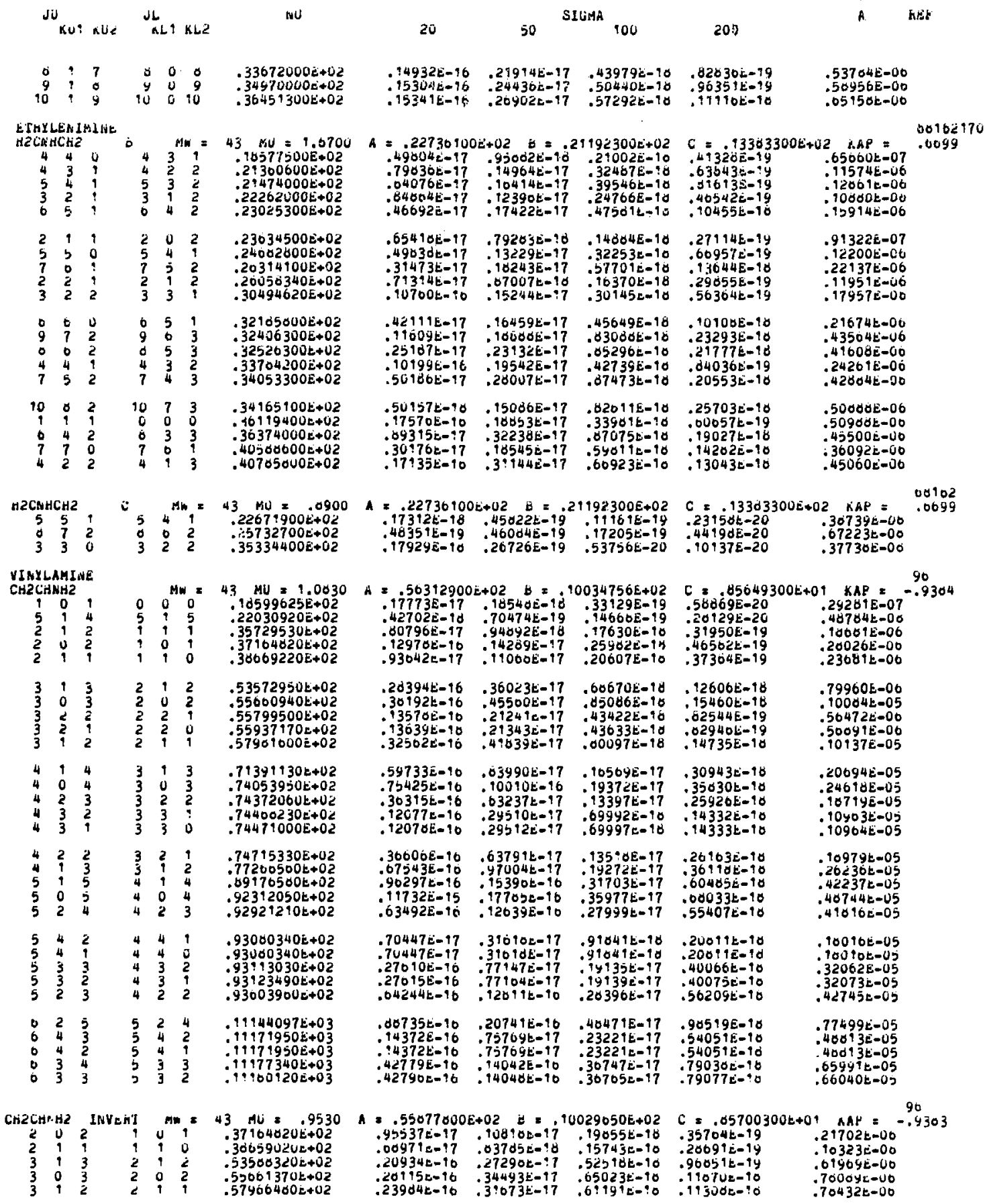


${ }_{\text {KU1 KU2 }}$ KL 1 KL2

$\begin{array}{lllllll}5 & 1 & 5 & 4 & 1 & 4 & .89202340 E+02 \\ 5 & 0 & 5 & 4 & 0 & 4 & .92315390 E+02 \\ 5 & 4 & 2 & 4 & 4 & 1 & .93079330 E+02 \\ 5 & 4 & 1 & 4 & 4 & 0 & .93079330 E+02 \\ 5 & 2 & 3 & 4 & 2 & 2 & .93601200 E+02\end{array}$

ตบ
20

50 S

SIGMA 100

.70909E-16 . .17667E-16

$.80360 \mathrm{E}-16 \quad .13465 \mathrm{E}=16$

$.52731 \mathrm{E}-17 \quad .24097 \mathrm{E}-17$

$.52731 \mathrm{E}-17$

$.47487 \mathrm{E}-16$. $24097 \mathrm{E}-17$
$.24245 E-17.46469 c-10$

$.27495 \mathrm{E}-17 \quad .52237 \mathrm{k}-1 \mathrm{~d}$

$.70418 E-10$. $10005 E-10$

10418E-18 . 16005t-10

$.43173 \dot{\varepsilon}=10$
- $32734 t-v s$

$.37740 \mathrm{t}-0 \mathrm{~s}$

$.13950 E-05$

$.13950 \mathrm{~b}=0 \mathrm{~s}$

$.33090 \mathrm{k}-\mathrm{Us}$

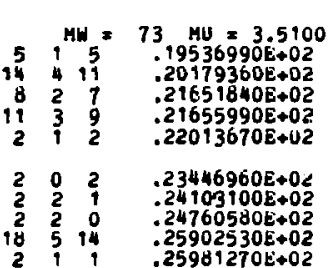

CITROETH YLENE

\begin{tabular}{|c|c|c|c|c|c|c|}
\hline $\begin{array}{r}5 \\
14 \\
11 \\
3 \\
3\end{array}$ & $\begin{array}{ll}1 \\
4 \\
2 \\
3 \\
1\end{array}$ & $\begin{array}{r}4 \\
10 \\
6 \\
8 \\
3\end{array}$ & $\begin{array}{r}5 \\
14 \\
8 \\
11 \\
2\end{array}$ & & $\begin{array}{l}5 \\
11 \\
7 \\
9 \\
2\end{array}$ & $\begin{array}{r}73 \text { HU }=3.5100 \\
.19536990 \mathrm{E}+02 \\
.20179360 \mathrm{O}+02 \\
.21651840 \mathrm{O}+02 \\
.21655990 \mathrm{O}+02 \\
.22013670 \mathrm{O}+02\end{array}$ \\
\hline $\begin{array}{l}3 \\
3 \\
3\end{array}$ & $\begin{array}{l}0 \\
2 \\
2 \\
5 \\
1\end{array}$ & $\begin{array}{r}3 \\
2 \\
1 \\
13 \\
2\end{array}$ & $\begin{array}{r}2 \\
2 \\
2 \\
18 \\
2\end{array}$ & $\begin{array}{l}0 \\
2 \\
2 \\
5 \\
1\end{array}$ & & $\begin{array}{l}.23446960 E+02 \\
.24103100 E+02 \\
.24760580 E+02 \\
.25902530 E+02 \\
.25981270 \mathrm{Q}+02\end{array}$ \\
\hline
\end{tabular}

A. 118910600005 (1)

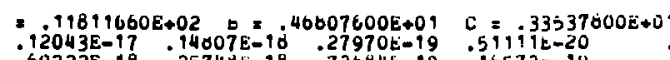

$.602228-18 \quad .25748 \mathrm{E}-18 \quad .73684 \mathrm{E}-19$

$.16931 \mathrm{E}-17 \quad .27796 \mathrm{k}-1 \mathrm{~b}^{\circ}$

$.12560 \mathrm{E}-17 \quad .31237 \mathrm{E}-18 \quad .74623 \mathrm{E}-19$

$.12684 \mathrm{E}-10$ :13799E-17

$.74623 E-19$

$.161728-16 \quad-17479 E-17$

$.93890 \mathrm{DE}-17 \quad: 10640 \mathrm{C}-17$

$.25365 \varepsilon^{-18}$

$.31629 \mathrm{E}-10$

$.10 j 39 \overline{10}-10$

$.173206-16$

$.19007 \mathrm{e}-17$

$.34709=-10$

$.16573 \mathrm{E}-19$

$.11007 \mathrm{E}-19$

1
$.44303 \mathrm{E}-19$

$0203 E-07$

$0.596295-07$

$.02456 \mathrm{E}-07$

$.60441 \mathrm{c}-07$

$.50109 \mathrm{e}-06$

WITROETHYLEME

CH2CHNO2

$\begin{array}{lll}5 & 2 & 3 \\ 4 & 2 & 2 \\ 3 & 2 & 1 \\ 6 & 2 & 4 \\ 5 & 1 & 4\end{array}$

$\begin{array}{lll}5 & 1 & 4{ }^{n+} \\ 4 & 1 & 3 \\ 3 & 1 & 2 \\ 6 & 1 & 5 \\ 5 & 0 & 5\end{array}$

73 MU $=1.1000$

-19437500E+02

$.19477940 E+02$

$.20340490 E+02$
$.20587660 \mathrm{E}+02$

$.21511380 \mathrm{E}+0 \mathrm{E}$

$\begin{array}{lll}2 & 2 & 0 \\ 2 & 1 & 2 \\ 7 & 2 & 5 \\ 2 & 2 & 1 \\ 5 & 1 & \end{array}$

$.21561490 E+02$

$.218733708+02$

$.23195560 \mathrm{E}+02$

$.25373770 \mathrm{t}+02$
$.25495380 \mathrm{0}+02$

404

$\begin{array}{lll}2 & 1 & 1 \\ 1 & 0 & 1 \\ 7 & 1 & 6 \\ 2 & 1 & 2 \\ 4 & 2 & 3\end{array}$

$.26055160 E+02$

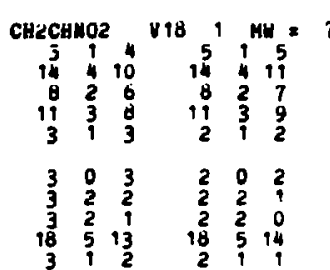

$73 \mathrm{MU}=3.5200$

$.19371150 E+02$

$.2000+3708+02$

.29484270E+02

$2 \uparrow 508520 \mathrm{*}+02$

$.22055450 E+02$

$23474860 \mathrm{E}+02$

$24127720 E+02$

$24780350 E+02$

$25773650 E+02$
$25989500 E+02$

$$
8
$$
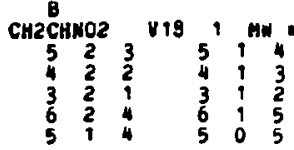

$73 \mathrm{MU}=1.1800$

$.19243580 E+02$

$.19279690 \mathrm{E}+02$

$.20132530 E+02$

$.20389480 E+02$
$.21321250 E+02$

220

$\begin{array}{lll}2 & 2 & 0 \\ 2 & 1 & 2 \\ 7 & 2 & 5 \\ 5 & 1 & 4\end{array}$

$\begin{array}{lll}2 & 1 & 1 \\ 1 & 0 & 1 \\ 7 & 1 & 6 \\ 4 & 2 & 3 \\ 3 & 1 & 3\end{array}$

$.21340240 E+02$

$.21826990 E+02$

$.22981890 \mathrm{2}+02$

$.26148550 E+02$

$A=.11811660 E+02 \quad B=.46807000 E+01$

$-137978-17 \quad .17446 \mathrm{E}-10$

$.06893 \mathrm{E}=98$.90469E-19

$.16491 \mathrm{E}-17 \quad .22353 \mathrm{E}-18 \quad .43690 \mathrm{E}-19$

$.11507 E-17$ :94948E-16 .26726E-19

$.56569 k-19$

$.33399 \mathrm{E}-19$

$.789066-00$

.51057 E-UG

$.11244 E-U D$

$.95622 k-00$

$.52103 E-16.56972 E-19.10349 E-19$.

$.8 ! 231 E-18 \quad .65614 E-19 \quad .15362 E-19$

$.19642 \mathrm{E}-17.29190 \mathrm{E}-18$. $50734 \mathrm{E}-19$

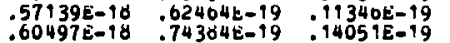

$02263 E-19$

$C=.33537800 E+01 \quad K A H=-.0002$

$1060 \mathrm{~d}=20 \quad .45097 E-07$

$\begin{array}{ll}46017 E-20 & .41745 E-07 \\ 32030 \mathrm{E}-20 & .3942 \mathrm{EE}-07\end{array}$

$\begin{array}{ll}.32030 t-20 & .39425 E-07 \\ .81291 t-20 & .55942 k-07\end{array}$

$.46637 \mathrm{E}-20 \quad .40255 \mathrm{E}-07$

$.16206 E-17$. $18305 E-18 \quad .33614 E-19$

$.10545 \mathrm{E}-20$

$.33050 \mathrm{~b}-07$

$.49972 E-67$

$.74510 \mathrm{E}=07$

$.42043 \mathrm{~b}=07$

$.25004 \mathrm{t}=07$

$.7200<k=07$

* $117370000+02 \mathrm{E}=.46791960 \mathrm{E}+01 \quad \mathrm{c}=.33633000 \mathrm{E}+01 \mathrm{KAF}=-.0051$ $.11802 E-17 \quad .14574 \mathrm{E}-18 \quad .2756 \mathrm{dE}-19 \quad .50493 \mathrm{E}-20 \quad .37510 \mathrm{E}-07$ $.59309 E-18$.25470E-10 .72973E-19.16422t-19 .59094E-07

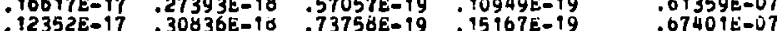
$\begin{array}{lllll}.126 d d E-16 & : 13864 E-17 & .25177 E-16 & .45111 E-19 & .078655 \mathrm{E}-00\end{array}$

$.16152 E-16 \quad .17535 E-17 \quad .31777 t-18 \quad .56875 E-19 \quad .78892 E=00$ $.89036 \mathrm{E}-17$ : $10197 \mathrm{E}-17$ :10615E-18 :33572E-19 . $46230 \mathrm{E}-00$ $.93783 E-17 \quad .10609 \mathrm{E}-17 \quad .19639 \mathrm{E}-18 \quad .35425 \mathrm{E}-99 \quad .52270 \mathrm{E}=00$

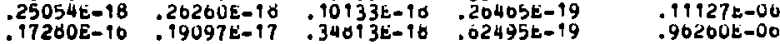

$A=.11737060 E+02 \quad B=.46791900 E+01 \quad C=.33633800 E+01 \quad$ AAP $=\$ .6057$ $.13077 \mathrm{E}-17 \quad .17617 \mathrm{E}-1 \delta^{\circ} .33635 \mathrm{E}-19 \quad .61793 \mathrm{E}-20 \quad .45096 \mathrm{E}-07$ $.19462 \mathrm{E}-17 \quad .13606 \mathrm{E}-18 \quad .25601 \mathrm{E}-19 \quad .40554 \mathrm{~L}-20 \quad .41909 \mathrm{~b}=07$ $.673 d 7 E-18$.99417E-19 . 18299E-19 .33009E-20 .39576E-07 $.16512 E-17.22581 E-18.44195 \mathrm{E}-19.02294 \mathrm{E}-20 \quad .55422 \mathrm{E}-07$ :11501E-17 :14300E-16 :27051E-10 :49460E-20

$.52369 E-10$.57511E-19 . 10401E-19 .18757E-20 .33175E-07 $.02946 E-10 \quad .07798 \mathrm{E}-19 \quad .15777 \mathrm{E}-19 \quad .28119 \mathrm{E}-20 \quad .3055 \mathrm{GE}-U 7$ $.19769 \mathrm{E}-17 \quad .29499 \mathrm{E}-10 \quad .59437 \mathrm{k}-19 \quad .91217 \mathrm{E}-19 \quad .74959 \mathrm{E}=07$ $.63069 E-10 \quad .77002 E-19 \quad .14733 E-19 \quad .20941 E-20 \quad .26595 E-07$ .16749E-17:18995E-10:34931E-19 .02902t-20

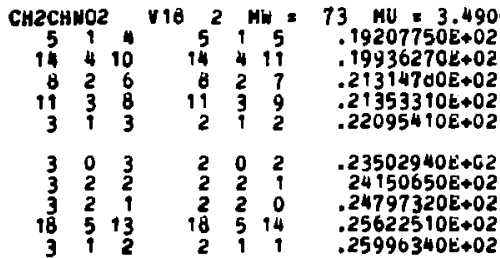

$A=.11067010 E+02 \quad B=.46774800 E+01$ $.11274 \mathrm{E}-17 \quad .14007 \mathrm{E}-18 \quad .26548 \mathrm{E}-19$ $\begin{array}{lll}.11274 E-17 & .14007 E-18 & .26548 E-19 \\ .56965 \mathrm{~L}-16 & .24575 \mathrm{E}-18 & .70520 \mathrm{E}-10\end{array}$ $\begin{array}{rrr}.56985 \mathrm{~L}-18 & .2457 \mathrm{LE}-18 \quad .70520 \mathrm{E}-19 \\ .15093 \mathrm{E}-17 & .20354 \mathrm{E}-18 \quad .54990 \mathrm{~L}-19\end{array}$ $.15893 \mathrm{E}-17 \quad .20354 \mathrm{~L}-18$ $.19832 E-17 \quad, 29703 E=10$ $.54990 \mathrm{~b}-19$

$=.337272000+01$ KAP $=-51$

$.15725 \mathrm{E}-16 \quad .17177 \mathrm{E}-17 \quad \cdot 31192 \mathrm{E}-16 \quad .55004 \mathrm{E}-19$ $.86700 \mathrm{E}-17.99092 \mathrm{E}-10 \quad .96267 \mathrm{E}-18 \quad .3297 \mathrm{DE}-19$ $.91282 E-17$. 10444E-17.19261K-18.34776E-19 $.24087 \mathrm{E}-10 \quad .25360 \mathrm{E}-18 \quad .90035 \mathrm{E}-19$.25624E-19 $.16797 \mathrm{E}-16$

$.253606-10$

$.34111 \mathrm{E}-18$ $.01295 \mathrm{E}-19$

$.35901 \mathrm{k}-0 \mathrm{~J}$

$.56949 t-07$

$.50096 \mathrm{~b}-\mathrm{u} 7$

$.04010 \mathrm{k}-07$
$.56171 \mathrm{~L}-0 \mathrm{0}$

$.770305=00$ $.47547 E-00$ $.51490 \mathrm{E}=00$ $10730 \mathrm{~b}-00$ $.94699 \mathrm{E}-06$ 
JU NUT NUE NL KL KL

NU

nLinnoz 492 HW =

$\begin{array}{llllll}5 & 2 & 3 & 3 & 1 & 4 \\ 4 & 2 & 2 & 4 & 1 & 3 \\ 0 & 2 & 4 & 0 & 1 & 5 \\ 5 & 1 & 4 & 3 & 0 & 5 \\ 2 & 1 & 2 & 1 & 0 & 1 \\ 7 & 2 & 5 & 1 & 9 & 0 \\ 5 & 1 & 4 & 4 & 2 & 3 \\ 4 & 0 & 4 & 3 & 1 & 3\end{array}$

73 Hu $x .0400$

$.19000400 \mathrm{t}+02$

$.19093070 \mathrm{k}+02$

$.20200730 \mathrm{t}+02$

$.21135050 \mathrm{E}+42$

$.21705270 \mathrm{E}+02$

$.22776 d 40 E+02$

$.25000030 E+02$

$.20235930 E+02$

HEIHYLTHIUCYANATE

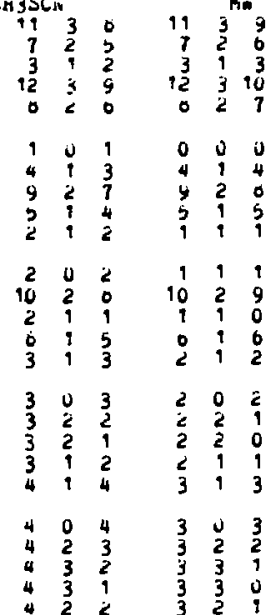

413312

MONUCHLUNOACETONI SHLLE

$\begin{array}{ccccc}3 & 1 & 3 & 2 & 9 \\ 4 & 1 & 4 & 3 & 1\end{array}$

$\begin{array}{llllll}4 & 0 & 4 & 3 & 0 & 3 \\ 4 & 2 & 3 & 3 & 2 & 2 \\ 4 & 3 & 2 & 3 & 3 & 1 \\ 4 & 3 & 1 & 3 & 3 & 0 \\ 4 & 2 & 2 & 3 & 2 & 1\end{array}$

$\begin{array}{lll}5 & 1 & 3 \\ 0 & 1 & 0\end{array}$

$\begin{array}{lll}4 & 1 & 4 \\ 5 & 1 & 5\end{array}$

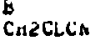

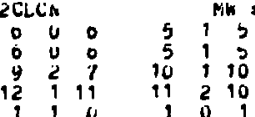

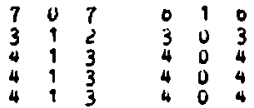

214305

$\begin{array}{llllll}6 & 1 & 5 & 0 & 0 & 6 \\ 7 & 1 & 6 & 7 & 0 & 7\end{array}$

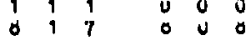

$\begin{array}{rrrrrr}9 & 1 & 0 & 9 & 0 & 9 \\ 10 & 1 & 9 & 10 & 0 & 10 \\ 2 & 1 & 2 & 1 & 0 & 1 \\ 11 & 1 & 10 & 11 & 0 & 11\end{array}$

$.15794490 k+02$

$.157 y 4540 t+02$

$10347330 \mathrm{E}+02$

$10400090 \mathrm{E}+02$

$.22551950 E+02$

$23021510 E+02$

$23021510 \mathrm{t}+02$

$.24625000 E+02$

$.25014000 \mathrm{Uet} 02$

$20001000 E+02$

$.26201000 E+02$

$.29031000 t+02$

. $31706000 k+02$

$.336185605+02$

$.33642000 E+02$
20

Silutid

50

900

200 ner

51

$110670108462 \quad=40774000 E+0$ $.00210 k-10$. $67109 k-19.16663 E-19$ $.50341 \mathrm{E}-10$ 6706UE-19 .12060E-19 $.81191 \mathrm{E}-10$.11160t-10 .21900E-19 $.50951 \mathrm{t}-18 \quad .707 \mathrm{~b} 3 \mathrm{t}-19 \quad .13411 \mathrm{k}-19$ $.41300 \mathrm{t}-16$. $44006 \mathrm{E}-19$.79342t-20

$.97241 E-18$. $14545 E-10$.29464E-19 $\begin{array}{rrr}.32063 \mathrm{E}-18 & .34033 \mathrm{E}-19 & .75501 \mathrm{E}-20 \\ .8+403 \mathrm{E}-18 & .96362 \mathrm{E}-19 & .17750 \mathrm{E}-19\end{array}$
$C=.33727200 \mathrm{E}+0$

$.306415-<4$

. $3000 t-20$

$40774 t-20$

$.2454 d t-20$

$.14955 \mathrm{E}-20$

$.55661 \mathrm{E}-20$

$.13020 E-20$
$73 \mathrm{ril}=4.0300$

$.33645000 \mathrm{E}+01$

$463301 \mathrm{iOOE}+0$ ?

$40072400 E+01$

$.737+1000 \mathrm{H}+01$

$.75099000 \mathrm{k}+\mathrm{C} 1$

$.10947400 \mathrm{de}+02$

$11905790 x+0<$

$.14979400 E+02$

$.15021120 \mathrm{e}+02$

$16724000 E+0$

$.21302720 E+02$

$.22309550 k+02$

$.22520650 \mathrm{E}+02$

$.22067040 t+02$

$23705300 t+02$

$.20354400 \mathrm{E}+02$

$.290443 \mathrm{AOC}+02$

$30047700 k+02$

$30197050 \mathrm{c}+02$

$30122090 \mathrm{E}+02$

$.30400940 \mathrm{E}+02$

$.31557420 t+02$

75 MU = $1.000 U^{\circ}$

$.17545000 E+02$

$2330>720$ U +02

$.23906230 \mathrm{E}+02$

$.24002000 t+02$

$24011000 \mathrm{E}+02$

$.24017000 E+02$

$.24036000 k+02$

$.29227240 E+02$

$35005700 \mathrm{E}+02$
$54000000 x+01$

$.000>4300 \mathrm{E}+01$

$.15300900 t+02$

90114

$=.15793000 \mathrm{E}+02 \quad b=.41555400 \mathrm{E}+01$ $.61213 x-19$

$18150 E-10$

$-18722 E-16$

$.337+4 E-10$

$14665 E-19$

$20579 e-19$

$.30705 \mathrm{E}-19$

$.54300 \mathrm{E}=19$

$.35237 E-20$

$.5304 t k-20$

$+37453 E-20$

.50644 -10

$.30644 E-10$
$.37253 t-10$
$.54053 t-10$

$.90407 \mathrm{E}-19$

$.11227 \mathrm{t}-19$

$.62353 E-10$

42851E-19

$.96049 \mathrm{E}-19$

$.90307 \mathrm{E}-19$

$.79101 \mathrm{t}-20$

$447935=17$

$47470-10$

$.20007 t-19$

$.14294 k-19$

$.1073 j t=17$

$.77003 \mathrm{E}-10$

$.19524 \mathrm{E}-18$

$53142 \mathrm{E}-11$.50575E-10

.2704k-10 $12070=-10$

.109005-16

$10535 \mathrm{E}-17$

$.21524 \mathrm{E}-10$

$.10023 \mathrm{k}-16$

. $33105 E-17$

Uद $710-10$

$12000-17$

$20765 \mathrm{E}-10.22045 \mathrm{k}-17$

$.39520 \mathrm{E}-16$. $44930 \mathrm{~J}-17$

.34909E-19

$.341300-19$

$.10540 E-10$

$.23204 \mathrm{E}-19$

$.33650 \mathrm{t}-10$

$.41006 k=10$

$.23100 \mathrm{E}=10$

$.23420 \mathrm{E}-10$

.4157 G

$.82717 \mathrm{E}-10$

$40854 E-1 \mathrm{t}$.52627E- 77

$32154 \mathrm{E}-10$.30741E-17

$10353 E-10$, $21492 E-17$

$.10350 \mathrm{E}=10 \quad .21499 \mathrm{E}-17$

$.96474 \mathrm{E}-18$

$.72699 \mathrm{E}-10$

$.41510 E-10$

$.41529 E-10$

$.74002 t-16$

$.47999 t-16 \quad .52212 k-17 \quad .10202 E-17$

$A=.23271300 t+02$ D. . $31510100 E+01$

$.72114 \mathrm{E}-10$
$.17033 \mathrm{E}-17 \quad .70410 \mathrm{E}-19$
$.19156 \mathrm{E}-10 \quad .14256 \mathrm{~d}-19$ $.17033 \varepsilon-17$

.20055E-17

$.12102 E-17$. 1511 1AE-18

$54433 \mathrm{E}-10$. $79300 \mathrm{E}-19$

$.12215 \mathrm{t}-17.15101 \mathrm{t}-10$

$.39845 E-19$

. $28722 E-19$

$.15894 \mathrm{E}-19$

$15094 t-19$

$.20003 t-19$

$\begin{array}{r}.31740 E-17 \\ 51040 E-17\end{array} .37239 E-10$

$.69432 t-19$
$.11943 t-10$

$75 \mathrm{HU}=1.0000^{\circ} \mathrm{A}$

$.22422500 E+02$

$.23192700 \mathrm{E}+02$

$.23029000 \mathrm{E}+02$

$.20120100 E+02$
$A=.25271300 E+02 \quad B=.31510100 E+01$ $.50090 E-10 \quad .01078 E-19$. $11475 E-19$

$.50096 E-10 \quad .01070 E-19$

$15503 E-18$.20203E-19

$22505 E-18 \quad .46283 E-19$

$.71020 \mathrm{E}-10$

$.46 \geq 83 E-19$
$.74075 E-19$

$11475 \mathrm{E}-19$

$.55085 E-20$

$.10430 E-19$

$13281 t-19$

$11535 E-17$

.15107 $\dot{-17}$

$.14702 \mathrm{E}-18$

$.20161 \mathrm{t}-19$

$.20410 \mathrm{k}-17$

$20410 \mathrm{e}-17$
$2 \mathrm{~T} 424 \mathrm{E}-17$

$.17040 \mathrm{E}=10$

$.23055 \mathrm{E}-10$

$.23055 \mathrm{E}-18$

$.32112 \mathrm{E}-19$

$.42440 \mathrm{E}-19$

. $42440 E-19$

$.42470 \mathrm{E}-19$

. $24920 E-17$

$27001 E-17$

.3093bc-17

$.74520 e-10$

$.33463 E-17$

$.53608 \mathrm{E}-19$

$34092 \mathrm{EN} 10$

$.409 \mathrm{dE}-10$

$.77691 k-19$

$.65961 \mathrm{E}-19$

$.79573 \mathrm{E}=19$

. $13927 \mathrm{t}-19$

$.94649 \mathrm{E}-19$

$.35437 t-17$

.30737E-17

$.15720 E-17$

$.54564 t-10$

.617 YjE-10

$.18667 \mathrm{e}-10$

$111395-10$

$.12992 \mathrm{~b}-10$

.30040E-19
C $=33541000 t+0$

$.72139 t-2$

$.10102 \mathrm{E}-20$

67 TOUE-2:

$.15969 \mathrm{E}-2 \mathrm{~V}$

$.21401 \mathrm{E}-20$

$.20429 k-20$

$14311 \mathrm{E}-20$

$.40060 \mathrm{E}-20$

$.20079 t-20$

. $15191 \mathrm{t}-19$

$.62002 E-20$

$.67633 \mathrm{t}-20$

$.16796 t-19$

$.42 Y 27 E-20$

$.00294 t-19$

$.74032 \mathrm{z}-19$

$.417720-19$

$.42363 \mathrm{E}-10$

$.74500 \mathrm{E}-19$

$.14921 \mathrm{t}-10$

$.17307 E=10$

$13241 t-10$

$.70710 \mathrm{E}-1 \mathrm{~g}$

$.76743 \mathrm{c}-1 \mathrm{y}$

.13590E-10

$.10439 \mathrm{E}-16$

C. $=28400000 E+01$

. $25557 \varepsilon-20$

$.63493 \mathrm{E}-20$

$.71475 \mathrm{E}-20$

$.52637 \mathrm{E}-20$

$.29910<-20$

2991 व -20

$.52705 E-20$

$.12005 \mathrm{E}-19$

$.21005=-19$

0167

$C=.20400000 E+01 \underset{\text { KAP }}{C}=-.9730$

$.20911 \mathrm{E}-20$

.2091 tE-20

$.10619 \mathrm{E}-20$ 


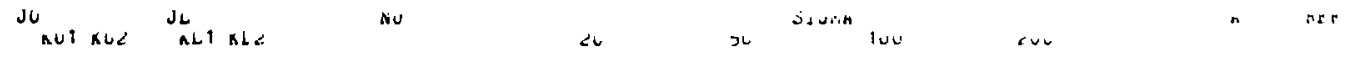

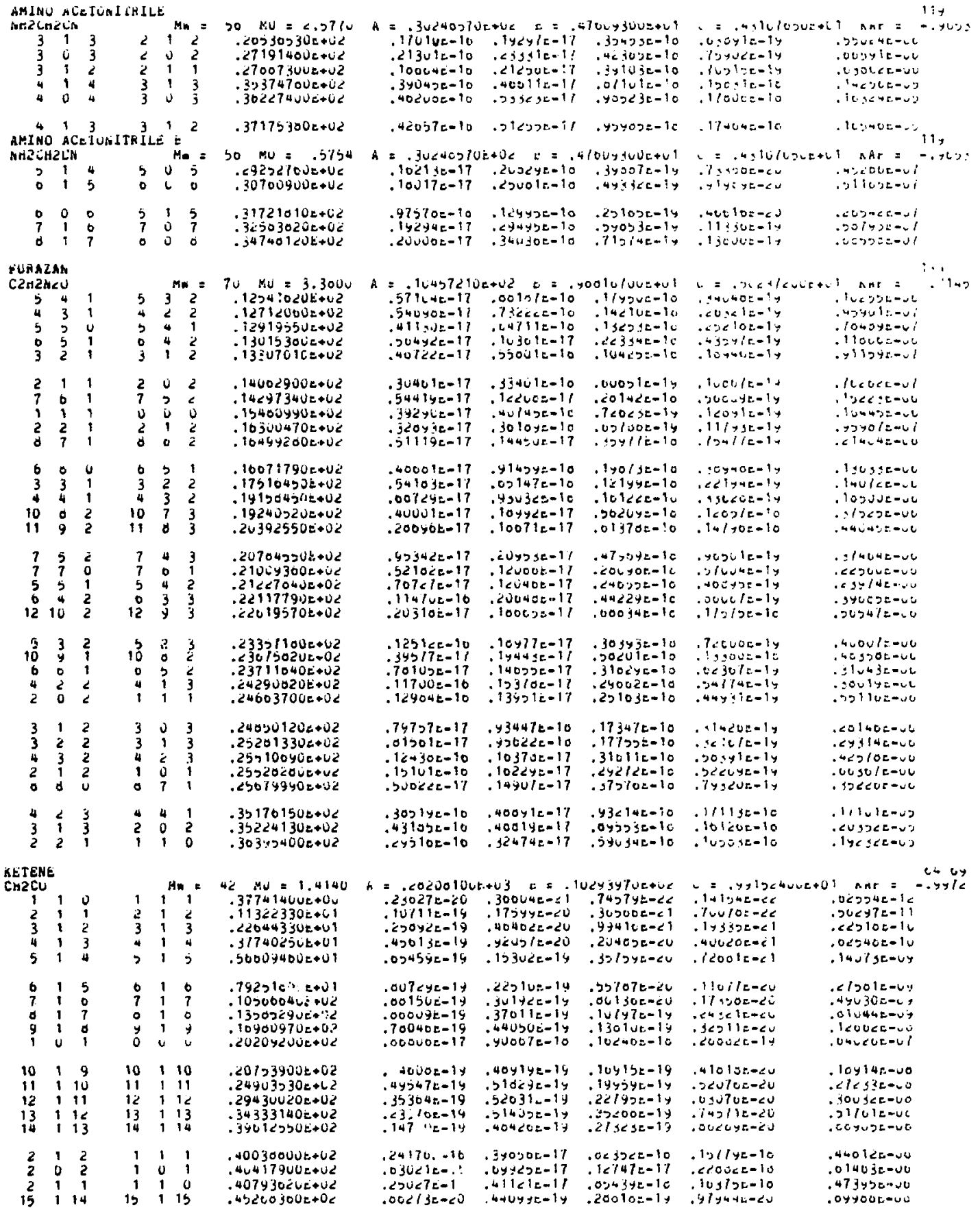




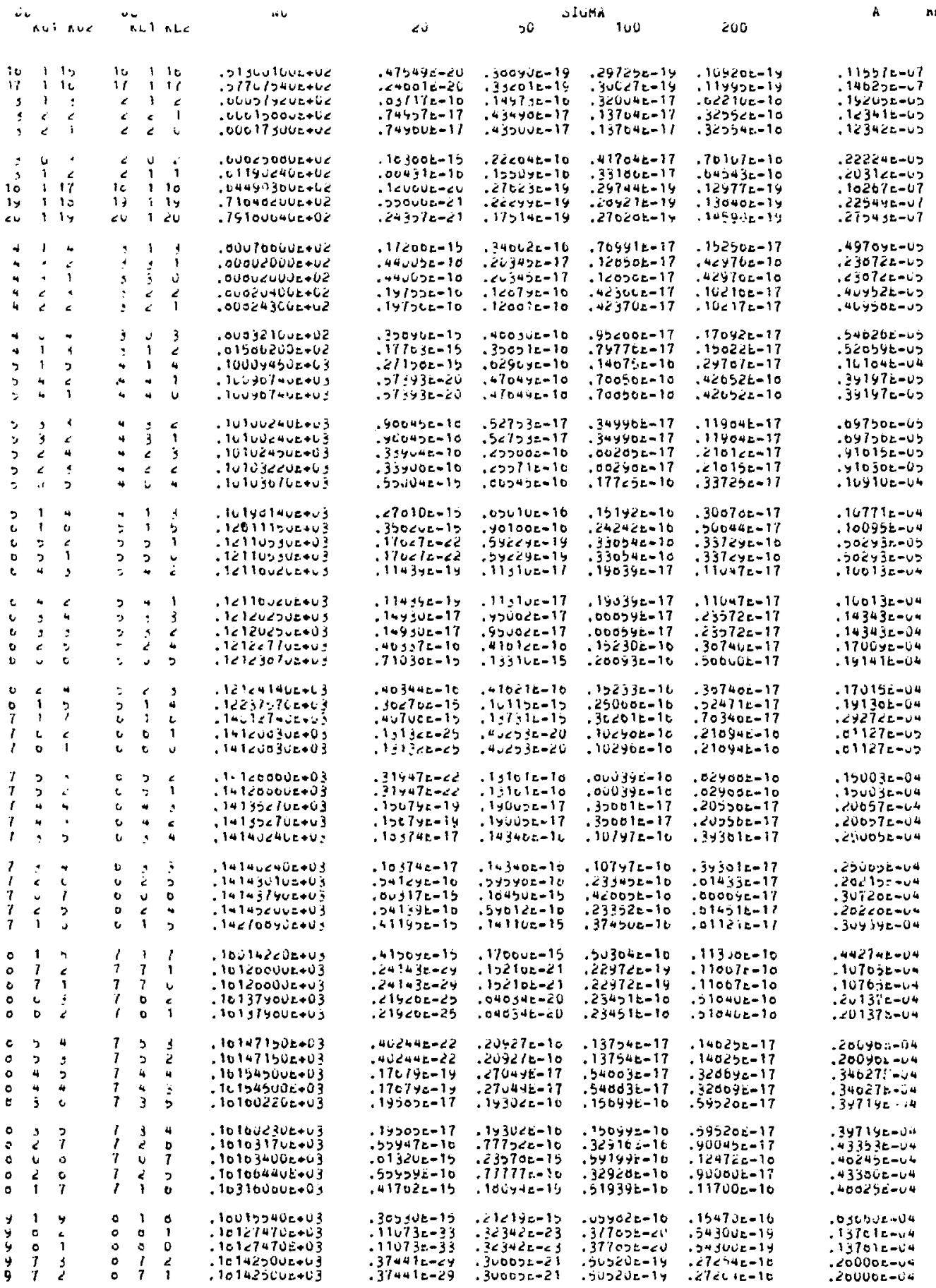




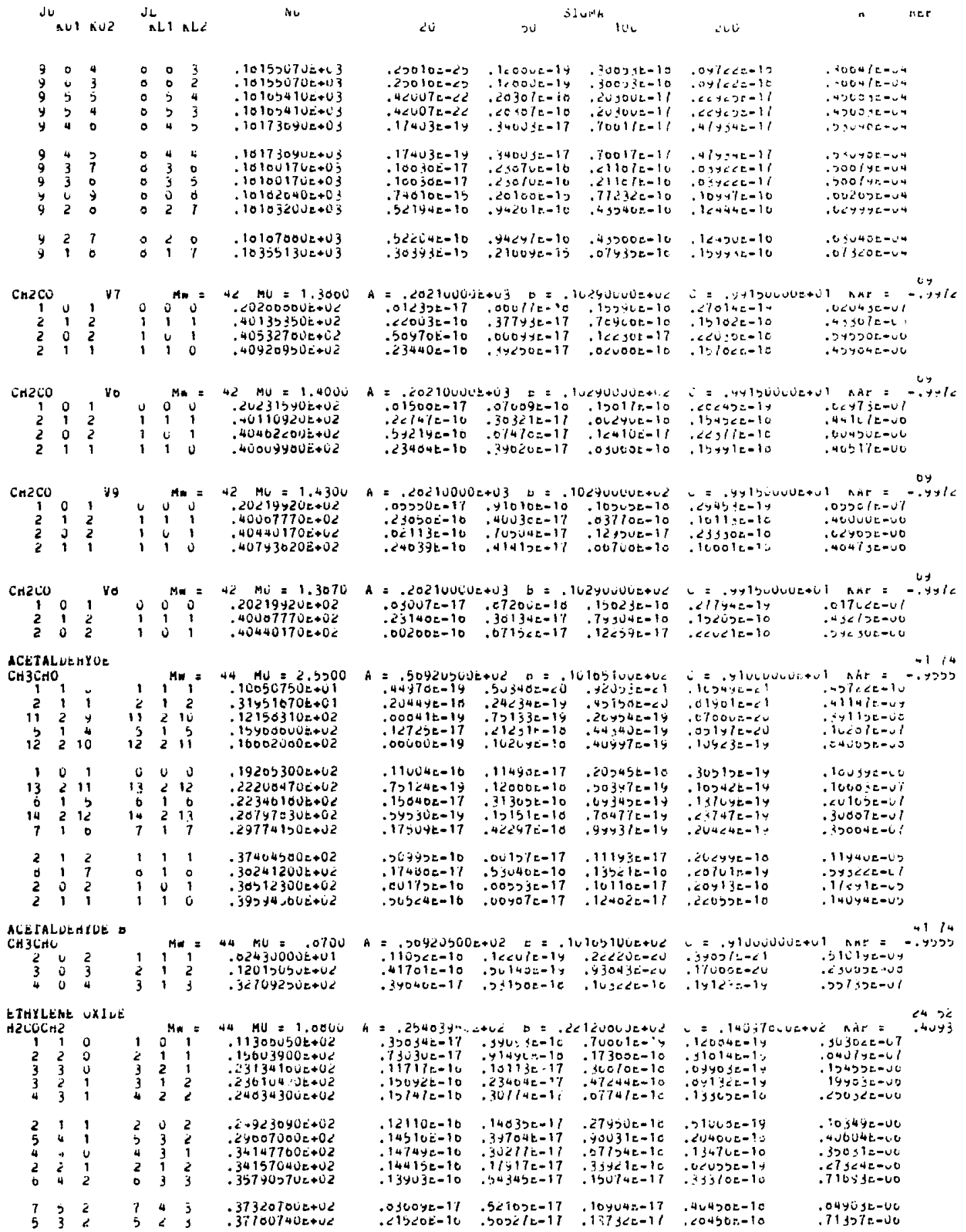




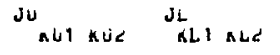

$\begin{array}{llllll}0 & 3 & 1 & 0 & 4 & 2 \\ 1 & 1 & 1 & 0 & 0 & 0 \\ 3 & 3 & 1 & 3 & 2 & 2 \\ 4 & 2 & 2 & 4 & 1 & 3 \\ 3 & 1 & 4 & 3 & 0 & 3 \\ 4 & 4 & 1 & 4 & 3 & 2 \\ 5 & 5 & 0 & 3 & 4 & 1 \\ 3 & 2 & 2 & 3 & 1 & 3 \\ 4 & 3 & 2 & 4 & 2 & 3 \\ 7 & 0 & 1 & 7 & 5 & 2 \\ 7 & 4 & 3 & 7 & 3 & 4 \\ 5 & 4 & 2 & 3 & 3 & 5 \\ 5 & 5 & 1 & 5 & 4 & 2 \\ 0 & 3 & 3 & 0 & 4 & 4 \\ 0 & 5 & 2 & 6 & 4 & 3\end{array}$

$\begin{array}{llllll}0 & 0 & 0 & 6 & 5 & 1 \\ 5 & 2 & 3 & 5 & 1 & 4 \\ 2 & 0 & 2 & 1 & 1 & 1 \\ 4 & 1 & 3 & 4 & 0 & 4 \\ 0 & 0 & 1 & 0 & 5 & 2\end{array}$

423414

$\begin{array}{llllll}5 & 3 & 3 & 5 & 2 & 4 \\ 7 & 0 & 2 & 7 & 2 & 3\end{array}$

$\begin{array}{llllll}1 & 4 & 3 & 0 & 3 & 4\end{array}$

$\begin{array}{llllll}7 & 7 & 3 & 7 & 4 & 4 \\ 7 & 7 & 4 & 7 & 0 & 1 \\ 7 & 7 & 1 & 7 & 0 & 2 \\ 7 & 3 & 4 & 7 & 2 & 5 \\ 0 & 2 & 4 & 0 & 1 & 5\end{array}$

$\begin{array}{lll}5 & 3 & 4 \\ 7 & 4 & 4 \\ 6 & 3 & 4 \\ 5 & 1\end{array}$

1511401510 ?

$\begin{array}{lll}2 & 2 & 4 \\ 2 & 1 & 1 \\ 2 & 0 & 3 \\ 3 & 1 & 3 \\ 4 & 1 & 3\end{array}$

$\begin{array}{lll}7 & 2 & 3 \\ 7 & 3 & 3 \\ 2 & 2 & 0 \\ 0 & 1 & 3 \\ 0 & 2 & 2\end{array}$

$\begin{array}{rrrrrr}3 & 1 & 2 & 6 & 2 & 1 \\ 2 & 2 & 0 & 5 & 2 & 3 \\ 3 & 2 & 6 & 5 & 1 & 1 \\ 11 & 2 & 0 & 11 & 4 & 7 \\ 4 & 4 & 4 & 3 & 1 & 3\end{array}$

$\begin{array}{rrrrrr}4 & 3 & 0 & 9 & 2 & 7 \\ 4 & 1 & 4 & 3 & 0 & 3 \\ 12 & 11 & 1 & 12 & 10 & 2 \\ 7 & 1 & 0 & 7 & 0 & 7 \\ 7 & 6 & 0 & 7 & 1 & 7\end{array}$

dULInIL ETKEh $(\cos 3)<0$

$\begin{array}{lll}13 & 0 & \\ 2 & 1 & 1 \\ 1 & 2 & 0 \\ 2 & 2 & 0 \\ 2 & 2 & 1 \\ 0 & 0 & 0 \\ 3 & 2 & 2\end{array}$

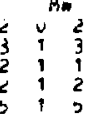

4

$$
\begin{aligned}
& .31100200 \mathrm{e}+02 \\
& 34602000002 \\
& \text { - } 220750 \\
& \text {. } \\
& \text {. 9u430100t } \rightarrow 0 \text { a }
\end{aligned}
$$

\begin{tabular}{|c|c|c|c|c|c|}
\hline $\begin{array}{l}30701050 \mathrm{E}+02 \\
.39501640 \mathrm{U}+02 \\
.39000050 \mathrm{E}+02 \\
.41579440 \mathrm{E}+02 \\
.45177530 \mathrm{E}+02\end{array}$ & $\begin{array}{l}.12217 E-16 \\
.2 y 432 E-16 \\
.21000 E-16 \\
.20333 E-16 \\
.20072 E-10\end{array}$ & $\begin{array}{l}.5 U 497 E-17 \\
.31739 \mathrm{E}-17 \\
.32443 \mathrm{E}-17 \\
.53420 \mathrm{E}-17 \\
.37601 \mathrm{E}-17\end{array}$ & $\begin{array}{l}.14209 E-17 \\
.57310 E-18 \\
.05954 E-10 \\
.11022 E-17 \\
.75014 E-18\end{array}$ & $\begin{array}{l}.31092 \mathrm{k}-10 \\
.10239 \mathrm{E}=10 \\
.12503 \mathrm{E}-16 \\
.22790 \mathrm{E}-10 \\
.14072 \mathrm{E}=10\end{array}$ & $\begin{array}{l}.74702 t-00 \\
.05037 \mathrm{t}-00 \\
.47330 \mathrm{t}-00 \\
.72746 \mathrm{t}-00 \\
.00020 \mathrm{t}-00\end{array}$ \\
\hline $\begin{array}{l}.47094930 e+02 \\
.47556090 \mathrm{c}+02 \\
.49000000 \mathrm{0}+02 \\
.51642390 \mathrm{0}+0 \mathrm{C} \\
.51391150 e+02\end{array}$ & $\begin{array}{l}.22115 E-10 \\
.14734 E-16 \\
.20035 E=10 \\
.34951 \mathrm{E}=10 \\
.09595 . .-17\end{array}$ & $\begin{array}{l}.45303 t-17 \\
.43245 E-17 \\
.41621 \mathrm{k}-17 \\
.00901 \mathrm{t}-17 \\
.00140 \mathrm{t}-17\end{array}$ & $\begin{array}{l}.10131 E-17 \\
.10902 E-17 \\
.03017 \mathrm{E}-10 \\
.14626 \mathrm{E}-17 \\
.19970 \mathrm{E}-17\end{array}$ & $\begin{array}{l}.201430-10 \\
.23010 k-10 \\
.15500 k-10 \\
.20754 E-10 \\
.40404 E=10\end{array}$ & $\begin{array}{l}.73030 \mathrm{E}-\mathrm{Ub} \\
.74010 \mathrm{E}-\mathrm{Jn} \\
.72192 \mathrm{E}-\mathrm{UD} \\
.11295 \mathrm{E}-\mathrm{US} \\
.14150 \mathrm{E}-\mathrm{US}\end{array}$ \\
\hline $\begin{array}{l}.54057510 E+0 z \\
.54099330 E+02 \\
.50202970 z+02 \\
.5943492 U E+02 \\
.002353 \text { UUE }+02\end{array}$ & $\begin{array}{l}.141 E \zeta E-16 \\
.30 ? \times 4 E-16 \\
.100 L 75=16 \\
.20502 E-10 \\
.21043 E-16\end{array}$ & $\begin{array}{l}.83597 \varepsilon-17 \\
.01043 \mathrm{E}-17 \\
.55151 \mathrm{E}-17 \\
.90307 \mathrm{E}-17 \\
.00791 \mathrm{t}-17\end{array}$ & $\begin{array}{l}.20600 E-17 \\
.20070 t-17 \\
.13900 \mathrm{E}-17 \\
.26025 \mathrm{t}-17 \\
.25020 \mathrm{t}-17\end{array}$ & $\begin{array}{l}.03195 \mathrm{~d}-10 \\
.41017 \mathrm{E}=10 \\
.29339 \mathrm{~b}=10 \\
.5009 \mathrm{~b}=10 \\
.5500 \mathrm{~b}=10\end{array}$ & $\begin{array}{l}.19004 E-U S \\
.15254 E-05 \\
.11140 E-U 5 \\
.20026 E-U 5 \\
.20359 E-U S\end{array}$ \\
\hline $\begin{array}{l}.01726790 E+02 \\
.03530120 E+02 \\
.03550750 t+02 \\
.65622410 \varepsilon+02 \\
.66040100 \mathrm{0}+02\end{array}$ & $\begin{array}{l}.11780 t-10 \\
.394 c 5 c-16 \\
.00130 E-10 \\
.30991 t-16 \\
.13357 c-16\end{array}$ & $\begin{array}{l}.53219 \mathrm{E}-17 \\
.90143 \mathrm{E}-17 \\
.94690 \mathrm{~L}-17 \\
.69247 \mathrm{t}-17 \\
.00322 \mathrm{t}-17\end{array}$ & $\begin{array}{l}.15491 E-17 \\
.23412 t-17 \\
.17629 t-17 \\
.14760 E-17 \\
.17556 t-17\end{array}$ & $\begin{array}{l}.35140 E-10 \\
.40079 E-10 \\
.31900 E-10 \\
.20060 E-10 \\
.34022 E-10\end{array}$ & $\begin{array}{l}.13335 E=05 \\
.20043 E-05 \\
.25903 E=05 \\
.14342 E=05 \\
.10310 E=05\end{array}$ \\
\hline $\begin{array}{l}.06753120 E+02 \\
.00005730 E+02 \\
.07604410 E+02 \\
.077179 y 0 E+02 \\
.07770020 E+02\end{array}$ & $\begin{array}{l}.39705 t-10 \\
.42316 z-10 \\
.13275 E-10 \\
.34191 t-10 \\
.11090 t-10\end{array}$ & $\begin{array}{l}.70754 t-17 \\
.10570 t-10 \\
.00170 t-17 \\
.11732 t-16 \\
.13122 t-10\end{array}$ & $\begin{array}{l}.15094 \mathrm{E}-17 \\
.2527 \mathrm{JE}-17 \\
.29452 \mathrm{E}-17 \\
.32127 \mathrm{E}-17 \\
.24430 \mathrm{E}-17\end{array}$ & $\begin{array}{l}.29312 \mathrm{E}-10 \\
.51943 \mathrm{E}=10 \\
.71322 \mathrm{E}-16 \\
.70006 \mathrm{E}-10 \\
.44341 \mathrm{t}-10\end{array}$ & $\begin{array}{l}.14075 t-05 \\
.22064 E-U 5 \\
.27459 E-05 \\
.20543 E-05 \\
.30425 E=U 5\end{array}$ \\
\hline $\begin{array}{l}.09029420 t+02 \\
.75562110 t+02 \\
.70013490 t+02 \\
.02000490 t+02 \\
.04202930 t+02\end{array}$ & $\begin{array}{l}.10020 E-16 \\
.75795 E-17 \\
.04150 E-17 \\
.20033 E-16 \\
.41404 E-10\end{array}$ & $\begin{array}{l}.114 \angle 0 E-16 \\
.50730 E-17 \\
.54487 E-17 \\
11425 E-10 \\
.14: 30 E-16\end{array}$ & $\begin{array}{l}.33074 E-17 \\
.1954 U E-17 \\
.2066 U t-17 \\
.44115 E-17 \\
.37441 E-17\end{array}$ & $\begin{array}{l}.07301 E-10 \\
.40214 t-10 \\
.50979 t-10 \\
.10324 E-17 \\
.01033 E-10\end{array}$ & $\begin{array}{l}.34940 \mathrm{t}-05 \\
.21115 \mathrm{E}-05 \\
.23050 \mathrm{t}=05 \\
.40500 \mathrm{E}-05 \\
.40001 \mathrm{t}-05\end{array}$ \\
\hline $\begin{array}{l}.04505350 t+112 \\
.04500200 t+02 \\
.050017 \text { yet+02 } \\
.05425420 t+02 \\
.05570500 t+02\end{array}$ & $\begin{array}{l}.14243 t-10 \\
.27229 E-16 \\
.42034 t-10 \\
.44143 t-10 \\
.32309 t-20\end{array}$ & $\begin{array}{l}.13442 c-16 \\
.14930 c-10 \\
.14343 c-10 \\
.16017 t-10 \\
.01520 E-10\end{array}$ & $\begin{array}{l}.50015 \mathrm{E}-17 \\
.40303 \mathrm{E}-17 \\
.30019 \mathrm{~s}-17 \\
.23179 \mathrm{E}-17 \\
.19532 \mathrm{E}-17\end{array}$ & $\begin{array}{l}12027 \mathrm{t}-17 \\
106 \mathrm{t}=17 \\
.02300 \mathrm{t}-10 \\
.40070 \mathrm{t}-10 \\
12711 \mathrm{t}-17\end{array}$ & $\begin{array}{l}.57610 e-u 5 \\
.50505 E-u 5 \\
.41071 e-u 5 \\
.25941 e-u 5 \\
.00455 e-U 5\end{array}$ \\
\hline $\begin{array}{l}.055 y 1020 t+0< \\
.90547000 t+0 z \\
.930001 \cup U t+02 \\
.9400440 U t+U z \\
.97201390 t+02\end{array}$ & $\begin{array}{l}.44273 E=10 \\
.10235 E-15 \\
.20570 E-15 \\
.27302 E-15 \\
.42732 E-17\end{array}$ & $\begin{array}{l}.1004 \text { yc- }-16 \\
.20004 E-10 \\
.35 y 00 z=10 \\
.37251=-10 \\
.75495 E-10\end{array}$ & $\begin{array}{l}.23254 t-17 \\
.42900 t-17 \\
.70107 t-17 \\
.72571 t-17 \\
.10212 t=10\end{array}$ & $\begin{array}{l}.47033 t-10 \\
.70495 t-1 t \\
13010 t-17 \\
.13400 t-17 \\
.31403 t-19\end{array}$ & $\begin{array}{l}.20079 \mathrm{E}-u S \\
.41023 \mathrm{E}-u S \\
.1130 \mathrm{E}=04 \\
11900 \mathrm{E}-04 \\
.23279 \mathrm{E}-00\end{array}$ \\
\hline $\begin{array}{l}10391530 t+03 \\
1040 j y 00 E+13 \\
10400500 E+03 \\
.10406170 E+03 \\
104006 y 0 \mathrm{1}+03\end{array}$ & $\begin{array}{l}.3542 b t-16 \\
.32502 E-10 \\
.12007 E-15 \\
.41503 E-10 \\
.41506 E-10\end{array}$ & $\begin{array}{l}.17200 t-10 \\
.17240 t-10 \\
15243 t-10 \\
12550 E-10 \\
.12555 E-10\end{array}$ & $\begin{array}{l}.51203 E-17 \\
.31406 E-17 \\
.29024 E-17 \\
.31936 E-17 \\
.31949 E-17\end{array}$ & $\begin{array}{l}.11773 \mathrm{E}-17 \\
.11602 \mathrm{E}-17 \\
.53177 \mathrm{E}=10 \\
.67732 \mathrm{E}=10 \\
.07701 \mathrm{E}-10\end{array}$ & $\begin{array}{l}.05974 E-05 \\
.60229 E-05 \\
.71445 E=05 \\
.40740 E-05 \\
.40776=-05\end{array}$ \\
\hline 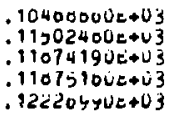 & $\begin{array}{l}.11257 t-15 \\
.20325 t-17 \\
.20042 t-15 \\
.19217 t-17 \\
.40424 t-15\end{array}$ & $\begin{array}{l}10209 \mathrm{c}-16 \\
.03137 t-10 \\
.35010 \mathrm{t}-10 \\
.10544 t-16 \\
.70200 \mathrm{c}-10\end{array}$ & $\begin{array}{l}.32309 t-17 \\
.20959 t-10 \\
.77019 t-17 \\
.70540 t-17 \\
.16170 t-16\end{array}$ & $\begin{array}{l}.00760 t=10 \\
.24240 E=19 \\
.14012 t=17 \\
.64203 t-17 \\
.30902 t-17\end{array}$ & $\begin{array}{l}.00000 \mathrm{k}-03 \\
.34413 \mathrm{E}-00 \\
.10339 \mathrm{E}-04 \\
.15179 \mathrm{e}-04 \\
.20207 \mathrm{E}-04\end{array}$ \\
\hline $\begin{array}{l}12220140 \mathrm{U}+03 \\
.12243030 \mathrm{U}+03 \\
.12243250 E+03 \\
1236130 v E+03 \\
.12301730 E+03\end{array}$ & $\begin{array}{l}.13519 t-10 \\
.40024 E-15 \\
.131<4 E-10 \\
.34002 E-10 \\
.34003 E-10\end{array}$ & $\begin{array}{l}.174202-16 \\
.70540 t=10 \\
.301910-17 \\
.142300-10 \\
.142390-10\end{array}$ & $\begin{array}{l}.74720 k-17 \\
.16<20 k-10 \\
.32573 k-17 \\
.40394 k-17 \\
.40300 k-17\end{array}$ & $\begin{array}{l}.20201 c-17 \\
.31012 i=17 \\
.1422 b t-17 \\
.9042 y e-10 \\
.90434 c-10\end{array}$ & $\begin{array}{l}.1<403 E=04 \\
.20400 E-U 4 \\
.107<1 t-U 4 \\
.50000 E-U S \\
.50093 E-U D\end{array}$ \\
\hline
\end{tabular}

2.JHYL ALCUnUL

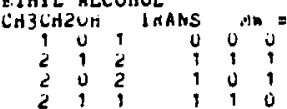

20

50

SIGMA

100

200

A KEE
$A=.30700500 x+02 \quad b=.10056000 \mathrm{e}+\mathrm{UL}$ $.12310 \mathrm{t}-10 \quad .14544 \mathrm{t}-17$ $.02743 e-10$. 1uduse-10 $.20418 t-10: 37110 \mathrm{~L}-17$ 29uUse-10 :3707j=-17 .0O0ClE-1b: $19549 t-10$ -20057b-10 $.20790 \mathrm{c}-19$ $73005=-10$ $.25083 c-17$

.4537 つt=10 .0430St-17

$.12719=-17$ $.71425 \mathrm{E}-10$

$C=.00009000 \mathrm{E}+49 \quad \times A r=-.4219$ $.40 \geq 23 c-19 \quad .2 y 090 z-10$ $.3035 y E-20 \quad .20102 E-U 7$ $13175=-10 \quad 2<3 b$ be-Us $.13440 E-10 \quad .237350-05$ .4Y350E-10 . $23702 t=10 \quad$.31ง0it-Uร

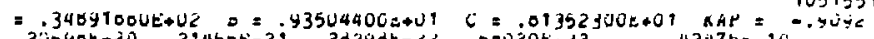
$\begin{array}{lllll}.20590 t-20 & .21452 t-29^{2} & .30290 t-2 L & .00030 t-23 & .42476 \varepsilon-10 \\ 10045 t-19 & .11397 \varepsilon-20 & .20931 t-21 & .37713 t-22 & .27747 t-04\end{array}$

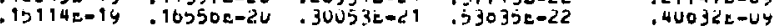
$.11313 t-19.929 u 3 s-20.2373 y \varepsilon-21.312011 \varepsilon-22.53003 t-v 4$ 
THBLE III .22

Ju KUI KUS JL KL KLL
Nu iluda
ETHXL ALCOHOL B

\begin{tabular}{|c|c|c|c|c|c|c|}
\hline $\begin{array}{c}\mathrm{CH} 3 \mathrm{CH} \\
2 \\
0 \\
3 \\
5 \\
2\end{array}$ & $\begin{array}{c}20 H \\
4 \\
1 \\
2 \\
1 \\
2\end{array}$ & $\begin{array}{l}2 \\
0 \\
1 \\
4 \\
1\end{array}$ & $\begin{array}{r}\text { TRAMS } \\
1 \\
5 \\
4 \\
4 \\
3\end{array}$ & $\begin{array}{l}1 \\
1 \\
2 \\
1 \\
2 \\
1\end{array}$ & $\begin{array}{l}1 \\
1 \\
3 \\
4 \\
3 \\
3\end{array}$ & $\begin{array}{l}\text { 40 HU }=1.4300 \\
.93002 \text { YUUE+U1 } \\
11710140 E+U 2 \\
.1404120 U E+02 \\
1701 \text { 1740E+UL } \\
.2230702 U E+02\end{array}$ \\
\hline $\begin{array}{l}1 \\
1 \\
3 \\
2 \\
2\end{array}$ & $\begin{array}{l}1 \\
1 \\
0 \\
1 \\
2\end{array}$ & $\begin{array}{l}7 \\
0 \\
3 \\
1 \\
0\end{array}$ & $\begin{array}{l}0 \\
1 \\
2 \\
2 \\
3\end{array}$ & $\begin{array}{l}2 \\
0 \\
1 \\
0 \\
1\end{array}$ & $\begin{array}{l}4 \\
1 \\
2 \\
2 \\
3\end{array}$ & 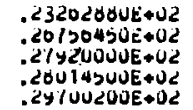 \\
\hline $\begin{array}{l}3 \\
4 \\
2 \\
6 \\
1\end{array}$ & $\begin{array}{l}1 \\
1 \\
1 \\
1 \\
1\end{array}$ & $\begin{array}{l}2 \\
3 \\
4 \\
2 \\
1\end{array}$ & $\begin{array}{l}3 \\
4 \\
3 \\
6 \\
j\end{array}$ & $\begin{array}{l}u \\
u \\
u \\
0 \\
0\end{array}$ & $\begin{array}{l}3 \\
4 \\
2 \\
0 \\
0\end{array}$ & $\begin{array}{l}.2 Y 41 \text { YOOUE }=02 \\
.32 / 43 \text { UUUE }-U 2 \\
.3041 / 1 \text { UUE } \rightarrow 02 \\
.411240 U J E+02 \\
.43020 \text { YUUE }+U 2\end{array}$ \\
\hline $\begin{array}{l}7 \\
0 \\
4\end{array}$ & $\begin{array}{l}1 \\
0 \\
1\end{array}$ & $\begin{array}{l}0 \\
0 \\
4\end{array}$ & $\begin{array}{l}7 \\
5 \\
3\end{array}$ & $\begin{array}{l}u \\
t \\
u\end{array}$ & $\begin{array}{l}7 \\
2 \\
3\end{array}$ & 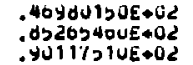 \\
\hline
\end{tabular}

DIMETHYLSULFOXY

(C)

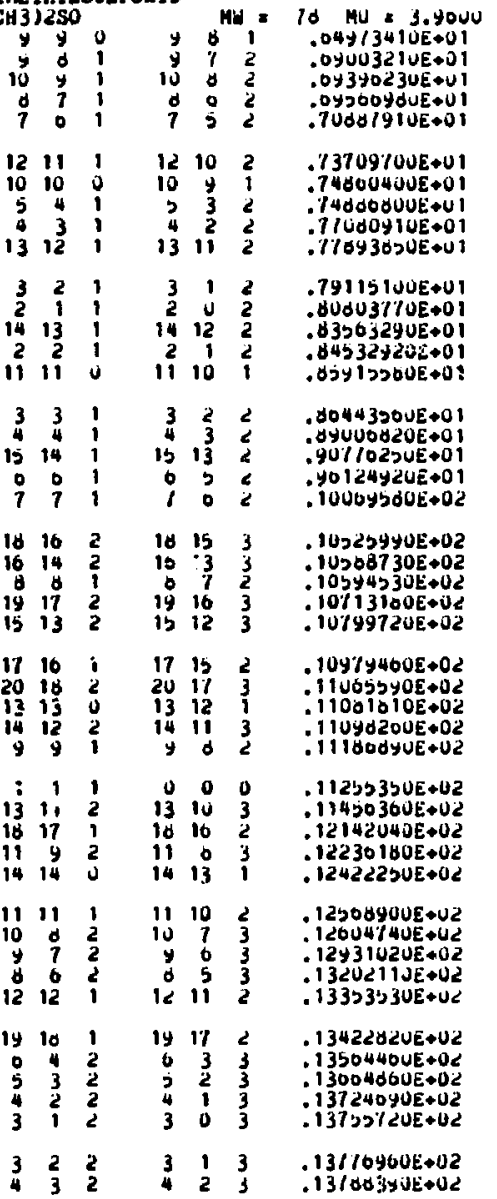

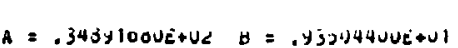
$.2 y l 0 d E-10.31 y / 4 E-1$.

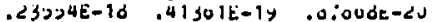

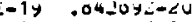
$.10 Y 1 / E-10.11 \rightarrow+0 E-10.2+4 Y O E=1 Y$

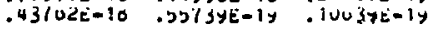

$.71103 E-10$. lovase-10 . SOUOAz-1y

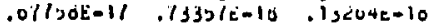
4/320E-1/ SU120t=10 BU44/E-1S

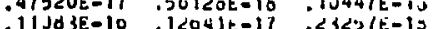

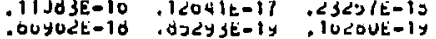

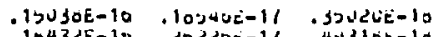
$.10432 E-10$. $23220 E-11$. 4 Y310E-10 .21U10E-10 .32/01E-1/ .00000 गरण illibe-10

$.22023 E-10 \quad 4$ YYOJUE-11 $.92321 \mathrm{E}-10 \quad .1241 / \mathrm{E}-10$

.11 jojtis 11

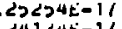

\begin{tabular}{|c|c|}
\hline 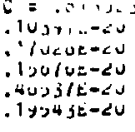 & 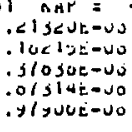 \\
\hline 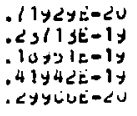 & 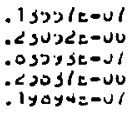 \\
\hline 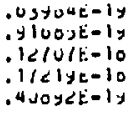 & 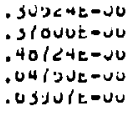 \\
\hline 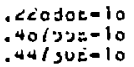 & 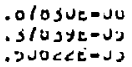 \\
\hline
\end{tabular}

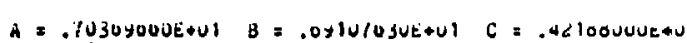

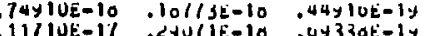

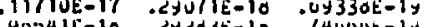
$.903411-10$. 2Y203E-10.1400UE-1y $.003018-10$ . UYYIUE-10 10,348-11 ISSISE-II $40 d y 4 E=10$

1 2S2YE-17 Tol $1458-i 0$ . 34 JुSE-10 , .0टा11E-10

1suY4till $.10413 k-17$ $.2400 d t-16$

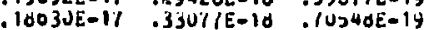
. $\angle 1 \angle 30 E=10$

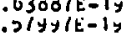

14c3re-1y

ceite-1y

iा $30=1 y$

lyflutily

$.21430 t=10$ :040J5E-1y . 11001E-1y

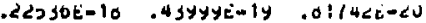

$.02110 \ddot{-2}<u$

.200गUE-10 : YLYIYE-1Y : $22 \angle 4 Y E=1 Y$

1 nht $=32$ sats

d1yu3E-1y .23UdyE=10.130<4E-10

$.22010 E-10$.343JวE-10 .1440/E-10

$: 1 / 2|2 E-1\rangle$

$.41074 E-19$

$.3012 \supset E-16$

30U4CE-10 . OI121E-1Y

.21 SOUE-10 .1SSOJE-10 .3YOdUE-10 1 130<Ji-10

$11003 E-10$

$2114 j E-14$

. $424 U 5 E-18$

Pग

$.23330 t=10$ . $2069 Y \mathrm{Y}=10$ $.4 \supset 13 / E-10$

$.124408-15$ ily/4E-10 . $10<25 E-10$

103010 $03943 E=10$

S1 $1404 E-1 ;$

704YJE-19

IUODSE-11

SIOI/E-10

.3303 YE $=1 y$

$.230<0 e^{-10}$

$.10743 \bar{E}-10$

iscuoetolo

- Oड1 $225-10$

1/20jt-io

I)

$274295=10$

Yojulti-1y

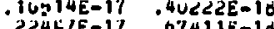

ORUपY 0 - 1 ?

PN 3 X $3 E=11$

.81yjik-15

$.07411 E-10$

-110d1E-10

- IT31E-10

$.10430 E-10$

IUUSIE-10
IDTOUE-10

$.14<4$ Jt-u

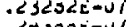

. 24 sooen-ut

. sclusz-ui

. Leu2je-ul

- Lysuzt-ul

- Iyjylizevi

icculuteul

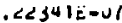

. 33 उUदE-U1

.LIIUEE-U1

. $11104+-01$

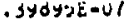

laglok-11

$.20143 i-01$

. ¿SlOSË-1Y

. $2110 \%=20$

. $<41+1 t-J i$

$.4 y 431 E-\leq u$

. 2 Cosutenl

.40şyt-ul

isty<lE-Ul

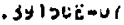

11JวOS-13

.ounuve-ui

. d19cuizul

.441 TDE-Ji

$.007 \partial J E-U 1$

.ocluat-ui

$.40200 E-1 Y$.22030E-10.1jO/4E-10 .4020Jt-1. i1†33E-10

4101 YE-1Y

10 IOSE-1?

. WIicse-1y

Injalkevi

iglusiti-ul

Hosule-ui

ojsoltend

. AyYjot-ul

$.00120 \mathrm{c}=\mathrm{v} / \mathrm{l}$

sotoje-vi

- islult-ul

2 (SOUL-U)

, DSIE-JI

is suse-ul

$\because 3 U E ̈-N U$

- Vivisje-UO

$|3 i-1, E-U|$

411 JUE-11 .02'J20E-10 .12'00<E-10 .23yJle-1y

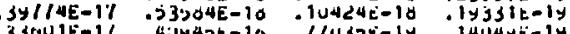

$33001 E=11 \quad .43 y 43 t=10$

.21 $200 E=11$

$.23414 E-16$

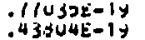

11 ic.4.

.10 itio-uo

yllogti: 0 -

$0>01+2-01$

$.011<1 .-j \mathrm{~J}$

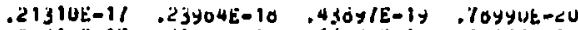

$.01340 t+t$

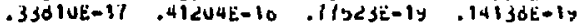

$.000<3 e-2$ - 


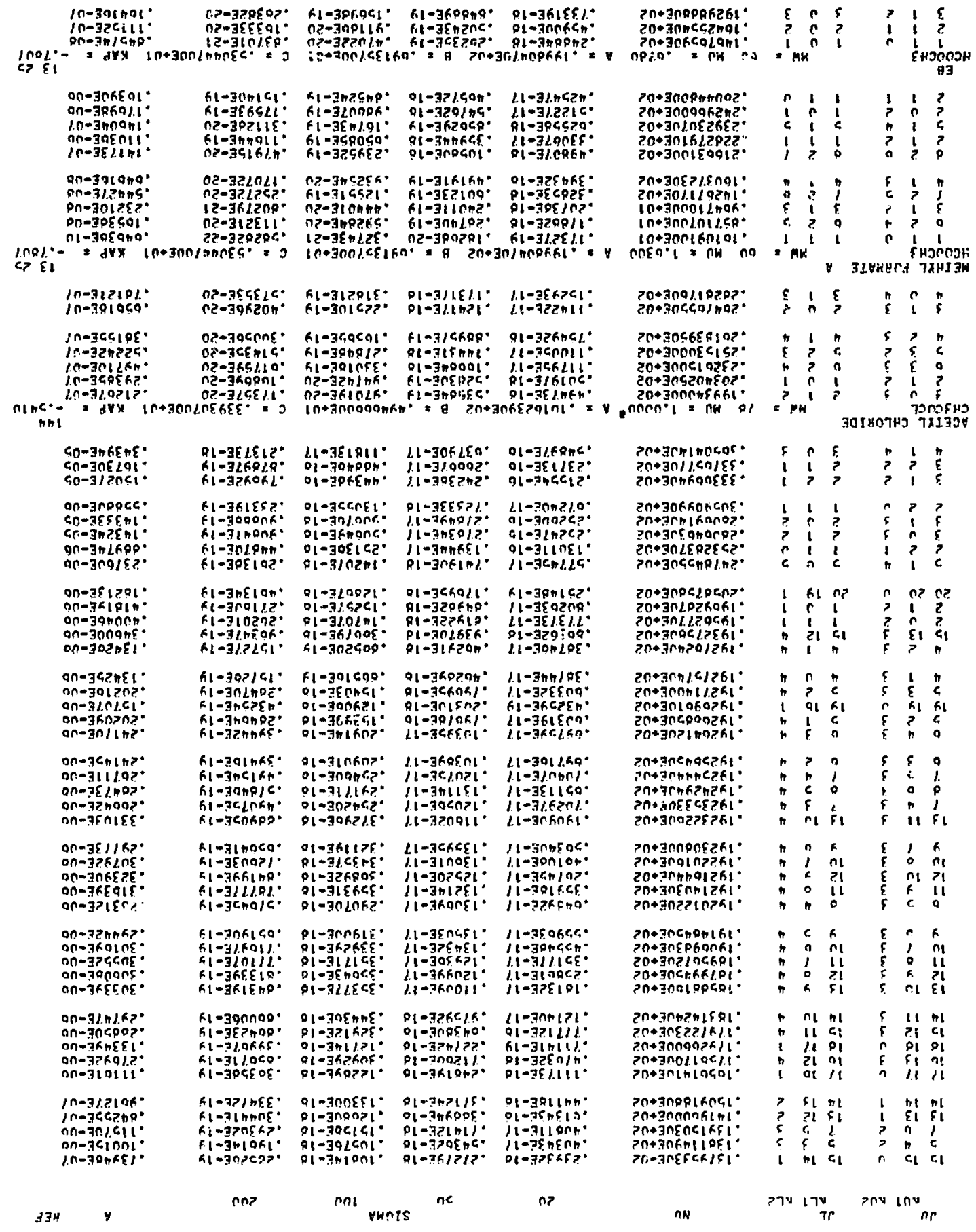


SABLE I11.24

${ }_{\text {KUI KU2 KLL KLL }}$

$\begin{array}{llllll}4 & 1 & 3 & 4 & 0 & 4\end{array}$

$.23050040 E+(12$

. 2Y4UIDUUE-UL

An

НСUосH 3

$\begin{array}{lll}1 & 1 & 0 \\ 0 & 2 & 4 \\ 3 & 1 & 2 \\ 1 & 0 & 1 \\ 1 & 2 & 3\end{array}$

$\begin{array}{lll}4 & 1 & 3 \\ 0 & 2 & 0 \\ 2 & 1 & 2 \\ 5 & 1 & 4 \\ 2 & 0 & 2\end{array}$

211

$A B$

HCOOCH 3

$\begin{array}{lll}1 & 1 & 0 \\ 2 & 1 & 1 \\ 3 & 1 & 2 \\ 4 & 1 & 3 \\ 5 & 1 & 4\end{array}$

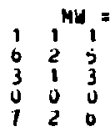

OO $\mathrm{HU}=1.0300$ $.16102200 E+03$ . Bל/ / 3 OUDE +0

- YOS34 IUUE +U

-122 IY $2 Y 0 E+02$

$.1427 y 190 E+02$

- rova7yoje+02

. 210 dudJ JuE +U2

.22d27 $110 E+0$

.2393901 UE +02
$.24295400 E+02$

$.2004 d 3 \zeta U E+02$

110

Oo $14=0 \%$ ov

. $1400113 \mathrm{JE}+\mathrm{UL}^{2}$

$.10431540 E \bullet 02$

$.23502300 \mathrm{0}+02$

.244173 PUE $\bullet 02$

VIHILFL JURIDE

Cen $3 F$

\begin{tabular}{cccccc}
$13 f$ & & & \multicolumn{1}{c}{${ }^{M A}=$} \\
3 & 1 & 2 & 3 & 1 & 3 \\
9 & 2 & 7 & 9 & 2 & $d$ \\
16 & 3 & 13 & 10 & 3 & 14 \\
17 & 3 & 14 & 17 & 3 & 12 \\
10 & 2 & $d$ & 10 & 2 & 9
\end{tabular}

to ins $=1$. cous

. $11113450 E+01$

- TOUUACGUE $\$ 02$

$.10312930 \mathrm{E}+02$

$.14419330 E+0$

$1473 \supset 420 E+U 2$

$\begin{array}{rrr}4 & 1 & 3 \\ 10 & 3 & 15 \\ 1 & 0 & 1 \\ 11 & 2 & 9 \\ 5 & 1 & 4\end{array}$

$\begin{array}{rrr}4 & 1 & 4 \\ 10 & 3 & 10 \\ 0 & 0 & 0 \\ 1 & 2 & 10\end{array}$

19316

$\begin{array}{r:r}12 & 10 \\ 6 & 5\end{array}$

$\begin{array}{rrr}6 & 1 & 5 \\ 20 & 17\end{array}$

$19 \quad 3 \quad 17$

12211

20310

$\begin{array}{llllll}2 & 0 & 2 & 1 & 3 & 1 \\ 2 & 1 & 1 & 1 & 1 & 0 \\ 6 & 1 & 6 & 5 & 1 & 5 \\ 0 & 0 & 6 & 5 & 0 & 5 \\ 0 & 2 & 5 & 5 & 2 & 4\end{array}$

$\begin{array}{llllll}6 & 3 & 4 & 5 & 3 & 3 \\ 6 & 3 & 3 & 5 & 3 & 2 \\ 6 & 2 & 4 & 5 & 2 & 5 \\ 6 & 1 & 5 & 5 & 1 & 4 \\ 6 & 1 & 8 & 7 & 1 & 1\end{array}$

$\begin{array}{lll}0 & 0 & 0 \\ 8 & 2 & 7 \\ 0 & 4 & 5 \\ 8 & 4 & 4 \\ 6 & 3 & 6\end{array}$

707

$\begin{array}{lll}1 & 2 & 0 \\ 7 & 4 & 4\end{array}$

835

$\begin{array}{lll}7 & 3 & 4 \\ 7 & 2 & 3\end{array}$

817

JHYLFLUORIDE

C2H3F

$\begin{array}{cccccc}12 & 2 & 10 & 13 & 1 & 13 \\ 13 & 2 & 11 & 14 & 1 & 14 \\ 20 & 3 & 10 & 19 & 4 & 13 \\ 11 & 2 & 9 & 12 & 1 & 12 \\ 13 & 2 & 11 & 12 & 3 & 10 \\ 14 & 2 & 12 & 15 & 1 & 13 \\ 13 & 3 & 10 & 14 & 2 & 13 \\ 16 & 2 & 15 & 15 & 3 & 12 \\ 10 & 2 & 0 & 11 & 1 & 11 \\ 1 & 1 & 1 & 2 & 0 & 2\end{array}$

. 1b182800E +02

.19674410E+02

- $19754920 E \$ 02$

$20795080 E+02$

$.22705,00 E+02$

$.2 \partial 309070 E+02$

.31852 100E+02

$.34272280 E+0$

. j19yilove+ve

- 34477 $770 E+02$

. 1 U2AS7OE+02

$11372120 E+03$

$.11743130 E+03$

$.1183 d 3 d 0 E+03$

$1186 y 3100+03$

- IId71540E+U3

. $11447460 E+03$

.15137 IUUE+03

. IBSA77O0E +03

$15764190 E+03$

$15026270 E+03$

$15026270 E+43$
$1582642 U E+43$

$.15036200 E+03$

$.15846400 \mathrm{E}+03$

$.10016320 E+03$

$.103410305+03$
$.12261340 E+03$

$46 \mathrm{MI}=2 \mathrm{~J}$

.979246UUE +O1

$.10422430 E+V L$

$.10430440 E+02$

100620LUE +UL

13 UOZdOJE+UL

$13340450 E+U C$

.1308 FYUUE +02

$1425>700 E+02$

$.14468 \% 20 E+02$
.1930 TSTUE*0

$.25243780 E+02$

20

SIMAA IU

20

200

NEF

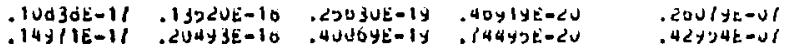

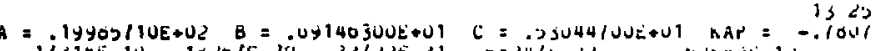

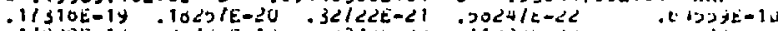

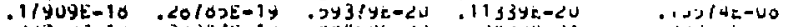

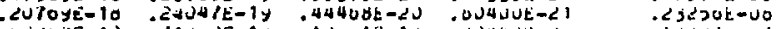

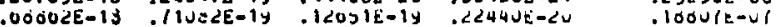

$.32 y 00 E-10$.0J230E-1t .12474E-1Y .2J310E-2U .24001E-LO

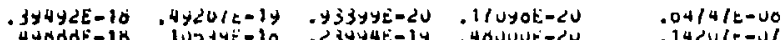

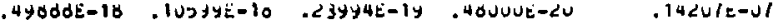

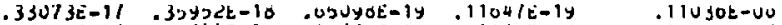

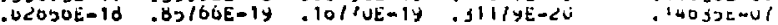

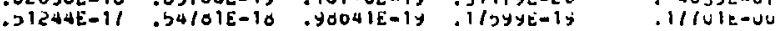

$.42507 E-11.40594 E-10$.04300E-1Y. 1214/E-1Y .10\$47E-JU

ISHdSIIUE+U2 $\quad H=.0+140300 E+U T \quad C=.33044 / U U E+U 1$ KAP $=$

$.249 J 4 E-1 d$.2620/E-1y .4/JOIE-20 .03/1UE-21 .040J2E-UO

43942E-1d .SU260E-1Y . प1209E-CU .10340E-2U .11104E-JI

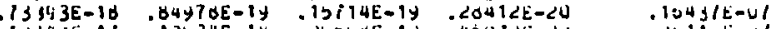

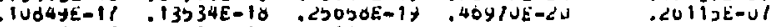

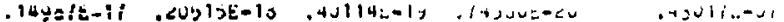

$=.043040 Y \mathrm{JE}+0 \angle \quad B=.1003000 \mathrm{NE}+\mathrm{V} 2$ $.20 Y 1 \angle E-10 \quad .32412 k-1 y \quad .00281 E-20$

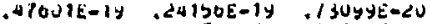

$.43244 E-21.4347 U E-20.3013+E=20$ $34533 E-21$
$.30001 E-1 Y$. 303 SUOE-1Y

$.3 D O Y E=2 U$
$.121 Y Y E-1 Y$

. $1401 E-18.101 S 1 E-19$

$.24 y d 2 E-21$. UYYd4E-20

$31134 E-11.3320 \mathrm{dE}=10$

$131395-11$

OQIOTE-10

. 53 उUटE-14

14JTOE-1Y

. Ju630E-2J

$2 Y 3 \zeta 3 E-19$

. $20511 E=19$

+4sfoe-1Y

. Ib 193E-21

$.1100<\mathrm{E}-10$

לכUSYE-19

$.73427 E-2 J$

. IJ23yE-1y

$39405-10$

$.73 \cup 2 d \bar{c}-19$

$30303 \mathrm{E}-14$

$171075-18$

1-licy-iu

100uvr

$30303 E=14$
$144115=19$

SIJUSE-To

$.230 \dot{0} 5-16$

$1029 y E-10$

luovurar

. $40 D O Y E=10$

$2168 b E=15$

.2 $1958 \mathrm{~B}=17$

. $30003 E-10$

$26174 \mathrm{E}-15$

$42332 t-10$

$93163 E-17$

$.13 y 37 E-15$

$.48199 \mathrm{E}-16$

- $32275 E-10$

$10403 E=10$

b $223 E-10$

- 2299OE-16

. $23000 E-16$

. $10342 t=16$

.62ybsti-17

$.23425 E-15$

$.20224 E-15$

445 सE 11

$3 U 311 E-15$

IIJUZE-I6

100Y9E-10

ifodJE=I?

. 6JUUYE-16

$21111 \dot{k}-16$

$2034 / E-10$.2030YE-10 .1UTOYE-10

203Y'E-10

$.016320-10$

$2030>E-10$

$14040 \mathrm{E}-10$

$.01701 t-10 \quad .47002 t-16 \quad .14004 E-10$

1/372E-15 .0/440E-10 idob2E-10

$.20009 E-15$

$.0 / 940 E=10$

22Uं山t-10

$A=.045 d 46408+02 \quad B=.10030600 E+12$

$.16672 E-20 \quad .24700 E-20$. IU $324 E-2 U$

$.10314 E-20$

$420075-23 \quad .10 / 0 j t-21 \quad .12 \% 02 t-20$

$.44440 E-20 \quad .46146 E-20 \quad .17134 E-20$

. $3544 \mathrm{Jt}-20$

$17134 E-2 U$
$3<530 E-2 U$

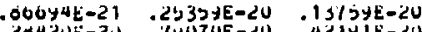

$.2842 U E-2 J$

(1)

1056je-1y

. วUवीगह-ट

$044 E-20$

$.18294 E-18$

. LUO'LE-IY

$3 / 45 Y E-20$
.31 3 $20 t=20$

$C=.+11004 J u t * 0$

120 UUE $=20$

TOTU/E-2U

$13112 E-20$

. LLYYOE-

. $3107 U E-2 U$

$.204+3 E-20$

$.30393 E-20$

. 1USZUE-1Y

. $222 \angle E-\angle U$

$.41415 E=\angle \mathrm{V}$

$.5 J 606 E-20$

$.02033 \mathrm{E}-2 \mathrm{~L}$

$10 \angle D J E=2 N$

- it $20<c=c$.

- $2 /<\cup<-1 y$

$.63450 E-1 y$

. $18370-1 \%$

¿djटाE-17

$1 / 430 \mathrm{E}-1 /$

-1364+E-17

$130,4 E-1$ ?

-Y17YUE-10

. $2120 D E-11$

$.41 J 43 E-11$

. 1 $\angle U \angle 3 E-U O$

$.01740 E-U Y$

. OD $1 \angle 2 E-U Y$

. 1

-LIUUAE-UO

IJjy

- 3400ut-0o

.4 YUJTE-UI

-

.15isut-jo

$.044012-00$

- IUUठUË-U1

$.14130 \mathrm{E}-\mathrm{U} /$

$.14 \leq 10=-01$

$.313 / 1 \mathrm{t}-40$

$.40 y<0 k=00$

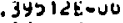

$.1 \geq 204 t-J 4$

.142 JUE-J4

- Beyldélua

$4429<k-11$

म.J4Yटt-1/

- codoí-11

- LlOdOE- il

- SOU/CE-17

$.11036 i=04$

$.11045 E-U 4$

. USYYयE-U'J

- 1)040E-J4

- उUUŻJE-UA

$.1303 / t=04$

. SCyวUt-J4

.200d1E-JU

- ¿UUOCL-UL

. JUOட‡E-UH

- juooot-u4

-34.3/2t-va 


$$
\text { JJut rive JLLKLe }
$$

nu

$$
\begin{aligned}
& 11, \geqslant \text { is }<1 \mathrm{~J} \\
& \text { is } 213 \text { is } 10 \text { 1 } 410 \\
& \begin{array}{llllll}
1 y & 9 & 10 & 10 & 4 & 10 \\
0 & 1 & 1 & 1 & 4 & 0 \\
y & 2 & 1 & 10 & 1 & 10
\end{array} \\
& \begin{array}{llllll}
11 & < & 10 & 10 & 9 & 13 \\
10 & 2 & 14 & 11 & 1 \\
0 & 2 & 0 & 1 & 1 & 1 \\
4 & 0 & 4 & 3 & 1 & 3 \\
12 & 13 & 4 & 13 & 5
\end{array} \\
& \begin{array}{rrrrrr}
3 & < & 4 & 0 & 1 & 3 \\
10 & 4 & 19 & 11 & 3 & 14 \\
10 & 5 & 17 & 11 & 3 & 14 \\
11 & 2 & 10 & 10 & 1 & 10 \\
2 & 1 & 3 & 4 & 0 & 4
\end{array} \\
& 010202
\end{aligned}
$$

\begin{tabular}{|c|c|c|c|c|c|}
\hline & & & & & $=$ \\
\hline 3 & 1 & 3 & 9 & 1 & 2 \\
\hline 1 & u & 1 & u & u & u \\
\hline 0 & $<$ & 2 & פט & 4 & 4 \\
\hline$y$ & s & 1 & $y$ & 3 & v \\
\hline 4 & 1 & 4 & 4 & 1 & 3 \\
\hline 2 & 1 & 2 & 1 & 1 & 1 \\
\hline 1 & $<$ & 0 & 1 & $<$ & ל \\
\hline 2 & 1 & 1 & 1 & 1 & 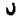 \\
\hline 2 & 1 & 2 & 3 & l & 4 \\
\hline IU & $\mathbf{J}$ & 0 & 10 & 1 & 7 \\
\hline
\end{tabular}

ETHYLENE SULFIJU CLIISS

SULFI UE DIMETRILL CCHSTLS

$\begin{array}{lll}1 & 1 & 2 \\ 3 & 1 & 2 \\ 2 & 1 & 1 \\ 4 & 0 & 2 \\ i & 1 & 1 \\ 4 & 1 & 0 \\ 3 & 1 & 2 \\ 0 & 2 & 2 \\ 4 & 1 & 3 \\ 1 & 1 & 1\end{array}$

$.124 \$ 1 \angle 1 \cup E+U \angle$ $10 / 2 \mathrm{~J} U \mathrm{YJE}+\mathrm{UL}$ I $\forall y>340 U E+U C$ . $U 10 \angle 2 U N E+U \angle$ . $\angle U \angle C O 3 S U E+0 \angle$

$24 Y 00200 E+U 2$ $2012421 U E+U$ $20344 * 4 U t+U \angle$ . $2 d 41 \angle Y D U E+U 2$ .20UOJULJE+UL

. SUU30770E+U2 . 3usY4 $200 E+U 2$ $.344 / y 300 E+U 2$ soyoluYuE +0́

$.14234 / 3 U E+U 3$

$.15 / 43180 t+03$

ov $M U=1.04 \mathrm{JU}$ $.10742 \angle U U E+U L$

$1 / 11$ gduUE +ue

l lod? $14 \mathrm{JUE}+\mathrm{UC}$

. 22Y1OUNUE +UL

$.2047320 U E+02$

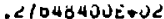

. J4YU JOUUE-UL - SSOIS SUUE-UL

. 4 UUUगUJE+UL

. 4UU 12UUUE+02

$.4 U d O P$ IUUE +UL

$02 \mathrm{HL}=1.20 \mathrm{JU}$ . 12UY 1O YUE +U2 $.12143320 \mathrm{~T}+U<$ .142 s/OUUE + JL $.10240340 \mathrm{UE}+U 2$ .1004 Y200E+U2

$.17 U 4023 U E+W 2$ - 17O/s 1YUE०Uz - ¿U7 UYDYUE+UL . LSSOS IUE+UL $.235214 \angle U E+U 2$

$.21 / 61400 E+42$ . $2103245 U E+U 2$ - $2013084 J E+U 2$ $.2 Y 4 J 3 \angle J U E+U E$

.304001 TUE - JUJ4204UE⿺02 - jUOUI I IUE⿺辶大 - jU0231/UE+UL .53211U2UE +U?

- 3400342UE-02 . उय †ण 2 OUE . 3020U/JUE + U2 . $302 / 45$ JUE 40 . $3100 / U \forall U t+02$

- 3y<7<030E+ 12 -34200U +UE +02 4 UUOSOXUE+U . 4 1 ju/1ue+ue

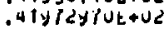

$.42302400 \mathrm{E}+\mathrm{UL}$ -4 $237<4 U U E+0$ $.43321 y 20 i+02$ $.43543 y 20 E+02$ $.4303 / O / U E+U E$ $.470>453 U E+U 2$
24

SIUHA

iv

11Yj/E-1Y . $>4>y t-21$

$.01132 \mathrm{~L}-22$

. I Y I $3 \mathrm{E}=10$

. गDण/E-1y उ山LYIE-2U . उपाIE-2 .0 IU/UE- 1 . . $201000^{\circ}-1 \mathrm{y}$

$.11 \% O / E+\angle U$ $.015 \angle 4 E=21$

luzule-io

$.10051 E-11$

- $1<4<2 D-1$ $.4473<t=25$ $.04341 k=1 y$ . $4 \angle 310 E-10$

- LCYUSËकIY

. 4 SOOLE-14

4ソ5 $16 E+16$

.9U0टाE-21

- yolu<t-21

$.44(24 E-\lambda)$

$10 \% 00 \mathrm{E}-10$ - $13 / 3 / t=14$ .10/YIE-1Y . $25042 E=2 U$

,$\forall \angle Y 0<E=\mid 1$

$.0 \angle O Y O E=10$

. ILL/OE-10

$A=, \angle 1 Y f 4 J U J E+U L \quad H=.10024 Y U U E+U \angle$ 121/1L-17

$.10834 E-10$

.3ЗUУOË-IT

. I $42 \mid E-11$

- y5todE-16

$14 n j 1 E-10$ $1 \supset$ IOUEE-18
$.34>4 U E-10$ . $\$ 34 / 1 E=10$ - Sos 1yE-10

. 273 Y $5 E-1 Y$ : $U 4$ SOE-1Y

. टरUTाE-1।

$.1203 / 3-10$

$.2>04 / E-11$

- LUTUOE-:U

. 2Yठ3UË-1d

. 10 y $3 E-1$

- $\rightarrow$ SUDE- 16

$.2 \angle 0 Y U E-11$

DIYLUE-IS

$.0109 / E-1 Y$

IUdbJE-10

$.3 / 140 E-1 y$

$.34304 E-1$

$.110 / 25-1 i$

$.02191 E-10.1 Y 3 J 2 E-10$

- $1103 \angle E-10$

$13010 E-10$

$41015 t-10$

$.104 / 1 E-16$

$A=.1604730 E+i J<\quad B=, 10<10 / 00 E+U 1$

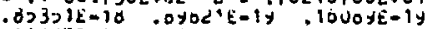
. I Ju2ie-18 $.10 / Y 2 E-11$ $.02134 \mathrm{E}-10$ $.73001 \mathrm{E}=1 \mathrm{Y}$

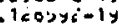

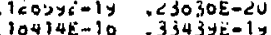
$.10414 k-10 \quad .3343+E=1 y$ $.013 y 1 E-1 y \quad .12134 t-1 y$ $.730012-14$

$.12 / 40 E=10$

$.200 / U E-11$

- 371 $120 E-10$

$43 \mathrm{j} / \mathrm{DE}-1 /$

. $1040 E-1:$

$12+24 E-1 y$

$+1 \geq 033:-1 y$ - jjuOle d ONUOUE-

$.103+1 \mathrm{k}-1$ : $.01303 \mathrm{E}-17$

. 13 ! vuE-1

.111 SUE- 17 $34 i 34 \mathrm{E}-1$ $.0 .3 \square \square E-17$ do/วyi, $=10$ $.1 / 34<c-18$

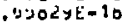
$130<3 t=17$

- 11YODE-10 $.434<2<-11$ $\therefore 314<2=11$

$.1+300 E-10$ - J10<3E-10 SOOOUE-10 $0,13 / E=17$ $. Y 1 \supset 01 E-11$ - $110,0 \mathrm{EE}-18$ .1 1 JUE-11

. $13000 E-11$ . visयi-11 - 1 JU/टE-lo

$.213015-10$ $13402 E-10$ .40ust-1y . SIVLAE-11 . $4 \angle 4 C / E=10$

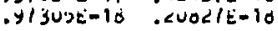

$.04005-11$ .400/iE-1? ODग:1E-11 .2गोite-io

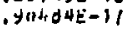

$100 / 1 \mathrm{E}-10$ . $2<v 0 y E-11$

1 jouve-11 . Y 7 प $E-7 y$ . $27002 \mathrm{E}-11$

$.03 \vdots 72 E-10$. 21UУसE-7o $.11 .25 E-10$.207ษ/E-11

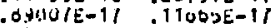
$.0<202 \bar{E}-11.20 y \$ 3 E-17$ $.2 \angle \partial 1<E-1 \sigma$. IUUJAE-1d

$.12840 E-10$.20050E-17 $.10 j 4 \angle E=10$.2101UE-11 $\because 11 U / E-1 / \quad 31<2 y E-17$

2uv

A H比

340UE-U6 . $1374 \mathrm{E}-\mathrm{UI}^{\prime}$ $.21305 \mathrm{E}-\mathrm{U}$ $.33 y 02 t-110$ I $314 \mathrm{E}-1 / 0$

.4 SULTE-JO $13130 \mathrm{E}-\mathrm{NO}$ - SOIIDE-UO . lodyue-Ul

1042yzi-uo

$.104 . j 7 \hat{c}-01$ . Y01411E-UO IUV3SE-Ul 2צYYSt-UO . St, OU $8 \mathrm{t}-\mathrm{U}$

. SUOOOE-U2

$.23300 E-10$

$c=.00 \angle 03 U U N E+01$ AAP $=-.240$

$.4 Y 04 J E=2 U$

$.142 U U E-1 Y$

$.10 Y 03 E-1 Y$

$.14410 E-1 Y$

$.24220 E-1 Y$

. INOO 1E- TY

$.25 ! 25 E-1 y$

$.2002 \mathrm{JE}-1 \mathrm{H}$

I $4 y 1$ ISE-1y

IYI/IE-IY

- THSYUE-UI

19 root-ur

.017 गyyc-ji

TOU37E-U/

$.201 d, E-G 1$

$43031 E-07$

$.2020 y \mathrm{t}-40$

- $>0$ TIEIEUI

- 10 dosoc-jo

,$y \rightarrow 2<<t-J 1$

$.14 / 01 E-J O$ $c=.2 / 17 T Y U U E+U 1$ nAP $=-0.0025$ .200दYE-2U .23151E-U? $.43147 E_{1}-21 \quad$. ISLlOE-UO . $\supset Y Y 13 E-2 U \quad$.34404E-U7 . ZIVUNE-2J .141YdE-U7 . 2U13UE-LI .12U/OE-UO

cutsoE-ve TUS? 2 LE $\rightarrow U 1$ 
jo

KUI KUL NLL KLL

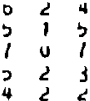

$\begin{array}{lll}5 & 3 \\ 4 & 3\end{array}$

$123 y$

$\begin{array}{lll}0 & 2 \\ 12 & 4 & 3\end{array}$

1025 ij 2

$\begin{array}{rrrrrr}2 & 2 & 1 & 1 & 1 & 0 \\ 11 & 4 & 1 & 31 & 3 & 0 \\ 0 & 3 & 4 & 0 & 2 & 3 \\ 1 & 2 & 0 & 1 & 1 & 1 \\ 0 & 1 & 1 & 0 & 0 & 0\end{array}$

$\begin{array}{rrrrrr}7 & 3 & 0 & 7 & 2 & 0 \\ 10 & 14 & 0 & 10 & 3 & 1\end{array}$

IMYL CHLORIDE

$\mathrm{CH} 2 \mathrm{CHCL}$

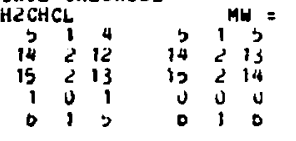

$\begin{array}{rrrrrr}16 & 2 & 14 & 16 & 2 & 13 \\ 7 & 1 & 0 & 1 & 1 & 8 \\ 17 & 2 & 13 & 17 & 2 & 16 \\ 3 & 1 & 2 & 2 & 1 & 1 \\ 4 & 1 & 4 & 3 & 1 & 3\end{array}$

$\begin{array}{lll}4 & 5 & 4 \\ 4 & 2 & 3 \\ 4 & 3 & 2 \\ 4 & 2 & 2\end{array}$

B

$\mathrm{CH} 2 \mathrm{CHCL}$

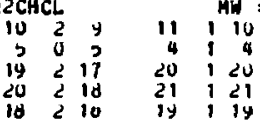

212

$\begin{array}{lll}2 & 1 & 2 \\ 11 & 2 & 15 \\ 10 & 3 & 10\end{array}$

3003

18118

$\begin{array}{ccc}14 & 2 & 17 \\ 5 & 1 & 5\end{array}$

$\begin{array}{lll}0 & 1\end{array}$

112
Nu

4117, $2, U E+U C$ $.40419430 E+02$ $\rightarrow$ OU 4UUULE +U - UU/Ýdlové+UC $.231 B Y 4 \angle U E+U C$

3443 YOYUE $+U$ 34 1 IY $23 U E \rightarrow 0$ L .0200300 UE $+0<$ .204OUSLUE+UC $.2100101 \mathrm{NE}+\mathrm{U} 2$

DYI4S SIUE+UC . $\forall+1621460+02$ - 2 Y $>00$ T $4 U E+U$ $.01,3070 U E+02$ $.015 Y 2310 E+02$

$.02435 \%$ DUE+UE . OJL3I3OUE +UL

$01 \mathrm{mu}=1.4200$ d7 byO $3 U$ UE +OI . 7 (Y) $3300 E+0$ ? 11 3SDSOUE+UC $.1147523 U E+02$ . 122BULUE+UL

$14414040 E+02$ 103033 OUE +U2 . 17 YYUAYUE+UC

. उלzYYS 10E+Uz

$.44 / 2 U$ UUUE +UC

$.45849000 E+02$

. 450 Y / OUUE $+U 2$

. 4डY13300E 02

$45947400 E+02$

.4TUSY RUOE+UL
$2 v$

SIUMA

wu

¿Uu

$.13 y>4 t=10 \quad .24013 E=17$

1UY3OE-1D BS/YIE-11

11)344E-10 10/3,E-11

.13 JOYE-10 : $21094 E-17$

.12100E-10 :1/3U<E-11

$.31420=10$

. $4 J 22 / E-10$

$.44190 \mathrm{E}-10$

. IUTSOE-10

$.10304 E-1 Y$

$.0410 j E-1 y$

$.10700 E-11$. LOSCOER-16

$.01 \angle U 0 E-1\} \quad 3430 Y E-1$

$120200^{-10}$

. वर्य,

$.131 \supset 1 E-16$

$.2 U 233 E-17$

.313ट3e-11

$.100 y 0$ E 1

4100

ICUUAE-11

$.200245=10$

. Oy $200 \mathrm{x}-1 \mathrm{y}$

$.0 y u$ lte-10. tycyuti=10

$.1 / 1 / 16-10$

$.03003 E-18$

- 1/YOUE-16

¿2U3U3E-11

4UYUOE-17

$.31414 E-1 \sigma$

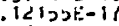

$.0122 y-1 y$

13 4 TUE -10

$-1$

$2402 . \mathrm{E}-1$

-2गYण

$.210308-10$

- ISUJUE-10

1042UE-10 .3/14ct-11

. d20jte-lo

$.110>3 E-10$. $44724 E-11$

$.12333 E-11$

$1030 / t=10$

$.21220 E-10$
.0ग1Y1E-1Y

i JUTYE-10

IUSTIE-1O

DJ2YUE-10 .11UगयE-10

$.010<0 t-00$ $.24040 E=-U 0$ . OOUUIE-UD .043 Je-vu

$.19410 t=00$

.14020 Li-J3 121YYE-vo 11 $3<0 E-U 2$ -ylosic-so

. leyube-us

.162 JUE-U. . 104Yวe-vo isusot-u' $.74003 E-U 0$

YULUYE-UO

. lbcivui-ua .2U4UYE-UD

$A=.200 j 57 / \mathrm{J} 4 \mathrm{E}+\mathrm{UL} \quad B=.002 y y \cdot 24 \mathrm{JE}+U 1$ . Y $3 y 10 E-19$.132L2E-19 .20UYOE-2U

$\because 8722 E-20$

. OU 2 Y $2 E-20$

3006E-10

. OUगO/E-2U

$21400 \mathrm{dE}-20$

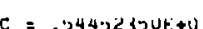

rat $=4<13 y$

$.43144 E-21$. ग21/UE-UY

. $3340 E-2+\quad$. $2 Y 1 Y Y E-U Y$

.1 YCYSE-2 $\quad$ ? $212 U E-U y$

.23y0yE-20 :11021E-J1

. $10343 E-0 B$

$.108025-20$

.13D10E-19

$.7304 E-2$

$.9 / 0 / 1 E-U Y$

Id $30 d E-v_{0}$

- 10OTOE-UQ

-3y $20 E-v 0$

$.07402 E-U 0$

$.14207 E=10$

. 2 YUT $\angle E-1 Y$

$.410 Y 1 t=20$

.113 ग1 $11-20$

ij $104 \mathrm{t}-20$

. วdyU3i-19

$.17244 E-11$.32347E-10

. ПつҢIE-1O

$.13104 \mathrm{E}-10$

1UVD3i-us

-1 JOLUE-UU

.4410 पEATlO

CoOOlE-jo

lutylíals

IUOdUE-10

. JUUว7E-1y

. IUTU/E-10

. $0070 \mathrm{TO}-18$

:3 $1047 E-16$

$10 y E-17$

TYU3ठE =10

.1 4 गОOE-10

NAT $=-42134$

$A=.50834704 \mathrm{E}+02 \quad D=.0024454 \mathrm{JE}+01$

$C=.24452300 \ddot{E}+U 1$

$.24 y 20 E-20 \quad .04202 E-21 \quad .2224 d E-<1$

i उUO $3 E-19$

$.11040 E-21$

.0573 je-jo

$.109 / 4 E-20$

. $3201 \mathrm{D} 1 \mathrm{dE-2}$

YUS 33 TUUE +0 I

$1 \rightarrow 00$ d $2 U G E+U_{c}$

$13448 Y d 0 E+U_{c}$

IDS/OY4UE+UE

$15823870 E+02$

. 1046 j $60 \mathrm{UE}+\mathrm{UL}$

17957 DUUE +02

$.21813130 E+02$

$. \supset 1 Y 03200 E+02$

$.22871010 E+02$

SY $Y$ YE- 1 Y

$3 / d>3 E-<1$

रपभा $4 \vec{E}-21$

$.00117 E-22$

$.01040 \mathrm{E}-22$

3UU $30 \mathrm{E}-10$

$.0613 y+-10$

,y $320 \dot{E}-10$

$.21<03 \mathrm{~s}-10$

.1 SUAYE-10

,2THCIOE-20 . SUU 34E-21

$.4014 y E-21$

$3614 / E-21$

$.0225 \% \mathrm{E}-1 \mathrm{t}$

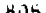

$.20 \% \angle 1 E-\angle 1$

. $1>0, y=-2$

$1 / 9.2 E-U Y$

1U4idE-JY

2ூO3.JE=UY

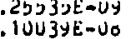

. $52313 E-U$

$.33+<3 E-0 \%$

VIAYLIUEHE CHLORIOE CH2CCLL

$\begin{array}{cccccc}2 C C L 2 & & & & W W & = \\ 0 & 3 & 5 & 0 & 2 & 4 \\ y & 5 & 0 & y & 2 & 1 \\ 2 & 0 & 2 & 3 & 1 & 3 \\ 3 & 3 & 2 & 5 & 2 & 3 \\ 10 & 3 & 1 & 10 & 2 & 0\end{array}$

$96 \quad 190=1.3400$

$.0215 \Rightarrow 5-18$

. $y$ YUUIE $=14$

$.1002 T$ KE- 1

$33+2 \cup t=20$

$A=\because 406800 U E+01 \quad B=.34113000 E+U 1 \quad C=.233$ YUUUUE+U1 KAP $=-2414 J$ $.06 / 32 E-16$. $11104 E-16$.21373E-1Y .3Y31/E-20

$.113 \supset 3 E=11$.11040E-18 .30360E-1Y

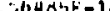
$\because y<y=-10$

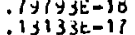

IHUT SUUE +02

. Id lusyuve+u

$.19747000 E+02$

$.2149 \angle 000 E+02$

$324 \geqslant 13.22310000 E+02$

SROSUOUNE +02

L2TUIZUUE+UL

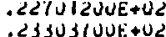

$.234<8,00 \mathrm{E}+0<$

$.08483 t-18$

$.13 \times 61 E-11$

$1214 y E-11$

.14 TUUE-1\%

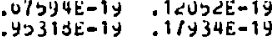

$.22400 E-10 \quad .4 / 4 / 2 E-1 Y$

LY 3 boE -20

.2 13 पण

.327vis-20

YIdÓlE-2U

. H 1 JOOE-U

$.32054 E-U 1$

$\therefore 10<1 \mathrm{~N}-\mathrm{U}$

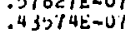

$.14 U$ UDE-UI

- $13113 E-10$. $230<0 E-1 Y$

1 IYUUE-1Y

, SYOUTtE-UI

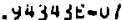

$.10047 E-U 1$

4 ClNUE-20

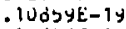

. Y. 24 SIE-UT

. $411 Y=16$.00211E-1Y .13140E-1Y

IUगว $25=30$

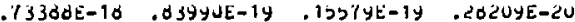

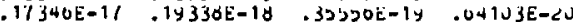

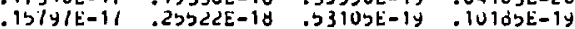

$.120 \cup 3 E-17.00073 E-10.30747 E-14.30 .349 E-20$

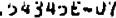

C4

.24 YOINUUE +02

.2 IllOUUE+U2

$.13420 E-11$.2YU02E-15

$.03044 E-14$

. $12 \cdot 322 t^{\prime}-1 y$

luglle-vo

$.10442 E-U C$ 


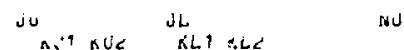

$\begin{array}{lllllll}2 & 1 & 1 & 3 & 0 & 2 & .13141970 k+02 \\ 4 & 0 & i & 3 & 1 & 3 & .13445000 k+0 L \\ 1 & 1 & 1 & 0 & 0 & 0 & .1300090 E+U 2 \\ 0 & 1 & 5 & 0 & 0 & 0 & .14900410 k+02\end{array}$

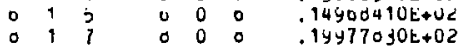

$\begin{array}{llllll}0 & 0 & 0 & 5 & 1 & 5\end{array}$

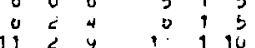

Cycluenuteat

\begin{tabular}{|c|c|c|c|c|c|c|}
\hline \multicolumn{3}{|c|}{ [i]114 } & \multicolumn{3}{|c|}{$M w=$} & $40 \mathrm{rU}=.4540$ \\
\hline 12 & 7 & 2 & 12 & 7 & 0 & $.19704200 \mathrm{E}+U 2$ \\
\hline 5 & 3 & 2 & ? & 1 & 3 & $.2154422 u v+02$ \\
\hline 14 & d & 0 & 14 & c & 7 & .22720 y40 \\
\hline 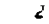 & 1 & 1 & 2 & I & i & $.24009570 E+02$ \\
\hline 10 & 9 & $r$ & 10 & $\dot{y}$ & \& & $.25593 y 00 t+02$ \\
\hline 7 & 4 & 3 & $l$ & 4 & 4 & .275 y $9740 \mathrm{t}+02$ \\
\hline 10 & 10 & $\varepsilon$ & 18 & 10 & 9 & $.20302210 t+02$ \\
\hline 20 & 11 & y & 20 & 11 & 10 & $.3109=940 k+02$ \\
\hline 9 & $j$ & 4 & y & 5 & 2 & $.33353410 \mathrm{c}+02$ \\
\hline 4 & 2 & 2 & 4 & 2 & 3 & $.34935330 \mathrm{t}+42$ \\
\hline 1 & 0 & 1 & u & 0 & $u$ & $.35021 \mathrm{a} 5 \mathrm{CE}+02$ \\
\hline 11 & $b$ & 2 & 19 & b & t & .35OOULOUE +02 \\
\hline 3 & 0 & 3 & $\dot{c}$ & 2 & 0 & $.3-\cup y 0420 \mathrm{e}+\cup 2$ \\
\hline
\end{tabular}

KMUFYLENE

injinctio

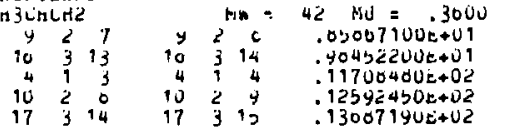

$\begin{array}{cccccc}1 & 0 & 1 & 0 & 0 & 0 \\ 5 & 1 & 4 & 2 & 1 & 5 \\ 11 & 2 & y & 11 & 2 & 10 \\ 10 & 3 & 15 & 10 & 3 & 10 \\ 12 & 2 & 10 & 12 & 2 & 11\end{array}$

$\begin{array}{rrrrrr}19 & 3 & 10 & 19 & 3 & 17 \\ 0 & 1 & 5 & 0 & 1 & 0 \\ 13 & 2 & 11 & 13 & 2 & 12 \\ 7 & 1 & 6 & 1 & 1 & 7\end{array}$

$\begin{array}{lll}7 & 1 & 6 \\ 2 & 1 & 6\end{array}$

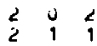

PRUPYLENE

CHJimint

$\begin{array}{lll}0 & 2 & 4 \\ 3 & 0 & 5 \\ 4 & 2 & 2 \\ 3 & 2 & 3 \\ 7 & 1 & 0\end{array}$

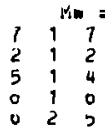

$42 \mathrm{MU}=.0500$

$.17439430 k+0 z$

$.17554970 \mathrm{t}+02$

$.17000220 \varepsilon+02$

$.10530010 E+02$
$.23934230 \mathrm{E}+02$

$.24500410 \mathrm{E}+\hat{C} 2$

$.24557050 E+02$

$.31430710 E+02$

$.32702090 \mathrm{E}+02$

$.33700000 \mathrm{t}+02$

$.34051330 \mathrm{E}+02$

$.94470100 t+01$

$10370000 \mathrm{t}+\mathrm{UL}$

$1090120 \mathrm{OE}+02$

$.21032430 E+U 2$
$25149040 E+U 2$

$\begin{array}{ccccccc}\ln 3 \text { intit2 } & v & 1 & & M w= \\ 1 & 0 & 1 & & 0 & 0 & 0 \\ 2 & 1 & 4 & & 1 & 1 & 1 \\ 2 & 0 & 2 & & 1 & 0 & 1 \\ 2 & 1 & 1 & & 1 & 1 & 0\end{array}$

$42 M U=.3000$

$.17429510 E+02$

.33700500e+02

. $34032200 E+02$

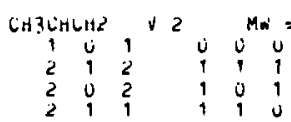

$42.80=.3000$

- 17400500 +02

. $33742200 \mathrm{e}+02$

$.34912400 t+02$

VIWIL CIALILE

$\begin{array}{rrr}20 i t i c & \\ 1 & 0 & 1 \\ 0 & 1 & 5 \\ 16 & 2 & 14 \\ 17 & 2 & 15 \\ 7 & 1 & 6 \\ 10 & 2 & 10\end{array}$

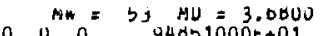

$.94051000 t+01$

$.90000700 \mathrm{e}+01$

. 10 luz2yue+uz

$.12042120 \mathrm{E}+02$

$.12042120 \mathrm{t}+02$
$.127 \pm 110 \mathrm{t}+\mathrm{UL}^{2}$

$.15504710 \mathrm{e}+02$
DIGHA

100

200

\begin{tabular}{|c|c|c|c|c|}
\hline $\begin{array}{l}34500 t-10 \\
15470 t-10 \\
90037 t-1 y \\
436 y 4 E-10 \\
.1150 y E-10\end{array}$ & $\begin{array}{l}\text { 37yyoc-1y } \\
10140 \mathrm{1}-19 \\
.95631 \mathrm{E}-20 \\
.51003 \mathrm{E}-1 y \\
.40092 \mathrm{E}-19\end{array}$ & 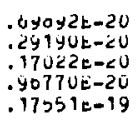 & $\begin{array}{l}.12003 \mathrm{k}-20 \\
.22103 \mathrm{t}-21 \\
.30145 \mathrm{t}-21 \\
.1757<\mathrm{c}-20 \\
.32427 \mathrm{t}-20\end{array}$ & $\begin{array}{l}.10919 t-07 \\
.20022 \mathrm{t}-00 \\
.47014 \mathrm{t}=00 \\
.14700 \mathrm{E}-07 \\
.20302 \mathrm{E}-07\end{array}$ \\
\hline $\begin{array}{l}.0<473 E-10 \\
.90401 \mathrm{E}-10 \\
.12504 \mathrm{E}-17\end{array}$ & $\begin{array}{l}.70032 t-19 \\
.11442 E-10 \\
.20<22 E-15\end{array}$ & $\begin{array}{l}.12409 t-19 \\
.21589 t-19 \\
.42314 t-19\end{array}$ & $\begin{array}{l}.23465 t-20 \\
.39429 t-20 \\
.01302 E-20\end{array}$ & $\begin{array}{l}.27519 t-U 7 \\
.53642 a-U 7 \\
.00037 t-U 7\end{array}$ \\
\hline
\end{tabular}

$A=.30061000 R+0 L E=.2102504 U t+02 \quad C=.13795030 E+02 \quad A A P=-.012 b$ $.50340 \mathrm{E}-21 \quad .92092 \mathrm{E}-20^{\circ} .00049 \mathrm{E}-20 \quad .30411 \mathrm{t}-20 \quad .39072 \mathrm{E}-00$ $.27024 \mathrm{E}-10 \quad: 74733 \mathrm{E}-1 \mathrm{y} \quad .10330 \mathrm{E}-19 \quad .30106 \mathrm{E}-20 \quad .51421 \mathrm{t}-00$ $.475 \supset 2 E-22.42204 \mathrm{E}-20 \quad .71415 \mathrm{E}-20.39059 \mathrm{E}-20 \quad .55275 E-00$ $.45301 E-10 \quad .55040 E-19 \quad .10536 E-19, .19242 E-20 \quad .55090 E=00$ $.24302 \mathrm{E}-23 \quad .15403 \mathrm{E}-20 \quad .50452 \mathrm{E}-20 \quad .30310 \mathrm{E}-20 \quad .74209 \mathrm{E}-00$

$.13571 E-10 \quad .04030 E-19 \quad .27109 k-19 \quad .04955 k-20 \quad .94331 E-00$ $.70717 \mathrm{E}-\mathrm{EJ} \quad .40170 \mathrm{E}-21 \quad .31592 \mathrm{E}-20 \quad .34743 \mathrm{E}-20 \quad .96760 \mathrm{E}-0 \mathrm{~d}$ $\begin{array}{lllll}1020 U E-20 & .11323 E-21 & .17005 E-20 & .29330 \mathrm{E}-20 & .12190 \mathrm{~L}-07\end{array}$

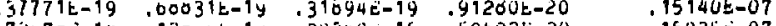
$.72070 E-10$. 130yot-10 . 30309t-1S. .59092E-20 . $150358=07$

$.14 Y 77$ E-17 .100SYL-10 .20942E-19 .510b0k-20 .3014לE-U7

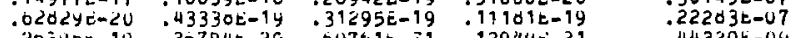
$\begin{array}{lllll}.20 j y 0=-19 & .35794 k-20 & .09701 E-21 & .12944 E-21 & .22203 E-07 \\ \end{array}$

$A=.4020040 U t+U 2 \quad D=.93052400 t+01 \quad C=.01341500 t+01 \quad K A P=-.9300$ $.31<25 t-20 \quad .1232 / t-20.3430 s t-21.70091 t-22 \quad .40477 t-10$ $.71131 t-22 \quad .30009 t-21.34032 t-2 ? .01790 t-22 \quad .44674 t-10$

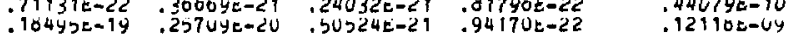

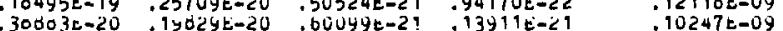

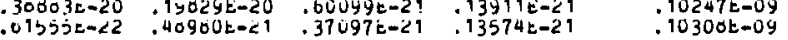
$.14735 t-10 \quad .15340 t-14 \quad .27397 t-20,40607 t-21$ $.27530 \mathrm{t}-19 \quad .43202 \mathrm{t}-20 \quad .06773 \mathrm{E}-21.10907 \mathrm{t}-21$ $.42701 \mathrm{t}-20 \quad .20433 \mathrm{E}-20 \quad .90340 \mathrm{E}-21 \quad .23377 \mathrm{E}-21$ $.40432 t-22 \quad .61000 \mathrm{t}-21.33444 t-21.21313 t-21$

$.34705 E-22 \quad .71303 \mathrm{~L}-21 \quad .74150 \mathrm{t}-21 \quad .31776 \mathrm{E}-21$ $.34955 \mathrm{t}-19$.04618t-20 $: 13905 \mathrm{E}-20 \quad .27295 \mathrm{E}-21$ $\begin{array}{llll}.34552 E-19 & .04610 E-20 & 13905 E-20 & .27295 E-21\end{array}$ $\begin{array}{llll}.37452 \mathrm{E}-20 & .4022<\mathrm{E}-20 & .19902 \mathrm{~L}-20 & .53750 k-21 \\ .3904 d \mathrm{t}-19 & .00297 \mathrm{t}-20 & .20270 \mathrm{t}-20 & .40834 \mathrm{k}-21\end{array}$ $.09004 t-10 \quad .00307 t-19 \quad .14049 t-19 \quad .26014 E-20$ $.200698-00$ $.27272 \mathrm{c}-0 \mathrm{~g}$ $.23140 \mathrm{k}-09$ $.22400 k-U 4$ . $45505 E-09$ $.23404 \mathrm{E}-0 \mathrm{Y}$ $.09726 k-09$ $.95174 \mathrm{k}-04$ $.17332=07$

$\begin{array}{lllll}.10022 E-17 & .11024 E-10 & .21510 E-14 & .30541 E-20 & .25537 k-U T \\ .740 j 2 E-10 & .91011 E-14 & .10440 E-19 & .30043 E-20 & .21201 E-U 7\end{array}$

$A=.40200400 \mathrm{E}+02 \quad E=.93052400 \mathrm{C}+U 1 \quad C=.013 \therefore 1500 \mathrm{E}+01 \quad \mathrm{AAF}=-.9300$

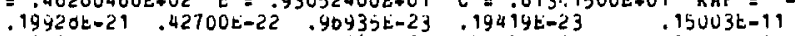

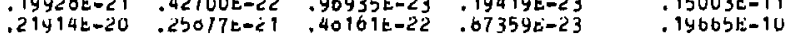

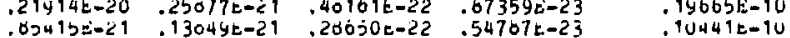
$.11403 E-20 \quad .21101 \mathrm{E}-21 \quad, 45530 \mathrm{E}-22 \quad .00944 \mathrm{E}-23 \quad .10131 \mathrm{E}-10$

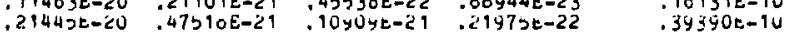

$A=.40200000 t+02 \quad B=.92939000 t+01 \quad C=.01309000 t+01 \quad \mathrm{AAF}=-.9394$ $.14509=-10 \quad .15241 \mathrm{k}-19 \quad .27<01 \mathrm{e}-20$ $.00097 \mathrm{e}-10$. $74090 \mathrm{E}-1 \mathrm{y}, 14795 \mathrm{E}-19$ $.10050 \mathrm{E}=17 \quad 11771 \mathrm{E}-10$ : $1420 \mathrm{E}-19$

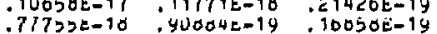

$.405200-21$

$.20768 t-20$

$1304335-20$

$.200<4 \mathrm{t}-00$

(1)

$.254352-0 ?$
$.21134 t-47$

$A=.40200000 t+C 2 \quad B=.92620000 t+01$ $.14390 \mathrm{E}-10 \quad .15227 \mathrm{k}-19 \quad .27324 \mathrm{E}-2 \mathrm{U}$ $.67 y 54 t-10 \quad .7 y 020 \mathrm{E}-19 \quad .1470 \angle \mathrm{L}-19$ $.1057 \mathrm{E}=17 \quad .11763 \mathrm{E}=10 \quad .21463 \mathrm{E}-19$

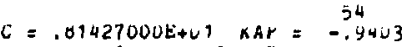
$.77207 \mathrm{t}-10$.90910E-1Y .10904E-19

$\begin{array}{ll}.40006 E-21 & .200030-00 \\ .2077 d \mathrm{dE}-20 & .17305 \mathrm{~L}-07\end{array}$ $.30547 \mathrm{E}-20 \quad .25012 \mathrm{t}-07$ $.30047 \varepsilon-20 \quad .21313 \varepsilon-0 \%$

$A=.49050700 k+02$ i $=.44712140 \mathrm{E}+01$ $\begin{array}{lll}.20332 E-17 & .27047 t-10 & .40067 t-19 \\ .47003 t=10 & .00174 t-19 & .13593 t-19\end{array}$ $.47003 \mathrm{E}-10 \quad .60174 \mathrm{E}-1 \mathrm{y}, 13593 \mathrm{E}=19$ $.40009 E-19 \quad .34004 E-19 \quad: 12401 \mathrm{E}-19$ .0143 JE-10 .90<71E-19 .20243E-19

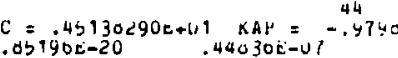

$.25510 \mathrm{t}-20$

$.25510 \mathrm{E}-20$
$.31401 \mathrm{t}-20$

$.31401 E-20$
$.442 \angle 7 E-20$

$.44227 t=20$
$.30029 t-20$

$.37040 E-19.52133 k-19$. 2zubue-19 .0u<94t-<0

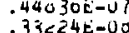

- $35<24 \varepsilon^{-1}=0$

$.22620 \mathrm{~L}-00$
$.39352 \mathrm{E}-00$

. $5900<0-40$

$.052100-40$ 
jU

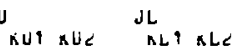

is

$\begin{array}{rlr}0 & 1 & 7 \\ 2 & 1 & \\ 19 & 2 & 1 \\ 2 & 0 & 2 \\ 2 & 1 & 1\end{array}$

119

$\begin{array}{rlr}19 & < & 10 \\ 1 & 0 & 1\end{array}$

$\begin{array}{lll}9 & 1 & 0 \\ 3 & 1 & 3 \\ 3 & 0 & 3 \\ 3 & 2 & \end{array}$

$\begin{array}{lll}9 & 1 & y \\ 2 & 1 & 2 \\ 2 & 0 & 2 \\ 2 & 2 & 1\end{array}$

$\begin{array}{lll}3 & 1 & 2 \\ 12 & 1 & 11\end{array}$

$\begin{array}{rrr}12 & 1 \\ 10 & 1 & 4\end{array}$

$\begin{array}{lll}10 & 2 & 9 \\ 10 & 4 & 7\end{array}$

1046

$\begin{array}{lll}10 & 3 & 0 \\ 10 & 3 & 7\end{array}$

1025

101

$\begin{array}{lll}11 & 1 & 11 \\ 11 & 0 & 11\end{array}$

11210

1140

$\begin{array}{rrrrrr}11 & 3 & 9 & 10 & 3 & 6 \\ 11 & 3 & 0 & 10 & 3 & 7 \\ 11 & 2 & 9 & 10 & 2 & 0 \\ 11 & 1 & 10 & 10 & 1 & 9 \\ 14 & 1 & 14 & 13 & 1 & 13\end{array}$

$\begin{array}{rrr}2 & 1 & 1 \\ 2 & 1 & 12 \\ 3 & 1 & 3 \\ 4 & 2 & 0\end{array}$

$\begin{array}{lll}y & 2 & 0 \\ 9 & 4 & 0\end{array}$

$\begin{array}{lll}4 & 4 & 3\end{array}$

$\begin{array}{lll}9 & 3 & 0 \\ 4 & 2 & 7\end{array}$

$\begin{array}{lll}9 & 1 & 0\end{array}$

10110

$\begin{array}{lll}10 & 0 & 10\end{array}$

$\begin{array}{lll}10 & 2 & 9 \\ 10 & 4 & 7\end{array}$

1040

414

1421

$\begin{array}{lll}14 & 5 & 10\end{array}$

145

14410

14312

$\begin{array}{lll}14 & 3 & 11\end{array}$

$\begin{array}{lll}14 & 2 & 12 \\ 14 & 1 & 13\end{array}$

$15 \quad 1 \quad 15$

$\begin{array}{lll}15 & 2 & 14\end{array}$

$\begin{array}{lll}15 & 4 & 12 \\ 15 & 4 & 11\end{array}$

15313

$\begin{array}{lll}15 & 3 & 12 \\ 15 & 2 & 13 \\ 15 & 1 & 14\end{array}$

$\begin{array}{lll}19 & 5 & 15\end{array}$

13013

13212

1355

$\begin{array}{lll}13 & 4 & 10\end{array}$

1349

$\begin{array}{lll}13 & 3 & 11 \\ 13 & 3 & 10\end{array}$

13211

13112

$\begin{array}{lll}14 & 1 & 14 \\ 14 & 0 & 14\end{array}$

14213

$\begin{array}{ll}14 & 4 \\ 4 & 10\end{array}$

$14 \quad 3 \quad 92$

$\begin{array}{lll}14 & 3 & 11 \\ 14 & 2 & 12\end{array}$

$\begin{array}{lll}14 & 2 & 12\end{array}$

10514

$15 \div 14 \quad 10=13$

$19410 \quad 10415$

$19415 \quad 16 \quad 414$

$\begin{array}{llllll}19 & 3 & 17 & 10 & 3 & 10 \\ 19 & 3 & 10 & 10 & 3 & 15\end{array}$

$19217 \quad 10<10$

19110

$10 \quad 17$

$\stackrel{\mathrm{B}}{\mathrm{CCHCi}}$

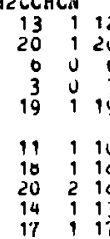

$\begin{array}{rrr} & & \\ 12 & 2 & 11 \\ 14 & 2 & 17 \\ 5 & 1 & 5 \\ 2 & 1 & 2 \\ 16 & 2 & 10 \\ 10 & 2 & 9 \\ 17 & 2 & 15 \\ 19 & 3 & 17 \\ 13 & 2 & 12 \\ 16 & 2 & 14\end{array}$
$1043 U \cup 10 E+U 2$

19 10

$1, j 9531900+02$

$.10900540 \mathrm{E}+0 \mathrm{OC}$

$.19427390 E+U 2$

$.20,55320 E+U 2$

$20440990 \mathrm{E}+0 \mathrm{C}$

$20457140 E+E 2$

.204 1000ue+02

$.291 .5970 \mathrm{k}+02$

$.35503920 \mathrm{k}+02$

$37019000 t+U 2$

$.54913160 t+02$

$.94913160 t+02$

$.94920710 \mathrm{E}+\mathrm{U2}$

$94941500 \mathrm{e}+02$

$95325490 \mathrm{U}+02$

$.10103720 \mathrm{E}+03$

$10357500 \mathrm{c}+03$

$10421200 E+0$

$.10441150 \mathrm{e}+0$

$.1044115 \mathrm{JE}+03$

$.10443200 \mathrm{c}+03$

$10445360 t+03$

$10496070 \mathrm{E}+0$

$.129<1920 \mathrm{E}+03$

$.131<0740 \mathrm{c}+03$

- $13252400 E+03$

. $132900000+03$

$.13290000 t+03$

$132916900+03$

$13293440 \mathrm{e}+03$

- $13295440 E+03$

$13303070 E+03$
$13402100 \mathrm{e}+U 3$

$.13553990 t+03$

$.13039520 x+03$

$.1419454 U E+03$

$.14242440 E+03$

$.14242050 \mathrm{t}+03$

$14247240 E+03$

$142572 y 0 \mathrm{e}+0$

$14375530 c+0$

$.14514150 t+03$
$.10041180 t+03$

$.10041100 t+U 3$

.10047 byoesu 3

100407 yuetu 3

$10053430 \mathrm{etu}$

$10005070 E+0$

$10204 y 40 \mathrm{c}+03$

$.10334300 c+03$ iv

2J

I Iurn

ivi

cus

$.7+310 t-10$.13CY4E-10

$129<3 c-1 j$

.33e27a-19 UJovoc-1

. $2 U 1 \angle U E-10$.2123:E-17

$.141045=10$

, 15y y4 $z=1$ ?

$204<3=-14$

$200+45-10$
$27400=-19$

$27 y 00 E=14$
$30,5 Y E-10$

. 2y321 $1 \mathrm{E}=1 \mathrm{c}$

$.04<<3 E-10$. 17ucot -10

$4034 y=-10$

Ecobye-10

ecoovt-10

$.50031 \mathrm{~A}-17$

3<Uvec-17

30 Jyok -14

$10>14 E-1 ?$

$.120735-17$

$.040 y i e-1$

$52045 x-10$

. 2 Y3 $320-10$

. $31032 \mathrm{t}-17$

D4by $7=-15$

$1405 ?$ E -15

$.11440 \mathrm{E}-15$

$.02<U \cup E-17$

$.11504 \mathrm{E}-1$ ?

13uvur -10

$.133<0 t-15$

$.04549 E-10$

$.11440 \mathrm{E}-15$

$.20405 E=15$

.0Y்4Yt-10

74700E-19

$.330200-10$

$.2<354 k-10$

2047 UL-72 10200t-15

. Oy443c-15

$13400 \mathrm{c}-13$
$1020 \mathrm{~s}=-12$

$223540-10$

$.2010 y+10$

- $20190 \mathrm{E}-10$

$3340 c-10$

OY93YE- 15

$10217 \mathrm{E}-1 \mathrm{~s}$

$.7901 \mathrm{SE}=15$

$.49930 \mathrm{c}-15$

$1<25$ UE- 15

19057t-15

$.10159 t-15$

$.00519 e-10$

$.270 y 0=-15$

$.125 \cup 1=-15$

$44140 E-10$

$40914 \mathrm{k}-10$

. 4टUY7e-10

$29230=-10$
$29230=-10$

270 YOE- 15

$30301 \mathrm{e}-1$

$.00795=-15$

1 रेvo

. $3030 \mathrm{CO}=10$

1940a $150054 \mathrm{c}-10$

$.200020=15$

.47 yu $1 \dot{c}-10$
$.70030 t-10$

$07029 c-15$

394 tot- 10

-3y410t-10

$.2625 y=-15$

B) $1335-10$

$.0 \cup 41 v=-10$

$.73 \supset 10 \mathrm{t}-10$

$.4175 y=-10$

$.4175 y=-10$

$1323 \mathrm{~b}=15$

1uyyje-15

$.109925-15$

$.534<0=10$

$.53424 c-10$

$24375 E-15$

$.243 y \cup=-15$

$.43230 c-15$
$.24620 c-15$

$.10027=-15$

$10043 \mathrm{E}-1$ ?
$240<0 \mathrm{E}-1 \mathrm{~b}$

in $1000=-10$

$74904=-10$

$0<3 y 7=-10$

- $530<0 t-15$

$37004 c-13$

$47107 \mathrm{E}-10$

$2004<\mathrm{E}-15$
$3 \mathrm{U}<70 \mathrm{c}-15$

. $20900 \mathrm{0}-10$

.

$.04027 t-10$

. $0: 004 t-10$

$.14303 \varepsilon-15$

$.21432 t-1 b$

.2010YE-15

$21+47 c-15$

. $37044 c-15$

$201325-15$

$.7470 \supset 5=16$

$.74749=-10$

$.004 c 1 c=10$

.1 $150 \mathrm{~s}-16$

.

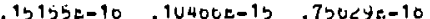

$41224 E-10$. 12073t-15 y4413c-10

$41222 \mathrm{E}-10$

पyण0y=-10

- $12073 \mathrm{E}-15$

$.15075 E-15$

oy $151 \mathrm{e}-10$

y44
$.11293 c-10$

11213ट-1?

$.130<00-1$

$21900=-15$

$.2004 z=15$

.310uje-12

. 1टy<4z-12

.1302 Uet 13

$A=.4 Y 050700 E+U L E=.44712140 E+U 1$

$.7104 y a-1 y \quad .270<y=-1 y \quad .70,1<z-20$

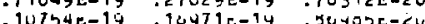

- 10734a-19 10971e-19 Toyose-<u

$.70430 t-10 \quad 0001<k-19$

$-39 y 53 E-14$
$.157036-1 y$

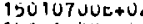

$13902540 E+U 2$
$10037470 t+U 2$

$.16<7$ 50UUt +02

17 y4 $\$ 310 E+U C$

$19202040=+42$

LUTU0O50E+U2
$40,30=-10$

$1102 y=19$

$.4 U \leq 44 \leq-19 \quad .4<4<2=-19$

CASDYE-19 bYULUE-1y

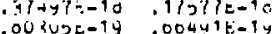

$.27100 \mathrm{t}-14$

$.9037 \cup z=14$

. $2 y y<<x-1 y$

$.210130-15$
.$<30 y+20-17$

$\rightarrow 203=-i j$

$4 / y 4 y t-1 y$

7yalie-2u

2Clooe-15

12050E-co

1 yutye-10 


\begin{tabular}{|c|c|c|c|c|c|c|c|c|c|c|c|c|c|}
\hline \multicolumn{2}{|c|}{ No } & \multicolumn{4}{|c|}{$J_{\text {NLI KLS }}$} & wo & \multicolumn{5}{|c|}{ sluith } & \multicolumn{2}{|c|}{$\dot{A} \quad$ NEF } \\
\hline $\begin{array}{r}1 \\
2 \\
2 \\
13 \\
11\end{array}$ & $\begin{array}{l}u \\
u \\
u \\
1 \\
u\end{array}$ & $\begin{array}{l}1 \\
5 \\
13 \\
11\end{array}$ & $\begin{array}{r}0 \\
1 \\
7 \\
12 \\
10\end{array}$ & $\begin{array}{l}\vdots \\
1 \\
1 \\
1\end{array}$ & $\begin{array}{r}0 \\
1 \\
7 \\
10 \\
10\end{array}$ & 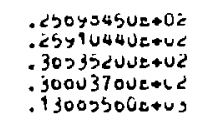 & & 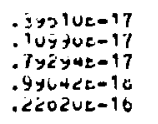 & 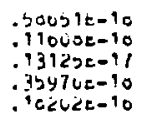 & $\begin{array}{l}.19703 E-10 \\
.20017 t-19 \\
.27340 E-10 \\
.97400 \mathrm{E}-19 \\
.04244 \mathrm{E}-17\end{array}$ & $\begin{array}{l}.22<10 c-19 \\
.37000 E-20 \\
.5240<c-14 \\
.21511 E-19 \\
.10047 L=17\end{array}$ & 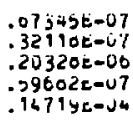 & \\
\hline $\begin{array}{c}1 \\
1< \\
\operatorname{cath}\end{array}$ & $\leqslant 1$ & $i<$ & $1 !$ & 1 & ${ }_{11}^{7}$ & $\begin{array}{l}1417417 \text { ved } 3 \\
.14275 \div 400+03\end{array}$ & & $\begin{array}{l}.09371 t-11 \\
.0240420-10\end{array}$ & $\begin{array}{l}\text { lovple-10 } \\
.20190 E-10\end{array}$ & $\begin{array}{l}.5219<E-17 \\
.04403 c-17\end{array}$ & $\begin{array}{l}.07<606-10 \\
.132--17\end{array}$ & $\begin{array}{l}12099 \varepsilon-u 4 \\
.15005 t-u 4\end{array}$ & \\
\hline $\begin{array}{l}1+\tan 2 \\
\cos 4 \sin \\
3\end{array}$ & $\operatorname{cis}_{1}^{1}$ & 3 & $<$ & estis & $2=$ & $\begin{array}{l}\text { bs Nu }=4.5200 \\
\text { 1070yotuetoe }\end{array}$ & A & $\begin{array}{l}=\text { luosudues } \\
.25 j \mathrm{j}=17\end{array}$ & $\begin{array}{l}E+4 z \quad E=.2 \\
.2 S 1 \text { yst-9c }\end{array}$ & 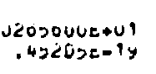 & 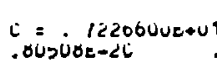 & $\begin{array}{l}n 45= \\
.11379=-40\end{array}$ & $\begin{aligned} 30 \\
-.4312\end{aligned}$ \\
\hline $\begin{array}{l}2 \\
3 \\
3 \\
3 \\
4 \\
4\end{array}$ & $\begin{array}{l}u \\
\dot{c} \\
\vdots \\
1 \\
j\end{array}$ & $\begin{array}{l}3 \\
\vdots \\
1 \\
2 \\
4\end{array}$ & $\begin{array}{l}5 \\
2 \\
5 \\
5\end{array}$ & $\begin{array}{l}0 \\
5 \\
1 \\
1\end{array}$ & $\begin{array}{l}\vdots \\
\vdots \\
\vdots \\
\vdots\end{array}$ & $\begin{array}{l}11221700 E+U E \\
11253000 E+U E \\
11205000 x+U 2 \\
11747400 E+W 2 \\
114290230 t+v 2\end{array}$ & & 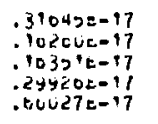 & 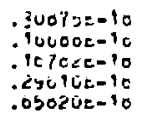 & $\begin{array}{l}.5307 E-1 y \\
.35537 E-1 y \\
.30504 E-1 y \\
.53174 E-1 y \\
.11030 E-10\end{array}$ & $\begin{array}{l}.90100 E-20 \\
.54503 t=20 \\
.340 \$ 2 E-20 \\
.94741 E-70 \\
.21139 E-19\end{array}$ & $\begin{array}{l}.14343 E-60 \\
.00094 E-07 \\
.01303 E-01 \\
.14530=-00 \\
.30040 E-00\end{array}$ & \\
\hline $\begin{array}{l}4 \\
4 \\
4 \\
4 \\
5\end{array}$ & $\begin{array}{l}1 \\
2 \\
2 \\
1 \\
1 \\
1\end{array}$ & $\begin{array}{l}4 \\
3 \\
5 \\
3\end{array}$ & $\begin{array}{l}j \\
3 \\
3 \\
3 \\
3\end{array}$ & $\begin{array}{l}1 \\
2 \\
2 \\
1 \\
1\end{array}$ & $\begin{array}{l}2 \\
2 \\
1 \\
2 \\
4\end{array}$ & 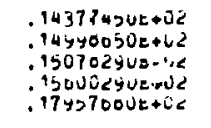 & & $\begin{array}{l}.0157+3 E-17 \\
.50140 E-17 \\
.300050-17 \\
.71903 E-17 \\
.11710=-10\end{array}$ & 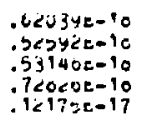 & $\begin{array}{l}.112 \text { OUt-10 } \\
.40290 E-19 \\
.47910 t-19 \\
.13170 t-18 \\
.22107=-10\end{array}$ & $\begin{array}{l}.24031 E-1 y \\
.17325 E-14 \\
.17504 E-14 \\
.23565 E=19 \\
.3 y 022 E-19\end{array}$ & $\begin{array}{l}.24442 E-U 0 \\
.20743 E-00 \\
.27172 E-v 0 \\
.37011 E-v 0 \\
.00074 E-i 0\end{array}$ & \\
\hline $\begin{array}{l}3 \\
b \\
3 \\
3 \\
0\end{array}$ & $\begin{array}{l}4 \\
2 \\
5 \\
1\end{array}$ & $\begin{array}{l}3 \\
\vdots \\
\vdots \\
0\end{array}$ & $\begin{array}{l}4 \\
4 \\
4 \\
4 \\
2\end{array}$ & $\begin{array}{l}0 \\
\vdots \\
1 \\
1\end{array}$ & $\begin{array}{l}4 \\
\vdots \\
\vdots \\
j\end{array}$ & 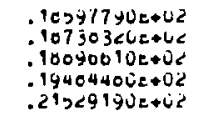 & & $\begin{array}{l}.13321=-10 \\
.104705-10 \\
.900408-10 \\
.157112-10 \\
.19351 E-10\end{array}$ & 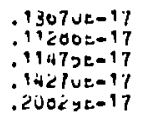 & $\begin{array}{l}.24031 t-1 c \\
.29052 E-10 \\
.2 ! 204 E-1 c \\
.20001 E-10 \\
.30351 E-10\end{array}$ & $\begin{array}{l}.44490 E-19 \\
.37005 c-19 \\
.30322 c=19 \\
.40027 \varepsilon=1 y \\
.0 y 191 E-1 y\end{array}$ & $\begin{array}{l}.09400 \mathrm{e}-00 \\
.59731 \mathrm{E}-00 \\
.08559 \mathrm{E}-00 \\
.70733 \mathrm{E}-00 \\
.10044 \mathrm{E}-60\end{array}$ & \\
\hline $\begin{array}{l}0 \\
\vdots \\
0 \\
0 \\
0\end{array}$ & $\begin{array}{l}2 \\
\vdots \\
\vdots \\
\vdots \\
\vdots\end{array}$ & $\begin{array}{l}5 \\
5 \\
5 \\
4\end{array}$ & $\begin{array}{l}3 \\
3 \\
3 \\
5 \\
3\end{array}$ & $\begin{array}{l}4 \\
2 \\
3 \\
3 \\
2\end{array}$ & $\begin{array}{l}5 \\
4 \\
3 \\
5 \\
5\end{array}$ & 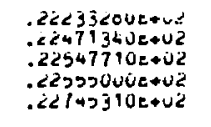 & & $\begin{array}{l}.29040=-10 \\
.90124 E-10 \\
19035 E-10 \\
.13004 c=10 \\
.105430-10\end{array}$ & 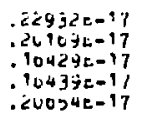 & $\begin{array}{r}.42102 E+10 \\
.37000 E-10 \\
.31330 E-10 \\
.313500-10 \\
.30504 E-10\end{array}$ & $\begin{array}{l}.75049 E-19 \\
.0044 j E-1 y \\
.57545 E-19 \\
.57502 E-1 y \\
.70117 E-1 y\end{array}$ & $\begin{array}{l}1<032 k-05 \\
11000 E-v 2 \\
.94362 k-00 \\
94454 \varepsilon-v 0 \\
.11470 \varepsilon=05\end{array}$ & \\
\hline $\begin{array}{l}0 \\
7 \\
7 \\
7 \\
1\end{array}$ & $\begin{array}{l}1 \\
1 \\
0 \\
2 \\
3\end{array}$ & $\begin{array}{l}3 \\
7 \\
7 \\
0 \\
3\end{array}$ & $\begin{array}{l}b \\
0 \\
0 \\
0 \\
0\end{array}$ & $\begin{array}{l}1 \\
i \\
c \\
\vdots \\
j\end{array}$ & $\begin{array}{l}4 \\
0 \\
0 \\
3 \\
4\end{array}$ & $\begin{array}{l}.2332754 U E+U E \\
.23090010 E+U L \\
.250 \angle 701 U E+0 C \\
.2019040 U E+U 2 \\
.20 \$ 170 U U E+U C\end{array}$ & & $\begin{array}{l}.220 j 0 e-10 \\
.<43570-10 \\
.32070=-10 \\
.<1010=-10 \\
. \angle 25 U U E-10\end{array}$ & $\begin{array}{l}.24304 E-17 \\
.3<434 E-17 \\
.3519<E-17 \\
.3<213 E-17 \\
.<77 \text { 1 } 0 E-17\end{array}$ & $\begin{array}{l}.449 y 0 e-10 \\
.004 y 0 e-10 \\
.65417 c-10 \\
.00920 t-10 \\
.53529 c-10\end{array}$ & $\begin{array}{l}.01307 e-1 y \\
.10979 t-10 \\
11050 e-10 \\
11140 E-10 \\
.90910 t-19\end{array}$ & $\begin{array}{l}.13590 E-05 \\
17102 E-05 \\
19050 E-U 5 \\
190315 e-U 5 \\
.10512 E-J 5\end{array}$ & \\
\hline $\begin{array}{l}7 \\
? \\
?\end{array}$ & 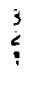 & $\dot{j}$ & $\vdots$ & $\begin{array}{l}3 \\
3 \\
1 \\
1\end{array}$ & $\begin{array}{l}5 \\
5 \\
3\end{array}$ & $\begin{array}{l}.2039370 e+U 2 \\
.26027110 k+02 \\
.27<15000=+02\end{array}$ & & $\begin{array}{l}.13303 t-10 \\
.20730=-10 \\
.330330-10\end{array}$ & $\begin{array}{l}.1030+E-17 \\
.33<21 \varepsilon-17 \\
.370 \mid<2-11\end{array}$ & $\begin{array}{l}.31050 E-10 \\
.024202-10 \\
.700000-10\end{array}$ & $\begin{array}{l}.30407 E-19 \\
.11509 E-10 \\
.12009 E-10\end{array}$ & $\begin{array}{l}.97090 e-00 \\
.192372-05 \\
.21090 E=09\end{array}$ & \\
\hline $\begin{array}{c}0.3 n t 5 \\
3 \\
\vdots \\
4 \\
4\end{array}$ & $\begin{array}{l}\ln i x \\
1 \\
j \\
1 \\
1 \\
0\end{array}$ & $\begin{array}{ll}1 \\
5 \\
3 \\
2 \\
4 \\
4 \\
4\end{array}$ & $\begin{array}{l}4 \\
2 \\
2 \\
3 \\
3\end{array}$ & $\begin{array}{l}1 \\
3 \\
1 \\
1 \\
0\end{array}$ & $\begin{array}{c}n_{2}= \\
\vdots \\
3 \\
3 \\
4\end{array}$ & 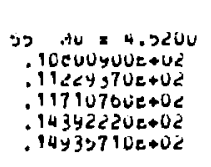 & A & 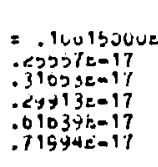 & $\begin{array}{l}=+020=10 \\
.25222 \varepsilon-10 \\
.5000 / E-10 \\
.29594 t-10 \\
.02107 t-10 \\
.71700 t-10\end{array}$ & 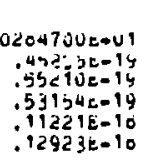 & $\begin{array}{l}c=.17251100 E+ \\
.00590 t-20 \\
.90140 E-20 \\
.94705 E-20 \\
.20053 E-19 \\
.23054 E-19\end{array}$ & $\begin{array}{l}1 \text { AAR }= \\
11414 E-60 \\
1442 C E=-60 \\
14540 E-00 \\
.29538 E=06 \\
.35174 k-60\end{array}$ & $\underbrace{30}-.9 \div 10$ \\
\hline $\begin{array}{l}4 \\
\vdots \\
5 \\
0\end{array}$ & $\begin{array}{l}1 \\
1 \\
0 \\
1 \\
1\end{array}$ & $\begin{array}{l}3 \\
5 \\
3 \\
4 \\
0\end{array}$ & $\begin{array}{l}3 \\
4 \\
+ \\
5\end{array}$ & $\begin{array}{l}1 \\
1 \\
\vdots \\
1 \\
1\end{array}$ & $\begin{array}{l}2 \\
4 \\
4 \\
3 \\
3\end{array}$ & 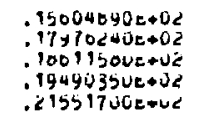 & & $\begin{array}{l}.71437 c-17 \\
.11707 c-10 \\
.13355 E-10 \\
.13700=-10 \\
.19>4 y=-10\end{array}$ & $\begin{array}{l}.72001 E-10 \\
.12100 E-17 \\
.130718-17 \\
.14205 e-17 \\
.2005<0-17\end{array}$ & $\begin{array}{l}.1317<E-10 \\
.22211=-10 \\
.24044 E-10 \\
.201152 E-10 \\
.30394 E=10\end{array}$ & $\begin{array}{l}.23557 E-19 \\
.39060 E-19 \\
.44520 t-19 \\
.40011 E-19 \\
0420 y E-19\end{array}$ & $\begin{array}{l}.37042 E-v 0 \\
.002016-40 \\
.09502 \mathrm{E}-00 \\
.700032-00 \\
.10077 \pm-60\end{array}$ & \\
\hline $\begin{array}{l}0 \\
6 \\
7 \\
1 \\
7\end{array}$ & $\begin{array}{l}\vdots \\
\vdots \\
\vdots \\
\vdots \\
1\end{array}$ & $\begin{array}{l}0 \\
3 \\
7 \\
7 \\
0\end{array}$ & $\begin{array}{l}3 \\
? \\
0 \\
0 \\
0\end{array}$ & $\begin{array}{l}3 \\
1 \\
1 \\
\vdots \\
i\end{array}$ & $\begin{array}{l}5 \\
4 \\
0 \\
0 \\
b\end{array}$ & 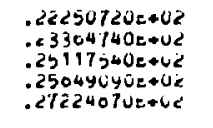 & & $\begin{array}{l}.210 y 02-10 \\
.2<0<4=-10 \\
.24303 t=-10 \\
.3<004 c=10 \\
.30-19=-10\end{array}$ & $\begin{array}{l}.22945 k-17 \\
.<4350 E-17 \\
.324 y>2=-17 \\
.35293 E-17 \\
.37001 E-17\end{array}$ & $\begin{array}{l}.421205-10 \\
.44 \% 84 k-10 \\
.00259 E-10 \\
.0940<E=10 \\
.700402-10\end{array}$ & 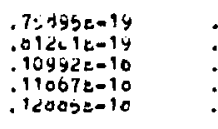 & 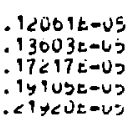 & \\
\hline 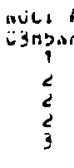 & $\begin{array}{l}\text { hutr } \\
\text { ric } \\
u \\
1 \\
u \\
1 \\
j\end{array}$ & $\begin{array}{l}1 \\
\vdots \\
i \\
i\end{array}$ & $\begin{array}{l}u \\
\vdots \\
\vdots \\
\vdots\end{array}$ & & $\begin{array}{l}0 \\
u \\
1 \\
1 \\
\vdots \\
2\end{array}$ & 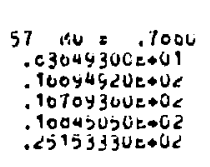 & A & 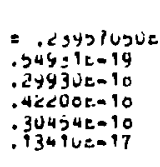 & $\begin{array}{l}.42 \quad 0=.4 \\
.502545-<0 \\
.32343 E-1 y \\
.4203 E-1 y \\
.3<07 y E-19 \\
.14000 E=10\end{array}$ & $\begin{array}{l}\angle 49000 k+U 1 \\
.95 y 13 t-\angle 1 \\
.30303 E-2 u \\
.7 y 203 E-2 u \\
.5441 E-2 u \\
.20436 z-19\end{array}$ & 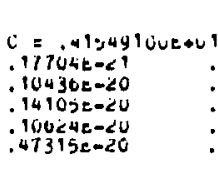 & 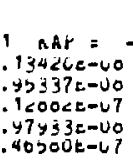 & $\begin{array}{c}16 \\
-.4 \times<4 \\
-4\end{array}$ \\
\hline $\begin{array}{l}3 \\
3 \\
3 \\
4 \\
4 \\
4\end{array}$ & $\begin{array}{l}2 \\
2 \\
1 \\
1 \\
0\end{array}$ & $\begin{array}{l}1 \\
2 \\
2 \\
4 \\
-5\end{array}$ & $\begin{array}{l}2 \\
4 \\
5 \\
5\end{array}$ & $\begin{array}{l}2 \\
2 \\
1 \\
1 \\
2\end{array}$ & $\begin{array}{c}u \\
1 \\
\vdots \\
\vdots \\
\vdots\end{array}$ & $\begin{array}{l}.25130 z 10 E+U 2 \\
.25150<14 E+U E \\
.2507140 E \rightarrow 62 \\
.33300470 E+U E \\
1335104 \angle U E+U 2\end{array}$ & & $\begin{array}{l}.01033 e-10 \\
.01033 E-10 \\
.11404 E-17 \\
.200110=17 \\
.24324 E-17\end{array}$ & $\begin{array}{l}.75137 t=1 y \\
.75137 z-19 \\
.12030 E-10 \\
.34530 t+10 \\
.13400 \varepsilon=10\end{array}$ & $\begin{array}{l}.14143 t-19 \\
.141432-14 \\
.234058-1 y \\
.207032-1 y \\
.01056 z-14\end{array}$ & $\begin{array}{l}.257 \text { yod-20 } \\
.<57906-20 \\
.422360-2 J \\
.10250 k=19 \\
.111230 .19\end{array}$ & $\begin{array}{l}.25007 E-07 \\
.25607 E-01 \\
41 y 6 y=-U 7 \\
.145 y 2 E-60 \\
1144 y E-v 0\end{array}$ & \\
\hline 4 & 1 & 3 & 3 & 1 & \& & . 33000 y 2 UE+UL & & $.204335-17$ & $.31007 t-10$ & $.21700 t-1 g$ & $.10474 E=1 y$ & $.10000 \mathrm{E}-U 0$ & \\
\hline
\end{tabular}



Ju
iv
26 jo sivat io
suv

MILI GLLYLAMINE ï3नjunट

30

40

$4: 3$

$2 !$

$\begin{array}{l:l:}3 & 1 \\ 4 & 3\end{array}$

$\begin{array}{lll}3 & 1 & 4 \\ 0 & 1 & 5\end{array}$

$\begin{array}{lll}7 & 1 \\ 0 & 1 & 9\end{array}$

910

$11: 10$

$\begin{array}{rrrrrr}5 & 0 & 5 & 4 & 1 & 4 \\ 12 & 1 & 11 & 12 & 0 & 12\end{array}$

$13 \quad 112 \quad 130013$

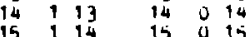

$10115 \quad 10 \quad 010$

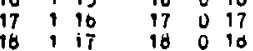

$\begin{array}{llllll}18 & 1 & 18 & 18 & 0 & 10 \\ 19 & 1 & 10 & 19 & 0 & 14\end{array}$

$i 1,00$ i

$\begin{array}{rrrrrr}20 & 1 & 1 y & 20 & 0 & 20 \\ 6 & 0 & 0 & 9 & 1 & ? \\ 2 & 1 & 2 & 1 & 0 & 1\end{array}$

GLT ALLYLAMINE $C$

C 3 H 5 hH2

\begin{tabular}{llllll}
$15 \mathrm{NH} 2$ & & \multicolumn{2}{c}{$M$} \\
20 & 1 & 20 & 20 & 0 & 20 \\
19 & 1 & 19 & 19 & 0 & 14 \\
18 & 1 & 10 & 10 & 0 & 10 \\
17 & 1 & 17 & 17 & 0 & 17 \\
16 & 1 & 10 & 16 & 0 & 10 \\
15 & 1 & 15 & 15 & 0 & 15 \\
14 & 1 & 14 & 14 & 0 & 14 \\
13 & 1 & 13 & 13 & 0 & 13 \\
12 & 1 & 12 & 12 & 0 & 12 \\
11 & 1 & 11 & 11 & 0 & 11
\end{tabular}

$\begin{array}{rrrrrr}1 . j & 1 & 10 & 10 & 0 & 10 \\ 9 & 1 & 9 & 9 & 0 & 9 \\ 8 & 1 & d & d & 0 & 0 \\ 7 & 1 & 7 & 1 & 0 & 7 \\ 0 & 1 & 0 & 0 & 0 & 6\end{array}$

$\begin{array}{llllll}5 & 1 & 5 & 5 & 0 & 5 \\ 4 & 1 & 4 & 4 & 0 & 4 \\ 3 & 1 & 3 & 3 & 0 & 3 \\ 2 & 1 & 2 & 2 & 0 & 2 \\ 1 & 1 & 1 & 1 & 0 & 1\end{array}$

$\begin{array}{llllll}1 & 1 & 0 & 0 & 0 & 0 \\ 2 & 1 & 1 & 1 & 0 & 1\end{array}$

ALLT
HSLCCint2

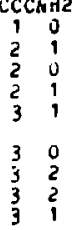

$\begin{array}{lll}1 & 0 & 1 \\ 2 & 1 & 2 \\ 2 & 0 & 2 \\ 2 & 1 & 1 \\ 3 & 1 & 3 \\ 3 & 0 & 3 \\ 3 & 2 & 2 \\ 3 & 2 & 1 \\ 3 & 1 & 2\end{array}$

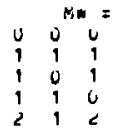

$27 \mathrm{Hu}=.0920$

$10089490 \mathrm{E}+\mathrm{U} 2$

. 10yúzane+0z

2007 UOOUE +UE

¿145 IOYUE +UC

$.25290000 \mathrm{t}+U \mathrm{U}$

$.24037700 k+02$ $.302700 \angle 0 E+U 2$

-30702120E+u2

. 321USTOUt+U2

NGLG

C 3HONH2

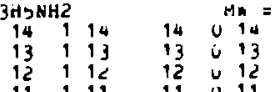

$27 \mathrm{nU}=.030 \mathrm{u}$

$.15329460 c+62$

$.129 y 2420 \mathrm{E}+42$

$.100<y 13 u e+02$

$.1723504 u t+02$
$A=.<j y>7 u>0 r+02 E=.422440 i v E+j 1$

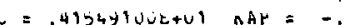

16

$10023=-1 y$. 19000e-cu

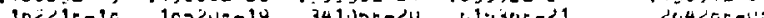

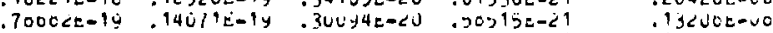

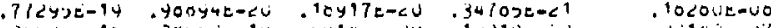

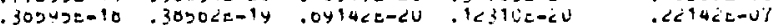

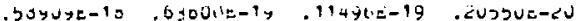

. $263 j 4 c-u 7$

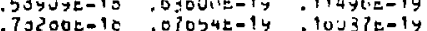

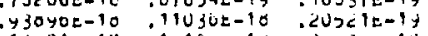

Y30yoe-10. $11030 \mathrm{l}-10$

112010

$191300-16$

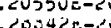

LCक $12+0$

< $1110=-17$

$230030-6$ ?

$\therefore+241_{E-U 1}$

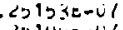

$2010 y c-0$.

$.<050,50-j \%$

. $\dot{y} y 71 \mathrm{E}-\mathrm{U}$ i

4L7<e-17. 1y3b7e-10.41407e-1y .0Jay4e-<

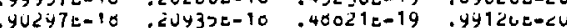

$\angle y+71=-4$

$.5 U Y 40 E-10$.0170לE-1Y

$.7 \times \supset<\mathrm{JE}=10,0132 \supset \mathrm{L}-10$

$11010 \mathrm{n}-1 \mathrm{y}$

.211 OUe-<0

$12 U 0 O E=U 7$

$.31042 \varepsilon-v i$

- $3503 y=-J 1$

- 35003E-UT

$.30107 E-v ?$

$2730>E-10 \quad .21345 E-10$

25304t-15

$.110<4 e-1 y$

$1<004 x-1 y$

$400400-07$

$37750 \mathrm{E}=10 \quad .20403 \mathrm{k}=10$

$.633 j 1 \mathrm{k}-19$

$.14013 \mathrm{c}-19$

$430940-67$

$47340=01$

$47340 \mathrm{U}-07$
$51190 \mathrm{E}-\mathrm{U} 7$

$.42<3 \supset E-67$

$170 d 2 \mathrm{E}-10 \quad$.17020e-10 .00000e-19:170y0e-19

$.10540 t-20$

-5つb10=-U?

3303 วE-U?

$\begin{array}{ll}12501 \mathrm{k}-10 & .1070 \mathrm{LE}-10 \\ 10021 \mathrm{e}-17 & 14110 \mathrm{e}-10\end{array}$

$.09700 \mathrm{E}-19 \quad .10930 \mathrm{E}-1 \mathrm{y}$

$.50105 \mathrm{~L}-<0$

$.02007 t-07$

$A=.23957050 E+02 \quad t=.42299000 t+01 \quad c=.41349100 t+L 1 \quad A A P=-.4924$ $07070 \mathrm{t}-2 \mathrm{U}$.0717วt-<0 .3011วt-2U $.10193 \mathrm{t}-19$ : $1049 \mathrm{de}-19$ :3yo35t-2u $.15079 e-1 y \quad .12265 e-19$ $.217110-1 y \quad 14<23 E-19$ . 3U415E-1y $16243 x-19$ $43401 t-20$ $400 y 3 E-<0$

$.91733 \mathrm{E}-\mathrm{c} 1$

$10327 t-20$

$11321 \mathrm{t}-<\mathrm{U}$

$1200 \mathrm{UL}-\mathrm{U} O$
$1434<\mathrm{E}-60$

1 joyot-vo

174 y 1 e-vo

$14133 c-40$

$.414455-19 \quad .10201=-1 y$

$.34915 e-19.20203 t-19.34 y 53 e-20$

$.7 \cup 714 E-19$.21YSoE-1y

$.00443 t=19 \quad .23: 14=-14$

$.107325=16$.24711E-14

SOS $34 \mathrm{E}=20$

$.57495 \mathrm{e}-<\mathrm{v}$

$.574 \dot{y 0}=2 \mathrm{~J}$

119)yt-zu

EUOUUE-UO

$.22477 \mathrm{c}=50$

$.<414>E-v 0$

. $27 \%$ Y

1 1टUंग๐-ट0

$11927 \varepsilon-20$

$.1105 y=-20$

$.12051 E-1=.204740-1) .50710=-\angle 0$

$14307 t-1 \pi$.2072yt-19 b3J27t-20

$.112 x 00-20$

730 ye-vo

$.15033 e-15 \quad .22411 t-19 \quad .52421 E-\angle 0$

$.16109 \mathrm{E}-10 \quad .24474 k-19.40045 \mathrm{t}-20$

$2 \pi-50$

. 20 yடye- 00

$.30391 t=00$

$10011 z-20 \quad . j 17 j y z-j 0$

$.91000 \mathrm{e}-21 \quad .33010 \mathrm{e}-00$

$.34163 \mathrm{E}-00$

$.16 j 90 \mathrm{e}=1 \mathrm{a} .20604 \mathrm{e}-19$. $39220 t-20$

1 1לट1t-10 17071k-19 $33210 \mathrm{t}-20$

$.12974 x-10 \quad 14517 t-14$.2055bt-20

$.94201 E-14$ 16713E-14 $19363 E-20$

$.02345 \mathrm{E}-19 \quad .05005 \mathrm{E}-20 \quad$.1177uri-20

. 3ว 151 e-vo

. 30002 e-ilo

$.30092 \mathrm{c}=40$

$.31219 \varepsilon-40$

$.37>73 \mathrm{e}-00$

$\begin{array}{rrr}.04042 t-19 & .04402 t-20 & .10030 t-20 \\ .20004 t-10 & .22304 t-19 & .40317 t-20\end{array}$

.20y00E-21

$\begin{array}{ll}.20 b 5 y c-21 & .73073 c-J 0 \\ .72000 t-21 & .14453 E-67\end{array}$

$A=.10107200 t+02 E=.30000340 t+01$

$C=.44041340 L+i 1 \wedge A E=-.10<0$

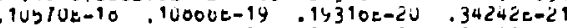

$.510 y 1 k=10$.55040E-19 .10033t-19

$.79514 t-10$. $04140 E-19$.15102E-19

$.00101=-10 \quad .71304=-19 \quad+12040=-19$

$17 y 11 \mathrm{t}-20$

$.20901 \mathrm{e}-20$

$.23 \cup 32 \mathrm{c}-2 \mathrm{c}$

$319 \angle 5 \mathrm{E}-\mathrm{NO}$

. 109u6t-u7

. $30070 \mathrm{t}-07$

. $<7011 \mathrm{E}-u$ ?

$.0041 \mathrm{UL}=u 7$

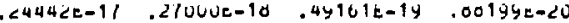

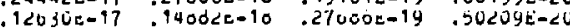

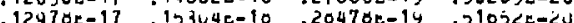

$.10547 \mathrm{E}-60$

$.01270 \mathrm{~d}=07$

$.042520-07$

$.04252 \mathrm{E}-07$
$.11744 \mathrm{e}-00$ $.24379 \dot{E}-17$.21532e-10 .50501E-14

Yư $37 \mathrm{e}-<\mathrm{U}$

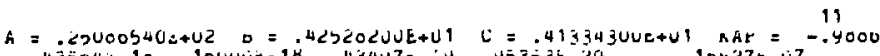

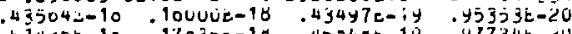

$.37430 \mathrm{E}-10 \quad 170330-10 \quad .43040 \mathrm{t}-19 \quad .97734 \mathrm{t}-\angle \mathrm{U}$

$.73340 \mathrm{y}=10 \quad, 19>01 t-10 \quad .47>41 E-1 y \quad .90040 t-\angle 0$

$10327 \mathrm{E}-\mathrm{L} ?$

10457e-u?

$.22400 E=U 7$ 
Ju SUT ULE KLL

$\begin{array}{rrrrrr}10 & 1 & 10 & 10 & 0 & 10 \\ 9 & 1 & 5 & 9 & 0 & 9 \\ 0 & 1 & 0 & 0 & 0 & 0 \\ 7 & 1 & 7 & 1 & 0 & 7 \\ 0 & 1 & 0 & 0 & 0 & 6 \\ 3 & 1 & 5 & 5 & 0 & 5 \\ 4 & 1 & 4 & 4 & 0 & 4 \\ 3 & 1 & 3 & 3 & 0 & 3 \\ 2 & 1 & 1 & 2 & 0 & 2 \\ 1 & 1 & 1 & 1 & 0 & 1 \\ 1 & 1 & 0 & 1 & 0 & 1 \\ 1 & 1 & 2 & 3 & 0 & 3 \\ 4 & 1 & 3 & 4 & 0 & 4 \\ 5 & 1 & 4 & 5 & 0 & 5 \\ 0 & 1 & 5 & 0 & 0 & 0 \\ 1 & 1 & 0 & 7 & 0 & 7 \\ 0 & 1 & 7 & 0 & 4 & 0 \\ 4 & 1 & 0 & 4 & 0 & y \\ 10 & 1 & y & 10 & 0 & 10 \\ 11 & 1 & 10 & 11 & 0 & 11 \\ 12 & 1 & 11 & 12 & 0 & 12 \\ 13 & 1 & 12 & 13 & 0 & 13 \\ 14 & 1 & 13 & 14 & 0 & 14 \\ 15 & 1 & 14 & 15 & 0 & 15 \\ 1 & 1 & 1 & 0 & 0 & 0 \\ 1 & 1 & 0 & 0 & 0 & 0 \\ 2 & 1 & 2 & 1 & 0 & 1 \\ 2 & 1 & 1 & 1 & 0 & 1\end{array}$

NGLE

\begin{tabular}{|c|c|c|c|c|}
\hline $\begin{array}{c}035 \\
14 \\
13 \\
12 \\
11 \\
9\end{array}$ & $\begin{array}{r}42 \\
1 \\
1 \\
1 \\
1 \\
1\end{array}$ & $\begin{array}{r}14 \\
13 \\
12 \\
11 \\
9\end{array}$ & $\begin{array}{r}1 \\
14 \\
13 \\
12 \\
11 \\
y\end{array}$ & $\begin{array}{ll} & M w \\
0 & 1 \\
0 & 1 \\
0 & 1 \\
0 & 1 \\
0 & \end{array}$ \\
\hline $\begin{array}{l}0 \\
7 \\
0 \\
5 \\
2\end{array}$ & $\begin{array}{l}1 \\
1 \\
1 \\
1 \\
1\end{array}$ & $\begin{array}{l}6 \\
7 \\
6 \\
5 \\
2\end{array}$ & $\begin{array}{l}0 \\
7 \\
0 \\
b \\
2\end{array}$ & $\begin{array}{l}0 \\
0 \\
u \\
0 \\
0\end{array}$ \\
\hline $\begin{array}{l}1 \\
1 \\
2 \\
3 \\
6\end{array}$ & $\begin{array}{l}1 \\
1 \\
1 \\
1 \\
1\end{array}$ & $\begin{array}{l}1 \\
0 \\
1 \\
2 \\
3\end{array}$ & $\begin{array}{l}1 \\
1 \\
2 \\
3 \\
0\end{array}$ & $\begin{array}{l}0 \\
0 \\
0 \\
0 \\
0\end{array}$ \\
\hline $\begin{array}{r}0 \\
9 \\
10 \\
11 \\
12\end{array}$ & $\begin{array}{l}1 \\
1 \\
1 \\
1 \\
1\end{array}$ & $\begin{array}{r}7 \\
8 \\
9 \\
10 \\
11\end{array}$ & $\begin{array}{r}0 \\
9 \\
10 \\
11 \\
12\end{array}$ & $\begin{array}{lr}0 & \\
0 & \\
0 & 1 \\
0 & 1 \\
0 & 1\end{array}$ \\
\hline $\begin{array}{c}13 \\
14 \\
15 \\
1 \\
1\end{array}$ & $\begin{array}{l}1 \\
1 \\
1 \\
1 \\
1\end{array}$ & $\begin{array}{r}12 \\
13 \\
14 \\
1 \\
0\end{array}$ & $\begin{array}{c}13 \\
14 \\
15 \\
0 \\
0\end{array}$ & $\begin{array}{ll}0 & 1 \\
0 & 1 \\
0 & 1 \\
0 & \\
0 & \end{array}$ \\
\hline
\end{tabular}

MULC

\begin{tabular}{|c|c|c|c|c|c|}
\hline $\begin{array}{r}03+51 \\
14 \\
13 \\
12 \\
11 \\
10\end{array}$ & $\begin{array}{r}42 \\
1 \\
1 \\
1 \\
1 \\
1\end{array}$ & $\begin{array}{l}14 \\
13 \\
12 \\
11 \\
10\end{array}$ & $\begin{array}{l}2 \\
14 \\
13 \\
12 \\
11 \\
10\end{array}$ & $\begin{array}{ll}19 w \\
0 & 14 \\
0 & 13 \\
0 & 12 \\
0 & 11 \\
0 & 10\end{array}$ & $\begin{array}{l}57 \text { MU }=0300 \\
.15440730 E+02 \\
.16090730 E+02 \\
116714050 E+02 \\
.17300160 E+02 \\
.17060200 E+02\end{array}$ \\
\hline $\begin{array}{l}9 \\
8 \\
7 \\
5 \\
5\end{array}$ & $\begin{array}{l}1 \\
1 \\
1 \\
1 \\
1\end{array}$ & $\begin{array}{l}9 \\
0 \\
7 \\
0 \\
5\end{array}$ & $\begin{array}{l}9 \\
0 \\
7 \\
0 \\
5\end{array}$ & $\begin{array}{l}0 \\
0 \\
0 \\
0 \\
0\end{array}$ & $\begin{array}{r}16391430 \mathrm{E}+02 \\
.18668040 \mathrm{E}+02 \\
19303120 \mathrm{0}+02 \\
19069400 \mathrm{t}+02 \\
.20025180 \mathrm{E}+02\end{array}$ \\
\hline $\begin{array}{l}3 \\
1\end{array}$ & 1 & 3 & 3 & $\begin{array}{l}0 \\
0\end{array}$ & $\begin{array}{l}.20536900 E+02 \\
.20025590 \mathrm{E}+02\end{array}$ \\
\hline
\end{tabular}

20

. $17007110 \mathrm{k}+02$

. $10339960 t+02$

$.10030370 \mathrm{t}+02$

$.192753000+02$

$.19670020 \mathrm{t}+02$

$.20013090 k+02$

$.20303440 \mathrm{C}=02$

$.20530090 \mathrm{E}+02$

$.20715100 \varepsilon+02$

$.20033700 k+02$

$.209527 u U t+02$

$+21254000 E+02$

.210u5j50E+u2

$.22170000 \mathrm{e}+02$

.22619090e+02

$.23131900 \mathrm{t}+02$

$.23717920 \mathrm{e}+02$

$.24301110 e+0 E$

$.25125310 \mathrm{E}+02$

$.25954450 \mathrm{k}+02$

$.26072690 \mathrm{k}+02$

$.27004510 E+02$

$.20992940 \mathrm{E}+02$

$.292190200+02$

. $29339290 k+02$

$.37406000 E+02$
$.37645200 E+02$

$57 \mathrm{~kJ}=.6300$ $.15407020 \mathrm{t}+02$ $160603100+02$
$10007200 \mathrm{c}+02$ $.17204250 a+0$ ? $10300000 \mathrm{E}+02$

. $18052090 \mathrm{~s}+02$ $.19209350 \mathrm{E}+02$ $.19077040 \mathrm{E}+02$ $.20015530 k+02$ $.20704140 \mathrm{E}+02$

$.20020600 \mathrm{E}+02$ $.20937910 \mathrm{k}+02$ $.210535702+02$ $.21233790 \mathrm{E}+02$ $.22140260 \mathrm{c}+02$

$.2307206 C t+02$ $.23649420 k+02$ $.24299240 \mathrm{E}+02$ $.25020240 E+02$ $.25840100 E+02$

$.26739000 \mathrm{E}+02$ $.27729290 \varepsilon+02$ $26615060 \mathrm{E}+02$ $.29346120 c+02$

$.15393 \mathrm{t}-17$

50401E-10

$.12035 \mathrm{E}-17$

$85030 \mathrm{E}=1$

$75094 k-18$
$.10074 \mathrm{t}-17$

$.14034=17$

.15 vio $1 E-17$

$.15405 E-17$

$.15107 t-17$

$.14010 \mathrm{c}-17$

91 Tuve-is

$.50130 \mathrm{E}-10$

$.50707 t-16$

$.12063 c=17$

-15270t-17

$.10403 e-17$

$.19049 \mathrm{t}-17$

$.10909 E-17$

$.103200-17$

$.17205 \mathrm{t}=17$

$.15741 E-17$

$.14049 \mathrm{E}-17$

$.12243 t-17$

$.10424 E-17$

$.00754 \mathrm{E}-10$

$.70241 E-10$

$.704448-18$

$.10005 E-17$

. $104175=17$
50

Sluma

100

200

$.219245-10.40017 c-19$ $.2250 E=10$. $40129 \mathrm{E}-19$ $.22530 \mathrm{E}-10.40499 \mathrm{E}-10$ $.21973 k-10 \quad .43910 \mathrm{~b}-19$ $.20772 E=16 \quad .40306 E-19$

$.10933 \mathrm{E}-18$ . $10470641 k=19$ 1.9463e-10 .24039k-19 $.01350 E-19$

11007

$.62051 \mathrm{E}-19$

.14205E-10

$.17903 e-10$

$.21556 E-10$

$.240038-10$

$.27925 \mathrm{E}-10$

$.3064 y=-10$

$.33023 E-10$

$.35020 k-10$

- $30634 \dot{E-10}$

- $37033 \mathrm{E}-1 \mathrm{O}$

. $30616 \mathrm{E}-10$

.309ó́t-10

$.30934=-10$

. $11132 \mathrm{E}-19$

.200U5E-19

.3346UE-19

$.40931 E-19$

$.55095 \mathrm{c}-19$

$.63370 \mathrm{E}-19$

$.70019 \mathrm{E}-19$

$.702190-19$

$.05540 \mathrm{E}-19$

$.92743 \dot{i}=19$

947 व $4 E-19$

$10061 E-10$

$.11315 \mathrm{E}-16$

.14434E=19

$.81110 z-19$

$.19549 \mathrm{E}-10$

$.14551 \mathrm{E}-19$

. $19916 \mathrm{E}-10$

$.30024 \mathrm{E}=19$
$.90040 E-20$

$9350 b=-20$

ธ0 $806 E-20$

$.02531 \mathrm{E}-20$

$.74014 E-20$

$.0577 d a-20$

$.555500-20$

$.44317 E-20$

$.32072 E-20$

.19600 ÉEO

$.19024 c-20$

$.46703 \mathrm{E}-20$

$.60044 \mathrm{E}-20$

$.749 d 9 k-20$

$.09700 \mathrm{E}-20$

$.10514 k-19$

$.12115 \mathrm{k}-19$

.13709E-19

$15541 x-19$

.17379غ-1y

$.19300 \mathrm{z}-15$

$.21327 E-19$

$.23442 \mathrm{E}-19$

$.25046 \mathrm{E}-19$

25913 kं 20

$.63204 \mathrm{E}-20$

$.64413 \mathrm{E}-20$
$.24365 t-47$

$.26273 \mathrm{t}-07$

20099c-07

$.29014 \mathrm{E}-07$

$.31301 \dot{0}=07$

$.32701 \mathrm{t}-\mathrm{T} 7$

$.33909 \mathrm{E}-U 7$

$.34400=-07$

$.35536 \mathrm{E}-07$

.30079E-07

.30222E- 07

$.39321 k-07$

. 4 Ü7 35E-07

$.4240 \mathrm{OL}=07$

.44007e-07

$.47130 k-07$

$.50124 E-07$

$.53020 \mathrm{U}-U 7$

$.57090 \mathrm{E}-07$

$.02407 \mathrm{E}-07$

$.67054 E-U 7$

$.74127 \mathrm{E}=07$

$.01323 \mathrm{E}-07$

$.06001 E-07$

$.07502 E-07$

$.12072 E-60$

. $13039 \mathrm{E}-00$

$A=.25003420 \mathrm{E}+02 \quad B=.42026100 \mathrm{E}+01$ $.43433 E-10 \quad .10001 E-10^{4} .43435 E^{\circ}-19$ $.57230 \mathrm{E}-10$

$.73000 E-10$

$.9040 \mathrm{bL}-10$

$17900 \mathrm{E}=1 \mathrm{~d}$

$.46150 \mathrm{E}-19$

$C=.41454200 E+01$

10

$.20945 E-18 \quad .40831 c-19 \quad .94132 E-20$

$.12499 E-17,22445 E-10$

$.40831 c-19$

.13956E-17

$.225416-10$

$.14 y 73 \mathrm{E}-17$

$.21900 E-10$

$.405995-19$

$.93044 k-20$

$20750 \mathrm{E}-10$

$.15074 \varepsilon_{-17}$

. $10913 \mathrm{E}-10$

$.43970 E-19$

$.89002 E-20$

$.74961 k-20$

$\begin{array}{ll}.35942 \mathrm{E}-19 & .05670 \mathrm{E}-20 \\ .16040 \mathrm{t}-19 & .32200 \mathrm{t}-20\end{array}$

$.01245 \mathrm{~L}-19$

.57704 Eato

$.01245 t-19$

$19610 \mathrm{k}-20$
$.19831 \mathrm{E}-20$

$.10239 E-10$

$11004 \mathrm{E}-19$

$.16547 E-19$. $33109 \mathrm{E}=20$

$.25952 \mathrm{E}-13 \quad .40770 \mathrm{E}-20$

$.09665 \mathrm{E}-20$

$.12571 E-17$
$.10315 E-17$

$.24700 \mathrm{E}-10$

$.40307 \mathrm{E}-19$

$.12004 \mathrm{E}-19$

$10759 \mathrm{E}-17$

$.30463 \mathrm{e}-16$

$.18102 \mathrm{E}-17$.32627

$.63145 E-19$

10901E-17

$.34707 \mathrm{~d}-16$

$.70534 \mathrm{E}-19$

$.15463 \mathrm{E}-19$

$.85063 \mathrm{E}-19$
$.9214 \mathrm{BE}-19$
$.17301 \mathrm{k}-19$

$.13630 \mathrm{E}-17$

$.37504 \mathrm{E}-10$

$.9214 B \mathrm{~B}=19$

.30241E-10

$.99057 \mathrm{E}-19$

. 34563E-18

$.10574 \mathrm{E}-1 \mathrm{~d}$

.21197E-19

$.23280 \mathrm{E}-19$

$.25452 \mathrm{E}-1 \mathrm{G}$

-11213E-10

.00407É-15

$.14573=19$

$.25705 \mathrm{E}=20$

$.25971 \mathrm{E}-20$

10

$A=.25097000 \varepsilon+02 B=.42715900 E+01$

$c=.41550500 E+01$

$.16742 \mathrm{E}-07$

$.1065{ }^{\circ} E=0$ ?

$.20007 \mathrm{E}-07$

$.22563 \mathrm{E}=07$

$.20335 \varepsilon-07$

$.20175 E-67$

$.29059 \mathrm{E}=07$

$.31403 t=07$

$.32770 \mathrm{E}=07$

$.356028=07$

-30106E-07

$.37309 t-07$

. $30113 \mathrm{E}-07$

$.422006=07$

$.46020 \mathrm{E}-07$

$.49750 \mathrm{E}-07$

$.53160 \mathrm{E}=07$

$617190=07$

$.67017 \mathrm{E}=07$

$.73114 \mathrm{E}-\mathrm{a}$ ?

$80110 \mathrm{E}-\mathrm{CT}$

$66742 \mathrm{E}-07$

$07549 \mathrm{k}=0$ ? 

JU
JL IL KL 2

เง

$\begin{array}{lll}2 & 1 & 1 \\ 3 & 1 & 2 \\ 6 & 1 & 5 \\ 7 & 1 & 6 \\ 0 & 1 & 7\end{array}$

$\begin{array}{lll}2 & 0 & 2 \\ 3 & 0 & 3 \\ 6 & 0 & 0 \\ 7 & 0 & 7 \\ 0 & 0 & 0\end{array}$

$\begin{array}{rrr}9 & 1 & 0 \\ 10 & 1 & 9 \\ 11 & 1 & 10\end{array}$

12110

$\begin{array}{lll}13 & 1 & 12\end{array}$

$\begin{array}{rrr}14 & 1 & 13 \\ 1 & 1 & 1 \\ 1 & 1 & 0\end{array}$

$\begin{array}{rrr}9 & 0 & 9 \\ 10 & 0 & 10\end{array}$

11011

$\begin{array}{lll}12 & 0 & 12\end{array}$

13013

NGL

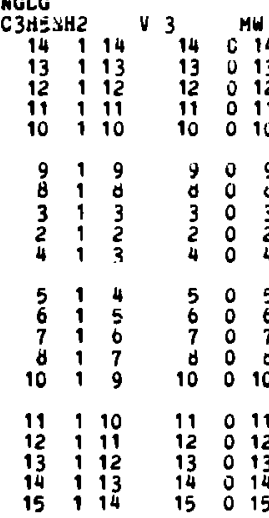

\section{METHYLEME CYANICE}

$\mathrm{CH} 2$ ( $\mathrm{CH}$ )

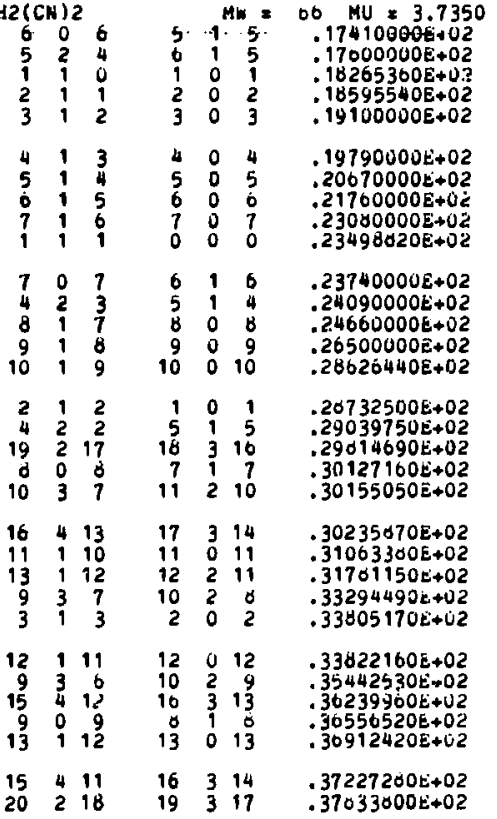

\begin{abstract}
$.21059510 \mathrm{E}+02$ $.21230430 \mathrm{t}+02$ $.2213772 u t+62$ $.22507060 \mathrm{c}+02$ $.23060000 t+02$

$.23 \mathrm{~b} 37290 \mathrm{t}+02$ $.24202950 \mathrm{E}+02$ $.25013520 t+02$ $.20706260 E+02$

$.27089420 \mathrm{CE}+02$

$.29252490 \mathrm{k}+02$
$.293091 \angle 0 \mathrm{E}+02$
\end{abstract}

$57 \mathrm{ML}=.0300$ $16100000 E+02$ $.17325500 c+02$ $.17000200 \mathrm{E}+\mathrm{L} 2$

$.16412500 \mathrm{E}+02$ . $16094700 \mathrm{E}+02$ $.20573720 E+02$ $.20747420 \mathrm{E}+02$ $.21516120 E+02$

$.21812040 E+02$ $.22105190 \mathrm{U}+02$
$.22016340 \mathrm{U}+02$ - $51200 \leq 0 E+02$

$.25075290 E+02$ $25867570 \mathrm{E}+02$ $26706790 E+02$ $27777140 \mathrm{E}+02$ $.20862800 t+02$ $.154370008+02$ $.16720300 \varepsilon+02$ $.24345910 E+02$
$\therefore$

30

slur.h IV

<UU

n net

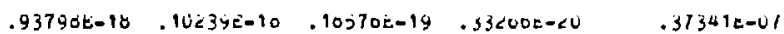

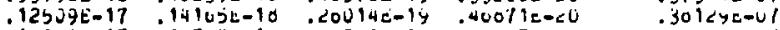
$.1020 j E-17.24703 e-10.40343 t-i y \quad .0+007 E-\leq u \quad .42<0 U E-U 1$ $.10739 c-17.2770 z e-10 \quad .5 j 171 e-1 y$.1uzuye-1y .44gu1a-ul

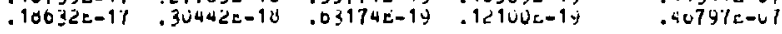

.17 YOAt-17 .32764k-16 .7U530e-19 .1375ye-1y $10040 t-17$ 3470ot-1d .77017:-14 .15495t-14 $.15377 E-17$ 30ट4YE-10.05203t-19 17307t-14

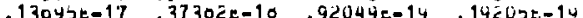

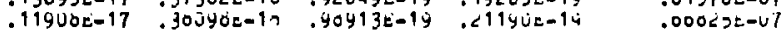

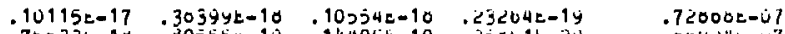

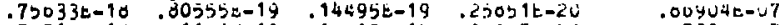
$.702105-10$.01109E-19 .14010E-19 .200J7E-20 .t770sE-Li

$A=.25100000 E+02 \quad b=.42010000 s+01 \quad C=.41050000 \mathrm{E}+C 1$ AAR $=-. \pm 004$

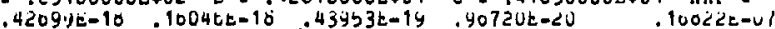

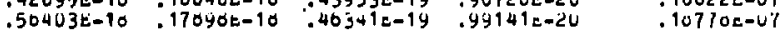

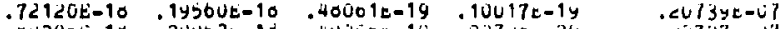

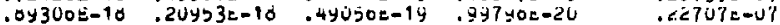

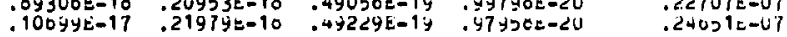

$.12305 \mathrm{E}-17.22547 \mathrm{t}-10.40507 t-19.945402-2 \mathrm{U} \quad .20543 \mathrm{~L}-u 7$ $.13825 E-17 \quad .22570 E-10 \quad .40042 E-19 \quad .03709 E-20 \quad .20351 E-U 17$ $.11001 \mathrm{E}-17$.13400t-10 .24705E-1y .44007t-20 .35150t-1U?

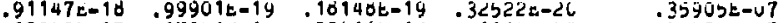
.15049e-17 :17900e-10 :33616e-1y :01112e-20

$.16959 \mathrm{E}-17 \quad .21541 \mathrm{E}-10 \quad .41104 \mathrm{E}-19 \quad .75493 \mathrm{E}-20 \quad .40032 \mathrm{e}-07$ $.10176 \mathrm{E}-17$. $24040 E-10$.40j90E-19.90341t-2U .42556t-U? :10707E-17 :27063E-10 .50006t-19:10574t-19 $.10590 \mathrm{E}-17 \quad .30553 \mathrm{E}-1 \mathrm{C} \quad .63520 \mathrm{E}-19 \quad .12177 \mathrm{E}-1 \mathrm{~g} \quad .47139 \mathrm{E}=07$ $.10005 \mathrm{E}-17 \quad: 34042 \mathrm{c}-10 \quad$.70276z-19 :1500Ue-14 $.15343 \mathrm{E}-17$. 30397t-10 . $55526 \mathrm{EE}-19$. $17421 \mathrm{k}-19 \quad .57507 \mathrm{E}-07$ $.130662 \mathrm{E}-17.37539 \mathrm{E}-10$.92640E-19.19349E-19 .02134E-07

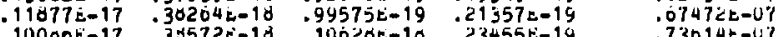

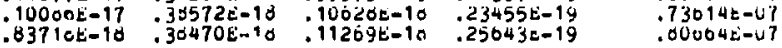

A $2.000 \partial 57506002$ a

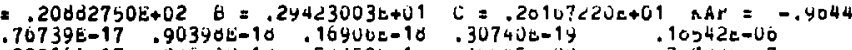

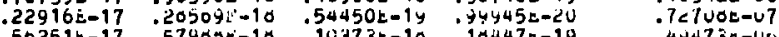
$.50251 t-17 \quad .57905 s-10 \quad .10373 t-10.10447 c-19 \quad .49473 c-10$ $.93004 \mathrm{E}-17$.90103i-10 .1700UE-10.514yze-1y .51735z-00 $.13097 \mathrm{E}-10 \quad .14009 \mathrm{E}-17$.2551hE-10 .4judot-19 .5529<E-J0

$.10795 \mathrm{E}-10.10040 \mathrm{~b}-17.34191 \mathrm{t}=10.01550 \mathrm{~d}-1 \mathrm{y}$.00500e-0u $\begin{array}{lllll}.20417 t-16 & .23620 t-17 & .43910 \mathrm{E}-10 & .79001 \mathrm{t}-19 & .07107 \mathrm{t}-00 \\ .23920 \mathrm{t}-16 & .29075 \mathrm{t}-17 & .54940 \mathrm{E}-10 & .10043 \mathrm{E}-10 & .76114 \mathrm{E}-00\end{array}$ $\begin{array}{lllll}.23920 \mathrm{t}-10 & .29075 \mathrm{E}-17 & .54940 \mathrm{E}-10 & .10043 \mathrm{E}-10 & .76114 \mathrm{E}-00 \\ .27246 \mathrm{E}-10 & .35001 \mathrm{t}-17 & .0750 \mathrm{E}-10 & .12472 \mathrm{E}-10 & .07725 \mathrm{E}-00\end{array}$ $.02117 E=17$.64000t-10 :11440E=10 :20350E-19 .70231E-U0

$.16005 E-16 \quad .20033 k-17 \quad .38109 t-10 \quad .70040 t-19 \quad .52330 t-40$ $.32454 E-17,36673 \mathrm{E}=10 \quad .73106 \mathrm{E}-19 \quad .13330 \mathrm{E}-19 \quad .10114 \mathrm{E}-6 \mathrm{O}$ $.30337 \mathrm{E}-16 \quad$ :41719E-17 :82153E-18 $: 15320 \mathrm{E}-10 \quad$.10276t-US $.33040 E-10 \quad .40930 E-17 \quad .90779 E-10 \quad .10657 E-10 \quad .12170 E-05$ $.35286 \mathrm{E}=1 \mathrm{~b} \quad .56724 \mathrm{E}-17 \quad$.11767E-17 $.22533 E-10 \quad$.14570E-U5

$.13574 E=16 \quad .1+206 t=17 \quad .25540 \dot{0}-10 \quad .45532 i-19 \quad .11525 t-05$ $.41070 E-17.49197 E .10 .92525 E-19$. $16071 \mathrm{E}-19 \quad .24500 \mathrm{E}-06$ $.24406 \mathrm{E}-17 \quad .12656 \mathrm{E}-17 \quad .30796 \mathrm{E}-16 \quad .90514 \mathrm{E}-1 \mathrm{~g} \quad .39011 \mathrm{~L}=00$ $.20075 \mathrm{E}-16$ :37200E-17 :12511E-10 :13449E-10

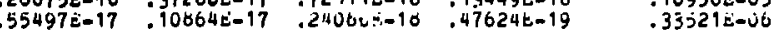

$.22999 \mathrm{E}-17 \quad .10347 \mathrm{k}-17 \quad .30246 \mathrm{k}-1 \mathrm{~d} \quad .00750 \mathrm{t}-19 \quad .35475 \mathrm{t}-U 0$ $.30964 E-16 \quad .05035 \mathrm{E}-17$.13y03E-17 .21220E-1d $.1026 E-10 \quad .23012 E-17 \quad .54504 k-10 \quad .10904 L-10 \quad .04303 t=00$

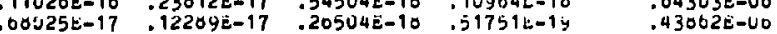
$.24207 \mathrm{E}=10 \quad .25931 \mathrm{k}=17$.40905E-10 $.04009 \mathrm{~d}-19 \quad .10003 \mathrm{E}=05$

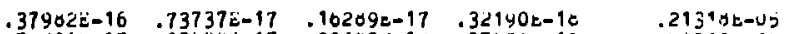
$.73026 E-17$.13699E-17 .29545E-10 .5709UE-19 . S20j0E-2t $37450 E-17 \quad .14020 \mathrm{E}-17 \quad .415 U 4 \mathrm{E}-10 \quad .92361 \mathrm{E}-19 \quad .59513 \mathrm{E}-100$ $43371 E-10 \quad .01010 E-17 \quad .12319 E-17 \quad .23123 t-10 \quad .20004 \mathrm{E}-05$ $.30279 \mathrm{E}-10$.02005E-17 :10921E-17 .30001E-10 .25927E-Ũ

$.393 y 2 k-17 \quad .15 j 00 k-17 \quad .43653 k-18 \quad .97134 s-19 \quad .04293 c=00$ $.32301 \mathrm{E}-17 \quad .19709 \mathrm{E}-17 \quad .04071 \mathrm{E}-10 \quad .15320 \mathrm{t}-10 \quad .03021 \mathrm{E}-0 \mathrm{~b}$ 
Je rege Ja nel NLe

is

$\begin{array}{ccc}4 & 1 & 4 \\ 11 & 1 & 1 \\ 0 & 3 & 0\end{array}$

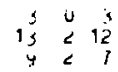

$.307<237 v e+L<$

$39<040340+62$

. 3 y $30<4 \div 0=0+6$

YNLFYAAL HCLU

$$
\begin{array}{ll}
1 & 1 \\
< & 1 \\
5 & 1 \\
4 & 1 \\
5 & 1 \\
0 & 1 \\
7 & 1 \\
1 & 4 \\
0 & 1 \\
y & 1
\end{array}
$$

3
1
3
4

$34 \quad \because 10=c .5 y u$ $4 \%+07$ Uuvictul 190 y $7160 \mathrm{u} a+01$ .3200U⿻上丨 $.405001 \mathrm{UUE+U}$ ?

00570500 t +11 $.41429000 E+01$ $.93250000 \mathrm{a}+1$

1175 sovueru2 $.14040130 \mathrm{c}+02$

. 17 yo $1320 x+v 2$

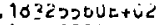
$1005 \mathrm{u} 35 \mathrm{u} c+\mathrm{U}$ loyioiouerida - E74uT40ue+ue

. 27y729juc+112 .27 youOOUE+0z $.27965530 \mathrm{i}+U 2$ $.20467150 \dot{0}+02$ $.30040250 \mathrm{c}+0 \mathrm{C}$

$.37290140 t+v 2$ $.3755459 \cup e+02$ $.45047690 \mathrm{t}+02$ $.40002070 \mathrm{E}+02$ $.47440450 t+02$

$.5490540 U t+U 2$ $.55909090 \mathrm{t}+02$ $.56424440 t+02$ $.041210 j U c+L^{2}$

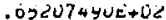

. COUUO 10EE+OZ $.73274320 \mathrm{c}+02$ $.7449402 \mathrm{U} x+02$ . $75050220 \dot{0}+U$ $.024<4$ y $10 E+02$

$.0377502 U z+U C$ $.63914790 \mathrm{C}+02$ $.039710901+02$ . O3y $7250 \mathrm{ULE} U \mathrm{UL}$ $.03901440 t+02$

$.84005700 \mathrm{e}+0 \mathrm{c}$ $.053012 \mathrm{Cut}+02$ $.91572510 t+L 2$ - $93443200 \mathrm{t}+02$ $.93230 j y u c+02$

$.93304340 t+U 2$ $.93300750 \mathrm{c}+02$ . $3313930 \mathrm{U}+U 2$ $.93313940 \mathrm{E}+0 \mathrm{~L}$ $.93437770 K+02$

$.94033680 t+02$ $.10071090 \mathrm{E}+\mathrm{C} 3$ $.10229000 \mathrm{t}+03$ $.10254390 \mathrm{~T}+03$ $.1020305 \mathrm{Jt}+03$

$.10204230 E+03$ $.10204000 t+03$

$.10264000 \mathrm{~L}+\mathrm{C} 3$

. $10206910 \mathrm{E}+03$

. 1027ufouiti:3

$<v$

24

slut 12

$.30033 z=10 \quad .42 U \angle Y E=17$

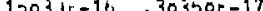

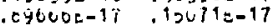

$.70445 \mathrm{c}=10$ $.91<17 E-1 \mathrm{c}$ $.31731 \mathrm{~L}=1 \mathrm{c}$
$.1304<\mathrm{E}-10$ . $01217 \mathrm{t}-19$
$.<0540=05$ $.12007 \mathrm{E}-U\rangle$ $.077300-0 \mathrm{c}$

$A=.0003524+12+6<\quad c=.40203614 \mathrm{c}+U 1$ $.14503 \varepsilon-2 u \quad .21945 t-2 i \quad .40205 \varepsilon-2 z$

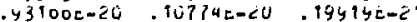
. $\angle 430<e-1 y$.2yjzse-<0 .j5U14e- 1 $.4 / 05 b x-1 j \quad .0004 j E-20 \quad .1157>0-20$ $.7 / y j 0 c-1 y$. 1uve<c-17 $.20742 c-<0$

$7235300551108+01$ AHE $=$ $72353 \mathrm{C}-2$

1 1 ن $14 \mathrm{E}-\mathrm{C}$ ,$\quad 1202 x-c 1$ $.30537 t-21$

$.1125 v e-10$. 1605ye-19 $.14750 \mathrm{~s}-10 \quad .24015 \mathrm{E}-1 \mathrm{y}$ $.1<3+5 x-17.12677 x-10$ . $<v 3 y 0=-10$. $410730-19$

$.33391 \varepsilon-\dot{z} u$ .497 U⿺ -20 $.2 \dot{c} \supset 20 t-1 \dot{y}$ . 0 syujt-20

$\begin{array}{ll}.01 y 4 j E-1 t & .51043 c-1 y \\ 50743 c-17 & .070945-10\end{array}$

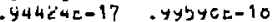

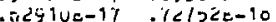
$.22 U 14 E-10 \quad .20440 E-17$ $.937305=2 \mathrm{U}$

$.62092 \mathrm{t}-2$

$.95222 \ddot{\mathrm{e}}-2$

- $3 \dot{y} y 25 x-20$

$13024 \mathrm{E}-20$

$.12100=-19$

$.12540 \mathrm{LE}-10$

.170 obl-10

$.1345 \cup e=10$

<y 7 y 1 t -10 . $32715 c-17$ पण $111 \mathrm{1}=17$

YU $14 \cup 0-1$ ?

$.203 y \geq c-16$

$.430200-1 T$

. $54459 c-10$ $.24 \mathrm{~L}: 2 \mathrm{t}-10$ . $292 i=-10$ - 330uoci-10 $.12164 t-17$

$.240<4 \mathrm{t}-20$ $+207 / E-1 y$ $.31793 \mathrm{c}-1 \mathrm{y}$ . 2451 Ut-1 .9011 4t-15

$04547=-10$ - $596290-10$ 902025-10 $.11207=-15$

.$? 47$ 4 At-17

$13037 c-17$ .95700 U -10 $.00<45 E-17$ $.13303 E-10$ $150=0 \mathrm{c}-17$ . $23796 t-17$ . 2०417E् $=17$

-100bor-10 ל5i $32=-19$ $.55757 t-14$ . $905 y 6 t-1 y$ $.22327 c-1 n$

$.13044 \mathrm{t}=13$ $.13040=-10$ $.25403 \mathrm{~B}-17$

$25024 \mathrm{t}-10$ . $\angle 3$ पूरU-10 $.44159 \mathrm{t}-10$ . $4 n 311=-10$ $.4 / 3000-10$ $.17012 E-1 S \quad .22054 t=10$ $.140 u \dot{s e-1}$.210u2z-16 $.10907 E-15$. 30ं4905-10 $.25 U$ SUE-15.34067z-10

$.40553 \mathrm{~L}=17$ $.44415 \mathrm{c}-17$

$.76255 \mathrm{~L}-10$ $.02324 \mathrm{E}-10$ $.43352 \mathrm{E}-17$.01653c-10 $.02900=17.12012 \mathrm{c}-17$ $.60313 \mathrm{t}-17 \quad .12062 \mathrm{E}-17$

$.199<2 t-15$ . $32422=-16$ $.23002=-15 \quad .42020 \mathrm{t}-10$ $.2000 u=-1, \quad .47307 c-16$ $.240092-15$ .27 YOOE-1j $.45970=-10$

- $55000-15$ $.50250 \mathrm{c}=16$

$.67174 \mathrm{t}-17$ $.91000<-17$ $.90317 \mathrm{E}-17$ $.97059 \dot{c}-17$ $.120045-10$

$.12050 \mathrm{~L}-17$

$.17701 \mathrm{E}=17$

$10045=017$ . $10 y 10 t-17$ $.047000=17$

$-15.0<100 \mathrm{c}-10$ $.17377 \mathrm{t}-1 \mathrm{~b} .404 \div 1 \mathrm{t}-10$

$.700150-10$. 3205yE-10

$.70017 \mathrm{a}-10$.3ácje-10

$.23004 \mathrm{t}-10$. $10907 \mathrm{t}=10$

$.13430 c-16$

$.262700-17$

$11345 \mathrm{t}-1 \mathrm{a}, 23582 \mathrm{t}-17$

$.91233 \mathrm{c}-17 \quad .20463 \mathrm{c}-17$

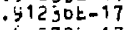

- 174 $\angle 2 E-15$

$.400700-10$

$.20474 c-15$

$.3007 c=-15$

$.405700-10$
$.55403 E-10$

. $65579 \mathrm{i}-17$

106 $11 \pm-17$

$11304 \mathrm{E}-10$ $.70010 \mathrm{e}-16$

$13316 \mathrm{k}-1 \mathrm{t}$

$.1644 y t=16$

$.1700 y t-10$

$.19241 \mathrm{c}-15$

$.50709=-16$

$.05330 t-10$

$.49100 \mathrm{k}-10$

. d53 $39 t-10$

$.4110 \mathrm{cL}-16$

$.15020 t-10$

$23065 \mathrm{E}-17$

204 YUe $=17$

$33332 \mathrm{c}=17$

. $35215 t-17$

. $3193 y t-17$

, $12252 \pm-16$

- $710<\mathrm{L}=16$

.19290E=15

$240245-16$

$.59015 \mathrm{Li}-10$

$.31719 \mathrm{E}-1 \mathrm{~s}$. .74050E-10 $.32319 t=15$ . $30201 t-15$ .20162E-15 $.90310 t-10$ $.05694 \mathrm{E}=10$ $.930 \mathrm{OLt}=10$ $.71409 c-10$

$90314 \mathrm{E}-10$ .2 $195 E-10$ $.29195 z-10$ . $6790 \sigma \ddot{-17}$ 
TAOLE II] 34

su KU1 KU2 JL KL1 KL2 in

$2 v$

jo

Sivin

ivo

zus

A MEE

$\begin{array}{rrrrrr}12 & 1 & 12 & 11 & 1 & 11 \\ 12 & 0 & 12 & 11 & 0 & 11 \\ 12 & 2 & 11 & 11 & 2 & 10 \\ 12 & 3 & 10 & 11 & 3 & 9 \\ 12 & 3 & 9 & 11 & 3 & 8 \\ 12 & 4 & 9 & 11 & 4 & 0 \\ 12 & 4 & 5 & 11 & 4 & 7 \\ 12 & 5 & 8 & 11 & 5 & 7 \\ 12 & 5 & 7 & 11 & 5 & 6 \\ 12 & 6 & 7 & 11 & 6 & 0 \\ 12 & 6 & 6 & 11 & 6 & 5 \\ 12 & 7 & 6 & 11 & 7 & 5 \\ 12 & 8 & 5 & 11 & 0 & 4 \\ 12 & 0 & 4 & 11 & 8 & 3 \\ 12 & 9 & 4 & 11 & 9 & 3 \\ 12 & 9 & 3 & 11 & 9 & 2 \\ 12 & 2 & 10 & 11 & 2 & 9 \\ 12 & 10 & 3 & 11 & 10 & 2 \\ 12 & 10 & 2 & 11 & 10 & 1 \\ 12 & 11 & 1 & 11 & 11 & 0\end{array}$

$.109057708+03$ $.11153910 E+03$ $11185430 \varepsilon+43$ $.1119 / 34$ Uete03 $.11197950 t+03$

.111900 UOe +03 .1119 ouldak+o $.112002000+03$ $11200<80 \mathrm{k}+03$ $.11203090 \mathrm{E}+0$ ?

$.11203090 t+03$ $.112000006+03$ $.11213100 E+03$ $11213100 x+03$ $.11218940 \mathrm{E}+03$

$.11218940 \dot{0}+03$ $.11221190 \mathrm{e}+\mathrm{C} 3$ $.11225540 \leq+03$ $11225540 \mathrm{E}+0$ .11232020t+03

$\begin{array}{lll}12 & 1 & 1 \\ 16 & 0 & 1 \\ 17 & 0 & 1 \\ 18 & 0 & 1 \\ 19 & 1 & 1 \\ 19 & 0 & 1 \\ 19 & 2 & 1 \\ 19 & 4 & 10 \\ 19 & 4 & 1 \\ 19 & 5 & 15 \\ 19 & 3 & 17 \\ 19 & 6 & 1 \\ 19 & 3 & 16 \\ 19 & 7 & 13 \\ 19 & 8 & 1\end{array}$

11110

150015

16016

$\begin{array}{lll}17 & 0 & 17 \\ 18 & 1 & 18\end{array}$

. $11370 b 50 t+03$

. $14834660 t+03$

.15750570E+C 3

$.16664700 E+03$

$.17372500 \mathrm{E}+03$

$16 \quad 010$

10415

18414

10514

17577U20E+03

.17092dd0e+03

$.17732750 \mathrm{E}+03$

$17732850 E+03$

$.17734230 E+03$

$16 \quad 316$

$18 \quad 315$

$18 \quad 712$

$.17734270 E+03$

$.17730490 \mathrm{t}+03$

$.17740380 k+03$

$177446208+0$

$.17752260 \varepsilon+03$

$19 \begin{array}{r}9 \\ 19\end{array}$

$\begin{array}{llllll}19 & 11 & 9 & 18 & 11 & 8 \\ 19 & 12 & 6 & 18 & 12 & 7 \\ 19 & 12 & 7 & 18 & 12 & 6\end{array}$

$\begin{array}{llllll}19 & 13 & 7 & 18 & 13 & 6\end{array}$

$\begin{array}{llllll}19 & 13 & 6 & 16 & 13 & 5\end{array}$

$1914 \quad 6 \quad 1614 \quad 5$

$\begin{array}{lrrrrr}19 & 2 & 17 & 10 & 2 & 16 \\ 10 & 15 & 5 & 10 & 15 & 4\end{array}$

$\begin{array}{lllll}19 & 1 & 16 & 18 & 117\end{array}$

$20120 \quad 19119$

$\begin{array}{llllll}20 & 0 & 20 & 19 & 0 & 19\end{array}$

$\begin{array}{llllll}20 & 2 & 19 & 19 & 2 & 10 \\ 20 & 4 & 17 & 19 & 4 & 16\end{array}$

$20 \quad 416 \quad 19 \quad 415$

$\begin{array}{llllll}20 & 4 & 16 & 19 & 4 & 15 \\ 20 & 5 & 15 & 19 & 5 & 14 \\ 20 & 5 & 16 & 19 & 5 & 15 \\ 20 & 3 & 10 & 19 & 3 & 17 \\ 20 & 6 & 15 & 19 & 6 & 14\end{array}$

$\begin{array}{llllll}20 & 6 & 14 & 19 & 6 & 13 \\ 20 & 3 & 17 & 19 & 3 & 16 \\ 20 & 7 & 14 & 19 & 7 & 13 \\ 20 & 8 & 13 & 19 & 8 & 12 \\ 20 & 9 & 12 & 19 & 9 & 11\end{array}$

$\begin{array}{llllll}20 & 10 & 11 & 19 & 10 & 10\end{array}$

201110

20012

$\begin{array}{lll}20 & 13 & 6 \\ 20 & 14 & 7\end{array}$

$2015 \quad 6$

$20 \quad 119$
$18 \quad 9 \quad 10$

1811 o

$177012200+03$

$.17771+10 E+03$

$17782720 E+03$

$177952806+0$

-17795200ن+03

$.170000602+03$

$17805000 \mathrm{O}+03$

$17623540 t+03$

$1702 y 540 k+03$
$1703931 C E+03$

$18283080 E+03$

$18467580 \mathrm{E}+03$

$.18620810 \dot{0}+03$

$.18666520 \mathrm{E}+03$

- $16566070 E+03$

$18667690 E+03$

$18667090 \Leftrightarrow+03$

$18668300 \mathrm{E}+03$

$.10671970 E+03$

$18671970 E+03$

$18686240 E+03$

. $186095020 E+03$

$18706310 E+03$

$10716220 E+03$

$18731340 E+03$

$18745640 E+03$

$.16701000 E+03$

1914 b

$\begin{array}{rrr}19 & 15 & 5 \\ 19 & 2 & 17\end{array}$

$16777650 \mathrm{E}+03$

$.10776600 E+03$
$.17985170 E+03$

$10676260 E+03$

$18670290 \mathrm{t}+03$

\section{$.32205 e-15$ \\ $.20184 \mathrm{E}-15$ \\ $20070 E-22$ \\ $2007 b t-22$ \\ $.61910 \mathrm{t}-24$ \\ $.32549 \mathrm{c}-15$ \\ $20130 t-15$ \\ $15997 \mathrm{E}-15$ \\ . 10950t-15 \\ $12310 \dot{2}-15$ \\ $.10309=-10$ \\ $10309 E-16$ \\ $.25540 E=17$ \\ .30492E-16 \\ $.403032-18$ \\ $.30447 \mathrm{E}-16$ \\ $.61061 E-19$ \\ $.59965 \mathrm{e}-20$}

$.3740<2=15 \quad$ iUY3UE-15

$20110=-10$. $03743=-10$

gu? $7 u=-10 \quad 54040=-10$

.90770 10 .5y052L-16

$.29070 \mathrm{E}-10 \quad-30443 \mathrm{E}-10$

$.70191 \mathrm{E}-17 \quad .19004 \mathrm{E}-10$

.7U191E-17.19d0ye-10

$.11957 k-17$ :9230JE-17

$.119 j 7 t-17$.9230se-17

$.14504 \mathrm{E}-10$.3000SE-17

$.12529 E-19$. 124T3E-1?

$.12529 E-19 \quad .12473 E-1$ ?

$.74301 E-21 \quad .34942 t=10$

$.74301 e-21$.34942i=1d

$.0413 d t=16$

$.76079 k-19$

$.700795-19$

$.10408 \mathrm{E}-15$

$.15309 c-15$

$.15023 i=15$

$.15900 \mathrm{E}-15$

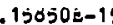

$.12302 \mathrm{E}-15$

$.57014 \mathrm{E}-16$

.57d1jE-10

. $32571 \mathrm{E}-10$

$.90302 E-16$

$.16140 s-10$

$.90345 \mathrm{k}-16$

$.70291 \mathrm{E}-17$

$.26801 \mathrm{E}-17$

$.42607 b-21$

.22083E-22

$.83294 \mathrm{E}-24$

.22791E-25

$.22791 \mathrm{E}-25$

$.45010 \mathrm{E}-27$

.45018E-27

$03716 \mathrm{E}-29$

$.06042 \mathrm{E}-10$

$.90153 b-10$

$.26473 k-1 a$

$.679045-19$

$.15160 k-19$

.15160t-19

$.29351 \mathrm{t}-20$

$.29351 \mathrm{E}-2 \mathrm{~J}$

$.40055 \mathrm{E}-\overline{\mathrm{C}}$

$.12400 \mathrm{E}-15$
$.69060 \mathrm{E}-22$

.10123E-15

9200?E-16

$.49405 E-16$

$.77107 t-17$

$.15042 \mathrm{E}-15$

.14S71E-15

$15453 \mathrm{E}-15$

$12005 \mathrm{E}=15$

$.50625 \mathrm{E}-10$

$.77100 \mathrm{EA} 17$

-19170k-17

.1917 LE- 17

$.22770 \mathrm{E}-16$

$.34940 E-10$

.31908E-16

$.31900 E-10$

HO264E-16

$.15907 t-1 b$

$.34940 E-10$ $.22779 \mathrm{E}-16$

$.46657 \mathrm{E}-19$

$.45629 \mathrm{E}-20$

$.32650 \mathrm{E}-21$

$15907 \dot{0}-16$

$.00312 \mathrm{E}-16$

$.69505 a-17$

$.26700 \mathrm{r}-17$

$.90300 \bar{E}-10$

$.17073 E-22$

$.05129 \mathrm{E}-24$

$.26775 k-10$

$10004 E-25$

$.36430 E-27$

. $69462 k-19$

$15744 E-19$

$.52907 E-29$

.31074E-20

$.53151 E-21$

$.5522 y t-31.70202 t-22$

4917dz-16 .12100t-15

$.74920 \mathrm{E}-16 \quad .14622 \mathrm{E}-15$ $27401 \mathrm{E}-10$ b $700 \mathrm{UE}-1$ ? $.23113=-10$.53000e-11 $140700-10$. $470<<E-1$ ? $.1900 \mathrm{UL}-10$.473<7e-17

- 1buvie-10 1)U01e-10

$10000 \mathrm{C}-10$

1000bL-10

. 6 บ 3UOE-17

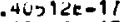

. $360>7 \mathrm{E}-17$

$32027=-17$

$.2524 u c-17$

$693085-17$ $.21936 \mathrm{E}-17$

$.21930 E-17$

$.10309=17$

- $2524 U E-1$

$.12232 \mathrm{E}=17$

$.12231 \mathrm{E}=17$

$.74440 \mathrm{E}-10$

$.10309 \mathrm{k}-17$

$.23051 \dot{E}-16$

$.40434 \mathrm{E}=10$

$.40434 E=10$

.11170E-TO

$.33342 E-11$

$.30900 t-10$

$30 y 00=10$

$.14020=-10$

$27205 x-10$

$.00514 \mathrm{k}-17$

$.11954 \mathrm{E}-10$

554 USE-10 $13700 \mathrm{E}-10$

$006210-10$

$.02007 \mathrm{e}=10$

$15090 t-10$

$.1706 / r-10$

$65421 \mathrm{E}-10$

$30979 \mathrm{t}-10$

$.1707<t-10$

$.10045 \mathrm{E}-10$

$.13457 \mathrm{t}-10$

$.13457 \mathrm{t}-10$

$.11431=-10$

$.20075 E-10$

पY211E- 10

$19990 \mathrm{E}-16$

$.4923 y=-10$

$12934 \mathrm{E}-10$

$.70069 \mathrm{E}-17$ $.93570 \mathrm{~b}-17$

$.15204 \mathrm{E}-10$

$73764 \mathrm{E}-17$

$.55930=-17$

$43921 \mathrm{E}-17$

$11173 \mathrm{t}-17$

$50245 E=10$

ऽU24 $25-10$

$.204000-17$

1905OE-17

-12100t-17 
JU SU1 KU2

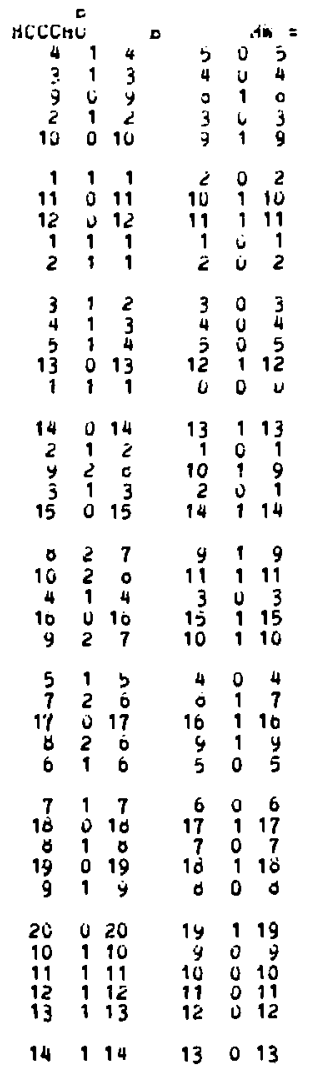

$24 \mathrm{MU}=.0000$ $.15140000 E+02$ $.25100050 c+02$ . $200740602+02$ $.34903040 k+0 a$ $.36093040 \mathrm{E}+02$

$.44550050 \mathrm{0}+02$ $.47410>9 u t+0 z$ $.56240 / 00 E+02$ $.03527010 E+02$ $.03855490 \mathrm{E}+02$

$.64350500 \mathrm{E}+02$ $.05015010 \mathrm{E}+0 \mathrm{~L}$ $.65052590 \mathrm{DE}+02$ $.09140340 E+02$ $.72520190 k+02$

$.60129330 k+02$ $.8152591 \mathrm{JE}+02$ $.07083330 \mathrm{E}+02$ $.90303030 E+02$ $.911712300+02$

$.98402180 e+02$ $.90971030 \mathrm{~b}+02$ $.99039070 z+02$ $.10220130 \mathrm{E}+03$ $.10625020 \mathrm{E}+03$

.107556606003 . $10950670 E+03$ $.113305506+03$

$.11375710 \mathrm{E}+\mathrm{C}$ . $11541910 \mathrm{E}+03$

$.12413110 \mathrm{E}+03$ $.12453240 \mathrm{E}+0$ - $13219000 \mathrm{~s}+03$ $13568700 \mathrm{e}+0$

$.14603700 E+03$ $.14792200 E+03$

$15559030 E+03$

$10315590 \mathrm{D}+\mathrm{D}$

$.170011006+03$

$.17797420 E+03$
$=.000352992+02 D=.440203018+02$ $.14092 E-17$.22100E-10 .44217e-19 $.30004 k-17$. $30759 k-10 \quad .9715 j e-19$ .210 eOt-17 .73161k-10 .19171E-10 b bouUe-17 $72005=-10,13407 \mathrm{E}-10$ $.34300 \varepsilon^{-17}: 14173 t-17 \quad .39732 t-16$

.57 dOGE=17 .62740t-10 .11206E-10 $.44283 \mathrm{E}=17 \quad .22073 \mathrm{E}=17 \quad .60356 \mathrm{E}-10$ $.560 j 4 k=17$.32235k-17.10470k-17 $15203 E-10.165 a 4 k-17.29785 k-10$ $.94410 \mathrm{E}-17$ $1320 \mathrm{CL}=17$

$.52463 \mathrm{E}-17$ $.32309 \mathrm{k}-17$ $.20575 t-17$ $.20573 \mathrm{E}-17$ $.13200 \varepsilon-16$

.90117 E- 10 $.70009 \mathrm{E}-16$ $.07175 \mathrm{i}-10$ $.42090 \mathrm{E}-17$

$.4 \mathrm{e} 704 \mathrm{k}-17$ $.21001 \mathrm{k}-10$ $30016 \varepsilon^{-17}$ $.309060-16$ $.45450 \mathrm{E}-17$

$.22931 t-19$

$.34740 \mathrm{E}-22$

$.0905 \mathrm{E}-10$

$.0051 \mathrm{i}-17$

$.10400 \mathrm{E}-21$

$.59710 E-16$

. $10751 E-10$

$.29430 \mathrm{E}-17$

$30971 \mathrm{E}-21$

$.66402 E-16$

.60013E-16

.22016E-1?

$.67304 E-16$

$16600 \mathrm{E}-17$

$.62630 \mathrm{E}-16$

$.11841 \mathrm{E}-17$

.5ว757E-16

$47000 \mathrm{c}-16$

. 3y2d2E-16

.24050É-10

$.25964 \mathrm{E}_{-10}$

$.20540 \mathrm{E}-10$

$10553 e-10$

$17411 \mathrm{E}-10$

$.1402 d \varepsilon-17$

$14414 \varepsilon-17$

$.25001 E-10$

$.5156 \subset \mathrm{C}=17 \quad .19773 \mathrm{t}-17$ 25032E-17 . .47700E-10 $.35500 \mathrm{E}-17$. 14030E-17 $.51159 \mathrm{E}-17.97420 \mathrm{E}-10$ $.60034 E-17$.25167E-17

190UGE-19.07213t-20

$19492 E-21$

$.72910 \mathrm{E}-17$

$.07015 \mathrm{e}-17$

$.13037 \mathrm{E}-21$

$.14544 \mathrm{E}=17$

$.30040 E-17$

$.35594 E-29$

$10200=-10$

$.04542 \mathrm{E}-17$

. $72100 E-17$

$.13167 t-10$

$.21302 \mathrm{E}-17$

$.20494 \mathrm{E}-17$

30629t-17

, $31553 \mathrm{E}-2$

$.20959 E-17$

$.16090 E-10$

$.37329 E-17$

$.42334 \mathrm{E}-17$

$18704 E-16$

$70435 E-17$

$21129 E-10$

$47707 \mathrm{E}-17$

$.55305 E^{-17}$

$75638 \mathrm{E}-17$

$.23034 \mathrm{t}-16$

$.24434 \mathrm{t}-10^{\circ}$

$.328206-17$

$.64572 E-17$

$.73600 E-17$

$.25296 \mathrm{t}-16$

$25023 \vec{E}-16$

$62231 \mathrm{E}-17$

.9020 EE-17

$.25437 \mathrm{E}-1 \mathrm{E}$

$.97542 \mathrm{E}-17$

FHOPATIUYL ALCOHOL

\begin{tabular}{|c|c|c|c|c|}
\hline $\begin{array}{l}\text { Lith } \\
1 \\
2 \\
2 \\
2 \\
3\end{array}$ & $\begin{array}{l}U_{H} \\
0 \\
1 \\
y \\
1 \\
1\end{array}$ & $\begin{array}{l}1 \\
2 \\
2 \\
1 \\
3\end{array}$ & $\begin{array}{l}0 \\
1 \\
1 \\
1 \\
2\end{array}$ & $\begin{array}{l}0^{M} \\
1 \\
0 \\
1 \\
1\end{array}$ \\
\hline $\begin{array}{l}3 \\
3 \\
3 \\
3 \\
4\end{array}$ & $\begin{array}{l}0 \\
2 \\
2 \\
1 \\
1\end{array}$ & $\begin{array}{l}3 \\
2 \\
1 \\
2 \\
4\end{array}$ & $\begin{array}{l}2 \\
2 \\
2 \\
2 \\
3\end{array}$ & $\begin{array}{l}0 \\
2 \\
2 \\
1 \\
1\end{array}$ \\
\hline $\begin{array}{l}4 \\
4 \\
4 \\
4 \\
4\end{array}$ & $\begin{array}{l}0 \\
2 \\
3 \\
3 \\
2\end{array}$ & $\begin{array}{l}4 \\
3 \\
2 \\
1 \\
2\end{array}$ & $\begin{array}{l}3 \\
3 \\
3 \\
3 \\
3\end{array}$ & $\begin{array}{l}0 \\
2 \\
3\end{array}$ \\
\hline
\end{tabular}

So NU $=1.1700$

$.49324800 E+01$

$.17397050 \mathrm{E}+\mathrm{O}$

- $17050900 k+v 2$

$10333130 E+02$

$.20091620 i+02$

$.26773030 \mathrm{e}+02$

.267900́00ट́+02

$.20821710 \varepsilon+02$

$27495720 \mathrm{E}+0$

$.34782160 \mathrm{E}+02$

$.356705900+02$

. $357206550 E+02$

. $35745010 \mathrm{~s}+02$

$.35745010 \mathrm{k}+\mathrm{C}^{\circ} \mathrm{a}$

$.35784770 k+02$

$.366537008+02$

$\begin{array}{ccccccc}\text { ACcC.H2UH } & \text { V } & & & & \\ 1 & 0 & 1 & & 0 & 0 & 0 \\ 2 & 1 & 2 & & 1 & 1 & 1 \\ 2 & 0 & 2 & & 1 & 0 & 1 \\ 2 & 1 & 1 & & 1 & 1 & 0 \\ 3 & 1 & 3 & & 2 & 1 & 2 \\ 3 & 0 & 3 & & 2 & 0 & 2 \\ 3 & 2 & 2 & & 2 & 2 & 1 \\ 3 & 2 & 1 & & 2 & 2 & 0\end{array}$

$50 \mathrm{HO}=1.1700$ $.89121800 E+01$

-17360100t+02

$170101500 \mathrm{ve}+02$

$10200650 E+02$

$.20040520 E+02$

$.20713770 k+02$

$26737700 \mathrm{~b}+02$

$.26759540 t+02$

$A=.31200270 k+02 \quad E=.47002900 k+01$ $.17039 k=10 \quad=10042 k-19$ $.90307 E-10 \quad 90603 \mathrm{j}-19$

$99950 \mathrm{~L}-16,10944 \mathrm{E}-16$ $.33919 \mathrm{E}-17$.30405E-10

$.42702 E-17.46 / 00 E-1 d$ $.10370 E-17$.23457E-10 $.10400 \mathrm{E}-17$.23497b-18 374 $.7>972 \dot{0}-17$

$.4202 z \mathrm{E}-\mathrm{O}$

$.92756 \mathrm{E}-17$. $1060 \dot{1 E-17}$ $.53954 E-17$.72510E-18 .25 LE -17 $22039 \dot{E}=17$ $37229 \mathrm{E}-10$ $54119 \mathrm{E}-17$

$.37229 \dot{E}-10$

$.72740 \mathrm{E}=16$

$.32142 E-20$ - $17906 \mathrm{t}-19$ $.25475 \mathrm{t}-19$ $.19071 \mathrm{E}-19$ $.70711 \mathrm{E}-19$

\section{$.847705-19$} $.44827 \mathrm{E}-19$ $.44902 \mathrm{E}-19$ $.70419 E-19$ $.17377 \mathrm{E}-10$

$19721 \mathrm{E}-10$ $.14090 \mathrm{E}-10$ $.77105 \mathrm{E}-19$ $.771 \mathrm{~d} 5 \mathrm{E}-19$

$.85021 E-17$

19253E-18

$A=.31350095 E+02 \quad E=.46842300 E+01$ $.17190 k-10.17$ ob $16-19$ $.80133 E-10$ $13179 k-17$ $97317 \dot{E-10} 10700 \mathrm{E}-10$ $33100 E=17$ $10700 E-10$
$.30019 E-10$ $31842 \mathrm{E}-20$ $25239 \varepsilon-19$

$19063 E-19$

$41645 \mathrm{E}-17$

$.40096 \mathrm{E}-18$

$17902 E-17$

$.2313 y=-18$

$.84000 k-19$

$.44474 k-19$

$C=.44495107 c+01$ iAt $=109$ $.0305 j E-\angle 0 \quad .70405 E-60$ $.17070 \mathrm{l}-1 \mathrm{y} \quad .31020 \mathrm{y}=07$ $41270 c-19 \quad .3<0^{\prime} 30 \mathrm{E}-47$ $24220 k-19 \quad .01964 c-67$ $.00471 E-19 \quad .92307 E-47$

$.20077 E-19$ $.15777 E-10$ $.25124 E-10$ $.53079 \mathrm{E}-1 \mathrm{Y}$ . 40304E-19

$.14246 E-06$ $.20134 k-00$ $.37033 E-00$ $.53713 k-0 b$ $.30777 \mathrm{e}-\mathrm{j} 0$

. $39956 \mathrm{t}-19$

$37044 k-19$

$37277 E-19$

$37 \cup 13 E-10$

$.461216-19$

.19153 E-UD

$.14954 E=06$

. 63 प 3 E 00

$.53204 \mathrm{E}-10$

$.51493 E-10$

$.00201 \mathrm{E}-1 \mathrm{~S}$

. $37120=-10$

$.10016 E-16$
$.00529 E-10$

$.99366 \dot{\varepsilon}-06$

$.60115 E=06$

$.12179 \Sigma-0 j$

$.1147 j=-05$

.14717 E-05

$.16608 \dot{t}-20$

$.44040 \mathrm{t}-22$

$.27310 \mathrm{k}-10$

$.00000 \mathrm{k}-10$

$05512 E-00$

$19492 \bar{E}-49$

$.15142 \mathrm{t}-45$

2udo7e-us

$.40941 E-10$

$.40507 E-18$

- 10974 E-17

$.57071 \mathrm{E}-10$

20032t-05

$.22545 k-05$

$.20505 \mathrm{k}=05$

$.48349 E-09$

$.26 d 95 \mathrm{c}=05$

$.75003 \mathrm{E}-10$

- $13348 \mathrm{E}-17$

$.33960 E-05$

$.37907 E-05$

$.41921 \mathrm{t}=05$

$.49301 \mathrm{E}=05$ 
JU KU1 KU2 JL ALI KLE

NU

$\begin{array}{llllll}3 & 1 & 2 & 2 & 1 \\ 4 & 1 & 4 & 3 & 1 & 3 \\ 4 & 0 & 4 & 3 & 4 & 3 \\ 4 & 2 & 3 & 3 & 2 & 2 \\ 4 & 3 & 2 & 3 & 3 & 1 \\ 4 & 3 & 1 & 3 & 3 & 0 \\ 4 & 2 & 2 & 3 & 2 & 1 \\ 4 & 1 & 3 & 3 & 1\end{array}$

$.27417220 \mathrm{E}+02$ $.3472490 \mathrm{Lict}$ उऽ592240e+02 $.35045400 t+0$ $.35003540 t+02$

$35003590=+02$ $.35700700 \mathrm{UL}+0 \mathrm{C}$ $.30549350 c+0<$
20

ju sturis tus

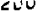

\begin{tabular}{|c|c|c|c|c|}
\hline $\begin{array}{l}30437=-17 \\
.70110 \mathrm{E}=17 \\
.90405 \mathrm{y}=17 \\
.32274 \mathrm{E}=17 \\
.22227 \mathrm{E}=17\end{array}$ & $\begin{array}{l}.4 \angle U \cup y E-10 \\
.910 y 4 t-10 \\
.10343 z-17 \\
.715 s<E-10 \\
.307 \cup y=-10\end{array}$ & 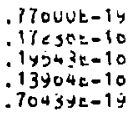 & $\begin{array}{l}140<5 e-1 y \\
. \pm 1 f 0 y e-1 y \\
. j 5 g i l e-1 y \\
.03 y 5 y-1 y \\
14000=-1 y\end{array}$ & 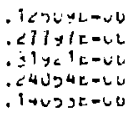 \\
\hline $\begin{array}{l}.22221 \mathrm{E}-17 \\
.32720 \mathrm{E}-17 \\
.03402 \mathrm{U}=17\end{array}$ & $\begin{array}{l}\text { 307ufe-10 } \\
.71740=-10 \\
.10130 t-17\end{array}$ & $\begin{array}{l}.7043 j E-14 \\
.14 \cup U 0 E-1 c \\
19 u \partial j E-1 c\end{array}$ & $\begin{array}{l}.14000=-1 y \\
.2001 y=-1 y \\
.34750=-14\end{array}$ & 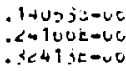 \\
\hline
\end{tabular}

HCCCHESH $\forall 2 \quad \mathrm{MH}=50 \mathrm{MU}=1.1700$ $\begin{array}{ccccccc}1 & 0 & 1 & & 0 & 0 & 0 \\ 2 & 1 & 2 & & 1 & 1 & 1 \\ 2 & 0 & 2 & & 1 & 0 & 1 \\ 2 & 1 & 1 & & 1 & 1 & 0 \\ 3 & 1 & 3 & 2 & 1 & 2\end{array}$

$\begin{array}{llllll}3 & 0 & 3 & 2 & 1 & 2 \\ 3 & 2 & 2 & 2 & 2 & 1 \\ 3 & 2 & 1 & 2 & 2 & 0 \\ 3 & 1 & 2 & 2 & 1 & 1 \\ 4 & 1 & 4 & 3 & 1 & 3\end{array}$

$\begin{array}{lll}4 & 0 & 4 \\ 4 & 2 & 3 \\ 4 & 3 & 2 \\ 4 & 3 & 1 \\ 4 & 2 & 2\end{array}$

$\begin{array}{lll}3 & 0 & 3 \\ 3 & 2 & 2 \\ 3 & 3 & 1 \\ 3 & 3 & 0 \\ 3 & 2 & 1\end{array}$

413

$.09426000 \pm+01$

$17411670 \mathrm{E}+02$

$.17079130 \mathrm{E}+02$

$10359450 \mathrm{k}+02$

$.20113460 \mathrm{E}+42$

$.20003430 E+02$

$.20029200 E+02$

$26052060 t+02$

$27534950 k+02$

. $340107205+02$

$.35709070 E+02$

$35766070 E+U 2$

$35705790 \mathrm{t}+02$

$35705790 E+02$

$.35020640 \mathrm{e}+02$

$.30705550 E+02$

5 TRAMS ACROLEL

CH2 ChilH

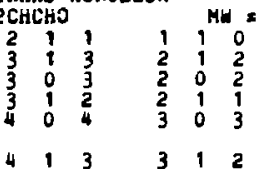

$56 \mathrm{HU}=3.0600$ $.18211010 \mathrm{E}+02$

$.26079470 \varepsilon+02$

$.26694370 E+02$

. $27329020 E+02$

$.35570420 t+02$

413

$.36435940 E+02$

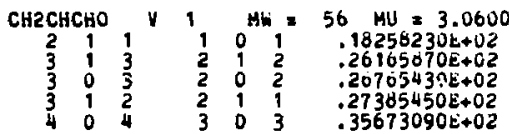

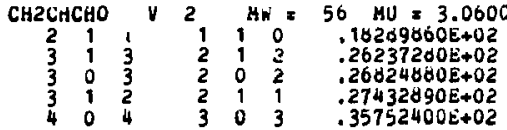

OXETANE

\begin{tabular}{|c|c|c|c|c|c|c|}
\hline $\begin{array}{r}\text { CH2 } \\
1 \\
8 \\
9 \\
9 \\
17\end{array}$ & $\begin{array}{r}30 \\
0 \\
7 \\
9 \\
8 \\
14\end{array}$ & $\begin{array}{l}1 \\
2 \\
1 \\
2 \\
3\end{array}$ & $\begin{array}{r}0 \\
8 \\
9 \\
9 \\
17\end{array}$ & $\begin{array}{r}0 \\
5 \\
7 \\
6 \\
14\end{array}$ & $\begin{array}{l}14 \\
0 \\
3 \\
2 \\
3 \\
4\end{array}$ & $\begin{array}{l}56 \quad 7001.9360 \\
.16464740 E+02 \\
.26724300 E+02 \\
.26726650 E+02 \\
.27303940 E+02 \\
.27763900 E+02\end{array}$ \\
\hline $\begin{array}{l}10 \\
11 \\
16 \\
10 \\
12\end{array}$ & $\begin{array}{r}9 \\
10 \\
13 \\
10 \\
11\end{array}$ & $\begin{array}{l}2 \\
2 \\
3 \\
1 \\
2\end{array}$ & $\begin{array}{l}10 \\
11 \\
16 \\
10 \\
12\end{array}$ & $\begin{array}{r}7 \\
0 \\
13 \\
8 \\
9\end{array}$ & $\begin{array}{l}3 \\
3 \\
4 \\
2 \\
3\end{array}$ & $\begin{array}{l}.20125190 E+02 \\
.29252500 t+02 \\
.29557300 E+02 \\
.300012 d C E+02 \\
.30749750 E+02\end{array}$ \\
\hline $\begin{array}{r}15 \\
2 \\
2 \\
14 \\
13\end{array}$ & $\begin{array}{r}12 \\
1 \\
0 \\
11 \\
12\end{array}$ & $\begin{array}{l}3 \\
2 \\
2 \\
3 \\
2\end{array}$ & $\begin{array}{r}16 \\
1 \\
1 \\
14 \\
13\end{array}$ & $\begin{array}{r}12 \\
1 \\
0 \\
11 \\
10\end{array}$ & $\begin{array}{l}4 \\
1 \\
1 \\
4 \\
3\end{array}$ & $\begin{array}{l}.31104510 \mathrm{E}+02 \\
.31925960 \mathrm{E}+02 \\
.32223230 \mathrm{E}+02 \\
.32407300 \mathrm{E}+02 \\
.32677360 \mathrm{E}+02\end{array}$ \\
\hline $\begin{array}{l}13 \\
11 \\
12\end{array}$ & $\begin{array}{r}10 \\
11 \\
9\end{array}$ & $\begin{array}{l}3 \\
1 \\
3\end{array}$ & $\begin{array}{l}13 \\
11 \\
12\end{array}$ & $\begin{array}{r}10 \\
9 \\
y\end{array}$ & $\begin{array}{l}4 \\
2 \\
4\end{array}$ & $\begin{array}{l}.334626 d 0 E+02 \\
.33752700 \ddot{0}+02 \\
.34282070 E+02\end{array}$ \\
\hline
\end{tabular}

$A=.3007134 j t+0 \hat{2} \quad=.4700340 j t+v 1$ $.17200 \mathrm{E}=10$

$.00472 \mathrm{t}-10$

$.13222 \mathrm{E}=17$

-90ujut-1a

. $332<7 t-17$

$.41793 \dot{c}-17$

. 10090e-17

$.10120 \mathrm{k}-17$

$36692 E-17$

$.70374 \mathrm{E}-17$

$.90709 \mathrm{E}=17$

$.22043 \mathrm{c}-17$

$.226435=17$

$.53272 \hat{E}=17$

$.03954 \varepsilon-11$

$.17 \circ 71 \mathrm{c}-1 \mathrm{~s}$

- $97477 \mathrm{t}=1 \mathrm{y}$

$.14043 \mathrm{t}-1 \mathrm{C}$

$.30013=-10$

. $31046 \mathrm{E}=<0$

$.17730=-1 y$

$.23240=-19$

1970OE-19

$.40133 t-10$

$.23224 \mathrm{i}-10$

$232030-10$

$.42149 \mathrm{E}-10$
$.91007 \mathrm{k}-10$

$.70030 \mathrm{t}-1 \mathrm{y}$

$.03 y 91 \mathrm{c}-19$

$.44404 \bar{E}-19$

$.44541 \mathrm{t}-19$

$.77702 \mathrm{e}-19$

1054 y $17-17$

$.71703 \mathrm{E}-10$

- 3090 Je-10

. $19537=-10$

$13902 \mathrm{E}-10$

$.70071 \mathrm{k}-1 \mathrm{y}$

$.72017 E-10 \quad .14026 E-10$

.10100t-17

19092E- 10

$\boldsymbol{A}$

$=.47639000 E+02 \quad B=46594300 E+01$ .79d37E-17 .09407E-18 .16375k-10 $.27423 E-16 \quad .31857 E-17 \quad .58995 E-10$ $.35747 \mathrm{E}-16$.39091E-17 .70940E-10 $.29935 E-16$ 34901E-17 .64704b-10

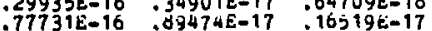
$.68556 E=16 \quad .04235 \mathrm{E}-17 \quad .15894 \mathrm{E}-17$

$\therefore=.4234450^{\circ}+U 1$ AAr $=-32641$

$.205<1 \mathrm{E}-\mathrm{c} 1 \quad .3740<\mathrm{E}-\mathrm{JU}$

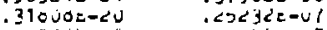

$.44990 \mathrm{E}-20 \quad .304<j E-U 7$

$.353530-\angle 0 \quad$. SY>OUE-U?

$.120 j+e-1 y$. Iuovoe-vo

$.15000 t-19$

$.13147 t-4 u$

$.73<02 \mathrm{~b}-\mathrm{U} 7$

1207100

$.2000 j \mathrm{t}-60$

140430

$.31322 t=1 y$

$.32<300-40$

$.24301 \mathrm{E}=0 \mathrm{U}$

$.14190=-U U$

$\begin{array}{ll}.14002 E-14 & .141306-00 \\ .20031=-14 & .244<3=-000\end{array}$

$\begin{array}{ll}.14002 E-14 & .141306-00 \\ .20031=-14 & .244<3=-000\end{array}$

$.347050-19 \quad .32030 z-100$

$C=.42427100 E+01 \quad \mathrm{AH}=-.4000$

$.29452 E-19 \quad .19747 t-0 t$

$.10674=-10 \quad .73044 E-40$

$.00040 \mathrm{E}-\mathrm{J0}$

$\begin{array}{ll}.117152-10 & .04751 E-v 0 \\ .290420-10 & .21013 E=U 5\end{array}$

$.29027 \dot{0}=10 \quad .2196000 \mathrm{US}$

100

$A=.47600000 \mathrm{E}+02 D=.46601900 \mathrm{E}+01$ $.79204 E-17.09641 E-10$. 16459E-10 $.27265 E-16$.31954E-17 .59350E-10 .35492E-16 .39159E-17.71201E-10 $.29091 E-16 \quad .34921 E-17$. 64935E-10 $.77160 \mathrm{E}-1 \mathrm{E} .89621 \mathrm{E}-17$. $16595 \mathrm{E}-17$

$C=.42596000 \mathrm{t}+01 \mathrm{KAF}=0.9012$ $.29031 t-19 \quad .1 Y 401 t-00$ $.10754 \mathrm{E}-10 \quad .74379 \mathrm{E}-\mathrm{U} \mathrm{l}$ $.12707 \mathrm{~L}-10 \quad .09557 \mathrm{~V}-0 \mathrm{O}$ $.11773 \mathrm{E}-10 \quad .05271 \mathrm{E}-00$ $.3002 b t-18 \quad .21900 \mathrm{c}-45$

100

$A=.47639000 E+02 \quad E=.46721000 E+01 \quad C=.42735000 E+01 \quad K A F=-.9010$ $.786175-17$. $09009 E-16.10504 c-16$ $270 d 3=16$ :32025ti-17:59057c-10

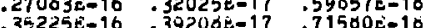
$\begin{array}{rr}.35225 \mathrm{E}-16 & -39200 \mathrm{E}-17 \\ .29430 \mathrm{E}-16 & .7154930 \mathrm{E}-17 \quad .65143 \mathrm{E}-10\end{array}$ $.70564 \mathrm{t}-10: 09720 \mathrm{~b}-17$ :10004E-17

$.29770 \mathrm{x}-19$

$.100200-10$

$12059 \mathrm{E}-10$

$11020 \mathrm{~b}=10$

$.30195 \mathrm{E}-10$

$.20004 t-00$

$.749 d 95-40$

.401 bUt -00

$.03715 \mathrm{t}-\mathrm{Ut}$

$.221350=05$

$A=.12045491 E+02 \quad B=.117340<1 E+02$ $.24710 \mathrm{E}-17 \quad .25700 \mathrm{~b}-1 \mathrm{t}$ $.23700 E-17$. do19ded-1d $.50530 E-16 \quad .24001 \mathrm{t}-10$ .15809E-17 .72030s-18 $.70374 E-20.13019 E-18$

$.977298-16 \quad .63454 \varepsilon-10$

$5010 \mathrm{db}=10 \quad .53193 \mathrm{e}=10$ $.21637 \mathrm{e}-19 \quad .21302 \mathrm{e}-10$ 29075F-10 .20091E-10 .30005E-18 $.20091 E-16$

$.55482 E-19$. $31769 t-10$ $.10255 \mathrm{E}-16$.11199E-17

$.1075 \Delta E-1 b \quad .1174 d E-17$

$.13201 E-18.45359 E-10$

$.15007 E \dot{-16}$

$.29104 E-16.02030 E-10$

$10507 t-18$, $10233 \mathrm{E}-10$
$.45992 E-19$ . $21200 \mathrm{c}-10$ $.712716-19$ $.21341 \mathrm{E}-10$ $.13644 E-18$

$c=.67300094 \mathrm{e}+0$ $.017000-20$ $.45024=19$ $.16309=-19$ $40560 \mathrm{c}-19$ $.57002 E-19$

$.200446-10$

$.50226 \mathrm{E}=19$

$.19816 \mathrm{E}-1 \mathrm{~B}$ 17407E-10 $.60774 \mathrm{E}-19$ $.10371 \mathrm{E}-10$

$.50053 t-19$

$.00033 \mathrm{E}-19$

$.10106 \varepsilon-19$ $.50494 \varepsilon-19$

$.21579 E-1 n$ $.20307 E-18$ $.21301 \mathrm{e}-18$ . 25966t-10 - 16b28t-18

$.74750 v-19$

. 36 $550 E-19$

. $36137 v-19$

$.620000-14$

$3020 d t-10$
$.01239 t-19$ $.49235 z-19$

AAL - YUYOCE=U? - 1 jóULE-UJ . 3340 Ut-ur $.16775 \mathrm{k}-06$ $.19251 \mathrm{c}-00$

$.1050 d z-v 0$ $.10902 t=00$ $.22040 \pm-60$ . ว $1076 t=0$ ? .17304two

$.20450 \mathrm{~b}-00$ $.42325 z-100$ $.44010 \mathrm{0}-00$ .29402 e $=06$ $.17967 \mathrm{E}-00$ $.93323 \mathrm{e}-14$

1523 $.0 \mathrm{C}<0$ . 34205E-10
. 34\$Y 1e-vo $.01030 \mathrm{t}=07$ . $35557 t-00$ 


$$
\text { Ju }
$$

$n \cup 1 n+<$

ALI ALC

¿̇u

ข0

31 UAA

100

ENu

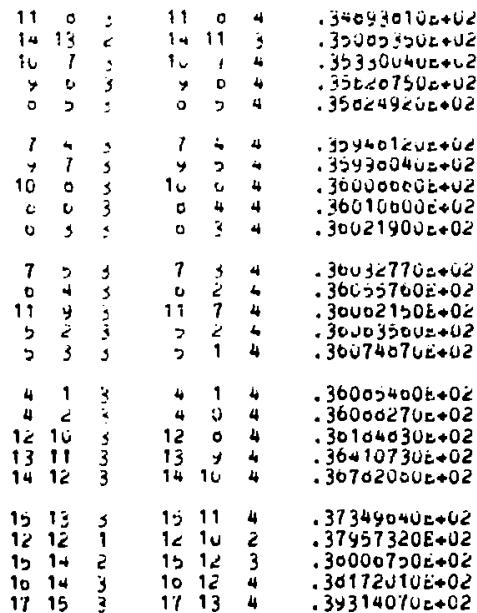

\begin{tabular}{|c|c|c|c|c|c|c|}
\hline 112 & 130 & & 1 & & $\omega=$ & so $d u=1.430$ \\
\hline $\begin{array}{r}1 \\
0 \\
9 \\
9 \\
10\end{array}$ & $\begin{array}{l}0 \\
7 \\
0 \\
9 \\
9\end{array}$ & $\begin{array}{l}1 \\
2 \\
2 \\
1 \\
2\end{array}$ & $\begin{array}{c}0 \\
0 \\
y \\
y \\
10\end{array}$ & $\begin{array}{l}0 \\
5 \\
0 \\
7 \\
7\end{array}$ & $\begin{array}{l}4 \\
3 \\
3 \\
2 \\
3\end{array}$ & $\begin{array}{l}.10490020 E+02 \\
.26006010 E+02 \\
.27332430 E+02 \\
.27561230 E+02 \\
.20279100 \varepsilon+02\end{array}$ \\
\hline $\begin{array}{l}16 \\
11 \\
15 \\
10 \\
12\end{array}$ & $\begin{array}{l}13 \\
10 \\
12 \\
10 \\
11\end{array}$ & $\begin{array}{l}3 \\
2 \\
3 \\
1 \\
2\end{array}$ & $\begin{array}{l}10 \\
11 \\
15 \\
10 \\
12\end{array}$ & $\begin{array}{r}13 \\
0 \\
12 \\
0 \\
9\end{array}$ & $\begin{array}{l}4 \\
3 \\
4 \\
2 \\
3\end{array}$ & $\begin{array}{l}.28330900 E+02 \\
.29575900 E+02 \\
.30055160 E+02 \\
.31102030 E+02 \\
.31295340 \mathrm{E}+02\end{array}$ \\
\hline $\begin{array}{r}14 \\
2 \\
2 \\
13 \\
13\end{array}$ & $\begin{array}{r}11 \\
1 \\
0 \\
10 \\
12\end{array}$ & $\begin{array}{l}3 \\
3 \\
2 \\
2 \\
3 \\
2\end{array}$ & $\begin{array}{r}14 \\
1 \\
1 \\
13 \\
13\end{array}$ & $\begin{array}{r}11 \\
1 \\
0 \\
10 \\
10\end{array}$ & $\begin{array}{l}4 \\
1 \\
1 \\
4 \\
3\end{array}$ & $\begin{array}{l}.31520240 E+02 \\
.32043110 \mathrm{U}+\mathrm{U} 2 \\
.32350430 \mathrm{E}+02 \\
.32737740 \mathrm{u}+\mathrm{J} 2 \\
.33493900 \mathrm{0}+02\end{array}$ \\
\hline $\begin{array}{r}12 \\
11 \\
10 \\
11 \\
4\end{array}$ & $\begin{array}{r}9 \\
0 \\
7 \\
11 \\
6\end{array}$ & $\begin{array}{l}3 \\
3 \\
3 \\
1 \\
3\end{array}$ & $\begin{array}{l}12 \\
11 \\
10 \\
11 \\
y\end{array}$ & $\begin{array}{l}9 \\
0 \\
7 \\
5 \\
0\end{array}$ & $\begin{array}{l}4 \\
4 \\
4 \\
2 \\
4\end{array}$ & $\begin{array}{l}33000070 t+02 \\
.34473830 t+02 \\
.34917390 \mathrm{0}+02 \\
.35143010 \mathrm{0}+02 \\
.35270000 t+02\end{array}$ \\
\hline $\begin{array}{l}6 \\
7 \\
9 \\
0 \\
0\end{array}$ & $\begin{array}{l}5 \\
4 \\
7 \\
b \\
3\end{array}$ & $\begin{array}{l}5 \\
5 \\
3 \\
3 \\
3 \\
3\end{array}$ & $\begin{array}{l}0 \\
7 \\
9 \\
0 \\
0\end{array}$ & $\begin{array}{l}5 \\
4 \\
5 \\
4 \\
3\end{array}$ & $\begin{array}{l}4 \\
4 \\
4 \\
4 \\
4\end{array}$ & $\begin{array}{l}35501000 E+02 \\
.35647000 E+02 \\
.35720330 E+02 \\
.35720530 t+02 \\
.35733740 E+02\end{array}$ \\
\hline $\begin{array}{r}10 \\
7 \\
0 \\
5 \\
5\end{array}$ & $\begin{array}{l}0 \\
5 \\
4 \\
2 \\
5\end{array}$ & $\begin{array}{l}3 \\
3 \\
3 \\
3 \\
3\end{array}$ & $\begin{array}{r}10 \\
7 \\
0 \\
5 \\
5\end{array}$ & $\begin{array}{l}0 \\
3 \\
2 \\
2 \\
1\end{array}$ & $\begin{array}{l}4 \\
4 \\
4 \\
4 \\
4\end{array}$ & $\begin{array}{l}.35742070 E+02 \\
.35750470 E+02 \\
.35775170 E+02 \\
.35702030 E+02 \\
.35790410 \mathrm{E}+02\end{array}$ \\
\hline $\begin{array}{r}4 \\
4 \\
11 \\
12 \\
14\end{array}$ & $\begin{array}{r}1 \\
2 \\
9 \\
10 \\
13\end{array}$ & $\begin{array}{l}3 \\
3 \\
3 \\
3 \\
2 \\
2\end{array}$ & $\begin{array}{r}4 \\
4 \\
11 \\
12 \\
14\end{array}$ & $\begin{array}{r}1 \\
0 \\
7 \\
0 \\
11\end{array}$ & $\begin{array}{l}4 \\
4 \\
4 \\
4 \\
3\end{array}$ & $\begin{array}{l}.35804220 \mathrm{E}+02 \\
.35011000 \mathrm{0}+02 \\
.3502020 \mathrm{OE}+02 \\
.35964700 \mathrm{0}+02 \\
.30224900 \mathrm{O}+02\end{array}$ \\
\hline $\begin{array}{l}13 \\
14 \\
15 \\
16 \\
20\end{array}$ & $\begin{array}{l}11 \\
12 \\
13 \\
14 \\
10\end{array}$ & $\begin{array}{l}3 \\
3 \\
3 \\
3 \\
4\end{array}$ & $\begin{array}{l}13 \\
14 \\
10 \\
10 \\
20\end{array}$ & $\begin{array}{l}9 \\
10 \\
11 \\
12 \\
10\end{array}$ & $\begin{array}{l}4 \\
4 \\
4 \\
4 \\
5\end{array}$ & 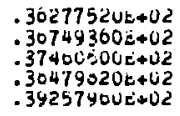 \\
\hline $\begin{array}{l}15 \\
12\end{array}$ & $\begin{array}{l}14 \\
12\end{array}$ & $\ddot{i}$ & $\begin{array}{l}12 \\
12\end{array}$ & $\begin{array}{l}12 \\
10\end{array}$ & $\begin{array}{l}3 \\
2\end{array}$ & $\begin{array}{l}39512130 \mathrm{j}+02 \\
.396535>0 \mathrm{0}+02\end{array}$ \\
\hline
\end{tabular}

\begin{tabular}{|c|c|c|c|c|}
\hline $\begin{array}{l}11301 c-17 \\
.0 y 430 c-1 y \\
.19505 c-17 \\
.31207 c-17 \\
.40019 c-17\end{array}$ & $\begin{array}{l}.10170 c-17 \\
.25277 k-10 \\
.121012-17 \\
.1305+2-17 \\
.145040-17\end{array}$ & $\begin{array}{l}.37350 t-10 \\
.14715 E-10 \\
.3 \div 4.3 t-10 \\
.40 U 75 E-16 \\
.35105 E-10\end{array}$ & $\begin{array}{l}.9519 y c-1 y \\
.47200 t-19 \\
.5+337 c-19 \\
.94015=-1 y \\
.03 y 9+c-19\end{array}$ & $\begin{array}{l}.374790-00 \\
.10057 t-00 \\
.30004 E-00 \\
.39123 E-00 \\
.301900-19\end{array}$ \\
\hline $\begin{array}{l}.01490 \mathrm{C}-17 \\
.31765 \mathrm{E}-17 \\
.20055 \mathrm{E}-17 \\
.40376 \mathrm{E}-17 \\
.73211 \mathrm{E-17}\end{array}$ & $\begin{array}{l}1522 y \mathrm{E}-17 \\
1400 \mathrm{E}-17 \\
12510 \mathrm{e}-17 \\
15104 \mathrm{z}-17 \\
114207 \mathrm{E}-17\end{array}$ & $\begin{array}{l}.30270 E-10 \\
.40722 t-10 \\
.40575 E-10 \\
.39422 c-10 \\
.31300 t-10\end{array}$ & $\begin{array}{l}.74447 c-1 y \\
.92000 \mathrm{c}-1 y \\
.97132 \mathrm{c}=19 \\
.04074 c-19 \\
.01074 \mathrm{c}-1 y\end{array}$ & $\begin{array}{l}.37204 E-00 \\
.40171 E-00 \\
.40002=-00 \\
.34235 E-00 \\
.34420 E-00\end{array}$ \\
\hline $\begin{array}{l}.01714 \mathrm{t}-17 \\
.73315 \mathrm{t}-17 \\
.1774<\mathrm{E}-17 \\
.74035 \mathrm{E}=17 \\
.7407 \mathrm{Ut}=17\end{array}$ & 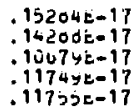 & $\begin{array}{l}.30412 c-10 \\
.31420 k-10 \\
.34247 \dot{1}=10 \\
.24131 \dot{i}=10 \\
.24143 E=10\end{array}$ & $\begin{array}{l}.747 c 4 t-19 \\
.01907 E-19 \\
.10004 E-10 \\
.45 \Rightarrow 00 E=19 \\
.40003 t=15\end{array}$ & $\begin{array}{l}.37512 E-i 0 \\
.34510 E-00 \\
.40714 E-10 \\
.29250 E-01 \\
.29274 E-U J\end{array}$ \\
\hline $\begin{array}{l}.54135 E-17 \\
.54141 E-17 \\
.04033 c-10 \\
.32074 c=10 \\
.755+7 \varepsilon=-10\end{array}$ & $\begin{array}{l}.72393 E-16 \\
.72402 E-10 \\
.07010=-10 \\
.09794 t=10 \\
.53720 t=10\end{array}$ & $\begin{array}{l}14044 \mathrm{t}-10 \\
.14045 \mathrm{c}-10 \\
.37009 \mathrm{E}-10 \\
.34119 \mathrm{E}-10 \\
.30015 \mathrm{t}-10\end{array}$ & $\begin{array}{l}.20007 E-19 \\
.20010 E-1 y \\
.10102 E=10 \\
10036 E-10 \\
.90119 E-19\end{array}$ & $\begin{array}{l}.19604 t=00 \\
.1960 d E-00 \\
.40641 E=00 \\
40490 E-00 \\
.40353 E=00\end{array}$ \\
\hline $\begin{array}{l}.05450 E-19 \\
.05040 t=19 \\
.30407=-19 \\
.29145 E=19 \\
.11445 E=19\end{array}$ & $\begin{array}{l}.40115 E-10 \\
.12759=-10 \\
.1045 U E-10 \\
.29070 E-10 \\
.20400 E-10\end{array}$ & $\begin{array}{l}.27304 \mathrm{E}-10 \\
.55207 \mathrm{E}-14 \\
.12755 \mathrm{E}-10 \\
.23755 \mathrm{E}=10 \\
.20305 \mathrm{E}-10\end{array}$ & $\begin{array}{l}.94700 E-19 \\
.15294 c-19 \\
.44500 t-19 \\
.90275 E-14 \\
.05030 t-19\end{array}$ & $\begin{array}{l}.40300 E-00 \\
.05433 E=07 \\
.9460 c-06 \\
.40494 E-U 0 \\
.4073 U E-U D\end{array}$ \\
\hline
\end{tabular}

$=.12050009 t+02 \quad 0=.11720502 t+02$ $.19159 \mathrm{t}-10$ $.4073 U E-U$

$.70143 \mathrm{E}-17$

$.45363 E-19$
.1939 st - 00

$.05745 E-07$ $.23944 \mathrm{k}-17 \quad .25240 \mathrm{t}=1 \mathrm{C}$ $.223406-17 \quad .77411 \mathrm{l}=10$

$.14079 \mathrm{E}-17$. 1 J190E-10

$.40979 \mathrm{E}=10 \quad .22912 \mathrm{E}-10$

$.91702 E-10$.01022E-10

$.45961 t-19$

$.20024 t-10$

$.20720 \mathrm{t}-10$

$.00415 \mathrm{k}-1$

$.20204 \mathrm{~L}=10$

$19253 \overrightarrow{\mathrm{E}}-19$

$.52603 \mathrm{E}-10 \quad .51021 \mathrm{E}-1 \mathrm{~d}$

$.49902=19 \quad .29250 \mathrm{c}-10$

$.27690 \mathrm{E}-18 \quad .19001 \mathrm{E}=10$

$.20001 E-10.410995-10$

$15902 \dot{c}-10$ . 2 UOUTE-10 $.63935 \mathrm{k}-19$ $.17706 \mathrm{E}-10$

$.11992 t-10.42100 t-10$ $.9 y 724 \mathrm{E}-17$ $1114 \mathrm{JE}-: 7$

$.10493 \mathrm{t}=10 \quad .11727 \mathrm{t}-17$

20604

$.13952 c-10$

$.50212 \mathrm{c}=18$

.31940 cis 10

$.24322 E-10$ $.20300 E-10$ $.21429 \mathrm{E}-10$ $.20040 \mathrm{E}-18$ $.15909 \mathrm{E}-10$

$i=.07722732 \mathrm{E}+u$

$.5464 t i=10$

$17.900450-1 d$

$.16252 \mathrm{t}-17 \quad .11651 \mathrm{t}-17$

$.1527 j E-10$. 15350i-10

$.29390 \mathrm{c}-17$

$13332 \mathrm{r}-17$

. 32615E-16 . $35072 \mathrm{E}-10$ . $30055 \mathrm{c}-10$ $.50569 \mathrm{E}-19$ . 30050E-10

$.43370 E-17$ $.56060 \mathrm{E}-17 \quad .14725 \mathrm{t}=17$ $.29954 \mathrm{E}-17 \quad .13553 \mathrm{E}-17$ $437 \mathrm{C} 4 \mathrm{E}-17,14600 \mathrm{E}-17$ .0924 dE- 17

$.13016 \mathrm{t}=11$

$.30029 t-10$ $.35355 k-10$ - 39625E-10 . 30400E-10 .30026k-10

. $10002 \mathrm{E}-17$

$.12007 \mathrm{E}-17$

$.50333 \mathrm{E}-17$

$.65370=-17$

$.70102 \mathrm{E}-17$

?3841 k-17

$.11309 \mathrm{t}-17$

$.11396 \mathrm{E}-17$

$.39420 E-10$ . 355160-10

. $30003 \dot{0}-15$

. $23576 \mathrm{E}=10$

$.23540 \mathrm{E}=10$

$.51290 \mathrm{E}-17$

$51305 E-17$

$11039 E-1$

$.001065-18$

$.70220 \mathrm{E}-18$

$13731 \mathrm{E}-10$

$70239 \mathrm{e}-18$

$.10200 \mathrm{e}-17$

$.13733 \mathrm{E}-16$
$.30004 \mathrm{E}=16$

-35000E-18

. 2390 je=10

i $14000 \mathrm{E}-10$

$.01900 \mathrm{E}-20$

$.447506-19$

.4735 be- 19

. $15719 \mathrm{E}-19$

$.40800 \dot{0}-19$

$.60761 \mathrm{E}-19$

$.69560 \mathrm{E}-19$

$.15560 \mathrm{E}-1 \mathrm{C}$

$.40062 E-19$

$.77609 \mathrm{E}-19$

$.36604 E-19$

$30515 \mathrm{E}-19$

Oे $4 i j E-19$

.47475 t-19

$.33011 E-16$

$.093335-19$

$91770 \mathrm{E}-18$

$.91445 \mathrm{E}-19$

$.15107 \mathrm{E}-19$

$.08203 e-19$

$.62004 \mathrm{E}-19$

$.72030 \mathrm{E}=19$

$.09953 \mathrm{E}-19$

$.62027=-19$

$.60626 \mathrm{E}-14$

$.04761 k-19$

$.73172 \mathrm{~L}-19$

$.00730 E-19$

. $45099 \mathrm{E}-19$

$.45126 \mathrm{E}-19$

.25527E-19

$.25531 E-19$

$90271 \mathrm{E}-1 \mathrm{~s}$

$.45355 e-19$

-3U50गE- 10

$.00991 \mathrm{k}-10$

$.14546 E-10$

$.51409 k-18$

. $2970 N E-10$

$.9742 y t-19$

OUdOOE-19 3d 3TTE-10

$.20332 \mathrm{E}=10$

$.95167 \mathrm{t}-19$

$91705 E-19$

$.67247 t-19$

by 290 E -21

$.03703 \mathrm{e}-19$

.22004

$.0501 \mathrm{t}-19$

. $12100 \mathrm{t}-10$

$.42700 t=1 y$

$.140900=19$

43

$91406=-07$

$.15405 t=00$

$.15799 \mathrm{E}-\mathrm{uO}$

$.53172 k-07$

$.10163 \mathrm{E}=00$

$.20100 \mathrm{t}=00$

.10000E-00

$.23005 \mathrm{E}-00$

$.56931 z-07$

$.17132 \mathrm{c}-06$

$.27369 t=00$

$.42793 E=06$

$.45470 E=06$

$.30656 \mathrm{E}-00$

$.1777 \mathrm{iE-U6}$

$.33467 E=00$

.356445-U6

.37084 e- 06

01077 e-07

. $37722 \mathrm{E}=\mathrm{J6}$

$.774 \$ 2 E-00$

- $36196 \mathrm{c}=00$

- 3890rk-00

-301u<E-Jo

$.33403 \dot{0}=40$

$.39545 \varepsilon-06$

.30400E-UO

$.33504 \mathrm{E}=0 \mathrm{D}$

$.20404=-06$

$.20513 t-00$

$19165 E-00$

19 logt-00

$39425 \mathrm{E}=00$

$39344 \mathrm{E}=00$

$10523 \mathrm{t}=0 \mathrm{0}$

- $39210 E-00$

$.39132 E-00$

- $39173 e-00$

$.3940<E-00$

$.500490-00$

$.20130 c-1 y .17453 c-10$

$.52807 t-19$ 
iv nut nue NL NLt KLe

$17 \quad 15$

17134

$.39000 \mathrm{C}$ บUE๋น2

$.100300=19$

วน

$\$ 10$

1ง১

cus

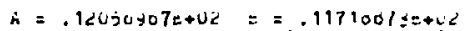

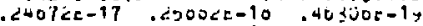
$.2<7+7 L=1$. T/4THL-10 e CUSEUE-10

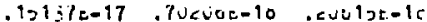
$14 i j 0^{\circ} 104072-10$ 10477 $10042 E=10$ 111 $117=-17$

$34903070=0$

.347127 YUL+ $+\mathrm{J}_{2}$ - 350YU $370 \mathrm{E}+0$ $.35 .330300 t+02$

$.354930 b 0 t+02$ $.35579710 \mathrm{E}+02$ $.35505020 E+02$ .35506170 it +02 . $35006250 E+02$

$.35000090 \dot{0}+02$ . 35031250Eं+02 $35630120 \mathrm{2}+02$ . 35653140E+0\% -3560́5210E+02

$\begin{array}{rrrrrr}4 & 2 & 3 & 4 & 0 & 4 \\ 11 & 9 & 3 & 11 & 7 & 4 \\ 11 & 11 & 1 & 11 & 9 & 2 \\ 12 & 10 & 3 & 12 & 6 & 4 \\ 13 & 11 & 3 & 13 & 9 & 4\end{array}$

$35060960 E+02$ . 350 ǴOலU5OE்+02 . $35746300 E+02$ . $35604590 E+02$

$\begin{array}{llllll}14 & 12 & 3 & 14 & 10 & 4 \\ 14 & 13 & 2 & 14 & 11 & 3 \\ 15 & 13 & 3 & 15 & 11 & 4 \\ 20 & 16 & 4 & 20 & 16 & 5 \\ 10 & 14 & 3 & 16 & 12 & 4\end{array}$

ISOPROP YL ALCONOL (Ch3) 2CHOH

$\begin{array}{ccccccc}\text { H3) } & 2 \mathrm{CHOH} & \text { y } & & & \mathrm{MW} & \text { * } \\ 5 & 4 & 1 & 5 & 3 & 2 \\ 0 & 5 & 1 & 6 & 4 & 2 \\ 4 & 3 & 1 & 4 & 2 & 2 \\ 7 & 0 & 1 & 7 & 3 & 2 \\ 3 & 2 & 1 & & 3 & 1 & 2\end{array}$

$.30732060 \hat{e}+02$ $.36736000 \mathrm{E}+02$ $37513200 \mathrm{E}+02$ $30571410 \ddot{2}+02$ .30627250E+02

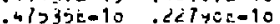

-i) $3<\tilde{y}+\varepsilon-1 c$.ovijae-10 $.4401 c=-1 y \quad .<00 / 1=-10$ $.53410=-10.504320-10$ .12u2verio +41sugc-10 $.20457 t-10.4047 U t=10$

.27yyze-10 .10yo9i-10 $.10<4 \mathrm{~J}=10.11310 \mathrm{l}-17$ $.20047=-10 \quad .77330=-10$ .5ว414t=10 .70ट30E-10

$.141<00-13$

. 1บวร4t-17

$.10521 \mathrm{E}-17$ .2y00 it-17 $.44 ? 34 \mathrm{E-17}$

$31791=-1 d$

ilogicoly

- cuvita-1: . $14+y 1=-10$ iyulle-10 . 2 sou $1 \mathrm{~s}-1 \mathrm{e}$ 17๖วue-10

- $03<U S E-1 Y$ . $2 u>\supset \$=-10$ $.201425=10$ 2105eE-io 3ं TODE-1E

1jOUAE-10 $.3 j 4 y d i=1 d$ $37744 \mathrm{k}-10$ . $300+3 \mathrm{E}-10$ $.37043 c-1 \mathrm{c}$ $30450=-17 \quad 13007 E-17$ $.4459: 4 E-17,14 \dot{1} 23 E=17$ $.70502 \varepsilon-17.15040 \mathrm{~s}=17$ $.34432 \mathrm{E}=17$.14 $142 \mathrm{Ut}=17$

$3521<E-10$ $.302520-10$ $3 \cup j 225=12$ $.35307=10$

$.15217=-17 \quad .12073=-17$

$.70097-17 \quad .15075 s-17$

$.71456 \mathrm{~b}-17 \quad .11410 \mathrm{t}-17$

71 1)

$.52300 \mathrm{e}=17$

. $11420 \mathrm{~L}-17$

7U420e-10

$.52329 \mathrm{E}=17$. 70430t-10

$.11230 \mathrm{E}-17$

15430s-16

$.01122 \varepsilon=10$

. $31069 \mathrm{E}-16$

$10201 \mathrm{t}=17$

$15304 \mathrm{E}-10$

$.04570 E=10$
$.06922 E-10$

$.14779 \mathrm{c}-10$

$.65463 \mathrm{t}-19$

.514 Uं $7 \mathrm{E}-10$

$65005 \mathrm{~s}-19$

.23041:- !o

$.30<90 \mathrm{t}-18$

$.60915 E-21$
$.27521 E-19$

$.017 \dot{\mathrm{E}} 1 \mathrm{\textrm {E }}-19$
- $39220 E-10$

- 30jdzi-10

$235 u$ ut- 10

$.255210-10$
$.135940-10$

$370006-10$

$.57000 E-19$

- 3 U $57 \mathrm{c}=10$

$29540 \dot{0} 10$

$.13914 E=10$

$.26110 \mathrm{t}-10$

$.15231 \mathrm{E}-10$

. 22059c-1d
$.13090 \mathrm{cos}$
$.27074 \mathrm{t}=16$

$5 y$ inu $=1.4000$

$.67275000 \mathrm{E}+01$

$.00005000 E+0$ ?

$.897000000+01$

$.92910000 \mathrm{e}+01$

$.93902900 \mathrm{0}+01$

$A=.04069900 \mathrm{~L}+01 \quad \forall=.00417500 \mathrm{E}+01$

$.41044 \delta-18 \quad .00950 s-19 \quad .143592-10$

$.30513 \mathrm{E}-1 \mathrm{~B} \quad .49143 \mathrm{~d}-19 \quad .93007 \mathrm{E}-2 \mathrm{C}$

$.40 \operatorname{coz}=10 \quad .79774 t-19 \quad .17355 t-19$

$.51972 E-10 \quad .37132=-19$.00736e-20

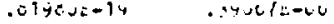

$\therefore=.0 / v+v+9 E+L 1$ nAF $=$

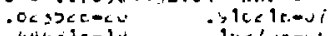

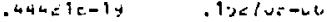

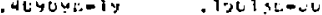

molpic-1y ix'

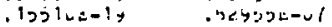

$.40+32 E-1 y$

$19 y+16-60$

40Jjle-1y

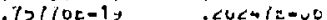

. $40<y j e=1$ y is

$.1534 . j=-1 y$

. $30 \mid 1<\varepsilon=4$

4cyabc-Ju

रि01

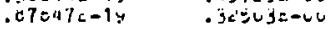

$.400450-19$

.yujzue-iy

$.4 U 430 z=1 y \quad .3032 i c=v 0$

. elijez=1y

. $01 j$ je-1y 3000 iteno

$.72 x+1=-1 . \quad$ spetsene

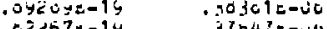

. $0 \geq 2575-14$.j754IE-NO

$.00252 c=1 y \quad .33 \mathrm{~J} 1 \mathrm{CL}-0 \mathrm{~V}$

$.7 \overline{103 E-19 \quad .359 \pm 4=-60}$

$.94 U i y=1 y \quad .3073 y=-U u$

. 0U57:z-14 . $\$ 1 \angle 4 E-6$.

$.4404 J E-1 Y$.2010 $2 E-V O$

$.4407 v e-1 y \quad .<0135 E-v 0$

.2ग300E-1y $109140-U$

. $253 y<x-1 y$

$.90031 x-19$

14yoot-1y

$.97379 \varepsilon-19$
$.904137 \mathrm{E}-19$

$\log 1 y \pm-00$

. olujue-ui

$.30730 \mathrm{vevu}$

-3oulueguo

$.941000-19 \quad .305010-10$

$.44090 \mathrm{e}=1 \mathrm{y} \quad .1043 y \mathrm{y}-40$

$.900 y 3 c-19 \quad .30045 t-10$

$.07421=-15 \quad .47004500$

. 00207e-1y .30ycye-1)

$\begin{array}{llllll}2 & 1 & 1 & 2 & 0 & 2\end{array}$

$.90727000 t+01$

- $102705102+02$

$11791300 \mathrm{v}+02$

$.11067090 \mathrm{E}+02$

$.12005900 \dot{02}$

$C=.47651900 \mathrm{U}+\mathrm{U} 1 \mathrm{nAr}=$

10
.7590

22000r,-20

$.27051=-20$

$.1724 y 0-20$

$.57134 \mathrm{E}-\mathrm{JO}$

$34025 \mathrm{~b}-20$

$.02314 \mathrm{y}=00$

$.35011=-00$

$.73063+-00$

. 52boyk-vo

$.2035 \partial t=18.22 v 31 t-19.37035 e-20$

$.3434 t e-10.920020-1 y \quad .21734 c-10$

$.37057 \varepsilon-10$ :1002je-10

$.30250 \mathrm{e}-10$. $42264 \mathrm{E}-19$

$.27244 \mathrm{E}-19$

$.71210 \mathrm{E}-21$

$46 y 53 c-10$.60290r-14.11540t-20

$.44204 \mathrm{E}=2 \mathrm{U}$

$.57403 \mathrm{e}-20$

$.1410 \cup e-20$

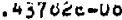

$.94044 \mathrm{E}-10$

.13 1Ube-U?

.7577 Ue -00

$.+0770 \mathrm{t}=00$

$.54011 \mathrm{E}=10 \quad .70277 \mathrm{E}-19$

. $90305 \mathrm{~s}-10 \quad 15934 \mathrm{E}-19 \quad$.2007E-19

$.94942 \mathrm{E}-17 \quad$ :15944E-18

.200yźc-19

. $2 \dot{y} \angle 00 \mathrm{E}-20$

$15 \mathrm{c} 33000 \mathrm{~s}+02$

$.15417000 \mathrm{E}+02$

$15654000 E+02$
$.22294000 E+02$

$10719 \mathrm{y}-17$

$75070 \mathrm{x}-1 \mathrm{y}$

$220 y 1000 \mathrm{t}+02$
$22704550 \mathrm{t}+02$

$.22704550 \mathrm{E} 402$

$.23404000 \leq 402$

$.24170000 \mathrm{~s}+02$

.13567 t-17 30090j-10

, 15753e-17

$.32000 E-19$

$.71504 \mathrm{E}-19$

$.04554 c-19$

$60144 \mathrm{E}-20$

. 30j07e-20

. $50540 t-20$

12172t-u?

2ट71OE-Ui

$.15324 \mathrm{E}-\mathrm{U} 7$

- $3015 \mathrm{t}-u 7$

$10740 t=14$

$.08037 t=07$
$.00953 E-07$

$57194 \mathrm{E}-2 \mathrm{U}$

$.00993 E-07$
$.74207 i-u 7$

. v00<つE-J?

$12050 \mathrm{~s}-1 \mathrm{y}$

$.03716 \mathrm{c}-u 7$

$.14007 \mathrm{j}-17.20494 \mathrm{E}-10$

$.402225=19$

$749900-20$

$.537,7$ t $-u 7$.

$.16650 \dot{0}-17$ 20501Ë-18 54700Ë-14 10430t-19

$.537276=07$

$.72477 \mathrm{~L}-07$

$.10240 \mathrm{t}-17$.30019t-10 :00015z-19:13004t=\$9

$.54140 \mathrm{E}-07$

$.24367200 t+02$
$.24401100 E+U 2$

$.140 / 1 \mathrm{~L}=11$ :20505E-10

$.40400 E-19 \quad .75252 E-20$ 
nut nes a.e nue

$\ldots$

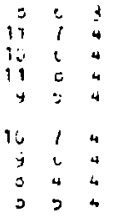

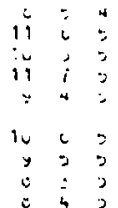

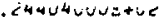

- ive ?ounuetus

- Evliquunetue

. Actounuuctue

- Pluajivitue

- siunoviune+ue

. 11 iqulJe+le

.11 yujuzitoc

. s1cavudatuc

\begin{tabular}{|c|c|c|}
\hline \multicolumn{3}{|c|}{ (ZH, IK-nur } \\
\hline$y$ & $y$ & $<$ \\
\hline 0 & $c$ & $<$ \\
\hline 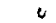 & $=$ & 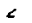 \\
\hline 1 & $i$ & $\bar{c}$ \\
\hline 0 & $i$ & $<$ \\
\hline$<$ & 1 & 1 \\
\hline$<$ & $<$ & لـ \\
\hline$<$ & $c$ & $?$ \\
\hline
\end{tabular}

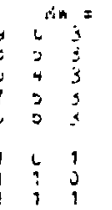

$\Rightarrow \quad \therefore u=.1900$ $.1350050 u a+l e$ - IL $116 b J e+v e$ - 10ट́ 14uvue+ue . 10754uvietul .195 ueviuetur

$.3<\mathrm{c} 14420 \mathrm{u}+\mathrm{UL}$ - $35104<6 u c+02$ .3马บUจ $2 C E+J 2$

- Inil rumiaze

$\begin{array}{lll}2 & 1 & 2 \\ 2 & \vdots & 4 \\ 2 & 1 & 1 \\ 3 & 1 & 5 \\ 5 & 4 & 1 \\ 3 & 2 & 5 \\ 3 & 4 & 1 \\ 3 & 1 & 2 \\ 4 & 2 & 1 \\ 4 & 2 & 3\end{array}$

$\begin{array}{lll}1 & 1 & 1 \\ 1 & 0 & 1 \\ 1 & 1 & 4 \\ 2 & 1 & 2 \\ 4 & 0 & 2 \\ < & < & 1 \\ 2 & 5 & 0 \\ 2 & 1 & 1 \\ 2 & 2 & 3 \\ 5 & 2 & \vdots\end{array}$

7c AU $=1.1000$ . 11457000 $.11075450 E+U \angle$ - 12 3 lulude+ula $.17101200 \mathrm{~s}+02$ .177 y4 100z+02

$.1702540 \mathrm{ve}+02$ $1705070 \mathrm{Ue}+02$

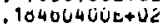
$.23009730=+02$ $.23701700 \mathrm{c}+02$

$.23059000 \mathrm{e}+02$

t

Crit

$\begin{array}{lll}2(L n u) 2 & \\ 7 & 2 & 2 \\ 2 & 0 & 5 \\ 1 & 1 & 4 \\ 2 & 1 & 1 \\ 3 & 1 & 5 \\ 4 & 1 & 3 \\ 5 & 1 & 4 \\ 0 & 0 & 0 \\ 5 & 2 & 3 \\ 0 & 1 & 5\end{array}$

$\begin{array}{lll} & \text { N* }= \\ 0 & 1 & 0 \\ 4 & 1 & 4 \\ 1 & 0 & 1 \\ 2 & 0 & 2 \\ 3 & 0 & 3\end{array}$

$72 \quad 11 \mathrm{~J}=1.0100$

$.13012200 t+02$

$.14107050 E+U$

- 17033050 a 42

- 1duod160z+v2

$.10734100 E+3 c$

$.19040000 t+v 2$

$20 d 35400 E+i$

$.20937000 \mathrm{t}+\mathrm{UL}$

. ¿145080ictoz

$.22320450 t+0 r$

$\begin{array}{lll}1 & 1 & 1\end{array}$

$.23149200 E+U 2$

VIMYLER Lameviale Ciñu 3

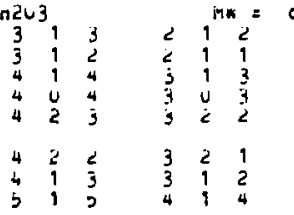

$00 \mathrm{HL}=4.5100$

$.19109020 \mathrm{U}+\mathrm{U} 2$

- $30370700+02$

$25355250 \mathrm{u}+42$

$20450130 \mathrm{u}+42$

.20151360 etuz

$.300192 .20 t+02$

$30409160 t+02$

$.31420000 \mathrm{t}+02$ $\dot{a}$

$x$

Stworat

Iis

$\because \longleftarrow j$

I4h JUE-1?

is $9=+10-1$ ?

isyoct-17

- 1ustye-i

$x<y+1 y x-10$

$.709 \vee 3 t-1 j$ $49,7<1 \mathrm{t}-10 \quad, \mathrm{ig}<\mathrm{it}-10$ $.40<0=10 \quad .1<04 y E-10$

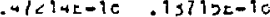

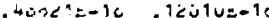

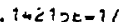

$10 \cup 44 \mathrm{E}-1$

viv $=-1$

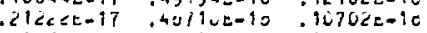

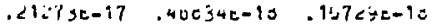

$A=.0400 \times y 000+012=.044175000+1$ $.140520-10 \quad .40304=-19$. 1U104E-13

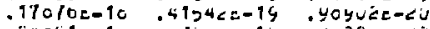

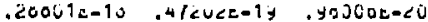

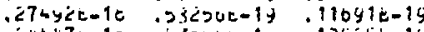
. $24 t+7 c-10$

$.900 j 4=-10$ $: 1<317=-17$ UUE-1,

$.135<5 t=14$

4h $900-10$

Yบ395E-17

$17700 \mathrm{us}-1 \dot{y}$ . $1430.12-10$

.10720 t-10

$A=.2034137 v c+02 t=.31041560 t+01$ . TY002E-10 . 2007ue-19 $.2 y 32 \cup 5=10$ $.220<0=-10$ . $35420 \mathrm{k}-10$ $.545 c 7 t=10$

\section{$30007 \mathrm{c}=15$}

. 3 Jotie-1y

$.013745-19$

$.94502 \mathrm{r}-1 \mathrm{y}$

$.3717 J E-20$

$.53000 \varepsilon-20$

$.42070 \mathrm{e}-<0$

$.147300-19$

$.17916 \mathrm{E}-19$

$.44022 a-12.51941 k-19$

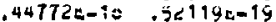

$.06535=-10 \quad .43590 \leq-19$

$.21112 \mathrm{c}-17$.22990r-10

$.900 ट 7+20$ $.90900 \mathrm{c}-20$ . $10991 \mathrm{t}-15$ $.4104 y t-19$

$.30>53 \mathrm{c}-19$

$10237 \mathrm{k}-10$

$.30753 c-19$

$.13505 k-17 \quad .10342 E-10$

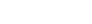

$A=.20391570 t+02 \quad b=.31041500 e+01$

$.922<15-19.12970=-19$

$.33+70 \mathrm{c}-10$.30U4OE-1Y

$.40310 \mathrm{E}-1 \mathrm{~d} \quad .41710 \mathrm{t}-14$

$.07047 t-1 \mathrm{t} \quad .71247 \mathrm{t}-1 \mathrm{y}$

.3j50UE-10 . 10j30E-10

$.25712 \mathrm{t}-20$

$.70221 \varepsilon-20$

$.74017 \pm-20$

$.12020 \mathrm{E}-14$

$.10707 \dot{\varepsilon}-19$

$.12414 \mathrm{E}-17$

. 1ל\$41t-17

$.13910 \mathrm{e}=10$

$07009 t-1$ s

$.20405 E-10$

.1797 Uer-10

.25565E-19

$.33500 \mathrm{E}-19$

$.19530 \mathrm{E}=19$

$.4090 y=-z$

1 CZOYE-17

$.25003 \mathrm{c}=19$

$.225420-10$

$.42000 \mathrm{E}-19$

$.40367 c=10$

$.47947 t-19$

$.05746 E-20$

$.15+4 c E-1 y$

$<y>34 c-1 y$

$<7<74=-15$

ijueje-iy

, $<J 44 E-1$

CO0:07E-15

$.<\mathrm{b}<0 \mathrm{t}-1$

$.<1537 \mathrm{e}-1=$

,$<13 y<2-1 y$

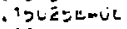

$.13040 \mathrm{~L}-\mathrm{NO}$

. i gyũe-lo

$i=.47001$ Y $=000+01$

. $21<33=-<0$

$.140 \% 7 \mathrm{i}-\leq 0$

1C77OL-EU

< $3431 L=<0$

$.27 b \dot{0} 7 \mathrm{~L}-<u$

$.31707 c-20$

$.43409 \mathrm{c}-20$

$.33544 E-20$

.70101 e... 7

. i

$.154 \mathrm{CZz}=\mathrm{UL}$

- 10sultave

14041 E

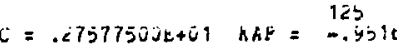

$.00271 \mathrm{a}-21$

$.93301=-21$

$704725-21$

$.20301 \mathrm{t}-20$

$.31950 \tilde{L}-2 \mathrm{U}$

$.17525 \mathrm{E}-20$

$.17503 t-20$

$.30437 t-20$

$.75057 \mathrm{E}-2 \mathrm{O}$

$.55724 \mathrm{~L}-\mathrm{CO}$

$.50009 t-20$

$.03554 r .40$

$.9434 \mathrm{dl}-\mathrm{ve}$

- 7colue-vo

$.27 \geq 10 \dot{\mathrm{i}}=\mathrm{u}$

- $33, y 9 \pm=07$

$.18994-07$

190 y $30-0$ ?

$.33752 \mathrm{e}-v$

$.03171 \mathrm{E}-\mathrm{u} 7$

$.0250<t-u 7$

$.030 U$ Ut-O?

$=.275775000+1) 1 \mathrm{khH}=-. .4510$

$.401 \dot{2} 4 c-21 \quad, 1 y 1000-00$

$12004=-20 \quad .09204 E-00$

$.13209 \mathrm{E}-20 \quad .32553 \mathrm{E}-\mathrm{U} 7$

$. \angle 2009 E-20 \quad .34500 \mathrm{k}-17$

$.330198=20$

$.370 \overline{3} 2 \mathrm{t}=07$

$46069 t-20$

$.00015 \mathrm{E}-20$

$.355932-20$

$.09993 \mathrm{E}-21$

$.70513 E-20$

$.42541 z-07$

$.49086, t-07$

$.2459<\dot{C}-37$

$7 \supset 007 E-50$

$.57^{75} 3 \mathrm{E}-07$

$49096 i t-07$

$A=.93407 y 00 E+J i \quad j=.41004600 E+01$

$.12004 t-10 \quad .13500=-17 \quad .24540 t-10$

$17903 \dot{E}-16 \quad, 16457 \mathrm{E}=17$

$24203=-10$ 32533c- 17

$33241 \mathrm{t}-18: 30037 \mathrm{E}-17$

$27321 \mathrm{e}=1 \mathrm{t}$

$.30537 \mathrm{~L}-17$

$.35204 \mathrm{E}-10$

$54496 \mathrm{E}-10$

$.07312 \mathrm{E}-10$

$.5015 \supset t-10$

$.31030=-16$

.35040:-17

$.004100-10$

$400790-10$

$.40035 t-17$

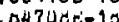

$.53240 \mathrm{H}-10$

.610 y $7=-17$

. $1: 492 \mathrm{E}-17$

$.152402-20$

71140

$=.20915400 E+01 \mathrm{nHA}=\ldots .59 \mathrm{C}$

4300 OE-19

. $3174 \mathrm{E}-1 \mathrm{H}$

$10097 \mathrm{t}-10$

- $12090 t-10$

10youk-05

$.154912=05$

$.10504 \mathrm{E}-0 \mathrm{~J}$

$.120200-10$

$.214205-1.2$

, $<740+16=0 ;$

$.3100 .4 c-v i$
AE IMILIAIOEIMYNE

$\begin{array}{cccccc}\text { CSCri3 } & & & \text { Rh } \\ 3 & 1 & 3 & 2 & 1 & \\ 3 & 0 & 3 & 2 & 4 & \\ 3 & 1 & 2 & 2 & 1 & 1 \\ 4 & 1 & 4 & 3 & 1 & 3 \\ 4 & 0 & 4 & 3 & 0 & 3 \\ 4 & 1 & 3 & 3 & 1 & 2 \\ 5 & 1 & 5 & 4 & 1 & 4 \\ 5 & 0 & 5 & 4 & 0 & 4 \\ 2 & 2 & 4 & 4 & 2 & 3 \\ 5 & 4 & 2 & 4 & 4 & 3 \\ 2 & 4 & 1 & 4 & 4 & 2 \\ 5 & 1 & 4 & 4 & 1 & 5\end{array}$

$72 \mathrm{AL}=1.0000^{\mathrm{a}}$ $.20050500 x+42$ $21550500 \mathrm{c}+02$ $22001700 \mathrm{E}+02$ $.2750500 U t+02$ $.20722350 \mathrm{E}+\mathrm{U2}$

$.3040726 L L+03$

$343244202 i+02$
$.35000200 z+02$

$30 \angle 2+050 E+02$

$303+1724 t+0<$

. 36́391720t+02

$.30014500 x+c 2$

$\dot{A}=.15953175 t+02 \quad B=.40027000 E+[1$

$.92935 \mathrm{E}-18.10446 \mathrm{t}-10$ 


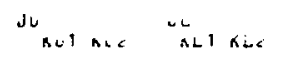

in

col

n ner

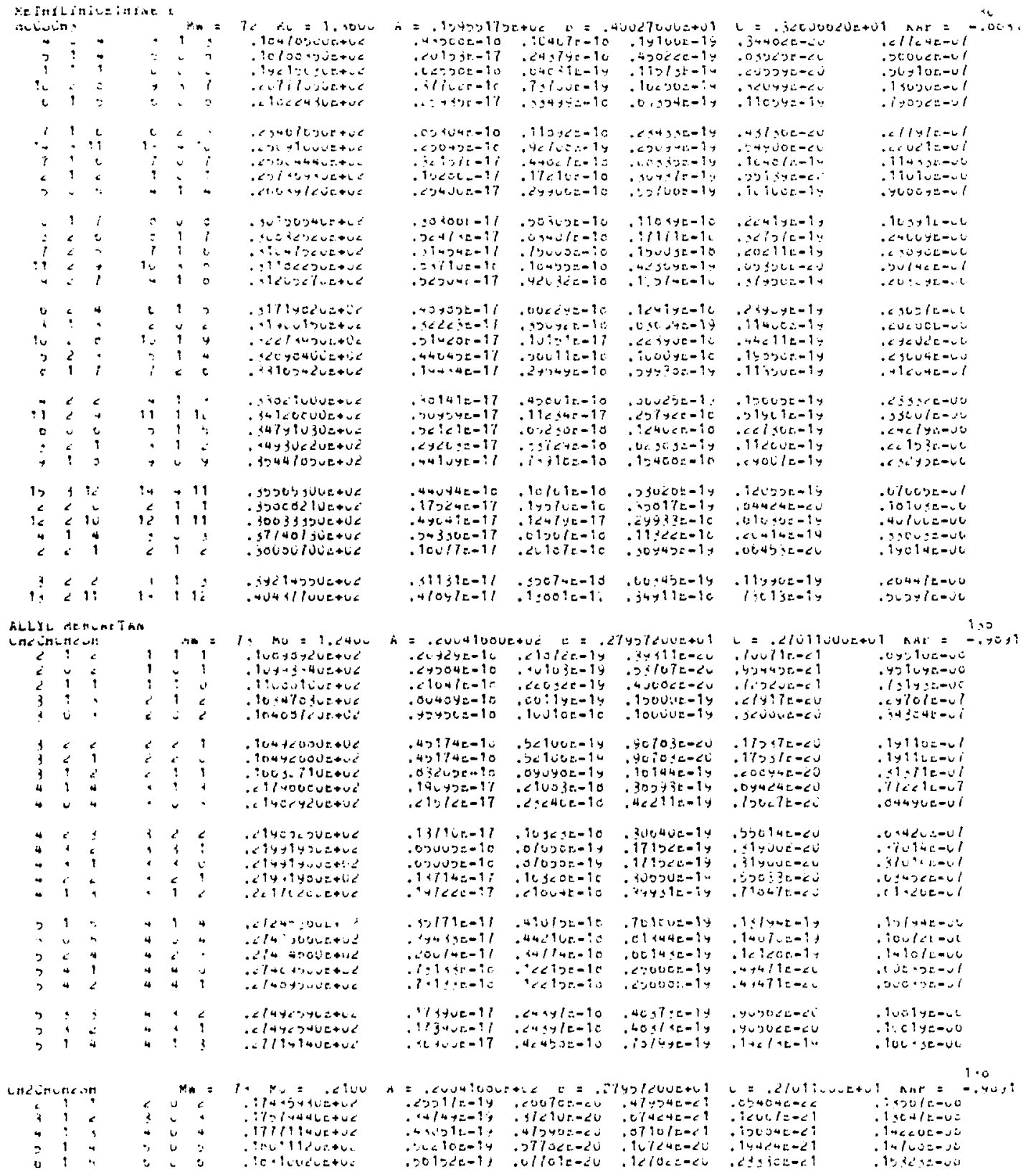




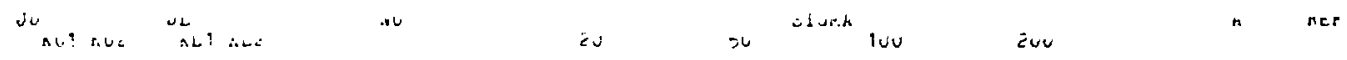

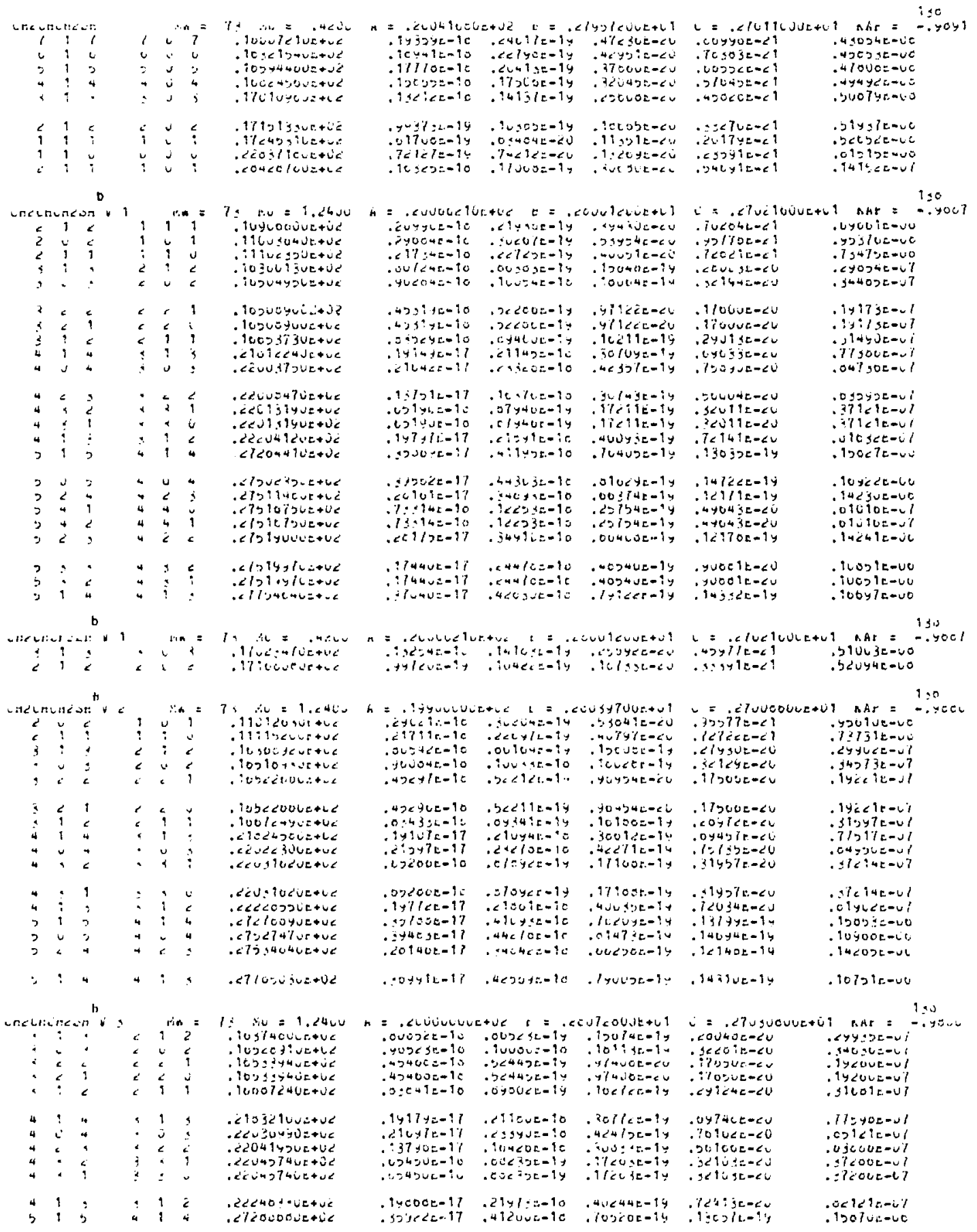




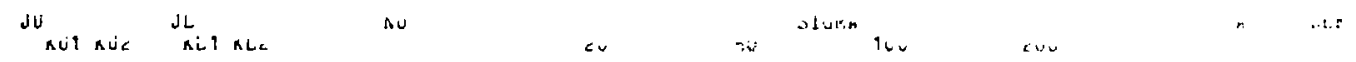

Me IMIL VINIL SULELLE

\begin{tabular}{|c|c|c|c|c|c|c|}
\hline \multicolumn{3}{|c|}{ H3sinens } & \multicolumn{3}{|c|}{$n *=$} & $7+\therefore L=1.14 \mathrm{C}$ \\
\hline & 1 & $t$ & 5 & 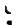 & 5 & 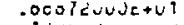 \\
\hline 2 & $v$ & 2 & 1 & 1 & 1 & . Tu<asyucteles \\
\hline 3 & $i$ & $c$ & ? & j & $=$ & $.197300 u v E+v 2$ \\
\hline 1 & 1 & 1 & $v$ & $v$ & $i$ & 19yidquivetue \\
\hline 4 & $:$ & $=$ & 4 & $u$ & 4 & 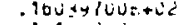 \\
\hline 4 & 2 & $c$ & 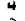 & $?$ & 3 & - $101<\angle \angle U U E+U<$ \\
\hline 3 & 2 & 1 & 3 & $i$ & $\leftarrow$ & . IOUUjouUtous \\
\hline ב & $<$ & $y$ & כ & $?$ & 4 & $.107270-v i c+02$ \\
\hline 2 & $\leqslant$ & $u$ & $<$ & 1 & 1 & .170 y>0JUEthe \\
\hline 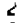 & 1 & $\dot{c}$ & $!$ & 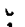 & $i$ & C L \\
\hline 2 & $c$ & 1 & $\dot{c}$ & 1 & 2 & $.<17<u$ buue $+u c$ \\
\hline 5 & $!$ & - & $?$ & $u$ & $?$ & .61716 JUQE+UC \\
\hline$j$ & $\leftarrow$ & $<$ & 3 & 1 & 3 & 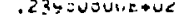 \\
\hline
\end{tabular}

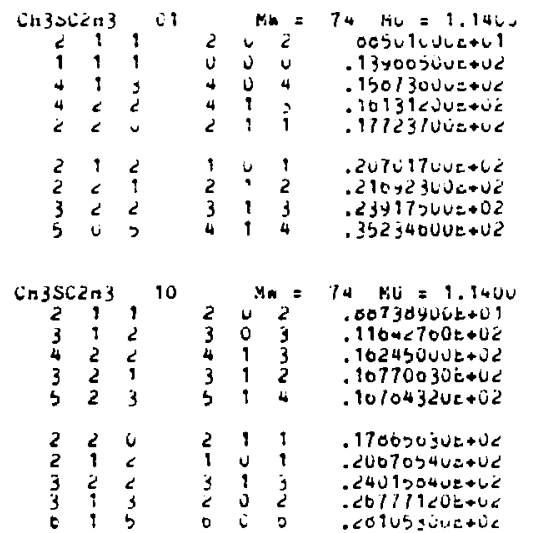

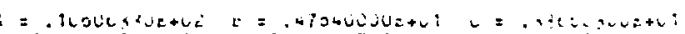

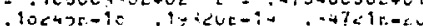

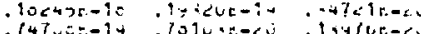

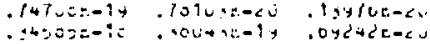
$.21 \angle 01 \mathrm{c}-1 \mathrm{c}$

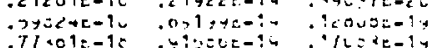

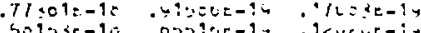

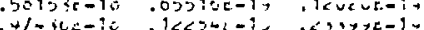

and : $:=-12$

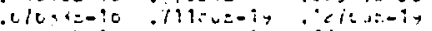

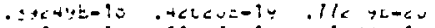

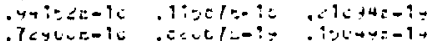

$A=$ luvyo7uvetud $2=.4104 /$ Luvatu:

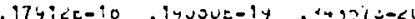

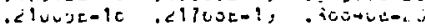
bo-7le-10 .0iyuze-1y itsoces

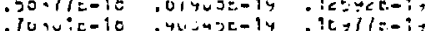

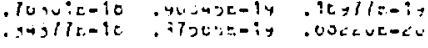

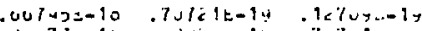
$.1007 / \mathrm{c}-10$, 4LCjuE-iy .707cil-co

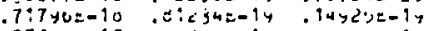
$.37100=-17$.45juas-10 enovecto

$h=.1002121 u c+42 E=.4730200 j e+U 1$

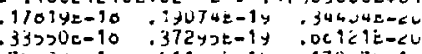

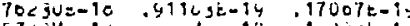

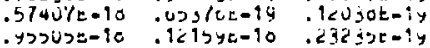

-3430ct-10 . 37740E-19 . UOU/4E$.0300 y=-10$

$.1150 y c-10$

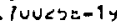

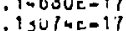

of 30ue-1y $14 y 77 \mathrm{t}=$

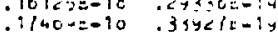

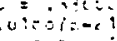

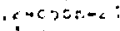

$\therefore$ acusecs

. $L$ yec ir $-x$ ?

riciteru

$\because$ ivatis $=-c$

$\leq i s+1 \mathrm{~L}-i \mathrm{C}$

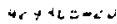

$1 c+1, z-c s$

$\therefore a t i t-c u$

$\therefore \because x>t=c$

c civtures
uUvite-ce

. 0 icyiciatel

: is $\therefore n r=-.2 n=$ icit 1.02

$\therefore \cdot a 1:-2$ $\therefore i, y, y=-i$ actiction $\operatorname{sen} 2-1$

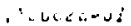
$c+c=0, i=0$ $c: 0+3 i=1 . i$ . $941=12=01$ a 4 ic : :

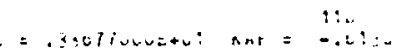
geveraten

$00 y=1 e-c i \quad i: \mid \cdot i c-u i$

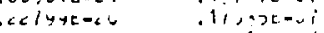

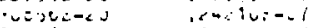

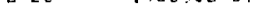

iresca-cu tavenic-ul

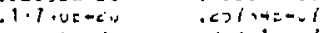

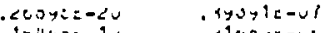
$124402-1, \quad .<100+5=60$

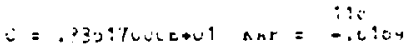

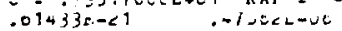
$.12<-1=2 \pi \quad$.00ulacele

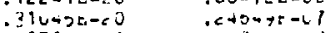
.$<17100-<3 \quad$.calueterl

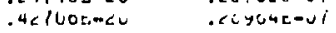

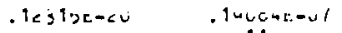

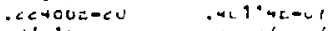

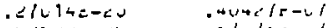

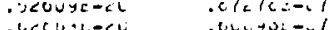

VIGYLALC IYLERE G 4 n

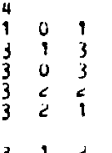

$\begin{array}{lll} & & M_{\text {A }} \\ 0 & 0 & 0 \\ 2 & 1 & 2 \\ 2 & 0 & 2 \\ 2 & 2 & 1\end{array}$

be $n=.4300$ - Yi $14120 v e+01$ ¿́o ouvunue $+0<$ $.2721<7$ ive+uc $.27220 v j u c+u 2$

$.2704030 v t+u c$

ME THYLALLELE

inzincine

$\begin{array}{lll}2 & 1 & 5 \\ 2 & 0 & 2 \\ 2 & 1 & 1 \\ 3 & 1 & 4 \\ 3 & 1 & 1\end{array}$

$A=. j 127 u j u i c i+c<E=.4144000 u j e+v$

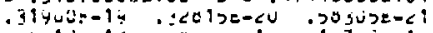
DEX12E-10. LOYJJE-1y. $1<70 j E-10$ $.713000-10$.0473un-1y .133yue-1y

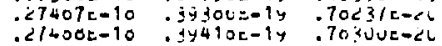

$1,0 y=-1 y$

$.04172 t=10.701075=14.039 y 7 c-1 y$

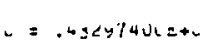

i $101-5$

- ing $350-<$ ?

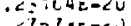

$1400: 20<0$

AHr $=-., 0<5$

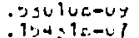

ic soue $=4$

1u $9412-01$

ivjore-ul

$1 / 1 j 1 c-21$

$H=.3300$ unduetue $0=.4 \angle U 120 u l e+v:$ . $81914 x-1 y$.0ys:9te-<u . 10<4ycaci iciles-1u icoyue-1y iez7ioc-2u

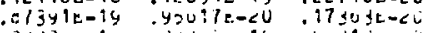

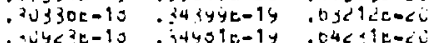
$.<>30 y c=<u$

$\mathcal{L}=.390011$ Uuetc1 nhr $=-.401$

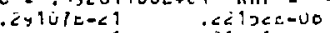

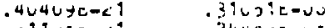

$\because 11 U C E-\angle 1 \quad .2440<2-U E$

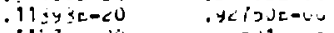

$.1157<\varepsilon-20 \quad .4+c y 1 c=0$

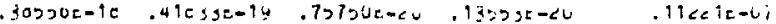

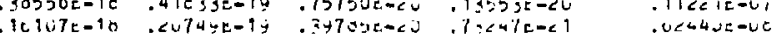

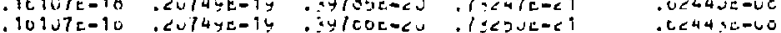

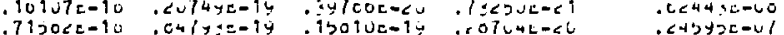

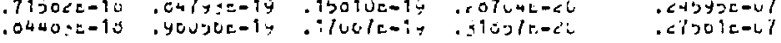

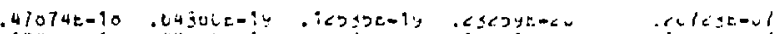

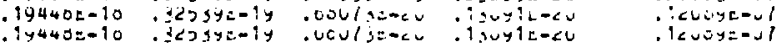

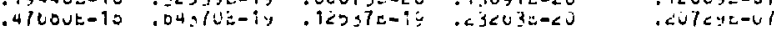

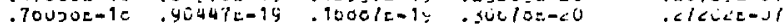

$32 \zeta 2, j 0 U i e+v$

. $3252 C T O L L+C L$

$=. \$ 1333<6 u e+01 t=.03017 \supset J u E+v 1$

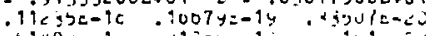

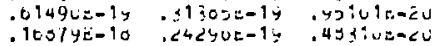

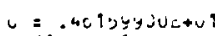

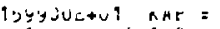

.Lj1+4L-C1 $j: 10 j L-U 0$

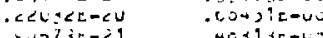

. =vilis-zi 
n. Les uni nes $\cdots$ $\therefore$

$.121-1+0 \times 20+0$

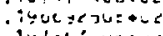
iylaciuvetue

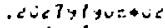
- cirluduatur

. $21411: 0+\infty$ - Civolusueve - Tulor sueve

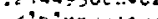
- C? Diyunustuc arizol bitene

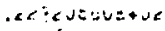
. $<x+102 y+2 t=2$ . ceपsicouctic . at tucturtur

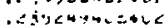

.

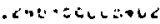

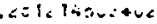

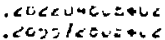

. celliveueque - टCIIDCluetue - cectuginetue

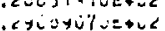

$. \angle y+w+0 y+0+v e$

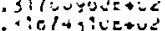
- iclativeruz
seuigyouetje

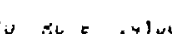

.11007 Curtal

$\therefore$ iclosyuctue

: ic la lavuetue

:77-7ove

. locavesucene

iyszi iytated

CUISAUL I

\begin{tabular}{|c|c|c|c|c|c|c|}
\hline & CL\$1 & C. 1 & & & $=$ & $\therefore=$ \\
\hline : & 1 & $:$ & $v$ & $v$ & עי & .131 yooyuetue \\
\hline o & $\dot{c}$ & - & 0 & 1 & $=$ & . igafoofulatuc \\
\hline ? & $<$ & $\dot{3}$ & כ & 1 & 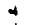 & 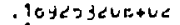 \\
\hline 4 & $<$ & $c$ & 4 & 1 & 3 & isughuzuetic \\
\hline ? & 6 & 1 & s & 1 & $<$ & 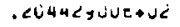 \\
\hline$<$ & $c$ & 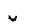 & $<$ & i & 1 & . Li1907'sueaus \\
\hline ; & 1 & 2 & c & $\therefore$ & 2 & . $\angle y y+\leq y \cup U E+\cup<$ \\
\hline & 6 & 5 & 4 & 1 & 4 & $.<5<5340 U E+J$ \\
\hline
\end{tabular}

cUIYiot-

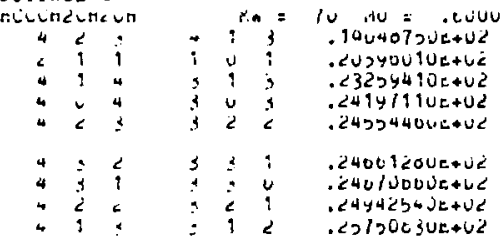
$\therefore: 0: u=-i$, - ivesic-is - icu?tt-1e

- intise-ic -ivoyie-10

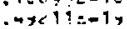

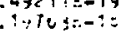

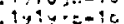

inctiz-ic - iceras-ic - CCE-Latic iyoutc-lo

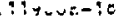

$.7 t / \cdot \pi=-17$

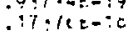
+ucese-i.

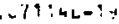
atidiselo

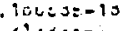
- 11 in: $10-10$

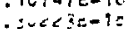
cousicoso

$.2010: 0-14$ य) $145=10$ $.00270 \mathrm{~T}-1 \mathrm{~g}$ - 4atusca-10 $\therefore 2$ ice-1s ju navin ing

Cosuve-1.

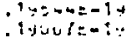

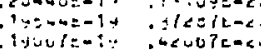
- Thagrat or itzoje-Lu

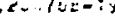

\section{- 1<x<atiy}

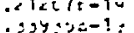
- $2951<=-1 y$

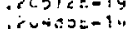
$\because 1 ; 735-1$

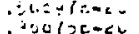
. InU/zL-1y $.45 z-9=-!$ $0+1012-: *$ - $4511:-i 4$

$.1<0 j y c e c 0$ isc17ate-i

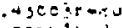
$.00 \operatorname{sultar}$ , 2501 y. $=c^{2}$

cosucus $=-1 y$

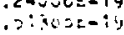

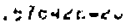
$+4 \times 0=25$

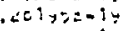
+4บวदz-1,

icy $y=-1 y$ Incive-is $0: 440 E=\angle J$ $.10 y 37 c-C$

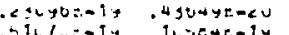
$.<1 / \angle 2=1 y+37 y+2-<1$ $901440-? y$

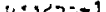
$137 y+2-20$ ingoits-iy

acac $1 \mathrm{c}-14$

: iviant-10 11990-iy $1: 4 i,\{2-1:$ 14ji:eti $.461 j i=-14$ incile-<u - UCUUCE-C

$\dot{n}=.1 u-00: v L+U 2 \quad D=.5903010 u t+w 1$

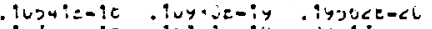

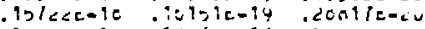
$1<a c+c-10 \quad .139 / 4=-1 y$.isysyl-ev

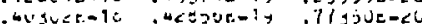

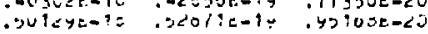

$.006045-10.26=00=-14$

$.4+0 O A=-60$

$n=$.iv4jujpuctue $r=.39000 / 000+4$ . OUUYUea in itudse-20

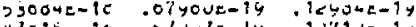

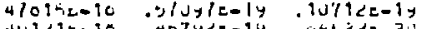

-

$.3940 c-1 y$. oivjje-cu

$.3950 c-1 y$

$.10 \mathrm{~s}<\mathrm{C}-10$

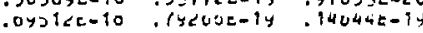

. UOA Tuc- i

- $54272-21$

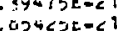

$.4072 y=-61$

in $\supset 0+c-<1$

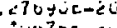

ivo7sece

$1: \rightarrow i C t-<0$

.$<0147 E-<y$

i $1 / 34$ je-co

$\because 1+1=-<1$

$.119 \$ t r=<u$

$<\lim >0-<v$

1 1you $u=-<v$

$i=7 u i c-c v$

. In gutencu

.7ylelcere?

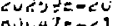

- ट3244lE-<

. Sugsuemo

- AwuTyt-cu

CथuYic-<u

$24,92 \mathrm{E}=<0$

. ग $1430-20$
$.34 / 260+5$

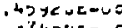

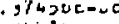

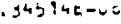
. HUJYCE $=0$

- J4 y0E-Jo . $5340 y 2-6$ - yeozoc-ue . ODHL $=5=U$ colpoe-ue

. Milie-uc .11 j’z=-x! . ivlege-jt . yutyutedt - गu<tut-vu

- $>=i L<$ L , $1<277=-21$ $.0 j y 30<-i 0$ $.110<y c=u t$

.77 gue $-6 \mathrm{c}$ $.1+j 4<2-01$ $.103135=u c$ ivilyeat $.1707 y c=u l$

- cultionar - $3 \cos c=-41$ $.33<152-0\}$ . COSY3E-U 7

$=270074$ iucoul aAr $=124$

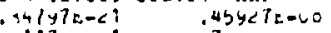

2117 YE- 1 .7UOCYE-VO 4iojilt-<i .0<3jus-vo

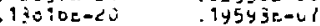
$10 y / 0=-20 \quad$ ingitit-ul

$.10 \div 23 t-c b \quad .<05 y 0 E-0 ?$ 124

$i=$. CTOUJAUUE+L? Rat $=-.0371$

, $25 \times 74 t-c 1$

$<$ ict<t-20 Iybu7c-ev iluyfor-cu

- custove-vo

194 sot-ci?

igitet-u

10 $14 \mathrm{E}-\mathrm{U}$ ?

$02541 \mathrm{e}=28$

isioue-L?

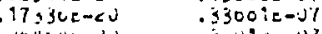
$.<0404 E-20 \quad: 3+49 \pm E-L^{2} 7$

A :.10430jjus+uE $E=.33002700 \dot{E}+U 1$ $.15170 \mathrm{e}-10.17307 \mathrm{t}-1 \mathrm{y}^{-314} .31+\mathrm{e}-\mathrm{cu}$ $.14212=-10$.1410uE-1y .20+77t-co

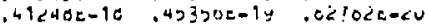

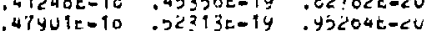

$.340<0 t=10 \quad .35404 t-1 y \quad .7<+302-\angle 0$

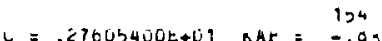

$100590=10$

$10073 \mathrm{c}-10$

3200je-ic

$.2244<E-19 \quad .4 \angle 2 U \angle E-\angle O$ $\dot{c}<430 \mathrm{E}-1 \dot{y} \quad .42 \dot{z} 34 \mathrm{k}-<\mathrm{C}$ $.4075 \div 10-14 \quad .750130-20$

$.57703 \mathrm{t}-28$

$.47110 \mathrm{E}-\mathrm{Cl}$

$14070 \mathrm{e}-<0$

$1704<2-20$
$13104 E-20$

-7UCTYE-UJ

Tu903e-J? CIYYUE-U? - $20<100-4\}$

$10947 \mathrm{E}-\mathrm{C} 1$

.11 Uuje-í

$.1<<<4 E-v i$

- lecsoest

- etc 7 utenil

ey 7 Ge-U?

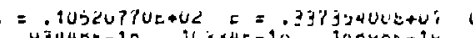
$=.212297$ ULE+01 nAT $=2.0404$

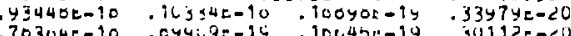

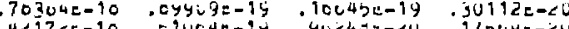

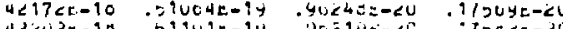

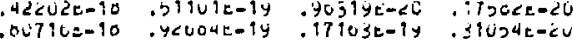

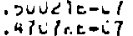

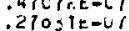
<7oúce-ut

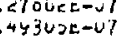

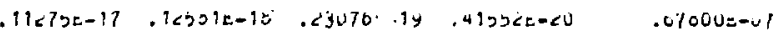




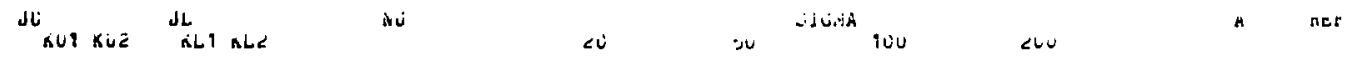

\begin{tabular}{|c|c|c|c|c|c|c|}
\hline \multirow{2}{*}{\multicolumn{3}{|c|}{ nCCCri2inann }} & $v 1$ & 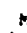 & $=$ & .0500 \\
\hline & & & 7 & 1 & o & $.100 j 717 u=+v<$ \\
\hline 0 & 2 & 4 & 0 & 1 & 3 & $.10727210 c+02$ \\
\hline 5 & 2 & 5 & j & 1 & 4 & $.19195070 \varepsilon+02$ \\
\hline 4 & 2 & $\dot{c}$ & 4 & $\mathbf{t}$ & 3 & $.19 y 1,1 j u n+U z$ \\
\hline$y$ & 2 & 7 & $y$ & 1 & 0 & . $2010040 v c+U c$ \\
\hline 3 & 2 & 1 & 3 & 1 & 2 & $.20720: 10 \mathrm{t}+\mathrm{U} 2$ \\
\hline
\end{tabular}

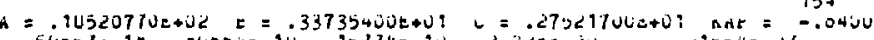

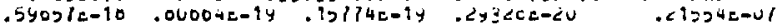

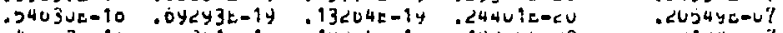

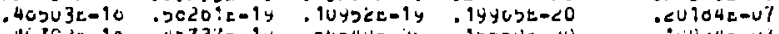

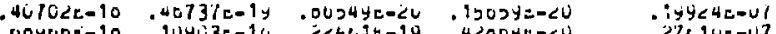

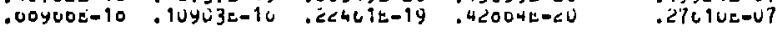

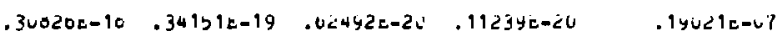

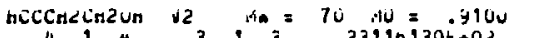

$A=.10004300 c+02 \quad t=.33015060 c+01$ $.92050=10 \quad .10239 c-10$.1070UE-19 $.771<1 c-10 \quad, 090<5 c-19 \quad .16,15=-19$

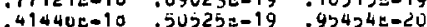
414

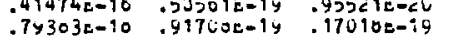
124

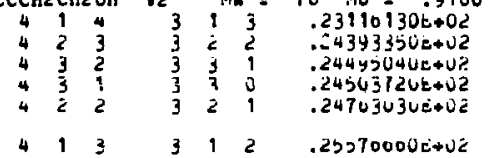

$C=.<7440 J U U k+U 1$ AAT $=-.0429$ $.4077 y=-07$

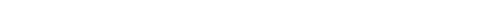

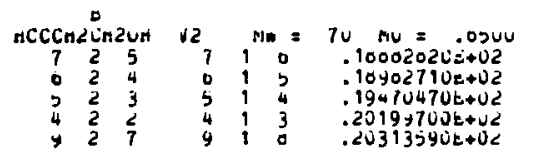

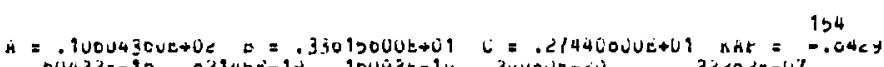

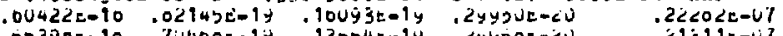

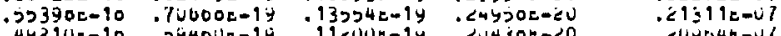

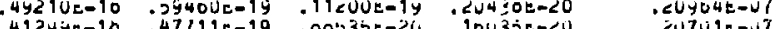

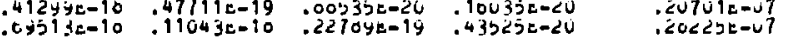

CROTONONI IRILE Cri3CHCHCN InAHS, Ha $=07$ al $=4.3500$

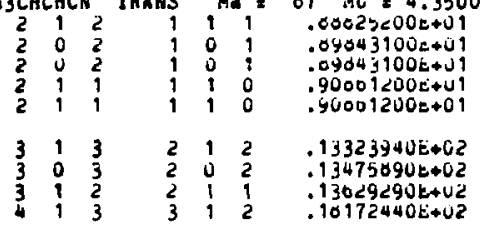

PYnIHIOENE C4N2 14

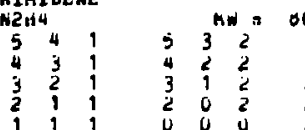

$110=2.3340$ $.81 \cup 11900 t \rightarrow 01$ $.03433700 t+01$ $.069302006+01$ $.04500200 \mathrm{E}+\mathrm{U1}$ $.09500200 \mathrm{E}+\mathrm{U} 1$
$.93013500 \mathrm{E}+01$

$\begin{array}{lllllll}2 & 2 & 1 & 2 & 1 & 2 & 99771700 t+01 \\ 4 & 4 & 1 & 4 & 3 & 2 & 90329440 z+02\end{array}$ $\begin{array}{llllll}4 & 1 & 4 & 3 & 2 & 103294402+02 \\ 5 & 1 & 5 & 4 & 2 & 10073150 e+02\end{array}$ $75:-743 \quad: 14015520 \mathrm{~s}+02$ $\begin{array}{llllll}2 & 2 & 5 & 3 & .14945500 k+02\end{array}$ $\begin{array}{lllllll}3 & 3 & 2 & 5 & 2 & 3 & .15175320 t+02 \\ 4 & 2 & 2 & 4 & 1 & 3 & 15310900 t+02\end{array}$

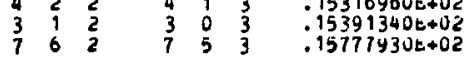

2 hHIHOPYNIUINE

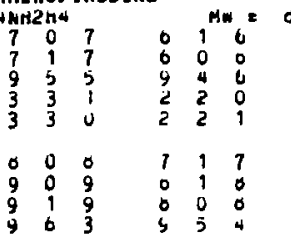

$62 \mathrm{NU}=.0000$ $.27710040 \mathrm{E}+02$ $.20030490 E+U 2$ $30502500 t+02$ $31128170 \mathrm{c}+02$ . $31310700 t+02$

$31511000 \dot{0}+02$ $35205 \% 00 \bar{t}+U 2$ $33330010 t+02$
$.37274100 t+02$

\begin{tabular}{|c|c|c|c|c|c|}
\hline NC4NGien 4 & \multicolumn{2}{|r|}{ i 1} & \multicolumn{2}{|c|}{$\mathrm{NH}=$} & \multirow[b]{2}{*}{ 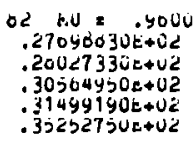 } \\
\hline $\begin{array}{ll}7 & 0 \\
7 & 1 \\
9 & 5 \\
0 & u \\
9 & 0\end{array}$ & $\begin{array}{l}7 \\
7 \\
? \\
0 \\
y\end{array}$ & $\begin{array}{l}0 \\
0 \\
9 \\
7 \\
0\end{array}$ & $\begin{array}{l}1 \\
0 \\
4 \\
1 \\
1\end{array}$ & $\begin{array}{l}0 \\
0 \\
0 \\
7 \\
0\end{array}$ & \\
\hline $\begin{array}{ll}9 & 1 \\
9 & 6\end{array}$ & $\begin{array}{l}y \\
3\end{array}$ & $\dot{8}$ & $\begin{array}{l}0 \\
5\end{array}$ & $\begin{array}{l}6 \\
4\end{array}$ & $\begin{array}{l}.35310330 E+02 \\
.37202940 E+C E\end{array}$ \\
\hline
\end{tabular}

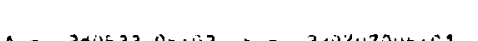
$=.300533,0 E+022=.2 \angle 97 u 70 u t+c 1$ $.20105 \mathrm{E}-17$.20001t-1C .4\%830t-1y $.19770 \mathrm{e}-17$ :207j0e-10 :375700-19 1977 ut- 17

.7327 ๖ะ 17 $.73275 E-17$
$.91528 E-17$
$.70500 \mathrm{~s}-17$ $.70500 z-17$ $207506=10$ - 37570 - 19

$70339 \mathrm{E}-10$ $.01922 t-10 \quad: 14932 t=10$ $.20120=17$ :37UU7E-10

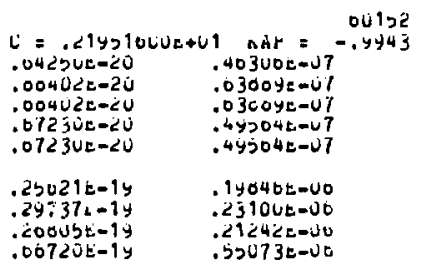

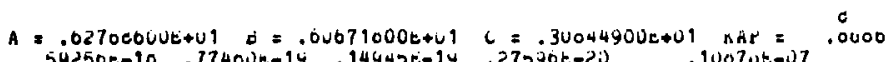
$.59250 e-10, .77400 k-19 \quad .14945 \varepsilon=1 y \quad .27590 k-20$ $.53007=-10.0417 b=-19.12020=-19$

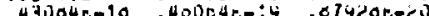
$.20720 E-10 \quad: 20273 E-19$. 500102-20 $.336325=10$

$.21071 k-20$ $10012=-<0$ $\rightarrow \forall 0056-<1$ $.100190-20$ 107005-J7 -14.23nc-0 7 $030445-U a$ $.173398-07$

$.27032 t=10 \quad .29264 t-19 \quad .52010 k-20.43004=-21$

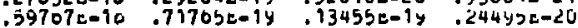
$.69410 \mathrm{t}-10 \quad .91109 t-19 \quad .17011 \mathrm{t}-1 \mathrm{y} \quad .32545 \mathrm{E}-20$ $.14245 t-17 \quad .23255 t-18.40243 t-19.92300 t-20$ $.14047 t=17 \quad .21340 \mathrm{E}-10 \quad .42479 x-14 \quad .79000 \mathrm{e}-20$

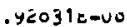
$140 j<\varepsilon-j ?$ $.17<200-U 7$ 4y $457 \mathrm{E}-u$ ? $.49003 c-u ?$

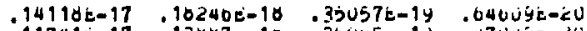
$.11741 \mathrm{z}-17 \quad .13007 \mathrm{E}-10 \quad .25405 \mathrm{E}-1 \mathrm{y} \quad .47042 \mathrm{E}-20$ $73234 t-10.000020-19$ 14094e $=14$ $.157075-17 \quad .25590 \mathrm{E}-10 \quad .53131 \mathrm{E}-19$

$203700-20$

47 ONOL -47 $.4<13<\varepsilon-U$ $.30163 E-07$ $.2 Y 43 \cup 5=07$

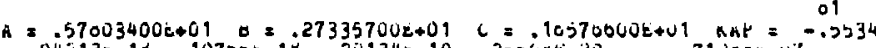

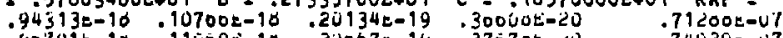

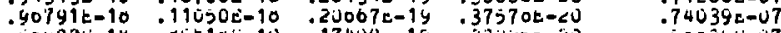 $.50002 E-10$.05010E-19 .17499L-19.332COE-20 .50025E-UT .j0050t-10 .59514E-19 .10020E-1Y .1941JE-2U

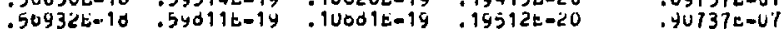 \\ $.13206 t-17,15072 t-10 \quad .30137 k-19 \quad .55215 k-20$ $.17540 E-17$.22057E-10 :4259Ge-1S :70710E-20 .17540E-17 .22057E-10 .4259GE-19 .ob912E-10 :102y2E-1a :2130dt-1y \\ $.10071 \mathrm{c}-50$ . $790495-00$ .4uy30ट-20
.OY}

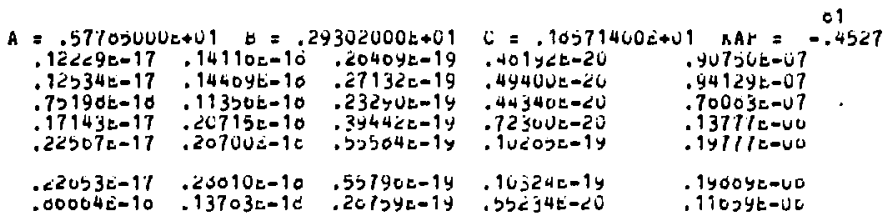


Ji nut kUE SL SLI ALE Ais

20

Sivitif 100 coi A net

IUAA LCMCUC2A

$\begin{array}{cc}3 & 4 \\ 10 & \text { io } \\ 4 & 3 \\ 3 & 5 \\ 2 & 1\end{array}$

$\begin{array}{rrr}5 & 4 & 2 \\ 10 & 10 & 3 \\ 4 & 3 & 2 \\ 3 & 2 & 2 \\ 2 & 1 & 2\end{array}$

Ue $M U=.0010$ . $1255275 U \mathrm{~L}+\mathrm{UL}$ $.12504040 E+02$ $.13030>900+02$ $.1343123 U \mathrm{UE}+\mathrm{CL}$ .13727 Ouva+ué

$\begin{array}{rrr}17 & 15 & 2 \\ 1 & 0 & 1 \\ 2 & 2 & 1 \\ 3 & 3 & 1 \\ 4 & 4 & 4\end{array}$

$1715 \quad 3$

c 0 u

i $1 \leq$

$.13914100 \mathrm{c}+0 \mathrm{Z}$ .13917700 Ue+02

$.14335340 \mathrm{e}+02$

140 objout +02

151 $1757 \mathrm{JE}+02$

10142

$\begin{array}{lll}2 & 2 & 1 \\ 0 & 0 & 1\end{array}$

1210

10143

$5 \mathrm{~d}$

12105

$.95230570 \mathrm{t}+0<$

$.157775 \times 0 \mathrm{t}+\mathrm{L} 2$

$.19011400 \mathrm{e}+02$

1 y 107 yojetoz

$.200<45400+02$

$\begin{array}{lll}11 & y & \\ i u & 0 & \\ y & 7 & 2 \\ 0 & 0 & \end{array}$

119

1003

y 73

० 10 i

$\begin{array}{rrr}0 & 0 & 3 \\ 10 & 0 & 2\end{array}$

$.20037710 \varepsilon+02$

$.21317 y$ ? Ut $+0<$

.219043 uve +02

224509 y $0 x+02$

$.2254 \mathrm{~J} \angle 70 \mathrm{U}+\mathrm{UL}$

$\begin{array}{lll}7 & 5 & 2 \\ 0 & 4 & 2 \\ 5 & 3 & 2 \\ 2 & 1 & 2\end{array}$

$\begin{array}{lll}7 & 5 & 3 \\ 0 & 4 & 3\end{array}$

$\begin{array}{lll}3 & 3 & 3 \\ 4 & 1 & 1\end{array}$

$.22010920 \mathrm{c}+1 \mathrm{de}$

$.23055000 \mathrm{~L}+\mathrm{L2}$

$.23213450 k+02$

$.23305000 \mathrm{0}+02$

$\begin{array}{lll}3 & 1 & 2 \\ 3 & 2 & 2 \\ 4 & 3 & 2 \\ 5 & 4 & 2\end{array}$

31

$\begin{array}{lll}3 & 1 & \\ 3 & 0 & 3 \\ 4 & 1 & 3 \\ 5 & 2 & 3\end{array}$

$.23352470 t+02$

$.23384400 \mathrm{E}+02$

$.2340253 \mathrm{UE}+02$

$.2345 \$ 130 \mathrm{k}+\mathrm{CL}$

$\begin{array}{lll}0 & 5 & \\ 7 & 0 & \\ 0 & 7 & \\ y & 0 & 2 \\ 10 & y & \end{array}$

- 33

. ¿3507710e+02

$2301900 \mathrm{Uc}+02$

$.23790730 \mathrm{U}+02$

$.24043000 c+02$

$24999770 \mathrm{~s}+02$

$1111 \quad 1 \quad 1192$

$\begin{array}{lllll}11 & 10 & 2 & 11 & 0 \\ 12 & 11 & 2 & 12 & 9\end{array}$

NiETHACRCLEIN

CinLCCH3LrJ

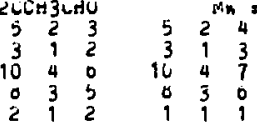

$.24707500 t+02$

$.24000970 x+02$
$.25041040 t+02$

$70 \mathrm{AL}=2.0700$

$.797053000+01$

$.05630000 \mathrm{E}+01$

$07773300 \mathrm{e}+01$

.114 éd $10 \mathrm{E}+\mathrm{UL}$

$.13290070 t+02$

$\begin{array}{lll}0 & 2 & 4 \\ 4 & 1 & 3 \\ 2 & 0 & 2 \\ 2 & 1 & 1 \\ 9 & 3 & 0\end{array}$

$\begin{array}{lll}0 & 2 & 5 \\ 4 & 1 & 4 \\ 1 & 0 & 1 \\ 1 & 1 & 0 \\ 9 & 3 & 7\end{array}$

$\begin{array}{llllll}3 & 1 & 3 & 2 & 1 & 2\end{array}$

$\begin{array}{llllll}7 & 1 & 3 & 2 & 1 & 2\end{array}$

$\begin{array}{llllll}5 & 1 & 4 & 5 & 1 & 3 \\ 2 & 0 & 3 & 2 & 0 & 2\end{array}$

$\begin{array}{llllll}3 & 2 & 1 & 2 & 2 & 0\end{array}$

$.13425000 t+02$

$.14005250 e+02$

$.14427130 \mathrm{k}+02$

$.1617+760 \mathrm{~V}+\mathrm{U}$

17yogjout+0z

$.19772240 c+02$

$20044500 \mathrm{E}+02$

$.20463410 \mathrm{E}+02$

$.2095084 \mathrm{Ne}+02$

$.22105040 t+C 2$

$.23259700 E+02$

$.24042520 t+C 2$

DIAETRYL RE'TEHE

\begin{tabular}{|c|c|c|c|c|c|}
\hline $\begin{array}{c}6031 \\
14 \\
17 \\
20 \\
\dot{4} \\
4\end{array}$ & $\begin{array}{c}24 c \\
5 \\
0 \\
7 \\
2 \\
1\end{array}$ & $\begin{array}{r}0 \\
4 \\
11 \\
13 \\
4 \\
3\end{array}$ & $\begin{array}{r}14 \\
17 \\
20 \\
6 \\
4\end{array}$ & $\begin{array}{lc} & \operatorname{man}= \\
5 & 10 \\
0 & 12 \\
7 & 14 \\
2 & 5 \\
1 & n\end{array}$ & 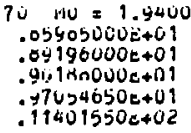 \\
\hline $\begin{array}{r}2 \\
2 \\
15 \\
2 \\
18\end{array}$ & $\begin{array}{l}1 \\
3 \\
5 \\
1 \\
0\end{array}$ & $\begin{array}{r}2 \\
1 \\
10 \\
1 \\
12\end{array}$ & $\begin{array}{r}1 \\
1 \\
15 \\
10\end{array}$ & $\begin{array}{rr}1 & 1 \\
0 & 1 \\
3 & 11 \\
1 & 0 \\
0 & 13\end{array}$ & $\begin{array}{l}12070040 E+02 \\
.13020100 \mathrm{0}+02 \\
.13937 \text { y0t }+02 \\
.14301190 t+02 \\
.14439400 \mathrm{U}+02\end{array}$ \\
\hline 3 & 1 & 3 & 2 & 1 & $.17930250 \mathrm{t}+02$ \\
\hline
\end{tabular}

$A=.94471001) t+01 \pm \pm .4<4000000+01$ $.13029 t-10$ 140ubt-2y $330730-20$

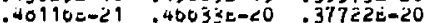
$.13491 \mathrm{E}-10 \quad .17759 \mathrm{E}-14 \quad 34274 E-200$ $.120 c 1 c-10 \quad .14203 c-1 y .205020-20$ .ouyyita-19 :00390t-2u $\$ 16025 t-20$

$.11011 \mathrm{~L}-20$.7UU4Uk-20 .40654E-2U $.109<3 E-10: 1129 y t-19$

$.02290 t-19 \quad, 40<13 \mathrm{E}-20$

$12544 c-10$ 1400Je-19

- ¿djoyc-ic

$.27102 \mathrm{E}-19$

$.24122 E-20$

. $10301 E-20$

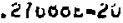

$.523 U \supset t-\dot{C}$

$.27157<-20 \quad$. 10<y

$.03720=1 y \quad .223 b 3 z-14$

$40747=19.3205<\bar{c}-13$

.0U54OE-1Y

200<ל⿺-18

$.60775 t=1 y \quad .3 y 952=-19$

$.100925-10$

$101+\angle E-10$

$2<294=-1 d$

$.41107 \mathrm{E}-19$

$.47111 \pm-1$

$.00933 \mathrm{k}-2 \mathrm{U}$

$43 y 30<-20$

$.11507 \mathrm{t}-1 y$

.12047 t-1C

$.13217 t-1 y$

. 14UJeE-19

- 57043

$.20524 \mathrm{E}-10$

$.33404 \mathrm{E}=10$

$.59712=-19$

$.5320 y 2020$

$353906=16$

$.43250 t-10$

$.501305=19$

$.13470 E-19$

$.12305 \mathrm{k}-1$

$104 y 0 t-19$

$.322192-10$

$.40200 \mathrm{E}-1 \mathrm{y}$

$.41617 \mathrm{E}-10$

$.03550=-20$

$.7901+E=20$

$.21649 \mathrm{E}-16 \quad .25110 \mathrm{c}-19$

$.21607 \mathrm{~L}-10$.25150E-1

$.32393 t-10$.41047e-19

$35840 c-10 \quad .52974 E-19$

$.46459 \pm-20$

$.45545 E-20$

$.00259 c-<0$

$449120-10$

. $40051 \mathrm{E}-19$

$.00540 E-20$

$34317 t-10$

297/7 $5-10$

$.54019 \ddot{E}-19$

$.0<400 k-19$

$125 \cdot 10-19$

$17000 \mathrm{t}-10 \quad .55321 \mathrm{E}=1 \mathrm{Y} \quad .1557 \mathrm{TEL}-19$

$.12502 t-10$

$.0463 \mathrm{CE}-10$

1 boyac-1

$20300 t=19$

$.50733 E-20$

$03434 t-19$. $40742 t-19$. $154545-19$

$.02003 E-1 Y \quad .42209 t-19 \quad .14919 k-19$

$h=. d b 123900 b+01 \quad=04030000 e+01$

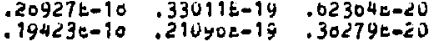

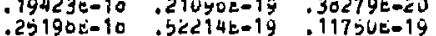

$.44920 k-10 \quad$ : $72100=19 \quad: 14901 t-19$

$.12505 t-17$. 13122t=1t .23407E-19

$.54907 \mathrm{E}-10$

-3ýं $14 t-10$

19541k-17

$.10425 \mathrm{E}-1\}$

$.72104 E-19 \quad .13953=-19$

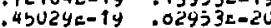

$.20357 E-10$. $30340 E-19$

.1 y331t-10 $.346725=19$

$70295 \mathrm{E}-1$

$.46974 \mathrm{t}-17$

$290000-19$

$.93289 t-10$

$60993 E-10$

$.57042 E-17$

$.503 \div 3 E-10 \quad .91000 t-19$

$132405-10 \quad 20347 \mathrm{t}=19$

$.02913 E-19$. 15500k-1Y

$.01002 \mathrm{E}-12$

$15500 \mathrm{E}-1 \mathrm{Y}$
$.11172 \mathrm{t}-10$

$.70702 k-19$

$7 c 502 t-14$

$67090 \mathrm{k}-17$

$.43052 E-10$

$13300=1$

$.73730 \mathrm{O}-10$

$=.02070320 c+0 j \dot{z}=.30041010 E+01$

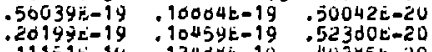

$.11151 \mathrm{E}-19,12400 \mathrm{~d}-19$

$1447 \mathrm{Ut}=10,10437 \mathrm{E}=19$

. $49365 \mathrm{t}-20$

$12077=-10$

. $13503 k=18$

- $3232 y=-20$

$.24773 \mathrm{~L}-20$

$.40735 t-10$

$.20+73 \mathrm{c}-19$

-

.

$223 \mathrm{c}-1$

$403126-15$

$.71467=-19$

- 1ubbyc-1y

127 y $1=-1 y$

$.103 d 3 E-17$

$.352436-19$
$54009=-20$

0

$=40700000 a+61$ AAR $=$

- $25051 E=10$

$.20349 \mathrm{e}-\mathrm{ve}$

.20004 - 20

.21 परे 7 E-UE

$40793 e-29$

$.20050 \mathrm{E}-\mathrm{E} 1$

$.30017=-00$

$.25304 \mathrm{E}-00$

.4067 ic-Uo

- $50013 \mathrm{E}-21$

$.43730 t-00$

$9700 k-20$

. 35011 r-uo

$.41010 t=C O$

. $80135 \mathrm{~L}-\mathrm{vo}_{0}$

$.00135 E=00$

-110

- $70750 \mathrm{~L}-0 \mathrm{O}$

$.100525-07$

$3 \neq 91$ ye -20
$34442 \mathrm{r}-20$

$.111 .40 t-17$

. $12525 t-u 7$

$.45504 t-60$

$.12008 \mathrm{k}-07$

. $12090 \mathrm{E}-\mathrm{U} 7$

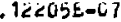

-1) 19de-U7

10090E-07

$.76020 \mathrm{k}-\mathrm{UO}$

.79003c-00

- 1 100Źc-UT

. $12400 \mathrm{c}=0$ ?

.20090k-U?

$.244105-20$

$.20112 E-20$

$.132610-07$

. 15741e-U7

- T4020E-0?

$.14231 \mathrm{E}-07$

1507 e-20

40107 telo

$14504 \mathrm{e}-\mathrm{T}$

$.14794 \mathrm{E}-07$

. $372045-c 0$ 


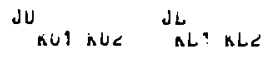

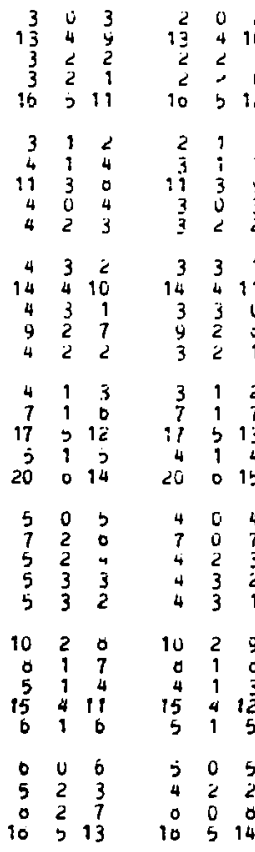

DIVINYL ETAEH

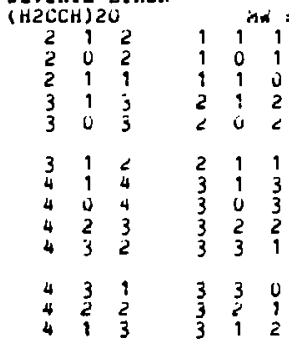

UIVINYL EIAES D (n2CCH) 20

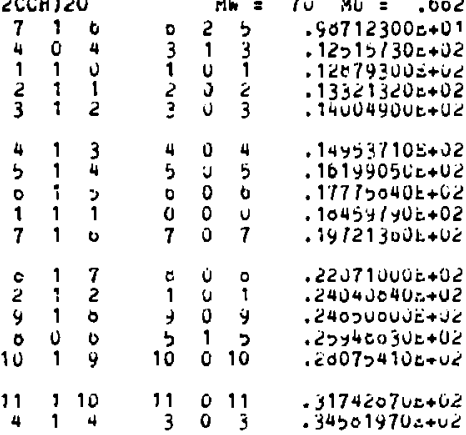

$.190703108+02$ $.1 y+22100 \mathrm{e}+0 \mathrm{U}$ $.1403011 \mathrm{ve}+\mathrm{J} 2$ $. \angle 0001130=+42$ $.20737300 \mathrm{~s}+\mathrm{C2}$

$.21434040 \mathrm{C}+02$ .237 yyuoúztü

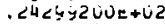
- $247339 i v a+b 2$ $. \approx 0294 y y 0 \mathrm{C}+\mathrm{ULC}^{2}$

20792 yyut +02 $\angle 00301006+02$ $269223400+42$

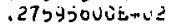
. zOU Túvene+v2

$.202905 y \mathrm{UL}_{L}+U 2$ $.2003000 \mathrm{je}+u<$ $.207000 L U E+02$ $.29450720 \mathrm{~V}+02$ . 3UU 144UUE+Cia

. $30143400=+02$ .32100300E+ +32 - 320ट 14YUE+0z

$.33551540 E+02$ $.33453000 \mathrm{E}+02$

$.34313500 \mathrm{0}+02$ - $34510>00 c^{2}+C 2$ - 34C4249UE+U2

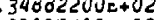
$.35107010 \mathrm{c}+02$

- $3540030 \mathrm{VE}+02$ . 35520000e+02 $.36523600 e+v 2$ $.374774 \cup 0 x+U 2$

$70 \mathrm{Ku}=.3490$ $.1159300 a+02$ $.12012200 e+02$ .1245:IUSOt+ue $.17301210 E+02$ .$: 7$ y $90010 \mathrm{C}+0 \mathrm{C}$

$.10073 \supset 70 t+v 2$ $.23101400 \mathrm{E}+0 \mathrm{C}$ $.2343532 \dot{U} x+U$ $.24037940 k+02$ .2400 yOYOL+UE

$.24070040 c+02$ $.24974000 x+02$ $.24004370 j+02$
¿ง

$3 t$

- Luria

ivo

Lu n.t.

\begin{tabular}{|c|c|c|c|c|}
\hline $\begin{array}{l}2230 z=-17 \\
.2 j 404=-1 c \\
1340 i=-17 \\
14442=-17 \\
13934=-1 c\end{array}$ & $\begin{array}{l}.24<0 j c-10 \\
.02470=-1 y \\
.14020=-10 \\
15100=-10 \\
.046 y 1=-1 y\end{array}$ & 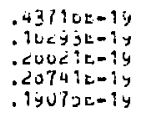 & 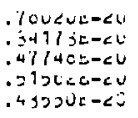 & 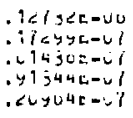 \\
\hline $\begin{array}{l}250 j 15-17 \\
4<005 E-17 \\
4 \angle 205 E-10 \\
47090 \therefore-17 \\
34002 \mathrm{E}-17\end{array}$ & $\begin{array}{l}.37 y<1 E-1 c \\
.4000<=-10 \\
.07 y<0=-19 \\
.5<374 E-10 \\
.4450 \supset E-10\end{array}$ & 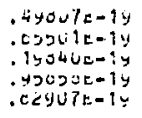 & 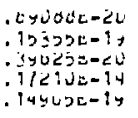 & 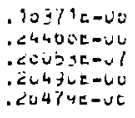 \\
\hline $\begin{array}{l}2<72 \partial E-17 \\
.3150 y E-10 \\
22 y 3 C E-11 \\
.25051 t=10 \\
.42054 E-17\end{array}$ & 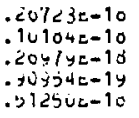 & 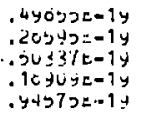 & 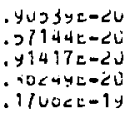 & 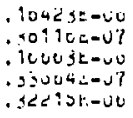 \\
\hline $\begin{array}{l}50311 c-17 \\
.40443 c-10 \\
11743 c-i 0 \\
77035 c-17 \\
.70410 \mathrm{t}-14\end{array}$ & 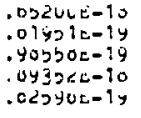 & 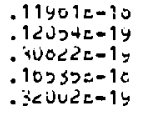 & 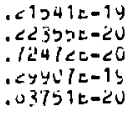 & 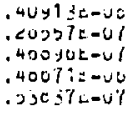 \\
\hline $\begin{array}{l}.0<4 y 4 z-17 \\
.54007 c-10 \\
70000 E-17 \\
00 \cup 73 \varepsilon=17 \\
.01500 t-17\end{array}$ & $\begin{array}{l}.94511 t-10 \\
.72490 E-19 \\
.45030 E=10 \\
.74140 \varepsilon=10 \\
.70040 E-10\end{array}$ & 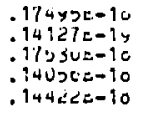 & 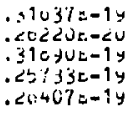 & 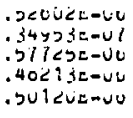 \\
\hline $\begin{array}{l}72735 c-10 \\
.00170 t-10 \\
10313 E-10 \\
.36535=-10 \\
1<245 E=10\end{array}$ & 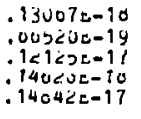 & $\begin{array}{l}.2007 \cup E-14 \\
.17201 L-1 y \\
.2201 y=-10 \\
.4 U 21 \zeta E-14 \\
.21 \times 345=10\end{array}$ & 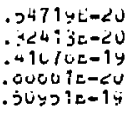 & 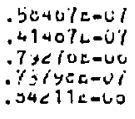 \\
\hline $\begin{array}{l}120<7 t=10 \\
.93454 c-17 \\
.0539>c-10 \\
.20701 c=10\end{array}$ & $\begin{array}{l}.1523 \partial c-17 \\
.11 i j 4 c-17 \\
.9420 j=-1 y \\
.13007 c-10\end{array}$ & $\begin{array}{l}.0007 c-1 c \\
.21612 E=1 c \\
.100 \angle 0 L=1 y \\
.40144 \delta-19\end{array}$ & 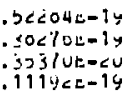 & 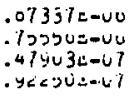 \\
\hline
\end{tabular}

$A=.1300 y 000 \mathrm{c}+02 \quad E=.3221200 \mathrm{~J}=+01$

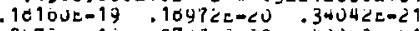
$.20752 c-19 \quad .2747<E-20 \quad .49009 E-C:$ $.20395 E-19.21071 \mathrm{t}-20.34207 \mathrm{E}-\mathrm{L}$ . oyoude-19 . .74je7t-2u .134yic-<u $.00170 \mathrm{e} 19$. YU011t-20.10341t-C

.7 Yó40L-19.05034E-Z̈0 $10441 \mathrm{E}-10 \quad, 10<09 \mathrm{c}=i 9$

ig 1ybe-10 .2uj30s-1y

$12005 e-10$ :15090e-1y $.64030 \mathrm{i}-19$

$.051 U \angle L=\angle 0$

$.1500 \overline{3}=-20$ $.33315 E=<6$ . $3010 j \mathrm{t}-\angle 0$ . $20101 \mathrm{t}-\mathrm{c} u$ , $15 y 7 \exists x-20$

$i=.21 \div u j 4 v i z+u 1$

$.040375-19 \quad .05104 E-2 C$

$1<9775=10$. i io701e-10 .2093ite-19

$15 y 7 y=-20$ $.304120-20$
$.0 \mathrm{~J} D 2 C L-\angle 2$

clusze-2i

QYYYJE-CZ

$.24120 \mathrm{E}-2$ :

. $2 y 14+E-21$

$.270355-21$

- $0 \ddot{y}$ y $14 \varepsilon-<1$

. 00इ亏ye-<1

. $: 1350-21$

. $\measuredangle$ ४⿻コ一

Cy $4040-21$

- DIDYOL-C? ivuouc-ci
LOC54 $2-U Y$

. YO2 JuE-Ly

. $C \angle 15 O E-U 4$

. $20357 \mathrm{c}-\mathrm{u}$

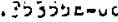

. 3 ว 100 $. T 33 c 4 c-v=$

. vosuoa-vo

. CoOj4E-cio

$.30443 E-v 0$

$.30444 E-U G$

- vUว32z-vC

- yiUiviL-ve

\begin{tabular}{|c|c|c|c|c|}
\hline 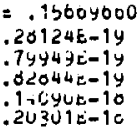 & $\begin{array}{l}+02 \quad D= \\
.3001 y=-20 \\
.01203 z-20 \\
.05170 z=20 \\
.14740 z=1 y \\
.2102 y z=1 y\end{array}$ & 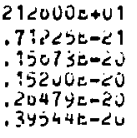 & 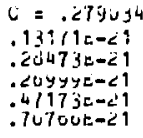 & 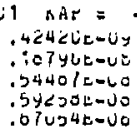 \\
\hline 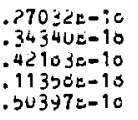 & 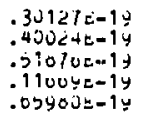 & $\begin{array}{l}.5233 t-20 \\
.74401 t-<0 \\
.95<00 \mathrm{E}-20 \\
.200<2 z-20 \\
.12103 \mathrm{E}-1 y\end{array}$ & 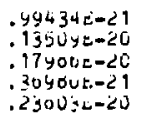 & 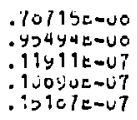 \\
\hline $\begin{array}{l}500030-1 c \\
2010<0-10 \\
06000=-10 \\
53504=-10 \\
73007 \varepsilon-10\end{array}$ & 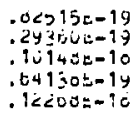 & $\begin{array}{l}10 j 40 E-19 \\
.220795-2 u \\
.20040 E-1 y \\
.12047 c-19 \\
.25713 E-19\end{array}$ & 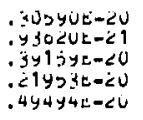 & 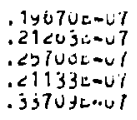 \\
\hline $\begin{array}{l}7 y 37 \leq-1 \varepsilon \\
x 157=-10\end{array}$ & 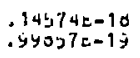 & $\begin{array}{l}.31 b 50 E-1 y \\
.10<0 \text { Ye-19 }\end{array}$ & $\begin{array}{l}.01741 c-20 \\
.320 j 0 E-<U\end{array}$ & $\begin{array}{l}.44122 L-U 7 \\
.00 U 0 C E-U 7\end{array}$ \\
\hline
\end{tabular}




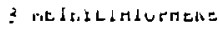

\begin{tabular}{|c|c|c|c|c|c|}
\hline$<$ & : & $<$ & 1 & $\therefore$ & \\
\hline 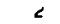 & $u$ & 2 & $i$ & $j$ & \\
\hline כ & $i$ & 4 & 3 & : & \\
\hline 5 & $\vdots$ & 1 & $?$ & 1 & \\
\hline & & & & & \\
\hline 1 & $u$ & 5 & $c$ & $\Psi$ & \\
\hline u & 1 & , & 0 & 1 & \\
\hline \pm & $<$ & $\leq$ & $<$ & $\varepsilon$ & \\
\hline & 1 & $\begin{array}{l}1 \\
4\end{array}$ & 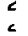 & $i$ & \\
\hline 4 & 1 & 4 & $\vdots$ & 1 & 4 \\
\hline 4 & is & 4 & $j$ & $u$ & \\
\hline 4 & $<$ & $y$ & ; & $\dot{c}$ & \\
\hline 4 & 3 & 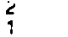 & 3 & $\frac{3}{3}$ & \\
\hline 4 & l & $\varepsilon$ & $\therefore$ & $<$ & \\
\hline 4 & 1 & 5 & 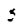 & 1 & \\
\hline$?$ & $i$ & " & 4 & 1 & 4 \\
\hline$j$ & $v$ & 2 & 4 & $\dot{v}$ & \\
\hline 2 & $<$ & 4 & 4 & 2 & \\
\hline כ & $=$ & $s$ & 4 & & \\
\hline 3 & 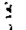 & $<$ & 4 & $\vec{j}$ & \\
\hline 2 & $a^{2}$ & $j$ & 4 & 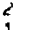 & \\
\hline$\stackrel{2}{2}$ & ? & - & 1 & & \\
\hline
\end{tabular}

Lon

\begin{tabular}{|c|c|c|c|}
\hline 1 & $<$ & 9 & 1 \\
\hline$\checkmark$ & $c$ & $i$ & j \\
\hline ! & 1 & 1 & 1 \\
\hline$i$ & 3 & $c$ & 1 \\
\hline 1 & $<$ & $<$ & 1 \\
\hline 1 & ه & 3 & 1 \\
\hline $\mathrm{J}$ & 4 & 3 & \\
\hline$i$ & $i$ & $\dot{j}$ & $\hat{u}$ \\
\hline
\end{tabular}

1 rendeh ins Cisiugatro

\begin{tabular}{|c|c|c|c|c|c|}
\hline & wat & & & & \\
\hline 2 & 1 & 2 & 1 & & \\
\hline$<$ & $u$ & $\therefore$ & 1 & $v$ & 1 \\
\hline & $i$ & $t$ & 1 & $i$ & \\
\hline & 1 & I & $c$ & 1 & \\
\hline & ১ & $\ddot{3}$ & 2 & v & \\
\hline & 1 & $a^{\prime}$ & $c$ & 1 & \\
\hline & 9 & 4 & 3 & 9 & \\
\hline & : & 3 & 3 & 1 & z \\
\hline & $?$ & כ & 4 & $?$ & \\
\hline & 1 & 4 & 4 & & \\
\hline & 1 & c & 2 & i & \\
\hline & 1 & 2 & $b$ & & \\
\hline & 1 & is & s & 1 & \\
\hline
\end{tabular}

LIIIE THYLALLEARE

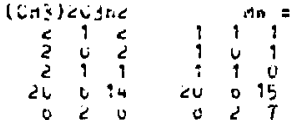

$17 \div 12 \quad 17>13$

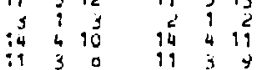

$\begin{array}{rrrrrr}11 & 3 & 0 & 11 & 3 & 7 \\ 3 & 0 & 3 & < & 0 & 2\end{array}$

$3 \div 5<25$

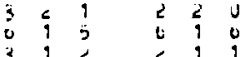

$\begin{array}{lllll}3 & 1 & 1 & 1\end{array}$

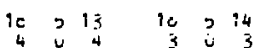

$2 v$

30

$i \%$

$2 i v$
Do $B 0=.7 \hat{200}$

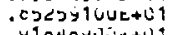

- H1040yuue+v?

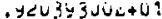

. liy 1 i $770 e+0 z$

.12044 couetuz

- ir?u?uluetie

. 1<07 ivivetuz

.13110ycuetue

$.137071500+02$

- ijuz jusuz+0z

. locueujuetuz

. 17124 NULE+LL

.17<louvenetue

itrigyoguetis

$.17 u s b y+u c t u a$

. 10270iviz+ve

- iguyucoutedie

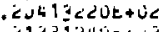

- c13:124uetua

- L1051buive+uz

. L17U40.Juetus

.2237 yeuvetue

. Ez719yourtue
$.790 .3<x u_{x}+c .9$

190

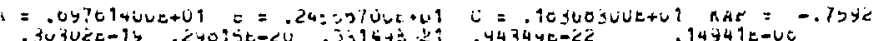

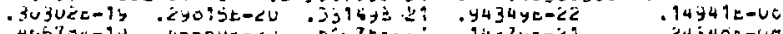

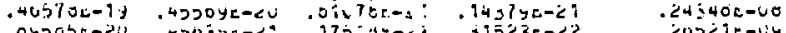

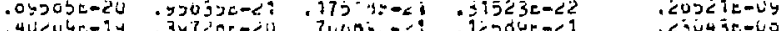

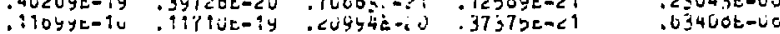

$.14030 c-i 0$ $14170 \mathrm{c}-9 y$ $.02<u$ ut 19 .ojloje-jy . 15400=-10

- $1+70<\mathrm{c}=19$

$.27 y$ Tla-ic . $32015 c-10$

.2buive-1c

. 1 . bulle-ic

$.14: 19 x=10$

$.12720=-26$

. ́20400lícic

15C<C4E=< $.04<20=-\angle 0$ . 15jloe-14

ij7oir-as

. 7 y Tye-zo

$$
\text { . ccoule-19 }
$$

$.510 j \vdots 0-20$

- $33<0<2-19$

- $20300=-1$

$: 1521 j=-19$
$\therefore=30<-14$

OUUวयt=cu

. 4oulet-zú

$.<0<52 E-20$

.2ut:oe-14

$.31247=-c u$

- $<0753 e-10$

$.30603 \mathrm{E}-10$

.jc7uje-ie

$.2000 \mathrm{t}=19$

$.532<3 \pm-19$

. $10120=-1 y$

$.11100=-15$

मीय

1)

$.217 \div 3=-16$

. JUUSER-19

$.00000 t-20$

.30zyee-1a

$.30 j+7 c-10$

$.42403 \mathrm{e}-19$

.80060te-20
- SúvuJe-<2

Li7jix-16

$.11374 c-19$

. OChaueric

. T100yt-3y

$.1 j 304=-19$

.47 if U Uc-2?

a? becaca

- L7، Tueazi

- cuitor $05-21$

$\therefore+0,4 t-\angle 1$

. Q4000E-VO

$.5 \angle 4015-U y$

. SUUłje-de

-j<073e-ve

. Y70 jet-ue

, y<27 1 $1 \mathrm{x}-21$

. 16⿺j5c-u?

. 1 y 7 y3t-u?

$.10470 \mathrm{c}=07$

- yoobje-10

- y $4<34 t-v 0$

. Suyog=-<

bivo7c-ci

. 1c 100e-u 7

. ¿4y y

. 3<72ue-u 7

. 34 21 1E-u?

. 30444E-vi

¿240<OL-UT

. $243<1=-01$

$.4 \angle 19$ L $=u$

.jo10be-u?

ov Bu = $=1.0460$

$.07 x+7300=+31$

opoyluuveti:

.13047 yuvitic

1135:0Jujetue

.77ýtoliulatul

. $7750 / 22 j e+6 e$

ir Tolduuctic

- cu3777puz+ú

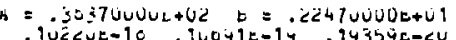

- 1UE<UE-10

. 1 0 y $1=-1 ;$

inover-1s

- Sycuse-10

- 1 गुण

$.20037 c-2 v$

. 4ट2:9E-14

zu<jo:-<i

$.413 c 4 c-1 i$

$.94 y 6<x-10$

inuver-iy

$.70 y 200-200$

$.1: \angle\{y=-17$

goygit-10

1uर 7 y -10

. Oujuocect

- i17ivive-10

, 1YUTUE-14

. दोisise-1y

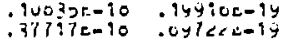

$\therefore 50040=17$

$00 \mathrm{mu}=.2790$

. Ocujolluietu

.02 voüsuvatui

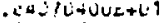

$.123 u c r 10-a b 2$

- izujoc oucaliz

$.1<01244 v E+c<2$

$.1649139 i \mathrm{l} c+v 2$

$.10013730 \mathrm{ut}+42$

,$\angle N=: 37<U E+0<$

- élulotuve $u$ ue

.24095 yutituz

$.2029 y 3 / U E+02$

$\dot{A}=.31$ juvuluetu

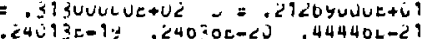

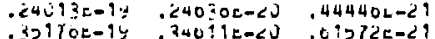

. $2=10 / 0-1 y$

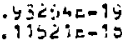

$.230>4 L-\angle U$. $4004 L E-<1$

$473 y i c-4 \nu, 17 L 74 c-20$

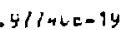

.2UObjE-CL

$.10<17=-19 \quad .102425-20$

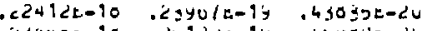

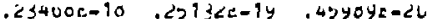

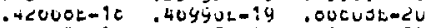

- Unulitit-10

. 4y<27L-19

$.9164 y=-26$

.7-30it-1c

oujige-1y. $120120-i y$

$.730 u i e-1 t .04134 k-1 y \quad .15741 t-19$

$.10457 t=17$

$154000=10$

$.24031 \mathrm{k}-19$

$14026=-20$

$\because \angle U \geqslant C E-\angle U$

$c=$. isjuatuutei

MAT $=-, y \times 47$

- $34034 E=21$

. 470ule...

- icijjzes

. 2407 UE-US

$.3425 \partial=-60$

. $\angle 0 j z$ uende

$.1605 j \mathrm{z}-67$

$. \because 442 \angle E-\angle 0$

$.11377 c-47$

$.34350 \mathrm{~L}-20$

$.27017 \mathrm{E}-47$

$.3044 v e-u$ I

. ¿y 4 yot $-u$ ?

$.30 y 01 k-20$

iscootitio

$\therefore=.2020 \$ 300 \mathrm{c}+U 1$ AAT $=-. .4+31$

$.8 y 371_{c-23} \quad .02 \dot{y} 07 \mathrm{c}-0$.

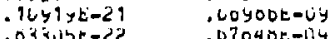

.03 Jubl-22 .0704OE-dY

$.39054 \pi-21 \quad .209 j 050-40$

- $20730=-29$

$.314516-v 6$

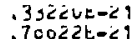

$.70022 t-\angle 1$

. $20 y y: x-00$

. $050+1 c=u c$

7 כIJYL-UO

$.14<y>c-07$

: $1045 y=-20$

. $1537 \leq x-u 7$

$.27<00 E-\angle 0$

$235 y=-47$

$.4410 j \mathrm{c}-0$ ?

$.47479 t-<0$

Do ru $=.2490$

$1153<0400+02$

$1250400 \mathrm{O}=02$

13401 YuUe +02

$154197 \mathrm{JU} U \mathrm{i}+\mathrm{J} 2$

$=.0<0407500+U 1 E=.301+1540 L+U 1$

- $3 j=2 c x-19.3 x<1$. $35-20$

. $5217<c=1$

. $3530 \mathrm{O}=<\mathrm{CU}$

$.0 Z$ QUJE-Zi

$.401425-9 y \quad: 47724=-20 \quad: 05437 c-<1$

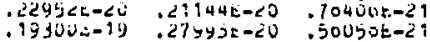

$\therefore 07 \div 1400 \mathrm{e}+02$

$17 \angle 181 Y 0 t+0<$

.17547 YUU:+Li

$179744 U U E+U e$

$.0 .5432 \mathrm{t}-20$

. $275 y=20$

$.99900 x-21$

-120?7e-10 ijocst-19

$130<2 L-19$
$30075=20$

$.242245-\angle 0$

. $94222 \mathrm{c}-2$

$104405-19$

$307>3 E-20$

.c: $: \square 11 E-21$

. 10109=-16 :i0970e-19

$.30=00 c=20$

$.10700130 \mathrm{U}+0 \mathrm{O}$

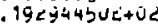

- 190607 yuetor

culcyululitua

$.91500 z-14 \quad .49505=-20$

$.90705 t=19$ : ivjoue-19

$.100 y 0 t-20$

$.19149 t-20$

$173456-10$

$334=4=-<0$ 
ju AU1 KUL KII KLE no

$\begin{array}{rrrrrr}4 & 2 & 3 & 3 & 2 \\ 4 & 3 & 5 & 3 & 3 \\ 4 & 3 & 1 & 3 & 3 & 0 \\ 4 & 2 & 2 & 3 & 2 & \\ 4 & 1 & 3 & 3 & 1 & \\ 0 & 2 & 5 & 0 & 0 & 0 \\ 5 & 1 & 5 & 4 & 1 & 4 \\ 5 & 0 & 5 & 4 & 0 & 4 \\ 3 & 3 & 10 & 13 & 3 & 1 \\ 5 & 2 & 4 & 4 & 2 & \\ 6 & 4 & 12 & 16 & 4 & 13 \\ 5 & 3 & 3 & 4 & 3 & \\ 5 & 3 & 2 & 4 & 3 & 1 \\ 5 & 1 & 4 & 4 & 1 & \\ 5 & 2 & 3 & 4 & 2 & \\ 0 & 1 & 0 & 5 & 1 & 5 \\ 0 & 2 & 5 & 5 & 2 & 4\end{array}$

BVnIUItue C5now

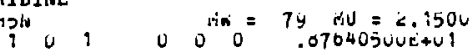

$\begin{array}{lll}1 & 0 & 1 \\ 2 & 2 & 1 \\ 9 & 7 & 2 \\ 0 & 0 & 1 \\ 1 & 7 & 1\end{array}$

$\begin{array}{rrr}10 & 13 & 3 \\ 5 & 3 & 2 \\ 4 & 2 & 2 \\ 2 & 1 & 2 \\ 3 & 1 & \end{array}$

322

432

$\begin{array}{lll}2 & 0 & 2 \\ 3 & 4 & 2\end{array}$

$\begin{array}{lrl}35 & 12 & 2\end{array}$

$\begin{array}{rrr}6 & 0 & 1 \\ 12 & 7 & 2 \\ 12 & 9 & 3 \\ 11 & 0 & 3\end{array}$

963

211

$\begin{array}{lll}0 & 5 & 3 \\ 7 & 3 & 5 \\ 4 & 1 & 3\end{array}$

63

06

$\begin{array}{ll}9 & 7 \\ 5 & 2\end{array}$

533

423

$\begin{array}{lll}3 & 0 & 3 \\ 7 & 2 & 5 \\ 10 & 94 & 4\end{array}$

$16 \quad 43$

17134

$16 \quad 12 \quad 4$

$\begin{array}{lll}15 & 11 & 4 \\ 17 & 15 & 3\end{array}$

1410 :

$\begin{array}{rrr}13 & 9 & 4 \\ 12 & 0 & 4 \\ 15 & 12 & 4 \\ 14 & 19 & 4\end{array}$

322

13104

1174

$10 \quad 134$

1104
$24 Y 00030 \mathrm{~L}+U 2$ $.232>0340 \mathrm{c}+U \mathrm{~L}$ . $532 y 05 U t+u 2$ $.20144320 \mathrm{c}+U L$ . $2004054 J 0+02$

$.20003700 \mathrm{c}+02$ $.2034270 v e+v e$ - gquioludue+ví - 3usu< 3uvetue . 3090R<70 c+uE

.313727 Uúctoz - $3102575 u z+U z$ . $31013 \supset 7 \mathrm{U} t+02$ .33141510e+U2

$.33700500 r_{0}+02$ $.309 u+y i d e+u z$

$.0704050 u \dot{0}+01$

$.12301490 \mathrm{t}+02$

$.12341190 \mathrm{t}+\mathrm{U}$

\$5060400e+0z

$.94499340 \mathrm{U}+02$

$.1440390 \mathrm{~J}=+02$

$.14054000 \mathrm{t}+02$

$.14002020 \mathrm{E}+0 \mathrm{E}$

$.1475302 \mathrm{JE}+02$

$.1402327 u c+42$

$.14003300 e+02$

$.14903130 c+02$

$.14947330 t+62$

$.1574047 \mathrm{Je}+\mathrm{U}^{2}$

$.15743010 t+02$

.15735 c10e+C

. 1070u9vob+Ci2

$.194 \mathrm{U} S 4 U 0 E+02$

$.20211200 E+02$

$.20373600 t+U 2$

$.20430000 \varepsilon+02$

$.204015002+0$

$.20594000 \mathrm{e}+\mathrm{C}$

$.200425700+02$

. ¿2000000ua+ua

.200\% $0506 a+0$

- 2uoy $100 u c+6$

. $20704350 \mathrm{cot}$

$.207097200+02$

.25710130e+02

$.20723190 \mathrm{C}+02$

.213945 Uu $c+0$ U

- $22854050 e+02$

$.25020000 \mathrm{c}+02$

$.24002000 \mathrm{c}+\mathrm{L}$

.251 yoy $00 t+u a$
$.2520 y 000 t+02$

. टगu4jivot+0z

.25 Y $0000 \mathrm{U}+02$ $.26102400 c+02$ - rozyuzuve +02 $.2029230 u t+02$ $.2029230 u t+02$
$.20242300 t+02$

$.20328 y 00 \mathrm{c}+02$ $.20330000 \mathrm{e}+02$ $.263+7500 \mathrm{~s}+42$

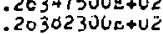
.20439 yoút, 2 ? - 32900UDUE+UL

.251 y y00t+ua ¿v

20

SI: NHA

16

cuv

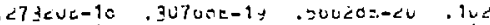

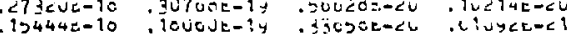

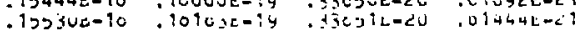

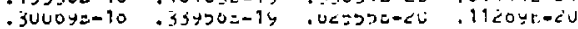

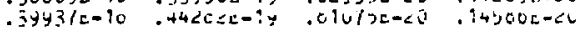

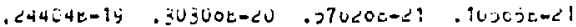

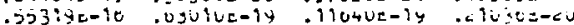

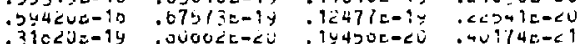

- Juovic-zi

$54723=\cdots 10$

.

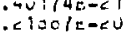

$.22530 \mathrm{~s}-<U$. $3 U \div 34 t-<$ ?

$190035-19$
$.4028=-10$

$.7 y / 00=-20$

. JUटUपL-1y

$410+1 \mathrm{c}=10$

$.71052=-10$

$.020 \$ 2 \mathrm{i}-1 \mathrm{c}$

- ju joy $=-14$

$.05342 c-1 y$
$.749915-14$

youy7c-civ

c $7943=-10$

. $10517=-15$

$.1254 j e-14$

$17.30=-<u$

- iluzurecs

.$<0 !$ IYLAL

$.<2<5 \div=-<0$

1 1y/4UE-14 . $251+\varepsilon-26$

- iqubie-cu

$19 y+u=-4$ ?

$H=.003 \dot{3} 3000 e+0 i \quad c=.3004 y 00 u i+01$ $.2351<0-10 \quad .24035-10 \quad .420032-2$

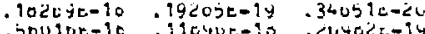

$30341=-10 \quad \because$ Thue-

$.3<941 t-10 \quad .33023=-1 y \quad .11104 c-1 y$

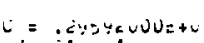

. $15091 \mathrm{E}-6$

. U1?

$.3+6<C E-\angle U$

- IYjoytez

. $1<U 4 L-20$

. 1U311t-10

.1004 Ue-10

$.37034 t-19$

$.4 / 64)=-<0$

- JuJsetio . 11510t-10

$45750 t-10$

- yũoote-1y

. $2<0 j y=-19$

$.1007 y t-1 y$

$.4 u>40 e-20$

- judielase

- 317 ele-ev

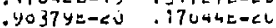

$.490208-10$

$707018-10$

-

$.11 /$ jue

$.244 v y c-1 y$

$.244 \sqrt{y c}-19$
$.4<301 \mathrm{e}-13$

$.9=603 a-20$

. 11 iy le-iy

1024 le $=17$

. 1007 $\leq x-10$

$1759+c=1 c$

1 icUUec- 15

19uyje-1y

$\because 11 y>z-20$

- j5y:2रe-cu

4 CCY4L-CU

$2015<c-10$

$0>905 \varepsilon-10$

$.03500=-10$

$.0770 y=-10$

$.52510 \mathrm{t}-1 \mathrm{y}$

$440 / 15=14$

$114 U y c-1 y$
$33435 \mathrm{c}-14$

-33035c-19

$.232115-10$

- $20140 \mathrm{t}=10$

14ub3e-17

. टY $2 y$ YE-10

60307e-1y

ooujitali

$\leftarrow<3>7=-c^{2} u$

. $00<\cot =\angle L$

. $1: 0<1=-1$ \%

14vuUe-iy

$.16002 \mathrm{E}-17$

. $150405=10$

102UUL-17

$.34<1 j c-1 y$
$.0<307 x-19$

UUyojt-Ci

$30204 \mathrm{t}-20$

$-10$

$03 y 23 t-2 ?$

$.43900=-21$

- lu7yoe-2e

.4040<E-19

$1<$ Jest-19

- $31 \zeta 92 \mathrm{E}-\angle \mathrm{j}$

. is 5 yor $-<2$

$.000050-\angle U$

$.10404 t-17$

$.2 y 5 L^{2}=-16$

$.43<44 t-19$

- $123 u \cup e-1 y$

$14537 t-17$

.1450 HE- 17

- $30329 \mathrm{c}-10$

- 10240e-io

vojolc- 15

.340yOE-19

$.130-4 t-1 y$

+0 sulece-

- 1ujuse-ly

$.30405=17$

.3ट721E=10

$.341350=1$
$.541100-1$

$.927050-10$

$3<540 \mathrm{~s}-7$

. $0>172 \mathrm{~L}-21$

- 1v112c-10

. 190000-19

$.20 \div 07 \mathrm{e}-20$

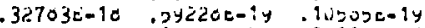

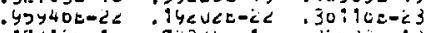

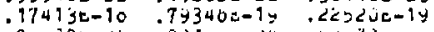

. SSyluc=L

$.17490-10$

- $20 j 05=-16$

. 331000-2ة

$.243520-1 y$

- Hiviu=-1y

.2ê110r-10

$.27100=10$

- $123>0 \mathrm{~J}-10$

$.14307 E-10$
$.37327=-10$

$.10104 \mathrm{E}-\mathrm{io}$

$.243526-1 y$

. 7 ijol-iy

$.10970 \mathrm{e}-14 \mathrm{y}$

ivooye-is

$.644020-10$

. $5 y+10 u=10$

. $73040-i y$

$.94270=-10$

$.17726-10$

$.274 U \nu=-14$

470 $4 \mathrm{E}-1$

$.45 j 02 E-10$

$274 J 5=-14$

- $200430-19$

. livues-iy

. $315<2 t-17$

-30000e-16

$.34 j 425=10$

12197

$.0<494 \varepsilon=19$

.20uj2E-19

Yo427t- 10

$.4<747 \varepsilon-10$

$.123 y 20 \dot{0}-10$

$.12014 \mathrm{E}-10$

- $11294 \mathrm{~L}=10$

. $25335 \mathrm{~s}-19$

- टyuपyz-1y

- Coy

$.204 y 00-19$ 


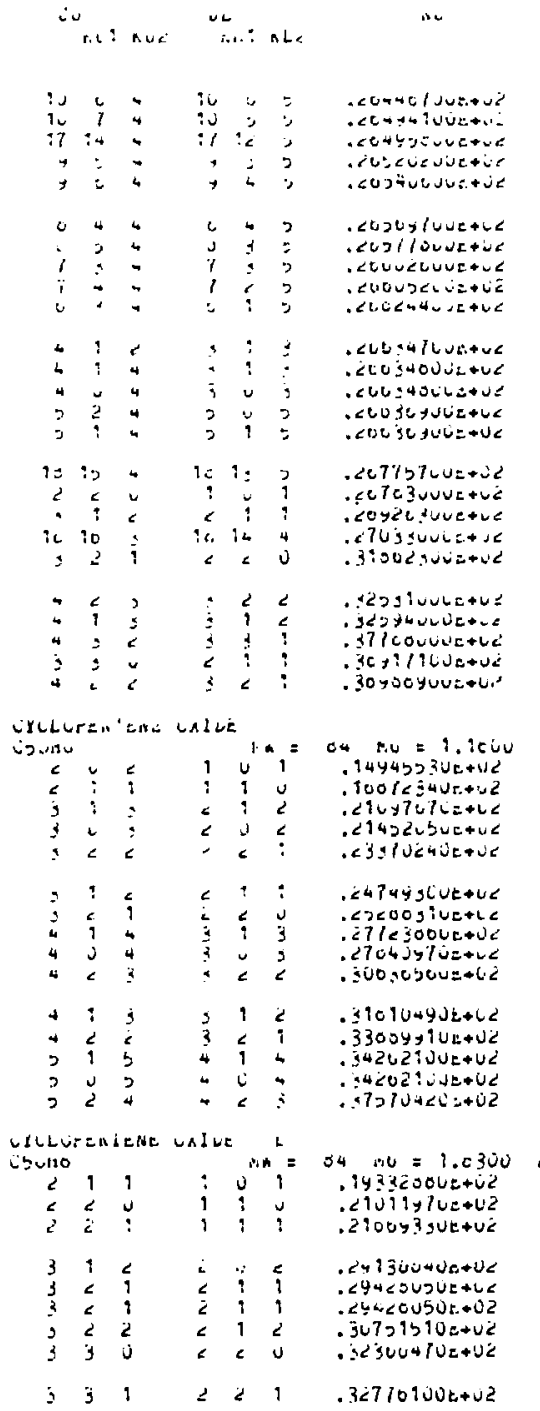

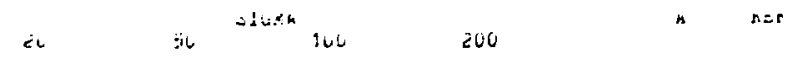

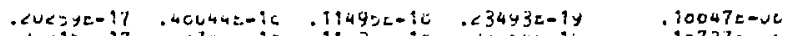

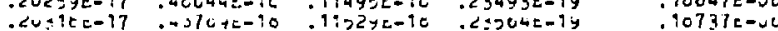

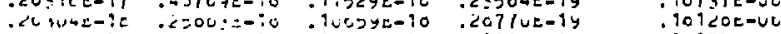

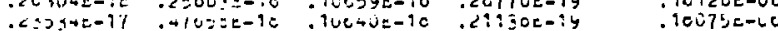

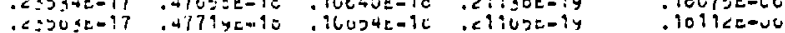

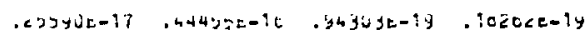

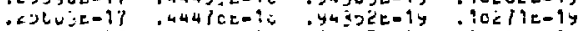

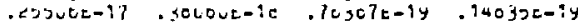
. Lz:ive-17 soucie-ic .7ojoue-1y .14037k-1y

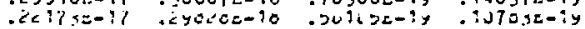

$17 \angle 44 E=60$
$.17650=-60$ $17450=-60$
$12900-60$ $.1330<2-40$ . $i<n=7 c-v e$

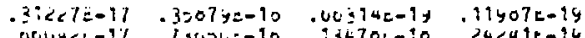
ocuact-17 .igosu=-10 .13470t-10 .24<4te-1y

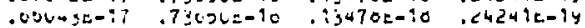

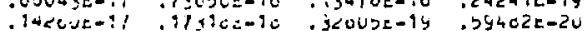

$.947730-00$ $.3 \times 70 y z-60$ $x y 7 \dot{y}=-u e$

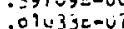
$014390-4$

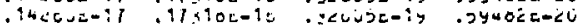

$.179072-40$

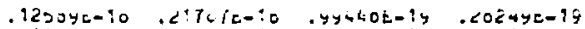

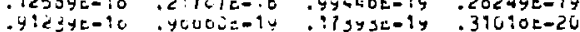

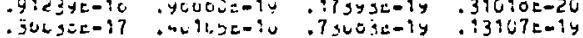

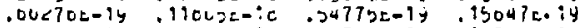
. $6137<E-U 1$ illutie-vo $.407435-U 6$

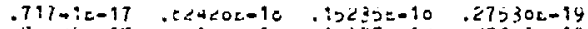

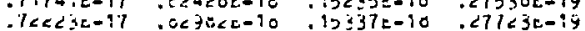

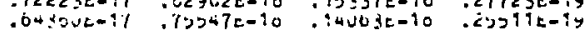

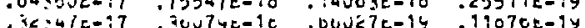

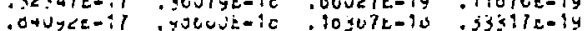

$.2340 j=-6 t$ $.55 y 0$ je-ve $-300890-30$
0

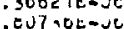

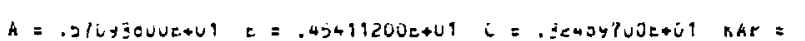
or

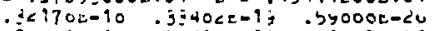

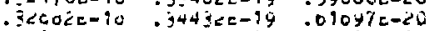
$.001 u<e-10$ :yetyue-1y :.80790e-1y .y997ye-10 :yoti1e-19::703y=-1y $.057100-16.1153 i 2-19$.12009-1s

$0, y \leq 37 a-v 1$ ¿<suec-uil $.037475-38$ $.5 y<0 z-01$ $.472402-07$

- ilcoye-11 .1<ejue-1c

.1<czue-1c .2<elae-1y

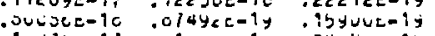
-19374E-11 .clothe-10

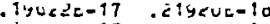
: $=45 y=-17$ in

$.2<077 t=11.2702 t-10 \quad .4743:=-14$

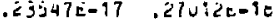

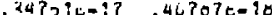
$34771=-11$

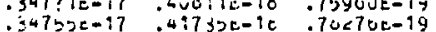

$.4 y 0 y 4 e-19$ . Toyice-19. 7.,yuJe-1y $.31000=-<0$

$.397002-<6$

$001402-47$

$.0313<2-u 7$ :30<1e*ve $13751 \mathrm{e}-\mathrm{Ne}$ $1400 / \mathrm{c}-\mathrm{Je}$ $.7 \angle 20 \dot{C}=-20$ $.09774 \mathrm{E}=<\mathrm{U}$

$.1007 j \mathrm{c}=2 \mathrm{cos}$

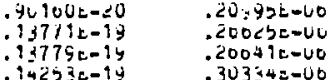

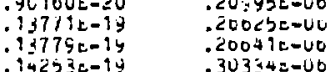

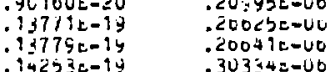

$A=.57393800 E_{E+01}=0.47411200 k+01$

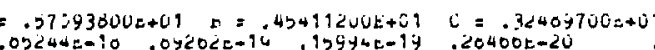

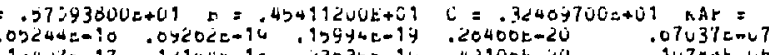

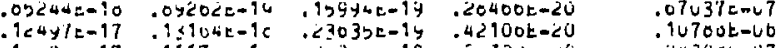

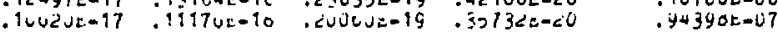

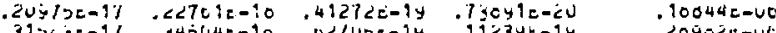

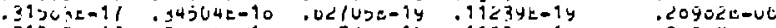

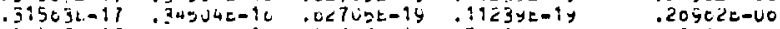

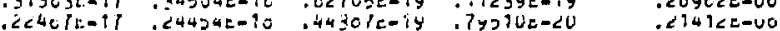

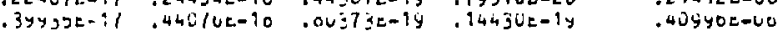
.2yjgge-11 .4j4iyiz-10 .7yle/te-1y. .1421jt-1y .40000e-wo $=02$

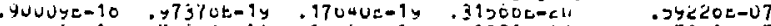

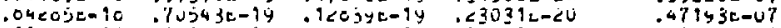

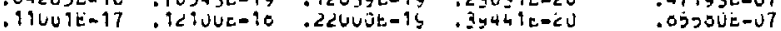

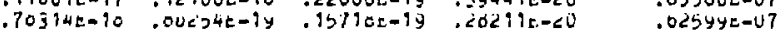

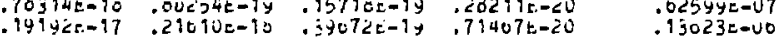

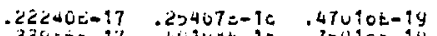
$.33505 e-17$. $401 y 3 t-10 \quad .15010 E-19$

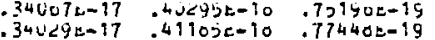

$.04 y 35 t-20$ 13e $135 E-99 \quad . \angle 6301 \mathrm{E}-60$ . $130440 E-10$ $.14121 k-19 \quad .30009 e-00$ $.34777<340+02$ $.374035000+02$

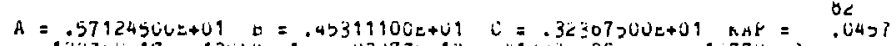

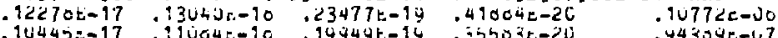

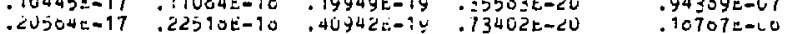


Jut ade AL: aLe

$\begin{array}{lllllll}3 & 2 & 2 & 2 & 1 & 2 & 3073070 j j+v 2\end{array}$

FULUEMe

Cono

$\begin{array}{lll}1 & 0 & 1 \\ 2 & 1 & 2 \\ 2 & 0 & 2 \\ 2 & 1 & 1 \\ 3 & 1 & 3\end{array}$

$\begin{array}{lll}5 & 1 & 4 \\ 3 & 0 & 3 \\ 3 & 2 & 1 \\ 4 & 2 & 3\end{array}$

$\begin{array}{lll}4 & 0 & 4 \\ 5 & 2 & 4\end{array}$

423

$\begin{array}{lll}4 & 3 & 2 \\ 5 & 0 & 5\end{array}$

CYCLONEXane OXIDE

$\begin{array}{cccccc}\text { O4 } & & 4 & & \text { M. } \\ 0 & 1 & 6 & 5 & 1 & 5 \\ 0 & 0 & 0 & 5 & 0 & 5 \\ 5 & 3 & 3 & 4 & 3 & 2 \\ 5 & 4 & 2 & 4 & 4 & 1 \\ 5 & 4 & 1 & 4 & 4 & 0 \\ 5 & 2 & 3 & 4 & 2 & 2 \\ 5 & 3 & 2 & 4 & 3 & 1 \\ 6 & 2 & 5 & 5 & 2 & 4 \\ 6 & 1 & 5 & 5 & 1 & 4 \\ 7 & 1 & 7 & 0 & 1 & 0\end{array}$

$\begin{array}{lll}7 & 0 & 7 \\ 6 & 3 & 4 \\ 6 & 2 & 4 \\ 6 & 4 & 3 \\ 6 & 5 & 2\end{array}$

$\begin{array}{lll}5 & 5 & 1 \\ 7 & 2 & 6 \\ 7 & 1 & 6 \\ 0 & 4 & 2\end{array}$

$\begin{array}{lll}0 & 4 & 2 \\ 6 & 3 & 3\end{array}$

$\begin{array}{lll}0 & 1 & 0 \\ d & 0 & 8 \\ 7 & 3 & 5 \\ 7 & 2 & 5 \\ 0 & 2 & 7\end{array}$

$\begin{array}{lll}0 & 1 & 7 \\ 7 & 4 & 4 \\ 7 & 6 & 2 \\ 7 & 6 & 1 \\ 7 & 5 & 3\end{array}$

$\begin{array}{lll}9 & 1 & 9 \\ 9 & 0 & 9 \\ 7 & 3 & \\ 7 & 5 & 2\end{array}$
$7 c$ He $=423 v$

$.03991000 x+09$

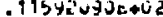

-1 1<201u7ucauc

$.1+0 u 4000 i+02$ $.10373900 e+02$ - ¿U0ट104UE+UL

. 2250ULOUE+UL $.22015370 E+02$

$.23152 y 700+02$ $.25292200 \mathrm{i}+02$ $.25420990 t+02$ $.23905920 z+02$ . 2 dod $4000 \mathrm{e}+\mathrm{L} 2$

$92 \mathrm{AU}=3.1500$ $.20000030 \bar{E}+C 2$ .2068gajuetua $.20902070 \mathrm{t}+02$ .270940305002 $.20191490 \mathrm{C}+0 \mathrm{C}$

$.26350220 \mathrm{E}+02$ $.29200920 \mathrm{t}+02$ .29379 C Jue+02 $.2946531, k+02$ .30909700E+02

-30909450e+0z .3:740youte+02 -320<910ué02 .330757 LOEL+LÉ .3325 Y120E+02

334guv10e+vz - $33025 y 00 k+02$ . $3360450108+02$ $.34737300 E+02$ -3jo i iubueacia

$.35131220 E+02$ . $35^{\circ}$ ? $1220 \mathrm{2}+02$ $.36<43250 t+02$ . $36595070 E+02$ . $37051200 k+02$

.37055350k+02 .302003705402 .30797490e+02 $.38004400 E+02$ . उ०93у ? UUE+02

- $39352550 \mathrm{se+02}$ $.39552550 e+02$ -39007340 $x+02$ -39899200s+ul
$1745 y 7000+b 2$
$2 \mathfrak{3}$

bJ
Sivitn ino cus n.:

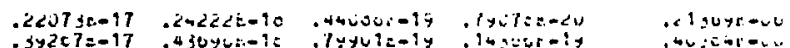

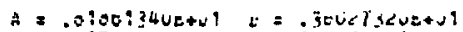
. $33017 e-<0$. $44 j u<=01$

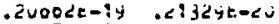

- \$C37<e-1y . $3 \leq 0 \partial 2-20$

$.2 y t+6 t-1 \neq .31<j=-26$

.71+31E-: 0 . OCAIJE-<J

$.10032 a-1 y \quad .20+10 n-c L$ . Ylo04l-1y ivguately

$.52700=15.00301=-x i$

$92010 \%=26$

$.100072=10$

$.20351=-10$

$14927 L-i \bar{y}$

$.171 u 4 c-10$

- yoduse-1y

उडuशce-10

(1)

. $2<<: 4=-14$

$.17010=-20$

$1 y \dot{x} y z=-19$

$.1151 \partial E-1 y$

.99040e-1y
- Icuidisers

- so inates

$.5+153=-2$

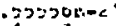

ineuptacis

icj:jate-c9

$1050:=-20$

$\because<+9 y<-<y$

?uvuy $=-<v$

- Nunje:ecc

- $3<+<0<-6$

?.)Jese-2

. 1 - 1 usesecu

$.735030-60$

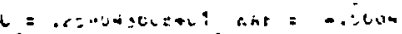

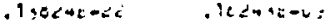

-uticsenser gludem.

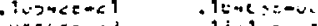

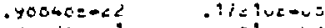

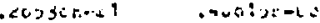

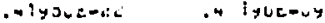

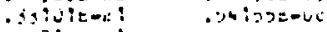

-nC3:Ge-5: PYYOAE-UE

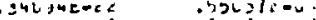

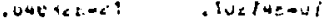

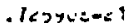

ingoseasi

. vis gu=ach

iO $06=-6$

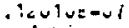

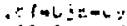

$.11+1$ ctevi

$.1:<c 1=-2 c$

.ex $y=-b 1$

$E=.30720050 \mathrm{~s}+41 t=.31273710 e+v 1$ $.14001 e-17.1043 y c-18$.3u433e-10 14003e-17: 10442e-10 3üsle-iy $.05 y 73 x-10 \quad .9200 y=-19 \quad .17000 z-1 y$

$.510430-20 \quad .503745-19 \quad .10033 e-1 y$

$.54345 z-16$.01<4as-1y

$113718-i y$

$.11000 \mathrm{E}-17$

$13101=-10$

10001द-17. 12120e-10

$.15147 \mathrm{E}-17 \quad . \$ 7327 \mathrm{E}=10$

. 1b<70e-17. $17407 e-10$

$.215320-17$

. $252000-10$

C1532t-17

$.15035=-17$

$10075 \mathrm{1}-17$

$12300=17$
$.0 y 4120-16$

.252UDE- 10

$.114940-10$

iync7e-10

istrice 10

. $817 y=-19$

. $24: y 0 t-1 y$

$.22453 c-17$
$.32320 \mathrm{c}-15$

$\therefore \cos 1 t-1 y$

.4 isyocely

$10401 t-10$

$.21934 t-17$

$.219720-17$

$145302-17$

$.0440 y c-19$

$.20341 t-10$

$.20301 \mathrm{k}-10$

- 10400k-1E

.47 juec-1y

. $3<0312-1 y$

- 3uvizle-1y

$.<103<e-1 y$
$.157010-1 y$

13s;je-su

. 1000

F.211400

. गु: $110=20$

$\therefore 1 y+1<-<0$

irveseeres

. CJyoesu

. $5 / 1$ I Y $20<\mathrm{J}$

- Gu<ve-ru

- 207uct-ciu

-5y 10ue-cu

cuntoencl

- 100u7e-19 $.4 y y+3 E-1 y$

- juútoe-19

$.32004 t-1$

syuvosecL

C7UJAte-6u

zuOsuz-cu

. $200450-60$

dicosyteí

- $914, j \mathrm{j}-<0$

$.9957<2-<i$

$.30 .77 .2-<J$

itiveoe-cu

.294Lic-17

$.294290-17$

$.22105 e-17$

$.22879 k-17$

$36232 E-10$
$.3023<E-10$

.0y2ditely

$.27205 e-10$

.20UDUt-10

$09201 k-1 y$

$.127300-14$

$.51945 e-19 \quad, 9 j 434 t-i \pi 0$

$.53504 c-19.95500 \mathrm{e}-20$

.29095e-?7

. 37525 E- 10

$.72454 c=1 y$

3 Bout-1s

. $29003 \mathrm{c}-17$

$.20961 \mathrm{ki}-17$

-37b 3 JE-10

$.72473=-19$

$.13504 t-4 y$

$.502200-19$. $92540 c=2 u$

$.21203 \varepsilon-19 \quad 343 y+c-20$

$.10951 \mathrm{e}-10$

- Bלsuse-10

$.10090 E-17$

$.110020-10$

$.20394 \mathrm{col} 1 \mathrm{a}$

$3+51 y=2 u$

. $3 y 372 c-19$.72733t-<0

- 37y $2 x=17$

$.3794 z=-17$

$20927 \mathrm{e}-17$

.45510E- 10

Yudo7t-9:

$17400 \mathrm{~d}-1 \mathrm{y}$

yous/e-19 :17;usre-14

il poosely

.7joticezu

$327705-10$

. oz 7 uje-1y

$.4100 y e-1 y$

* 30720osut+01 b $=.31573 / 1 u n+u 1$

$c=21100096 e+v$

$40703 \mathrm{e}-15$

ijuyserecu

. 14 iste-1.

$.03<i<5 e-<u$

$560+1 e-1 y$

$50041 \mathrm{c}-1 \mathrm{y}$

? $120 \mathrm{E}-17$

.25G5UE-17

$30973=-10$

$.27159 \mathrm{x}-10$

. $35200 \mathrm{t}-17$

.3017 Y

$.35304 \varepsilon-17$

- 302u1t-10

$.09925 \dot{-19}$

$.90+43 \mathrm{k}-20$

- $1250 \cos ^{2}-1 y$

$.12540 \mathrm{t}=1 \mathrm{y}$

- i7soge-1y

i41410-94

$94271=-14$

$.70200 t-1 y$

$36108 i c-17$

$.42375 t-10$

$.22740 \mathrm{e}-17$

$.249310-10$

$.450002-19$

.c2093t-20

.2CO51E- 17

$.25003 c-1 c$

$.40104 t-19$

$.0313 y=-20$

$.14707 \mathrm{e}-14$ 
de nul nus ol: hes

1.4

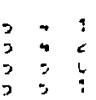

$\begin{array}{lll}4 & \vdots & 1 \\ 4 & 5 & 6 \\ 4 & 4 & 1\end{array}$

$.30010100 \mathrm{~d}+\mathrm{NL}$

. 3ذ719700E+LE

. 37 sež $400=+v 2$

$.51023+80 c-u 2$ 2u

50

$\checkmark$

$.55450=-17$ $.223490=2$

$.04072 e-17$

$.04950 \mathrm{x}-17$

$002400-10$

- SEy34c-10

$16107 t=10$
$142020-10$

$11905=-10$

130 cive- 10

$.13037 \mathrm{co} 10$
$.2 v 2 t 2 c-1 y$

$.190112=19$

$.2509<\mathrm{c}-19$

$.2 b i 2 j c-i y$
$.72<40 \mathrm{C}-U \mathrm{~L}$ .7टU44ट-U0 . Z5 COE-LO $.404052-v 0$

\begin{tabular}{|c|c|c|c|c|c|c|}
\hline \multicolumn{2}{|c|}{ actids } & \multicolumn{2}{|c|}{ A tc.ul } & \multicolumn{2}{|c|}{ in $=$} & $92 \mathrm{Mu}=1.1500$ \\
\hline 0 & $i$ & 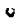 & ว & 1 & 2 & $.20071920 E+U E$ \\
\hline$j$ & $u$ & $c$ & 2 & 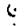 & 3 & $.20073040 \mathrm{Jat}$ \\
\hline$b$ & $\dot{5}$ & $\vdots$ & $\dot{\lambda}$ & $i$ & 5 & 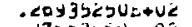 \\
\hline 2 & 4 & 4 & 4 & 4 & 1 & 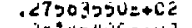 \\
\hline 2 & 4 & 1 & 4 & 4 & i & $. \angle O T 454 Y C E+U 2$ \\
\hline$=$ & $\epsilon$ & $d$ & 4 & $?$ & $\leqslant$ & $.20330710 L+U 2$ \\
\hline 7 & , & $c$ & 4 & 3 & 1 & .29224 buUetí \\
\hline 0 & 5 & \% & כ & 2 & 4 & 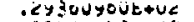 \\
\hline 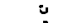 & : & 3 & 3 & 1 & 4 & $. \angle 9440 Y \sum O C+U E ̈$ \\
\hline$P$ & 1 & $\tau$ & $:$ & 1 & e & 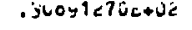 \\
\hline 7 & $\dot{v}$ & $I$ & $\therefore$ & 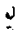 & $b$ & - jud914iut+U2 \\
\hline$u$ & 3 & 4 & 3 & $\vdots$ & 3 & $.31722410 t+U 2$ \\
\hline $\mathbf{0}$ & $\dot{z}$ & 4 & $b$ & 2 & 3 & $.3<014900 E+02$ \\
\hline $\mathbf{b}$ & 4 & 3 & 3 & 4 & c & - $3303070005+32$ \\
\hline 0 & $\Rightarrow$ & $c$ & $j$ & 5 & 1 & 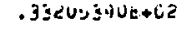 \\
\hline 4 & $?$ & 1 & 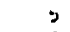 & $\$$ & $v$ & . $33430440 \mathrm{u}+02$ \\
\hline 7 & 2 & 0 & 0 & $\dot{c}$ & 3 & - $33005530 t+U 2$ \\
\hline 7 & 1 & $?$ & U & 1 & $?$ & - $336<543 \cup L+U 2$ \\
\hline 0 & 4 & 2 & 5 & 4 & : & $-34079020 \mathrm{C}+02$ \\
\hline$\theta$ & g & 5 & 3 & 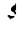 & 2 & $.34 y 74 u 30 \mathrm{O}+42$ \\
\hline 0 & 1 & $\theta$ & 7 & 1 & 7 & - $35110100 k+02$ \\
\hline 0 & $u$ & 0 & 7 & 4 & $i$ & 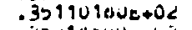 \\
\hline 7 & 3 & 3 & $v$ & 7 & 4 & - 30 $140 U U=+v E$ \\
\hline 7 & 2 & 2 & 0 & 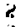 & 4 & - $305700 J U_{t}+U 2$ \\
\hline 0 & $<$ & 7 & 1 & 2 & 0 & $.37020430 E+02$ \\
\hline 5 & 1 & 7 & 7 & 1 & 0 & - $37032700 c+02$ \\
\hline 1 & 4 & $\downarrow$ & 0 & 4 & 3 & $.3017030 \mathrm{JE}+02$ \\
\hline 7 & 0 & ? & $e$ & $v$ & $i$ & - 307 ब2090t \\
\hline$?$ & 5 & 1 & 6 & $\$$ & $u$ & - $10027220 \mathrm{E}+0 \mathrm{E}$ \\
\hline 7 & 3 & 3 & 0 & 5 & 4 & . 3000012UE\$U2 \\
\hline y & 1 & $y$ & 0 & 1 & 0 & $.3+3<900 L^{\prime} z+02$ \\
\hline 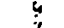 & U & $y$ & 0 & 0 & 4 & - 39329000k+CZ \\
\hline 1 & 3 & $<$ & 0 & 3 & 1 & ¿92ZUË+U2 \\
\hline 7 & 3 & 4 & 0 & 3 & 3 & . 3904ź 9uUE+02 \\
\hline
\end{tabular}

Lourt

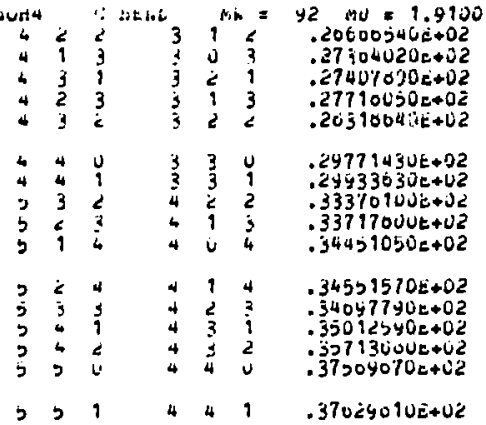

$A=.3073295 \mathrm{j} s+0 t \quad b=.31532410 \mathrm{E}+\mathrm{J1}$ $.144 y 4 c-17$

.144 ye cal 7

.040 yer-10

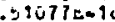

$.754 y-2=10$

.11atue-17

. 1000uea!?

$149500=17$

- isjozeng?

. $\angle$ IC5TE-17

- $212500=17$

$.14043 t-17$

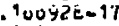

. $12 \vdots \vdots<\dot{L}-17$

.50300e-1s

- by37jக́-10

- cijecenalt

$.21700 \mathrm{t}-17$

$.14104 \mathrm{E}-17$

. 1yites-17

$.29347 \varepsilon-17$

-2yb47k-17

.21บ12e-17

.2<b Jute:7

$.29<<7=-17$

$.+9235 t-17$

- Luvose-17

- 0j050t-10

- OACUUE-10

$37510 t-17$

- $37510 \mathrm{c}=17$

- $10790 \mathrm{E}-17$

$103300-10$

- 0 $039 \mathrm{z}-10$

$.94 y 10=-19$
$.57051 \mathrm{~s}-19$

. ovodez-1y

- ijus5t-16

- ieveze-io

- 17225z-10

- Ij jooe-10

.250JUE-iO

$.1730 y=-10$

- Iyว41z-10

. $2 \angle 47$ LE- 19

$.03717=19$

$.20103 t-10$

$.202 \dot{2} 4 \mathrm{i}=15$

$.10021 \mathrm{t}-10$

-30J4วt-10

-30uUze-io

. $27044 c-10$

$.27907 \varepsilon-15$

.37300E-10

. $37310 e-10$ ¿s477e-10

-10030ะ-10

- 1UYŨ⿱一⿻上丨-10

$.20<20 \mathrm{~L}-10$

. $44200 \mathrm{C}-10$

$.29407 \mathrm{e}-10$

.32037 E-10

30329c-1y

$30534 k-15$

. 11<4ia-15

$24120=14$

2c 3uoz-is

- $3<20 j \mathrm{e}-19$

. 32471e-1y

$47203 e-1 y$

$3270<\mathrm{c}-1 \mathrm{y}$

$3075 y=1 y$

$277010-19$

$.15001 a-1 y$

$15900 \mathrm{c}-19$

$4 \$ 754 E-19$

. पYेo31E-19

- T?22E-14

4zusie-10

$.009900=19$

$.00990 e-1 y$

- $1752 \mathrm{E}-1 \dot{0}$

$.53400 t-19$

.7C176E-19

$.72 \% 90 c-19$

$.4 y+97 \mathrm{em} 1 \mathrm{~s}$

$.211202-19$

$.21222 t-19$

gous $30-19$

$.90093 c-10$

$.495000-19$

$.02770 \mathrm{c}-19$
.224 y $20-16$

$.44200 E-10$

17 hook-15

$=.30732150 z+04 t=31532410 t+01$ - 1Y508E-32 $17303 \mathrm{E}-23^{3} .53230 \mathrm{~L}-21$ $.347100-20$. 10015e-1\% . 9y055E-10 - 1919yc-j2 - 1147 c-32 $.204400-52$ $2109 \mathrm{de}-31$ $.100796-23$ $.23104 \mathrm{E}-31$

$.72474 \mathrm{E}-25$ $.57471 \mathrm{c}-21$

10994E-00 .11111E-42 $17100 \mathrm{col} .1 \% 290 \mathrm{~d}-\mathrm{di2}$ $20312 c-52$. $33407 t-31$ $.3<945=-j 2$. 3022je-23 $.61290 \mathrm{j}=2 \mathrm{j} \quad .3110 u t=1 d$

$.34547 \varepsilon-32.31094 t-23$ .30013E-52 .30170E-31 - ¿2́S97e-0u .23730E-42 $.25014 \varepsilon-0 J \quad .24095 k-42$

$15296-110$

. 10110k-50

$.77371 c-<5$

$.25526 \mathrm{E}-30$ $.25005 E-50$ $.133213-24$ .9417 IE-4 .300म1E-10

$.907558-21$ $.12235 \mathrm{E}-2$ - $35000 \dot{-}-30$ - $37309 \mathrm{y}-30$ .20uote-37

$.15046-116$

.11150=-50

$.20177 t-37$ $c=.210 y 5520 z+0$ .54 प4

$.3+454=-20$

$.31011 \mathrm{E}-\mathrm{eO}$

. 1ெjoze-zo

$.2 \cup 477 \mathrm{E}-<0$

$.43041 E-20$

$.4039 \pm E=20$

. 50ว40t-20

. $59035 E-20$

$.001400-20$

$.00150=-20$

- $50<5=-20$

. $00479 \dot{ }=20$

. 50030E-<0

$.20095 \mathrm{a}-20$

. $24135 \dot{0}-20$

- $911000-20$

50 UL-zo

$.77533 \mathrm{e}-20$

$.12700 \mathrm{E}-19$

$.12700 E-19$

- צेंढje-2

. $40219 \pm-20$

$.13349 E=15$

$.13353 c=19$

$.92223 \mathrm{E}-20$

. 39104t-20

. $39503 i-20$

$.17054 t-19$

$.17054 c-1 y$

$.76003 E-20$

$.11574 t=1$
.285 $12037 \mathrm{E}-60$

$.1204 u \in-v 0$ $.07 \geqslant 10 \mathrm{r}-07$ $.50430 \mathrm{c}-07$ .507yye-u?

. 12504e-UL - Jeúcor-ue 14076=-Ũo - Ijúljo=ur . 20u37e-0u

$.20037 a-j 0$ $.10410 \mathrm{em}$ Jo . $10957 \mathrm{c}-\mathrm{ve}$ - $14 b j a ̈ c-U 0$ $.03100 E-07$

$.04970=-67$ $.23109 t=00$ . $23220 \dot{200}$ $.17507 \mathrm{E}-46$ . $23573 \mathrm{E}=00$

$.29072 \mathrm{c}=00$ $2 \rightarrow 07 \angle r i=06$ $.20754 \mathrm{E}-0 \mathrm{O}$ - 27256E-00 . $339960-00$

$.34009 t-60$ $20707 \mathrm{E}-06$

$11010 \mathrm{E}-06$

$.11094 E-00$

. $21475 \mathrm{E}-\mathrm{Ub}$

$.42451 \mathrm{k}=00$ $.42491 \mathrm{c}=06$ $.23309 \mathrm{E}-00$ $.35077 t-0 b$

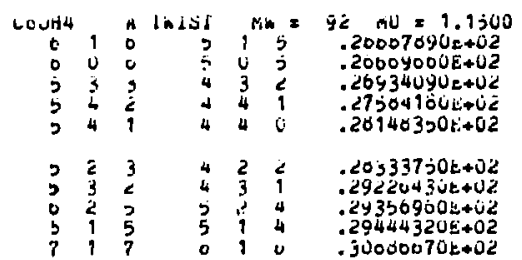

$C \pm .210955200+01 \times A H=-.9995$

. $34201 \mathrm{ic}-20$

$.09344 \mathrm{t}-19$

$.55690 \mathrm{E}-22$

$.42304 \mathrm{~L}-20$

$.59453 \dot{z}-22$

$13109 E-24$

$13252 \mathrm{c}-24$

$07543 \mathrm{c}-22$

$.0 y 0910-20$

$.16073 \mathrm{k}-10$

$.73293 \mathrm{t}-20$

$94013 \varepsilon-2 z$

TC5 $32 \mathrm{E}-24$

$.19201 k-24$

$.02219 E-\angle 0$

$.02410 \mathrm{t}-20$
$10700 k-00$
$241630-00$

$.25501 t=00$

- $10030 \mathrm{E}=00$

$.20120 \dot{2}=00$

$.43575 E-06$

. 4429 IL-U0

$.40 ; 33 t=00$

$.31193 \dot{c}-00$

.47332 b́-00

$.33434 c-00$

.4515 ye-vio

.5Y05UE-UO

$.03302 t=00$

$.92110 \mathrm{E}=0 \mathrm{O}$

$.92550 c-00$

H = 30710700e+01 E = 31533310e+41 $+143505-17$ . $140205=10^{\circ}$ $.54029 E-10$
$.500225-10$ $.53020 t .10$

. 9u2́ay 
Ju nU1 nUe dita bu

$30000070 \mathrm{e}+\mathrm{J}<$ $31719040 z+02$

- $320 J 0450 E+U L$

$3030700 \mathrm{e}+\mathrm{Uc}^{\mathrm{C}}$

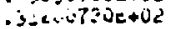

.35441100 E⿺辶- $33031000 e+02$ - 34e=2 sbiletu2

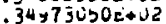
. 3j 1049CJe+de

- 3ิ 10+40uk+ù2

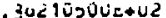

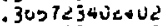
$.30572340 \mathrm{c}+02$
$.3022901 \cdot 0-02$

$.3752 \angle 900 \mathrm{C}+\mathrm{UL}$ $.37027100=+02$ $.30100510 \mathrm{t}+42$ $.3014470 u c+v 2$ - Jad 2 yoyje+u<

$.300 .19120 \mathrm{c}+0 \mathrm{C}$ . $333<3100 \mathrm{t}+02$ $.39323100 E+02$ . $34034430 \mathrm{e}+02$ . $39037100 E+02$

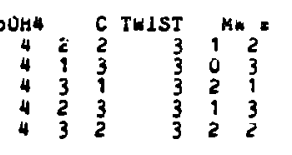 \\ $\begin{array}{lll}4 & 4 & 0 \\ 4 & 4 & 1 \\ 5 & 3 & 2 \\ 5 & 2 & 3 \\ 5 & 1 & 4\end{array}$ \\ $\begin{array}{lll}5 & 2 & 4 \\ 5 & 3 & 3 \\ 5 & 4 & 1 \\ 5 & 4 & 2 \\ 5 & 5 & 0\end{array}$}

551

NITROXYL

HNO

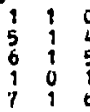

NITAOXYL

HNO $\begin{array}{lll}7 & 0 & 7 \\ 4 & 1 & 4\end{array}$

NITUIC ACIO iñ

\begin{tabular}{rrrrrr}
1403 & & & \multicolumn{1}{c}{1} \\
7 & 0 & 1 & 7 & 0 & 2 \\
12 & 10 & 2 & 12 & 10 & 3 \\
6 & 5 & 1 & 6 & 5 & 2 \\
11 & 9 & 2 & 11 & 9 & 3 \\
5 & 4 & 1 & 5 & 4 & 2 \\
4 & 3 & 1 & 4 & 3 & 2 \\
10 & 0 & 2 & 10 & 8 & 3 \\
3 & 2 & 1 & 3 & 2 & 2 \\
2 & 1 & 1 & 2 & 1 & 2 \\
1 & 0 & 1 & 0 & 0 & 0 \\
9 & 7 & 2 & 9 & 7 & 3 \\
8 & 6 & 2 & 0 & 0 & 3
\end{tabular}

$92194=1.9100$ $2730: 900 t \rightarrow 0^{2}$ $2740<190 \mathrm{c} \rightarrow 0$ $.27714 j 00 e \dot{0}+02$ $.253120 u 0 z+c a$

$.29701950 \mathrm{k}+02$ $2942449 \mathrm{Jt}+02$ 33372910 ive $.33190530 E+02$ $.34447560 \mathrm{E}+02$

$34547300 \mathrm{e}+02$ $34692510 \mathrm{t}+02$ $35003020 E+02$ $.35704090 \mathrm{E}+02$ .375574 YنL 102

$.37617740 \mathrm{0}+02$ cu

jo

$=1$ UN

Iuo xat

\begin{tabular}{|c|c|c|c|c|}
\hline $\begin{array}{l}.210 j 4 E-17 \\
14724 \mathrm{E}-11 \\
145<0 E-17 \\
12<29 \mathrm{E}-17 \\
.0170 \partial E-10\end{array}$ & 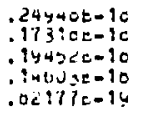 & 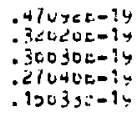 & 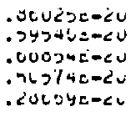 & 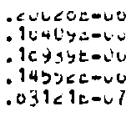 \\
\hline $\begin{array}{l}c 17 w E-10 \\
19 y 5=-17 \\
1490-17 \\
10034 .=17 \\
207 / 5 t-11\end{array}$ & 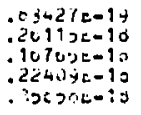 & 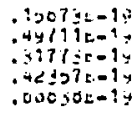 & 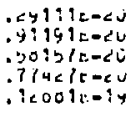 & 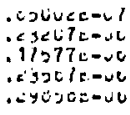 \\
\hline 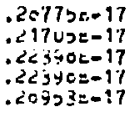 & 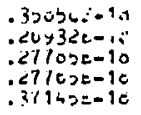 & 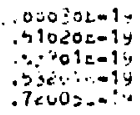 & 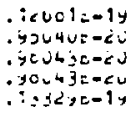 & 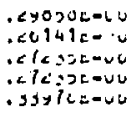 \\
\hline 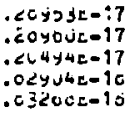 & $\begin{array}{l}.37145=-10 \\
.37152 E-10 \\
.220745=10 \\
.16010=-10 \\
.10001=-10\end{array}$ & 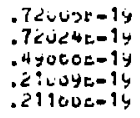 & 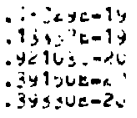 & 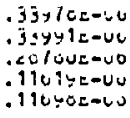 \\
\hline $\begin{array}{l}15715=-17 \\
37106 t-17 \\
37100=-17 \\
10054 E-17 \\
25790=-17\end{array}$ & $\begin{array}{l}.20154 E-10 \\
.44005=-10 \\
.49005 E-10 \\
.21390=-15 \\
.3240 y=-10\end{array}$ & 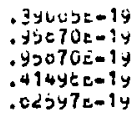 & 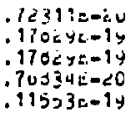 & 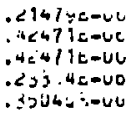 \\
\hline
\end{tabular}

$A=.30710100 c+01 t=.31533310 t+c 1$ . $3504 \mathrm{c}-17 \quad$. 25401E-10 $.14400=-17.15400=-10$ $.20251 \mathrm{c}-12.3 u 0 j 0 \mathrm{z}-10$ $.14794 \mathrm{k}-17$. $15034 \mathrm{E}-10$ $.25307 \mathrm{E}-17$.27477t-17 $40+0 j c-19$ $.20257 \mathrm{t}-19$ . $20094 \mathrm{t}=1 \mathrm{~s}$ . $) \quad 3 \angle 0 \mathrm{O}=19$

$0<$

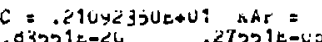
$.03501=-<6 \quad .27551 E-40$

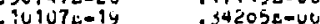
D $10900-20 \quad .177052=00$

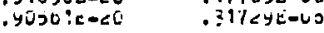
$.34520=-17$.37012c-10 .095j2t-19 . 1253ce-1y $.34552 \mathrm{t}-17 \quad .3703 \mathrm{tt}-1 \mathrm{c}$ $4000 y=-17$. $3034 t=10$ $.3733<c-17$ :492CE=10 .र<230b-17 .2406ye-10 $.09726-19: 120436-19$
$.900775-19: 170048-1 y$ $172 y=-10$ 14073x-1y $.45550=-19 \quad .02 \$ 17=-<u$

$20<2-U 0$ $.40 j 4 U E-U U$ $.0 U D E<t=U R$ $.40350 \mathrm{~L}-\mathrm{W}$ $.2002 y c=U 0$

$.22353 e-17.24003 e-10.45000 \varepsilon-1 y .0277 U c-60$ $.30726=-17 \quad .43572=-10$ $52207=-17$ :540?1 $.51200 i-17 \quad .503342-10$ $.03495 \mathrm{E}-17 \quad .73405 \mathrm{E}-10$ 60647t-14:1404<2-1Y 11111E-1e :́uluoc-1y $10070=10 \quad: 1 y 7290=19$ $.137440-10$.25004E-19

- $<Y \cup 7<x-\cup 0$ $.51 \% 0 \div c-v 0$ . $72105=-00$ . 19 y4:-ve . 40133 c-ve

$.03579 \varepsilon=17 \quad .73449 c-16 \quad .13701 \varepsilon-10 \quad .45030=-15 \quad .90407 t=60$

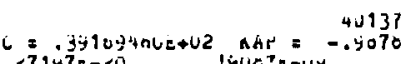

$31 \mathrm{HU}=1.0300$ $.31305000+01$ $.47009330 \Sigma+02$ $.05004620 \dot{0}+02$ $014774908+02$ $.07027600 \mathrm{E}+02$

$A=.55 \$ 90300 \varepsilon+43 \quad B=.4 \angle 300520 E+02$ . $25022 E-10$. ju100E-19 $1324 \mathrm{Ge}-19$ $.77000 \mathrm{e}-10$.10101k-17 $.30050 \mathrm{E}-10 \quad: 10350 \mathrm{E}-17$ $.35594 \mathrm{E}-15.413268-10$ $.410105-10$ $.54455 k-10$ $.74450=-17$ .14060e-10 :91901t-10

$.03503 e-10$

C $<7147=-<0$

$.1131<E-10$

$.10003=-10$

1900? $=-0 y$

4 ey<j=-07

$.041012-07$

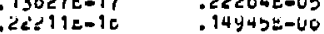

$A=.5534030 \mathrm{JE}=03 \quad \ldots=.42300520 \mathrm{C}+02$

40137

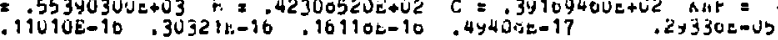

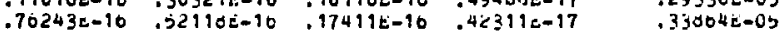

$.00623170 t+02$
$.90416350 t+02$

$\dot{A}=.13011150 \mathrm{v}+\mathrm{U} 2 \quad \mathrm{e}=.1209+y$ y $30 \mathrm{C}+\mathrm{C2}$

c<luo

63 $\mathrm{MU}=1.9000$ $.505300-10$

$.124 Y J E=18 \quad, 3 y 37+4 t-19$

$C=.0<000000 t+v 1$ Rat $=$

.7300

$.110942-17 \quad: 334942-10 \quad .53091 z-1 y$

$92455600 E+U 1$

$10344230 E+02$

$.12317350 E+02$

$.1030 \% 5-10$

$.10446=-10 \quad .720330-14$

$100050-14$

10 Wut -1 .

$.12007 E-U 7$

$.17992 \dot{i}-17$

$.2+491=-10$.02902e-1y

. 1999ge-99

$10090 \varepsilon=07$
$20373 c-47$

.2049 ye-u 7

$14534250 c+02$

$156209906+02$

$162155900 i+02$

$.17517510 \mathrm{e}+02$

$.22329 \mathrm{E}-17$

$.3204 d \mathrm{~d}-10 \quad .03653 \mathrm{~d}-19$

$43 c 00=10$.319j0E-10 . 1ư740e-10

. ¿35jट́e-17

.319jose-10 : 1u740E-10

$.11927 e-1 y$

.$<02002-14$

$.10161 \mathrm{i}-19$

$20347=-17$

$214=7=-10$.

$05277 \leq-20$

$30104 c-47$

$.475750-07$

$.430 y 2=-07$

.41130 ULU 7

$.94715 k-07$

$.90920 \mathrm{t}-1 \mathrm{~d} \quad .40237 t-10 \quad .14415 c-10 \quad .33121 \mathrm{e}-19$

$.70014 E-U$ ?

$16964500 E+02$

: Yo69oE-17 .0023tE-10 : $17773 E-10$

$.30715 t-1 y$

$.90=796-06$ 


\begin{tabular}{|c|c|c|c|c|c|c|c|c|c|c|c|c|}
\hline \multirow{2}{*}{\multicolumn{2}{|c|}{ su }} & \multirow{2}{*}{\multicolumn{4}{|c|}{$J_{L}: M L C$}} & \multirow[t]{2}{*}{ ט. } & \multirow{2}{*}{\multicolumn{4}{|c|}{ 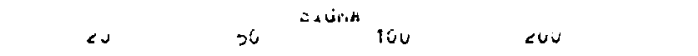 }} & \multirow{2}{*}{\multicolumn{2}{|c|}{ 's $\quad r E F$}} \\
\hline & & & & & & & & & & & & \\
\hline $\begin{array}{l}7 \\
4 \\
? \\
2\end{array}$ & $\begin{array}{c}2 \\
4 \\
\vdots \\
i\end{array}$ & $\begin{array}{l}c \\
c \\
<\end{array}$ & $\begin{array}{l}7 \\
0 \\
? \\
i\end{array}$ & $\begin{array}{l}3 \\
\vdots \\
\vdots \\
\vdots \\
\vdots\end{array}$ & $\begin{array}{l}3 \\
3 \\
3\end{array}$ & 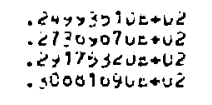 & $\begin{array}{l}31220 \mathrm{c}-17 \\
.40 \cup \cup J E-17 \\
.300 \angle 3 E-17 \\
.902 \forall=-16\end{array}$ & 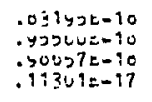 & 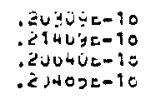 & $\begin{array}{l}.4219 u e-1 y \\
.4070 b t-1 y \\
.340 y 1 f=1 y \\
.30070 c-1 y\end{array}$ & 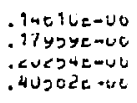 & \\
\hline $\begin{array}{c}01 \ln 30 \\
0+1 \\
\vdots \\
\vdots\end{array}$ & 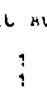 & 11.5 & $\vdots$ & $j$ & $\because$ & 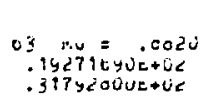 & 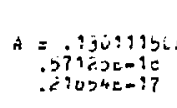 & $\begin{array}{l}x+u 2 \quad E=1 \\
5=19 E-1 y \\
.<30<4 E-10\end{array}$ & 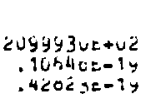 & 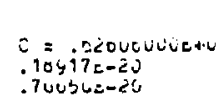 & $\begin{array}{l}1 \text { nat: } \\
.24003 \mathrm{E}-4 ? \\
.01<9<0=01\end{array}$ & $\begin{array}{l}\text { celle } \\
7.750 \mathrm{~s} \\
7\end{array}$ \\
\hline $\begin{array}{c}\text { N Linpir } \\
\text { watewne } \\
\vdots \\
4 \\
5 \\
\vdots\end{array}$ & $\begin{array}{l}\text { IL } \\
2 \\
\vdots \\
\vdots \\
\vdots \\
2\end{array}$ & $\begin{array}{l}\vdots \\
\vdots \\
\vdots \\
\vdots\end{array}$ & $\begin{array}{l}i \\
\vdots \\
\vdots\end{array}$ & $\begin{array}{l}m \\
\vdots \\
\vdots \\
j\end{array}$ & $\begin{array}{c}2= \\
\vdots \\
3 \\
3 \\
1 \\
3\end{array}$ & 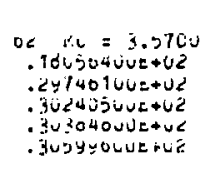 & 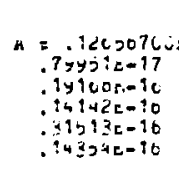 & 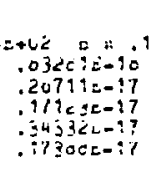 & 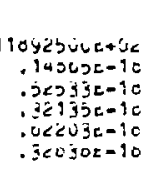 & 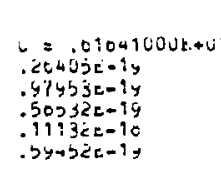 & 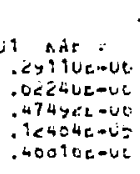 & $\begin{array}{l}163 \\
0.7040 \\
0 \\
0\end{array}$ \\
\hline $\begin{array}{l}4 \\
< \\
2 \\
0 \\
7\end{array}$ & $\begin{array}{l}: \\
\vdots \\
\vdots \\
i\end{array}$ & 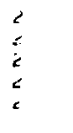 & $\begin{array}{l}4 \\
i \\
i \\
7\end{array}$ & $\begin{array}{l}1 \\
4 \\
2 \\
3 \\
4\end{array}$ & $\begin{array}{l}r \\
1 \\
\vdots \\
\vdots \\
\vdots \\
\vdots\end{array}$ & 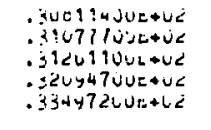 & 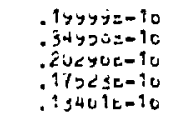 & 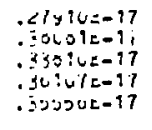 & 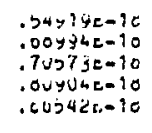 & 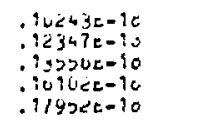 & 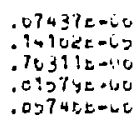 & \\
\hline $\begin{array}{c}\text { ar.enue } \\
4 \\
2 \\
2 \\
4 \\
2 \\
2\end{array}$ & $\begin{array}{l}i \\
i \\
i \\
i \\
i\end{array}$ & $\begin{array}{ll}2 & 11 \\
2 & \\
2 & \\
< & \end{array}$ & $\begin{array}{l}\vdots \\
\vdots \\
4 \\
1\end{array}$ & & $\begin{array}{c}7 n= \\
7 \\
\vdots \\
3 \\
3 \\
1 \\
1\end{array}$ & 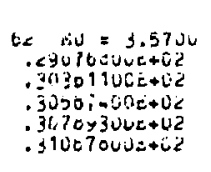 & 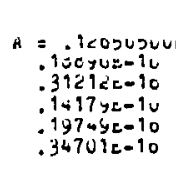 & 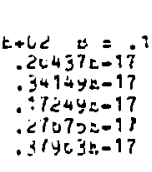 & 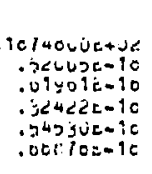 & 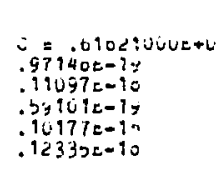 & 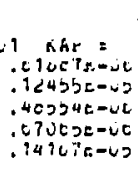 & 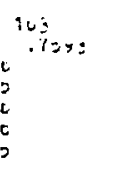 \\
\hline $\begin{array}{l}j \\
\vdots \\
7\end{array}$ & $\begin{array}{l}4 \\
0\end{array}$ & $\dot{s}$ & $\begin{array}{l}? \\
\imath \\
7\end{array}$ & $\begin{array}{l}5 \\
\vdots \\
4\end{array}$ & $\begin{array}{l}3 \\
3\end{array}$ & $\begin{array}{l}.3120110 u c+0 i \\
.3213400 u t+02 \\
.3306010 u=+W 2\end{array}$ & $\begin{array}{l}.20034 c-16 \\
: 172002-10 \\
: 132070-10\end{array}$ & $\begin{array}{l}.33007 c-17 \\
.35730 E-17 \\
.35173 c-17\end{array}$ & $\begin{array}{l}.70034 c-10 \\
.00<20=-10 \\
.05110=-10\end{array}$ & $\begin{array}{l}.13402=-10 \\
13470=-10 \\
177 y i=-10\end{array}$ & $\begin{array}{l}.730906-v e \\
.011 e y t-v 0 \\
.05307=-6.0\end{array}$ & \\
\hline $\begin{array}{c}\text { nowinge } \\
\text { nivs } \\
1 \\
4 \\
4 \\
4 \\
4\end{array}$ & $\begin{array}{l}20 I L \\
0 \\
1 \\
3 \\
4 \\
<\end{array}$ & $\begin{array}{l}1 \\
4 \\
\vdots \\
\vdots\end{array}$ & $\begin{array}{l}u \\
\vdots \\
3 \\
3 \\
3 \\
\vdots \\
3\end{array}$ & & $\begin{array}{c}4_{n}= \\
u \\
3 \\
1 \\
\vdots \\
2\end{array}$ & 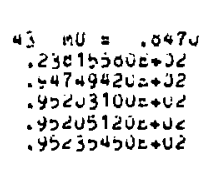 & 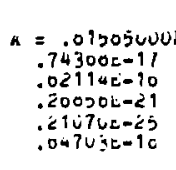 & 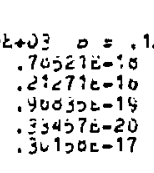 & $\begin{array}{r}12034150 E+U 2 \\
.14007=-1 C \\
.56447 E=17 \\
.26124 E-10 \\
.60071 E=19 \\
.19004 E=17\end{array}$ & $\begin{array}{l}\tau=11701310 c+U \\
.23033 c-16 \\
12213 E-17 \\
.10040=-10 \\
.13011 E-10 \\
.02001 E-10\end{array}$ & 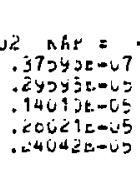 & $\begin{array}{l}173: 72 \\
?-.4+76 \\
5 \\
5 \\
5\end{array}$ \\
\hline $\begin{array}{l}4 \\
4 \\
4 \\
5 \\
3\end{array}$ & $\begin{array}{l}2 \\
3 \\
1 \\
1 \\
4\end{array}$ & $\begin{array}{l}5 \\
\vdots \\
\vdots \\
3 \\
1\end{array}$ & $\begin{array}{l}3 \\
3 \\
3 \\
3 \\
4 \\
4\end{array}$ & & $\begin{array}{l}1 \\
3 \\
2 \\
2 \\
3 \\
2\end{array}$ & 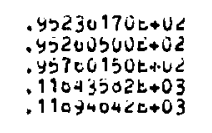 & $\begin{array}{l}.04704 E-1 c \\
.2044 Y E-15 \\
.03002-10 \\
.05470 E-10 \\
.1270 y E-20\end{array}$ & 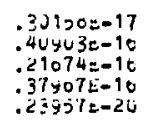 & $\begin{array}{l}.19095 k-17 \\
.01223 c-17 \\
.07240-17 \\
10057 c-10 \\
.520050-19\end{array}$ & 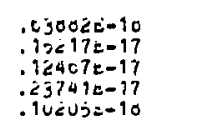 & 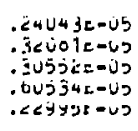 & \\
\hline $\begin{array}{l}3 \\
3 \\
3 \\
5 \\
5\end{array}$ & $\begin{array}{l}4 \\
3 \\
3 \\
2 \\
2 \\
2\end{array}$ & $\begin{array}{l}2 \\
3 \\
2 \\
4 \\
3\end{array}$ & $\begin{array}{l}4 \\
4 \\
4 \\
4 \\
4\end{array}$ & & $\begin{array}{l}1 \\
\vdots \\
1 \\
\vdots \\
2\end{array}$ & 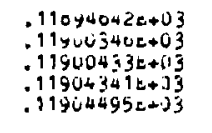 & $\begin{array}{l}.1276 y E-25 \\
.57050 E-21 \\
.57057 t=? 1 \\
.10054 \mathrm{~S}=17 \\
.10034 \mathrm{E}-17\end{array}$ & $\begin{array}{l}.23357 \mathrm{k}-20 \\
.2407 \% \mathrm{E}=10 \\
.24070 \mathrm{E}-10 \\
.50341 \mathrm{E}-17 \\
.5004<\mathrm{E}=17\end{array}$ & $\begin{array}{l}.52003 E-19 \\
.70498 E-10 \\
.70492 E-10 \\
.39447 E-17 \\
.39440 E-17\end{array}$ & $\begin{array}{l}.10205 E-10 \\
50051 \mathrm{E}=10 \\
.000920=10 \\
.135000=17 \\
130002=17\end{array}$ & 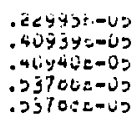 & \\
\hline $\begin{array}{l}5 \\
5 \\
5 \\
0 \\
0 \\
0\end{array}$ & $\begin{array}{l}0 \\
1 \\
1 \\
b \\
3\end{array}$ & $\begin{array}{l}3 \\
4 \\
2 \\
1 \\
2\end{array}$ & $\begin{array}{l}21 \\
4 \\
3 \\
5 \\
3\end{array}$ & & $\begin{array}{l}4 \\
3 \\
5 \\
4 \\
1\end{array}$ & 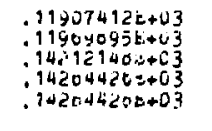 & $\begin{array}{l}.41747 E-15 \\
.94010=10 \\
.11029=15 \\
.20539=-31 \\
.20539=-31\end{array}$ & $\begin{array}{l}.712 j J E-10 \\
.30040 \mathrm{E}-16 \\
.57904 \mathrm{E}-16 \\
.10574 \mathrm{E}-22 \\
.10574=-22\end{array}$ & $\begin{array}{l}.14,002=-10 \\
.10000=-10 \\
17420 E-10 \\
.52413 E-20 \\
.52413 E-20\end{array}$ & $\begin{array}{l}.20003 E-17 \\
.24220 E-17 \\
.40121 c-17 \\
.3 y 100 E-19 \\
.34100 E-19\end{array}$ & 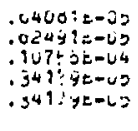 & \\
\hline $\begin{array}{l}1 . \\
t \\
0 \\
0 \\
0\end{array}$ & $\begin{array}{l}4 \\
4 \\
3 \\
3 \\
3 \\
6\end{array}$ & $\begin{array}{l}\vdots \\
\vdots \\
\vdots \\
\vdots\end{array}$ & $\begin{array}{l}3 \\
j \\
5 \\
j \\
j\end{array}$ & & $\begin{array}{l}1 \\
2 \\
\vdots \\
j \\
4\end{array}$ & $\begin{array}{l}14 \hat{2} 73+00 t+03 \\
114<73400 c+03 \\
14200307 c+03 \\
14200307 k+03 \\
14<05103 c+03\end{array}$ & $\begin{array}{l}.24040 E-2 b \\
.24040 E-23 \\
.0<040 t-21 \\
.0<04 t E-? 1 \\
.137 \text { yot } 17\end{array}$ & $\begin{array}{l}.550925-20 \\
.536 y 2 E-20 \\
.430404 E-10 \\
.43504 E-12 \\
.93797 E-17\end{array}$ & $\begin{array}{l}: 2 y 03=-10 \\
: 12 y 035-10 \\
.133202-17 \\
.13320=-17 \\
.07340=-17\end{array}$ & $\begin{array}{l}.20290 \mathrm{~L}-1 . \\
.20290 \mathrm{c}-10 \\
.90099 \mathrm{1}-10 \\
.40093 \mathrm{c}-10 \\
.239 y 4 \mathrm{c}-17\end{array}$ & 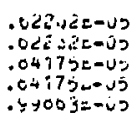 & \\
\hline $\begin{array}{l}0 \\
0 \\
0 \\
7 \\
7\end{array}$ & $\begin{array}{l}2 \\
\vdots \\
1 \\
1 \\
3\end{array}$ & $\begin{array}{l}4 \\
0 \\
2 \\
7 \\
2\end{array}$ & $\begin{array}{l}5 \\
5 \\
2 \\
0 \\
0\end{array}$ & & $\begin{array}{l}3 \\
5 \\
4 \\
0 \\
1\end{array}$ & $\begin{array}{l}14<05304 t+03 \\
142000924+03 \\
143037445+03 \\
10500033 E+43 \\
10041070=+03\end{array}$ & 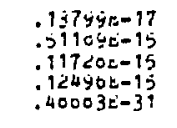 & 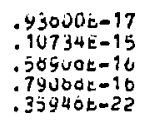 & 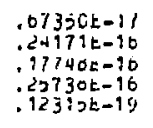 & $\begin{array}{l}.23495=-17 \\
.40220=-17 \\
.44900=-17 \\
.617335=-17 \\
.930305-19\end{array}$ & 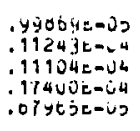 & \\
\hline $\begin{array}{l}7 \\
7 \\
7 \\
7 \\
7\end{array}$ & $\begin{array}{l}3 \\
4 \\
4 \\
3 \\
3\end{array}$ & $\begin{array}{l}3 \\
3 \\
3 \\
4 \\
4 \\
5\end{array}$ & $\begin{array}{l}0 \\
0 \\
0 \\
0 \\
0\end{array}$ & & $\begin{array}{l}2 \\
2 \\
2 \\
3 \\
3 \\
4\end{array}$ & $\begin{array}{l}106+1070 t+03 \\
16652279 E+03 \\
1005 c 27 y E+03 \\
10000203 E+03 \\
10000203 x+03\end{array}$ & $\begin{array}{l}.400031-31 \\
.310020-25 \\
.310230-25 \\
.95401 E-21 \\
.55901 E-21\end{array}$ & $\begin{array}{l}.35 y 40 e-22 \\
.191340 c-20 \\
.913400-20 \\
.04150 E-10 \\
.04135 t=1 c\end{array}$ & $\begin{array}{r}.12315 b-19 \\
.23034 z-10 \\
.23034 c-10 \\
.21203 c-17 \\
.21203 c=17\end{array}$ & $\begin{array}{l}.95030 t-19 \\
.400330-10 \\
.400330-10 \\
.10270 c=17 \\
.10274 t=17\end{array}$ & $\begin{array}{l}.07 y u b=-U S \\
.121110=-i 4 \\
.1<1110 E-j 4 \\
.147100-U 4 \\
.14710 x-U 4\end{array}$ & \\
\hline $\begin{array}{l}7 \\
7\end{array}$ & $\frac{2}{2}$ & $\begin{array}{l}7 \\
5\end{array}$ & v & $\frac{1}{2}$ & 3 & $\begin{array}{l}106057000+03 \\
.10000205 L .03\end{array}$ & $\begin{array}{l}15171 E-1 T \\
.15171 E-17\end{array}$ & $\begin{array}{l}131120-10 \\
-13112=-10\end{array}$ & $\begin{array}{l}\text { Iu fyýz-10 } \\
\text { 1uz00e-10 }\end{array}$ & $\begin{array}{l}.3702 c=-17 \\
.37022=-17\end{array}$ & $\begin{array}{l}.105052-0 . \\
.10507 \mathrm{k}-0-\end{array}$ & \\
\hline
\end{tabular}




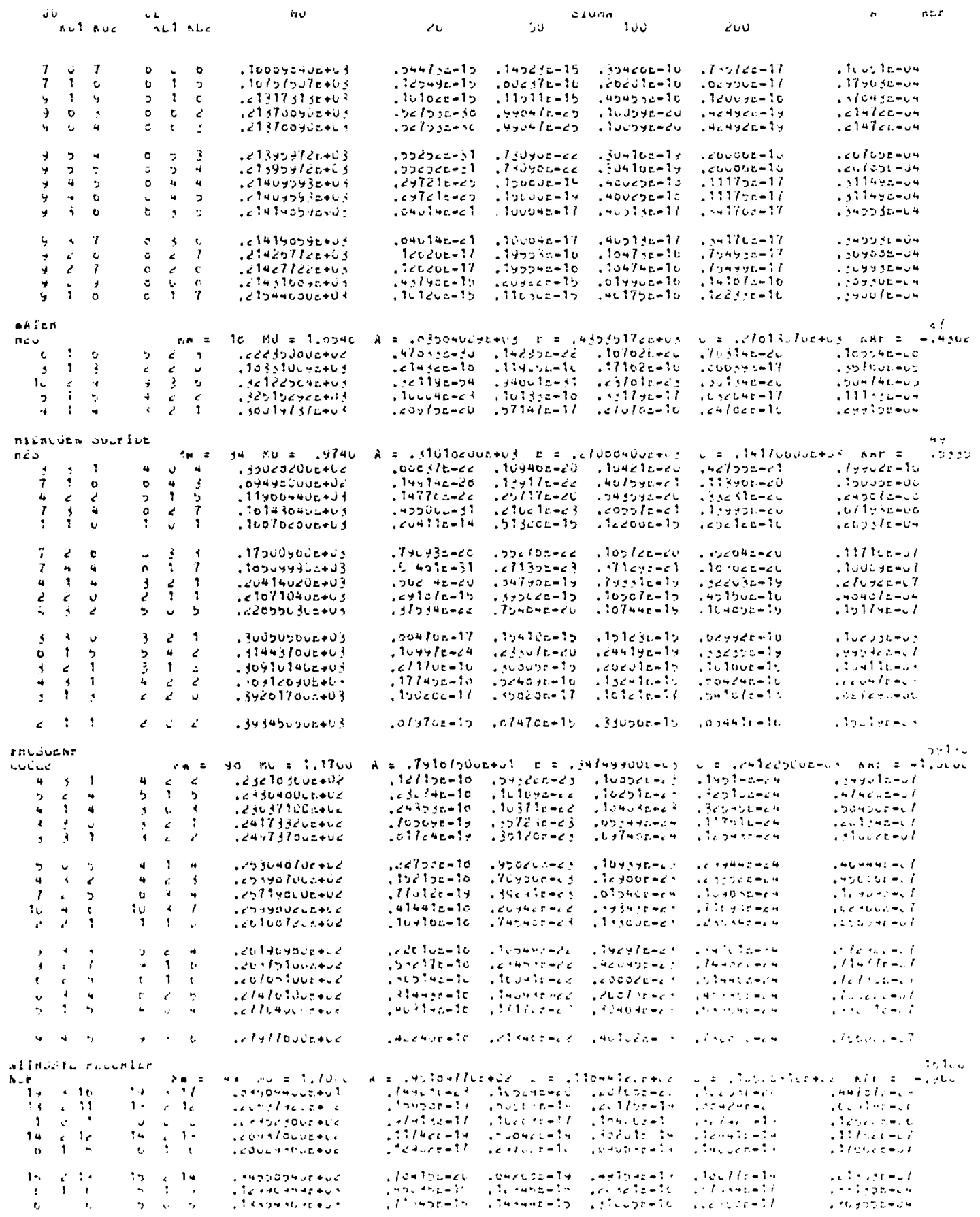




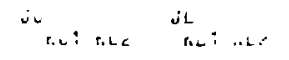

$\begin{array}{ll}5 & 5 \\ i & \vdots \\ 2 & \vdots \\ 0 & \vdots \\ 0 & \vdots \\ 1 & \vdots \\ 7 & \vdots \\ 1 & \vdots\end{array}$

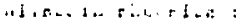

ar

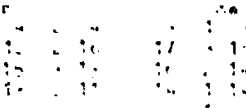

$=\quad-i=$

$\begin{array}{cccccc}\vdots & \vdots & \ddots & \vdots & , & \\ 1 & \vdots & \vdots & \vdots & \vdots \\ \vdots & \vdots & \vdots & \vdots & \vdots & \vdots\end{array}$

$\begin{array}{cccc}\because & \vdots & \vdots & \vdots\end{array}$

$\because+\frac{\vdots}{\vdots}+\vdots \quad \vdots \quad \vdots$

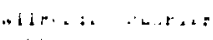

....

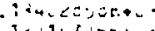

$\therefore 34 !=34=5+c$

inticisotas

$.9-1 \leq \leq 100+2$.

$\therefore+3, \leq+c+p+1:$

$\because<i+1<0: 2+0$

IVivivete

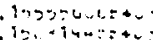

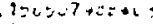

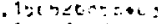

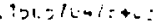

$\therefore>1<0: y+a t$

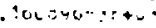

. 1 1694 $140+\ldots$

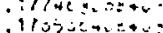

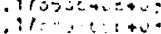

, $770: 4, \ldots+\ldots$

$.972011+0+5+6$

$170 \cdot 0.00+2+0$

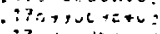

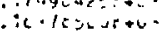

. inacoirleanat:

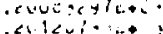

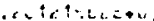

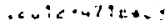

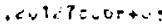
sul recuet.

ivisinctetc.

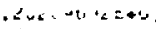

.21 46 ?: $2: 45$

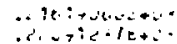

cer mane

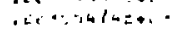

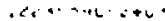

.x.

icterergets

ortation

ox
$\therefore-1$

anc $4: 2+4 t+6$

$\therefore$. $\therefore, \therefore<P+4$.

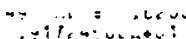

.

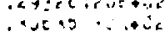

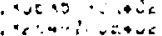

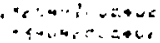

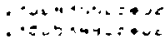

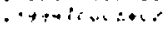

.8 $362 \ldots$

. $19.0 .9 \mathrm{~s}$.

$\therefore 1,1+11, \cdots$

$\because 1+2,01: \ldots$

$\theta, n=2, \cdots 6$

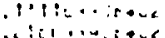

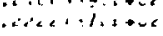

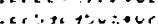

Stur

is

end

ner

$<06:+i=13$
$1+207: 5=17$

$3 x>2 x-10$

$\therefore 14+4 L=50$

$\therefore<1<2=-12$

. $x<t_{t}=-10$

$-<+<a+4-10$

. $2 / 10 \mathrm{~d}-17$

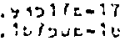

. $5 e u<C L-1$

. $17 x<>c-17$

. $<=5\{4 c-1\}$

$\because 370=0$

$\csc \ln .-i^{\circ}$

. $26: 2, x=20$

. Ic $7 \geq 2 \mathrm{c}-\mathrm{il}$

- $4 a s<t=10$

zacle -12

-lac $=-i s$

$\because \because \operatorname{lin}:=1$

. $444<12-i=$

. $נ<45 y<-i ?$

$\therefore$ \&Oe 7 .

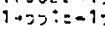

icuyter

$-4<4 y+010$
He $71-5=1$

1.: ?:

itsous=12

orfic -17

- cevujelit

$\Leftrightarrow \rightarrow y>1:-17 \quad$. $20, i r-16$

1 4 $4000-10$

ins $: 1 c-9$

- Enac: c-:?

c $\angle 1 \geq 4=-1$

$8: 0+15=:$

$1+x+5=1$.

- Provia-le.

$.207225-11$

- Celle-il eucese-17

- Re-ce-1e

- juinstat?

, ivention

$.00>4=-10$

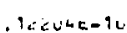

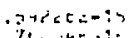

Thunte

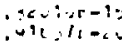

$c c+2 r_{1}-9$

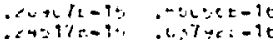

$c \geq 200 x-10$

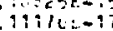

- $1.9746:-10$

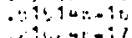

. izc:e-1c

. 11,04L-ic

$.5724 \times-i t$

oviul:=18

<x+10e=:?

- wivest anisie-10

- Vicipers.

$\therefore 1+2=-1 ?$

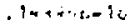

$\therefore: 10: s,: 17$

ccerez=-1)

$\because y=4$ is $-i$

- $1914 \cdot--11$.

$\therefore 10=-i 1$

, $x+7 c 1 \mathrm{r}-17$

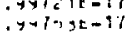

ic iecelio

indue-je

$\because+4: 15+2 ?$

. xil $=:=$

$: 7<1: s-10$

- $1401+8-16$.

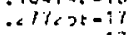

-3ucone-17

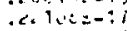

- isman

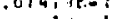

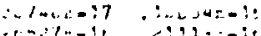

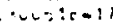

or 146crai:

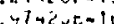

. $7: 16 y+-17$

, il.ye+t=tl

ulinisute

. $\therefore<=:=-$ ?

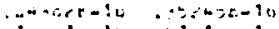

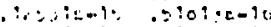

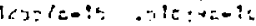

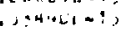

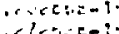

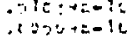

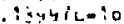

$.14 v u+1:=16$

ivicitell

$\therefore y \div 910-10$

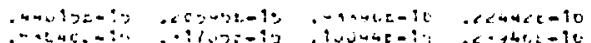

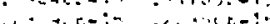

,$\rightarrow 12 \therefore=-i$

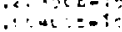

10unate?

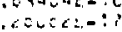

$x+1+1,=10$

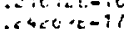

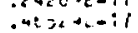

1 12.26015

.

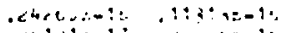

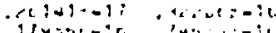

$.13+4 i=-20$

istacoset:

$1100 ;=16$

$\because 4: 28=i 1$

- $94,: 11-7 ;$

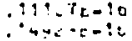

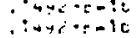

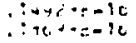

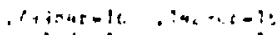

$\therefore \therefore+c .=-$

$\therefore+C+C=18$

$0 \div+1+5=-2$
$\therefore+101=9$

cxchats

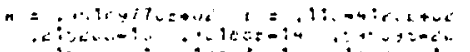

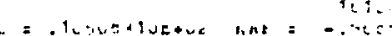

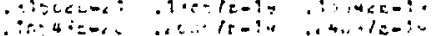

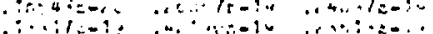

0.

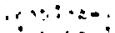

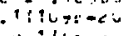

$\because+1 \cdot i=c-c$

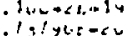

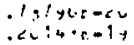

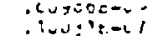

iigulate-

ic: $9442=$

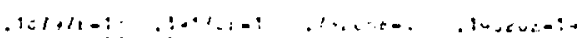

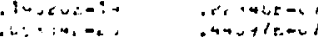

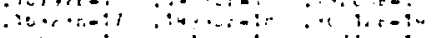

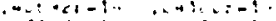

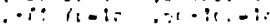

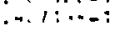

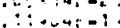

i.

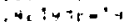

icuruces

in inge.

iोist:-

... i. $<-\because, \quad, \cdots \ldots-i t$

: $1,191=$

are tratir

$\therefore a(1, a)=$

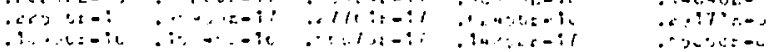

$\sim=$

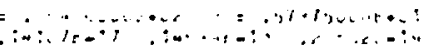

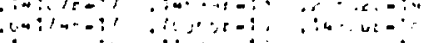

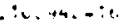
$\therefore \therefore:=-1$

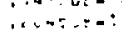

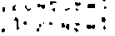

$\therefore \therefore ル 4+\cdots$

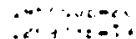

$\therefore a+b=-4$ ir ise-t.
औ.

-110:-20.5.

$\because 10:=0$
1 tne $=-\ldots$ 


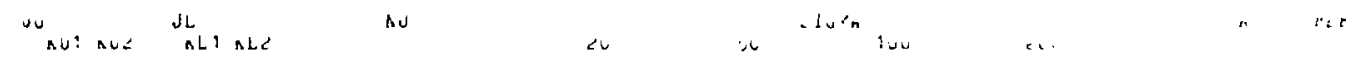

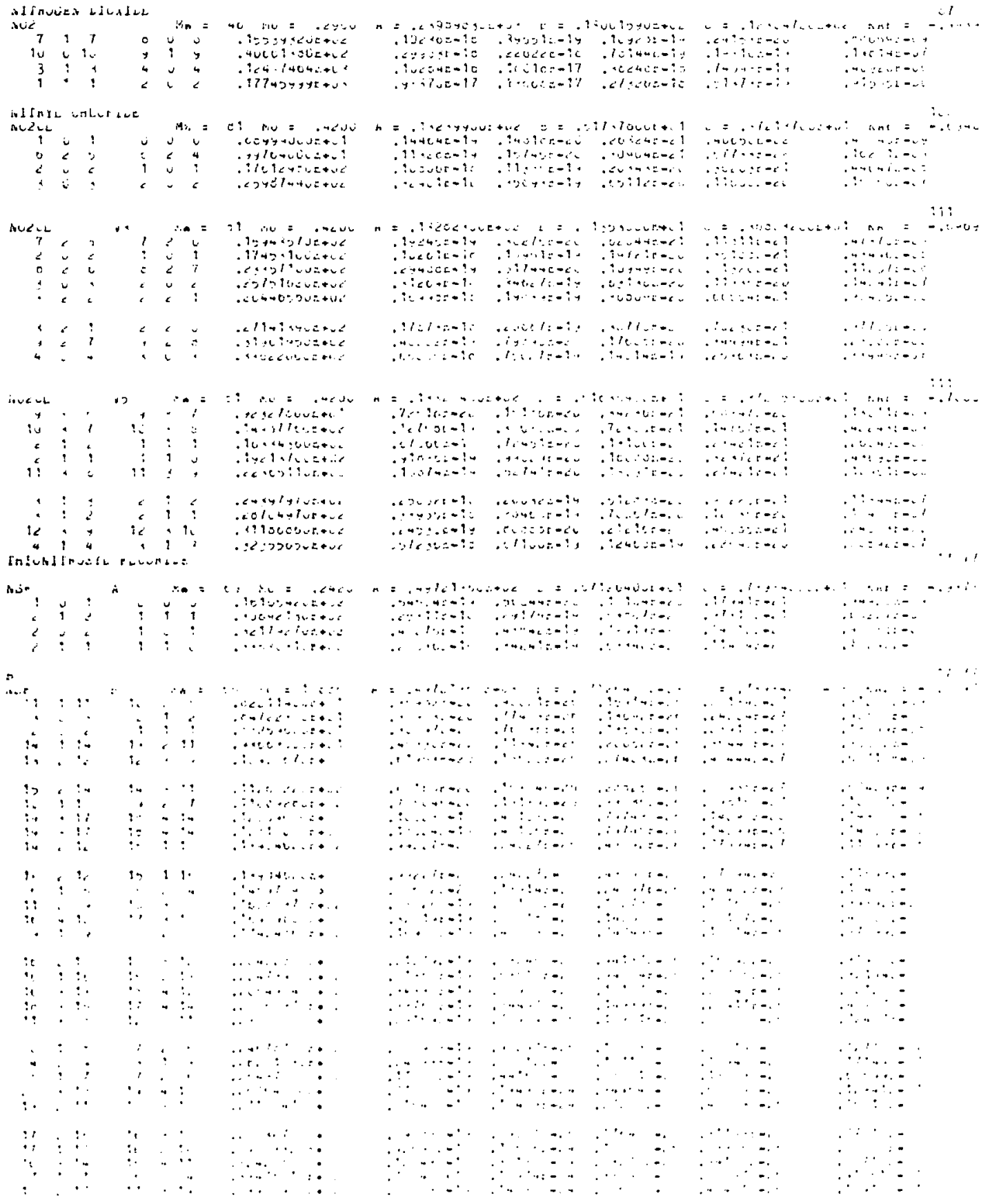




\begin{tabular}{|c|c|c|c|c|}
\hline 3 & 1 & 4 & 4 & $<$ \\
\hline Iu & $c$ & $\dot{u}$ & 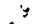 & : \\
\hline 11 & $r$ & Iu & it & $\vdots$ \\
\hline ب ? & 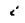 & 1. & ت 1 & ; \\
\hline 17 & 6 & $: 0$ & $1 x$ & $\vdots$ \\
\hline$<0$ & 6 & $i y$ & $i y$ & $\leq$ \\
\hline & $\vdots$ & $i$ & $?$ & $v$ \\
\hline & $?$ & ${ }^{\prime}$ & 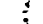 & $\therefore$ \\
\hline & $i$ & 2 & 7 & $*$ \\
\hline & i & a & $c$ & \\
\hline
\end{tabular}

$34 / 50 j=v e+j C$

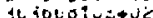
$370<030 v e+12$ - joubcruiovic . 313y+4j $5 v+j c$

$.5777 \% 45 u e+b e$ $42 x+44 t u=40$ $.54 \mathrm{~s}$ juvun $+\mathrm{du}$

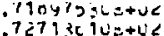

. coto7te-1y .71937e-c5 19lute-10

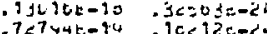
$-7<7 y+t-8 y$
$.7<171 r-10<12 c-2 \alpha$

.704 dec-iy :i90<clec4

7yjouc-1y .19009t-ach $.40071 x-10$ :1944t-ce

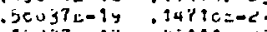

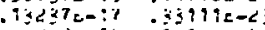

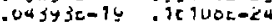

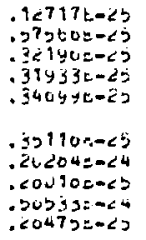

$224012-<0$ $10177 \mathrm{r}-\dot{x}$ it $>100-<0$ $.30450 \mathrm{x}=<0$ $.6134 y=-<0$

bejoje-ic $320>7 \mathrm{C}=\mathrm{CS}$

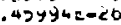
$10 \div 47 \varepsilon-24$ $.04337=-<0$

$.2 E \cup 5 Y=-U 7$ $24 \dot{1} 77=-\Delta 7$ $.204<3=047$ $.170 \dot{0} 7=-67$ .$<142 j E-47$

$.90000 \mathrm{e}-\mathrm{T} 7$ iseyise-us

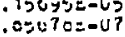
- holuic-us . ickzes -40

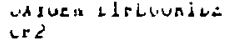

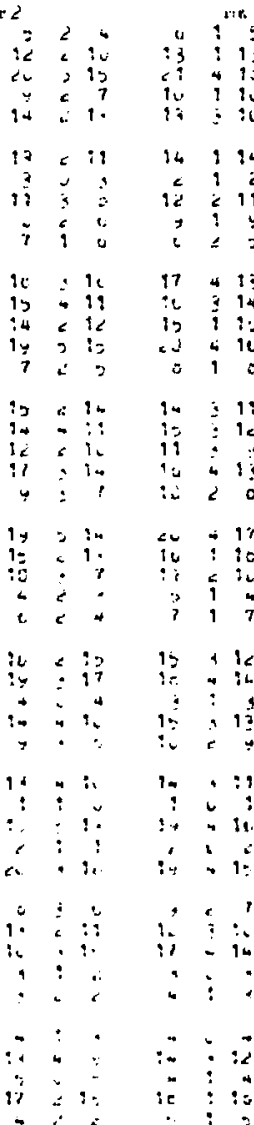

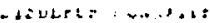

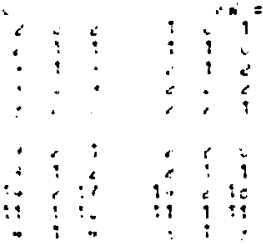

$=2 x=.6 y>$

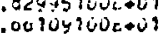
.c7d2 Y $1 \mathrm{JCL}=+\mathrm{V} 1$ gujb7t<letue 12702=yJe+Li

.135751005 .002 Gougúguetú isuenczurave $.1577144 \dot{s}+0$ a . 1735G7lvítue

10uysut $72+U 2$ - iyufaloúz-0z - <isa 4 ve Jritus ¿2strajuetwe cegoncyeutave

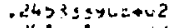

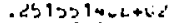
. Csगe7civetic ulycizzjexua $.274 y 20040+16$

$. \angle y z=3030=0+02$ . $91401470 \mathrm{n}+U \mathrm{C}$ . j1114suvatce .947 :ocuzade Ind4angutell

0.1429 ! TuUzala - jejpojuetla - geveop?utrotic - adwoocise+uce $.4 c+1.91 \mathrm{u}$ tous

$\rightarrow \rightarrow 1<0<: \mu_{2}+\infty$

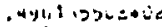

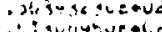
.21147 yivrecer

iscuchunatue $\therefore$ ipueozus ave o jogrivisave -541:Pugucas $\therefore 7$ t7lulatie

.7750906206 Doisgeustos

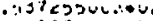

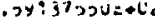
- osuleve =

ou $\therefore=. e 73$. $\because \forall i<c+4 v=e x$

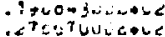
ic: $7: 49 w 2+42$ . $\angle 00 y ;<k+z+u<$

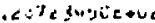

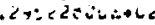

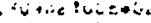

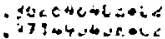

$A=$ ju7urujuttue $1=.14 \times 4045 i c+42$

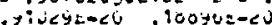
A

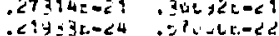

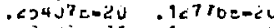

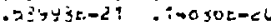

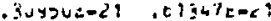
Dylcosely .035yte-20

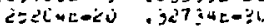

luvuse-1y jestocted

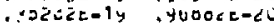

$.33+20 y=-2<$ $.17 v$ jejec 1 - JUAClE-z:

$.3>4<4$ c. -23

. $3<\mathrm{Cuc}-1 \mathrm{C}$

$.9201 x-61$ $.11304=-20$ . 47707 tét -j1470e-c1 totise-15

-yysicic-z? - Prytur-e?

7n>Voe-cj

$13700=-c$ ?

-1910ye-1y

$.4120 \dot{2}=-2 b$ $\therefore 7 y$ yicasu Qiviele-14 . cunsze-ci

$.0104 z-23$ .cooiy $=-21$

$.101 y y 0-1 y$

icotur-10.

cresyee-1y

cylule-29

$.90024 \pi-20$

- isenge-1y

- $25 e v 0=-1$.

$.<1+41<-1$,

$4043 x+42$
$4244 y=-2 y$

DDsoci=-<1

- Igecle-r

$.3054 j \mathrm{jr}-<$ ?

$.29<37 c-<$ ?

$12030=-20$

$13>3 \cup 5-2$

INO4:E-cL

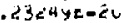

10y $77=-20$

14 ¿ोjद-<ل

. b30yyt-a 1

$.10207=-<0$

$.22010<-20$

- $20033 c-20$

- $2035=00$

$.41947 \mathrm{e}-2$

.44500:-

$17174 \mathrm{E}=<\mathrm{C}$

$.00 y 04=-C^{\circ}$

$.30 \%$ yuem<o

- 4yous $5:-<0$

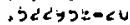

yox $13 y<-c 1$

- vaverero

. $4<0 ; 3=-<v$

-

-1.cace-ru

वर $10-2$

oujuce

nuty<e+<

gloutely

$\therefore x^{2}<y<0<x$

.171 jutel

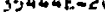

- Reudice-1y

$.6\left\{47_{E}=00\right.$

$.13127_{c}-1 y$

. Buesze-14

i yx $x=-1 t$

- isolueso

. Tacuuz-co

daver:a-17

- $4 x+4 \cos 2<$

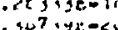

$07494=-1 y$

$.1+7 y 20-1 y$

. $434 y, .=29$

asuluts-17

culjecte

-jeyse-it

7410 jo-du

"tintelt

$\therefore$ जu=-tec?

entajar-1.

$\because 9114 x-9 x$

$\therefore 46<25-60$

$.3747 v E-1 c$

$.43 .5 \times 1 .-14$

aprzeselic andue-15 crivureme

. ienulemer

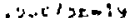

$20,7 u x=1 y$ - bosis-a $.4 x+c^{2} x=1$ $.0447 y=-20$

$\therefore a t s 7=-14$ icomic-1y - $402<05.060$ Taljote-1y

$x, y, c e c e-50$

$.00 .07 r_{r-1}$

$\therefore+445-14$ guchireis .10,ugiece icicuis:y

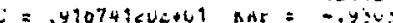

edyyus-ce .52139te-1u

$4 ! 70 \leq 5-20 \quad$ ieju7tell - yU4joz-cL ipyour-iu

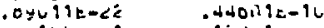

.$<4104$ LEC

$.04000<-22$

cojosen-

äou $3\{-<1$

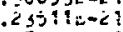

$470002-<1$

. $7521 t-29$

$5+173 \mathrm{c}-\mathrm{c} 1$

Poot 4 ces?

$.00 y 000-2$ ?

$.54030 \mathrm{e}-\mathrm{Cl}$

$.04435 \mathrm{CL}-\angle 1$

$.70005=-\dot{c} 1$

$.1141120<v$

ivua $76-20$

- luegue-so

. issoltevy

$.24701 e-10$

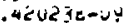
- ¿7ulae-uy - iy 7 ic $.4077 \leqslant 5-4$ C

$10 \geq 0>E-<U$ . दो love-cl $.1450+5-20$ . $400>7=-21$ $11734=-20$

. $4280 j 32-u y$ $. j 100 y=-4 y$ , $13500 \mathrm{~J}=-v_{y}$ .0j146f-uy .75007t-Ly

guible-uy levalando Tey yez $=v c$ - $193330=00$ . $15770=-v e$

1yajocuse .4U73 Je-Uy icsloge-so coster-vo ¿xos<c-ua

. 15<11Lecu $11450<-20$ . $\rightarrow 77$ OefeC संO4Ye-iov - jeveorelu

- $230 u z=-\pi 0$ 


\begin{tabular}{|c|c|c|c|c|c|c|}
\hline \multicolumn{3}{|c|}{ Ju nut nue } & \multicolumn{3}{|c|}{ JLL } & \multirow[b]{2}{*}{ 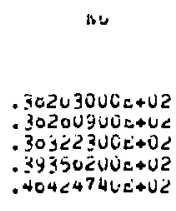 } \\
\hline $\begin{array}{l}4 \\
4 \\
4 \\
4 \\
5\end{array}$ & $\begin{array}{l}0 \\
2 \\
2 \\
1 \\
1\end{array}$ & $\begin{array}{l}4 \\
3 \\
2 \\
3 \\
5\end{array}$ & $\begin{array}{l}3 \\
3 \\
3 \\
3 \\
3 \\
3\end{array}$ & $\begin{array}{l}u \\
2 \\
2 \\
1 \\
1\end{array}$ & $\begin{array}{l}3 \\
3 \\
2 \\
1 \\
1 \\
2 \\
4\end{array}$ & \\
\hline $\begin{array}{l}5 \\
3 \\
5 \\
3 \\
5\end{array}$ & $\begin{array}{l}0 \\
2 \\
4 \\
3 \\
3 \\
2\end{array}$ & $\begin{array}{l}5 \\
4 \\
1 \\
3 \\
3 \\
3\end{array}$ & $\begin{array}{l}4 \\
4 \\
4 \\
4 \\
4\end{array}$ & $\begin{array}{l}0 \\
0 \\
2 \\
4 \\
3 \\
2 \\
2\end{array}$ & $\begin{array}{l}4 \\
3 \\
0 \\
0 \\
2 \\
c\end{array}$ & $\begin{array}{l}.477 U 70 Z U E+C Z \\
.4751004 U E+U L \\
.47044 \angle J U+U 2 \\
.47004040 E+U 2 \\
.47940040 E+U 2\end{array}$ \\
\hline $\begin{array}{c}13 \\
0 \\
130 \\
10 \\
10\end{array}$ & $\begin{array}{l}1 \\
1 \\
\vdots \\
0 \\
i\end{array}$ & $\begin{array}{r}12 \\
6 \\
5 \\
4 \\
4\end{array}$ & $\begin{array}{r}13 \\
5 \\
9 \\
9 \\
9\end{array}$ & $\begin{array}{ll}1 & 1 \\
1 & \\
0 & \\
0 & 7\end{array}$ & $\begin{array}{l}15 \\
5 \\
4 \\
3 \\
3 \\
3\end{array}$ & 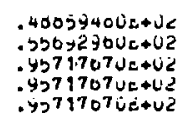 \\
\hline $\begin{array}{l}10 \\
10 \\
10 \\
10 \\
10\end{array}$ & $\begin{array}{l}7 \\
0 \\
0 \\
b \\
b\end{array}$ & $\begin{array}{l}3 \\
2 \\
3 \\
6 \\
5\end{array}$ & $\begin{array}{l}\dot{y} \\
y \\
y \\
z \\
\vdots \\
\vdots \\
y\end{array}$ & $\begin{array}{l}7 \\
0 \\
0 \\
5 \\
5\end{array}$ & $\begin{array}{l}2 \\
1 \\
2 \\
2 \\
4\end{array}$ & $\begin{array}{l}.9571707 G c+0 E \\
.7572217 U E+U 2 \\
.95722170 E+U 2 \\
.557240 U U E+02 \\
.95724 J U U E+U 2\end{array}$ \\
\hline $\begin{array}{l}10 \\
10 \\
10 \\
10 \\
10\end{array}$ & $\begin{array}{l}4 \\
4 \\
3 \\
3 \\
2\end{array}$ & $\begin{array}{l}7 \\
0 \\
5 \\
7 \\
0\end{array}$ & $\begin{array}{l}y \\
y \\
y \\
y \\
y \\
y\end{array}$ & $\begin{array}{l}4 \\
4 \\
3 \\
3 \\
2\end{array}$ & $\begin{array}{l}0 \\
j \\
7 \\
0 \\
7\end{array}$ & 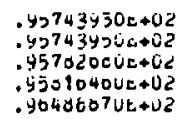 \\
\hline $\begin{array}{ll}11 \\
11 \\
11 \\
11 \\
11\end{array}$ & $\begin{array}{l:}1 \\
0 \\
2 \\
7 \\
7\end{array}$ & $\begin{array}{l}1 \\
11 \\
14 \\
5 \\
4\end{array}$ & $\begin{array}{l}10 \\
10 \\
10 \\
10 \\
10\end{array}$ & $\begin{array}{ll}1 & 1 \\
0 & 11 \\
2 & \\
7 & \\
7 & \end{array}$ & $\begin{array}{l}10 \\
10 \\
4 \\
3 \\
3\end{array}$ & $\begin{array}{l}10100330=+03 \\
10342310 E+03 \\
1050117 J e+03 \\
10529200 E+03 \\
10529200 E+03\end{array}$ \\
\hline $\begin{array}{l}11 \\
11 \\
11 \\
11 \\
11\end{array}$ & $\begin{array}{l}0 \\
0 \\
0 \\
0 \\
0\end{array}$ & $\begin{array}{l}0 \\
5 \\
4 \\
3 \\
7\end{array}$ & $\begin{array}{l}10 \\
10 \\
90 \\
90 \\
10\end{array}$ & $\begin{array}{l}1 \\
0 \\
0 \\
0 \\
2\end{array}$ & $\begin{array}{l}5 \\
4 \\
3 \\
2 \\
0\end{array}$ & $\begin{array}{l}1052944 d E+U 3 \\
10529440 E+63 \\
10529050 E+113 \\
10529050 E+43 \\
.10530450 E+63\end{array}$ \\
\hline $\begin{array}{l}11 \\
11 \\
11 \\
11 \\
11\end{array}$ & $\begin{array}{l}3 \\
3 \\
3 \\
2 \\
2 \\
1 \\
1\end{array}$ & $\begin{array}{l}0 \\
\dot{y} \\
y \\
y\end{array}$ & $\begin{array}{l}10 \\
10 \\
10 \\
10 \\
10\end{array}$ & $\begin{array}{ll}5 & 5 \\
3 & 5 \\
3 & 5 \\
2 & 5 \\
1 & 5\end{array}$ & $\begin{array}{l}j \\
9 \\
z \\
0 \\
y\end{array}$ & $\begin{array}{l}10330450 t+03 \\
10535010 e+03 \\
10543490 t+03 \\
10030000 t+03 \\
110704530 k+03\end{array}$ \\
\hline 15 & $\sim 1$ & & 14 & 014 & 14 & $.94045020 c+03$ \\
\hline
\end{tabular}

o

520

\begin{tabular}{|c|c|c|c|c|c|c|}
\hline $\begin{array}{l}20 \\
12 \\
15 \\
17 \\
11 \\
12\end{array}$ & $\begin{array}{l}2 \\
3 \\
3 \\
2 \\
1\end{array}$ & $\begin{array}{r}70 \\
13 \\
14 \\
9 \\
11\end{array}$ & $\begin{array}{l}13 \\
10 \\
10 \\
15 \\
\text { it }\end{array}$ & $\begin{array}{l}1 \\
1 \\
2 \\
2 \\
1 \\
2\end{array}$ & $\begin{array}{l}16 x \\
13 \\
14 \\
17 \\
12 \\
10\end{array}$ & 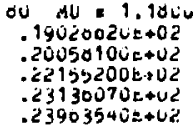 \\
\hline $\begin{array}{c}0 \\
7 \\
10 \\
10 \\
20\end{array}$ & $\begin{array}{l}0 \\
2 \\
2 \\
3 \\
4\end{array}$ & $\begin{array}{r}6 \\
6 \\
13 \\
10\end{array}$ & $\begin{array}{l}2 \\
6 \\
11 \\
17 \\
19\end{array}$ & $\begin{array}{l}1 \\
1 \\
1 \\
2 \\
3\end{array}$ & $\begin{array}{r}5 \\
7 \\
11 \\
10 \\
17\end{array}$ & 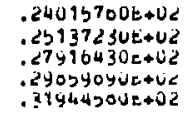 \\
\hline $\begin{array}{r}14 \\
9 \\
7 \\
13 \\
6\end{array}$ & $\begin{array}{l}3 \\
6 \\
4 \\
1 \\
2\end{array}$ & $\begin{array}{r}12 \\
7 \\
7 \\
12 \\
3\end{array}$ & $\begin{array}{r}12 \\
10 \\
0 \\
12 \\
7\end{array}$ & $\begin{array}{l}? \\
1 \\
1 \\
\vdots\end{array}$ & $\begin{array}{r}11 \\
14 \\
0 \\
11 \\
0\end{array}$ & 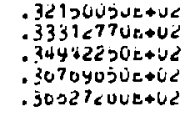 \\
\hline $\begin{array}{c}1 \\
15 \\
2 \\
3 \\
0\end{array}$ & $\begin{array}{l}1 \\
3 \\
3 \\
1 \\
1 \\
2\end{array}$ & $\begin{array}{r}0 \\
12 \\
1 \\
2 \\
u\end{array}$ & $\begin{array}{r}1 \\
10 \\
2 \\
3 \\
y\end{array}$ & $\begin{array}{l}u \\
\dot{2} \\
j \\
u \\
j\end{array}$ & $\begin{array}{r}1 \\
13 \\
2 \\
3 \\
4\end{array}$ & 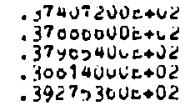 \\
\hline $\begin{array}{r}4 \\
5 \\
14 \\
1 \\
14\end{array}$ & $\begin{array}{l}1 \\
1 \\
3 \\
1 \\
1\end{array}$ & $\begin{array}{r}3 \\
3 \\
11 \\
13\end{array}$ & $\begin{array}{r}4 \\
5 \\
15 \\
15\end{array}$ & $\begin{array}{l}0 \\
0 \\
\vdots \\
u \\
\vdots \\
2\end{array}$ & $\begin{array}{r}4 \\
3 \\
14 \\
\vdots \\
12\end{array}$ & 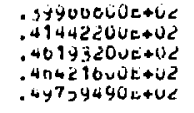 \\
\hline 9 & 1 & 0 & $y$ & $u$ & 4 & $.2100 \mathrm{~J} 2 C^{\prime} L=+0 \angle$ \\
\hline
\end{tabular}

20 so sivisin no no ner

$.72004=-17$

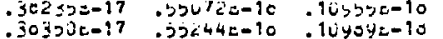

-

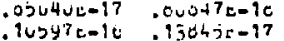

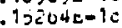

$.20057 \mathrm{c}=10$

$.<0<>35-1 y$

$\angle U>4<E-1 y$

cंنouos-1y

$-2750 t \leq-1 y$
$+412<0-1 y$

$\therefore<<J 7 U a-L U$

$.100 g 7 e-4 c$

LuTíc-ua

$.220345-v 0$

.30×نosalo

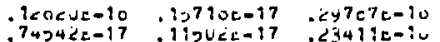

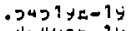

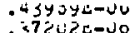

i isyorive

- isyoutave

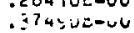

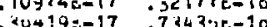

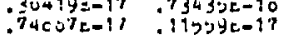

- $1110=-15$

. $10342 \mathrm{c}-10$

$17124=-10$

$\therefore<40 y=-1 y$

.0uybie-19 . L SOYCE-1Y

$.10<14 \mathrm{E}-10 \quad .2<y=<E-17$

. iv474t-17.1כ250E-17

- iva7atit

. uTCOUt:-cu

. $45: y 12-1 c$

$\therefore$ DOUวYe-La

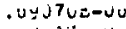

- <scitie-us

$.<3017+-i=$

. loy ye-Jo

. $201 y 0=-10 \quad .77070=-10$

$.04071 \mathrm{col}$

- 105yว=-10

. 131 Y

$.4050<2-16$

$.40502 \mathrm{E}-19$

$.00505 \mathrm{E}=17$

:3<7<3E-17

$32<03=-10$

$.131 y 7 c-10$

. 1 ¿y7ye-l’

$.133 y+E=U ?$

1jyyye-va

1 jyyyedva
.$<7$ yice-d:

- C7y ice-vy

$.0171 y_{i}-17$

-

$.<0 y j<t-17$
$.20 y \supset<\varepsilon-17$

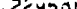

Y5O0ec-10

Y YOOCL-10
. Yot $00-10$

- Disuce-iy

$.2405 y+2=10$
$.246 .5 y c=10$

- 1 1<cuévo

$017750=17 \quad .410<3 c=17$

$\begin{array}{ll}.017 y=-17 & .410<3=-17 \\ 1055=-10 & , 59914=-17\end{array}$

.10ว5 $10-10$

.2751vei=10

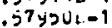

$.1<011=-17$

$12011=-17$
$15409=-17$

. Cyidose-14

. $2 y$ ivos-10

$.3367 \mathrm{c}=10$

. 1549ye-i7 oj301E-10

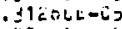

.35y.j5-L:

-3ituyiarj

.3usyu=us

- $37207=-10$

$.73 y 30=-17$

$10107 t-17$

$37074 \mathrm{c}-10$

$.4400 y 0-05$

$.47705 E-U_{4}$

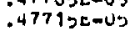

. $<y<0<a-J$

isyouji-us

315 tOr $=10$

. I1วY0k-10

- Iv295e-10

.07472017 17

$10 \times 320-17$

$.110000-17$

.20) $10 \mathrm{C}=17$

$.0<739 \mathrm{c}-10$

- 20015

$.40<4<c=10$

- 1 y $13040-1$

- yju $7 k-10$

. CO4YIE-10

$.34 y>x<+\sqrt{5}$

. इसपडरह

.$<344 J 5-\dot{S} 5$

$.25+40 \div-0 ;$

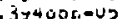

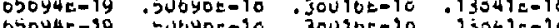

$.37790 \mathrm{C}=17$

$34364=-17$

izojace 17

isuves-10

$.3 y 4000-u 3$

.357 jue-17 . 34304=-17 . 1<03j=-17

33 tivor-10

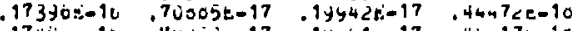

174uye-10 .7uyjue-17 .19y012-17.44j17k-ir

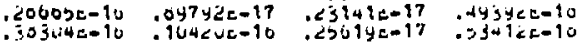

. $401030-\mathrm{i}$

$.40<37 \mathrm{C}_{0}-v^{2}$ ?

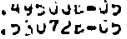

$.303 j 1 c=10 \quad .157492=10 \quad .40030=-17 \quad .191 j 4 c-17$

$.19 y i<z=v 4$

$h=.41915440 \mathrm{c}+42 \quad t=.20590600 t+i 1$ $.1745+c-16$.04bj5c-1y .17b1je-1y :YU475E-19 .77TU4t-19

.j29u4t-19 :71040b-19

- $22944 \mathrm{k}-19$

. $00<50 E-10$

$71040 \mathrm{~b}=19$
$10574=-? 0$

$.2135<2=10$

$.20023 e-1 y$

- Juं $19<2-19$

1 $27250=-1 y$

$.27250 e-1 y$
$5255 \mathrm{~J} e-1$

1919

$.279060-17$. 370025-10

$.9022 T_{t}-10 \quad .177$ Out -10

$.737200-14$

$3037 \cup L-1$

- 4u4jyti-1y

. $204 c^{2}+2-i y$

$14>35 \dot{y} t=10$

$1 ? 54 \div 0=1$

ititoz-is

300570072

$30057 E-<0$
$7 U 77$
$0 E-<U$

$0220+5-2 u$

$30104 E-<0$

11915e-1y

.7usouz=:

- 30035c-10

-cuyoitc-10

- $v 4$ i $z=17$

$.24434 \mathrm{c}-10$

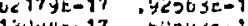

$1 y 432 k=17$
.190

- गن $0 y<2-10$

. $271275-14$

, $150010-15$

$.744005-C 0$

$.0410<2=20$

$.140024-1 y$

$.200+50-1 y$

- j350ue-17 . o7asue-1a

. $2 y 7,1 \mathrm{~s}-3 \mathrm{c}$

$1:$ voye-10

- $25300=10$

$.10010 t-10$

$17<<U$ e $-1 y$

$0015 y=-7 y .13104 c-1 y$

$90730.5=10$

- 10400l-ir

- $107<10-1 y$

17 voyr=10 :3931<e-14

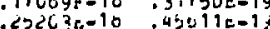

.70zojz-1y isloje-1s'

$.14<77 t-16$ -34uvue=10

.0ugrok-1y

$.10321 c-10$

$<1010 \mathrm{~L}=17$

- $3920<-10$

$00620 i=19$
$17<b b=1 y$

$417 y 1 c-10 \quad: 17<b b=-1 y$
$1171 y=-10 \quad: 12470 \leq-17$

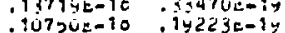

$540>0 E-17$
$19 P C U E-17$

$.59420 \pm-10$
$.4<U \supset \supset E-10$

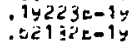

$.10004 c-10.37773 c-17.04124 c-10.10092 x-1 c$

$.05011 \mathrm{k}=00$

$.19745 E-U 1$

$144 C 4 E-U ?$ $1200 y e-6 ?$
$.24000-67$

.4704 Ue- 07 . 24 yjue-67 -c 3ubte-u'?

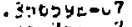
. testormu?

$.4731: E-U 7$ -40055 $5=-47$

1000je-vo $.94094 c-u ?$

$.7<23<\mathrm{c}-u>$

$.4<490=-00$ $44 v 13 c=0$

- 4losule-ve

.7 UUYE-U?

4yำ $25-v 0$

- 34vo4c-vo

- $1321 \supset=-6.6$

. $2404<5-00$

. yu4 $40 z-U u$ 


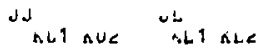

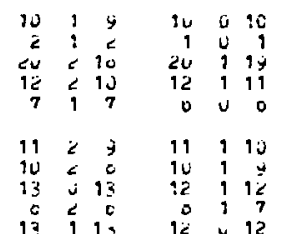

$.54540000 t+02$ $35435900 \mathrm{t}+\mathrm{U}$ $.904 c 474 u \dot{c}+u 2$
$.9002+100 c+0$

$.97930330 \mathrm{u}+\mathrm{CL}$ - $94 \div 2722 v e+v e$ - 10evyl10e+0z - 1uas? louto +0 . 14LUGétJe+u 3 zu

S1Gith - $90404010 \mathrm{u}+0$
100
200

$.909040=10$

$17840=-10 \quad .41544 E-17$

.11222 $=-10: 12407=-17$

$.22934 \mathrm{c}-17.34310 \mathrm{c}-17$

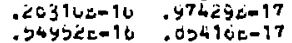

$.27799 \mathrm{i}-10$

$.27404 E-17$

$.20549 \mathrm{~L}-17$
$.17420 \mathrm{~L}-17$

-317UAE-10 .9431<5-17

- $703 y z-10$

. $99500=-17$

$.25594 t-17$

$.24379 L-17$

. $20534 \Sigma-17$

$.45007=-10$

.

$.9420 y 5=17$
$.20 y 21 E=10$
$.19703 \mathrm{t}-10 \quad .105 t<t-U 5$

$404302-19 \quad .02039 \mathrm{E}-00$

$.02110 E^{2}-10 \quad .50043 E-05$

$.50<716-10 \quad .50147 \leq-U 5$

.33099E-10

$.54029=-10 \quad .50444 L-U S$

$.50724 E-10 \quad .50017 \mathrm{E}-05$

$.02070 t-10 \quad .59069 \mathrm{k}-0 \mathrm{~J}$

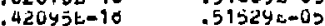

:14252 $\mathrm{E}-04$

1 nAt $=$ 15o

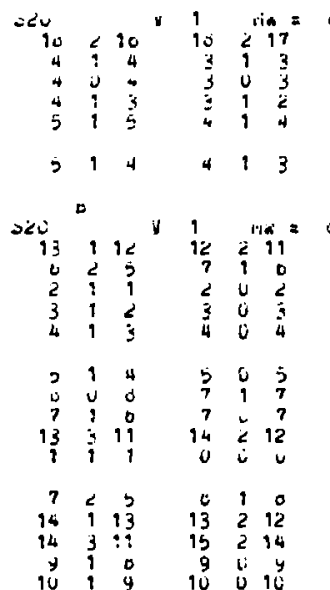

oc $\quad .4=.07 j u$

- 2 cujo35ut $30 \mathrm{UL}$

- $3711<300 \mathrm{c}+42$

- 30 1dUULUe+U2

$.09347<30 e+42$

$A=.424777 J U t+02 \quad E=.50297000 t+U 1$

-3<U72E-20 .72443E-20 . Judodt-Z̈0

$.57005 E-17$.790720-10 :13012z-10

7uguétir .0soyje-10 .15025e-10

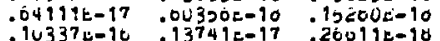

$C=.45009000 t+0$

$.49979940 \mathrm{c}+42$

$.1135 y t-10 \quad .15322 t=17 \quad .24793 E-10$

. $83900 \mathrm{x}-21$

$.249000-19$

.20951L-19

$.279 u 1 t-19$
$.4923 y=-1 y$

$.103320-00$

10Y79t-Ub

- ŻंU3YE-VO

$.22019 i-00$

$.30791 \mathrm{E}-\mathrm{U} 0$

$.55237 \varepsilon-19$

$.402302-10$

ou $\quad$ iv $=1.1000$

$35301500 \mathrm{E}+\mathrm{U}$

$3040 y$ yut +ua

- 3ç4е74 Je+0z

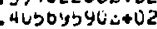

$.420033005+02$

$4540031 \mathrm{UEE}+U 2$

$401220200+C 2$

407932 luet

$40 y 7$ y 4 ouetcz

$.47033510 \mathrm{Ne+02}$

$403304 \mathrm{CuE}+U 2$

$4914720 \mathrm{UL}+02$

$\$ 17$ y josictur

$533 c^{2} 2000 \mathrm{c}+0 \mathrm{C}$

Crlatide rosuadle

LL2

$\begin{array}{rrrrrr}10 & 2 & y & 11 & 1 & 10 \\ j & 0 & y & 0 & 1 & 0 \\ y & 2 & 0 & 10 & 1 & y \\ 14 & 0 & 10 & j & 1 & 9 \\ 1 & 1 & 0 & 1 & 0 & 1 \\ 2 & 1 & 1 & 5 & 0 & 2 \\ 3 & 1 & \vdots & 1 & 0 & 3 \\ 4 & 1 & j & 4 & 0 & 1\end{array}$

$00 \mathrm{nu}=1.0 \mathrm{und}$

$=.4 \angle 477704 z+v i 2 \quad 0=.50597000 k+01$

.2U030t-1 :35e77e-10 :74<95E-19

$.073+1 c-17$. $4913 j=-10 \quad .10214 t-10$

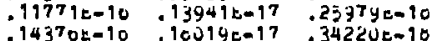

$c=.45009000 \mathrm{t}+0 \mathrm{har}=-.47 \%$

$.03074 \mathrm{~L}=U \mathrm{~T}$

- $43250-19$

-32b2ue-19 .40052c-00

$.4715 j c-19 \quad .4004 y c-00$

.522 is $7 E-V O$

$.10403 \mathrm{i}-90 \quad .2694 y c-17$

$.10303 \mathrm{~b}-10 \quad .173+10 \mathrm{~b}=17$

$.10055 E-10$ :30495e-17

TaYi3t-10 453ULE-10

$.43000 k-10$

4 jं उULE- 10

- 30404L-10

$.79040 \mathrm{E}-19$

$.579000=04$

- $30540 \varepsilon-00$

$71553 \mathrm{t}-40$

$14157 \mathrm{c}-\mathrm{j}$

- 120j3t=10

. $3416 y \mathrm{E}-19$

$.50094 \mathrm{c}-\mathrm{LO}$

$.23310 t-17$. H77UOE-16. 10379E-16

$.17 y 550-17.00314 c-10 \quad .25042=-10$

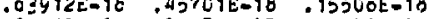

$.10003 \mathrm{E}-10$.3007\$E-17.00641t-10

$.20305 t=19$

.5r7女25i-19

$.37902 \mathrm{~b}-19$

$12001 E-00$

$.22003 E-40$

$.159210-06$

$.944595=00$

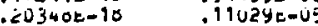

$h=.42044000 t+02 \quad$ i: $=.30020000 t+01$ 03

$.7<930=-10$. 10004E-10.3000\%t-14

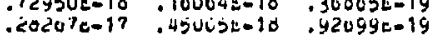

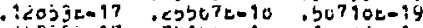

$.44329 E-17$.701U4=-10 .10040E-10

$.3 \cup 545 c-17 \quad .320 y<=-10 \quad .5 y 46 y c-19$

$C=.33000000 \mathrm{c}+0$

$.74252 \mathrm{k}=20$

$.17004 \mathrm{t}-19$

$.11232 \mathrm{E}-19$

$.32293 x-19$

. $1003<E-19$

2740 AR $=$

$.27409 \mathrm{c}=07$

$.73947 t-07$
$.59060 E-U 7$

$.59060 \mathrm{e}-07$
$.150390-00$

$.33635 \mathrm{t}-40$

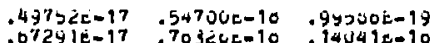

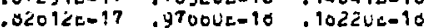

$17000 \mathrm{E}-1 \mathrm{~s}$

$.25320 c-19$

$.34297 c-00$

-3ग311E-0i

$404041905+4 c$

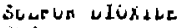

sus

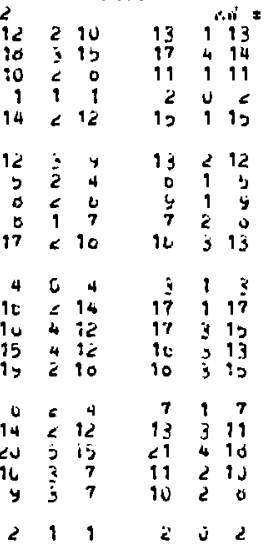

$04 \mathrm{MU}=1.03 \mathrm{UU}$

$.04203000 \mathrm{c}+\mathrm{U} 1$

Q40 $320 \mathrm{Cus}+\mathrm{U}$

$117000005+0<$

12दbuotuetud

- 14bC7 OUUEtUC

- $20335410 z+\dot{2}$ $.23494<4 U E+U 2$

$.240034700+U 2$

$.2534<010 \mathrm{~L}+02$

.$<00 j 7$ yyue+ce

$.295<13010+42$

- उúvis $30 \mathrm{c}+02$

- $3192210 \mathrm{t}+02$

- 3E510 $10 \mathrm{UL}+02$

$.44 v>2020+02$

$.47513540 \mathrm{C}+02$

. $1730540=+12$

5ru? $7790 \mathrm{e}+4 \mathrm{c}$

- उड उंj jovitoz

.53520 Yuvidotu2
$A=00778 b i 4 t+02==.10317905 E+02$

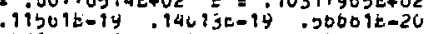
- 17501E-19 . 140i3c-19 . 26601E-20

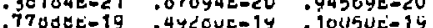

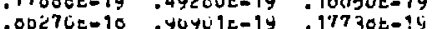

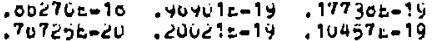

$.0 y 277 c-19$. 15627c-10 .6yU20E-19 $.20031 \mathrm{c}-17$. $42970=-10.40322 z-1 y$ .d130sc-10.3v193c-10.02310z-15

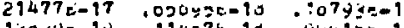

190<1c-14 $11407 \mathrm{t}-10$

.9u'10e-19

$.11701 \mathrm{t}-10.12755 c-17$

.j7503t-c0 . 3c321c-1y .2503t-1y

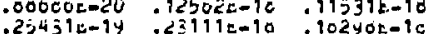

$000 y=-20 \quad: 12200 t-10$

$1: 0350-10$

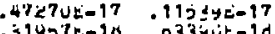

- j1957E-10

.033 yut -10

- 273 39t-1c

$.433005-10$

- Juvbe-zi

- UTंu

THUABE-?

- 1<0u<E-17

151010

$.404 \mathrm{U} 2 \mathrm{~L}-1 \mathrm{O}$

ic0uor-10

$.70255 t-10 \quad .03747 L-17 \quad .15037 L-17$
$.33004 k-1 y \quad .3070 u z=40$

$c=.07990040 t+01$ Riat $=04120$

$15857079000 t+01 \quad$ nAP $=-.7410$

$.15157 t-20 \quad .5771 y t-0 y$

$\begin{array}{rl}.49291 E-20 & .7 y 37<E-00 \\ 30592 r-20 & .29934 E-00\end{array}$

-31900e-É $\quad$.y

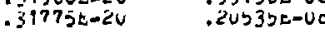

$.1+j 30 \mathrm{x}-1 \mathrm{y} \quad, 19021 \mathrm{y}-\mathrm{J}$

$191742-19 \quad .3435<=-47$

$.10070 k-19 \quad .23701 k-u 7$

$.15090 \mathrm{c}-15 \quad .47925 x-07$

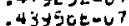

$.50000=19$

. $0 y+30 t-\angle 0$

$.1444 U E-U C$

. $1 \angle 21<$ LEU?

$.705 \angle 7$ ᄃ年7

$13424 c=u c$

$00453 k-10$

$.1043 u e-v e$

- Lotyje-vi

$.32407 t=00$

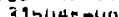

Jje?te-vo

$.2330<[-40$ 


$$
\text { Aut nue JLI ALE }
$$

ab

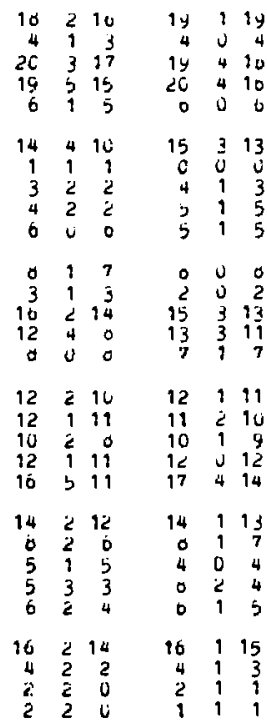

$.54033400 \mathrm{t}+02$ - $272 \times 4040 \mathrm{c}+02$ .01535 youe + Ũ $.07040010 \mathrm{e}+\mathrm{u}^{7}$

$.09404070=+02$ $.07575900 \mathrm{E}+02$ .096534 yut+Uc $7013444 U E+0<$ 727502 UOE + OC

$.53057080=+U 2$ $.1040<943 \mathrm{E}+03$

$.10403353 E+03$

$.10704355 E+03$ 110 youdoetoj

$.12000 \supset 16 \mathrm{e}+\mathrm{Cj}$ - $12 y 10572 x+43$

. 12951400e+03 $.131014706+03$ ij127400t+U3

$.132744 d 3 r i+i 3$

$.134004 d 0 x+03$

$.135095704+03$

$.13935470 z+03$
$.14030597 z+C 3$

$.14305094 t+03$

- $14000536 \mathrm{~L}+03$

. $13137067 \varepsilon+03$ . 00y $72160 \mathrm{a}+02$ $<u$

20

iv

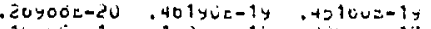

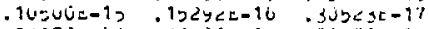

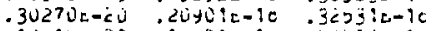

$.11133 \mathrm{e}-1 \mathrm{z}$

$.145335-10$

$.5321=c-10$

$.150<0=-10$

$.7 y 4 \div 45-16$

. $y<44 j t=10$

. 1 y Jut -1 b

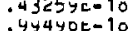

$.9 y 4 y 0 t-10$
$.15 y 30 z-15$

$+34174 \mathrm{t}-1 \mathrm{t}$

- 1000je-10

- jojoue-10

$.44934 \mathrm{t}-19$

: I Iyygélu

.1533ye-15

$.34224=15$

$.21143 t-10$
$.22740=-15$

$22<010-10$

.4 पर 1UE-17

ayuaye-10

$.59774=-17$

1 >UYLE -17
$234335-17$

$.14340=-10$

$.014 \sqrt{2}=-10$

$10 y 42 z-17$
$8 y+10=-10$

. 5 10ye-10

$.307440-17$

$.20277 \mathrm{~T}-1 \mathrm{C}$

$.2+U \supset 3 E-16$
$.27 E Y J E-17$

$-27 \leq y ว E-17$
$.20313 L-17$

. $32002-10$

$.7<203 c-17$

. $4900 \mathrm{v}-17$

$40935-17$
$.15<<2 x-17$

. 1 ivelue-10
.

$.41421 t-1 t$

.107 ว

. $01<y 7 z-17$

- juyout-16

- 32010 to-10

$.12310=-11$

- louvos-il

$:\{2403=-10$

.1457 ye-17

- $31310 t-10$

$.1035 j \mathrm{c}-10$

. 1ว51วL-1t

$.115 r 3 c-10$
$13 y 91 \mathrm{t}-17$

$.13100 \mathrm{e}-1$

$.52530 \mathrm{u}=10$

- 3ऽस1 IE- ?7

$.244 y<E-15$

.374ว4=-15

$.15054=10$

$.22343 \mathrm{c}-10$

1 $200745=10$

$.502700-10$

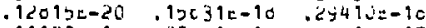

. Y4 35 पE $=$ ?

cus

\begin{tabular}{|c|c|}
\hline $\begin{array}{l}1077 \cdot 2 E-14 \\
.57330=-1 c \\
17 \cup 44 E-10 \\
10=01 E-10 \\
.47411 E-10\end{array}$ & 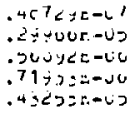 \\
\hline 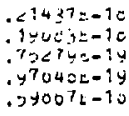 & 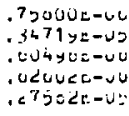 \\
\hline 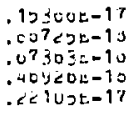 & 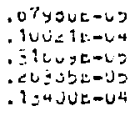 \\
\hline $\begin{array}{l}2+E \cup 4 E-17 \\
100 y 3 c-17 \\
3 y=4 L-17 \\
3295 y c-17 \\
03507 \varepsilon-10\end{array}$ & 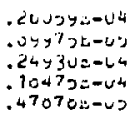 \\
\hline 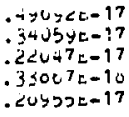 & 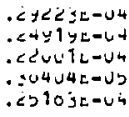 \\
\hline 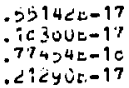 & 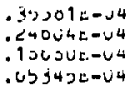 \\
\hline
\end{tabular}

44756

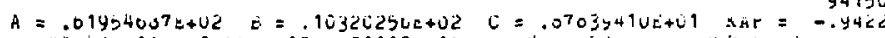

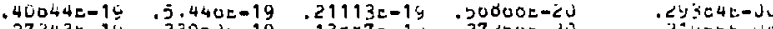

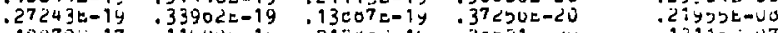

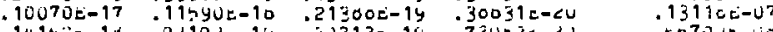

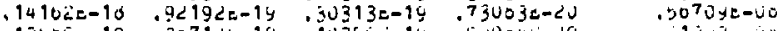

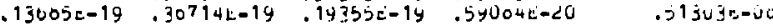

$13004100 \mathrm{C}+\mathrm{U}$ $136041000+0$ $.13457500 \mathrm{c}+\mathrm{J}$ $.161 \angle 00 y 0 E+U C$ $.1900113 \cup=+0 \dot{2}$

$\begin{array}{rrrrrr}10 & 2 & 0 & 11 & 1 & 11 \\ 14 & 2 & 12 & 15 & 1 & 15\end{array}$

$\begin{array}{rrrrrr}17 & 1 & 7 & 7 & 2 & 5 \\ 12 & 2 & 16 & 16 & 3 & 13 \\ 5 & 3 & 4 & 13 & 2 & 12 \\ 4 & 2 & 4 & 6 & 1 & 5\end{array}$

$.2200>730 \mathrm{~L}+02$ $.22733990 E+v i c$

$.20411790 \mathrm{C}+\mathrm{UC}$

$.20050 j 00 \mathrm{~s}+\mathrm{UL}$ $.2013030 \mathrm{UE}+0 \mathrm{~L}$

$.20173400 \mathrm{U}+62$ $.3505 j$ dout +02 $1300,70002+02$ .40. 13 UOUe+iz

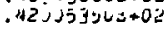

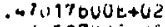
$\therefore-1$ H5OYYUE+UE $.53040000 E+U 2$ $.34730000 e+c i 2$ $.5610104 \cup e+02$

$.59019 y 3 \cup c+\cup 2$ $.0049073 \cup E+02$ $.70347950 x+U 2$ $.7073592 L t+0<$ $.71027040 \mathrm{c}+02$

$.73251300 \mathrm{c}+02$ $.13153051 t+u j$ - $13254439 c+c 3$ - 133uv355t+03 . $1332710 \mathrm{cE}+03$

- $135531 \geqslant 06+23$ $.130075 j 4 \mathrm{~L}+\mathrm{Uj}$

$.15000 \mathrm{E}-\mathrm{I}^{\mathrm{P}}$. 49137E-10 .12002=-10 $.77307 \varepsilon-20$.71603t-19 .jobujt-19

:1433je-10 :25330E-10 :11030t-10

$.00<03 E-17$.53025e-10 :1203be-1c

. 10531e-10 - $5025 \mathrm{~L}-10$ $12036=-16$
$.201530-10$

$.270251 .-1 y$ $21<2,0=1 y$ $33130 \mathrm{0}-1 \dot{3}$ . $\triangle 2 \div 77 \pm-1 Y$

.10770 a 17 .411לUe-10. .11320E-10

$.7 y 72 J E-20 \quad .517$ UUE-1y .3057 CE-19

$.30301 E-20$.912J0e-1y.10127e-10

$.1320<2-19$. $19003 E-10$

$.23025 t-10 \quad 03302 E=10$

- $10 \ddot{2} 02 \dot{2}=10$

$.24 y 04=-1 y$

. $2430=-1 y$

$440070-15$

$74<C+E-1 y$
1UEUVE-1C

- 32317e-19 . 33002 $=10$

$270705-10$ $.3 \angle 4 Y U=-10$ 24U3Ht- 10

. $1370 z-20$. 15200t-18

$4 c-10.00 y 37 c-17$

$166250-17$

$15549 \mathrm{c}-17$

$.10305 x-17$
$.2700 y+-10$

$.10<114-10$

$.0005 j e-1 y$

.1207ac-10

.240335-10

$.14774 \mathrm{E}-1 \mathrm{~s}$

$.31370 \mathrm{~L}-47$

- ¿̇uzti-i7

- 417 VOE-U?

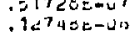

$.22027 c-17 \quad .1774 U c-17$

$.100<2=12 \quad .12051 \mathrm{~L}=10$

$11241 c-1 j \quad+22900=10$

7400 í- 10

. $\operatorname{liv} 11 \mathrm{t}=: 0$

.1407 DE- 10

- iv10ue -16
$.102 \cup u e-17$

$.5132 j \mathrm{~L}-17$. 102 UUL-17

. $00<13 k-10$

, jo $7 \mathrm{C}=\backsim=?$

. $2 \cup 05<L-\cup 7$

$.7073 \ddot{z}=-v^{7}$

-124jula-jo

- $\angle 44730-U D$

- $\angle 1<01$ E-ve

. 3c $207=-v 0$

$.245000-03$

$.430745-U U$

$.10474=-10 \quad .503570-17 \quad .173000-17 \quad .46007=-10$

2y7 $00=1$

. 31yo7L-UJ

JyOUE-UJ

30<7ur-u

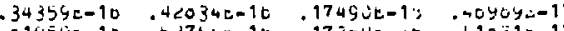

$.010590-10$.327ULE-10 .17330E-:0 .41031E-17

-3<5OUE-10.33ว0วE-10

$23073=-17$

$.1350 \mathrm{Je}-\mathrm{J}_{2}$

$.27037_{0}-74$

$.200000-64$

1 1Y $432 \mathrm{~L}-\cup 4$

$.47 .3005-05$

$.1204 y=-10 \quad .323<7 \varepsilon-10 \quad .17 \cup 42:=10 \quad .32023 r-17 \quad .31147 \varepsilon-04$

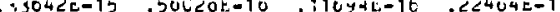

. $C 470=-14$

¿๐UYYOE-V4

. OUDUO=-U5 - $13 ?<34 j 1 c i+0 j$

$\begin{array}{rrrrrr}0 & 2 & 5 & 0 & 1 & 7 \\ 16 & 2 & 11 & 17 & 4 & 14\end{array}$

$.142044 \sqrt{ } y c+b 3$
$470210-1 y$.

$10290 \pm-10$
$.10<0 y 0-17$
$2,77,3 \mathrm{c}-17$

$.74013 \mathrm{c}-10$

7150
-7414

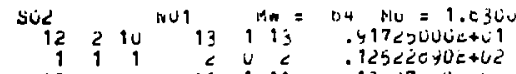

$1: 00010503 E+U 2 \quad 2=, 94200<2 Y t+U$

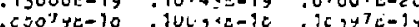

$=.07,73$

nar $=-. y 414$

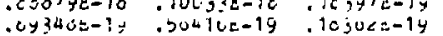

$120035=<0$

is $1<1 \mathrm{e}-<\mathrm{L}$

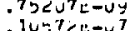

iugki,c-ue 


\begin{tabular}{|c|c|c|c|c|c|c|c|c|c|c|c|c|}
\hline & \multicolumn{4}{|c|}{$J L L 1$ nLC } & is & \multicolumn{4}{|c|}{ glulin luv } & \multicolumn{2}{|r|}{$t$} \\
\hline $\begin{array}{c}1_{4} \\
i_{c} \\
j \\
0 \\
0\end{array}$ & $\begin{array}{l}5 \\
\vdots \\
\vdots \\
\vdots \\
\vdots\end{array}$ & $\begin{array}{l}14 \\
y \\
7 \\
0\end{array}$ & $\begin{array}{r}12 \\
13 \\
0 \\
1 \\
4\end{array}$ & $\begin{array}{ll}1 & 1 \\
2 & 1 \\
1 & \\
\dot{2} \\
1\end{array}$ & $\begin{array}{l}1 \% \\
14 \\
5 \\
0 \\
y\end{array}$ & 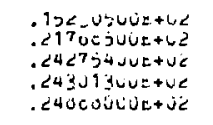 & 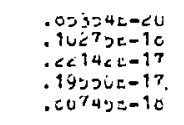 & 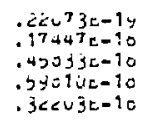 & 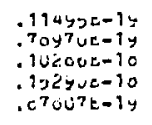 & 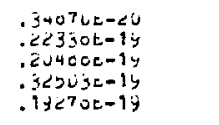 & 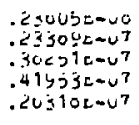 & \\
\hline $\begin{array}{l}11 \\
4 \\
10 \\
16 \\
15\end{array}$ & $\begin{array}{l}5 \\
j \\
c \\
4 \\
4\end{array}$ & & $\begin{array}{l}10 \\
\vdots \\
11 \\
17 \\
10\end{array}$ & $\begin{array}{ll}\vdots & 1 \\
1 & 1 \\
1 & 1 \\
3 & 1 \\
3 & 1\end{array}$ & $\begin{array}{l}13 \\
\vdots \\
17 \\
1 \% \\
19\end{array}$ & 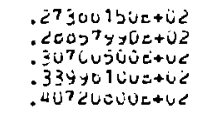 & 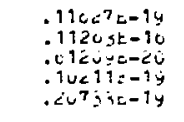 & 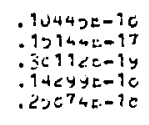 & 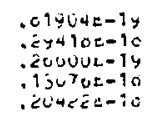 & 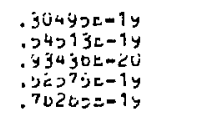 & 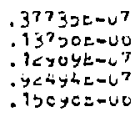 & \\
\hline $\begin{array}{l}1 y \\
19 \\
14 \\
i 0 \\
4\end{array}$ & $\begin{array}{l}c \\
2 \\
\vdots \\
\vdots \\
1\end{array}$ & $\begin{array}{c}10 \\
4 \\
16 \\
7 \\
1\end{array}$ & $\begin{array}{r}10 \\
7 \\
13 \\
11 \\
4\end{array}$ & $\begin{array}{ll}\vdots & 1 \\
\vdots & \vdots \\
\vdots & \vdots \\
\vdots & 1\end{array}$ & $\begin{array}{l}9 \\
7 \\
1 \\
13 \\
1 \\
c\end{array}$ & 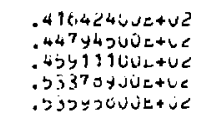 & 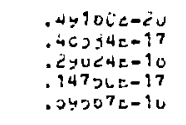 & 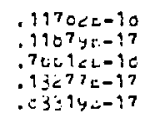 & 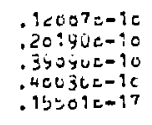 & 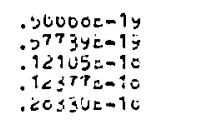 & 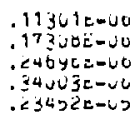 & \\
\hline $\begin{array}{l}4 \\
6 \\
1 \\
3 \\
4\end{array}$ & $\begin{array}{l}1 \\
1 \\
\vdots \\
2\end{array}$ & $\begin{array}{l}3 \\
\vdots \\
\vdots \\
\vdots\end{array}$ & $\begin{array}{l}4 \\
j \\
j \\
j\end{array}$ & $\begin{array}{l}u \\
\dot{u} \\
0 \\
i \\
i\end{array}$ & $\begin{array}{l}4 \\
\vdots \\
\vdots \\
\vdots\end{array}$ & 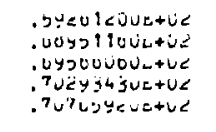 & 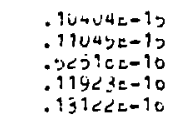 & 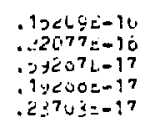 & 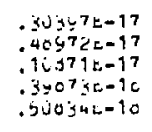 & 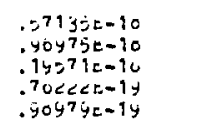 & 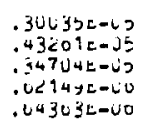 & \\
\hline 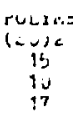 & $\begin{aligned}= & n \\
& 7 \\
\vdots & \vdots\end{aligned}$ & $\begin{array}{c}-4 L r \\
y \\
0 \\
12\end{array}$ & $\begin{array}{l}10 \\
10 \\
10\end{array}$ & $c_{4}^{3}$ & & 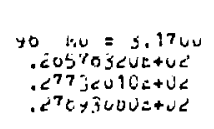 & 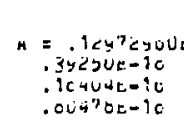 & 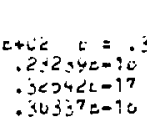 & $\begin{array}{l}1400 \text { YUUUE+Ui } \\
.74 j 20=-19 \\
.095045-16 \\
.100175-1 c\end{array}$ & 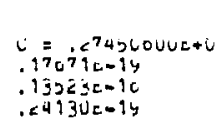 & 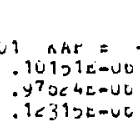 & $\begin{array}{l}y, y \\
0 \\
0 \\
0\end{array}$ \\
\hline $\begin{array}{c}3 \\
2 \\
14 \\
14 \\
14\end{array}$ & $\begin{array}{l}1 \\
\vdots \\
3 \\
\vdots \\
\vdots\end{array}$ & $\begin{array}{r}7 \\
2 \\
17 \\
5 \\
19\end{array}$ & $\begin{array}{r}c \\
2 \\
10 \\
5 \\
13\end{array}$ & $\begin{array}{ll}i & 1 \\
i & 1 \\
4 & 1\end{array}$ & & 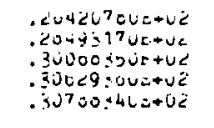 & 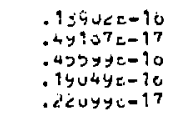 & 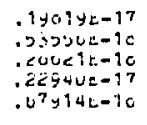 & 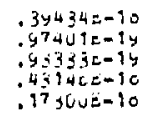 & 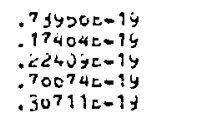 & $\begin{array}{l}.0333 y c-v 0 \\
.03<04 z-v e \\
.1900 y=-v 0 \\
.45<4<E-v 0 \\
.<330>-00\end{array}$ & \\
\hline $\begin{array}{c}1 y \\
\vdots \\
0 \\
14\end{array}$ & $\begin{array}{l}4 \\
5 \\
3 \\
1 \\
1\end{array}$ & $\begin{array}{c}10 \\
\vdots \\
3 \\
7 \\
7\end{array}$ & $\begin{array}{r}9: \\
\vdots \\
\vdots \\
\vdots \\
13\end{array}$ & $\begin{array}{ll}3 & 1 \\
1 & 1 \\
4 & 1 \\
2 & 1 \\
1 & 1\end{array}$ & & 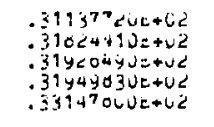 & 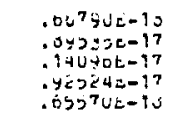 & 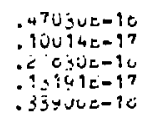 & $\begin{array}{l}15+36=-10 \\
10377=-10 \\
010179 E-19 \\
.20245 i=16 \\
.90903=-10\end{array}$ & 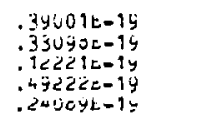 & $\begin{array}{l}.21475 E-v 0 \\
.70370=-v 0 \\
.1200 y e-60 \\
.40007 \varepsilon-v e \\
.10030=-00\end{array}$ & \\
\hline $\begin{array}{r}17 \\
13 \\
? \\
3 \\
14\end{array}$ & $\begin{array}{l}3 \\
3 \\
3\end{array}$ & $\begin{array}{l}10 \\
10 \\
4 \\
?\end{array}$ & $\begin{array}{c}10 \\
16 \\
\vdots \\
\vdots \\
14 \\
14\end{array}$ & $\begin{array}{ll}7 & 1 \\
1 & 1 \\
3 & 1 \\
0 & 1 \\
2 & 12\end{array}$ & $\begin{array}{l}11 \\
1 \\
1 \\
4 \\
12\end{array}$ & 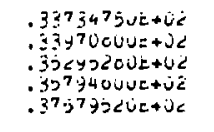 & 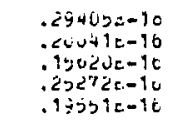 & $\begin{array}{l}.209 y-1-10 \\
.44050=-17 \\
.10 y 30=-17 \\
.24121 i=17 \\
.064 y 4 c-17\end{array}$ & 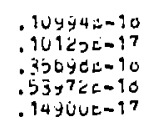 & $\begin{array}{l}.20400 r-19 \\
.2040 y e-10 \\
.05100 e-1 y \\
.970 y 2 k-19 \\
.5109 y=-10\end{array}$ & 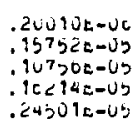 & \\
\hline $\begin{array}{l}127 \\
10 \\
13 \\
10\end{array}$ & $\begin{array}{l}3 \\
? \\
? \\
? \\
1\end{array}$ & $\begin{array}{l}3 \\
3 \\
4 \\
? \\
3\end{array}$ & $\begin{array}{l}96 \\
0 \\
19 \\
14 \\
16\end{array}$ & $\begin{array}{lll} & 1 \\
4 & 4 \\
3 & \\
0 & 4 \\
0 & 14\end{array}$ & & 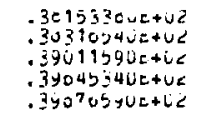 & 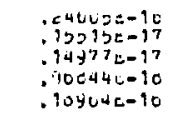 & 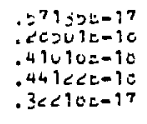 & $\begin{array}{l}.13575 E-17 \\
001700 E-19 \\
.161450-94 \\
.12431 E-10 \\
.0790<E-10\end{array}$ & 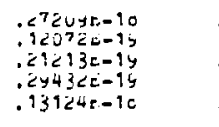 & 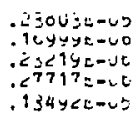 & \\
\hline $\begin{array}{r}10 \\
7 \\
10 \\
2 \\
14\end{array}$ & $\begin{array}{l}0 \\
\dot{c} \\
j \\
\dot{c} \\
i\end{array}$ & & $\begin{array}{l}17 \\
7 \\
10 \\
1 \\
14\end{array}$ & $\begin{array}{ll}7 & 1 \\
i & 1 \\
5 & 1\end{array}$ & $\begin{array}{l}17 \\
7 \\
14 \\
1 \\
3\end{array}$ & 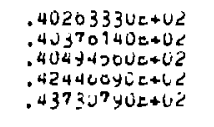 & 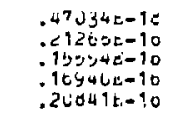 & 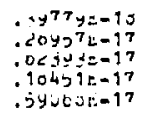 & 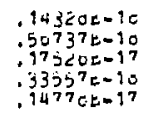 & 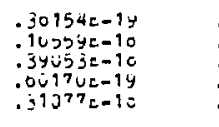 & 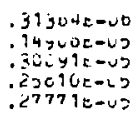 & \\
\hline $\begin{array}{l}0 \\
8 \\
7 \\
y \\
7\end{array}$ & $\begin{array}{l}c \\
2 \\
3 \\
1 \\
2 \\
2 \\
2\end{array}$ & $\begin{array}{l}0 \\
3 \\
7 \\
0 \\
2\end{array}$ & $\begin{array}{l}y^{\prime} \\
\vdots \\
\vdots \\
\underline{z}\end{array}$ & $\begin{array}{l}1 \\
2 \\
2 \\
4 \\
1 \\
1\end{array}$ & $\begin{array}{l}7 \\
0 \\
0 \\
y \\
1\end{array}$ & 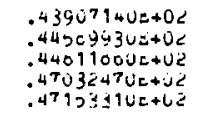 & 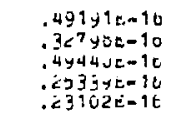 & 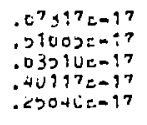 & 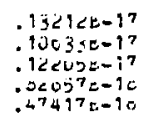 & 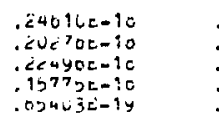 & 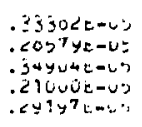 & \\
\hline$\iota$ & $\vdots$ & 3 & $e$ & $<$ & 4 & $.47 \dot{3} 77 y 2 u x+U z$ & $.3 u^{7} v^{7} x-10$ & $.+\dot{C} \angle \dot{C} c=-17$ & $.0<y$ ye- 10 & $.93+7<2-10$ & 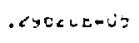 & \\
\hline $\begin{array}{c}(\text { sule } \\
\text { (su)k } \\
10 \\
0 \\
14 \\
2 \\
19\end{array}$ & $\begin{array}{l}i \\
i \\
\vdots \\
i \\
i\end{array}$ & $\begin{array}{l}0 \\
7 \\
11 \\
10\end{array}$ & $\begin{array}{l}1 \\
14 \\
c \\
1: \\
6\end{array}$ & $\begin{array}{ll} & \\
1 & \\
2 & \\
1 & \\
= & 1\end{array}$ & $\begin{array}{c}= \\
\vdots \\
\vdots \\
\vdots \\
\vdots\end{array}$ & 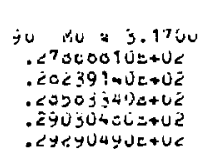 & 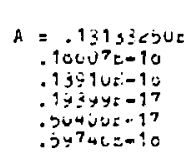 & 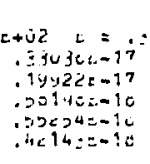 & 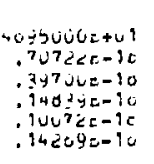 & 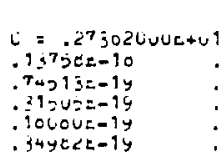 & 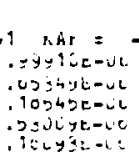 & 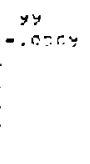 \\
\hline $\begin{array}{r}0 \\
0 \\
3 \\
10 \\
12\end{array}$ & $\begin{array}{l}u \\
1 \\
\vdots \\
\vdots \\
4\end{array}$ & $\begin{array}{r}U \\
7 \\
\ddot{Z} \\
14 \\
10\end{array}$ & $\begin{array}{c}3 \\
3 \\
3 \\
16 \\
16\end{array}$ & $\begin{array}{ll}1 & \\
2 & \\
1 & \\
2 & 1 \\
1 & 1\end{array}$ & $\begin{array}{c}5 \\
0 \\
\vdots \\
3 \\
1\end{array}$ & 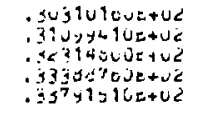 & 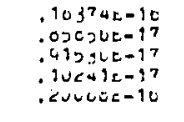 & 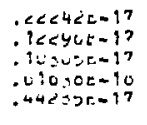 & 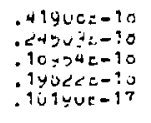 & 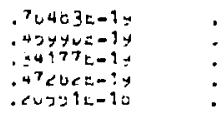 & 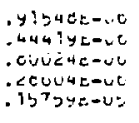 & \\
\hline ? & 2 & 4 & 2 & $:$ & 3 & . $5 g^{7} y j e+u c+u e$ & $.123 x<10-10$ & $.1 Y 4<, z-17$ & . 50uyze-1c & $. c^{2} v>25-9 y$ & . $119406-4:$ & \\
\hline
\end{tabular}




\begin{tabular}{|c|c|c|c|c|c|c|c|c|c|c|c|}
\hline \multicolumn{3}{|c|}{ JU AU1 nue } & \multicolumn{3}{|c|}{$J_{\text {KLI ALC }}$} & WU & \multicolumn{4}{|c|}{ ülin } & $n=5$ \\
\hline $\begin{array}{l}7 \\
14 \\
12 \\
10 \\
10\end{array}$ & $\begin{array}{ll}1 & \\
3 & 1 \\
\vdots \\
1 \\
\vdots \\
\vdots & 1\end{array}$ & $\begin{array}{l}3 \overrightarrow{3} \\
y \\
y \\
13\end{array}$ & $\begin{array}{l}4 \\
14 \\
12 \\
10 \\
10\end{array}$ & & $\begin{array}{l}4 \\
12 \\
14 \\
10 \\
14\end{array}$ & 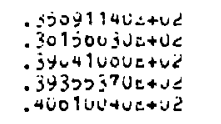 & 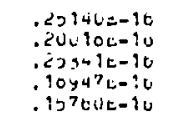 & 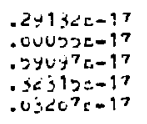 & 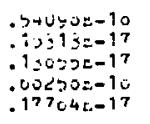 & 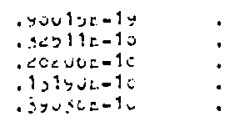 & 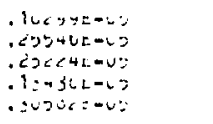 \\
\hline $\begin{array}{r}7 \\
2 \\
10\end{array}$ & $\begin{array}{l}2 \\
2 \\
3\end{array}$ & $\begin{array}{c}\mathrm{t} \\
0 \\
15\end{array}$ & $\begin{array}{c}7 \\
1 \\
10\end{array}$ & & ? & 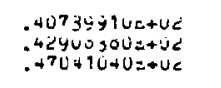 & 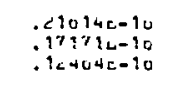 & $\begin{array}{l}.<y 2 y 3=-17 \\
040002 t=17 \\
.0935:-17\end{array}$ & $\begin{array}{l}.201400-10 \\
\text { 34 } 39<-10 \\
.<104+2=17\end{array}$ & 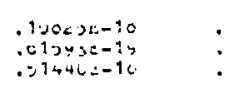 & 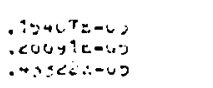 \\
\hline $\begin{array}{l}\text { Uzove } \\
\text { U3 } \\
20 \\
10 \\
4 \\
10 \\
10\end{array}$ & $\begin{array}{ll}3 & 1 \\
1 & \\
0 & \\
3 & 1 \\
2 & 1\end{array}$ & & $\begin{array}{r}21 \\
y \\
3 \\
14 \\
17\end{array}$ & $\begin{array}{ll}2_{2 n}^{n i n} \\
2 \\
2 \\
2 \\
2 & 1 \\
1 & 1\end{array}$ & $\begin{array}{c}n= \\
20 \\
0 \\
3 \\
10 \\
17\end{array}$ & 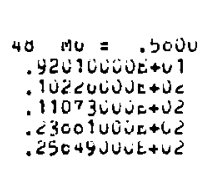 & 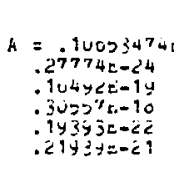 & 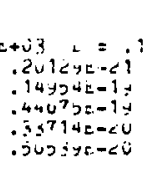 & 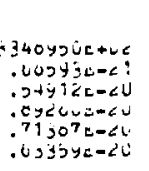 & 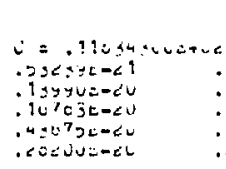 & 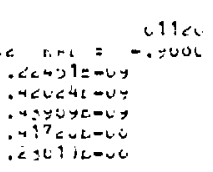 \\
\hline $\begin{array}{c}15 \\
14 \\
10 \\
10 \\
1\end{array}$ & $\begin{array}{ll}3 & 1 \\
2 & 1 \\
< & 1 \\
2 & 1 \\
1 & \end{array}$ & & $\begin{array}{r}10 \\
15 \\
19 \\
17 \\
2\end{array}$ & & $\begin{array}{l}14 \\
15 \\
12 \\
15 \\
25\end{array}$ & 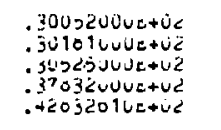 & 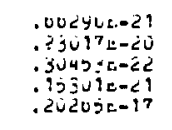 & 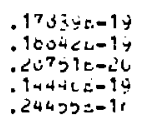 & 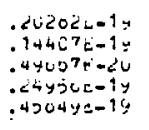 & 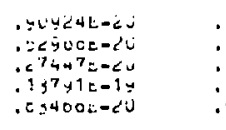 & 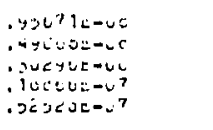 \\
\hline $\begin{array}{r}12 \\
2 \\
4 \\
1\end{array}$ & $\begin{array}{ll}2 & 1 \\
1 \\
1\end{array}$ & $\begin{array}{l}10 \\
1 \\
3 \\
1\end{array}$ & $\begin{array}{r}13 \\
2 \\
4 \\
u\end{array}$ & $\begin{array}{ll}1 & 1 \\
0 & \\
0 & \\
0 & \end{array}$ & $\begin{array}{l}13 \\
23 \\
4 \\
0\end{array}$ & 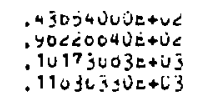 & $\begin{array}{l}.2007 j<-1 y \\
.430000-10 \\
.20000-10 \\
.300100-10\end{array}$ & 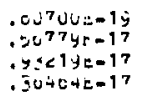 & $\begin{array}{l}.44201=-1 y \\
: 104<1 E=17 \\
11 y=04=-17 \\
.00304=-16\end{array}$ & 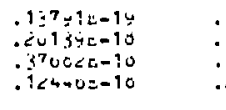 & 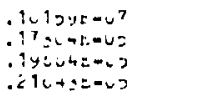 \\
\hline $\begin{array}{l}E I L I C C \\
\text { SIr } \\
10 \\
13 \\
0 \\
14 \\
14\end{array}$ & $\begin{array}{ll}C & 4 \\
2 & 1 \\
4 & \\
3 \\
0 \\
0\end{array}$ & $\begin{array}{l}12 \\
y \\
3 \\
y \\
0\end{array}$ & $\begin{array}{l}\text { und } \\
12 \\
12 \\
7 \\
15 \\
15\end{array}$ & $\begin{array}{ll}0 & \\
& \\
0 & \text { in } \\
3 \\
2 \\
5 & 1 \\
5 & 1\end{array}$ & $\begin{array}{l}n= \\
3 \\
0 \\
0 \\
10 \\
11\end{array}$ & 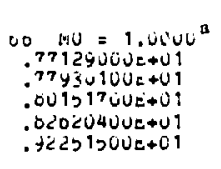 & 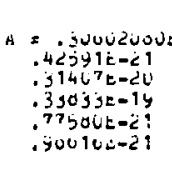 & 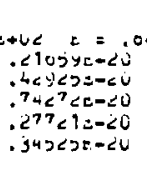 & 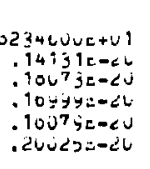 & 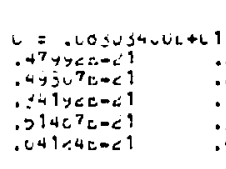 & 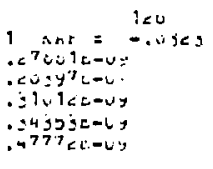 \\
\hline $\begin{array}{r}2 \\
10 \\
11 \\
0 \\
11\end{array}$ & $\begin{array}{l}0 \\
0 \\
5 \\
5 \\
4 \\
5 \\
=\end{array}$ & $\begin{array}{r}2 \\
11 \\
7 \\
? \\
\dot{0}\end{array}$ & $\begin{array}{r}1 \\
15 \\
12 \\
9 \\
12\end{array}$ & $\begin{array}{ll}1 & \\
0 & 1 \\
4 & \\
3 & \\
4 & \end{array}$ & $\begin{array}{r}1 \\
10 \\
0 \\
0 \\
y\end{array}$ & $\begin{array}{l}93900200 e+0 \\
.95049700 \mathrm{e}+01 \\
10521310 t+02 \\
.12141000 t+02 \\
.12^{7} 22130 t+02\end{array}$ & 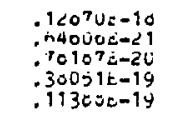 & 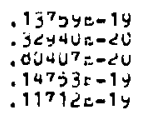 & 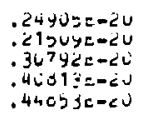 & 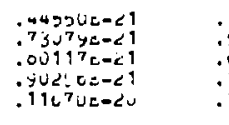 & 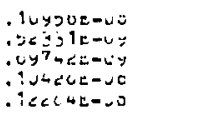 \\
\hline $\begin{array}{r}2 \\
9 \\
10 \\
3 \\
0\end{array}$ & $\begin{array}{l}2 \\
2 \\
2 \\
2 \\
4\end{array}$ & $\begin{array}{l}1 \\
c \\
1 \\
1 \\
4\end{array}$ & $\begin{array}{r}3 \\
0 \\
15 \\
4 \\
4\end{array}$ & $\begin{array}{ll}1 & \\
3 & \\
3 & 1 \\
1 & 1 \\
3 & \end{array}$ & $\begin{array}{r}2 \\
5 \\
12 \\
4 \\
7\end{array}$ & 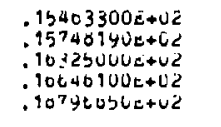 & 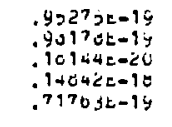 & 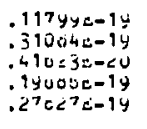 & 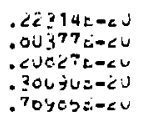 & 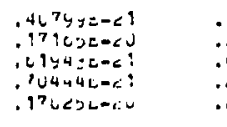 & 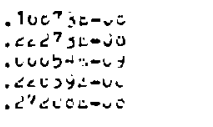 \\
\hline $\begin{array}{r}14 \\
5 \\
1 \\
5 \\
11\end{array}$ & $\begin{array}{l}4 \\
3 \\
1 \\
1 \\
2\end{array}$ & $\begin{array}{l}1 \\
2 \\
3 \\
4 \\
y\end{array}$ & $\begin{array}{r}13 \\
0 \\
1 \\
4 \\
12\end{array}$ & $\begin{array}{l}j \\
2 \\
i \\
i \\
1\end{array}$ & $\begin{array}{c}0 \\
3 \\
1 \\
3 \\
1<\end{array}$ & 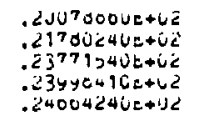 & 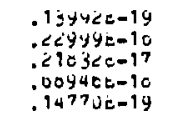 & $\begin{array}{l}.201 y \cup 5-19 \\
.444+25-19 \\
23+27=-10 \\
.1434 y=-15 \\
.00020=-20\end{array}$ & 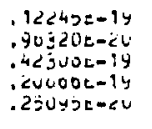 & 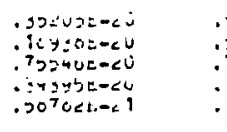 & 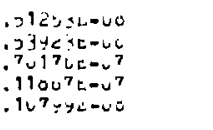 \\
\hline $\begin{array}{r}17 \\
10 \\
2 \\
13 \\
13\end{array}$ & $\begin{array}{ll}2 & 1 \\
2 & \\
1 & \\
0 & \\
0 & \end{array}$ & $\begin{array}{l}13 \\
9 \\
1 \\
0 \\
7\end{array}$ & $\begin{array}{r}16 \\
9 \\
2 \\
14 \\
14\end{array}$ & $\begin{array}{ll}0 & 1 \\
j & \\
4 & \\
5 & \\
5 & 1\end{array}$ & $\begin{array}{r}10 \\
0 \\
2 \\
y \\
10\end{array}$ & 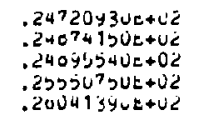 & $\begin{array}{l}.<5320-20 \\
.170100=-10 \\
.352000-17 \\
.100920-19 \\
.11300=-19\end{array}$ & 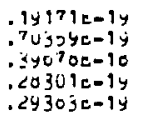 & 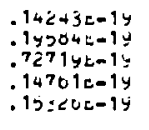 & 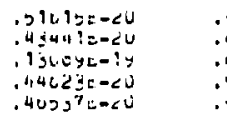 & 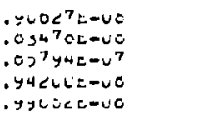 \\
\hline $\begin{array}{r}15 \\
3 \\
14 \\
12 \\
10\end{array}$ & $\begin{array}{ll}2 & 1 \\
0 & \\
4 & 1 \\
3 & 1 \\
3 & \end{array}$ & $\begin{array}{l}14 \\
3 \\
0 \\
0 \\
0\end{array}$ & $\begin{array}{r}14 \\
2 \\
13 \\
11 \\
11\end{array}$ & $\begin{array}{ll}3 & 1 \\
1 & 1 \\
3 & \\
4 & \\
4 & \end{array}$ & $\begin{array}{r}11 \\
2 \\
y \\
7 \\
7\end{array}$ & $\begin{array}{l}.200 c 20<u E+02 \\
.20525330 t+v c \\
2710 y 430 c+0 c \\
.2709190 u c+02 \\
.2000104 v e+02\end{array}$ & 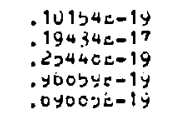 & 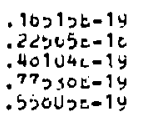 & 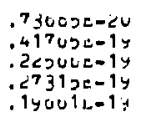 & 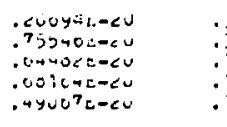 & 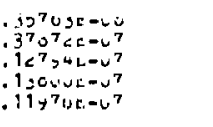 \\
\hline $\begin{array}{r}8 \\
3 \\
11 \\
4 \\
14\end{array}$ & $\begin{array}{l}2 \\
3 \\
2 \\
3 \\
3 \\
2\end{array}$ & $\begin{array}{l}0 \\
2 \\
10 \\
2 \\
3\end{array}$ & $\begin{array}{r}7 \\
3 \\
10 \\
5 \\
13\end{array}$ & $\begin{array}{ll}3 & \\
0 \\
3 \\
3 \\
5 \\
3 & 1\end{array}$ & $\begin{array}{r}2 \\
3 \\
7 \\
3 \\
16\end{array}$ & 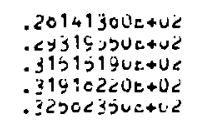 & 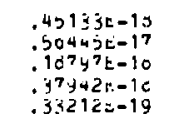 & $\begin{array}{l}.12000 c-10 \\
.003050-10 \\
.443405-1 y \\
.04000-19 \\
.3079 c-19\end{array}$ & 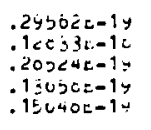 & 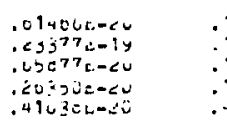 & 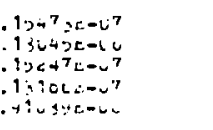 \\
\hline$\stackrel{7}{12}$ & $\begin{array}{l}4 \\
2 \\
2 \\
1\end{array}$ & $\begin{array}{l}3 \\
1 \\
2 \\
1\end{array}$ & $\begin{array}{r}1 \\
11 \\
12 \\
6\end{array}$ & 辛 & $\begin{array}{l}0 \\
0 \\
y \\
i\end{array}$ & 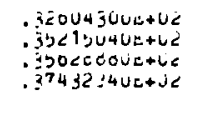 & $\begin{array}{l}.27000 c-90 \\
.140650=10 \\
.100300:-19 \\
.30<630=17\end{array}$ & 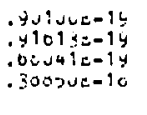 & 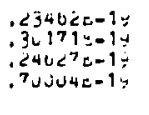 & 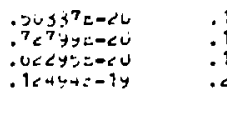 & 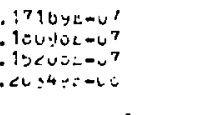 \\
\hline $\begin{array}{r}152 \\
4 \\
3 \\
1 \\
1\end{array}$ & $\begin{array}{l}u \\
\vdots \\
1\end{array}$ & $\begin{array}{l}2 \\
1 \\
i\end{array}$ & $\begin{array}{l}1 \\
4 \\
1 \\
1\end{array}$ & $\begin{array}{l}1^{\operatorname{lin} n} \\
1 \\
c^{2}\end{array}$ & $\begin{array}{l}1= \\
1 \\
4 \\
1\end{array}$ & 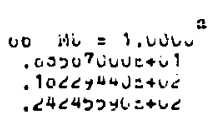 & 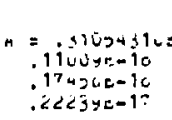 & 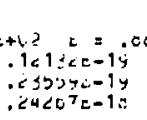 & 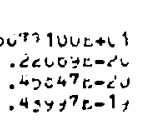 & 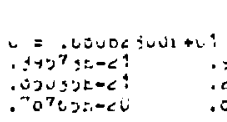 & 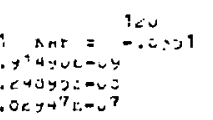 \\
\hline
\end{tabular}


InLLL LA , CS

\begin{tabular}{|c|c|c|c|c|c|c|}
\hline iv & five & rise & d & 1 & nte &.$u$ \\
\hline 5 & L & $=$ & 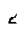 & 1 & 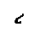 & 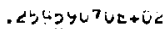 \\
\hline . & $i$ & $i$ & 6 & $\checkmark$ & $<$ & $. \angle U D^{\circ}+C O U D+L<$ \\
\hline 4 & $?$ & 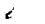 & $\therefore$ & ، & $g$ & 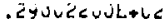 \\
\hline 4 & 1 & $:$ & i & v & 4 & $\therefore 34^{7} \approx 4+4 \cup \cup x+\cup 2$ \\
\hline$i$ & $i$ & $i$ & $i$ & J & $v$ & . jootéc luc.tue \\
\hline
\end{tabular}

el

SIU.ंA

LUL

h ner

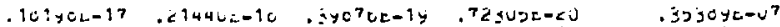

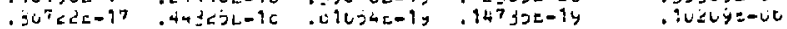

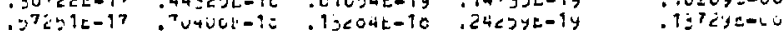

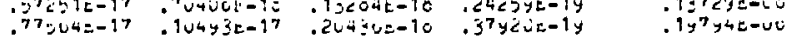

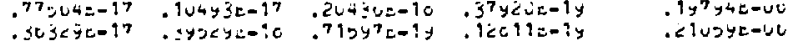


1. AMBLE. Ė. hHD DAILEY. B. P., 1950, J. CMEM. FaYS. 10, 1422

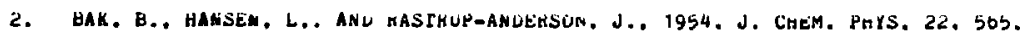

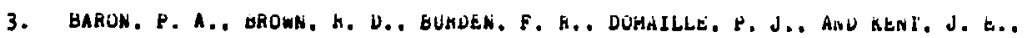

1972. J. MUL, Śrei. 43, 401.

4. beard, C. I.. ANd DaIlezy, B. P., 1950, J. Chem. PHYS, 1d, 143?, 1951, J. Cheh. PhYS. 19, 9?5

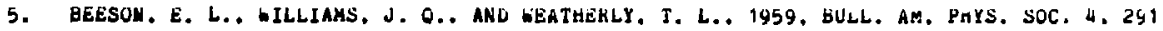

6. BEt.LET. J.. SAHSON. C.. STEENGECKELIEKS. G.. ALL WERTHEIHER. K.. 19?1. J, HOL. STKUC. Y. 4Y.

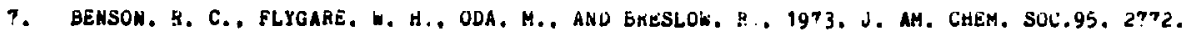

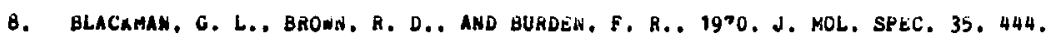

9. BLOKIS. U.. DASAL. P., AND HEYEAS. R., 1903. J. CHEM. PIIYS. 30, 2753.

10. BOTSKOR. I.. RUUOLPH. H. D.. AND ROUSSY. U.. 1974, J. MOL. SPEC. 52, 45?.

11. BOTSKOR, I.. MULOLPH, H. D., AHD MOUSSY, G.. 1974, J. MUL, SPEC. 53, 15.

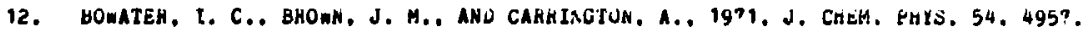

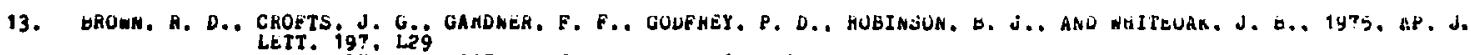

14. BURKUS. C. A. AHD GURDY. W.. 1957. J. CHEH. HHYS. 20.391.

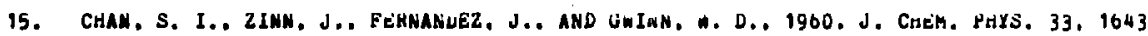

16. COOK. H. L.. 1905. J. CHEH. PHYS. 42. 2927

17. COOK, R, L., ANL LIRChHOFF, W, H., 1967, J. ChEM, PHYS, 47, 4521

18. COOK, R, L., WALDEN, R. T., ANU JONES, G. E., 1974. J. MOL, SPEC, 53, 3?0.

19. COOK, R, L., WINNEWISSER, G., ANU LINDSEY, U. C., 1973, J, MOL. SPEC. 46, 276.

20. costaIN, C. C.. AHD DOmLING, J. H., 1960, J. CHEN. PHYS. 32, 150.

21. Cox, A. P.. ANd ESBItT, A. S., 1963, J. CHEH. PHYS. 30̄, 1636.

22. COX, A. P.. AND RIVEROS, J. H., 1965, J. CHEH. PHYS. H2, 3106

23. ChESHELL, R. A.. AND MILLS, I. M., 19?4, J. MOL. SPEC. 52, 392.

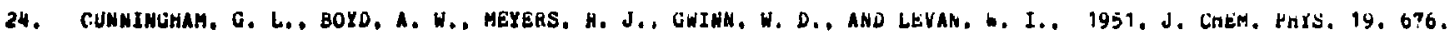

25. CURL. R. F.. 1959. J. CHEN. PHYS. 30. 1529

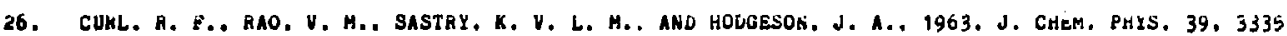

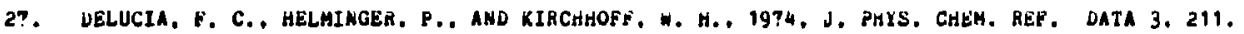

26. DEMAISON, J., AND FULOLPH, h. D., 1971, J. MOL. SFEC. 40, 445.

29. DEMORE, B. B., WILCOX, W. S., ANU GOLUSTEIN, J. H., 1954, J. CHEH. HHYS. 22.

30. DENENGELSEN, D., 1967, J, MOL. SPEC. $22,420$.

31. DEZAFAA, A, G., 1971, AP, J, 170, 165.

32. DIXON, W. B., ANL WILSOA, E. B., 1961, J. ChEH. PHYS. 35, 191

33. DAEIZLEA, H, AND DENOL, G., 1964, ZS, MATUAF, 19A, 512

34. DREIZLER, H. AND DENDL, G., 1965, ZS. NATUKF, 20A, 30.

35. EalandsSON, 5.. 1953. AKKIV bySIK 6. 491.

36. FaYero, P.. MIAhI, A. M., AND BAKE,, J, G., 1900, nuovo CIH. 17. 740

3?. FlyGare. W. H. ANL HOWE. J. A., 1962, J. CHEM. PHYS. 36, 440

36. EORO, R, G., 19:4, J, MOL, SPEC, 49, 117.

39. FORD, R, G., ANU SZALANSKY, L. B., 1972, J. MOL, SPEC. 42, 344.

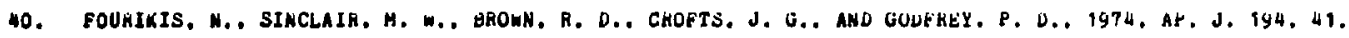

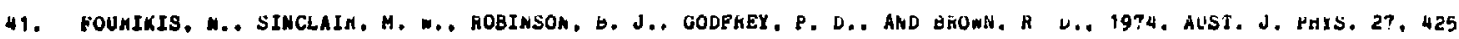

42. GERKY. H. C. L.. 19?1, CAN, J. CHË. 49, 255

43. GEARY, H, C. L.. 1973, J. HOL. SPEC. 45, 71. 
44. GERKY. H, L. L., ANU WIANEWISSEM, G., 19?3, J. MOL, SPEL, 4h, 1.

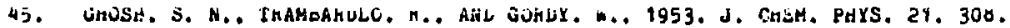

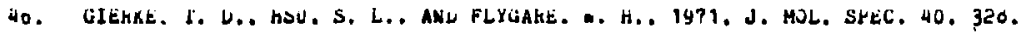

47. vOITLIED, C. A., PALMEK, P.. HICMAKL. L. J.. AMD ZULKEGHAN, b.. 1973, AP. J. $102,699$.

40. GAAYDEAL, J. L... 1960, J. CHEK. PHYS. 32. 1250

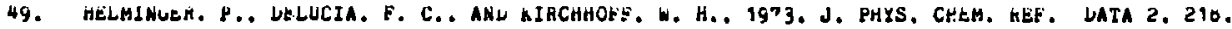

50. Hefizblimi, C. Aal makSAY, D. A.. 1956, HRÜ. ROY. SOC, LONDON 233.34

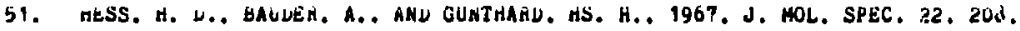

52. HIhOSE. C.. 1974, AP. J. 189. L145.

53. HIhUSE. C.. ANL CUHL. H. F.. 1974, J. MOL. SHEC, 3\%. 350.

54. HIHOTA. E.. 1900. J. CHEM. PAYS. 45. 3904.

55. MIHOTA, E.. 1960, J. MOL. SPEC. 20.335.

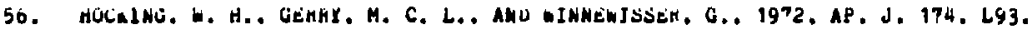

57. HOEFI. J.. 1905. 2S. HAIUAF 20A. 132"

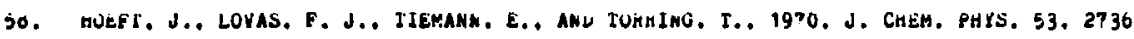

59. HOEE. J. A.. ANע Flygahe, H. H.. 1962, J. CHEM. PHYS. 36, 650

00. HSU. S. L.. ANL FLIUAAE. W. H., 19?1, J. MOL. SFEC, 3?, 92,

67. HUlines, H. H., 1953, J. CHEM. PHYS. 21, 959

02. IKEUA, I. . MEULEY, H, AKن CURL, R, F., 1972, J. MOL, SPEC, 44, 459.

63. JACKSUN. H. H.. AND MILLEN. D. J.. 1959. PROC. CHEH. SOC. 10

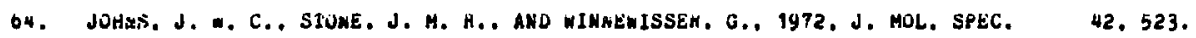

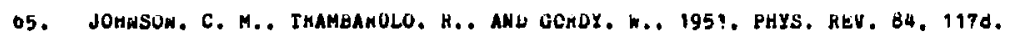

b6. JOHESUN. D. H.. AND LONAS. E. J.. 1971. AP. J. 109. 019.

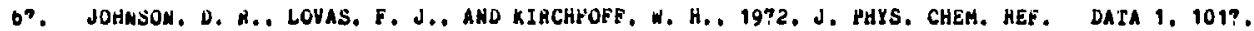

60. JOHASOd. H. U.. HYEKS. K. J., ANO GWIAK. H. D., 1953. J. CHEH. PHYS. 21.1425

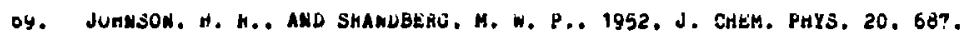

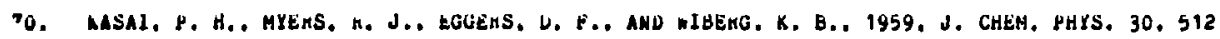

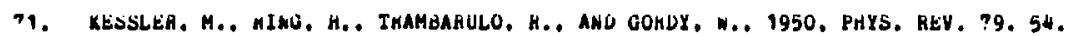

72. KEnLEY. H.. SASTKY. K.. AND WIMNEWISSEf, H., 1963. J. MOL. SPEC. 10. 416.

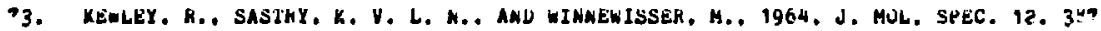

74. KILE, H, W., LIN, C, C.., AHD WILSON, E. B., 195?, J, CHEH, PHYS, 26, 1695

75. KIM. H.. KELLEH, H.. ANO GKINH. W. U.. 1962. J. CHEK. PHYS. 37. 2?90.

?6. KIRCHHUKE, W. H., JUHASUM, J. H.. AND LOVAS, F. J.. 19?3, J, PHYS. CHEM. HEF, DATA 2 , 4.

77. KIKCHHOFF, W. H. AHD NLLSON. E. H. 1963. d. AH. CHEM. SOC. 65, 1726

70. KOEdo. S., AND HIhOTA, É.. 19?0. J. MOL. SPEC. 34, 97.

79. KkUGH. W. D., AND GULd, L. P.. 1974. J. HOL. SPEC. 49. 423.

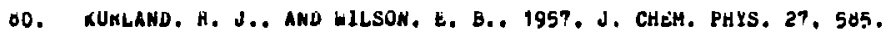

61. XYOD, R. A.. AND MILLS, I. M.. 1972, J. MOL, SPEC, 42, 320.

82. LAFEEATY, W, J.. 1970, J. HOL, SHEC, so. 84.

03. LaGRIE. V. W.. 1961, J. CHEM. FHYS. 34, 1516

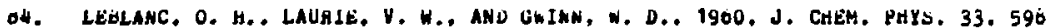

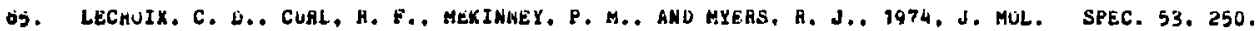

06. LEES, R. M.. 1973, AP. J. 184, ?63. 
6?. LEES. R. H., CURL. R. E., ANU BAKER. J. G., 1906. J. CHEM. FHYS. 4j. 2037

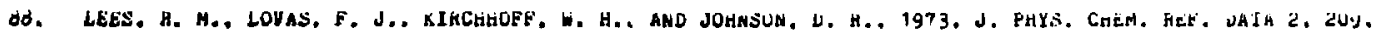

8. LERNEH, R. G., DALLEY. B. P., AND FHLEND, J. P., 1957. J. CHEM. PHYS. 20, OdU.

90. LETT, R. G., ANO ElYGaHE. W. H., 1967, J. CMEH. PHYS. 47, 4730

91. LEUIME, I. W.. 1962, J. MCL. SPEC, 8, 276

92, LIDE, D. R., AND MAMM, D. E., 1957, J. CHEH, PHYS. 2?. O66

93. LIDE. D. A.., AMD MAKH, D. E.. 195?, J. CHEH. PHYS. 27. 874

94. LIMDFORS, K. K.. ANU COKMELLL, C. D., 1965, J. CHEW. PHYS. 42, 149

95. LOVAS, S. J.. 1974, AP. J. 193. 265.

96. LoVAS, F. J.. CLAKK, F. O.. AND TIEMANM, E.., 1975. J. CHEM, PHYS. 62, 1925

97. LOYAS. F. J.. AMD KKUPEMIE. P. H.. 19?4. J. PHYS. CHEM. REF. JATA 3. 245.

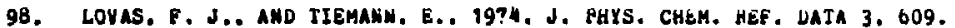

99. LOYAS, F. J., TIEMAMI, E., AND JOHWSON, D. R., 1974, J. CHEM, PHYS, DO, 5005.

100. HaGnuson, D. W.. 1951, J. Chem. PHirs. 19, 1071

101. MAXI, A. 6., 1967. J. MOL. SPEC. 23. 110.

102. MAKI, A. G., 1974, J. PHYS, CHEM, REF, DATA 3. 221.

103. MAXI. A. G., ANU JOHASOM, D. R.. 1973. J. MOL. SPEC. 4?, 22 b.

104. HESCHI, D. J., KND MYEAS, R. J.. 1959. J. MOL, SPEC. 3. 405

105. HICHIELSEN-EFFIMGEA, J., 1964, BULL. ACAD. ROX. BELL. 50, 645.

106. MILLEA, D. J.. AND HORTOW. J. R.. 1960, J. CHEM. SOC. 1523

107. HILLEN, D. J., AND PANGELL, J., 1961, J, CHEM, SCC, 1322

108. MILLEN, D. J., AND SINWOTT, K. H., 1958, J. CHEH. SOC. 350

109. HIRRI. A. M.. OURHWIERI, A., AHU FAYERO. P., 1961. HUOYO CIM, 19, 1109

110. hoRgAn, H. W., AND GOLDSTEIN, J. H., 1952, J. CHEM, PHYS, 20, 19d1

111. MUAINO, X.. AHd TAMAKA, T.. 1965, J. MOL. SHEC. 16. 179

112. MYERS, R. J.. AMD GWINH. W. D., 1952. J. CHEM, PHYS. 20.1420

113. MAIA, K. P. R.. RUUULPA, H, D.. HWU DREJZLER, H.. 19?3, J. MUL, SPEC. WÓ, ST1,

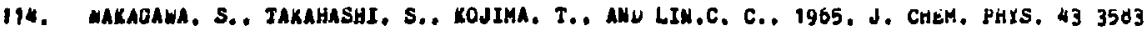

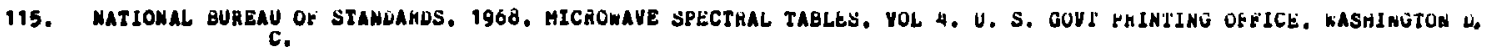
116. COATA. T., ANU LOZIMA. K.. 1972, J. MOL. SPEC, 42, 38.

117. PEARSOM, R., SUllivaM. T., ANL FHENKEL, L., 1970, J. MOL. SPEC. 34, 440.

118. PEN, D. E., ANU CURL, R. F., 1967. J. MOL. SPEC, 24, 235.

119. PICXETT. H. M.. 1973, J. HOL. SPEC. 46. 335.

120. PIERCE, L.. 1950, J. CHEM, PHYS. 24, 139

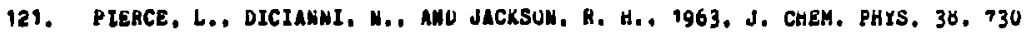

122. PIERCE. L.. AND dOHYMS. V., 1962, J. AN. CHEH, SUC. 04. 2052

123. PIERCE, L., aMu haYASHI. H.. 1961. J. CHEM. PHYS. 35, 479

124. PIERCE, L., J.CKSOM, R., AMU DICIAHKI, N., 1961, J. CHEM, PHYS. 35. 2240

125. RAO, У. H.. AND CURL, H, F., 1964, J. CHEM, PHYS, 40, 3688

126. HAO. У. H., AND CURL, H. E.., 1966, J. CrEH. PHYS. 45, 2032

127. RAYMONDA, J. W.. MUEHTER. J. S.. AMD KLEHPERER. W. A., 1970, J. CREM. 8MYS. 52, 3458.

126. AtGuEM, J. S., ANU BUtChER, S. S., 1964, J. CHEM. PHYS. 40. 2109

129. RING. H., EDLARUS. H., KESSLER, M., AHU GOHDY, H., 1947. PHYJ, HEV, 72, 1262,

130. ROHINSON, G. W., 1953, J. CHEM. PHYS. 21, 1741 
131. HOUSSY, G.. DEMAISUN. J.. GOTSKOK, I.. AND KUDULPH, H. L.. 1971, J. MUL, SPLC. $38,535$.

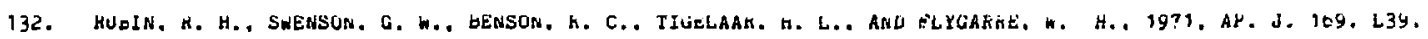

13j. hUVOLYH, H. L., 1902, 2S, MATUHF, 17A, 208.

134. SAEuEBAKT. E.. hNLL COX. A. P., 1965. J. CHEM. HHYS. 43. 166

135. SAITU. S., 19?2, AP. J. 170, L95

130. SAIJO, S.. ANL aMANO, T., 1970, J. MOL. SHEL, 34, 303.

137. SAIJO, S.. AND TAKAGI. K., 1973. J, MUL, SPEC, 4?, 99.

130. SASTKY, A.. LASS. S. C. BROUKS. W.. ANL BHANMIK. A.. 1969. J. MOL. SFEC. 31. 54.

139. SAYARIRAJ, G. A., 1966, ANN. SOC. SCI, bRUXELLES OO. do

140. SEKINO, S., AND NISHIKAMA, T, 195\%, J. PHYS. SUC, JAF, 12, 43

141. SHIHIZU. T. . ANU TAKUMA, H.. 1960, J. PHYS, SOC. JAP. 15, 640

142. SHCOLEHY. d. A.. SHULMAN,R. C.. AND YOST, U. M., 1951. J. CHEM. PHYS. 19. 250

143. Shulgan. M. G.. UAIley, E. P., ANi TUnNes, C. H. . 1950. PHYs. MEV. 7d, 145

144. SIMNUIT. K. M.. 1901, J. CHEM. PHYS. 34. 851

34. SInYEIL. M. H.. 1951. J. CHEM. PHYS. 19. 1609

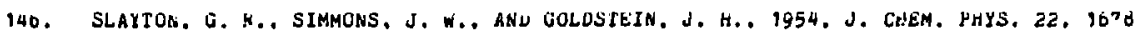

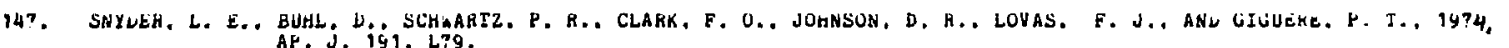

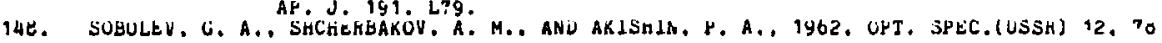

149. SORENSE.V, L. O., 190?, J, MOL, SPEC, 22, 32b.

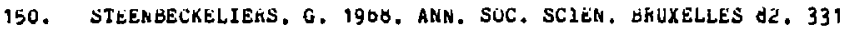

151. StiglianI. *. M., Laukie. Y. W.. ANb LI. J. C., 1975. J, CHEM. PHYS. 62, 1890

152. SULUKI, M., AND KOZIMA, K., 1970, J. MOL. SPEC. 33. 307.

153. SULUKI. M.. ANU KUZIMA, K., 1971, J. MOL, SPEC, 36, 314.

154. SLALANSKI, L. B., AN FOKD. R. G.. 1975. J. MOL. SPEC. 54, 148.

155. TaKano, M., SASaya, Y., AND SATOH. T., 1906, J. MOL, SPEC, 26, 15 ?.

156. TANMEgaum, E., MYEHS, R, J., ANu GWINN, H, D., 1956, J, CHEM, PHYS, 25, 42

157. TLEMANA, E.. 1974. J. HHYS. EHEM. RËF. DATA 3. 259.

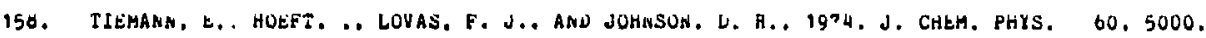

159. Thambakulo, K.. Clakk. A.. aho heakns, C., 1950, J. Chék. Phys. 20.736.

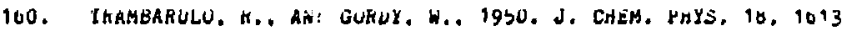

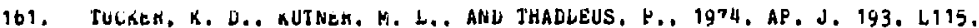

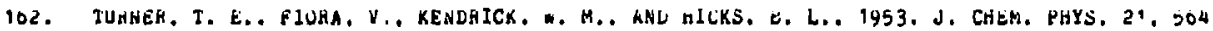

163. TYLEK. J.K., 1963, J. MOL. SPEC. 1..39

164. TYLER. J, K., ANL SHEFIUAN, J., 1903, THANS, HAFABAY SLC. 59, 2661

1h5. TYLeH, J. K.. SHËIUAN, J., AND COSTAIM. C. C.. 1972, J. MOL. Stec. 43, 240.

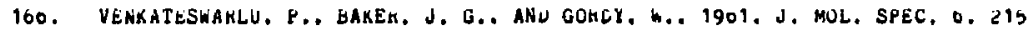

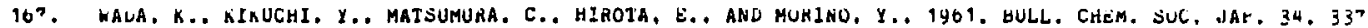

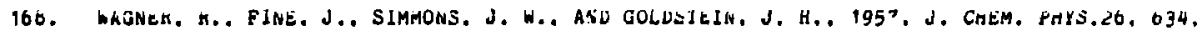

109. WEKTHEIMEH, h.. 1956, CUHPTES RENDUS 242, 1551.

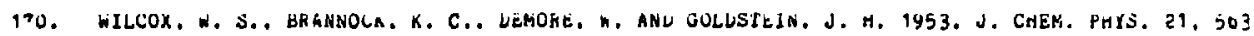

171. MINNEWISSEK. C.. 1973. 3. MOL. SPEL, 40. 10.

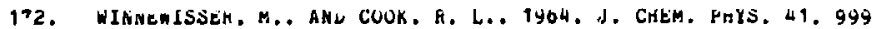

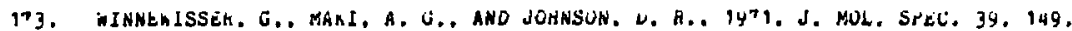


nefEngMisi, ?

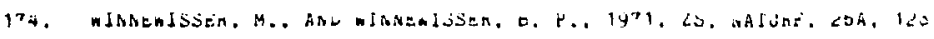

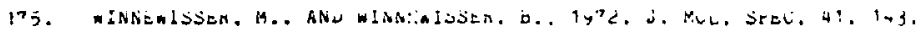

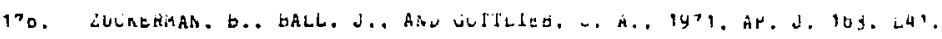

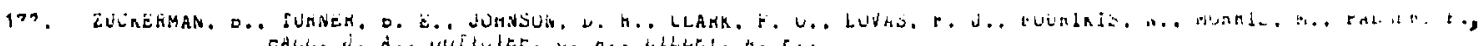

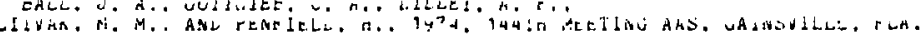


TABLE IV

LINE FREQUENCIES 


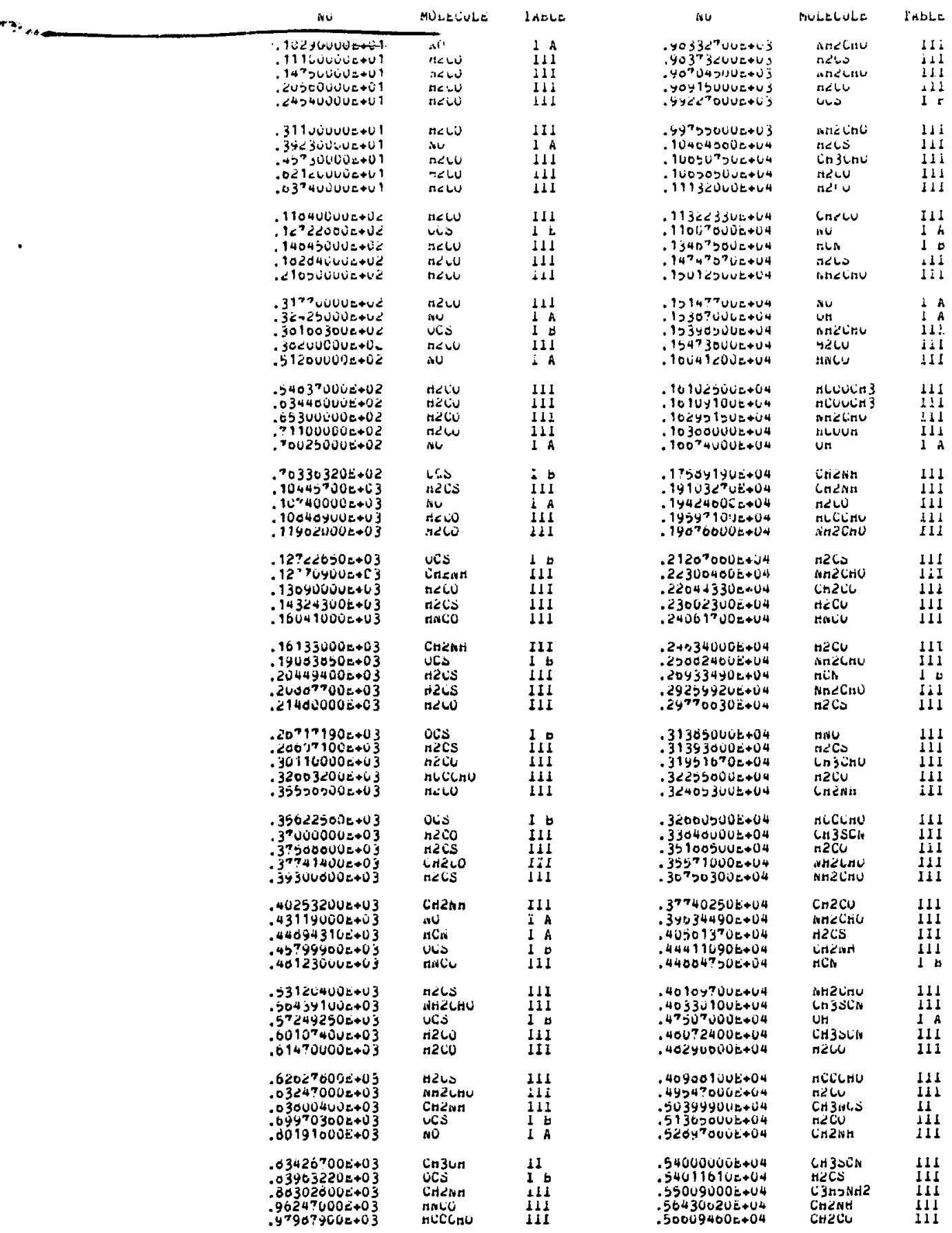


av

$.50777010 \vdots+64$

$.30^{7} d^{7} 130 L+24$

.oc $35:$ :.ju-ju+

- oc onsuita

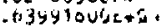

$.049734105+14$

$.673109 \mathrm{l}$ that 34

- Oeg7at.

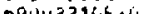

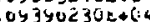

$.043005000+04$

$7052 u^{7} 00 t+14$

$70007910 x+v$

- cosoluvietol

- 37v0súne+ua

? 3709700e+04

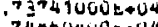

$.74000400 \mathrm{c}+\mathrm{J} 4$

$.740000000+0$ a

"juvgyouve+út

$70179000 c+0$

$77000510 \mathrm{t}+0$

$77129000 \mathrm{e}+0$

775930 toce 44

?7950100t+04

$.70201000=004$

$78411040 E+04$

.70920300e+si

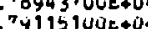

$.79251<00 \mathrm{k}+04$

1 y44330ue 404

?506 25 Jotola

.97053602+04

$.000543000+04$

$.80151700 E+04$

$00030900 \varepsilon+0$

$808037^{7} 0 x+04$

.049750 U0e+0

$.81011900 \mathrm{E}+1 / 4$

$.01459000 e+04$

. $81526000 t+04$

. $1090000 \mathrm{E}+04$

$.82058700 \mathrm{E}+04$

. 6 ¿211400t+04

.024300005 .04

$02020400 \mathrm{E}+0$

$82995100 t+04$

83006400e+04

$.83060800 t+04$

$.034164006+04$ $.83433200 t+04$

$.83563290 \mathrm{E}+04$

$83589400 E+04$

$.0304930 v e+04$

$63933700 E+0$

$84070400 \mathrm{e}+04$

$.4203000 \mathrm{t}+04$

$.84532920 E+04$

. B4 P12010E+04

$.84722300 k+04$

$.05259100 t+04$

ds $346000 \mathrm{c}+04$

.65710700t +04

$.857 ? 3500 \mathrm{e}+04$

$858360005+04$ $8506 ? 1000+04$ 8506 $1000+04$

$05915500 \mathrm{c}+04$ $.65905000 \mathrm{E}+04$
Mitelule

Intule

tiv

Nutelute

IhEL:

HCLAV II

inclais ill

$n<4=$

lono II

(cni)

$\mathrm{HCA}$.

111

Lnjplest j!

(n3)des 11

पing

(cinj) $<0$

inn

11

रn $j$

(Lnj) 2Si

Lasica

(Cirj)< $<0$

(n) $<5$

Lnsolit

Ats

$(\cos )<\infty 0$

(in 3$)<0$

site

On

atident

HeCO

Ans 3

( $\mathrm{CH} 3$ ) 430

cinelo

neche

CnjSCN III

SIf

(C)

( $\ln 3) 2$

C4M2n4

III

III

Con3uniz

Ans

$\mathrm{CH}$

CH3C3nCH2

111

Chicnu

SIFe

CHE

CH उC उHCH2

CHJUA

CHECHCL

(Cn3) 250

NOF

C3nshH2

C4N2H4

CHзC3HCh2

(CH3) 230

HH2 CHO

MSE

CHSrio

$\mathrm{HCOUCH}$

CH2CCH $3 \mathrm{LH}$

CM3CHLHZ

( $\mathrm{CH} 3$ ) 250

(CA 2 ) $2 \mathrm{CCO}$

Ii

III

III

II

III

II

III

118

111

III

ILI

111

111

III
III
III
III

$12 I$
III
III
III
- ODLSLOVIE+UA

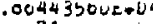

. Dot lavluetua

. Dolcululletua

$.00430<0 u E+04$

.07 Guuvuūe $\rightarrow 04$ . $0^{7} e^{7}$ ovuverual .0" UR व? UCe+O

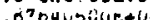

77 culuetua

. 7 bylizuUk+Ua

. 0 ??"y zuUL

क7

$.0^{7} 02$ y 1 Uut

$.0^{7} y+? 3 u v e+04$

$.000450040+04$

.00\% 5 juUUE+UA

.0025 gouve+0

$.002^{7} 479 u E+U 4$

$.0050120 U k+04$

$.0050^{7} 0$ Uúeiou

$.80730 \mathrm{j00 \textrm {e }}+\mathrm{u} \alpha$

$00025200 i+c$

$000700=004$

$000^{7} 20$ Uverua

$00090000 z+04$

$05999000 \mathrm{c}+u$

- O9600020 e+0a

. 0y $121000 b+04$

. OY I YOUGUE+OL

$.0920<400 E+00$

oj 3 z $400 \mathrm{ONE}+114$

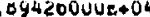

oyb00200E+04

0 grovo00tava

. 69? 1180 uetua

$.0 y 0431$ Uut +0

oyoug10We+OA

- 9vioovlut + va

$.40331300 \mathrm{C}+\mathrm{CL}$

$.9033^{77}$ uuciand

gosutouve+04

$9 v^{7} 4 ?<00 \mathrm{~T}+0$

70770 20004

g000 1200 cou

9U0O $1200 E+04$

.90983000 t +04

.911134 UUe $\$ 0$
$. \$ 1313090 \mathrm{t}+0$

$.91429000 \mathrm{E}+04$

$.91724500 E+04$

$.91725000 E+04$

- 910454 (ick +04

$.92010000 \mathrm{~b} \rightarrow 0 \mathrm{~d}$

$.92 C 39300 E+04$

$.9<251500$ écó

$.92327000 \mathrm{~d}+0 \mathrm{~d}$

$.92455000 e+04$

.9253 y400e+04

$.92 ? 21000 \mathrm{E}+0 \mathrm{~A}$

$92030500 E+04$

9291000NE+OA

$.930504106+04$

$.93250000 c i+u 4$

$.93013500 E+04$

$930639008+04$

$9063908+04$

(3)

$.939029000+104$
$.93900200 \mathrm{E}+04$
nLU

(Lins)ios il

Litisu dt

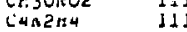

LSHO I11

ionsi

ang

Cićlnut II

Grallonsils II

vie

Esto II

(cm3)zinu $11 \mathrm{i}$

Anj

Licinn lid

site 11

CnjडC2n3 II

injutiden II

20

injscen 3

Gono 12

NüCL 11

(const256 II

(i) II

Cenjor

nLLCTEUA HI

MCLĩnizun II

Cundth III

(inz)acino III

aLUUy

LAZCRLCLN III

LA3GRCALA 11

CHECMCL

nets I1

(4nis II

(Ln3)2S0 liI

LE3CHLnLIV III

dC 3 N I

C2435 If

$\begin{array}{ll}\text { nacito } & 111 \\ \text { nicino } & 111\end{array}$

Nus

so2

C4sho II

13 11

$\begin{array}{ll}\text { SIF2 } & 111 \\ \text { NOLLL } & \text { III }\end{array}$

NHeCno III

Citibl 111

ing 11

Narchiso II

accith

C4N2 $\mathrm{N}^{4}$ III

पis

CH3CH2UH

(CHI) $2 \mathrm{CHO}$

Sitie 
Fn=-venul whi 3

av

\begin{tabular}{|c|c|}
\hline 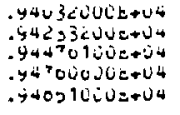 & 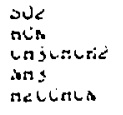 \\
\hline 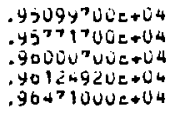 & 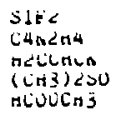 \\
\hline 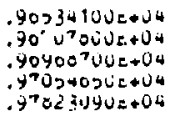 & 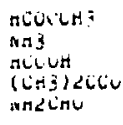 \\
\hline 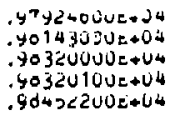 & 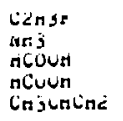 \\
\hline $\begin{array}{l}9055<9100 e+04 \\
.907123000+04 \\
.90727000 E+04 \\
.99004000 E+04 \\
.100010000+65\end{array}$ & 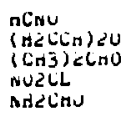 \\
\hline $\begin{array}{l}10000460=+05 \\
10057720 e+05 \\
10050300 e+05 \\
10009500 \varepsilon+05 \\
10079390 e+05\end{array}$ & 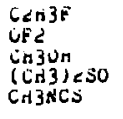 \\
\hline $\begin{array}{l}10009990 e+05 \\
10102900 e+05 \\
1132200005+05 \\
10244900 e+05 \\
.10270510 e+05\end{array}$ & 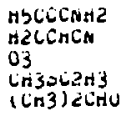 \\
\hline $\begin{array}{l}10293400 E+05 \\
10312430 t+05 \\
10329440 E+05 \\
103442300+05 \\
10397120 E+05\end{array}$ & 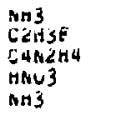 \\
\hline $\begin{array}{l}1042243 U E+05 \\
1042670 U E+05 \\
10430440 E+05 \\
10463 y^{2} U E+05 \\
104020 \angle 0 E+05\end{array}$ & $\begin{array}{l}\text { cenjo } \\
\text { anj } \\
\text { cenjo } \\
\text { Hecis } \\
\text { ing }\end{array}$ \\
\hline $\begin{array}{l}1052131 \mathrm{U}=+05 \\
1052594 \mathrm{UE}+05 \\
1052 ? 600 \mathrm{0}+05 \\
10536300 \mathrm{0}+05 \\
10566040 \mathrm{E}+05\end{array}$ & 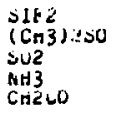 \\
\hline $\begin{array}{l}10500 ? 30 E+05 \\
10594530 \varepsilon+05 \\
10060000 e+05 \\
100 ? 2060 e+05 \\
100 d 2040 e+05\end{array}$ & 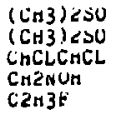 \\
\hline $\begin{array}{l}1070 \text { dodOE }+05 \\
10713100 t+05 \\
10^{7} 54560 E+05 \\
10^{7} 59020 t+05 \\
10 ? 70550 E+05\end{array}$ & $\begin{array}{l}\text { NH2CHU } \\
(\mathrm{CH}) 2 \text { SO } \\
\text { NH3 } \\
\text { NA3 } \\
\text { nit2CHO }\end{array}$ \\
\hline $\begin{array}{l}107090011 z+05 \\
10799720 E+05 \\
10000900 E+05 \\
10030100 E+05 \\
100^{2} 3150 E+05\end{array}$ & $\begin{array}{l}\text { CH3HCCHN } \\
(\mathrm{Cn} 3) 2 S O \\
\text { Cn3HCCHN } \\
\text { OH3 } \\
\text { C4N2H4 }\end{array}$ \\
\hline $\begin{array}{l}10090920 \mathrm{e}+05 \\
10900 \mathrm{~b} ? 0 \mathrm{t}+05 \\
10900000 \mathrm{~d}+05 \\
109474 d 0 \mathrm{e}+05\end{array}$ & 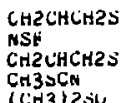 \\
\hline
\end{tabular}

IV.

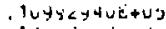

1Uyy $1940 x+\sqrt{3}$

- 11uveinvetuj

- inveroetus

livutoxued

$.1101<016$ etu

- 11uejoyuetos

- 11utzojuvet

$11001016 \mathrm{ta}$

11 joo 1uve tu

$.110 y 0<0 \mathrm{CE}+0 \mathrm{UL}$

111 UL joverut

$.11115<$ juetus

$.1111033 \mathrm{Je}+$ Us

.11132 Tunetus

$11100040 E+U 5$

11 c1350uetun

-112दituutou

11<2937UEஷU

-11255000n+us

$1120030 \mathrm{C}$

$11205000 \mathrm{sev}$

.11 sozo0uctus

$.113^{7} v 440 \mathrm{den}$

$.11300000 \mathrm{c}+0 \mathrm{~J}$

$.11420210 \mathrm{~b}+05$

$.114305306+05$

$11430300 t+45$

$1145^{7} 000 \dot{0}+05$

$1147,230 t+05$

$1153<0400+05$

$11504210 k+U$

$11592090 E+05$

.11592u90et+05

$115 y 2300 \mathrm{t}+05$

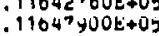

$.1100^{7} 400 \mathrm{t}+45$

$.110^{7} 3$ loue

-117074 Jueals

11? U0400 + Us

$.11710700 e+U 5$

$11730000 t+u$

$11735000 t+U 5$

-11735000E+05

$11723130 \mathrm{c}+05$

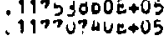

.11700000E+Uj

. $11791300 \mathrm{E}+05$

$.1103334 \mathrm{JE}+\mathrm{US}$

$1100^{7} 0 y 0 e+03$

$.110^{7}$ > Y5UE+Cb

$.11009200 \mathrm{u}+0 j$

$110 y$ da $24 \mathrm{U}+05$

1191377 Ue +05

$.11947140 \mathrm{e}+\mathrm{VS}$

$1194 ? 140 E+05$
$119 ? 1700 E+05$

$11902090 k+U 5$

$11902090 k+05$
$11905790 \mathrm{k}+05$

$11905790 \mathrm{~b}+05$
$12012260 \mathrm{O}+05$

$12015050 \mathrm{t}+05$

$1201750 u t+05$

. $120179040 b+0 j$

- 12091 أ $90 \mathrm{~b}+\mathrm{U} 5$

- $12101520 c+05$

-12141000E+US
Hilegule bete

sinjuiss it

alnivili:

slasuls: 11

LnCLHLI:CS lat

Cnckun $\quad$ I1

incincias

(umb)

(Unj)LE lij

Lituntines 1J

(cis)

Gile LnLA SA iil

vinzirill

ins

(Lthis)esu II

ancine did

cristichs

Cingnclan 111

imsalina 11

$\left.\operatorname{lin}_{n \rightarrow 5} 3\right)<0$ ild

unsucina lid

CHeCHLL 111

nlwo I

(Cnj) LLLO III

Cacicigun 11 i

MLCs 111

(CH3) $2 \mathrm{SU} 11$

$\mathrm{CH}(\mathrm{CHO}) 2$ III

CHaCricl 11

Chenum

Cono

Chiscins

HCU:

HCLSHECHL 111

NAS

CA3HCCA 11

CnjCnCHe II

CHLLLHCL III

CH3SL2H 3 III

neCO III

accrid

SO2 III

(CH3) $2 \mathrm{CHO}$

Lnjull

NSP

nnacho 111

Cusno III

CH 30 CL II

IHZNUH III

CH3SLN 11

(H2 CCh) 20 III

Ciscto

III

( $\mathrm{CH} 3) 2 \mathrm{COO}$ III

( $\mathrm{CH}$ ) 25 III

IF2 
nu

$.121435200+03$

$121503100+0$

$.121629700+05$

$.12230100 \mathrm{c}+0$

.122b1400k+ub

$.12252450 \mathrm{0}+05$

-12250b40L+U5

$12301490 \mathrm{E}+05$

$.1230-400 k+05$

$1230 d^{7} 10 c+05$

$12310000 t+0$

$12317340 t+05$

$.12330400 \mathrm{E}+\mathrm{O}^{\circ}$

$12341170=05$

$.12369500 \mathrm{c} \rightarrow 05$

$.12300000 \mathrm{c}+0$

$.12422250 \mathrm{r}+05$

$.12450600 \mathrm{E} \rightarrow 05$

$.12461040 \mathrm{E}+05$

$.12511000 \mathrm{E}+05$

$.12515^{7} 30 \mathrm{E} \rightarrow 05$

.12522090 LOS

$12540900 \mathrm{E}+05$

$.12541620 \mathrm{t}+05$

$.12552^{4} 50 e+05$

$.125023200+07$
$.125646400+05$

. $12560900 k+05$

$.125016 ? 00+05$

$.12592450 \mathrm{~s}+05$

$.1259^{7} 690 \mathrm{E}+05$

. 12000500E+05

$12604740 E+05$

$.12609402 E+0 j$

$.12642120 i+05$

$.12044280 \mathrm{E}+05$

$.12674120 E+05$

12707010E+05

$12722130 \mathrm{E}+05$

$12752900 \mathrm{E}+05$

$.12782 j 90 t+05$

$12 ? 9 ? 7105+05$
$.128059005+05$

$-128059005405$

$11609600 E+05$
$12010000 \mathrm{0}+05$

$1267790000+05$

. $13879300 E+05$

$.12918150 \mathrm{1}+05$

$12919550 t+05$

$12923100 \mathrm{0}+05$

$.12931020 k+05$

$1295: 2200+05$

$1 \times y+34 j D E+05$

13015 out +05

.13023 ? $10 \mathrm{E}+05$

$.130261000+05$

$.13034590 \mathrm{E}+05$

$.13043700 E+05$

$.13047900 E+05$

13062800E+05

$.13084100 \mathrm{E}+05$

$.13110920 E+05$

$.13113340 \mathrm{E}+05$

$.13147170 \mathrm{OE}+05$

$.13147170 E+05$
$.13198090 E+05$

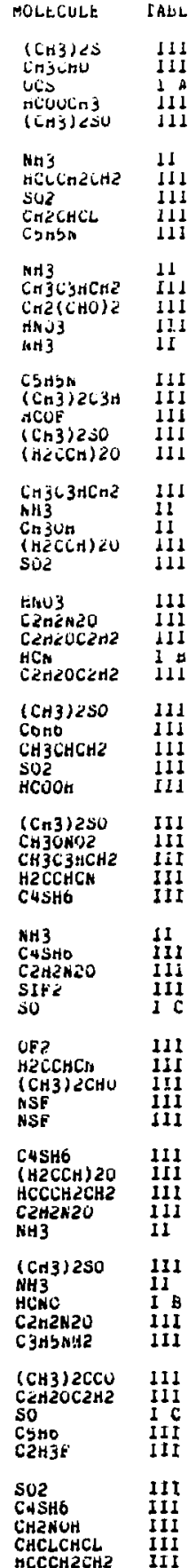

su

1 govic110e+ub

$132903^{7} 05+0$

$132 y^{2} 4 y 02+03$

$.9320^{7}$ viuetub

$.133<1320 \mathrm{e}+0 \mathrm{~s}$

$.153<3440 \tilde{e}+05$

. 1933000úb+os

$135400=+40$

$.134<2 d 2015+0:$

$.131125000 E+0 \%$

$13451<50 \mathrm{t}+0$

$1344140 u=00$

. $3545000 \mathrm{E}+16$

$.1343^{7500 t+1: 2}$

$134^{7}, 0 \times 0 \mathrm{Et}$

-1340190JEAd:

. 1340y: $066^{\circ}$

.132505luetós

$.13504400 \mathrm{k}-05$

-1357U0GLituz

$.135^{2}$ s 1tok+45

. $1350529 \mathrm{j} t+05$

. 13000 guive+us

$.1 \% 012300 \mathrm{E}+0 \mathrm{~s}$

$13612500 \mathrm{c}+05$

1,13012 sude +05

$1300+000 \mathrm{e}+05$

$1300 \% 90 \mathrm{e}+05$

$.1364^{7} 940 E+05$

$13700900 \mathrm{E}+0$

$.13719510 \mathrm{E} .05$

$13 ? 240904+05$

13727d0uetub

$13755 ? 20 \varepsilon+05$

$.13747130 \mathrm{z}+05$

$137 \pi 7130 \mathrm{k}+05$
$137 ? 0960 \mathrm{E} \rightarrow 0 \mathrm{~S}$

$137 d 0390 \mathrm{e}+05$

$.13795330 \mathrm{t}+05$

$.13304030 k+05$

$-13611490 \mathrm{c}+05$

$139446200+05$

$13904620 E+05$

$13914100 t+05$

$13915030 \mathrm{D}+05$

$13917700=+05$

$13937900 E+05$
$13900500 e+05$

$139729005+05$

$.13974540 \mathrm{E}+05$

$.13990000 E+05$

$.139952408+05$

$.14004900 \mathrm{~b}+05$

.140029008405

$.14005250 E 405$

-1410d

$.14123730 \mathrm{E}+05$

$.14167050 E+05$

$.14190000 x+05$

- 14211000 E405

$.14210250 \mathrm{E}+05$

. $14224740 \mathrm{E}+05$

$.14237000 E+05$

$.14259700 \mathrm{E}+05$

$.14261900 E+05$

$142071706+05$

$.1420 ? 1 ? 0 E+05$
$.14260200 E+05$
MULHLJLE IAELE

(6ris) 250

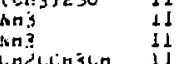

cencuzis III

$($ HeLthi) Idi

Lnsciacnua d1!

(Lnj)

(cons) 11 is

craccosin 111

canzúzzía $11 \downarrow$

Un I

su2 111

Cricirinch 111

IOAZCHC IIJ

(Cnj) 2 cas III

(Ch3)2su 111

c) उntin?

Uirecs lld

CnCLLHLL 11 I

un

Cnz $(\mathrm{CHU}) 2$ II

GazGachea lid

(Cn3)ZSU III

Cistor

Nn 3

Mis

(in3)2So ilf

( $\mathrm{CH} 3$ ) $25 \mathrm{O}$

CuSnt

(CA3) 250

(cn3) 250

(CM3) $\angle \mathrm{SO}$

(Cr13) 250

CSASN

NSE

NSt

C2n2UC:2n2

( $\mathrm{CH} 3) 2 \mathrm{ZSO}$

C2H2UCZn

$(\mathrm{CH}) \mathrm{COCO}^{2} \mathrm{CCO}$

$\mathrm{CH} 3 \mathrm{sC2H} 3$

CH3SC2H3

$\mathrm{An}_{3}$
( Ch3) $2 \mathrm{CHC}$

C3n5MH2

COHó

( $\mathrm{H} 2 \mathrm{CCH}) 20$

C2H $2 M 20$

CH2CCHJCH

C3HSHH2

NHECHO

$\mathrm{CH} 2(\mathrm{CHO}) 2$

(CH3) 2 SO

( $\mathrm{CH} 3$ ) $2 \mathrm{CHO}$

CH $35 \mathrm{CN}$

NH 3

(Cn3)2S

$12 \mathrm{H} 3 \mathrm{t}$

MCOOCh3

NACH2

\section{III}

III 


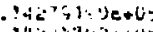
$14 x+463000$ -14द2 $2090+2$ $142 y^{\prime \prime 2}+14 .+1$
14320912.15

$14535340 \mathrm{y}=0$. $.143^{7} 050 u c+0 j$ $.143^{7+4}$ bletu $.1430119 \mathrm{UE}+0 \mathrm{O}$ $143 y 2 z<U e=0$ ?

$.1440^{2} 110 t+10$ $14414040 E+0$ ? $14419 ; 300+03$ $.1441+340 z+U$ $.14427130=+45$

$.1443 y 400=+03$ $14463 y<0 e+05$ $.1440 d^{7} \angle 0 E+0 y$ $.14400^{7} 00 \mathrm{e}+\mathrm{Ub}$ $.145342005+02$

$.1450^{7} 000=+05$ $.146155202+05$ $140340020 t+u 5$ $14005550 E+05$
1400505

140705YUt+05 $14001130 \mathrm{E}+05$ $.14602020<+05$ $.14000450 e+05$ $14090150 \mathrm{c}+0$

$.147242^{7} 0 \mathrm{E}+0 j$ $.1472 b^{7} 40 x+05$ $.14735920 \mathrm{E}+05$ $147530200+05$
$.14770250 E+05$

$.14003350=05$ $.14422^{\circ} 00 \mathrm{E}+05$ $.14023270 E+05$ $.14863300 E+05$ $.1409^{7500 x}+05$

$.1484^{7} 090 t+05$ $.14 y 03130 \mathrm{t} \rightarrow 05$ $.14435 \geqslant 10 s+05$ $.14945530=405$ $.149455008+05$

.149473360405 $.14953710 x+03$ -1440841UE+0s $.14 y^{7} 9400 x+05$ . 1490?700E+05

$.149906500+05$ $.15010700 \mathrm{x}+05$ 150223006005 $.15033000 \mathrm{E}+\mathrm{U}_{3}$ $1505 c=n+05$

$150782900 \div 05$ $.150^{2} 9420 \mathrm{E}+\mathrm{OJ}^{2}$ 15 fiodoet0s $151050400^{\circ}+45$ $.15124^{7} 20 \hat{e}+05$

$151375^{\prime \prime J E+05}$ . $15145510 \mathrm{E}+05$ $.15146000 k+05$ - $1517,3520 E+05$ $.15102000 t+05$

$15193540 \varepsilon+05$ $15195400 \mathrm{e}+05$ $152305006+05$ $15230570=05$ $.15233360 \mathrm{E}+05$

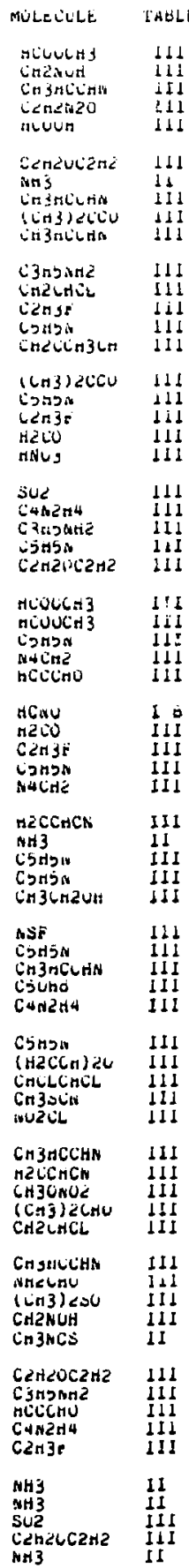

mo

. 13<Ubscuctus $13<00<4 j t+u$ - 1Jjosiove+los $.12 j<y+10 u=+U$ ijjuoyde +uj

$\because \xi y 154 v e+U=$

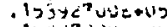
$.154 \omega^{2}$ U2uetus .1541<jcueal 13417 uuverus

$1241 y^{7}$ Uue Ub $.1545^{7}$ buvetuly $.1244 U^{7} 3 \mathrm{C} t+25$ - 154asyouetus . 12403 juUt+Uj

.15400 y yū $.12401210 E+U 5$ - Isj 14 UUUet0j - 15b3400e+ci - 1503 y $3200+15$

.15b51500e+05

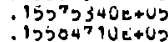
15 oudazoue+ub 1500jyú́s+Us

$.156 \mathrm{v}+0 y 0 \mathrm{0}+\mathrm{U}^{3}$ - $15024020 t+05$ - 15020190e+0j $.1503<00 t i t+U$ 150jyouvetus

$.15040140 E+00$ $.15040120 E+05$ $.15730410 t+0$ - 15? $157404^{7} 05+05$
$15^{7} 43016 e+05$

.15740190t+ob . $15735290 E+05$ $.157714400+05$ $.15^{777} 590 \mathrm{E}+05$ .15777930s+Uל

$.15^{7} 94490 \mathrm{0} 05$ . $15 ?$ Y4540 $\rightarrow 05$ 15021120E0 - 100<jo7ve+05 .1502 Jy JuetUS

1 1505900Ue+US - 15ugyoyue+us -150? juUue+L's $.1590^{7} 5^{7} 0 \mathrm{E}+\mathrm{UJ}^{2}$ . $15933320 \mathrm{l}+\mathrm{Ub}$

$.159435^{7} 0 t+05$ - $12400000 E+05$ $.1590<240 E+U 5$ $.1590^{7} 840 \mathrm{E}+\mathrm{U}^{\circ}$ -159y24 2Uetus

$.1000 ? 210<+05$ $.100 j " 30 \mathrm{~s}+\mathrm{Ub}$ $.100 j{ }^{2}<30 e+d s$ $.10039700 \mathrm{c}+0 \mathrm{Co}$ $.10039^{7} 00 \mathrm{OL}+05$

$.16000310 E+05$ $.100003^{7} 0 \mathrm{t}+\mathrm{U} \mathrm{J}$ $.10090 ? 30 \mathrm{E}+05$ $.10100600 t+05$ $.16105420 E+05$

$.10115740 E+05$ $.10122200 \mathrm{~L}+05$

$.10120090 \mathrm{~s}+05$ $.16131200 \mathrm{E}+05$

\begin{tabular}{|c|c|}
\hline ELULE & $\triangle A O L$ \\
\hline 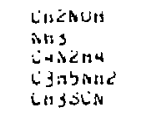 & $\begin{array}{l}\text { II } \\
11 \\
\text { II }\end{array}$ \\
\hline 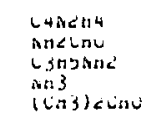 & 1 \\
\hline 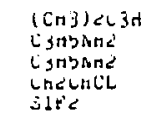 & 11 \\
\hline 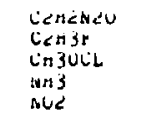 & $\begin{array}{ll}11 \\
11 \\
11 \\
11\end{array}$ \\
\hline 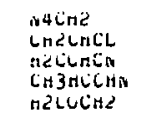 & \\
\hline $\begin{array}{l}\text { inzricena } \\
\text { Ur2 } \\
\text { nNus } \\
\text { Ans } \\
\text { ins } 3\end{array}$ & $\begin{array}{l}11 \\
11 \\
11\end{array}$ \\
\hline 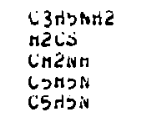 & \\
\hline 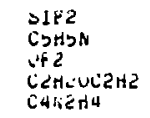 & I \\
\hline $\begin{array}{l}\text { GRZLLCN } \\
\text { CHLCLCN } \\
\text { Ch3SLN } \\
\because n 2 C H C L \\
\text { CHSHO }\end{array}$ & $\begin{array}{l}11 \\
11 \\
11 \\
11 \\
11\end{array}$ \\
\hline 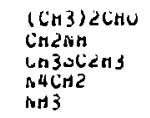 & $\begin{array}{l}11 \\
11 \\
11 \\
11\end{array}$ \\
\hline $\begin{array}{l}\mathrm{NO} 2 \mathrm{CL} \\
\mathrm{CH} 3 \mathrm{Cnu} \\
\text { n2CCnLL } \\
\text { CH3CnCCH2 } \\
\text { C3 } 35 \mathrm{NH} 2\end{array}$ & $\begin{array}{l}\text { II } \\
\text { II } \\
\text { II }\end{array}$ \\
\hline 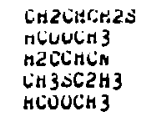 & 11 \\
\hline 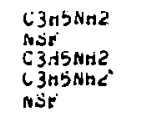 & II \\
\hline 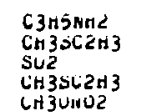 & I. \\
\hline
\end{tabular}


no

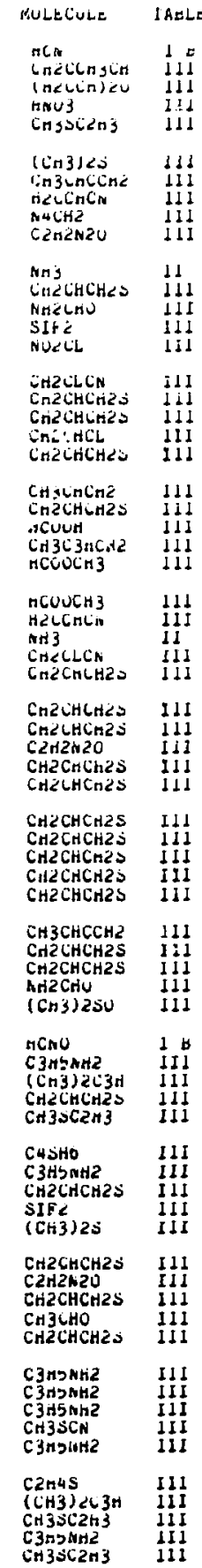

in $35 \mathrm{SC} 2 \mathrm{a}$ triv

$.10^{7} 043 \angle 0 \mathrm{E}+U 5$

$10^{7}$ yousuralj

$10^{7}$ yoe $000+0$

. 100ujauveelis

1001,?aveitoj

$.100<+500 \mathrm{e}+U \mathrm{O}$

$10041100=000$

1004 JusUe $\rightarrow 15$

$1000<3+J e+U$

. JuYcizudieug

10yuydoue+Uj

loyeutadetus

$104002700+05$

1090⿰2005e+U5

injuvockét

$170104008+05$

$17025470 \varepsilon+U$

$17027000 E+45$

$17040530=+05$
$.17124000 \mathrm{U}+U 5$

17151j30cets

1710000ue+us

17101<U6e+sis

$.17216190 \varepsilon+0$

$17230 j 40=+U 5$

.12 àusglueavj

$172029 y d e+U z$
$17 \sum^{7} 000 u e+U b$

$1720425 v e+05$

$17<00100$ e

$.17291540 \mathrm{U}+\mathrm{US}$

$.172950000+05$

172 yyoyuarus

- $17300100 i+05$

$16499200 \mathrm{E}+0$ J

$.165049500+05$

$16500900 E+05$

$.16510934 E+05$

$.10522600 \mathrm{t}+05$

16522060E+05

$10528910 \mathrm{E}+05$

$.16529 ; 00 \mathrm{t}+05$

$.16533440 E+0$ D

$16533940 \mathrm{E}+05$

$.16556650 t+05$

$.10501410 t+05$

$.1650<000 t+05$

$.16569 d 50 t+05$

$.165943402+05$

$.16594460 \mathrm{E}+05$

$16603000 \mathrm{t}+05$

$.16600000 \mathrm{E}+05$

$.16024130 \mathrm{E}+05$

$.16632710 \mathrm{E}+05$

$.166461000+0$

$.16649200 \mathrm{c}+05$

$.16653^{7} 30 \mathrm{t}+05$

$10672490 \mathrm{t}+05$

$10682000 \mathrm{E}+05$

$16687290 t+05$

$16694920 \mathrm{t} * 05$

16?14850t+05

$.16724000 \mathrm{t}+05$

$.16728300 \mathrm{E}+05$

$.16742400 \mathrm{E} \bullet 05$

$.16751400 \mathrm{t} \rightarrow 05$

$.1675 ? 500 t+05$

$16769380 t * 05$
$.17354710 E+05$

. $17300100 \mathrm{e}+05$

-1? 3? $0140 \mathrm{c}+05$

$.17301210 \mathrm{t}+05$

$.17396000 \mathrm{c}+05$

$.17397050 \mathrm{e}+05$

$.17405070 \mathrm{e}+\mathrm{U}$

$17410000=005$

$174116^{\circ} 0=005$
$17424000 \mathrm{O}$

$.174200000+45$

$.17429510 \mathrm{k}+45$

$17435 y 30=+05$

$17434430=+05$

$17439 ? 00 \mathrm{~d}+05$

$17453100 E+U 5$

$17409240 \mathrm{~d}+U \mathrm{U}$

$.17470900 \mathrm{E}+05$

. 175igdjudetus

.17513350c+us

$.17517510 \mathrm{E}+05$

17 यsJuve+US

$.17547900 \mathrm{t}+05$

$.175549^{7} 0 t+05$

- 17559300t+Us

1750U840E*05

$.17565920 E+05$

$.17579400 t+15$

$.175^{7} 9440 E+05$

.1758?020E+05

. 17 ovv000t+u5

17012900e+us
174005U0t-15

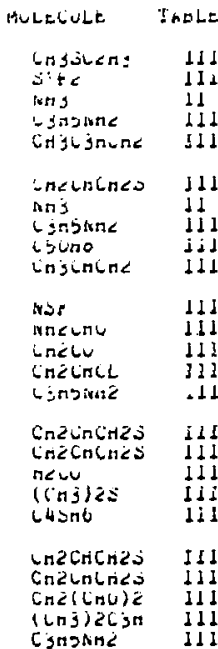

Checinlnas It

Cono

cono

Cusno

núun

$1+1$
$1+1$
11
11

$\mathrm{ni}$

Cojcrieun it

C4Sart II

çrisnne 11]

vite 11

It

$\begin{array}{ll}\text { (n2LCri) } 20 & \text { Itd } \\ \text { C5nO } & \text { IIJ }\end{array}$

MCLCHZUH 111

C3njtina 11

$\mathrm{Cr} z(\mathrm{CH}) 2$ Ij

Hicinzun Ill

जn 3 Cu 11

enticrichic III

cinzcritizs lid

injencaz II

NO2CL

Ln 3incent

Cn30LL III

nccincine 111

CH3OH

LCHLN2U IfD

LISCLLCN III

(CH3)2Lin III

Cnjlicitia III

iscitz III

(inj)zin

$\mathrm{n} 2 \mathrm{CS}$

$\begin{array}{lll}1 & 11 \\ \text { I1 } & 1 \\ 1 & 11 \\ 11 & 1\end{array}$ 


\begin{tabular}{|c|c|c|c|c|c|}
\hline no & MULELULE & ABOLE & ML & MULELULE & lntis: \\
\hline 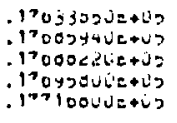 & 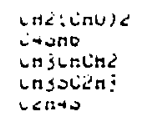 & $\begin{array}{l}11 \\
111 \\
111 \\
111 \\
i 1\end{array}$ & 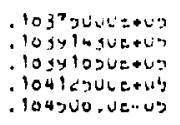 & 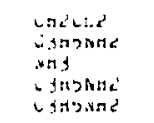 & $\begin{array}{l}111 \\
11 \\
11 \\
11 \\
11\end{array}$ \\
\hline 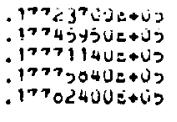 & 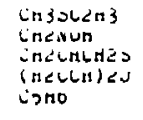 & $\begin{array}{l}111 \\
111 \\
111 \\
111 \\
111\end{array}$ & 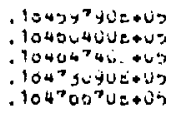 & 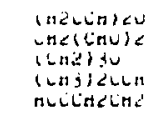 & $\begin{array}{l}1 \\
1 \\
1 \\
1 \\
1 \\
1 \\
1 \\
11\end{array}$ \\
\hline 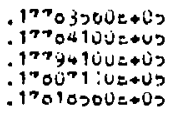 & 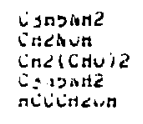 & $\begin{array}{ll}11 & 1 \\
11 & 1 \\
11 & 1 \\
11 & 1 \\
1 & 11\end{array}$ & 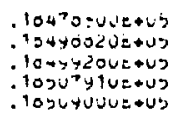 & 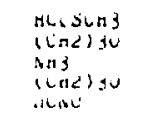 & $\begin{array}{l}1 \\
1 \\
1 \\
11 \\
1 \\
1\end{array}$ \\
\hline 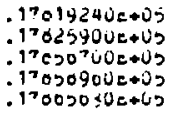 & 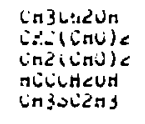 & $\begin{array}{l}111 \\
111 \\
111 \\
111 \\
111\end{array}$ & 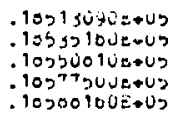 & 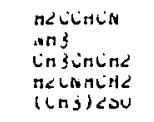 & $\begin{array}{l}11 \\
11 \\
11 \\
11\end{array}$ \\
\hline 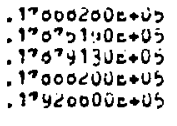 & 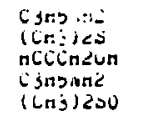 & $\begin{array}{l}111 \\
111 \\
111 \\
111 \\
111\end{array}$ & 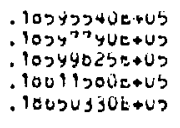 & 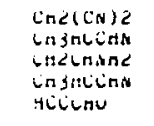 & $\begin{array}{l}11 \\
11 \\
11\end{array}$ \\
\hline $\begin{array}{l}17949310=+05 \\
17951320 E+05 \\
17957000 E+05 \\
1790900 E+05 \\
1790920050+45\end{array}$ & 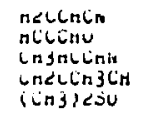 & $\begin{array}{l}111 \\
111 \\
111 \\
111 \\
111\end{array}$ & 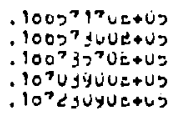 & 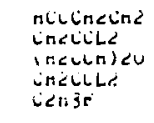 & $\begin{array}{l}11 \\
1 \\
1 \\
1 \\
11 \\
11\end{array}$ \\
\hline $\begin{array}{l}17970240=+05 \\
17907000 e+05 \\
17990010 c+05 \\
17990<20=+05 \\
17990490=+05\end{array}$ & 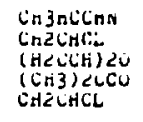 & $\begin{array}{ll}1 & 11 \\
1 & 1 \\
1 & 1 \\
1 & 1 \\
1 & 1 \\
1 & 1\end{array}$ & $\begin{array}{l}10^{7} 2^{7} 21 J E+U z \\
10^{7} 3 U^{7} U U E+U 5 \\
10^{7} 3410 U E+U 5 \\
10^{7} 30 y 4 U E+U J \\
10^{7} 3032 U E+U S\end{array}$ & 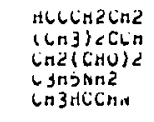 & $\begin{array}{l}\text { DI } \\
\text { II } \\
\text { II } \\
\text { II }\end{array}$ \\
\hline $\begin{array}{l}18011120 \mathrm{E}+05 \\
.10017420 \mathrm{~b}+05 \\
.10056400 \mathrm{e}+05 \\
.10000000 \mathrm{E}+05 \\
10000100 \mathrm{0}+05\end{array}$ & $\begin{array}{l}\text { Cn2LHCHZS } \\
\text { NHS } \\
\text { WLNUZ } \\
\text { SIS } \\
\text { Un2(CnU) }\end{array}$ & $\begin{array}{l}1 \\
1\end{array}$ & 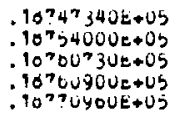 & 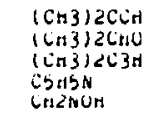 & $\begin{array}{lll}1 & 1 & 1 \\
1 & 1 & 1 \\
1 & 1 & 1 \\
1 & 1 & 1 \\
1 & 1 & 1\end{array}$ \\
\hline 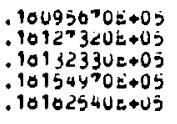 & $\begin{array}{l}\text { Ure } \\
\text { wnj } \\
\text { Ljabnte } \\
\text { sis } \\
\text { uns }\end{array}$ & $\begin{array}{l}1+1 \\
11 \\
\text { II } \\
1 \\
11 \\
11\end{array}$ & 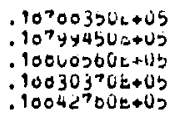 & $\begin{array}{l}\text { nLCSCH3 } \\
(\mathrm{Cn} 3)<00 \\
\text { ing } \\
\text { i3ribNH2 } \\
\text { na }\end{array}$ & $\begin{array}{l}11 \\
11 \\
11 \\
11 \\
11\end{array}$ \\
\hline $\begin{array}{l}10172440=+05 \\
191779900+09 \\
1101900000+05 \\
182110100+05 \\
10214000 c+05\end{array}$ & 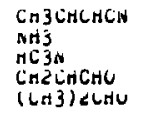 & $\begin{array}{l}111 \\
11 \\
11 \\
111 \\
111\end{array}$ & $\begin{array}{l}1002140 U E+05 \\
1005<0 y 0 z+05 \\
10000040 \mathrm{US} \\
10002020 \mathrm{05}+05 \\
100047000+05\end{array}$ & 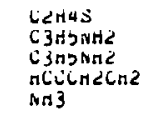 & $\begin{array}{l}11 \\
11 \\
111 \\
11 \\
11 \\
11 \\
11\end{array}$ \\
\hline $\begin{array}{l}1022^{7} 250 E+05 \\
.10229440 E+05 \\
.102502300+05 \\
.102053000+05 \\
.102^{7} 0100 e+05\end{array}$ & 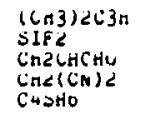 & $\begin{array}{l}\text { III } \\
\text { III } \\
\text { III } \\
\text { III } \\
111\end{array}$ & $\begin{array}{l}10094700 E+05 \\
10040010 E+05 \\
10900240 E+05 \\
10925320 \mathrm{0}+05 \\
.10953140 \mathrm{O} 55\end{array}$ & 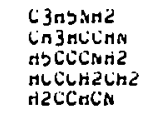 & $\begin{array}{l}11 \\
11 \\
11 \\
11\end{array}$ \\
\hline $\begin{array}{l}10200050=+05 \\
102000300+05 \\
10201000 e+05 \\
10205550=+05 \\
10209000=+05\end{array}$ & 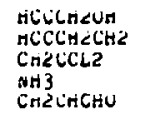 & $\begin{array}{l}111 \\
111 \\
111 \\
11 \\
111\end{array}$ & $\begin{array}{l}10950100 e+05 \\
.10450 ? 20 t+05 \\
10400540 e+U 5 \\
18904500 E+05 \\
10 y>0700 t+05\end{array}$ & 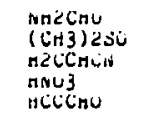 & $\begin{array}{l}11 \\
11 \\
11\end{array}$ \\
\hline $\begin{array}{l}16310 u 20=+05 \\
10314240=+05 \\
10325300 E+05 \\
10333130=+05 \\
16339900 \mathrm{O}+05\end{array}$ & 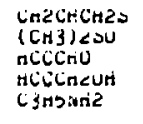 & $\begin{array}{l}111 \\
111 \\
111 \\
111 \\
111\end{array}$ & 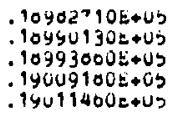 & 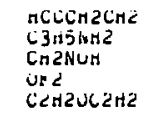 & $\begin{array}{l}111 \\
111 \\
111 \\
111 \\
111\end{array}$ \\
\hline $\begin{array}{l}10330450 e+0 j \\
.10359450 e+00 \\
10560400 e+05 \\
.103000 \mathrm{Je}+05 \\
.18373400 \mathrm{C}+05\end{array}$ & 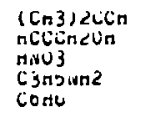 & 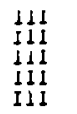 & 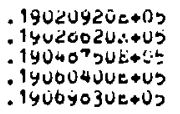 & 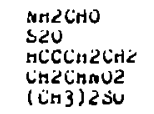 & $\begin{array}{lll}1 & 1 & 1 \\
1 & 1 & 1 \\
1 & 1 & 1 \\
1 & 1 & 1 \\
1 & 1 & 1\end{array}$ \\
\hline
\end{tabular}


no

$.190^{2} 0310 k+02$

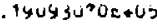

14 1 Guvuetu?

1y 1 420

191<0400tout

$.19140<5 U E 004$

. Iy1504300005

- 1916yozuzeas

191950? 140005

$.1920^{77}$ zue us

14209040 eut

$14213^{2} 0 u e+0$ 2

$142140206+05$

1y<156.

$.1921644 i t+05$

. 1 y $210500 t+05$

$.19220100 \mathrm{cos}$

.192262 que+0s

$.19232200 t+05$ $.19235330 \mathrm{E}+05$

. $9240520 \mathrm{t}+05$

192429402005

$.19243500 \mathrm{e}+0 \mathrm{~b}$

$19254440 t+05$

- 1925500لé05

- 19250450e+05

.19204120 éts

- 19205300ea0s

$.19208850 E+05$

. 14209010t+0b

$.19271400 \mathrm{E} 005$

$.19271090 \mathrm{UE}+05$

$.19275300 \mathrm{E}+05$

19275?40t+05

$19270240 t+05$

$19279090 \mathrm{t}+05$

$192850406+05$

. $192693506+05$

$.19294450 \mathrm{t}+0$

$19290000 \mathrm{t}+0$

$19303120 \mathrm{c}+0$

$.1930^{\circ} 5^{+} U E+05$

. $1432^{7} 500 \mathrm{E}+05$

$.19332080 t+0$

$.14351190 \mathrm{t}+05$

$19371150 t+0$

$19393000 t+05$
$19405400 \mathrm{t}+0 \mathrm{~S}$

$.19412370 \mathrm{t}+05$

$194221000+05$

$.19427590 t+05$

$.19437500 t+05$

$19477940 E+05$

$.19404400 E+05$

$19490350 \mathrm{E}+0$

$.19490350 E+05$

$19506000 E+05$
$.195087905+05$

$.19536990 E+05$

$19540 \mathrm{~d}$ ? $\mathrm{E}+0$

$1960840 n E+0$

$.19034650 \mathrm{E}+05$

$.19648600 \mathrm{E}+05$

$.19652430 E+05$

$19669250 \mathrm{t}+05$

$196 ? 0020 e+05$

$.1967040 \mathrm{~T}+05$
Muleluec

TAELE

(Lnj) 0 Co

trathrovis

seu

(Cing)

Cancneos

C jneus

MLLLricla

Críchive

Cunolune

MueCL

(cnis)asu

nccsciss

(Cns) $<00$

anj 3

(CH3) 650

Cnzhun

(CH 3 ) 250

(CH3) 250

(Cn3) 600

C2nánzu

( Cn3) 250

CHELHAUE

$(C H 3)<s o$

iccuun

( Cn3) $<0$

(CH3) $<50$

Cnsino

(CH3) 250

CH $<20$

Hध山F

C3nblone

$(\mathrm{C}+3) 250$

(CH3) 250

CHZCHNOZ

$\mathrm{H} 2 \mathrm{CHCN}$

:3A5AH2

( $\mathrm{CH} 3)_{2}(\mathrm{C} 3 \mathrm{n}$

HCOCCh 3

C Jnsint

HCouch 3

(cH3)

C5unto

HCCCHLCha

CHZChNOZ

C 3noniza
COHON

FCCn

(CH3) $2 \mathrm{Cco}$

HaCCHCN

CH2CHNO2

$\mathrm{HCCCH} 2 \mathrm{CH} 2$

CH2CHMO2

CHJ ICCHN

Crincha

( $\mathrm{Cn}$ ) $2 \mathrm{C} 3 \mathrm{n}$

CHZCHAOZ

C 3nSAHE

(Ch3) 25

HCCCH2CH2

11!

ill

111

,

III

It

iti

111

II

III

II

III

$+11$

1:1

I:I

III

ill

III

if

II

II

III

III

III

111

CH2 ( $\mathrm{CHO} 22$

(c) 35 hits 2

C3HSNH2
C2 $\mathrm{H} 3 \mathrm{r}$ nu

1y97704je+do

1400119tue+uj

Tyoca guve+u?

lyooy40uent

1yoyucoue+us

- 1405207Uz+Us

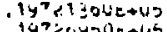

- 19720y30e+45

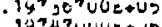

$.9 y^{2} 4010 U E+U 5$

$.1 y^{2} y^{4} y^{\prime} u e+u b$

i. 14, 74 UUE+L?

$1 y^{7} 0^{n}$ you ${ }^{2}+65$

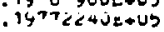

$.19^{2} 0420 \mathrm{U} t+\mathrm{US}^{2}$

. 197 yUuUUietus

- 190019y0E+O?

. 14050410E+0j

1y:73e0z+Uj

$.19915150 \mathrm{E}+\mathrm{U}$ ?

$.19434000 \mathrm{c} 005$

$.149302^{7} 0 \mathrm{E}+05$

$.154534800+05$

1940? 300t+Lt

$.199^{77} 030$ UEUS

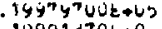

- $1999 ? 3^{7} 0 t=0$,

19y4510uet2h

.20013090E+US

$.20015530 \mathrm{t}+05$

$.20021040 \mathrm{U}+05$

$.20025180 E+05$

$.20044500 E+05$

$.20050100 E+05$

$.20004370 \mathrm{t}+65$

$.200^{7} 06605+05$

.20078000 Eats

$.200^{\circ 7} 8000 \mathrm{E}+0 \mathrm{US}$

$.20129040 E+05$

$.20132530 \mathrm{E}+25$

$.20143920 \mathrm{c}+05$

.20174300E+US

$.201004006+05$

.20101400 e + U $.20199^{7} 00 \mathrm{E}+05^{\circ}$ $.20200730 \mathrm{t}+05$

. $20200730 E+05$ $.20209200 E+05$
$.20211200 E+05$

$.20219920 E+05$

$20219920 c+05$

$.202315 y 0 e+05$

2U200000E+US

2U270 330 étút

21י>79790t+05

$.2029^{7} 090 \mathrm{E}+05$

$.20303940 \mathrm{C}+0$

$.20313590 \mathrm{E}+15$

$.20313590 E+L 5$
$.20333550 \mathrm{E}+05$

$.20335410 \mathrm{t}+05$

$.20335600 \mathrm{E}+0$

$.20340250 \mathrm{e}+05$

$.20340490 \mathrm{E}+0$

$.203^{71} 14600+05$

. $20373000 \mathrm{E}+05$

.2030 y4dOE+0

.20392550

$.20413220 \mathrm{t}+05$

$.20413220 t+0$

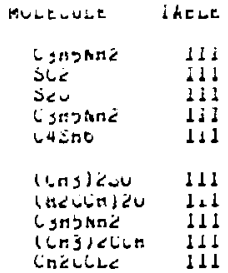

nuécru Iti

LCr:St 111

ivr 3 bl

CerEuLERE 111

$63 n 4$

III

ijnonaz il:

(cinj) Ecio id

Cymsklines 111

ALCCH2CH2 11

CHACnive

Cit30n 11

aries is

Cungione lil
nridin

Consmila 11

cjobiune its

Cono

Sristutia

Crach $3 \mathrm{Cm}$

sico

En<innue

cecinta

Silt 2

(CH3) $2 \mathrm{C} 3 \mathrm{H} \quad 11$

CaCCHNOC

cingionz

Cazcinoz

HCCCH2ChE

HCN

$\mathrm{HCCCH} 2 \mathrm{CH}$

CHICANUZ

CHZCO

Lim2Cu

inecu

in 200

CH2CO

( ing) 2 Citi

acoun

çnלme

HCCCHecha

inemun IJI

SU2

C 3 n5 Nna

CnjCUCL

CA2 CANU2

at 3

C5HSN

$\mathrm{CH} 2 \mathrm{CHNO2}$

C2M $2 N 20$

C2 $2 \mathrm{H}<\mathrm{NO}$

MSF" 


\begin{tabular}{|c|c|c|c|c|c|}
\hline$x u$ & $\therefore U L E L U L E$ & InELE & mo & $\because \because U E L U L E$ & intie. \\
\hline 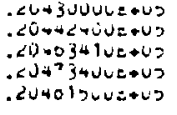 & 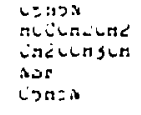 & $\begin{array}{lll}1 & 1 & 1 \\
i & 1 & 1 \\
1 & i & 1 \\
1 & 1 \\
1 & 1\end{array}$ & 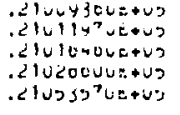 & 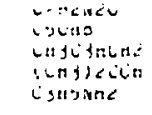 & 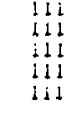 \\
\hline 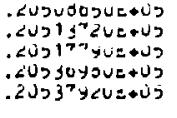 & 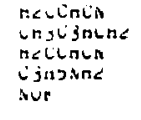 & 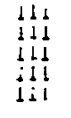 & 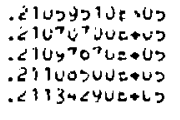 & 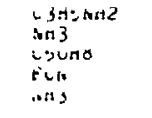 & $\begin{array}{l}111 \\
11 \\
121 \\
1 \\
11\end{array}$ \\
\hline 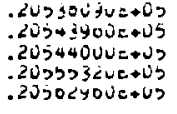 & 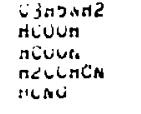 & $\begin{array}{lll}1 & 1 & 1 \\
1 & 1 & 1 \\
1 & 1 & 1 \\
1 & 1 & 1 \\
1 & 1 & 1 \\
1 & 0\end{array}$ & 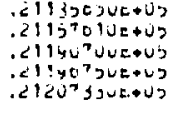 & 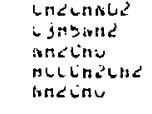 & $\begin{array}{lll}1 & 1 & 1 \\
1 & 1 & 1 \\
1 & 1 & 1 \\
1 & 1 & 1 \\
1 & 1 & 1 \\
1 & 1 & 1\end{array}$ \\
\hline 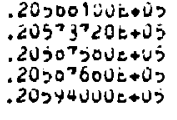 & 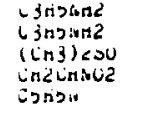 & 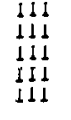 & 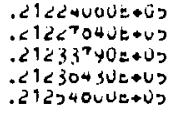 & 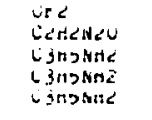 & $\begin{array}{lll}1 & 1 & 1 \\
1 & 1 & 1 \\
1 & 1 & 1 \\
1 & 1 \\
1 & 1 \\
1 & 1 & 1\end{array}$ \\
\hline $\begin{array}{l}.205960 \text { IUE+05 } \\
.20501130=+05 \\
.20024340=005 \\
.20037910 E+05 \\
.20045502+05\end{array}$ & 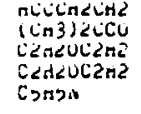 & 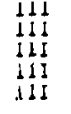 & 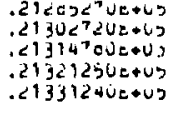 & 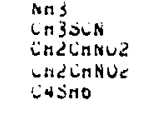 & $\begin{array}{ll} & 1 \\
1 & 1 \\
1 & 1 \\
1 & 1 \\
1 & 1 \\
11 & 1\end{array}$ \\
\hline $\begin{array}{l}.20049040 \mathrm{de}+05 \\
.20650500 \mathrm{0}+05 \\
.200003^{7} 0 \mathrm{0}+05 \\
.20000000 \mathrm{0}+05 \\
.200^{2} 0000 \mathrm{0}+05\end{array}$ & 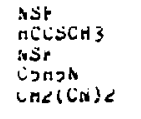 & $\begin{array}{l}111 \\
111 \\
111 \\
111 \\
111\end{array}$ & 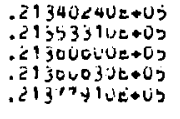 & 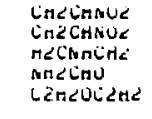 & $\begin{array}{lll}1 & 1 & 1 \\
1 & 1 & 1 \\
1 & 1 & 1 \\
1 & 1 & 1 \\
1 & 1 & 1 \\
1 & 1 & 1\end{array}$ \\
\hline 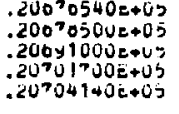 & 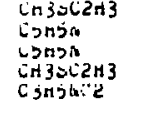 & $\begin{array}{l}111 \\
111 \\
111 \\
111 \\
111\end{array}$ & 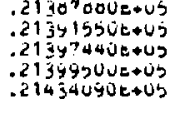 & $\begin{array}{l}\text { cound } \\
\text { naz } \\
\text { nics } \\
\text { conzan } \\
\text { ingideco }\end{array}$ & 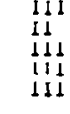 \\
\hline $\begin{array}{l}.20704300 \mathrm{l}+05 \\
.20705200 \mathrm{0}+05 \\
.207097 j 0 \mathrm{0}+05 \\
.20715100 \mathrm{E}+05 \\
.20710130=+05\end{array}$ & 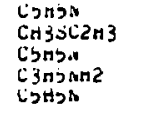 & $\begin{array}{l}111 \\
111 \\
111 \\
111 \\
115\end{array}$ & 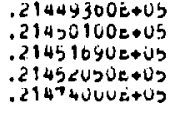 & 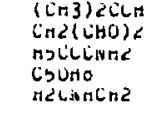 & $\begin{array}{ll}1 & 11 \\
11 & 1 \\
1 & 1 \\
1 & 1 \\
1 & 1 \\
11 & 1\end{array}$ \\
\hline $\begin{array}{l}.20714210 c+05 \\
.20723190 E+05 \\
.20720110 E+05 \\
.20735440 E+05 \\
.20737300 E+05\end{array}$ & 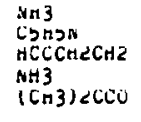 & $\begin{array}{l}11 \\
111 \\
111 \\
11 \\
111 \\
111\end{array}$ & $\begin{array}{l}.21404 \sum^{7} U E+U 5 \\
.414+40 U U E+U S \\
.2149003 U E+U 5 \\
.2150020 E+0 D \\
.21511500 E+U j\end{array}$ & 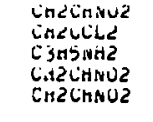 & $\begin{array}{l}111 \\
111 \\
111 \\
111 \\
112\end{array}$ \\
\hline $\begin{array}{l}.2074^{7} 420 E+05 \\
.20^{7} 53900 E+05 \\
.20^{7} 00^{7} 00 \mathrm{0}+05 \\
.20^{7} 0^{7} 10 \mathrm{~T}+05 \\
.20^{7} 09590 \mathrm{E}+05\end{array}$ & $\begin{array}{l}\mathrm{C} 3 \mathrm{HSNH} \\
\mathrm{CH} \mathrm{CO} \\
(\mathrm{Cr}) 2 \mathrm{CCH} \\
(\mathrm{CH}) \leq \mathrm{CH} \\
(\mathrm{Cn} 3)_{<S}\end{array}$ & $\begin{array}{l}\text { III } \\
121 \\
131 \\
\text { III } \\
\text { III }\end{array}$ & $\begin{array}{l}.21516120 \mathrm{E}+05 \\
.2151940 \mathrm{U}+05 \\
.21519400 \mathrm{E}+05 \\
.21529190 \mathrm{E}+05 \\
.21544220 \mathrm{U}+05\end{array}$ & 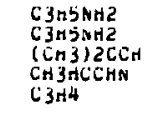 & 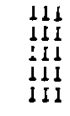 \\
\hline $\begin{array}{l}.20 ? 77650 \varepsilon+05 \\
.20784550 e+05 \\
.20 ? 95600 \mathrm{k}+05 \\
.26004030 e+05 \\
.20020000 E+05\end{array}$ & 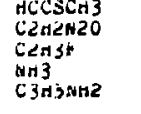 & $\begin{array}{l}111 \\
\text { IIII } \\
\text { III } \\
\text { II } \\
\text { III }\end{array}$ & $\begin{array}{l}.21551700 E+U S \\
.21561490 E+U 5 \\
.2163056 U E+05 \\
.21631500 t+U 5 \\
.21631640 E \rightarrow 05\end{array}$ & 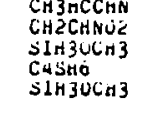 & 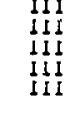 \\
\hline $\begin{array}{l}.200243406+05 \\
.200255 y 0 E+05 \\
.2003370 E+05 \\
.20035400 E+05 \\
.20041560 \varepsilon+05\end{array}$ & 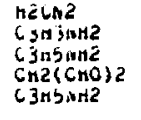 & $\begin{array}{l}\text { III } \\
\text { IIII } \\
\text { III } \\
\text { IIII } \\
\text { III }\end{array}$ & $\begin{array}{l}.21632430 E+05 \\
.21051640 e+05 \\
.21055940 e+05 \\
.21050540 E+05 \\
.21003100 e+05\end{array}$ & $\begin{array}{l}\text { Cn3CHCH2 } \\
\text { CHLCHAUL } \\
\text { CHLCHAOZ } \\
\text { HCLSEn3 } \\
\text { MLUUCH3 }\end{array}$ & 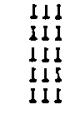 \\
\hline $\begin{array}{l}.20052510 e+45 \\
.20937000 E+05 \\
.20937910 E+05 \\
.20950640 e+45 \\
.20952700 E+05\end{array}$ & 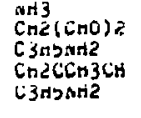 & 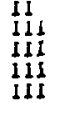 & 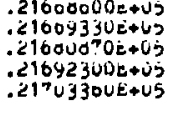 & 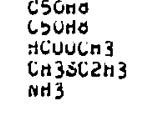 & $\begin{array}{l}11 \\
111 \\
11 \\
111 \\
111 \\
11\end{array}$ \\
\hline $\begin{array}{l}.20902100 E+05 \\
.20992020 E+05 \\
.20993060 E+05 \\
.20994610 E+05 \\
.21000^{7} 00 E+05\end{array}$ & 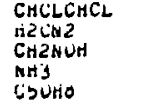 & $\begin{array}{l}\text { III } \\
\text { III } \\
\text { III } \\
\text { II } \\
\text { III }\end{array}$ & $\begin{array}{l}.21704000 E+15 \\
.21720000 \mathrm{C}+05 \\
.21700000 \mathrm{U} \\
.21700500 \mathrm{U}+05 \\
.21770500 \mathrm{U}+\mathrm{US}\end{array}$ & 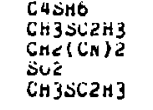 & $\begin{array}{lll}1 & 1 & 1 \\
1 & 1 & 1 \\
1 & 1 \\
1 & 1 \\
1 & 1 \\
1 & 1 \\
\text { III } & 1\end{array}$ \\
\hline
\end{tabular}


ai

. 17oúusetos $-21705{ }^{7} U=005$ - Th youbuens?

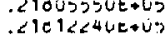

$.2101513 \mathrm{jc}+05$ $.21010040 \mathrm{c}+05$ $.10224325+05$

$.21024500 \mathrm{e}+0$ 's

. Lic LUYYULitos

$.2103 \angle$ luUetos . $200 Y$ YOUE.U? $.210^{7} 35^{3} 0 \mathrm{Oe} 0 \mathrm{O}$ - Tiyudzotie-65 $.2191740 u e+45$

$.214227300+03$ iy ju370 $x+05$ . $143 y 9700+05$ . $1977000+05$ $.21901460 \mathrm{~L}+0 \mathrm{~S}$

. $19019^{2} 0 t+05$ $.21902420 \mathrm{t}+05$ $.299043000+05$ $.21900 \angle 302+05$ $.21991900 t+05$

$.21991400 \mathrm{c}+0 \mathrm{~S}$ $.2199190 \mathrm{de}+05$ $.29900130 \dot{0}+05$ $.22040134 k+05$ $.22000136 \mathrm{~L}+05$
$.22003+50 \mathrm{E}+05$

$.220004 \mathrm{TH}+02$ $.22093190 \mathrm{E}+05$ $.22013190=+05$ $.22013670 E+05$ $.22022300 t+05$

$.220223^{\top} 0 E+05$ $.22030920 \mathrm{E}+05$ $22031400 \mathrm{E}+05$ $.22031020 \mathrm{c}+05$ $.2 ? 031020 \mathrm{~L}+05$
$.22031020 \mathrm{~L}+05$

$.22030990 k+05$ .22041 Y $50 E+05$ $.22045740 \mathrm{E}+05$ $.22043740 E+05$ $.22052130 E+05$

$.22055450 \mathrm{E}+05$ $.22065730 E+05$
$.22071000 E+05$ $.22095410 k+05$ $.22095410 E+05$

$.22117 ? 90 k+05$ $.22120050 \mathrm{E}+05$ $.22137720 E+05$ $.22140260 k+05$ $.22147000 \mathrm{E}+05$

$.22155200 \mathrm{E}+05$ $.22150000 \mathrm{E}+05$ $.221762005+05$ $2217 \square 660 \mathrm{E}+05$ $.22985190 \mathrm{E}+05$

$22204120 E+05$ $22200470 \mathrm{E}+05$ $.22208470 \mathrm{E}+05$ $.2222737 \mathrm{OL}+05$ $.22233260 \mathrm{E}+05$

$.22234530 E+05$ $.22235080 \mathrm{E}+0$ $.22240330 E+05$ $.22250^{7} 20 \mathrm{E}+05$
$.22262000 \mathrm{E}+05$

\begin{tabular}{|c|c|}
\hline E & \\
\hline 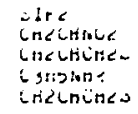 & $\begin{array}{l}11 \\
11 \\
\text { iI } \\
11\end{array}$ \\
\hline 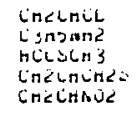 & $\begin{array}{l}1 \\
1 \\
1 \\
1 \\
1 \\
1\end{array}$ \\
\hline 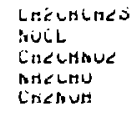 & $\begin{array}{ll}1 & 1 \\
1 & 1 \\
\text { il } \\
1 \\
1 \\
1\end{array}$ \\
\hline 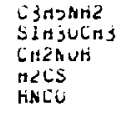 & $\begin{array}{lll}1 & 1 & 1 \\
1 & 1 & 1 \\
1 & 1 & 1 \\
1 & 1 & 1 \\
1 & 1\end{array}$ \\
\hline 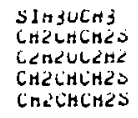 & $\begin{array}{l}1 \\
111 \\
111 \\
111 \\
11 \\
1\end{array}$ \\
\hline 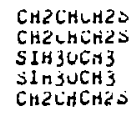 & $\begin{array}{l}11 \\
11 \\
12 \\
11 \\
11\end{array}$ \\
\hline 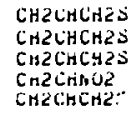 & $\begin{array}{lll}1 & 1 & 1 \\
1 & 1 & 1 \\
1 & 1 & 1 \\
1 & 1 & 1 \\
1 & 1 & 1\end{array}$ \\
\hline $\begin{array}{l}\text { CHZNUM } \\
\text { CHZCHNIAZ } \\
\text { HCNO } \\
\text { CHZCHLHZS } \\
\text { CnZCHCHZS }\end{array}$ & $\begin{array}{lll}1 & 1 & 1 \\
1 & 1 & 1 \\
1 & 1 \\
1 & 1 & 1 \\
1 & 1 & 1\end{array}$ \\
\hline 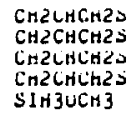 & $\begin{array}{lll}1 & 1 & 1 \\
1 & 1 \\
1 & 1 & 1 \\
1 & 1 & 1 \\
1 & 1 & 1 \\
1 & 1 & 1\end{array}$ \\
\hline $\begin{array}{l}\text { CH2CHNOZ } \\
\text { SU2 } \\
\text { (H2CCH) } 20 \\
\text { CH2CHNO2 } \\
\text { CH2CCH3 Cri }\end{array}$ & 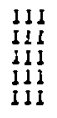 \\
\hline $\begin{array}{l}\text { C2H2N2O } \\
(\mathrm{CH}) 2 \mathrm{CCH} \\
\text { C3HSNH2 } \\
\text { C3HSNH2 } \\
\text { HNO3 }\end{array}$ & $\begin{array}{l}\text { III } \\
\text { IIII } \\
\text { IIII } \\
\text { IIII } \\
\text { IIII }\end{array}$ \\
\hline $\begin{array}{l}\text { S2O } \\
\text { HCUF } \\
\text { CH2CHCH2S } \\
\text { C } 3 \text { HSWH2 } \\
\text { C } 3 \text { nOHL }\end{array}$ & $\begin{array}{l}\text { III } \\
\text { III } \\
\text { III } \\
\text { III } \\
111\end{array}$ \\
\hline $\begin{array}{l}\text { CH2CHCH2S } \\
\text { CH3CHO } \\
\text { NOCL } \\
\text { CH2CHCH2S } \\
\text { CH3HCCHN }\end{array}$ & $\begin{array}{l}\text { III } \\
1 \text { II } \\
\text { iII } \\
\text { III } \\
\text { III }\end{array}$ \\
\hline $\begin{array}{l}\mathrm{NH} \\
\mathrm{H} 2 \mathrm{O} \\
\mathrm{CH} 2 \mathrm{C} \\
\mathrm{CH} 3 \mathrm{H} \\
\mathrm{H} 2 \mathrm{CN}\end{array}$ & $\begin{array}{ll}\text { II } & \text { II } \\
\text { III } \\
\text { III } \\
\text { III }\end{array}$ \\
\hline
\end{tabular}

\begin{tabular}{|c|c|c|}
\hline nu & RuLEu-t. & lnt: $-\mathrm{L}$ \\
\hline.$<<<b+5 \cup v e * u b$ & $i=5 ; i<\backsim+n$ & $1 i t$ \\
\hline 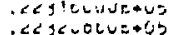 & 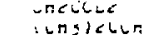 & 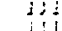 \\
\hline $.2 \angle 1 \leq 0<U E+O S$ & 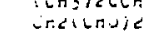 & i! \\
\hline . $\angle A$ SAYYSUEQUD & & $11:$ \\
\hline 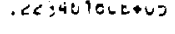 & -rginu & 2to \\
\hline . $2 \angle$ SOCgOJER & $\ln x$ & 111 \\
\hline 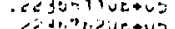 & $n \cup<6$ & \\
\hline 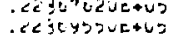 & 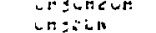 & \\
\hline 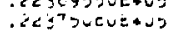 & cosines & $\therefore i$ \\
\hline $.229^{7}>20 v=065$ & - Arjuns & $1 \vdots 1$ \\
\hline . $\angle \angle:^{7} U Y O L E+U D$ & singutr: & d: \\
\hline$. \angle C 3^{7} c 53 L E+U D$ & $u r c$ & $1:=$ \\
\hline 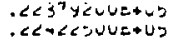 & $\begin{array}{l}\text { LAine } \\
\text { EneuLufi }\end{array}$ & $\begin{array}{ll}j+1 \\
1 \\
1\end{array}$ \\
\hline 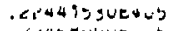 & Crishto & $\therefore 1$ \\
\hline . $<<4>d y$ y ULA & L $<$ niculane & ta \\
\hline$. \angle Z 4^{7} \mid 1 C L E+U D$ & xw口 & it \\
\hline 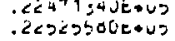 & 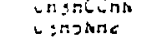 & Iit \\
\hline$. \angle \angle D<C O B U z \cdot U b$ & ingsus & \\
\hline $.2\left\langle b 4 L L^{\top} U L+U S\right.$ & i $\angle \pi 2 U$ L CnE & 1 \\
\hline $.2<b 471$ Ivenv & Enjoctono & iil \\
\hline $.2 \angle 2 * 14 b U E+U 5$ & $\sim n<i+[\lambda$ & d! \\
\hline 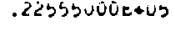 & Lostucing & is \\
\hline $.2250^{7}$ OOLELLS & $\operatorname{con} 2 \mathrm{Nan}$ & dil \\
\hline 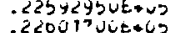 & nolín & 11) \\
\hline $.2<01094 J E \cdot 05$ & stionne & \\
\hline $.22019 b^{2} \dot{\Delta} 2+0 b$ & cónenzi & \\
\hline $.22019 c+0 \mathrm{E}+U \mathrm{~L}$ & $\sin \sin 2$ & 111 \\
\hline $.2 \angle 05 U 00$ UEtUS & Hriclle & \\
\hline$-22003000 E+0 D$ & $\sin 3$ & if \\
\hline $\begin{array}{l}2200 U 200 E+05 \\
.220^{9} 14 U U E+05\end{array}$ & $\begin{array}{l}\text { cunt } \\
\text { nźnnuse }\end{array}$ & Iil \\
\hline $.2200^{\circ} 040 \ddot{0}$ & Lngsx & iil \\
\hline $.2 \angle 000290 \mathrm{U}+00$ & $n n^{3}$ & \\
\hline 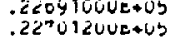 & 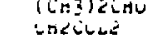 & iil \\
\hline $.22^{7} 11900 c+05$ & itsino & $i \mathrm{i}$ \\
\hline $.22^{2}<4310=65$ & nutes & \\
\hline $.22^{7}<034 u E+v>$ & 4354 & \\
\hline - $\angle Z^{7} j 2+s u E+U 2$ & tor:s & 11. \\
\hline $\begin{array}{l}.22733 y 900+U 5 \\
.22+45310=+05\end{array}$ & $\begin{array}{l}\operatorname{sL} 2 \\
\operatorname{Cn} 3 H \cos N\end{array}$ & $\begin{array}{l}111 \\
11 \mathrm{i}\end{array}$ \\
\hline $.22^{2} 05900 \mathrm{E}+\mathrm{US}$ & $\operatorname{con} 3 \mathrm{~F}$ & $11 \downarrow$ \\
\hline$. \angle 2^{7} 0040 E+15$ & Cnćnauz & \\
\hline $.22010920 t+05$ & 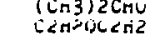 & 111 \\
\hline $.220153^{7} 0 E+05$ & conto & \\
\hline $.2<022020 \mathrm{t}+05$ & $(\mathrm{CH} 3) 2 \mathrm{C} 3 \mathrm{ri}$ & 111 \\
\hline $.2202^{2} 910 E+05$ & MLUUCH 3 & i \\
\hline $.22027910 E+05$ & $\mathrm{nCOCOCH}^{3}$ & \\
\hline $.2203400 U E+0 S$ & $\cos 2 \operatorname{sis}$ & \\
\hline $.22037100 \mathrm{~L}+\mathrm{U}_{5}$ & CnzCHCnzS & 111 \\
\hline $.220^{\circ} \mathrm{D} 290 \mathrm{E}+05$ & [3 & 111 \\
\hline $\begin{array}{l}.22001^{2} 00 E+0 b \\
.22924440 E+0 b\end{array}$ & "ICLSCtis & i \\
\hline $.229300^{\circ} 0 E+05$ & $\begin{array}{l}\text { ans } \\
\text { neso }\end{array}$ & \\
\hline $.22905710 E+U 5$ & $\mathrm{~h} 2 \mathrm{Cu}$ & 111 \\
\hline $\begin{array}{l}22970400 E+05 \\
22970590 E+05\end{array}$ & C2A4S & 111 \\
\hline $.22901280 \mathrm{E}+05$ & $(\mathrm{CH} 3)_{2 \mathrm{CC}}$ & 111 \\
\hline $.22901090 \mathrm{E}+05$ & & \\
\hline $.229926^{7} 0 E+05$ & Cn2NOn & III \\
\hline $.22990000 \mathrm{U}+0 \mathrm{~s}$ & MCNO & 1 \\
\hline $\begin{array}{l}.23004000 \mathrm{E}+\mathrm{u}^{2} \\
2302530 \mathrm{ct}+0 ?\end{array}$ & $\begin{array}{l}\text { MH } 3 \\
\text { MLCNOCHE }\end{array}$ & II \\
\hline $.23020000 t+05$ & מCNO & \\
\hline
\end{tabular}




\begin{tabular}{|c|c|c|c|c|c|}
\hline su & NLELLLt & InLLe & no & RuLCAUt & $1+1=$ \\
\hline 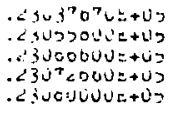 & 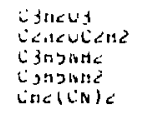 & 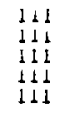 & 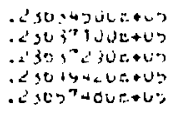 & 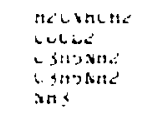 & $\begin{array}{ll}1 & 1 \\
1 & 1 \\
1 & 1 \\
1 & 1 \\
1 & 1\end{array}$ \\
\hline 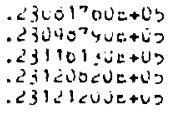 & 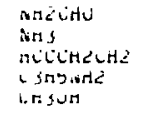 & $\begin{array}{l}111 \\
11 \\
111 \\
111 \\
11\end{array}$ & 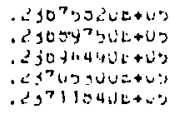 & 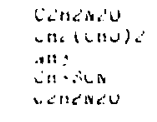 & $\begin{array}{ll}1 & 1 \\
1 & 1 \\
1 & 1 \\
1 & 1 \\
1 & 1\end{array}$ \\
\hline 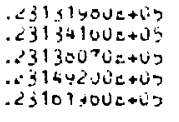 & 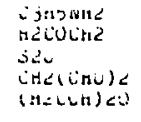 & $\begin{array}{l}111 \\
111 \\
111 \\
111 \\
111\end{array}$ & 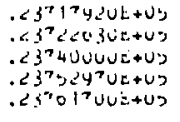 & 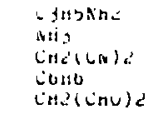 & $\begin{array}{ll}1 & 11 \\
11 & 1 \\
1 & 1 \\
11 & 1 \\
11 & 11\end{array}$ \\
\hline 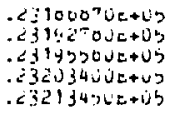 & 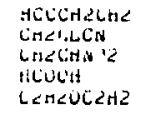 & 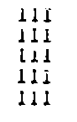 & 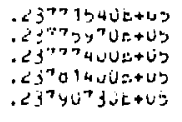 & 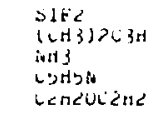 & $\begin{array}{lll}1 & 1 & 1 \\
1 & 1 & 1 \\
1 & 1 \\
1 & 1 \\
1 & 1 \\
1 & 1 & 1\end{array}$ \\
\hline 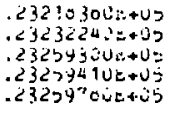 & 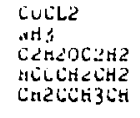 & $\begin{array}{l}111 \\
11 \\
111 \\
111 \\
111\end{array}$ & 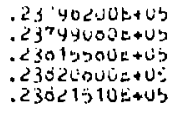 & 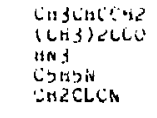 & $\begin{array}{ll}1 & 1 \\
1 & 1 \\
1 & 1 \\
1 & 1 \\
1 & 1 \\
1 & 1 \\
1 & 1\end{array}$ \\
\hline $\begin{array}{l}.23201500 E+15 \\
.23202050 E+05 \\
.233 U 370 U E+05 \\
.2330560+05 \\
.233 \cup 5 d 0 E+U 5\end{array}$ & 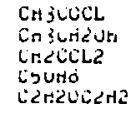 & $\begin{array}{l}111 \\
\text { I1I } \\
111 \\
\text { III } \\
\text { III }\end{array}$ & 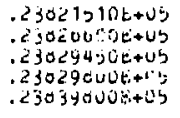 & 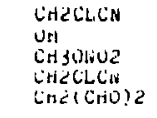 & 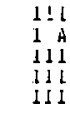 \\
\hline 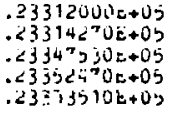 & 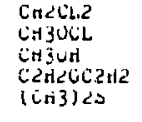 & $\begin{array}{l}111 \\
111 \\
11 \\
111 \\
\text { III }\end{array}$ & $\begin{array}{l}.2304 \alpha^{2} 340 t+05 \\
.030010004+02 \\
.23070+35 t+02 \\
.23054000 n+45 \\
.23917500 t+05\end{array}$ & 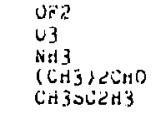 & $\begin{array}{l}\text { III } \\
\text { III } \\
\text { II } \\
\text { III } \\
\text { III }\end{array}$ \\
\hline 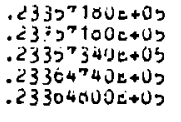 & 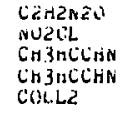 & 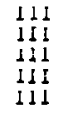 & $\begin{array}{l}.239230^{7} U t+05 \\
.2392040 u t+05 \\
.23932320 t+45 \\
.23936010 t+05 \\
.2394340 u t+135\end{array}$ & $\begin{array}{l}\text { HCUUCA3 } \\
\text { CH3UCL } \\
\text { (HICCH)ZO } \\
\text { HCOUCH3 } \\
\text { HCCCHZCHE }\end{array}$ & 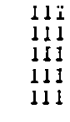 \\
\hline $\begin{array}{l}.233^{7} \cup 240 E+05 \\
.23304400 E+U 5 \\
.23307^{2} 20 E+05 \\
.23402530 E+05 \\
.23404000 E+115\end{array}$ & 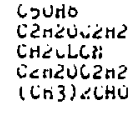 & $\begin{array}{l}111 \\
111 \\
111 \\
111 \\
111\end{array}$ & 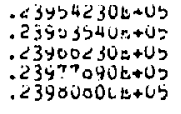 & $\begin{array}{l}\mathrm{CH} 3 \mathrm{CHCH} 2 \\
\mathrm{~S} 2 \mathrm{U} \\
\mathrm{CH} 2 \mathrm{CLCH} \\
\mathrm{CH} 3 \mathrm{CHCCH} 2 \\
\text { CHSSCLA3 }\end{array}$ & $\begin{array}{lll}1 & 1 & 1 \\
1 & 1 & 1 \\
1 & 1 & 1 \\
1 & 1 & 1 \\
0 & 1 & 1 \\
1 & 1\end{array}$ \\
\hline $\begin{array}{l}.23414240 c+U 5 \\
.23424300 E+U 5 \\
.23420420=+0 n \\
.23420 \% 00 E+0^{2}= \\
.23440000=+0.3\end{array}$ & 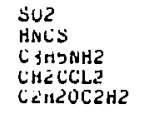 & $\begin{array}{l}\text { III } \\
\text { ind } \\
i 11 \\
111 \\
111\end{array}$ & $\begin{array}{l}.23394640 \mathrm{~d}+05 \\
.2349 \mathrm{~d} 410 \mathrm{0}+05 \\
.24002000 \mathrm{0}+05 \\
.24019000 \mathrm{0}+05 \\
.2401 .000 \mathrm{0}+03\end{array}$ & $\begin{array}{l}(\mathrm{CH}){ }^{\prime} \mathrm{CCH} \\
\mathrm{H}+2 \\
C H 2 \mathrm{CLCN} \\
\mathrm{CHCCLCN} \\
\mathrm{CH} 2 \mathrm{CLCN}\end{array}$ & $\begin{array}{l}1 \\
1\end{array}$ \\
\hline $\begin{array}{l}.23444620=+05 \\
.234464005+05 \\
.23493130 z+05 \\
.23430000 t+05 \\
.2340^{7} 650 t+05\end{array}$ & 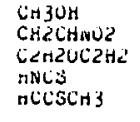 & $\begin{array}{l}11 \\
111 \\
111 \\
111 \\
111\end{array}$ & $\begin{array}{l}.24015 ? 00 t+05 \\
.24195045 E+05 \\
.24033040 E+05 \\
.24030004 E+05 \\
.240 .7940 E+05\end{array}$ & 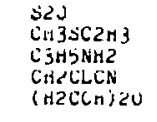 & $\begin{array}{lll}1 & 1 & 1 \\
1 & 1 & 1 \\
1 & 1 \\
1 & 1 & 1 \\
1 & 1 & 1 \\
11 & 1\end{array}$ \\
\hline $\begin{array}{l}.234 " 3700 E+U ? \\
.2347400 U E+0 b \\
.234 y 0040 E+105 \\
.23490 d 20 L+U b \\
.234 y 9200 E+U 3\end{array}$ & 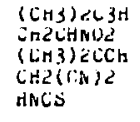 & $\begin{array}{l}\text { III } \\
\text { III } \\
111 \\
\text { III } \\
115\end{array}$ & 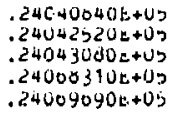 & 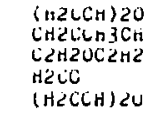 & $\begin{array}{l}111 \\
111 \\
111 \\
111 \\
111\end{array}$ \\
\hline $\begin{array}{l}.235024400+05 \\
.235077100+05 \\
.23524440 e+05 \\
.235274202+05 \\
.23530700 x+05\end{array}$ & 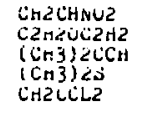 & 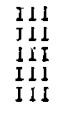 & $\begin{array}{l}.24070040 t+45 \\
.240034 .0 \mathrm{U}+05 \\
.24009570 \mathrm{U}+05 \\
.24090000 \mathrm{U}+05 \\
.24090100 \mathrm{U}+05\end{array}$ & $\begin{array}{l}\ln 2(C N) 20 \\
\text { SU2 } \\
\text { C } 3{ }^{4} 4 \\
\ln 2(C N) 2 \\
\text { CnCLCHCL }\end{array}$ & $\begin{array}{l}1 \\
1 \\
I\end{array}$ \\
\hline $\begin{array}{l}.23550040 t+05 \\
.23553800 t+05 \\
.23502300 \mathrm{t}+05 \\
.23010420 t+05 \\
.23619000 \mathrm{t}+05\end{array}$ & 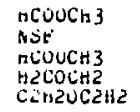 & $\begin{array}{l}111 \\
111 \\
111 \\
I 1 I \\
1\end{array}$ & $\begin{array}{l}.241031 u u t+05 \\
.24127720 t+05 \\
.2413940 \varepsilon+0 b \\
.24139420 E+05 \\
.24147000 i+05\end{array}$ & $\begin{array}{l}\text { CHEEHNUZ } \\
\text { CH2CHINC } \\
\text { HH } \\
\text { CHCLCHCL } \\
(H 2 C C H) 20\end{array}$ & $\begin{array}{lll}1 & 1 & 1 \\
1 & 1 & 1 \\
1 & 1 \\
1 & 1 & 1 \\
1 & 1 & 1\end{array}$ \\
\hline
\end{tabular}




\begin{tabular}{|c|c|c|c|c|c|}
\hline ML & MULELULE. & $\therefore \times L$ L.L. & Ac & Autrothe & はேニした \\
\hline 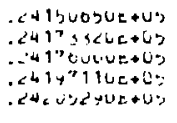 & 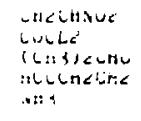 & $\begin{array}{l}1 i ! \\
i+1 \\
0 i \\
i 1 \\
j i\end{array}$ & 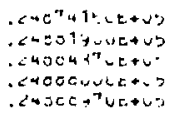 & 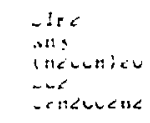 & 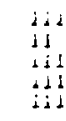 \\
\hline 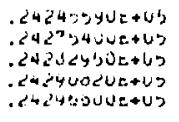 & 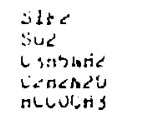 & 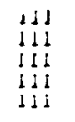 & 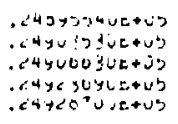 & 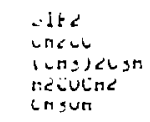 & $\begin{array}{lll}1 & 1 & 1 \\
1 & 1 & 1 \\
1 & 1 & 1 \\
1 & 1 & 1 \\
1 & 1\end{array}$ \\
\hline 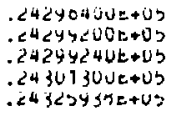 & 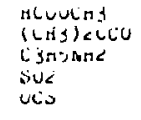 & $\begin{array}{lll}1 & 1 & 1 \\
1 & 1 & 1 \\
1 & 1 & 1 \\
1 & 1 & 1 \\
1 & h\end{array}$ & 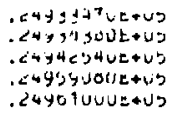 & 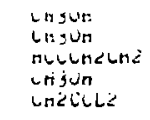 & $\begin{array}{ll}11 \\
11 \\
11 \\
11 \\
11\end{array}$ \\
\hline 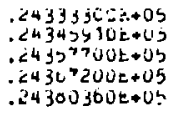 & 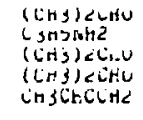 & $\begin{array}{l}111 \\
111 \\
111 \\
11 \\
115\end{array}$ & 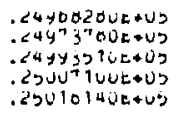 & 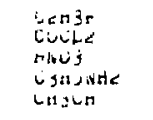 & $\begin{array}{lll}1 & 1 & 1 \\
1 & 1 & 1 \\
1 & 1 & 1 \\
1 & i & 1 \\
1 & 1\end{array}$ \\
\hline $\begin{array}{l}.24391110 x+05 \\
+24342000 e+15 \\
.24333200 e+05 \\
.24343350 E+07 \\
.4^{4} 3 y^{7} y^{7} 0=+05\end{array}$ & 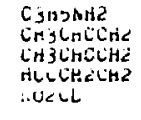 & 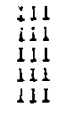 & 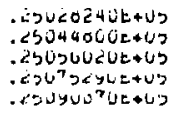 & 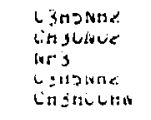 & 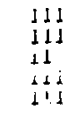 \\
\hline $\begin{array}{l}.24399770 E+05 \\
.24401100 E+05 \\
.24404000 t+05 \\
.24472230 E+05 \\
.24495040 E+65\end{array}$ & 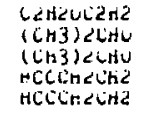 & 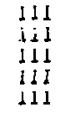 & 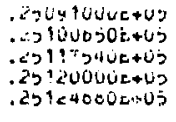 & 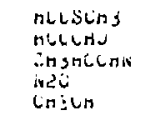 & $\begin{array}{l}111 \\
11 \\
1: 1 \\
1=1 \\
11\end{array}$ \\
\hline $\begin{array}{l}.24503720 t+0 b \\
.24532500 \mathrm{c}+05 \\
.24533340 z+05 \\
.24554460 t+05 \\
.24550410 \mathrm{0}+02\end{array}$ & 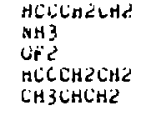 & 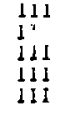 & 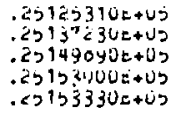 & 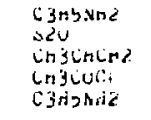 & 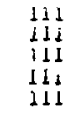 \\
\hline $\begin{array}{l}.24657850 \mathrm{t}+05 \\
.24506040 \mathrm{E}+05 \\
.24500900 \mathrm{U}+05 \\
.24570990 \mathrm{E}+05 \\
.24500070 \mathrm{t}+05\end{array}$ & $\begin{array}{l}\mathrm{CH} 3 \mathrm{CHCH} 2 \\
\mathrm{CH} 30 \mathrm{OCL} \\
\text { HCUUH } \\
\text { HCLCH2CHZ } \\
\text { HCLCH2LH2 }\end{array}$ & $\begin{array}{l}111 \\
111 \\
111 \\
111 \\
111\end{array}$ & 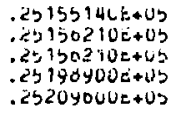 & 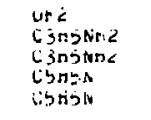 & $\begin{array}{lll}1 & 1 & 1 \\
1 & 1 & 1 \\
1 & 1 & 1 \\
1 & 1 & 1 \\
1 & 1 & 1\end{array}$ \\
\hline $\begin{array}{l}.24602000 z+05 \\
.24015900 z+05 \\
.24625000 t+05 \\
.24634000 t+05 \\
.24000000 t+05\end{array}$ & 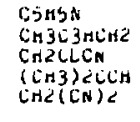 & 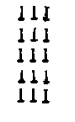 & 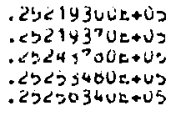 & $\begin{array}{l}\text { isune } \\
\text { bnjisiline } \\
\text { íhjr } \\
\text { nïLineone } \\
\text { icn3tecin }\end{array}$ & $\begin{array}{lll}1 & 1 & 1 \\
1 & 1 & 1 \\
1 & 1 & 1 \\
1 & 1 & 1 \\
1 & 1 & 1\end{array}$ \\
\hline $\begin{array}{l}.246003105+05 \\
.24661200 E+05 \\
.24663^{7} 00 E+05 \\
.24664240 E+05 \\
.246 ? 0000 t+05\end{array}$ & $\begin{array}{l}\text { HCA } \\
\text { HCC HACHE } \\
\text { CLA2N2O } \\
\text { SIF2 } \\
\text { HCCOHZCHE }\end{array}$ & 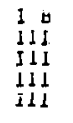 & $\begin{array}{l}.25207140 E+03 \\
.202013 j 0 z+05 \\
.20200 \$ 10 t+05 \\
.2546200 E+05 \\
.25294410 \varepsilon+05\end{array}$ & 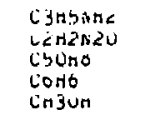 & 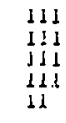 \\
\hline $\begin{array}{l}.24670^{766 E+05} \\
.24680100 \mathrm{0}+05 \\
.24602000 \mathrm{0}+05 \\
.24689000 \mathrm{0}+05 \\
.24693990 \mathrm{0}+05\end{array}$ & $\begin{array}{l}\text { MSE } \\
\text { an3 } \\
\text { H2LNHCH2 } \\
\text { CSUnd } \\
\text { C jubith2 }\end{array}$ & $\begin{array}{l}\text { II I } \\
\text { II } \\
\text { III } \\
\text { I1 } \\
\text { III }\end{array}$ & $\begin{array}{l}.25304070 t+02 \\
.25320370 t+05 \\
.25324050 t+0 y \\
.2535289 u k+05 \\
.25373 ? 70 e+0 b\end{array}$ & 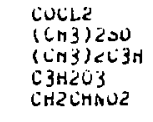 & $\begin{array}{lll}1 & 1 & 1 \\
1 & 1 & 1 \\
1 & 1 & 1 \\
1 & 1 & 1 \\
1 & 1 & 1\end{array}$ \\
\hline $\begin{array}{l}.24720930 t+05 \\
.24733960 \mathrm{E}+05 \\
.24739000 \mathrm{t}+05 \\
.24799300 \mathrm{t}+05 \\
.24760580 \mathrm{t}+05\end{array}$ & $\begin{array}{l}\mathrm{SHZ}^{2} \\
\mathrm{CH} 3)<\mathrm{CCO} \\
\text { CHECCL2 } \\
\text { C5UMd } \\
\text { CH2CHWOZ }\end{array}$ & $\begin{array}{lll}1 & 1 & 1 \\
1 & 1 & 1 \\
1 & 1 & 1 \\
1 & 1 & 1 \\
1 & 1 & 1\end{array}$ & $\begin{array}{l}.25392010 t+115 \\
.25390^{7} U U E+U j \\
.25414010 t+U 5 \\
.25420940 E+U 5 \\
.25445360 E+05\end{array}$ & 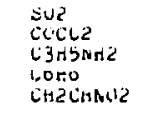 & $\begin{array}{lll}1 & 1 & 1 \\
2 & 1 & 1 \\
1 & 1 & 1 \\
1 & 1 & 1 \\
1 & 1 & 1\end{array}$ \\
\hline $\begin{array}{l}.24763030 E+05 \\
.24707500 E+05 \\
.24700350 t+0 ' \\
.24704550 E+05 \\
.24797320 E+05\end{array}$ & 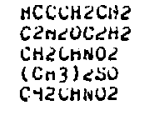 & $\begin{array}{lll}1 & 1 & 1 \\
0 & 1 & 1 \\
1 & 1 \\
1 & 1 & 1 \\
1 & 1 & 1 \\
1 & 1 & 1\end{array}$ & 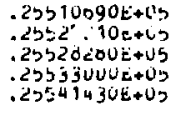 & 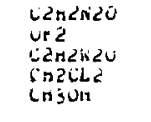 & $\begin{array}{lll}1 & 1 & 1 \\
1 & 1 & 1 \\
1 & 1 & 1 \\
1 & 1 & 1 \\
1 & 1\end{array}$ \\
\hline $\begin{array}{l}.24830000 E+05 \\
.240343005+05 \\
.2465 C 120 t+05 \\
.24050800 t+05 \\
.24051000 t+05\end{array}$ & $\begin{array}{l}\text { HCOUH } \\
\text { A2COCHE } \\
\text { c2n2k20 } \\
\text { (H2CCH)2U } \\
\text { nCCOALCH2 }\end{array}$ & $\begin{array}{l}111 \\
111 \\
111 \\
111 \\
111\end{array}$ & 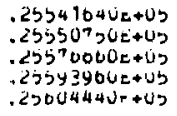 & 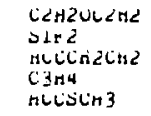 & $\begin{array}{lll}1 & 1 & 1 \\
1 & 1 & 1 \\
1 & 1 & 1 \\
1 & 1 \\
1 & 1 & 1 \\
1 & 1 & 1\end{array}$ \\
\hline
\end{tabular}




\begin{tabular}{|c|c|c|c|c|c|}
\hline $\begin{array}{l}\text { III } \\
\text { III } \\
\text { III } \\
\text { III } \\
\text { III }\end{array}$ & 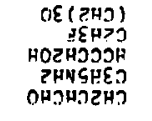 & 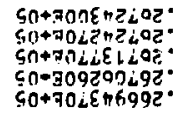 & $\begin{array}{l}\operatorname{liT} \\
\text { IIT } \\
\text { ITT } \\
\text { IIT } \\
\text { TIT }\end{array}$ & 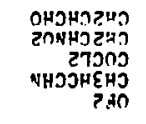 & 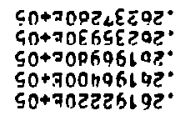 \\
\hline $\begin{array}{l}\text { III } \\
\text { III } \\
\text { III } \\
\text { III } \\
\text { III }\end{array}$ & 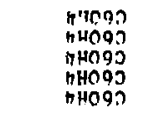 & 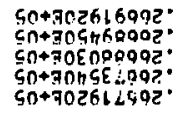 & $\begin{array}{l}\operatorname{IIT} \\
\text { III } \\
\text { IIT } \\
\text { III } \\
\text { III }\end{array}$ & 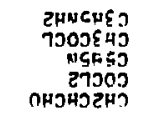 & 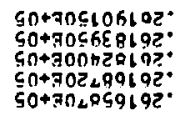 \\
\hline $\begin{array}{l}\text { IIT } \\
\text { III } \\
\text { III } \\
\text { III } \\
\text { III }\end{array}$ & 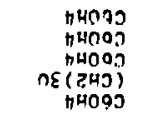 & 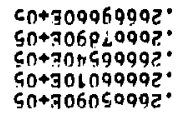 & $\begin{array}{l}\text { II I } \\
\text { III } \\
\text { III } \\
\text { III } \\
\text { II }\end{array}$ & 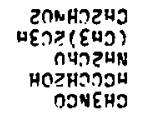 & 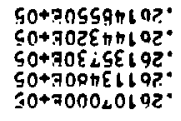 \\
\hline 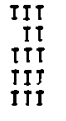 & $\begin{array}{r}\text { HONZHS } \\
\text { EHN } \\
\text { WFOP(EHO) } \\
\text { EHOSOSH } \\
\text { NGHCJ }\end{array}$ & 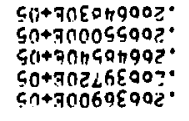 & $\begin{array}{l}\text { II I } \\
\text { III } \\
\text { III } \\
\text { I I I } \\
\text { I I I }\end{array}$ & 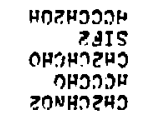 & 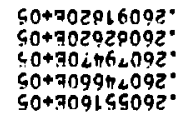 \\
\hline $\begin{array}{ll}\operatorname{ltg} \\
\operatorname{lit} \\
\operatorname{lit} \\
\operatorname{lit} \\
\operatorname{lit} \pi\end{array}$ & $\begin{array}{l}\text { NGHSO } \\
\text { NGHGS } \\
\text { NGTGS } \\
\text { NGHCD } \\
\text { OE(टHD) }\end{array}$ & 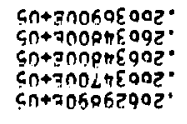 & $\begin{array}{l}\text { ITI } \\
\text { III } \\
\text { III } \\
\text { III } \\
\text { III }\end{array}$ & 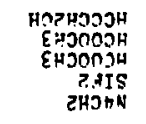 & 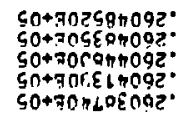 \\
\hline $\begin{array}{l}\text { tIt } \\
\text { TIt } \\
\text { TIt } \\
\text { tit } \\
\text { tit }\end{array}$ & 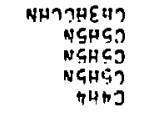 & 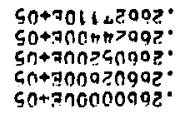 & $\begin{array}{l}\text { IT } \\
\text { IT } \\
\text { IIT } \\
\text { IIT } \\
\text { II }\end{array}$ & 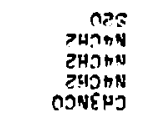 & 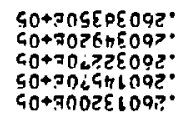 \\
\hline 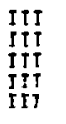 & $\begin{array}{l}\text { NNRWD } \\
\text { Z(OS) } \\
\text { NGHSD } \\
\text { NGHSD } \\
\text { NSHSD }\end{array}$ & 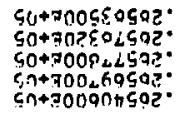 & $\begin{array}{l}\text { III } \\
\text { III } \\
\text { III } \\
\text { III } \\
\text { III }\end{array}$ & 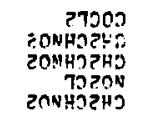 & 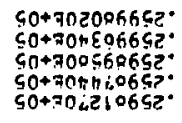 \\
\hline 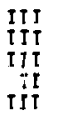 & 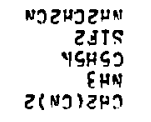 & 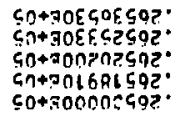 & $\begin{array}{l}\text { III } \\
\text { III } \\
\text { III } \\
\text { III } \\
\text { IIT }\end{array}$ & 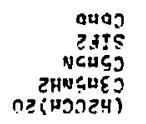 & 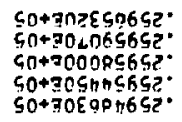 \\
\hline 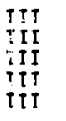 & 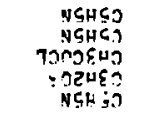 & 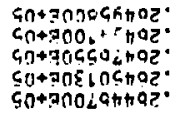 & $\begin{array}{l}\text { III } \\
\text { III } \\
\text { II I } \\
\text { II I } \\
\text { II }\end{array}$ & 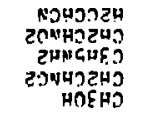 & 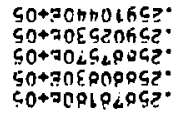 \\
\hline 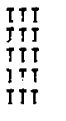 & 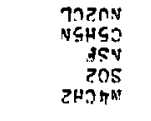 & 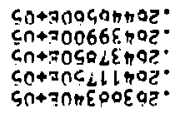 & $\begin{array}{l}\text { ITT } \\
\text { ITI } \\
\text { III } \\
\text { ITI } \\
\text { III }\end{array}$ & 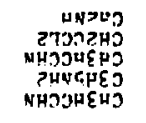 & 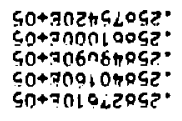 \\
\hline 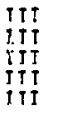 & 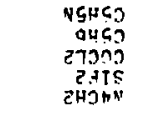 & 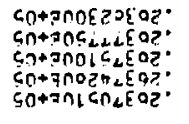 & 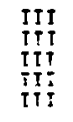 & 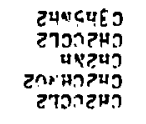 & 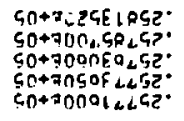 \\
\hline 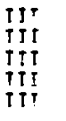 & 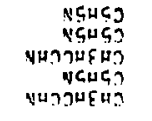 & 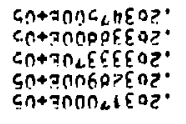 & $\begin{array}{l}\text { IT } \\
\text { IIT } \\
\text { IIT! } \\
\text { ITI }\end{array}$ & 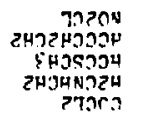 & 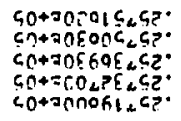 \\
\hline 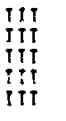 & 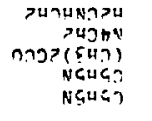 & 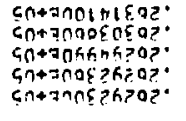 & 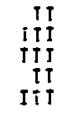 & 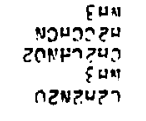 & 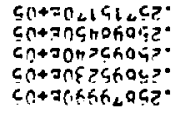 \\
\hline 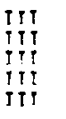 & 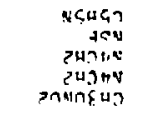 & 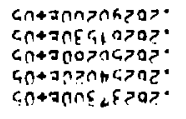 & $\begin{array}{l}\text { IIT } \\
\text { IT } \\
\text { IIT } \\
\text { TIT } \\
\text { TII }\end{array}$ & 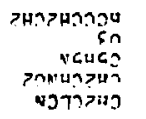 & 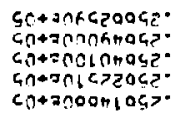 \\
\hline פ רךา & 37?ח. & וג & วาवस & 2?กวมากd & $\eta n$ \\
\hline
\end{tabular}

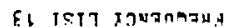


lot

$-\angle 0^{7} \leq 005 U \mathrm{ctus}$

$.20^{7} \mathrm{j}^{7} \cup\left(\mathrm{VE}+\mathrm{UJ}^{2}\right.$

. $0^{7} 2 y<0 u x+0$ J

$20^{3} 50450 \mathrm{D}+05$

$.20704200 \mathrm{C}+0 \mathrm{~b}$

.20? as 100e+os

$.26702430 i c+05$

$.26773030 \mathrm{~L}+05$

$.20772^{7} v 0 \mathrm{~L}+05$

- $6777120 \ddot{\mathrm{E}}+0$;

$2 b^{7} 03000 E+C 5$

$.20^{7} 80^{7} j u t, 05$

. $26 ? 92990 t+05$

$.20790060 \mathrm{e}+05$

$.260010000+05$

$.2600343 \mathrm{je+05}$

$20024000 t+05$

$.20029200 k+05$

.26030 1000t-05

$20050300 i+0$

$26052000 \mathrm{x}+\mathrm{C}$

$20063760 \mathrm{E}+0$

200726y0E+05

$.20922340 t+0$

. 20Y24050E-05

.2692b300e+Ds

$20934090 \mathrm{E}+\mathrm{C}$

$26935250 v+05$

$26937000 \mathrm{E}+\mathrm{C}$

$269026 ? 0 \mathrm{O}+0$

20973200E+O

$.26999140 \mathrm{x}+05$

$.27033000 \overline{0}+05$

.27102470E + 65

$.27141390 \mathrm{~b}+0$

$.2 ? 1914 E 0 E+05$

. 27212n10غ+05

2?215860E+95

$27224870 E+0$

.2722 bu $30 b+05$
$.2723 ? 090 \mathrm{~s}+05$

. $240300 \mathrm{~T}+05$

$.272044100+05$

$.272^{7} 00908+05$

? $200600 \mathrm{E}+05$

$.27303140 \mathrm{E}+05$

- 27 $324020 \mathrm{E}+05$

27332430405

. 7375005

.2? 301960k+05

.27 364020E+05

$273090^{\circ} 0 t+0$

$.27305450 \mathrm{k}+05$

.27300150e+05



.2?402790t+0

.2? $41 ? 220 t+05$

$.27417220 E+05$

.27432090e+05

$274756604 \div 05$

$.274 ? 6100$ in 05

$.27470000 \mathrm{c}+05$

$.27404680 E+05$
$.274074005 \div 05$ multcult

I ADLE

nU

(cie) su 111

ijholuti 111

icicine

MSF

CUCLL

inzlycins

HCLCHLOA

C5boti

in $3 \mathrm{sC} 2 \mathrm{ing}$

ConiN

C 3 Houn2

(Cri3) $<\mathrm{CCO}$

HCCCHzUn

CHÉLCH

CCcirizun

accenzOH

(Cd3) atco

Soz

nCCCHzOH

$\left(\mathrm{C}_{3}\right) 2 \mathrm{C} 3 \mathrm{H}$

C3HSAH2

( $\left.\mathrm{CH}_{3}\right) \mathrm{eCO}$

NH2Ctiv

CSnSk

COCHA

Court 4

$N O \vec{F}$

Cout4

CH2H4S

C3HSt+12

CSTiBn

NSF

HU2Cl

NH2CH2CN

C4n4

Ca3HCCHN

ChinCerin

C4H 4

C4 44

CH2LHCH2S

Carlichis

CHECHCH2S III

Chalschás

$\mathrm{HC} 3 \mathrm{~N}$

(CH2) 30

ChzChCHO

( Сн2 ) 30

(CH2) 30

CoOs 4

C6OH4

HAO3

CH2CHCHO

SU2

$\mathrm{CbOH} 4$

C60H4

CoOH 4

HCcChion

Chachintes

COCL?

Nis

CH2 Lh:H2S

ACCCHO
11
11
11
11
II

III

III

III

111

III

III

II

III

III

III

III

III

III

III

III

III

III

III

III

sit

II

II

III

III

III

II:

III

I

$11 \frac{1}{2}$

III

III

IfI

III

III

1.

III

III

III

III

II

II
$.27409500 \mathrm{E}+0$ S

$.27409500 \mathrm{c}+\mathrm{U}$

- 27 4y $2590 \mathrm{~L}+\mathrm{US}$

. 27452590i

79

. 27523 Juetus

. 27 OCSLDOE+US

$.27511900 t+0_{3}$

.27, $13030 \mathrm{r}+\mathrm{US}^{2}$

$.27516 ? j 6 \varepsilon+U s$

.27510750t+05

.27519000e+05

$.27519 y 70 \mathrm{C}+05$

. $275199 ? 0 \mathrm{E}+05$

$.27327470 E+05$

-2753404CE+DS

- $27534950 E+05$

$.27543550 t+05$

.27504160E+05

.27545 bUUE+05

.27599? JWE +05

$.27019650 \mathrm{C}+05$

$.27009+20 E+05$

- 27695030E+05

. 777 1C440 40

. 777 Ca

. $774300+05$

27? $19140 \&+05$

$.27 ? 191406+09$
$.277 \times 3000 x+05$

$.27 ? 296305+05$

- 27?32010E+0

.2?7347 30E $\rightarrow 05$

$.27751570 t+05$

. 27754040E+05

$.27750480 E+00$

$.277040005+05$
$.2776 ? 320 E+05$

$.27769230 E+05$

-27?72520 405

.27? O3900E+C

. $27705030 t+C 5$

$.2778 ? 400 E+05$
$.27032450 E+05$

$.2704097 \mathrm{VE}+05$

i? $045354 e+05$

2? 06 ?000écus

.270600 TUE'+ 15

-27002300k+05

2? $004510 \mathrm{H}+0$

$.27057300 E+05$
$.27591900 E+05$

.27 \$93060t +05

$.27910430 \mathrm{E}+\mathrm{US}$

$.27920000 \mathrm{E}+0$

$.27921930 E+0$

$.279366 ? 05+05$

.27972130E +05

.2797700DE+05

.2790udbosios

. $27900000+05$

$20010000 \mathrm{E}+05$

$26014500 \mathrm{E}+05$

. $20014500 \mathrm{E}+05$

$.28027 .330 E+05$
$.20029300 t+05$

$.20029300 E+05$
$.20030700 E+05$

$.20030490 \mathrm{k}+05$

MULLCULE TAELE

CH2CHCH2S

CH2CHinds II

Hi2CHCH2S II

HCCCAzOr

Uti 111

Ch5chChISA II

Cischl I11

$\begin{array}{ll}\mathrm{CH} 2 \mathrm{CHCH} & \mathrm{I}+1 \\ \mathrm{~N} 2 \mathrm{CnO} & \mathrm{I} i \mathrm{i}\end{array}$

CHi2hCHOS II

CHeChCH2S 111

त2CHCH2S III

CnzCaches did

CHaCnCHas 11

त2CHCHaS Ill

III

$\begin{array}{ll}\text { (Ch2) } 30 & \text { III } \\ \text { CoOr } 4 & \text { III }\end{array}$

Cóch 411

(Cn3) $2 \mathrm{cco}$ III

C 3 सi

CoOn4

C2H4S

C3H5NH2

CANAZHA

NC4HH2 HA

Cónlu

C60r4

$\mathrm{CH} 2 \mathrm{CHCH} \mathrm{SS}$

C5OH6

c3n5nita

( $\mathrm{SO}) 2$

CoOH4

C5OHO

CH2ChCH2S

hCOO'i

COCLÉ

$\mathrm{H} 2 \mathrm{CCHCW}$

( $\mathrm{CH} 2) 30$

INA3

C3HSHHa

$\begin{array}{ll}\text { ( } \\ \text { CHE } 30 & \text { III } \\ \text { CHECHCHIS } & \text { III }\end{array}$

(CH3) 25 11L

(CH3) IS II

esutro

520

(50) 2 
NU

$.20050340 E+05$ $.28004630 \mathrm{E}+05$ $.200691=0 E+05$

$.20075410 k+05$

$.20001640 j+05$

$.28003000 E+05$ $.280003600+05$ $.28105300 E+05$ $281110900+25$ $.26120100 E+05$

$.28121480 \mathrm{0}+05$ $.281251906+05$ $.26130300 E+05$ $.26141360 t+05$ $.20145490 t+05$

$.28146350 z+05$ $.26151300 t+05$ $.26173030 E+05$ .2ة 17 $3400 \mathrm{E}+05$ $.28187040 E+05$

$.28191490 \mathrm{E}+05$ $26201000 \mathrm{E}+05$ $28220480 E+05$ $23239140 \mathrm{t}+05$ $.282 ? 9100 \mathrm{E}+05$

$.28<817605+05$ . 2d2yida 90 Ex05 $.20296000+05$ $.283090^{\prime} O E+05$ $.2631280 \mathrm{c}=05$

$.20316030 \mathrm{E}+05$ $.28316640 E+05$ $.28316640 E+05$ $28330980 E+05$ $.28333750 E+05$

$.28335510 E+05$ $.28336710 E+05$ $.28342020 E+05$ $28342760 \mathrm{E}+05$ $.28344540 \mathrm{~b}+05$

$.28358220 E+05$ $.28359400 E+05$ $.28382 \pi 1 \mathrm{iE}+05$ $28412350 \mathrm{E}+0$ $.26420700 E+05$

.74420700k+05 $28440990 \mathrm{E}+05$ $2845 ? 340 \mathrm{E}+0$ $284577005+0$ $28461330 \mathrm{E}+05$

28467150E 05 $.38470600 \mathrm{C}+05$ $.26462050 \mathrm{c}+0$ $244076505+05$ $.28407650 \mathrm{E}+05$

$28557280 e+05$ $.28557280 e+05$ .205033402405 $.285912700+05$ $.23604 ? 30 E+05$

$.286366005+05$ $28673990 \mathrm{0}+05$ $.28685020 \mathrm{E}+05$ $28699300 \mathrm{E}+05$ $.28 ? 04970 E+05$

$28708600 E+05$ $28722350 \mathrm{E}+0$ $.28722350 \mathrm{E}+05$ .28723950E+05 $.28732500 E+05$
$.2873 d d 4 O E+05$
MOLECULE TAOLE

HacincH2

( $\mathrm{CH} 3) 250$

$(\mathrm{CH} 3)<50$

SIF2

CL2O

$\mathrm{HCOOH}$

Cr $3 \mathrm{SC}_{2} \mathrm{H} 3$

C3 thut?

ChzCLCA

(CH3) $2 \mathrm{CCn}$

(CH2) 30

$\mathrm{SO2}$

$S I 52$

C6OH 4

C6OH4

CHêt

SO2

C3нbnh2

CoOll:

(CH3) $2 \mathrm{CC}$

( $\mathrm{CH} 3$ ) $2 \mathrm{CCH}$

(CHa) 30

$\mathrm{CH} 3 \mathrm{COCL}$

( $\mathrm{CH} 3$ ) acco

H5CCCNH2

C2H3E

COUH4

CH3OH

Cound

(CH2) 30

$\mathrm{CoOH} 4$

NH2CHO

C6OH4

(CH2) 30

( $\mathrm{CH}$ ) $2 \mathrm{C}_{3 \mathrm{i}}$
$\mathrm{C} 2 \mathrm{~d} 3 \mathrm{~F}$

C6OH 4

CH3SCN

C 3 H 4

C 2 HF

(SO) 2

ChzLHCH2S

$\mathrm{H} 2 \mathrm{CCHCN}$

$\mathrm{H} 2 \mathrm{CCHCN}$

IH3OCH3

SIH 3 UCH 3

HCCCHO

HZCCHCM

SIL $30 \mathrm{CH} 3$

SIh $30 \mathrm{CH}$
(s0) 2

( $\mathrm{Ca} 3)_{2 \mathrm{CH}}$

SO) 2

$\mathrm{HH} 2 \mathrm{CHO}$

sili3

$\mathrm{CH} 2(\mathrm{CH}) 2$

( $\mathrm{CH} 3$ ) $2 \mathrm{CCO}$

520

C2A3F

S20

(C)

HCISCH 3

SĹJ

CHE (CN)

(CH3)
NU

$.20 \% 1600 E+05$

$.20^{77} \mathrm{jo}^{77} 0 \mathrm{e}+05$

- 209 ? $30 \varepsilon+0$

$.20615000 \mathrm{E}+\mathrm{U}$

$.20010030 v^{2}+05$

$.20530010 i+05$ $.20032910 t+05$

$20021310 \varepsilon+05$

2007 $740 t+0$

.

.20050dantitus

$.2000=0000+0$ S

$20606410 t+0$ ?

$.200040000+U 5$

$.20 .9102600+05$

$.26930000 E+05$ $.26902220 \mathrm{e}+05$

.20909 Y00E $\rightarrow 05$

3074000E+05

$.26942940 \mathrm{a}+0$

.29013320E-Uj $.29030400 t+05$ $.29039750 E+05$ $.2903970 \mathrm{c}+05$ $.29001140 E+U 5$

$.29001640 \mathrm{k}+\mathrm{UJ}$ $.29009070 \mathrm{E}+\mathrm{US}$ $.29091600 \mathrm{E}+0 \mathrm{~S}$ . $29130040 E+U 5$ .29136970 e+05

$.291 ? 3320 E+05$ $.29193400 E+05$ $.292190<0 \mathrm{E}+05$ $.29224600 E+05$ $.29220430 \mathrm{E}+05$

$.292272408+05$ $.29220000 \mathrm{e}+05$ $.29252490 \mathrm{E}+05$ .29252500E+05 $.29252760 b^{\prime}+05$

. 29260920k +05 $.29290490 \mathrm{E}+05$ $.29293210 \mathrm{E}+05$ $203195506+05$ $.29321300 E+05$

$.29326120 E+05$ $.29339290 \mathrm{E}+05$ . $29340120 t+05$ $.29356460 \mathrm{E}+\mathrm{O}$ $.29300900 t+05$

$.29309120 \mathrm{E}+05$ . $29379690 t+03$ $.29401500 \mathrm{t}+05$ $294043905+05$ $.29404390 E+05$

2942194 Uё+05 .2942174 Jet0s $.29420050 E+05$ $.29420050 t+05$ $.29430020 t+05$
$.29430050 E+05$

.29 $444320 \mathrm{E}+0$ $.29446920 \mathrm{t}+05$ $.29453230 E+05$ $.29465310 \mathrm{c}+05$ $.29496720 \mathrm{E} \rightarrow 05$

$29522000 \mathrm{E}+0$ . $29533050 E+05$ 2955 300 $.29567000 E+05$
$.29575960 \mathrm{E}+05$

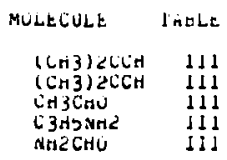

(CH3) $2 \mathrm{ClH}$

SinjuCs 3 111

(Ln3)LCLA 111

Sue 111

S1H30Ch 3 II

Cinowa2 II

Callint

$\begin{array}{ll}\text { Cono } & \text { I1 } \\ \text { C3njnat } & 111\end{array}$

$\mathrm{CH} 2 \mathrm{CL} 2$

CHENH

HeCu

C3H5NH2 Ill

Nis?

( $\mathrm{sO}) 2$

$\mathrm{it2}(\mathrm{CN}) 2$ III

( $\mathrm{CH} 3) 2 S$ III

(CH3) $2 \mathrm{C} 3 \mathrm{H}$

(CH3) $2 \mathrm{CCH}$ II

C5OHO III

rechics II

anu 3

CHRCL2

Ctund

CH2CLCN $\quad 111$

C3H5MH2

(CH2) 30 1II

CoOH4 III

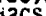

SIF

IrOF

C3HSNHZ

C3H5NH2

Côn 4

C6OH 4

C3H5NA2

$\mathrm{CbOH} 4$

$\mathrm{HCOOCH}$

SIH3OCH

$\mathrm{HCOOCH} 3$

CH3C3nCn2

SOHo

Csund

CH2CO

SIH $30 \mathrm{CH} 3$

C60n4

$\mathrm{COOH} 4$

(CH3) $2 S$

Cót 4

$\begin{array}{ll}\text { CoOH4 } & \text { III } \\ \text { (CH3) } 2 \text { CCO III }\end{array}$

520

UF 2

(CHiz) 30

(CH2) 30
III

III

III

III

III

III

$1 \mathrm{I}$

1 II

III

111

II I

11

III

III 


\begin{tabular}{|c|c|c|c|c|c|}
\hline int & MULEG GLE & CADLr. & nu & MuLELULe & IADLE \\
\hline 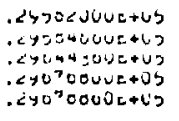 & 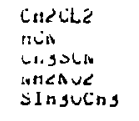 & 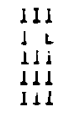 & 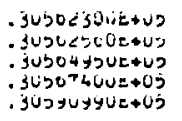 & 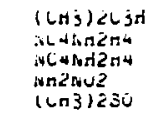 & $\begin{array}{lll}1 & 1 & 1 \\
1 & 1 & I \\
1 & 1 & I \\
1 & 1 & 1 \\
1 & 1 & 1\end{array}$ \\
\hline 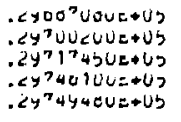 & 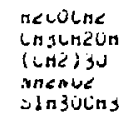 & $\begin{array}{l}\text { ill } \\
\text { III } \\
\text { III } \\
\text { III } \\
\text { III }\end{array}$ & 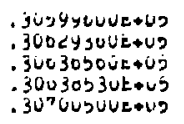 & $\begin{array}{l}\text { Nn2mu2 } \\
\text { (su)C } \\
\text { Csond } \\
\text { nuf } \\
\text { su2 }\end{array}$ & $\begin{array}{l}\text { III } \\
\text { III } \\
\text { III } \\
\text { dI } \\
\text { dil }\end{array}$ \\
\hline 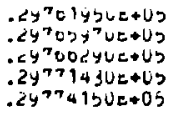 & 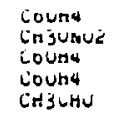 & $\begin{array}{l}\text { III } \\
\text { III } \\
\text { III } \\
\text { III } \\
\text { III }\end{array}$ & 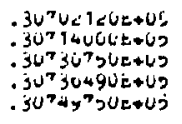 & 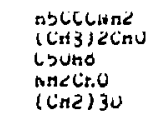 & 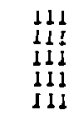 \\
\hline 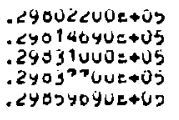 & 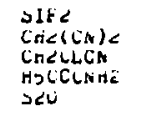 & $\begin{array}{l}\text { III } \\
\text { III } \\
\text { III } \\
\text { III } \\
\text { III }\end{array}$ & 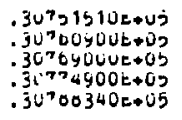 & $\begin{array}{l}\text { CoUno } \\
\text { nnzLinzCh } \\
\text { CniLL2 } \\
\text { t:COU: } \\
\text { (SU)Z }\end{array}$ & 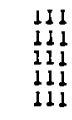 \\
\hline 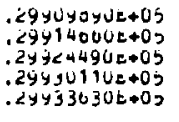 & $\begin{array}{l}(\operatorname{lin} 3)<C C n \\
\ln 3 \\
\text { Count } \\
\text { count } \\
\text { Cound }\end{array}$ & $\begin{array}{l}111 \\
11 \\
111 \\
111 \\
111\end{array}$ & $\begin{array}{l}.30+09300 E+05 \\
.3000 ? 110 E+05 \\
.30011400 E+05 \\
.30011000 E+07 \\
.30023170 E+05\end{array}$ & 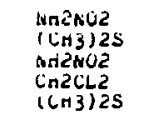 & $\begin{array}{l}\text { IIII } \\
111 \\
\text { III } \\
\text { III } \\
\text { III }\end{array}$ \\
\hline 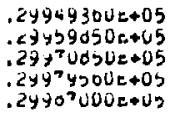 & $\begin{array}{l}\text { So } \\
\text { Cn3OCL } \\
\text { SIH3UCnis } \\
\text { LH3LH2Un } \\
\text { CL2O }\end{array}$ & $\begin{array}{l}11 C \\
111 \\
111 \\
1 d I \\
11 I\end{array}$ & $\begin{array}{l}.300325200+05 \\
.30001090 t+05 \\
.300000^{7} 0 E+05 \\
.300000^{7} 0 E+05 \\
.300912^{7} 0 E+05\end{array}$ & $\begin{array}{l}\text { nccsons } \\
\text { nous } \\
\text { Cound } \\
\text { Coun } 4 \\
\text { coOnd }\end{array}$ & $\begin{array}{l}\text { III } \\
\text { III } \\
\text { III } \\
\text { III } \\
\text { III }\end{array}$ \\
\hline 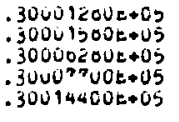 & 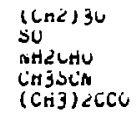 & $\begin{array}{l}11 I \\
11 C \\
11 I \\
\text { III } \\
\text { III }\end{array}$ & $\begin{array}{l}\text { 300y1430t+05 } \\
.300 \text { y2 } 130 E+05 \\
.30 y 0 y 00 t+05 \\
.3090 y \text { y } 50 E+05 \\
.30 y 3400 E+05\end{array}$ & $\begin{array}{l}\operatorname{logn} 4 \\
\text { ASE } \\
\text { CoOn4 } \\
\text { CoOn4 } \\
(\mathrm{Ct} 3)<\mathrm{Cri}\end{array}$ & $\begin{array}{l}\text { III } \\
\text { III } \\
\text { III } \\
\text { III } \\
\text { III }\end{array}$ \\
\hline 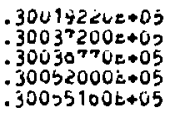 & 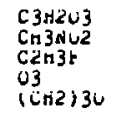 & $\begin{array}{l}111 \\
11 \\
111 \\
\text { III } \\
111\end{array}$ & $\begin{array}{l}30962 L^{7} \text { UE+U5 } \\
.30902100 E+05 \\
.310040 U 0 E+05 \\
.31047200+05 \\
.31040000 t+05\end{array}$ & $\begin{array}{l}(\mathrm{CH} 3) 2 \mathrm{C} 3 \mathrm{H} \\
52 \mathrm{O} \\
(\mathrm{CH}) 2 \mathrm{CHO} \\
\mathrm{HCCSH3} \\
(\mathrm{CH} 3)_{2} \mathrm{CHO}\end{array}$ & $\begin{array}{l}111 \\
\text { III } \\
111 \\
\text { III } \\
\text { IIJ }\end{array}$ \\
\hline $\begin{array}{l}30000350 E+05 \\
.30105000 E+05 \\
.30117020 E+05 \\
.301220 y 0 E+05 \\
.30127100=+05\end{array}$ & $\begin{array}{l}(\mathrm{SO})_{2} \\
\mathrm{Cn} 2 \mathrm{CL} 2 \\
\mathrm{CH} 3 \dot{\mathrm{C} N A} \\
\mathrm{Cn} 3 \mathrm{SCN} \\
\mathrm{CH} Z(\mathrm{CN}) 2\end{array}$ & $\begin{array}{l}111 \\
\text { III } \\
\text { III } \\
\text { III } \\
\text { iJI }\end{array}$ & $\begin{array}{l}.31003300 E+05 \\
.31007000 E+05 \\
.31077700 t+U 5 \\
.3100 y 500 t+05 \\
.31095940 E+05\end{array}$ & $\begin{array}{l}\mathrm{CH} 2(\mathrm{CH}) 2 \\
\text { NH2NU2 } \\
\text { NA2NU2 } \\
\text { C3HSNHE } \\
\text { C } 3 \mathrm{H}_{4}\end{array}$ & $\begin{array}{l}I I I \\
I I I \\
1 I I \\
I I I \\
I I I\end{array}$ \\
\hline $\begin{array}{l}30143400 E+05 \\
.30155050 E+05 \\
.30150540 E+05 \\
.3017901 U E+05 \\
.30101000 E+05\end{array}$ & 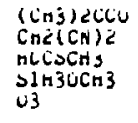 & $\begin{array}{l}\text { III } \\
\text { III } \\
\text { III } \\
\text { III } \\
\text { III }\end{array}$ & $\begin{array}{l}.31009410 \mathrm{C}+05 \\
.31102030 E+117 \\
.31104510 \mathrm{O}+05 \\
.31100200 \mathrm{0}+05 \\
.31111130 \mathrm{0}+05\end{array}$ & $\begin{array}{l}(\mathrm{Su}) 2 \\
(\mathrm{CH}) 30 \\
(\mathrm{CHE}) 30 \\
(\mathrm{CH}) 20 \\
(\mathrm{CHE}) 30\end{array}$ & $\begin{array}{l}\text { III } \\
\text { III } \\
\text { III } \\
\text { III } \\
\text { IIII }\end{array}$ \\
\hline $\begin{array}{l}.30205520 E+05 \\
.302 \cup 0340 E+05 \\
.30235070 t+05 \\
.30<40500 \mathrm{0}+05 \\
.302^{7} \text { UU2UE+05 }\end{array}$ & $\begin{array}{l}\text { SU2 } \\
\text { SIn3UCH3 } \\
\text { CH2 (CN) } \\
\text { nH2Nu2 } \\
\text { nOCCNn2 }\end{array}$ & $\begin{array}{l}111 \\
\text { III } \\
\text { III } \\
\text { IIII } \\
\text { III }\end{array}$ & $\begin{array}{l}31120170 \mathrm{U}+05 \\
.3113^{77} 20 \mathrm{t}+05 \\
.31140000 \mathrm{0}+0 \mathrm{U} \\
.3114 y 420 \mathrm{0}+0 ? \\
.31109150 \mathrm{0}+05\end{array}$ & $\begin{array}{l}\text { SC4AH2H4 } \\
\text { (SOS2 } \\
\text { (CH3) } 2 \text { CHO } \\
\text { CH3ONU2 } \\
\text { SIH3OSH3 }\end{array}$ & $\begin{array}{l}111 \\
11 I \\
11 I \\
1+I \\
11 I\end{array}$ \\
\hline $\begin{array}{l}302^{7} 0000 t+05 \\
.30310100 t+05 \\
.30361100 t+05 \\
.30304600 t+05 \\
.30394200 t+05\end{array}$ & $\begin{array}{l}(\mathrm{CH} 3) \angle C H U \\
(\mathrm{SU}) 2 \\
\text { Nn2NU2 } \\
\text { NH2hUZ } \\
C, 2 n 3 E\end{array}$ & $\begin{array}{l}\text { III } \\
\text { IIII } \\
\text { III } \\
\text { III } \\
\text { III }\end{array}$ & $\begin{array}{r}31170100 E+05 \\
.31102250 E+05 \\
.31180000 E+05 \\
.31190000 E+05 \\
.31195900 E+05\end{array}$ & $\begin{array}{l}\text { SIH3OLH3 } \\
\text { HCCSCH3 } \\
\text { HOZCL } \\
(\text { IH3) } 2 \mathrm{CHO} \\
\text { SIH3OCn3 }\end{array}$ & $\begin{array}{l}\text { III } \\
\text { III } \\
\text { III } \\
\text { III } \\
\text { III }\end{array}$ \\
\hline $\begin{array}{l}.30400190 \mathrm{t}+05 \\
.30400940 \mathrm{E}+05 \\
.30409100 \mathrm{t}+05 \\
.3043150 \mathrm{E}+05 \\
.30467200 \mathrm{t}+05\end{array}$ & $\begin{array}{l}(\mathrm{CH}) 2 \mathrm{LS} \\
\mathrm{CH} 3 \mathrm{SCN} \\
\mathrm{C} 3 \mathrm{H} 2 \mathrm{U} 3 \\
\text { SIH3OCH3 } \\
\mathrm{MCCSCH} 3\end{array}$ & $\begin{array}{l}\text { III } \\
\text { III } \\
\text { III } \\
\text { III } \\
\text { III }\end{array}$ & $\begin{array}{l}.31205270 E+05 \\
.31230000 E+05 \\
.31261100 E+05 \\
.31201100 E+05 \\
.31203340 \mathrm{E}+05\end{array}$ & $\begin{array}{l}\text { HLCSCH3 } \\
\text { (CH3) 2CHO } \\
\text { Mr2NO2 } \\
\text { nH2NCL } \\
\text { (CH2) } 30\end{array}$ & $\begin{array}{l}\text { III } \\
\text { III } \\
\text { III } \\
\text { III } \\
\text { III }\end{array}$ \\
\hline $\begin{array}{l}.30494620 L+05 \\
.30521650 E+05 \\
.30523100 \pm+05 \\
.30525000 E+05 \\
.3054204 U E+05\end{array}$ & $\begin{array}{l}\text { n2 CNnCH2 } \\
\text { nCOUn } \\
\text { nCUOH } \\
03 \\
(\mathrm{CH} H) 25\end{array}$ & $\begin{array}{l}\text { III } \\
\text { III } \\
\text { IIII } \\
\text { III } \\
\text { III }\end{array}$ & $\begin{array}{l}.31310700 E+05 \\
.31372700 E+05 \\
.31420000 E+05 \\
.314249^{7} 0 E+05 \\
.31436710 E+05\end{array}$ & $\begin{array}{l}\mathrm{MC} 4 \mathrm{NH} 2 \mathrm{~A} \\
(\mathrm{CH}) 2 \mathrm{CH} \\
\mathrm{C} 3 \mathrm{H} 2 \mathrm{O} 3 \\
\mathrm{NH} \\
\mathrm{CH} 3 \mathrm{CHCH} 2\end{array}$ & $\begin{array}{l}\text { IIII } \\
\text { III } \\
\text { III } \\
\text { II } \\
\text { III }\end{array}$ \\
\hline
\end{tabular}


nu

$.314610^{7} 0 \hat{e}+05$

$.31499190 \mathrm{t}+05$

$.31511600 t+05$

$.31515190 t+05$

$.31520240 \mathrm{e}+05$

. $31536030 \mathrm{e}+05$

$.31557420 \mathrm{E}+05$

.315770008*05

$.315797300^{\circ}+05$
$.31625 ? 50 \mathrm{c}+05$

. 316200 U0itoj

$.31633090 E+05$

.31652050E+05

-31607460e+05

$.31 ? 00000 E+05$

.31719040 + +05

.31719 d20 +05

- $31721210 \mathrm{c}+05$

. $31722410 \mathrm{E}+05$

.31?35500E+05

.31735930E+05

$.3174 d 9 d 0 z+05$

. 31 ? $00900 \mathrm{c}+05$

. $317 ? 4500 E+05$

.317d1150z+05

$.31792800 \mathrm{E}+05$

- $31010490 \mathrm{~d}+05$

$.31824410 E+05$
$.31052100 E+05$

.31862300E+05

. $318735706+05$

$.31074310 E+05$

$.31900150 E+05$

$.319102200+05$

$.31922210 E+05$

$.31925960 \mathrm{~B}+05$

- $31926490 \mathrm{t}+05$

$.31926490 E+05$

.31949830t+05

$.31961950 \varepsilon+05$

.31967670 $t+05$

. $32015950 \mathrm{E}+05$

$32043110 \mathrm{E}+05$

. $32005960 \mathrm{E}+05$

-32094?00\& +05

. $32105700 E+05$

- $3210 \delta 500 \mathrm{e}+05$

$.32134800 E+05$

$32179270 E+05$

. $32105000 E+05$

. $32217680 \mathrm{~b}+05$

$32223230 E+05$
$.32273950 E+05$

.32290190E+05

$.323148006+05$

- $323556505+05$

- 3235 व $30 E+05$

$.32366470 E+05$

. 32309520E+05

- $32366230 E+05$

.32391470E+05

$.32406300 E+05$
$.3240 ? 360 E+05$

$32409020 \mathrm{E}+05$ .324131?0E+05

32425850E+05

$.32442000 E+0$

$.32461300 \mathrm{E}+05$
MOLECULE IABLE

UE 2

NC4Mn2n

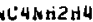

SIr 2

(CH2) 30

111

$\begin{array}{lll}1 & 1 & 1 \\ 1 & 1 & 1 \\ 1 & 1 & 1\end{array}$

(Cr2) 30

Cn3SC:

CH2CL 2

(Cri2) 30

Nid 2 CN

Chizauli

CH3ONOL

Cnina

CnzClCa

COUH4

MCCSCH3

$\mathrm{COOH} 4$

Cjund

ChInn

( $\mathrm{H} 2 \mathrm{CCH}) 2 \mathrm{O}$

COUH4

(CHJ) $2 \mathrm{Ccm}$

OF 2

CH2 ( $\mathrm{CN}) 2$

HNO3

C5OHO

(SO) 2

C2+3 3

Cons

(CH3) $2 \mathrm{CH}$

$+C \mathrm{CSCH}$

SIF 2

$\mathrm{SO2}$

(CH2) 30

(SU) 2

S20

(SO)?

$\mathrm{NO2CL}$

CH3CHCCH

( $\mathrm{CH} 3$ ) $2 \mathrm{CCH}$

(CH2) 30

MHENO2

hSCCCNHE

( Ca3) $2 \mathrm{CCO}$

(Cत3)

$\mathrm{S} 2 \mathrm{O}$

MSE

h2CHACH2

$5 \mathrm{IH} O \mathrm{OCH}$

(CA2) 30

$\mathrm{HCCSCH} 3$

NH2CHO

(SO) 2

NO2CL

( 12$) 30$

C50hy

SIH3OCn

(CH2) 30

(CH2) 30

(CH2) 30

(CH2) 30

SIH3OCH3

NSE

S IH3OCH3 niv

.3<490200E-0j

- $32525000 \mathrm{t}+05$

.32525UUDE

$.32525000 \mathrm{u}+05$

-3252030uetus

.325201 uUt+us

. $32531000 \mathrm{E}+0 \mathrm{~S}$

$.3254000 \mathrm{det}+05$

. $3250235 \mathrm{vetut}$

$.32594000 \mathrm{~s}+05$

$.32004300 E+45$

-320v045Ub+U5

$.32014100 \mathrm{etu}$

.3201442Ue+Us

. 32021490k+0?

-320291uvétus

- उ<077jouetos

- 320youvuetus

- उ270 $2090 \mathrm{E}+\mathrm{US}^{2}$

. 3270y250e+U5

$.32743000 \mathrm{e}+\mathrm{Us}$

-32? byuout + 5

-327010uetus

$.32^{7} 02250 v+05$

- 32d49700e+0s

- 32932 s0e+us

-329042U0eitos

- 32900000e+05

. 329, U510e+Us

-329yas 10t+os

$.33029620 \mathrm{E}+05$

.33030? UUE+US

. $33036 ? 00 \mathrm{t}+05$

.3305 YO $4 \mathrm{UL}+05$

-33075?00t+05

$.33104220 t+05$

. $33141510 \mathrm{e}+05$

$.33147800 E+05$

.33156950E+OS

. $33105420 c+02$

. $33105330 E+05$

. $3320>390 \mathrm{t}+05$

- 3320072Ue+U5

.33211020t +06

.33251120t+05

. $33251000 e+05$

$.33251000 E+05$
$.332 ? 0590 t+05$

. $33294490 \mathrm{E}+0 \mathrm{~J}$

$33294490 E+05$
$.33312770 E+U 5$

. $33312770 t+05$

$.33353410 c+0 j$

$.33366440 E+U 5$
$.33372910 E+U 5$

$.333 ? 6100=+05$

$.33306470 E+05$

$.33300 ? 00 \mathrm{c}+0 \mathrm{O}$

. $33401320 x^{2}+05$

$.33402090 \mathrm{E}+0 \mathrm{~S}$

$.33430440 \mathrm{E}+05$

$.33441104 E+05$

-334

- $3340<000+05$

$.33407450 E+U S$

$.33490010 t+05$

$.3349390 \mathrm{UE}+\mathrm{OS}$

. 33497200 E

$.33500 ? 20 E+0$

$.33530310 e+U 5$

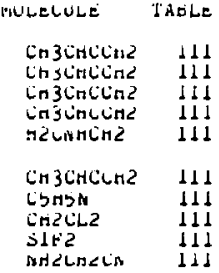

C5M5O

Slrz 111

Coun'4 11!

Coun4 111

(cn3) $2000 \quad 111$

Coun 4

(CH2) 30 III

nLiscis III

Lisincres

chjlinu

(Lin2) 30

Lin 3Lincon

Couta

111

111

SInjucng II

IVUF

Cin3ùvu2

$\begin{array}{ll}\text { Sinjultis } & \text { III } \\ \text { cnjunve } & \text { III }\end{array}$

III

(CH3) 2 L3H 111

SInjOLH3 111

SIH $30 \mathrm{CH}^{3} \quad 111$

CIINAH

CoOn 4

Couschecas

111

$\begin{array}{ll}\text { (CH3) } 2 \mathrm{CHN} & 111 \\ (\mathrm{CH} 3) 2 \mathrm{C} 3 n & 111\end{array}$

(Su)z HI

Nits

nicscin 3

singuinz 3

Coun4 III

(Ln3) 25

Uf 2

CHizICN)Z

520

304

(Cri3) $2 \mathrm{SO}$

CoUn 4

CoOn 4.

C3Hsarie

(SO) 2

(Cn2) ju

LOUt14

COOH4

Cout 4

(CnL) 30

cm3cnzol

cound

(CHE) 30

NH2MOL

( 


\begin{tabular}{|c|c|c|c|c|c|}
\hline 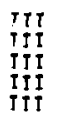 & 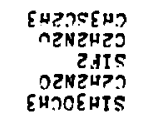 & 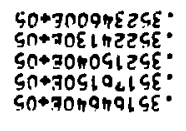 & $\begin{array}{l}\text { III } \\
\text { III } \\
\text { III } \\
\text { III } \\
\text { IIt }\end{array}$ & $\begin{array}{c}\text { HHO9S } \\
\text { OE(ZHJ) } \\
\text { HOODH } \\
\text { SOZZH } \\
\text { OJZHO }\end{array}$ & 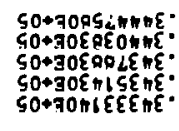 \\
\hline 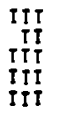 & 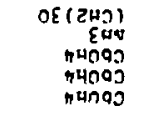 & 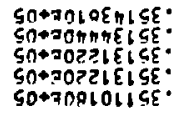 & 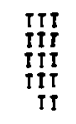 & 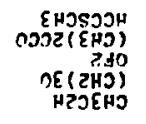 & 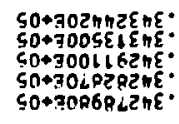 \\
\hline 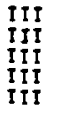 & 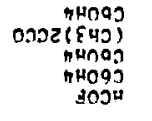 & 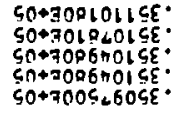 & $\begin{array}{l}\text { II } \\
\text { III } \\
\text { IIT } \\
\text { III } \\
\text { IIT }\end{array}$ & $\begin{array}{r}\text { HZJEHS } \\
\text { AEM?D } \\
\text { OHOSD } \\
\text { PHDSP } \\
\text { OHJZHA }\end{array}$ & 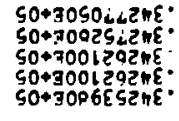 \\
\hline 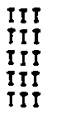 & 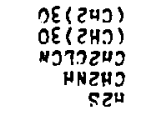 & 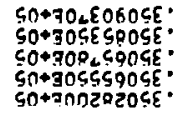 & $\begin{array}{l}\text { II } \\
\text { III } \\
\text { III } \\
\text { III }\end{array}$ & 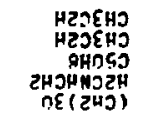 & 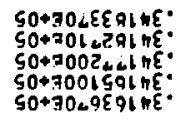 \\
\hline 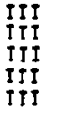 & 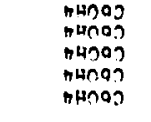 & 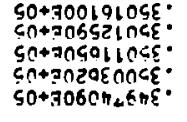 & $\begin{array}{l}\text { III } \\
\text { III } \\
\text { III } \\
\text { III } \\
\text { III }\end{array}$ & 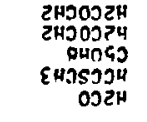 & 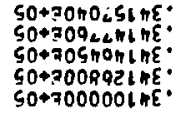 \\
\hline 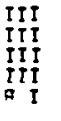 & 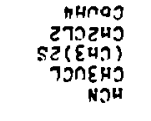 & 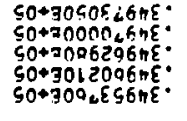 & $\begin{array}{l}\text { ITI } \\
\text { ITI } \\
\text { III } \\
\text { III } \\
\text { ITI }\end{array}$ & 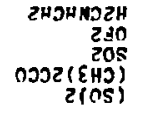 & 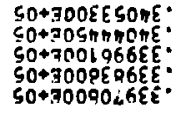 \\
\hline 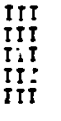 & 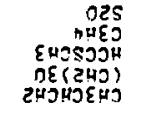 & 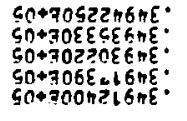 & $\begin{array}{l}\text { III } \\
\text { III } \\
\text { III } \\
\text { IIT } \\
\text { III }\end{array}$ & $\begin{array}{r}\text { SON } \\
\text { JSH } \\
\text { 2ONOEHS } \\
\text { PHOSS } \\
\text { EODEHIS }\end{array}$ & 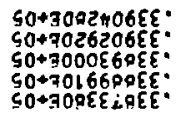 \\
\hline $\begin{array}{l}\operatorname{lit} \\
\text { III } \\
\text { III } \\
\text { III } \\
\text { III }\end{array}$ & 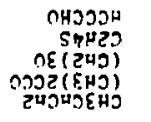 & 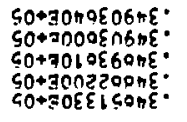 & $\begin{array}{l}\text { III } \\
\text { III } \\
\text { IIT } \\
\text { III } \\
\text { III }\end{array}$ & 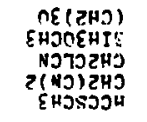 & 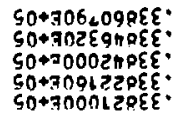 \\
\hline 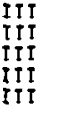 & 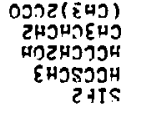 & 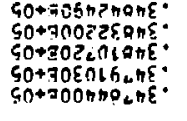 & $\begin{array}{l}\text { III } \\
\text { III } \\
\text { III } \\
\text { III } \\
\text { III }\end{array}$ & 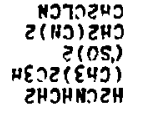 & 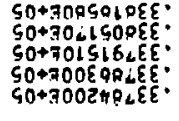 \\
\hline 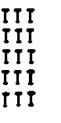 & 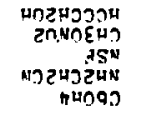 & 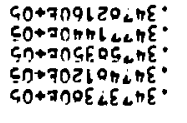 & $\begin{array}{l}\text { III } \\
\text { III } \\
\text { III } \\
\text { III } \\
\text { III }\end{array}$ & 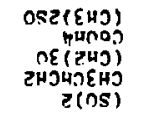 & 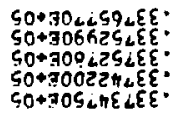 \\
\hline 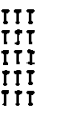 & 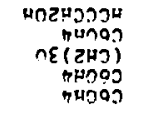 & 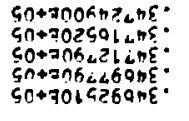 & $\begin{array}{l}\text { III } \\
\text { III } \\
\text { III } \\
\text { IJI } \\
\text { III I }\end{array}$ & 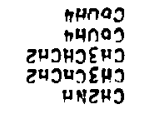 & 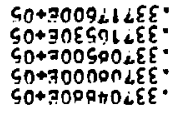 \\
\hline 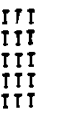 & 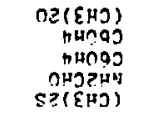 & 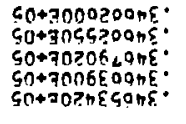 & $\begin{array}{l}\text { III } \\
\text { III } \\
\text { III } \\
\text { III } \\
\text { ITI }\end{array}$ & 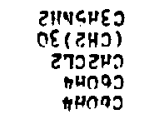 & 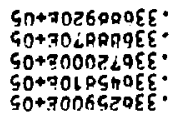 \\
\hline 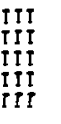 & 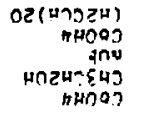 & 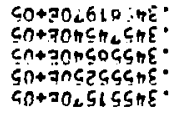 & $\begin{array}{l}\operatorname{lit} \\
\operatorname{III} \\
\operatorname{III} \\
\operatorname{III} \\
\operatorname{tit}\end{array}$ & 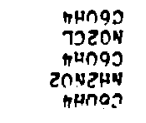 & 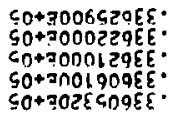 \\
\hline 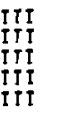 & 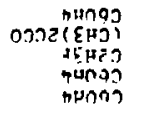 & 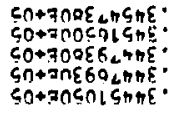 & 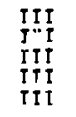 & 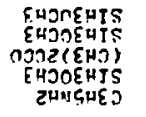 & 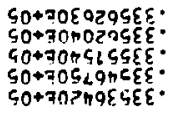 \\
\hline $270 \mathrm{H}$ & $37 n ว=7 n$ - & nN & วาดท I & จากงวา & ח!N \\
\hline
\end{tabular}




\begin{tabular}{|c|c|c|c|c|c|}
\hline$w$ & PIULECULE & $1 \mathrm{I} \forall \mathrm{LE}$ & lnu & NULELLLE. & IAELE \\
\hline 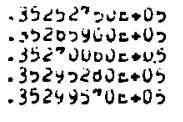 & 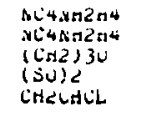 & 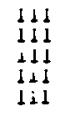 & 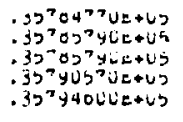 & 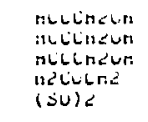 & $\begin{array}{l}1 \\
\vdots \\
\vdots \\
1 \\
1 \\
1 \\
1 \\
1\end{array}$ \\
\hline $\begin{array}{l}.35301500 E+05 \\
.35310330 e+05 \\
.35330640 e+05 \\
.35330010 e+05 \\
.33334400 e+05\end{array}$ & 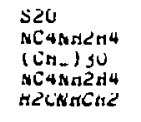 & 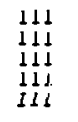 & 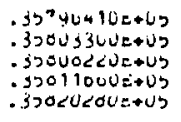 & 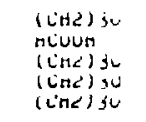 & $\begin{array}{l}111 \\
111 \\
111 \\
111 \\
111\end{array}$ \\
\hline 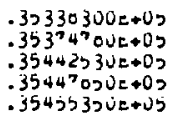 & 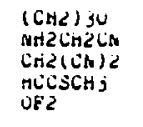 & $\begin{array}{l}111 \\
111 \\
111 \\
1 \perp 1 \\
1 \perp 1\end{array}$ & 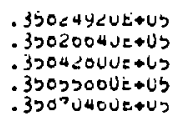 & 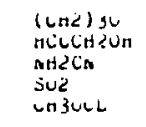 & 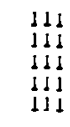 \\
\hline $\begin{array}{l}35401400=+45 \\
.35400300 E+05 \\
.35493050=+05 \\
.3520100 u t+05 \\
.35515300 E+115\end{array}$ & 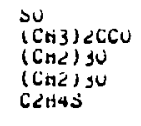 & $\begin{array}{ll}1 & c \\
1 & c \\
1 & 1 \\
1 & 1 \\
1 & 1 \\
1 & 1\end{array}$ & 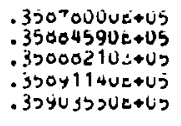 & 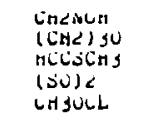 & 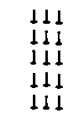 \\
\hline $\begin{array}{l}\text {.35520 UUE+0J } \\
.35563 y<U t+U 5 \\
.3550530 u E+05 \\
.35507000 t+05 \\
.355^{7} 0420 t+05\end{array}$ & 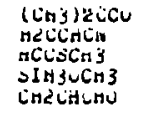 & 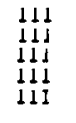 & 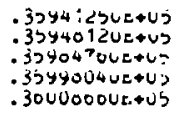 & 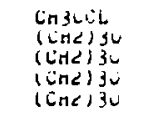 & $\begin{array}{lll}1 & 1 & 1 \\
1 & 1 & 1 \\
1 & 1 & 1 \\
1 & 1 & 1 \\
1 & 1 & 1\end{array}$ \\
\hline 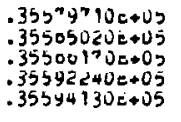 & 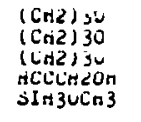 & 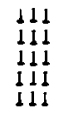 & 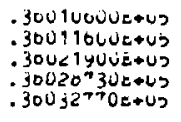 & $\begin{array}{l}(\cos ) 30 \\
\operatorname{cosincine} \\
\operatorname{lin} 2) 30 \\
\cos \\
(\cos ) 30\end{array}$ & $\begin{array}{lll}1 & 1 & 1 \\
1 & 1 & 1 \\
1 & 1 & 1 \\
1 & 1 & 1 \\
1 & 1 & 1\end{array}$ \\
\hline $\begin{array}{l}.3500025 U t+02 \\
.3500009 u E+05 \\
.3502125 U t+05 \\
.3562030 U t+05 \\
.3502000 U t+05\end{array}$ & 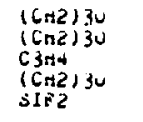 & $\begin{array}{l}111 \\
111 \\
111 \\
111 \\
111\end{array}$ & $\begin{array}{l}.30045500 e+05 \\
.30044000 E+U 5 \\
.30055700 E+L 5 \\
.30002150 E+05 \\
.30003500 E+U 5\end{array}$ & $\begin{array}{l}\text { nur } \\
\text { Cnjuncme } \\
\text { (Criz) } 30 \\
\text { (Criz) } 30 \\
\text { (Cत2) } 30\end{array}$ & $\begin{array}{lll}1 & 1 & 1 \\
1 & 1 & 1 \\
1 & 1 & 1 \\
1 & 1 & 1 \\
1 & 1 & 1\end{array}$ \\
\hline $\begin{array}{l}35031250 E+0 j \\
.35030120 E+0 j \\
.35045460 E+0 j \\
.35047000 E+05 \\
.3565314 U E 415\end{array}$ & $\begin{array}{l}(\mathrm{cn} 2) 30 \\
\text { (Cn2) } 30 \\
\text { nCLLn<Un } \\
(\mathrm{CH}) 3 \mathrm{U} \\
(\mathrm{Cn} 2) 3 \mathrm{U}\end{array}$ & $\begin{array}{l}111 \\
111 \\
111 \\
111 \\
\text { dII }\end{array}$ & $\begin{array}{l}\text { 30074070e+05 } \\
.3000540 U E+U 5 \\
.30000270 E+45 \\
.30112^{7} 00 E+05 \\
.30114+00 E+65\end{array}$ & $\begin{array}{l}(\mathrm{Cn} 2) 30 \\
(\mathrm{Cn} 2) 30 \\
(\mathrm{CH} 2) 30 \\
\text { inzincne } \\
\text { naisnine }\end{array}$ & $\begin{array}{lll}1 & 1 & 1 \\
1 & 1 & 1 \\
1 & 1 & 1 \\
1 & 1 & 1 \\
1 & 1 & 1 \\
1 & 1 & 1\end{array}$ \\
\hline 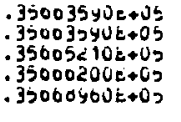 & 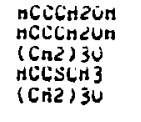 & $\begin{array}{l}1111 \\
111 \\
111 \\
111 \\
111\end{array}$ & 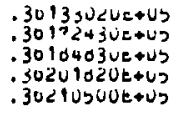 & 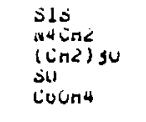 & 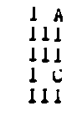 \\
\hline 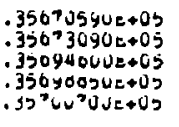 & 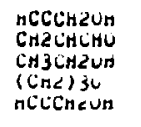 & $\begin{array}{l}111 \\
111 \\
111 \\
111 \\
111\end{array}$ & 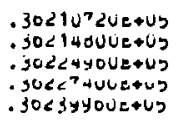 & 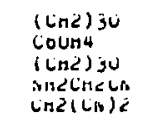 & $\begin{array}{lll}1 & 1 & 1 \\
1 & 1 & 1 \\
1 & 1 & 1 \\
1 & 1 & 1 \\
1 & 1 & 1\end{array}$ \\
\hline 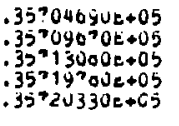 & $\begin{array}{l}\text { CoOH4 } \\
\text { nCCCH2On } \\
\text { Coun } \\
\text { COUn4 } \\
\left(\mathrm{CH}_{2}\right) 30\end{array}$ & $\begin{array}{l}111 \\
111 \\
111 \\
111 \\
111\end{array}$ & $\begin{array}{l}30240250 e+U 5 \\
.30242000+05 \\
.30200^{2} 00 e+U 5 \\
.302^{7}+300 t+05 \\
.302^{7} 720 E+05\end{array}$ & $\begin{array}{l}\text { Coun } \\
\text { aciscin } 3 \\
\left(C_{13}\right) 2 S \\
(\ln 3) 2 S \\
(\operatorname{CH} 2) 30\end{array}$ & $\begin{array}{lll}1 & 1 & 1 \\
1 & 1 & 1 \\
1 & 1 & 1 \\
1 & 1 & 1 \\
1 & 1 & 1\end{array}$ \\
\hline $\begin{array}{l}3 b^{7} 2055 u c+05 \\
3 b^{7} 2053 u t+05 \\
35>29530 e+05 \\
35>33240 e+05 \\
35^{7}=33^{7} 40 c+05\end{array}$ & 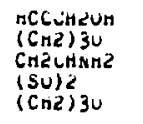 & $\begin{array}{l}\text { IIJ } \\
\text { iII } \\
\text { III } \\
\text { III } \\
\text { III }\end{array}$ & $\begin{array}{l}\text { 30204040t+U5 } \\
.3030 y^{7} 2 v E+U 5 \\
.30300014 E+U 5 \\
.3037400 u t+05 \\
.30300520 t+U 5\end{array}$ & $\begin{array}{l}\text { S2O } \\
\text { Sis } \\
\text { MSr } \\
\text { M2CNMCH2 } \\
\text { CH } 3 \cup C L\end{array}$ & $\begin{array}{ll}1 & 1 \\
1 & 1 \\
1 & \hat{1} \\
1 & 1 \\
1 & 1 \\
1 & 1\end{array}$ \\
\hline $\begin{array}{l}35^{7} 42070 e+05 \\
35 ? 45090=+05 \\
35745010=+05 \\
35^{7} 4030 \cup c+05 \\
5750470 E+05\end{array}$ & 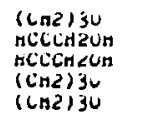 & $\begin{array}{l}111 \\
111 \\
111 \\
+11 \\
111\end{array}$ & 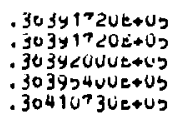 & 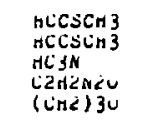 & $\begin{array}{lll}1 & 1 & 1 \\
1 & 1 & 1 \\
1 & 1 \\
1 & 4 \\
1 & 1 & 1 \\
1 & 1 & 1\end{array}$ \\
\hline 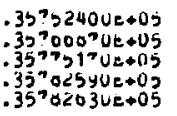 & 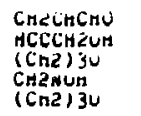 & 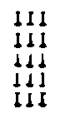 & 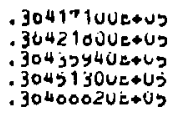 & 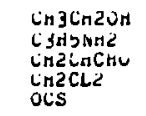 & $\begin{array}{ll}1 & 1 \\
1 & 1 \\
1 & 1 \\
1 & I \\
1 & 1 \\
1 & 1 \\
& A\end{array}$ \\
\hline
\end{tabular}


MU

. 36504140k+05

- 3052 jo0ue+05

305352 UOE+OS

$.30549350 t+05$

$.30550520 t+05$

$.365+2340 \mathrm{E}+05$

. $365,2340 \mathrm{E}+05$

. $36570000 k+05$

$.30595070 E+05$

$.36646060 \mathrm{k}+05$

$.3664 B 2500+05$

$.36653^{7} 00 \mathrm{E}+05$

$36693040 \mathrm{t}+05$

$.36705550 \mathrm{E}+05$

$.367320 E O E+05$

36? 36880E+05

.36744800E+05

.3674 y $300 \mathrm{E}+05$

.36769650t: i,

.367 d20d0E +05

-307940d0k +05

. $36795300 \mathrm{E}+05$

- 368037 d0E+05

$.36818030 \varepsilon+05$

.36827260E+05

$.36633350 \mathrm{~b}+0$

. 36857000t+05

36909990E+05

$.36912420 E+05$

. $36956000 E+05$

-3690?090t+05

.36́9 $9400 \mathrm{E}+05$

$.37019000 E+05$

- 37020, $v 0 E+05$

.37023770E+05

- 370237 TOE+05

-3707? $112300 E+05$

-37 $140040 \mathrm{0}+05$

$37149040 \mathrm{E}+05$
$.37164820 \mathrm{E}+05$

. $37164820 E+05$

$37175300 \mathrm{E}+0$

-3722?280E+05

$.37242150 E+05$

.3726 $1440 \mathrm{E}+05$

$.37274100 E+05$

$37290140 \mathrm{E}+\mathrm{C}$

$37320700 E+05$
$.37349640 E+05$

$.373851605+05$

- $37407200 \mathrm{E}+0$

. $37432240 E+05$

-37460600E +05

$.37463500 \mathrm{E}+05$

. 374645d0E+05

37477400E 405

$.37486800 E+05$

$37513260 E+05$

- $37557490 \mathrm{E}+05$

.37562400E+05

- $37567250 E+0$

- $37567810 E+0$

$37569670 E+05$
$.37570420 E+05$

375T9520E+05

. $37594320 E+05$

$.37617740 E+05$

$.37623400 E+05$

.37623610E+05
Mulacult

IABLE

(CH3) cso

(CH3) $26 \mathrm{CO}$

recefcs

CHa(CN)L

Cover 4

C60H4

COUH4

CoOnd

hriecinu

C 3H5th2

$\mathrm{HCCCH} 2 \mathrm{OH}$

heCCnO

HCCCHZOH

(CH2) 50

(CH2) 30

CL2O

(CHe) 30

111

111

$(\mathrm{CH} 2) 30$

$\mathrm{Cn} 3 \mathrm{CH}$
$\mathrm{CH} 3 \mathrm{CN}$
$\mathrm{H} 2 \mathrm{CCHCN}$

H2CCHCN

AcoOd

S20

$\mathrm{HCCSCH}$

$\mathrm{SO} 2$

( $\mathrm{CH} 3)_{2} 2 \mathrm{CH}^{3}$
$\mathrm{CH}(\mathrm{CH})^{2}$

HCOF

C2겨

OH

H.2CHCN

SF

CHJOCL

S1H3UCA3

S2O

CH2CHNH2

CH2CHNH2

$\mathrm{HH} 2 \mathrm{CH} 2 \mathrm{CH}$
$\mathrm{CH} 2(\mathrm{CN}) 2$

$\mathrm{M} 4 \mathrm{CH} 2$

HH2CHO

MCUMHEN 4

HCCCHO

( $\mathrm{H}$ (H2) 30

$\mathrm{NH}_{\mathrm{H}} 3$
$\mathrm{~S} 2 \mathrm{O}$

SIF?

(CH2) 30

CHzcho

(CH3) $2 \mathrm{CCO}$

c3HbH2

(CH2) 30

CoOH

c60H4

SIH3OCH3

NH2CHO

CbOH 4

c50h8

(so)?

SIH3OCH 3

CoOH 4

ClyH4

Contry iro

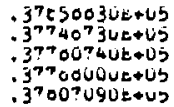

$.37022900 t+05$

. $3702290 \mathrm{UL}+\mathrm{U} 5$

- 3702? 100E+Ut

-3?020430E+05

. 37032? UuË+05

. 37033000e+05

$.37045200 \mathrm{~L}+05$

3705120UE+05

. 3705b350E+0s

.37002210k+05

370652050

$.37006000 t+i 5$

. 37954590k+05

. 3795? 320e+05

.37905400E+US

-3? 9 y12dOK+05

.30006? 50E+05

.300143UUe +05

$30000^{7} 00 t+05$

.361b;300é+05

.301506 31E+05

. $3010>4506+05$

. 3010dj10k+05

-30170300E+05

. $3017<010 \mathrm{x}+05$

- 30100000E +05

- $30203000 \mathrm{E}+155$

$.30200370 E+05$

.30241200E+05

.36260900E+05

. $30293500 E+0$

- 30316540E+US

$.38322300 k+05$

.36452690E+05

$.30409450 \Omega+0$

.30479020E+05

. 305 $12300 E+05$

$.36516210 i+05$

. $385427406+05$

30571410E+05

.30579950E+05

30027250040

-30653490t+05

36659020E+05

$36663200 \mathrm{E}+05$

. $36669220 E+05$

.3070 1050E+05

$30706690 \mathrm{E}+05$

3872237OE+05

. 6743 QUE 05

3d74700E 05

J

$38614000 \mathrm{~s}+05$

. 30824040E+05

$30827220 \mathrm{E}+0$

3082 . 490

-38029690 +0

-30060280 E+05

$.30804460 E+05$

.3088B120E+05

30d80120E+0

- 0017 100E+05

.38939180 $\$ 05$

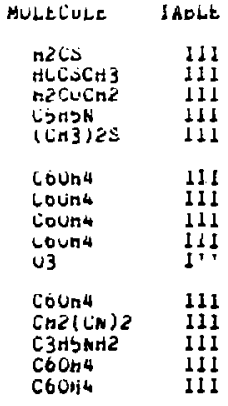

coun4 111

(a) $(\mathrm{LN}) 2$

c60h4

C60iju

SIt?

"Ss"

520

HCCCHO

520

C2H3F

II

(Su)2 III

(su)z

CLIÓ

Cound

CoOnt

$\$ 20$

20

CH3Crio

520

$\mathrm{CH} 30 \mathrm{H}$
(SO) 2

S20

S20

CH3OH

(CH2) 30 III

CHICHO III

520

(Ch2) 30

hCoun

(CH2)
NUF

CH2CHAHZ II

CH2CHAHZ II

$\mathrm{H} 2 \mathrm{CuCH2}$ III

CHe(CN) 2 III

$\mathrm{C}_{6 \mathrm{OH}} 4$ II

CoOnd III

C6OH4 III

S20

$\mathrm{ECCH}$

C60t1 4

$\mathrm{NH} 2 \mathrm{CH}$

CoOn 4

C $3+44$

C6OH4

L6OH4

CoOHA

C5HSN
CoOH4

II

11

11

12

11

11

III

III

11 
wu

SOYOOUAJERU

- 3eyooyunetus

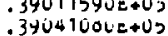

- 34u+4 3uve+us

- 39001250e+0?

-340yo420t+0?

-371 U23ue+l:

$.3922^{2} 400 \mathrm{c}+05$

$.39 e^{7} 2030 c+0 ?$

$.342^{7}>300 e+05$

- S42048 3uetus

- $342000400+05$

$.39<97010 \mathrm{e} \rightarrow 0$ 2

$.393140^{7} 0 e+05$

. $393231 \mathrm{vO}=+05$

. $39323100 t+05$

. 3932yUUNe+05

.3932y000k $\$ 05$

$.3934^{7} 230 \mathrm{c}+05$

$.3 \rightarrow 3525 j \mathrm{U} c+05$

. $393525000+05$

- $39352500+05$

3y 335 $370=+05$
3Y $3502 U U E+05$

$.393024 \div 0 t+05$

.39 $344750 \mathrm{E}+05$

- 5y4URLLUE+0D

.394242UUitos

$.394^{79} 90 \mathrm{O}+\mathrm{CS}$

.39512130e+05

$.3953^{7} 900 c+05$

- $39501040 \mathrm{U}+05$

- $59594000 \mathrm{~d}+05$

. $39012550 t+05$

.39645340E+05

. $39653550 \mathrm{c}+05$

-39070590 E+05

$.39000050 e+05$

-39725000k+05

-39029220t+05

-39034430E+05

-39037 100e+05

.39d4á 100u-05

- उyo00000t +05

$3900^{7} 340$ e 40

-390006500405

- 3909 yźuUe 05 .39910120e+0

$.39941540 E+05$

$.39947000 \mathrm{e}+05$

$.39950500 \mathrm{e}+05$

.39966000t+05

$39990000 \mathrm{t}+05$

$40030000 E+05$

$40041150 E+05$

$.40060090 \mathrm{0}+05$

.4000777 uet05

$40007790 \mathrm{x}+0$

$.40110920 E+0$

$.40135350 \mathrm{E}+05$
$.40205200 \mathrm{t}+05$

402 t0270e+05

$40211160 t+05$

$40253000 \mathrm{E}+\mathrm{C}$

$40263330 \mathrm{C}+0$

$.40313000+05$
Mulelule table.

ilez

tongn

(J)

$(20) 2$

sive

ili

I $1 \mathrm{i}$

d!

Singucins

Lint

singuCas

nciseng

ind Ju

$\begin{array}{ll}1 & 1 \\ 1 & 1 \\ 1 & 1 \\ 1 & 1\end{array}$

$(\mathrm{Cn} 3)<S$

S<u

$\mathrm{Cn}$ ( $\mathrm{CN})$

sinjuins

(Ch2) 30

Colint

cound

CoOn4

Court

$\$ 20$

Coun4

COOH4

(SU)
S2u

CHL (CM)

NST

SLU

c2njs

(CH2) Ju

HCOOA

n2 COCH2

Cris $\mathrm{CHO}$

Chico

(SU) 2

(CH2) 30

(Su) 2

HELCCH2

Mri2 CN

$\mathrm{CSO}$

$\operatorname{CoOH} 4$

Colits

Count

CoOA 4

SIthjuCn 3

Cound

SInjuCH3

Couna

Cilchs

and

NHECH

S2O

NH2CN

CH2CO

CL2O
(CH3) 2.5

CH2CO

Cn2CO

CH2 CO

CH2 CO

$\mathrm{NH} 2 \mathrm{CN}$

CH3NC

CH3NC

Mri2CH

(SU) 2

$\mathrm{SO} 2$
NU

$.40320000 \mathrm{v}=0$

4uj 37 ol 4 Ue

$40 j 44000+0$

.40417 4uve

. 4utgotuUe?tis

$.4 U 44 U 17 \forall E Q U S$

$.4044017 v e$ UU

-.U40<20Ue⿻us

4unvonuve us

$.40532^{7}$ OUceUs $40304550=005$ 4usooouveus

$401004 j \mathrm{e} u$ S

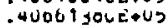

$.400 ?$ LOUOECUn

$.40^{2} 20000 \mathrm{t}+\mathrm{US}$

$40^{2} 1$ y $10 \mathrm{10}=0 \mathrm{O}$

$.40700 y 00=+05$

$.4 u^{7} y 3020=045$ $.40^{7}$ y $30200+05$

. 400uyo0cous

.4000 1 1 JUE $\$ 0$ S

.4007 4y $10 e+05$

.4UYzoysue-US

. 40 y 3 y32Ue+Uל

- $410<05$ vecus

.41 USASJUE+0J

$.410,49902+05$

$.4112400 u t+05$

$.41442200 t+05$

$.4153 U^{7} 10 \mathrm{UL}$ J

$41534 y 00 E+05$

.41534 y

$.416424000+05$

$.41642400 e+05$

$.499^{\circ} 297$ UEt05

. $42005350 E+45$

$.426033605+05$

$.4 \angle 099350 E+05$

$.422106405+05$

$.42324460 k+05$

$.42362900 E+05$

$.42306070 E+05$

$.424400 y 0 e+05$ $.425193000+05$ $.4257<900 \mathrm{C}+05$ .426 CUSOUÉ+U $.42032010 e+03$

. $42900300 t+12$ . 42950 OUt + 05 .43010 20UE+US $.43020900 t+05$ $.43020130 t+05$

.430 dOUUOĖ015 $.43122000 E+05$ $.43200^{7} 20$ Ut 45 $.43303710 \mathrm{E}+05$ $.43321920 E+05$

$.43423800 k+05$ $.43543920 E+0$ $.4303 \%$ CTOE+05 $.43054000 E+0$ $.43730 ? 90 \mathrm{t}+05$

$.43794020 t+05$ $.43030500 \mathrm{e}+\mathrm{U}$ $.43007000 E+05$ $.43907140 \mathrm{E}+05$ $.43954470 \mathrm{E}+05$

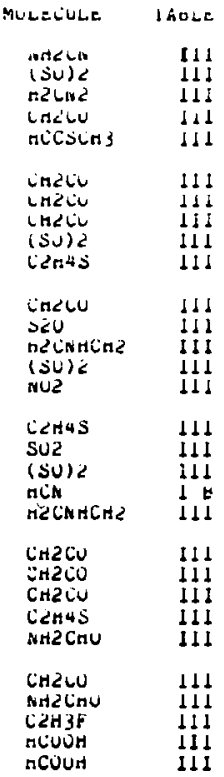

Ch3CHiuh

520

( $\mathrm{CH} 3$ ) $2 \mathrm{~S}$

H3NOZ

2 CuCtiz

su2

( $\mathrm{CH} 3$ ) $2 \mathrm{~S}$

SU2

CHaHON

Crizmur

NSE

(CH3) 25

NH2 CHO

$(\mathrm{sO}) 2$

( $\mathrm{CH}$ ) $2 \mathrm{~S}$

ن3

(SU) 2

SUL

ChjChzOn

nizCMO

SIOt

Sio

NH2CHO

$\mathrm{HCOOH}$

(Cit3) 25

SIU

(CHJ) 25

(CH3) $2 S$

03

(50)2

CHenOH

CHENOH

SIN

( $\mathrm{SO}) 2$

HH2 CHO

111

I A

II

III

III

\section{$1 A$}

Iิ

III

IA

III

I11

III

III $\quad .43907140 \mathrm{E}+0$


bKEUUKNCY LISJ 24

\begin{tabular}{|c|c|c|c|c|c|}
\hline ab & HULELULE & IABLE & nu & MULECULE & IABLE \\
\hline $\begin{array}{l}.43402^{77} 0 t+05 \\
.44052020 E+05 \\
.44060000 t+05 \\
.44296000 t+05 \\
.44550000 t+05\end{array}$ & $\begin{array}{l}\text { nNCÖ } \\
\text { SUC } \\
\text { Cn } 3 \text { Un } \\
\text { HCUF } \\
\text { aCcino }\end{array}$ & $\begin{array}{l}111 \\
111 \\
\text { II } \\
\text { III } \\
\text { III }\end{array}$ & $\begin{array}{l}.479: 0990 e+05 \\
.40204 .) 20 E+05 \\
.40334400 E+05 \\
.40349000 E+15 \\
.48372000 E+U 5\end{array}$ & $\begin{array}{l}\text { SUL } \\
n 2 \mathrm{Cu} \\
\$ 20 \\
\text { nnLLRO } \\
\text { CnjOH }\end{array}$ & $\begin{array}{ll}1 & 1 \\
1 & 1 \\
1 & 1 \\
1 & 1 \\
1 & 1 \\
1 & 1\end{array}$ \\
\hline $\begin{array}{l}.44509930 t+05 \\
.44720000 t+05 \\
.44762090 E+05 \\
.44760190 t+05 \\
.44794500 t+05\end{array}$ & $\begin{array}{l}(\mathrm{SU}) 2 \\
\mathrm{CH} 2 \mathrm{CHCL} \\
(\mathrm{CH} 3) 2 \mathrm{~S} \\
\text { NH2CHU } \\
\text { SU2 }\end{array}$ & $\begin{array}{l}111 \\
111 \\
111 \\
111 \\
\text { III }\end{array}$ & $\begin{array}{l}.403^{7} ? \text { U90E+05 } \\
.40419430 E+U 5 \\
.40012700 E+05 \\
.46035400 E+05 \\
.4005140 U E+05\end{array}$ & 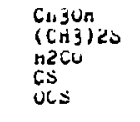 & $\begin{array}{ll}1 & I \\
1 & 1 \\
1 & I \\
1 & 1 \\
1 & A \\
1 & A\end{array}$ \\
\hline $\begin{array}{l}.44011000 c+05 \\
.4491150 E+05 \\
.45003100 t+05 \\
.45177530 t+05 \\
.45208300 t+05\end{array}$ & $\begin{array}{l}\text { ( SU) } \\
\mathrm{HCOOH} \\
\mathrm{n} 2 \mathrm{CO} \\
\mathrm{n} C \mathrm{COCH} 2 \\
\mathrm{CH} 2 \mathrm{CO}\end{array}$ & $\begin{array}{l}111 \\
\text { IIII } \\
1 \\
111 \\
111 \\
\text { III }\end{array}$ & $\begin{array}{l}.40010 y 10 e+05 \\
.40059400 \mathrm{0} 05 \\
.40419170 \mathrm{0}+05 \\
.40 y 910006+05 \\
.49000000=+05\end{array}$ & $\begin{array}{l}\operatorname{Mn} 2 \mathrm{Cn} \\
\sin \\
\text { ur } 2 \\
\mathrm{CS} \\
\mathrm{H} 2 \mathrm{CuCn} 2\end{array}$ & $\begin{array}{l}11 ! \\
1 ! ! \\
11 ! \\
1 \\
1 ! 1\end{array}$ \\
\hline $\begin{array}{l}.45400310 t+05 \\
.45490000 x+05 \\
.455420402+05 \\
.455904602+05 \\
.456573002+05\end{array}$ & 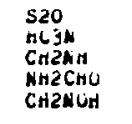 & 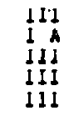 & $\begin{array}{l}.49020240 t+05 \\
.44147200 E+05 \\
.49171940 E+05 \\
.49100210 E+05 \\
.49550290 E+05\end{array}$ & $\begin{array}{l}\text { nCOUH } \\
\text { SLU } \\
\text { SLU } \\
\text { Ut2 } \\
\text { CH2hH }\end{array}$ & $\begin{array}{ll}1 & 1 \\
1 & 1 \\
1 & 1 \\
1 & 1 \\
1 & 1\end{array}$ \\
\hline $\begin{array}{l}.45001010 \mathrm{t}+05 \\
.45007690 \mathrm{0}+05 \\
.45043300 \mathrm{t}+05 \\
.45649600 \mathrm{t}+05 \\
.456700^{7} 0 \mathrm{E}+05\end{array}$ & $\begin{array}{l}\text { HCOOn } \\
\text { HCCCHO } \\
\text { CH3On } \\
\text { CH2CHCL } \\
\text { HCNO }\end{array}$ & 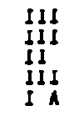 & $\begin{array}{l}44013520 E+05 \\
.49759490=005 \\
.50250000 E+05 \\
.50343230 E+05 \\
.50540050 E+05\end{array}$ & $\begin{array}{l}042 \\
520 \\
120 \\
0 F 2 \\
10 n 3125\end{array}$ & 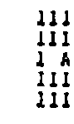 \\
\hline $\begin{array}{l}.45897600 \mathrm{t}+05 \\
.45911100 \mathrm{t}+05 \\
.45913300 \mathrm{t}+05 \\
.45947400 \mathrm{0}+05 \\
.4599200 \mathrm{E}+05\end{array}$ & $\begin{array}{l}\text { CH2CHCL } \\
\text { SU2 } \\
\text { CH2CHCL } \\
\text { CH2CHCL } \\
\text { HCHO }\end{array}$ & $\begin{array}{l}111 \\
111 \\
111 \\
111 \\
1\end{array}$ & $\begin{array}{l}.50053020 E+05 \\
.500959^{7} 0 E+05 \\
.50^{7} 96100 E+05 \\
.51042390 t+05 \\
.51000220 E+05\end{array}$ & $\begin{array}{l}\text { nCOUn } \\
\text { nneCnu } \\
(\operatorname{lin} 3) 2 S \\
\text { nocucnc } \\
\text { S2O }\end{array}$ & $\begin{array}{ll}I & I \\
I & I \\
I & I \\
I & I \\
1 & I \\
\text { III }\end{array}$ \\
\hline $\begin{array}{l}.46017230 t+05 \\
.46056000 \mathrm{0}+05 \\
.46002 \% 00 \mathrm{0}+05 \\
.46122820 \mathrm{0}+05 \\
.46193200 \mathrm{0}+05\end{array}$ & $\begin{array}{l}\text { HCHO } \\
\text { HCHO } \\
\text { HCNO } \\
\text { S2O } \\
\text { S2O }\end{array}$ & $\begin{array}{ll}1 & 0 \\
1 & 0 \\
1 & 0 \\
1 & 1 \\
1 & 1 \\
1 & 1\end{array}$ & $\begin{array}{l}.51200500 t+05 \\
.512^{7} 0000 E+J 5 \\
.512^{7} 3^{7} 60 E+05 \\
.512^{7}+750 t+05 \\
.51300100 E+05\end{array}$ & 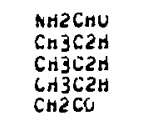 & $\begin{array}{l}115 \\
11 \\
11 \\
11 \\
111\end{array}$ \\
\hline $\begin{array}{l}.46233940 k+05 \\
.46378610 E+05 \\
.46421600 E+05 \\
.46424740 E+05 \\
.46501220 E+05\end{array}$ & $\begin{array}{l}\text { HCNO } \\
\text { S2O } \\
\text { S2O } \\
\text { SटO } \\
\text { HCOOH }\end{array}$ & $\begin{array}{l}\text { I B } \\
\text { IIII } \\
\text { III } \\
\text { III } \\
\text { III }\end{array}$ & $\begin{array}{l}.51300950 t+05 \\
.51391150 t+05 \\
.51415350 E+05 \\
.51416230 E+05 \\
.51416 ? 50 t+05\end{array}$ & $\begin{array}{l}\text { of } 2 \\
\text { n2CuCn2 } \\
\text { Cn3C2n } \\
\text { Cn3C2H } \\
\text { CH3C2n }\end{array}$ & $\begin{array}{l}111 \\
111 \\
11 \\
11 \\
11\end{array}$ \\
\hline $\begin{array}{l}.46541400 E+05 \\
.46602070 t+05 \\
.46713210 t+05 \\
.46979460 t+05 \\
.46960150 t+05\end{array}$ & $\begin{array}{l}\mathrm{HCDOH} \\
\mathrm{HCCChO} \\
52 \mathrm{U} \\
520 \\
\mathrm{CH} 3 \mathrm{CH} 2 \mathrm{OH}\end{array}$ & $\begin{array}{l}111 \\
111 \\
111 \\
111 \\
111\end{array}$ & $\begin{array}{l}.51730590 t+05 \\
.51747 \text { y0uE+U5 } \\
.5179 y 000 E+05 \\
.51933200 E+05 \\
.52051710 t+05\end{array}$ & $\begin{array}{l}\text { SU2 } \\
\text { OF } 2 \\
\text { S2U } \\
\text { CH2CHCL } \\
\text { SO2 }\end{array}$ & $\begin{array}{l}11 I \\
1 I I \\
11 I \\
1 I I \\
\text { IIJ }\end{array}$ \\
\hline $\begin{array}{l}.47017600 E+05 \\
.47023200 E+05 \\
.47032470 E+05 \\
.47041040 E+05 \\
.47059700 E+05\end{array}$ & $\begin{array}{l}-\mathrm{SO} 2 \\
\mathrm{HCH} \\
(\mathrm{SO}) 2 \\
(\mathrm{SU}) 2 \\
\mathrm{CH} 2 \mathrm{CHCL}\end{array}$ & $\begin{array}{l}111 \\
110 \\
111 \\
111 \\
111\end{array}$ & $\begin{array}{l}.52302400 t+05 \\
.52077610 t+05 \\
.52000030 E+05 \\
.53015400 E+05 \\
.53040000 t+05\end{array}$ & $\begin{array}{l}\text { Of2 } \\
\text { Cn'CHCL } \\
\text { CH2HA } \\
\text { SU2 } \\
\text { SO2 }\end{array}$ & $\begin{array}{l}11 J \\
\text { III } \\
\text { IJI } \\
11 I \\
11 I\end{array}$ \\
\hline $\begin{array}{r}47067730 \mathrm{E}+05 \\
.47066330 \mathrm{t}+05 \\
.470690 \mathrm{E} 0 \mathrm{t}+05 \\
.47094930 \mathrm{t}+05 \\
.47153310 \mathrm{t}+05\end{array}$ & $\begin{array}{l}\mathrm{H} 2 \mathrm{CS} \\
H \mathrm{NO} \\
\text { H2CHO } \\
\mathrm{H} 2 \mathrm{COCH2} \\
\text { (SO)2 }\end{array}$ & $\begin{array}{l}111 \\
111 \\
111 \\
111 \\
111\end{array}$ & $\begin{array}{l}.53189420 E+05 \\
.53251920 E+05 \\
.53206950 E+05 \\
.53370900 E+05 \\
.53528900 E * 05\end{array}$ & $\begin{array}{l}(\mathrm{CH} 3)_{2 S} 25 \\
\mathrm{HCOOH} \\
\mathrm{HCOOH} \\
\mathrm{SO} \\
\mathrm{SO} \\
\mathrm{SO}\end{array}$ & $\begin{array}{l}1 ! 1 \\
1 ! d \\
1 ! 1 \\
1 ! 1 \\
1 ! 1\end{array}$ \\
\hline $\begin{array}{l}47377920 E+05 \\
.47418590 \mathrm{t}+05 \\
.47440450 E+05 \\
.47554530 \mathrm{0}+05 \\
.47556890 \mathrm{0}+05\end{array}$ & $\begin{array}{l}(\mathrm{SO}) 2 \\
\mathrm{HCCCHO} \\
\mathrm{HCCCHO} \\
\mathrm{HCOOH} \\
\mathrm{H} 2 \mathrm{COCH}\end{array}$ & $\begin{array}{l}\text { III } \\
\text { III } \\
\text { III } \\
\text { III } \\
\text { III }\end{array}$ & $\begin{array}{l}.535 ? 2950 E+05 \\
.5350 d 320 E+05 \\
.53595600 E+05 \\
.53700050 E+05 \\
.53729 ? 00 \mathrm{0}+05\end{array}$ & $\begin{array}{l}\text { Chi2 ChnHe } \\
\text { Ch2 Chnti2 } \\
\text { SU2 } \\
\text { Or2 } \\
\mathrm{HCH}\end{array}$ & $\begin{array}{l}111 \\
111 \\
11 \\
11 ! \\
1 \\
1\end{array}$ \\
\hline $\begin{array}{l}.47633510 E+05 \\
.47641490 E+05 \\
.47054250 E+05 \\
.47707620 E+05 \\
.47775750 E+05\end{array}$ & $\begin{array}{l}\mathrm{S} 2 \mathrm{O} \\
(\mathrm{CH}) 2 \mathrm{~S} \\
(\mathrm{CH} 3)_{2 S} \\
\mathrm{~S} 2 \mathrm{O} \\
(\mathrm{CH}) 2 \mathrm{~S}\end{array}$ & $\begin{array}{l}\text { lII } \\
\text { IIII } \\
\text { IIII } \\
\text { IIII } \\
\text { III }\end{array}$ & $\begin{array}{l}.53739100 E+05 \\
.54036000 E+05 \\
.54057510 E+05 \\
.54061090 E+05 \\
.54137050 E+05\end{array}$ & $\begin{array}{l}\text { WE2 } \\
\text { HSF } \\
\text { H2COCH2 } \\
\text { NHECHU } \\
\text { OEL }\end{array}$ & $\begin{array}{l}\text { III } \\
\text { III } \\
\text { III } \\
\text { III } \\
\text { III }\end{array}$ \\
\hline $\begin{array}{r}.47816040 E+05 \\
.47849270 E+05 \\
.47054040 E+05 \\
.47913340 E+05 \\
.47940840 E+05\end{array}$ & $\begin{array}{l}\mathrm{s} 20 \\
\mathrm{~s} 20 \\
\mathrm{~S} 20 \\
\mathrm{~s} 02 \\
\mathrm{~s} 20\end{array}$ & $\begin{array}{l}111 \\
111 \\
111 \\
111 \\
111\end{array}$ & $\begin{array}{l}.54439090 E+05 \\
.54519230 E+05 \\
.54540660 E+05 \\
.54500200 E+05 \\
.54033400 E+05\end{array}$ & $\begin{array}{l}(\mathrm{CH}) / 2 \mathrm{~S} \\
(\mathrm{CH} 3) 2 \mathrm{~S} \\
\mathrm{~S} 2 \mathrm{U} \\
\mathrm{nC} 3 \mathrm{~N} \\
\text { SO2 }\end{array}$ & $\begin{array}{l}\text { J1I } \\
111 \\
111 \\
11 \\
\text { III }\end{array}$ \\
\hline
\end{tabular}




\begin{tabular}{|c|c|c|c|c|c|}
\hline 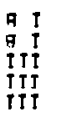 & 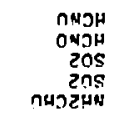 & 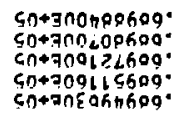 & 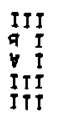 & 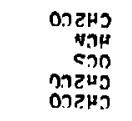 & 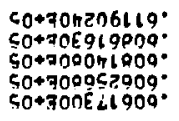 \\
\hline $\begin{array}{ll}\operatorname{IrT} \\
\operatorname{lit} \\
y \\
\text { IIT } \\
\text { IIT } \\
\text { ITt }\end{array}$ & $\begin{array}{l}\text { OHOSHN } \\
\text { HOSOH } \\
\text { ONJH } \\
\text { HONOH } \\
\text { SOSH }\end{array}$ & 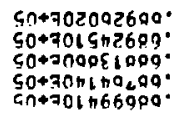 & 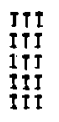 & 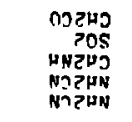 & 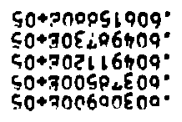 \\
\hline $\begin{array}{ll}9 & T \\
\text { IIT } \\
\text { IIT TI } \\
\text { TIT } \\
\text { TIT }\end{array}$ & 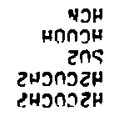 & 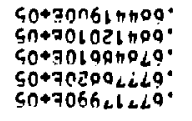 & $\begin{array}{l}\text { IIT } \\
\text { III } \\
\text { III } \\
\text { III } \\
\text { tII }\end{array}$ & 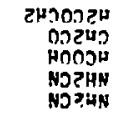 & $\begin{array}{l}50+509 E S E 2090^{\circ} \\
50+7026<5000^{\circ} \\
50+7019<0000^{\circ} \\
50+300 \angle 50665^{\circ} \\
50+70002 \angle 665^{\circ}\end{array}$ \\
\hline 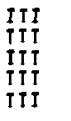 & $\begin{array}{r}2 H O N O 2 H \\
\text { SDZH } \\
\text { HONOH } \\
\text { HกOSH } \\
\text { HOOSH }\end{array}$ & 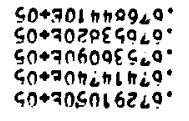 & $\begin{array}{l}\text { III } \\
\text { itI } \\
\text { III } \\
\text { iII } \\
\text { tII }\end{array}$ & 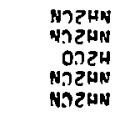 & 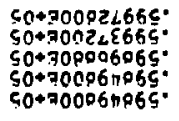 \\
\hline 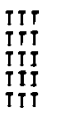 & 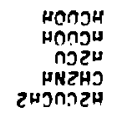 & 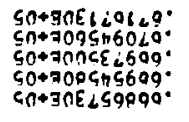 & $\begin{array}{l}\text { iII } \\
\text { III } \\
\text { III } \\
\text { III } \\
\text { III }\end{array}$ & 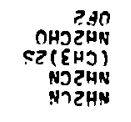 & 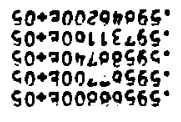 \\
\hline 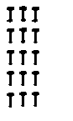 & 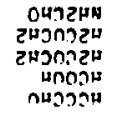 & 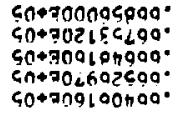 & $\begin{array}{l}\operatorname{trt} \\
\operatorname{tit} \\
\operatorname{tit} \\
\operatorname{tit} \\
\operatorname{tit}\end{array}$ & 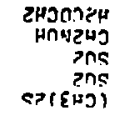 & 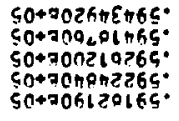 \\
\hline 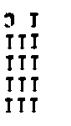 & 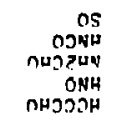 & 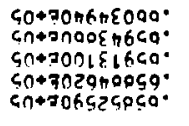 & $\begin{array}{l}\text { TII } \\
\text { IIT? } \\
\text { IIT } \\
\text { IIT } \\
\text { TIT }\end{array}$ & 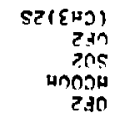 & 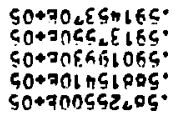 \\
\hline $\begin{array}{l}\text { Tit } \\
\text { TIT } \\
\text { Tit } \\
\text { Tit } \\
\text { Tit }\end{array}$ & 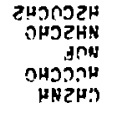 & 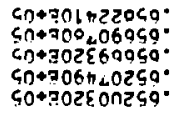 & $\begin{array}{l}\operatorname{lT}_{I T I} \\
\operatorname{III} \\
\text { III } \\
\text { IIT }\end{array}$ & 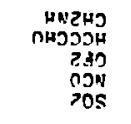 & 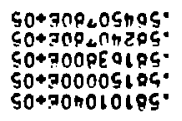 \\
\hline 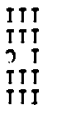 & 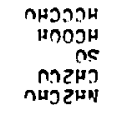 & 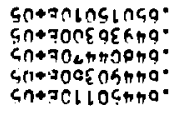 & 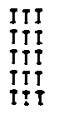 & 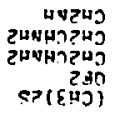 & 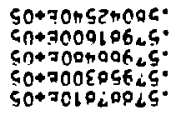 \\
\hline $\begin{array}{l}\text { ITI } \\
\text { ITI } \\
\text { ITI } \\
\text { ITI } \\
\text { ITI }\end{array}$ & 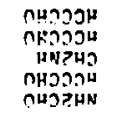 & 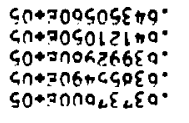 & 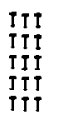 & 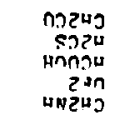 & 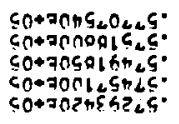 \\
\hline 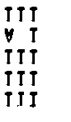 & 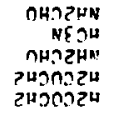 & 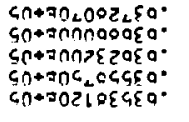 & $\begin{array}{l}\operatorname{TIT} \\
\text { IIT } \\
\text { II I } \\
\text { TIT } \\
\text { ITI }\end{array}$ & 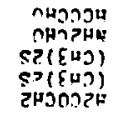 & 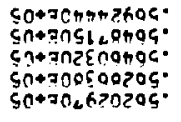 \\
\hline $\begin{array}{ll}0 \\
y^{2}\end{array}$ & 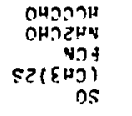 & 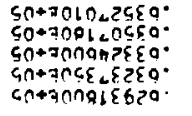 & $\begin{array}{l}\text { y T } \\
\text { IT T } \\
\text { IIT } \\
\text { ITI } \\
\text { IT I }\end{array}$ & 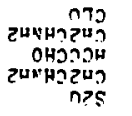 & 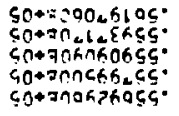 \\
\hline 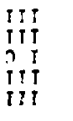 & 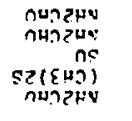 & 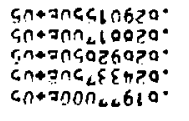 & $\begin{array}{l}\text { TIT } \\
\text { TIT } \\
\text { TIT } \\
\text { ITT } \\
\text { IT }\end{array}$ & 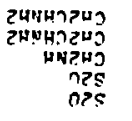 & 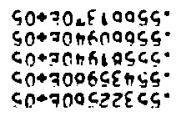 \\
\hline $\begin{array}{ll}\text { IIIT } \\
\text { III } \\
\text { TIT } \\
\text { TIT }\end{array}$ & 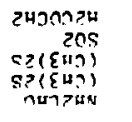 & 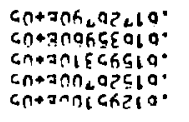 & $\begin{array}{l}\operatorname{lit} \\
\text { IIT } \\
\text { iTf } \\
\text { itT } \\
\text { ITt }\end{array}$ & 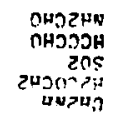 & 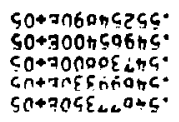 \\
\hline ㄱาจํ. 1 & 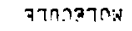 & กN & गาธะ! & כากราก & ทข \\
\hline
\end{tabular}


Bu

$.09002000 \mathrm{e}+05$

. 90100102005

$.69025010 \mathrm{E}+05$

$.69061000 E+05$
$.6907 y 600 \mathrm{t}+05$

$.0900 t^{7} 00 t+05$ $.69043400 \mathrm{O}+05$ $.69112400 \mathrm{E}+05$ $.059403400^{\circ}+05$ $.04<44500 x+0$ )

$.093500^{2} 06405$ $.09411400 \mathrm{e}+0 \mathrm{~S}$ $.094640^{\circ} 0 \mathrm{E} \rightarrow 0$ $.69566060 \mathrm{t}+0$ $.695 \% 5900 \mathrm{~L}+0 \mathrm{~S}$

$.09053490 E+05$ $.09746720 E+0$ $.09829420 \mathrm{k}+0 \mathrm{~S}$ $.69029020 \mathrm{E}+05$ $.09652030 t+05$

$.09854370 E+05$ $70134440 \mathrm{t}+05$

? $7161010 t+05$

$70162590 \mathrm{t}+05$ $.70293430 \mathrm{E}+05$

$.70347950 \mathrm{t}+05$ $.70735920 E+05$

$.70765920 E+05$

$.71324890 \mathrm{t}+05$

$.713911308+0$

$.71453110 k+05$ $.7162^{7} 640 \mathrm{t}+05$

$71646200 t+05$

$.71646200 E+05$ $.72023230 \mathrm{E}+05$

$.724091005+05$ $.72526190 \mathrm{E}+05$ $72 ? 13610 \mathrm{E}+0$ $.72758200 E+05$

.

$.72837970 E+05$

$73231500 \mathrm{E}+05$

$.73274320 \mathrm{E}+05$

? $4053950 \mathrm{E}+05$

$14372060 \mathrm{E}+05$

$74451680 \mathrm{~T}+05$

$.7446 B 230 E \div 0$

$.74471000 E+05$
$.74496020 E+05$

$.747153308+05$

$.74877110 k+05$

$.75370000 E+05$

$.75562110 k+05$

.75655220E+05

$.76461430 E+05$ $.76575540 E+05$

$.76648580 E+05$

$.77266560 \mathrm{E}+05$

$.70013490 E+05$

$.70633590 E+05$

$.79180640 E+05$

$.79280720 \mathrm{E}+0$

$798221502+05$

$.80076600 E+05$

.B0129330E+05

$.80802000 \mathrm{E}+05$

$80802000 E+05$

$.80820400 \mathrm{t}+05$

$.00820400 E+05$

\section{MOLELULE}

TABLE

nS

H2CS

ricko

MCHO

$\mathrm{HCNO}$

HCas

HChu

acocas

कियेo

MCNU

MS

Sü

Su2

SO2

neCs

M2 COCH2

$\mathrm{NH} 2 \mathrm{CHO}$

$\triangle \mathrm{COOH}$

HCOUH

$\mathrm{SO2}$

CrizNH

aHaCHO

SUE

$\mathrm{Su2}$

$\mathrm{SO} 2$
$\mathrm{SO2}$

tiCOUH

ChaChint

NOF

$\mathrm{SU} 2$

CHaCO

NH2CHO

Hece

$\mathrm{HCCCHO}$

NSF

HC 3N

$\mathrm{H} 2 \mathrm{CO}$

Sc

HCC CHO

Chrectinta

Ch2CHNH2

NHẼCHO

CH2CHNH2

CH2CHNi2

HCCCHO

CH2 CHNH2

HH2CHO

N2O

$\mathrm{H}$ COCHO
$\mathrm{HCCCHO}$

$\mathrm{HCN}$

NH2CHO

CH2CHNH2
H2COCH2

NH2CHO

$\mathrm{CH} 2 \mathrm{CO}$

CH2H

$\mathrm{NH} 2 \mathrm{CHO}$

$\mathrm{CH} 2 \mathrm{CO}$

$\mathrm{HCCCHO}$

$\mathrm{CH} 2 \mathrm{CO}$

$\mathrm{CH} 2 \mathrm{CO}$

CH2CO
NO

$.0003<100 \mathrm{~L}+\mathrm{O}$

. O110stútelos

.01490 uUutalj

$.01477440 c+U_{3}$

$.01525 y 100+05$

$.01541000 k+05$

.015002 Gut +UJ

.616Y354UE+ 63

.01979d0ue +0s

ocovaryuetus

$024249105+45$

$.0254437 \mathrm{UE}+05$

$.025 ? 0440 \mathrm{C}+155$

$.0300^{\circ} 000=+45$
$.037750<0<+05$

.039147 yútuj

$.03979090 k+U 5$

$.035^{7} 2500 \mathrm{E}+05$

$.03901440 E+05$

$.04062 \% 00 \mathrm{t}+05$

$.040^{7} 3^{7}$ bUt $+U 5$

$.0420<y 30 \mathrm{E}+05$

$.043047 \% 0 \mathrm{~L}+05$

$.04505350 \mathrm{c}+05$

$.045424002+05$

$.84500200 E+U 5$

$.64779000 \mathrm{e}+05$

.04607 y $40 \mathrm{k}+45$

$.8408 \$ 150 E+05$

$.240 y 1150 E+02$

$.64519200 \mathrm{e}+05$

$.05401740 \mathrm{c}+05$

$.05093300 x+05$

$.05093300 \mathrm{C}+05$

$.0530320 u e+U 5$

$.85 ! 25420 \mathrm{t}+05$

$05431340 \mathrm{E}+0$

$.85442010 E+05$

$.05450780 \ddot{E}+05$

$.8545729,1 E+05$

$.35570500 \mathrm{t}+05$

$85040300 E+U$

Dou

$.000935502+0$

$.60117 \mathrm{JOUE}+05$

$.66226+30 \mathrm{E}+0$

$.66243300 \mathrm{E}+0$

$.36302870 t+0$

$.06545900 \mathrm{E} \rightarrow 05$

$.00670550 E+05$

.068 -0OLCE+05

$.87317000 \mathrm{E} \rightarrow 05$

. $07402100 E+05$

d7527450 $t+05$

b7546000k+05

$875972406+05$

. $070276000+05$

$.07848930 E+0$

. $07800520 E+05$

. $47067000 \mathrm{E}+05$

.d? da $3330 \mathrm{~g}+05$

$.67458530 \mathrm{E}+05$

$.67090530 t+05$

$.67925240 E+05$

$.80027200 E \div 05$

$.00225400 t+05$

$.80254050 \mathrm{E}+\mathrm{US}$

$.823570 \mathrm{O}+\mathrm{U}$

.80631600 t +55
Mulziule

TAELE

crizco

neivn

$\mathrm{CJ}$

nicino

तiz 65

nदc

nacino

zécuna

receres

actru

ne $\ln$

$\begin{array}{ll}\text { Sce } & 111 \\ \text { accitic } & 11]\end{array}$

ALCORU 11

ACELitio

nCCinO

CCCAO

nCCCinc II

Mríte 11

AZLilne 111

nineche III

位

H2Cucine

Anz CHU

ARELHO

$\ln 2 \operatorname{Er} 0$

ancinu

$\mathrm{nCH}$

riscicas

añint

Crijcinzis

HeCinu

necuche

$\mathrm{CH} 3 \mathrm{C}_{2 \mathrm{H}}$

CH3C2n

CH 3 C $2 \mathrm{ri}$

$\mathrm{CA} 3 \mathrm{C} 2 \mathrm{H}$

$\mathrm{CH} 3 \mathrm{C2H}$

$\mathrm{H} 2 \mathrm{COCH} 2$

ri2 COCH2

510

$510+$

(Cri3) 20

SIU

Nitzino

rcoun il.

$\mathrm{nCO}$

SIU I

C2H I A

CHENM IIS

SIn $I A$

anco II

Anachu

inco

MINCC III

isccino II

MNCi

HAd 2 Lino

ANCi)

HNU
HCN 
RMLUUEACY LISt È

\begin{tabular}{|c|c|c|c|c|c|}
\hline nu & MOLECULE & TABLE & NU & MULEC:ULE & IABLE \\
\hline $\begin{array}{l}.09007700 E+05 \\
.09170500 E+05 \\
.09202340 E+05 \\
.0949000 x+05 \\
.09504900 E+0 j\end{array}$ & 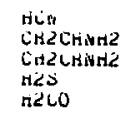 & $\begin{array}{l}\text { Jin } \\
\text { III } \\
\text { III } \\
\text { III } \\
\text { III }\end{array}$ & $\begin{array}{l}.94247010 \mathrm{E}+05 \\
.943130 ? 0 \mathrm{U}+U 5 \\
.94604400+05 \\
.94749420 \mathrm{E}+U 5 \\
.94700030 \mathrm{E}+05\end{array}$ & 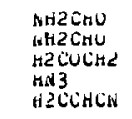 & $\begin{array}{lll}1 & 1 & 1 \\
1 & 1 & 1 \\
1 & 1 & 1 \\
1 & 1 & 1 \\
1 & 1 & 1\end{array}$ \\
\hline $\begin{array}{l}.09579200 x+05 \\
.0 y^{7} 01300 E+0 j \\
.09001500 x+05 \\
.09 y 40300 x+0 j \\
.9 y 400300 x+05\end{array}$ & 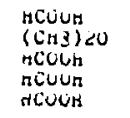 & $\begin{array}{l}\text { III I } \\
\text { III } \\
\text { III } \\
\text { III } \\
11 I\end{array}$ & $\begin{array}{l}.94633000 E+U j \\
.94913100 E+05 \\
.94913100 t+U 5 \\
.94920^{7} 10 \varepsilon+U 5 \\
.9494150 u e+U 5\end{array}$ & 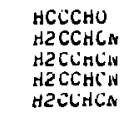 & $\begin{array}{lll}1 & 1 & 1 \\
1 & 1 & 1 \\
1 & 1 & 1 \\
1 & 1 & 1 \\
1 & 1 & 1\end{array}$ \\
\hline $\begin{array}{l}.90117510 \mathrm{~L}+0 j \\
.40104500 E+05 \\
.90303030 \mathrm{~L}+05 \\
.90410350 \mathrm{c}+05 \\
.90547500 \mathrm{0}+05\end{array}$ & 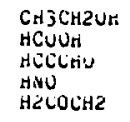 & $\begin{array}{lll}1 & 1 & 1 \\
1 & 1 & I \\
1 & 1 & 1 \\
1 & 1 & 1 \\
1 & 1 & 1\end{array}$ & $\begin{array}{l}.95100200 E+05 \\
.9510444 U E+02 \\
.95203100 E+U 5 \\
.95203120 E+05 \\
.95235450 E+U 5\end{array}$ & 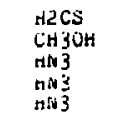 & $\begin{array}{l}117 \\
11 \\
111 \\
111 \\
111\end{array}$ \\
\hline $\begin{array}{l}\text { 9u705ó:0E+05 } \\
.30930100 c+05 \\
.91171200 E+05 \\
.914 ? 053 \cup E+05 \\
.915 ? 2510 t+05\end{array}$ & $\begin{array}{l}\text { nCOF } \\
(\operatorname{In} 3) 20 \\
\text { HCLCHO } \\
(\mathrm{CH} 3) 20 \\
\text { nCCCHO }\end{array}$ & $\begin{array}{l}11 I \\
11 I \\
\text { III } \\
\text { III } \\
\text { III }\end{array}$ & $\begin{array}{l}.95230170 t+05 \\
.952005000+05 \\
.95300050 E+05 \\
.953254900+05 \\
.95334160 E+05\end{array}$ & $\begin{array}{l}A N 3 \\
4 N 3 \\
C H 2 N H \\
H 2 C C H C N \\
H C O E\end{array}$ & $\begin{array}{l}\text { III } \\
111 \\
11 \\
\text { III } \\
\text { III }\end{array}$ \\
\hline $\begin{array}{r}.917,1320 \varepsilon+05 \\
.91959260 z+05 \\
.91 y 91350 \varepsilon+05 \\
.91974100 t+05 \\
.81980000+05\end{array}$ & $\begin{array}{l}\text { HCNU } \\
\text { CHZCN } \\
\text { CHZCN } \\
\text { HCNU } \\
\text { CHBCN }\end{array}$ & $\begin{array}{l}\text { I } A \\
\text { II } \\
\text { II } \\
\text { I } B \\
\text { I I }\end{array}$ & $\begin{array}{l}.95717070 E+05 \\
.95717070 \mathrm{O}+05 \\
.95717070 \mathrm{O}+05 \\
.95717070 \mathrm{0}+05 \\
.95722170 \mathrm{E}+0 .\end{array}$ & $\begin{array}{l}\mathrm{s} 20 \\
520 \\
520 \\
5<0 \\
520\end{array}$ & 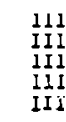 \\
\hline $\begin{array}{l}91903500 E+05 \\
.91905350 k+05 \\
.91907070 E+05 \\
.92033000 E+05 \\
.92001000 t+05\end{array}$ & $\begin{array}{l}\text { HCNO } \\
\text { CHJCN } \\
\text { CHJCN } \\
\text { ACtiO } \\
\text { ACNO }\end{array}$ & $\begin{array}{ll}I & B \\
I I \\
I \\
I & b \\
I & B\end{array}$ & $\begin{array}{l}.95 ? 22170 E+05 \\
.95724000 E+05 \\
.95724000 E+05 \\
.95743950 E+05 \\
.95743950 E+05\end{array}$ & $\begin{array}{l}520 \\
520 \\
520 \\
520 \\
520\end{array}$ & $\begin{array}{l}\text { III } \\
\text { III } \\
\text { IIII } \\
\text { IIII } \\
\text { III }\end{array}$ \\
\hline $\begin{array}{l}.92105500 E+05 \\
.92114000 E+05 \\
.92124600 E+05 \\
.92149400 \varepsilon+05 \\
.92309020 t+05\end{array}$ & $\begin{array}{l}\text { HCNO } \\
\text { HCNU } \\
\text { HCNO } \\
\text { ICNO } \\
\text { HCNO }\end{array}$ & $\begin{array}{ll}1 & 6 \\
I & B \\
I & B \\
1 & E \\
I & B\end{array}$ & $\begin{array}{l}.95700150 t+05 \\
.95 ? 02600 E+05 \\
.95010400 t+05 \\
.959142904+15 \\
.96220040 t+05\end{array}$ & $\begin{array}{l}\mathrm{H}+3 \\
520 \\
520 \\
\mathrm{CH} 30 \mathrm{H} \\
03\end{array}$ & $\begin{array}{l}\text { III } \\
\text { III } \\
\text { III } \\
\text { II } \\
\text { III }\end{array}$ \\
\hline $\begin{array}{l}.92312050 t+05 \\
.92315590 t+05 \\
.92435710 c+05 \\
.9240^{7} 000 t+05 \\
.92673910 \mathrm{c}+05\end{array}$ & $\begin{array}{l}\text { CH2LHNHZ } \\
\text { CH2CHNH2 } \\
\text { NH2CHO } \\
\text { HCNO } \\
\text { CLO }\end{array}$ & 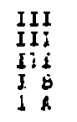 & $\begin{array}{l}.90404010 \mathrm{E}+05 \\
.96464 ? 40 \mathrm{0}+05 \\
.96406670 \mathrm{0}+05 \\
.96629700 \mathrm{0}+05 \\
.90741420 \mathrm{E}+05\end{array}$ & $\begin{array}{l}\$ 20 \\
\$ 20 \\
\$ 20 \\
\$ 20 \\
\text { CH3OH }\end{array}$ & $\begin{array}{l}\text { III } \\
\text { III } \\
\text { III } \\
\text { III } \\
\text { II }\end{array}$ \\
\hline $\begin{array}{l}92921210 t+05 \\
.93043280 t+05 \\
.93079330 E+05 \\
.930 ? 933(1 E+05 \\
.93000340 t+05\end{array}$ & $\begin{array}{l}\text { CH2CHNH2 } \\
\text { HCCCHO } \\
\text { CH2CHNH2 } \\
\text { CH2CHNH2 } \\
\text { CH2CHNH2 }\end{array}$ & $\begin{array}{l}\text { IIII } \\
\text { IIJI } \\
\text { IIII } \\
\text { IIII } \\
\text { IIII }\end{array}$ & $\begin{array}{l}.96744580 \mathrm{c}+05 \\
.90755510 E+05 \\
.96982490=05 \\
.97201390 \mathrm{E}+05 \\
.97301190 \mathrm{0}+05\end{array}$ & $\begin{array}{l}\mathrm{CH} 30 \mathrm{OH} \\
\mathrm{CH} 30 \mathrm{H} \\
\mathrm{H} 2 \mathrm{CCHCN} \\
\mathrm{H} 2 \mathrm{COCH} 2 \\
\text { OLS }\end{array}$ & $\begin{array}{l}\mathbf{I I} \\
\mathbf{I} I \\
\mathbf{L I} I \\
\mathbf{I} I \mathbf{I} \\
\mathbf{I} \mathrm{A}\end{array}$ \\
\hline $\begin{array}{r}.93000340 E+05 \\
.93093350 E+05 \\
.93090500 E+05 \\
.93113050 E+05 \\
.93123490 E+05\end{array}$ & $\begin{array}{l}\mathrm{CH} 2 \mathrm{CH} H \mathrm{H} 2 \\
\mathrm{NH} 2 \mathrm{CHO} \\
\text { HCOOH } \\
\mathrm{CH} 2 \mathrm{CHNH2} \\
\mathrm{CH} 2 \mathrm{CHNH2}\end{array}$ & $\begin{array}{l}\text { IIII } \\
\text { IIII } \\
\text { III } \\
\text { III } \\
\text { III }\end{array}$ & $\begin{array}{l}.97582830 E+05 \\
.97938330 E+05 \\
.99981000 E+05 \\
.98502160 E+05 \\
.90971030 E+05\end{array}$ & $\begin{array}{l}\mathrm{CH} 3 \mathrm{OH} \\
\mathrm{S} 2 \mathrm{O} \\
\mathrm{CS} \\
\mathrm{HCCCHO} \\
\mathrm{HCCCHO}\end{array}$ & $\begin{array}{l}\text { II } \\
\text { III } \\
\text { II } \\
\text { III } \\
\text { III }\end{array}$ \\
\hline $\begin{array}{r}.93164040 z+05 \\
.93230590 t+05 \\
.93304340 z+05 \\
.93300750 t+05 \\
.93313930 t+05\end{array}$ & $\begin{array}{l}\mathrm{HCOK} \\
\mathrm{HCCCHO} \\
\mathrm{HCCCHO} \\
\mathrm{HCCCHO} \\
\text { HCLCHO }\end{array}$ & $\begin{array}{l}\text { III } \\
\text { III } \\
\text { III } \\
\text { III } \\
\text { III }\end{array}$ & $\begin{array}{l}.99039070 E+05 \\
.99299870 E+05 \\
.99340760 \mathrm{0}+05 \\
.99527220 E+05 \\
.10002964 E+06\end{array}$ & $\begin{array}{l}\text { HCCCHO } \\
\text { SO } \\
\text { NH2CHO } \\
\text { S2O } \\
\text { SO }\end{array}$ & $\begin{array}{l}I I I \\
I C \\
I I I \\
1 I I \\
I C\end{array}$ \\
\hline $\begin{array}{l}.933^{1} 3940 E+05 \\
.93437770 t+05 \\
.93447500 t+05 \\
.93001200 t+C 5 \\
.93603900 E+05\end{array}$ & $\begin{array}{l}\text { HCCCHO } \\
\text { HCCCHO } \\
\text { CLO } \\
\text { CH2CHNHZ } \\
\text { CH2CKNH2 }\end{array}$ & $\begin{array}{l}\text { III I } \\
\text { III } \\
\text { II } \\
\text { III } \\
\text { III }\end{array}$ & $\begin{array}{l}10007640 E+06 \\
10009450 E+06 \\
10049174 t+06 \\
.10057240 E+06 \\
.10071690 t+06\end{array}$ & $\begin{array}{l}\text { HCBN } \\
\text { CH2CO } \\
\text { N2O } \\
\text { NH2CHO } \\
\text { HCCCHO }\end{array}$ & $\begin{array}{ll}I & A \\
\text { III } \\
\text { I } & A \\
\text { III I } \\
\text { III }\end{array}$ \\
\hline $\begin{array}{l}.93660100 E+05 \\
.93692760 E+05 \\
.93013050 E+05 \\
.93029910 E+05 \\
.9303200 E+05\end{array}$ & $\begin{array}{l}\text { H2 COCH2 } \\
\text { HNCS } \\
\text { HCN } \\
\text { HNCS } \\
\text { HACS }\end{array}$ & $\begin{array}{l}\text { III } \\
\text { III } \\
\text { II } \\
\text { III } \\
\text { III }\end{array}$ & $\begin{array}{l}10003005 E+06 \\
.10096740 \mathrm{E}+06 \\
.100567405+06 \\
10100240 \mathrm{E}+06 \\
.10100240 \mathrm{0}+06\end{array}$ & $\begin{array}{l}\mathrm{HCOF} \\
\mathrm{CH} 2 \mathrm{CO} \\
\mathrm{CH} 2 \mathrm{CO} \\
\mathrm{CH} 2 \mathrm{CO} \\
\mathrm{CH} 2 \mathrm{CO}\end{array}$ & $\begin{array}{l}\text { IIII } \\
\text { III } \\
\text { III } \\
\text { IIII } \\
\text { III }\end{array}$ \\
\hline $\begin{array}{l}930632 d 0 \mathrm{~d}+05 \\
.93671730 \mathrm{0}+05 \\
.93075200 \mathrm{0}+05 \\
.93875280 \mathrm{0}+05 \\
.93994900 \mathrm{c}+05\end{array}$ & $\begin{array}{l}\text { HNCS } \\
\text { WHECHO } \\
\text { HNCS } \\
\text { HNCS } \\
\text { HNES }\end{array}$ & $\begin{array}{l}\text { IIII } \\
\text { IIII } \\
I I I I \\
I I I I \\
\text { IIII }\end{array}$ & $\begin{array}{l}.101024 j 0 E+06 \\
.10103220 E+06 \\
.10103070 \mathrm{0}+06 \\
.10129930 E+00 \\
.10133290 \mathrm{0}+00\end{array}$ & $\begin{array}{l}\mathrm{CH} C \mathrm{CO} \\
\mathrm{CH} 2 \mathrm{CO} \\
\mathrm{CH} 2 \mathrm{CO} \\
\mathrm{NH2CHO} \\
\mathrm{H} 2 \mathrm{CO}\end{array}$ & 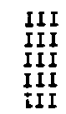 \\
\hline
\end{tabular}


HEUUENCY LIS' 20

Nu

$.10147760 E+06$

. $11155036 E+00$

$.10163 ? 20 \mathrm{e}+06$

$.10173683 \mathrm{E}^{\circ}+06$

$.10186330 \bar{\varepsilon}+06$

$.10198140 \mathrm{E}+06$

$.10201970 \mathrm{i}+05$

$.10206440 \mathrm{E}+06$

$.10209110 \mathrm{E}+06$

$10221760 t+06$

$.10220130 t+06$

$.10229400 \mathrm{E}+06$

$10254390 \mathrm{E}+06$

$.10263650 E+06$

$.10264230 E+06$

$.10264680 E+06$

$.10264680 \mathrm{E}+\mathrm{C6}$

$.10266910 E+06$

$102 ? 0100 E+06$

$.102740908+06$

$.10278800 E+06$

$.10281960 t+06$

$10284+80 t+06$

$102071808+06$

103030ग0E+06

$.10304020 E+06$

$.0305180 \mathrm{E}+06$

$10344590 \mathrm{E}+06$

$.10352440 \mathrm{E}+06$

. $10391530 \mathrm{~L}+06$

$10392370 \mathrm{E}+06$

$101029435+01$

$10463353 E+06$

$.10405960 \mathrm{E}+06$

$.10408580 \mathrm{E}+0 \mathrm{O}$

$.10421200 \mathrm{E}+06$

$.10430220 \mathrm{E}+06$

$.10441150 E+06$

$.10441150 E+06$

.10443280 L +06

$.10445380 E+06$

$.104617008+06$

$.10466170 E+06$

$10468690 \mathrm{OE}+06$

$.10468860 E+U 6$ $.10496070 \mathrm{0}+06$

-10501170E+06

$.10529280 E+06$
$.10529280 E+06$

$.105294408+06$

$.10529440 \mathrm{E}+06$

$10529650 E+06$

.10529650E+06

$.10530450 \mathrm{E}+06$

$10530450 \mathrm{E}+06$

$10538010 \mathrm{E}+06$

$10540363 \mathrm{E}+06$

$.105434906+06$

.10546430E+06

10555x08E+06

$.10559561 E+06$

.10560918E+06

$.10574377 E+06$

$.105794108+06$

10597270E406

. 10597270E+06

$.10610820 E+06$
MOLECULE TABLE

III

$\mathrm{H} 2 \mathrm{CS}$

$\mathrm{H} 2 \mathrm{CCriCh}$

03
520

III

III

$\mathrm{CH} 2 \mathrm{CO}$

NH2 CHO

$\mathrm{Nn} 2 \mathrm{CHO}$

NHZCHO

II!

III

HCCCHO

HCCCHO

HCCCHO

$\mathrm{HCCCHO}$

$\mathrm{HCCCHO}$

ACCCHO

II.CCHO

HCCCHO

HCCCHO

HCCCriO

HCCCHO

$\mathrm{HCCCHO}$

CCCHO

$\$ 20$

H2CS

H2 CS

Nit2CHO

NHE CHO

H2 CChCN

$\mathrm{H} 2 \mathrm{COCH} 2$

$\mathrm{S} 2 \mathrm{C}$

$\mathrm{SOC}_{4}$

H2COCH2

$\mathrm{H} 2 \mathrm{COCH} 2$

$\mathrm{2} \mathrm{CCHC}$

$\mathrm{HCCCHO}$

$\mathrm{H} 2 \mathrm{CCHCH}$

H2CCHCN

$\mathrm{H}$ CCHCW

$\mathrm{H} C \mathrm{CHCH}$

$\mathrm{H} 2 \mathrm{CS}$

Hecoch2

$\mathrm{H} 2 \mathrm{COCH} 2$

H2COCH2

$\mathrm{H} 2 \mathrm{CHCN}$

520

$\mathbf{5 2 0}$
$\mathbf{5 2}$

s20

S20

320

S20

520

S2O

S20

NH2CHO

HNCS

HNCS

HNCS

HHCS

CH2NH

NH2CHO

NH2CHO

NH2CHO

III

III I
NII

$.10613460 t+00$

$.306141602+00$

- $10625020 t+06$

. $10030000 t+06$

$.10654160 \mathrm{E}+66$

$10664140 \mathrm{E}+06$

$.10677500 E+60$

$.10701365 E+00$

.107302 ? $1 E+06$

$.10755060 \mathrm{E}+40$

1070301Ut+Ut

-10? $84375 t+06$

107 C $950 \mathrm{E}+06$

. $1001 \% 6$ ? E +U6

$.10832940 \mathrm{E}+06$

- $10917360 \mathrm{E}+00$

- 10925<10E+D0

$.10940670 k+06$

$10950070 \mathrm{t}+00$

$.10970940 \mathrm{E}+136$

- $10975360 \mathrm{E}+00$

- 1097. 33 E+06

$10977894 t+06$
$.10900770 t+06$

$.10963345 \mathrm{E}+00$

$.10983384 E+06$

$.10985770 \mathrm{E}+06$

$.10967260 t+06$

. $109892002+06$

$.109905 \mathrm{d5E}+06$

$.11029802 \mathrm{E}+00$

$.11033079 E+06$

$.11034010 E+06$

$.11034968 E+06$

$.11036452 E+U 6$

- $11037501 E+06$

$.11030139 E+06$

$.11036347 E+0 B$

$.11058317 E+06$

$11083630 E+06$

$110096908+06$

11128062E+06

$.11128962 E+06$

$.19153910 E+06$

. $11153910 \mathrm{E}+06$

.11171950E+06

-111 1950E+06

.17777340E+06

$.11180120 \mathrm{E}+06$

$.11185430 E+06$

$.111973405+06$

$.17197950 E+00$

$.11198000 \mathrm{E}+06$

.11198000E +06

. $11200280 \mathrm{E}+06$

$11200280 \mathrm{E}+0$

$11203690 E+06$

$.11203690 \mathrm{t}+06$

$.11208000 E+06$

$.11213100 E+06$

$11218940 \mathrm{E}+06$

.11218940E+06

$1122119 \mathrm{CE}+06$

$.11225540 \mathrm{E}+06$

$-11225540 E+0$

-11225540e+0

$.11228 ? 10 \mathrm{E}+06$
$.11232820 \mathrm{E}+06$
NUI,ECULE TADL

M2Cho 111

Ni2ino 111

111

WH2CHO $11 \mathrm{I}$

HeCOACH III

$\mathrm{CH} 3 \mathrm{OH}$

MCCCHO III

ACUOH 111

SU2 111

$\$ 20$ III

KHECHO III

AC3N 1

ASECHO III

nacc

$\begin{array}{ll}\text { HaCS } & \text { III } \\ \text { NH2CHO } & \text { III }\end{array}$

HaCO II

$\begin{array}{ll}\text { HINCU } & \text { III } \\ \text { SitheCHU } & \text { III }\end{array}$

HNCO 111

HNEO III

$\mathrm{HCCCHO}$ III

HWCO III

CH2NH III

INCO II

$\mathrm{CH} 3 \mathrm{Ch}$ II

$\mathrm{COOH}$

$\mathrm{CH} \mathrm{CN}$ II

$\mathrm{CH} 3 \mathrm{CN}$

$\mathrm{CH} 3 \mathrm{CN}$

MOF

$\mathrm{CH} 2 \mathrm{NH}$

CH2NH III

$\mathrm{Cn} 2 \mathrm{CHNH2}$ II

HCCCHO

CH2CHNH2 II

HCOOH

CH2CHNH2

HCCCHO

HCCCHO

$\mathrm{HC}$ ЕCHO

HCCCHO

$\mathrm{HCCCHO}$

HCCCHO

HCCCHO

HCCCHO

HCCCHO

HCCCHO

HCCCHO

$\mathrm{HCCCHO}$

$\mathrm{HCCCHO}$

HCCCHO

$\mathrm{HCCCHO}$

$\mathrm{HCCCHO}$

HCCCHO

HCCCHO


III

III

III

III

III

III

III 


\begin{tabular}{|c|c|c|c|c|c|}
\hline 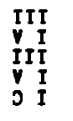 & $\begin{array}{r}\text { zOS } \\
\text { OIS } \\
\text { NOHगJ्टH } \\
\text { +OIS } \\
\text { OS }\end{array}$ & 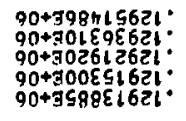 & $\begin{array}{l}\mathbf{I} \mathbf{I} \mathbf{I} \\
\mathbf{I} \mathbf{I} \\
\mathbf{I} \mathbf{I} \\
\mathbf{I} \mathbf{I} \\
\mathbf{I} \mathbf{I}\end{array}$ & 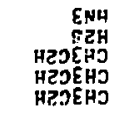 & 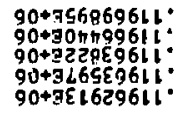 \\
\hline $\begin{array}{l}\text { III } \\
\text { III } \\
\text { II } \\
\text { II } \\
\text { II }\end{array}$ & $\begin{array}{r}2 D S \\
\text { OHDZHN } \\
\text { NDEHJ } \\
\text { NDEHJ } \\
\text { NOEHJ }\end{array}$ & 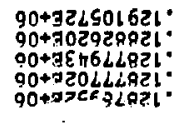 & $\begin{array}{l}\text { II } \\
\text { II } \\
\text { II } \\
\text { II } \\
\text { III }\end{array}$ & 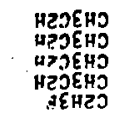 & $\begin{array}{l}90+3<9<19611: \\
90+329109611: \\
00+321109611: \\
90+300955611: \\
90+300616611:\end{array}$ \\
\hline $\begin{array}{l}\text { II } \\
\text { II } \\
\text { II } \\
\text { II } \\
\text { III }\end{array}$ & $\begin{array}{l}\text { NOEHO } \\
\text { NOEHHO } \\
\text { NOEHO } \\
\text { NOE HO } \\
\text { ZOS }\end{array}$ & 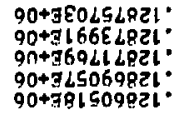 & $\begin{array}{l}\text { III } \\
\text { III } \\
\text { III } \\
\text { III } \\
\text { III }\end{array}$ & $\begin{array}{r}\text { OHOZHN } \\
\text { JOJH } \\
\text { ENH } \\
\text { ENH } \\
\text { ENH }\end{array}$ & 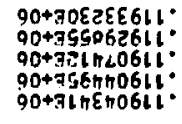 \\
\hline $\begin{array}{l}\text { YII } \\
\text { IIT } \\
\text { III } \\
\text { III } \\
\text { III }\end{array}$ & $\begin{array}{r}\text { OIS } \\
\text { OHSEHA } \\
\text { HNZHS } \\
\text { OHOZHN } \\
\text { OHDZHN }\end{array}$ & 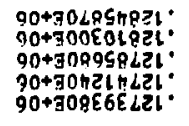 & $\begin{array}{l}\text { III } \\
\text { III } \\
\text { III } \\
\text { III } \\
\text { III }\end{array}$ & $\begin{array}{r}\text { ENH } \\
\text { ENH } \\
\text { ENH } \\
\text { ENH } \\
\text { ZHJONZH }\end{array}$ & 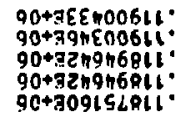 \\
\hline $\begin{array}{l}\text { III } \\
\text { III } \\
\text { III } \\
\text { III } \\
\text { III }\end{array}$ & $\begin{array}{l}\text { OHOZHN } \\
\text { OHOZHN } \\
\text { OHOZHN } \\
\text { OHOZHA } \\
\text { OHOZHA }\end{array}$ & 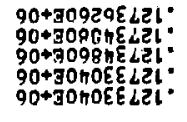 & $\begin{array}{l}\text { III } \\
\text { III } \\
\text { III } \\
\text { III } \\
\text { III }\end{array}$ & 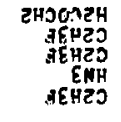 & 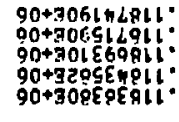 \\
\hline $\begin{array}{l}\text { III } \\
\text { III } \\
\text { IIII } \\
\text { III } \\
V I\end{array}$ & $\begin{array}{l}\text { OHDZHN } \\
\text { OHOZHN } \\
\text { OHDZHN } \\
\text { OHOZHN } \\
\text { OZN }\end{array}$ & 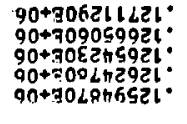 & $\begin{array}{l}\text { III } \\
\text { III } \\
\text { IIII } \\
\text { III } \\
\text { III }\end{array}$ & $\begin{array}{r}\text { OHล2 HN } \\
\text { EO } \\
\text { OHכटHN } \\
\text { SONH } \\
\text { נ5HZJ }\end{array}$ & 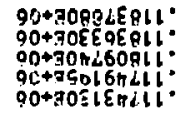 \\
\hline $\begin{array}{ll}y & I \\
V & I \\
V & I \\
V & I \\
V & I\end{array}$ & $\begin{array}{l}n Z N \\
0 Z N \\
0 Z N \\
\text { OZN } \\
0 Z N\end{array}$ & 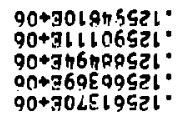 & $\begin{array}{l}\text { III } \\
\text { III } \\
\text { III } \\
\text { III } \\
\text { ITI }\end{array}$ & $\begin{array}{l}\text { soNH } \\
\text { sONH } \\
\text { sONH } \\
\text { sONH } \\
\text { sONH }\end{array}$ & 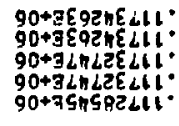 \\
\hline $\begin{array}{l}\operatorname{IIT} \\
\text { VI } \\
\text { II } \\
\text { III } \\
\text { III }\end{array}$ & $\begin{array}{r}\text { SOZH } \\
\text { OLN } \\
\text { पOEHS } \\
\text { OHכOכH } \\
\text { ZON }\end{array}$ & 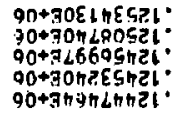 & 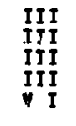 & $\begin{array}{r}\text { SOMH } \\
\text { टnS } \\
\text { ODटH } \\
\text { HOODH } \\
\text { SH }\end{array}$ & 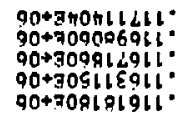 \\
\hline $\begin{array}{l}\text { III } \\
\text { III } \\
\text { II } \\
\text { III } \\
\text { III }\end{array}$ & 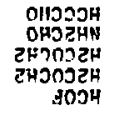 & 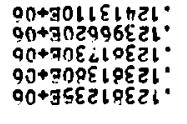 & 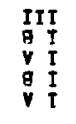 & $\begin{array}{r}\text { OHOOJH } \\
\text { ONOH } \\
\text { SN } \\
\text { ONOH } \\
\text { OJ }\end{array}$ & 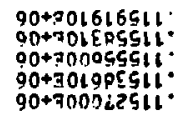 \\
\hline $\begin{array}{l}\text { III } \\
\text { III } \\
\text { IIII } \\
\text { IIII } \\
\text { III }\end{array}$ & $\begin{array}{r}\text { OHDZHN } \\
\text { AEHEJ } \\
\text { HNEHO } \\
\text { ZNONOEH } \\
\text { ZHOOOZH }\end{array}$ & 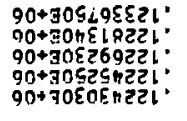 & 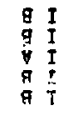 & $\begin{array}{l}\text { ONOH } \\
\text { ONOH } \\
\text { SH } \\
\text { ONOH } \\
\text { ONOH }\end{array}$ & 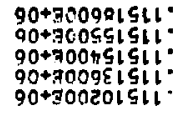 \\
\hline $\begin{array}{l}\text { III } \\
\text { III } \\
\text { III } \\
\text { III } \\
\text { IIT }\end{array}$ & 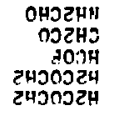 & 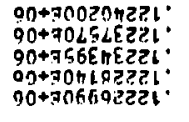 & $\begin{array}{l}\text { g I } \\
\text { IIT } \\
\text { IIT } \\
\text { g T } \\
\text { I I }\end{array}$ & 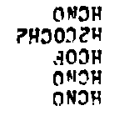 & 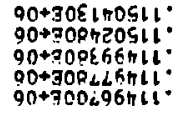 \\
\hline 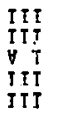 & 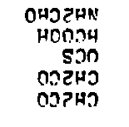 & 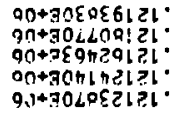 & $\begin{array}{l}\text { yI } \\
\text { III } \\
\text { III } \\
\text { IIT } \\
\text { III }\end{array}$ & 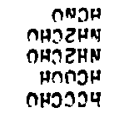 & 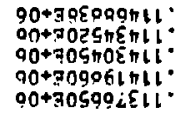 \\
\hline $\begin{array}{l}\text { IIT } \\
\text { III } \\
\text { I II } \\
\text { III } \\
\text { III }\end{array}$ & 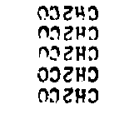 & 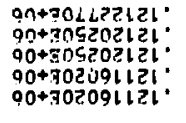 & $\begin{array}{l}\text { ITI } \\
\text { ITI } \\
\text { UII } \\
\text { ITI } \\
I T I\end{array}$ & 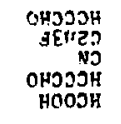 & 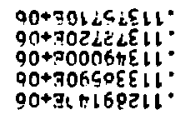 \\
\hline $\begin{array}{l}\text { III } \\
\text { III } \\
\text { IrII } \\
\text { III } \\
\text { III }\end{array}$ & 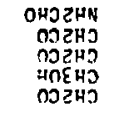 & 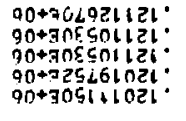 & $\begin{array}{l}\text { III } \\
\text { III } \\
\text { III } \\
\text { III } \\
\text { III }\end{array}$ & $\begin{array}{c}\text { OHOZHN } \\
\text { HNOOH } \\
\text { nOOOH } \\
\text { HOOJH } \\
\text { HOOJH }\end{array}$ & 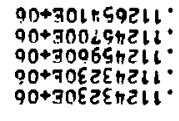 \\
\hline ราดหม & รากวราดห & אח & जาดหม & งากอมา & nN \\
\hline
\end{tabular}


Nu

.129671005406 $12490460 E+0$ .12996959E+06 $.13026 \mathrm{~B} 40 \mathrm{E}+0$ $.13090020 E+00$

$.131014 ? 6 \mathrm{E}+06$ $.13120700 t+06$ $13126740 k+06$ $171274885+06$ $.13153051 E+06$

$15161000 \mathrm{E}+06$

$15167720 E+00$

.13219er10E+0

$\$ 13252460 E+06$

$.13262194 E+06$

$.132744036+06$

$.13290000 E+06$

$13290009 E+06$

$13291780 E+06$

$13291890 E+06$

. $13295940 E+06$

$13300355 E+0$

$133030705+06$
$.133271685+06$

$13327210 \bar{E}+06$

$.133543695+06$

$.133734908+06$

$133767108+06$

$134021805+06$

$134020545+06$

$13405458+06$

$113414755+06$

$13415845 E+06$
$.13418596 E+06$

.13419216E+0

$13458232 E+06$

13468640E+06

$.13489696 \mathrm{E}+06$

$.134920208+06$

$134928508+06$

$13493850 \mathrm{E} .06$

1343050206

-13500905 +06

, $13503050 \mathrm{E}+06$

$.135297808+06$

$.13537676 \mathrm{E}+06$

$.13553158 \mathrm{E}+06$

$.1353990 E+06$

$.1356957 \mathrm{aE}+06$

$13571760 k+06$

1370606

$.136286808+06$

$136600158+06$

$136606208+06$

$136634035+06$

$13666274 \mathrm{E}+06$

$13667554 E+06$

$.13668619 \mathrm{E}+06$

$.13669356 \mathrm{E}+06$

-3670440E+06

13671760E+06

$130725368+06$

$136727932+06$

$13685560 \mathrm{E}+06$

23723131E+06

$.13736920 \mathrm{E}+06$

$13736920 E+06$
$.13737110 E+06$
MOLECULE TABLE

$\begin{array}{ll}\text { ACOOH } & \text { III } \\ \text { CHENH } & \text { III } \\ \text { NOF } & \text { III } \\ \text { SIO } & \text { IIA } \\ \text { HCONH } & \text { III }\end{array}$

SUE

$\mathrm{H} 2 \mathrm{CCHCN}$

$\mathrm{SOZ}_{\mathrm{SO}}$
$\mathrm{SO}$

NHACHO

$\triangle \mathrm{H} 2 \mathrm{CHO}$

HCCCHG

$\mathrm{HZCCHCN}$

$\mathrm{CH} 3 \mathrm{OH}$

502

H2CCHCN

HËCCHCN

$\mathrm{HZCCHCN}$

$\mathrm{HZCCHCN}$

$\mathrm{H} 2 \mathrm{CCHCM}$

$\mathrm{H} 2 \mathrm{CCHCN}$

S02

CH2NH

NOF

$\mathrm{HCCOH}$

S02

H2CCHiN

NOF

MOF

NOF

NOF

MOF

$\mathrm{CH} 3 \mathrm{OH}$

$\mathrm{HCOOH}$

HCOOH

$\mathrm{HCCOH}$

ACOOH

$\mathrm{H}_{\mathrm{H}} \mathrm{CO}^{\mathrm{COH}}$

HeCS

$\mathrm{CH} 3 \mathrm{OH}$

$\mathrm{SO} 2$

$\mathrm{H2CCHCN}$

$\mathrm{SO} 2$

$\mathrm{NH} 2 \mathrm{CHO}$

HCOOH

CH2CHO

CH2NH

$\mathrm{CH} 3 \mathrm{C} 2 \mathrm{H}$

$\mathrm{CH}_{3} \mathrm{C2H}$

$\mathrm{CH} 3 \mathrm{C2H}$

NoF

Chacent

CHicen

CH

H2CCHCH

$\mathrm{SO2}$

H2Cs

H2CS
III
III
III

III

IA

III

11

III

III

III

II

III

III

III

III

III

III

III

III

III

III

III

III

III

II

III

III

III

III

III

III

III

11

III

II

III

II

III

III

in

III
III
III
III
III
NU

. $13730200 E+06$

$.13740010 E+06$

- 13 ? $1100 c+00$

$.137654902+06$

$.1379 ? 110 \mathrm{0}+06$

$.137972838+06$

$130040006+46$

$13012300 \mathrm{k}+00$

$.130140002+00$

$.136176050 t+00$

$.13010490 k+06$

.13 322200k +56

. $13 d 24040 \varepsilon+06$

$.13639520 \hat{E}+06$

$.13846210 \mathrm{E}+00$

$.130690700 \div 06$

- $13000040 E+00$

. 1309063லE+06

$.13931300 E+06$

.13935470 a +00

$13948170 E+06$

139402005406

.14012000 OQU6

$.14012740 E+06$

.14030597e+06

$.14042950 \mathrm{E}+06$

$.14045 \dot{200}+06$

$.14046240 E+06$

$14058740 E+06$

$.14081030 E+00$

$140010300+00$

$.14120030 \mathrm{E}+06$

$.14120030 \mathrm{E}+06$

$.14120000 E+00$

$.14128060 \mathrm{E}+06$

$14135270 \mathrm{E}+06$

$.14135270 \mathrm{E}+\mathrm{OC}$

$.14140240 \mathrm{E}+06$

$.14140240 E+06$

$.14143010 E+06$

$141437908+06$

$14143790 E+06$

$: 14174170 \mathrm{E}+00$

.1417\%000E+06

$.14194540 E+06$

. $74200020 \dot{1}+06$

$.14204409 E+00$

$.142235702+06$

$.14234730 \mathrm{E}+06$

$142424408+06$

$14242650 \mathrm{E}+06$

$.14246244 t+06$

$142402448+06$

$142472402+06$

14257290E+06

$.142644268+06$

.14270150E+06

142734060600

$.14273466 \mathrm{E}+06$

$14275406+00$

$14280367 E+06$

$14280367 E+00$

$14285103 E+06$

14

$14285384 E+06$
$.14200692 E+06$

MOLECULE

Table

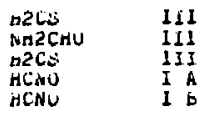

HCNO

NUF

metio

HCro I

so 20

HCriv I

HCAU If

H2CCnCN IIS

$\mathrm{HCHO} I$

HCHU I I

HCOE II

MHICCOO II

302

H2CS III

HCCCHO III

CHECO

SO2 $\mathrm{H}$ III

S20 III

MHzCHU III

CHINA IIJ

CH2CO

CH2CU III

CHaco

CHECO III

$\mathrm{CH} 2 \mathrm{CO}$ III

CH2CO III

CHicu III

Chi2Co III

CHaCU III

H2CCHCN II

MaCHO III

$\mathrm{H} 2 \mathrm{CCHCN}$ III

$\mathrm{HN} 3$

$2 \mathrm{ChO}$

II

$\mathrm{H} 2 \mathrm{CCHCN}$

H2CCHCN III

HCOE

$\mathrm{H} 2 \mathrm{CCHCH}$

HECCHCN

HN3

MH2CHO

$4 N 3$

HAM 3

HeCCHCH

HN3

HN3
HN3
HN3

HN3 


\begin{tabular}{|c|c|c|c|c|c|}
\hline 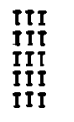 & 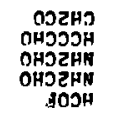 & 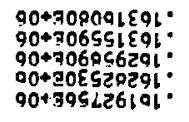 & $\begin{array}{l}\text { III } \\
\text { III } \\
\text { III } \\
\text { II } \\
\text { II }\end{array}$ & 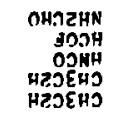 & 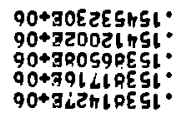 \\
\hline $\begin{array}{ll}\text { IIIT } \\
\text { III I } \\
\text { d I I } \\
\text { III } \\
\text { III I }\end{array}$ & $\begin{array}{l}\text { YOOH } \\
\text { OHOEHN } \\
\text { ONDH } \\
\text { ONZH5 } \\
\text { ODZHJ }\end{array}$ & 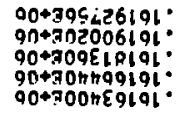 & $\begin{array}{l}\text { II } \\
\text { II } \\
\text { II } \\
\text { II } \\
\text { II }\end{array}$ & 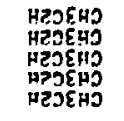 & 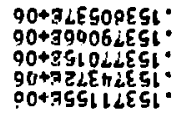 \\
\hline $\begin{array}{l}\text { III } \\
\text { III } \\
\text { III } \\
\text { III } \\
\text { III }\end{array}$ & 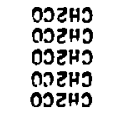 & 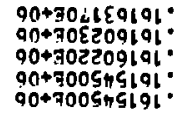 & $\begin{array}{l}\text { III } \\
\text { III } \\
\text { III } \\
\text { III } \\
\text { III }\end{array}$ & 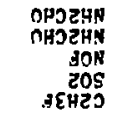 & 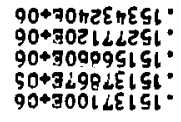 \\
\hline $\begin{array}{l}\text { å I } \\
\text { III } \\
\text { III } \\
\text { III } \\
\text { III }\end{array}$ & 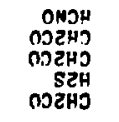 & 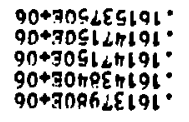 & $\begin{array}{ll}y & I \\
y & I \\
y & I \\
y & I \\
\text { IrI }\end{array}$ & $\begin{array}{r}\text { OEM } \\
\text { OZN } \\
\text { OZN } \\
\text { OZN } \\
\text { OHOZHA }\end{array}$ & 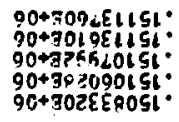 \\
\hline $\begin{array}{l}\text { III } \\
\text { III } \\
\text { III } \\
\text { G I } \\
\text { I I }\end{array}$ & $\begin{array}{l}\text { OOZFS } \\
\text { OJZHS } \\
\text { OOZHS } \\
\text { ONOH } \\
\text { ONOH }\end{array}$ & 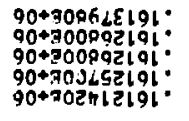 & $\begin{array}{ll}y & I \\
y & I \\
\text { ZII } & I \\
y & I \\
V & I\end{array}$ & 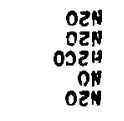 & 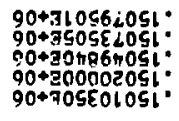 \\
\hline 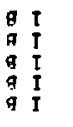 & $\begin{array}{l}\text { ON.OH } \\
\text { ON.H } \\
\text { ONOH } \\
\text { ONOH } \\
\text { ONOH }\end{array}$ & 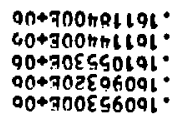 & $\begin{array}{l}\text { III } \\
\text { III } \\
\text { III } \\
\text { III } \\
\text { III }\end{array}$ & $\begin{array}{l}\text { SOSH } \\
\text { OYOZH4 } \\
\text { OHOZHN } \\
\text { OHDZHN } \\
\text { OSZH }\end{array}$ & 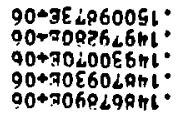 \\
\hline $\begin{array}{l}\text { IIT } \\
\forall T \\
\text { III } \\
\text { III } \\
\text { III }\end{array}$ & $\begin{array}{l}\text { SON } \\
\text { ONSH } \\
\text { JEHZS } \\
\text { OJZHO } \\
\text { OHOZH }\end{array}$ & 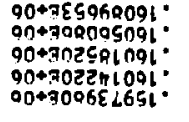 & $\begin{array}{l}\text { III } \\
\text { III } \\
\text { IIII } \\
\text { III } \\
\text { III }\end{array}$ & $\begin{array}{l}\text { NPDEHN } \\
\text { OHOZHN } \\
\text { OHOZHN } \\
\text { OHOZHY } \\
\text { OHDZHN }\end{array}$ & 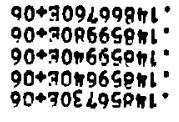 \\
\hline 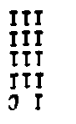 & 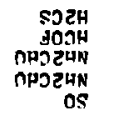 & 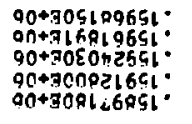 & $\begin{array}{l}\text { III } \\
\text { III } \\
\text { III } \\
\text { III } \\
\text { III }\end{array}$ & $\begin{array}{l}\text { OHOZHN } \\
\text { OHOZHN } \\
\text { OHOZHN } \\
\text { OHOSOH } \\
\text { OHOPHW }\end{array}$ & 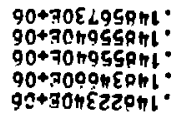 \\
\hline $\begin{array}{l}\text { ITI } \\
\text { III } \\
\text { III } \\
\text { III } \\
\text { III }\end{array}$ & 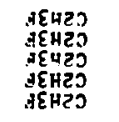 & 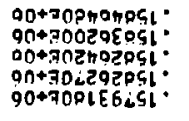 & $\begin{array}{l}\text { III } \\
\text { III } \\
\text { II } \\
\text { II } \\
\text { II }\end{array}$ & 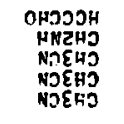 & 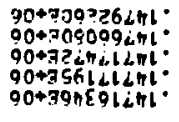 \\
\hline 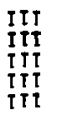 & 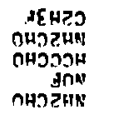 & 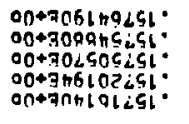 & $\begin{array}{l}\text { II } \\
\text { II } \\
\text { II } \\
\text { II } \\
\text { II }\end{array}$ & $\begin{array}{l}\text { NOEHS } \\
\text { NOEHS } \\
\text { NOEUS } \\
\text { NDEHS } \\
\text { NOEHS }\end{array}$ & 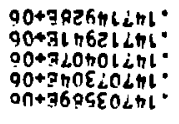 \\
\hline $\begin{array}{l}\text { TIT } \\
\text { TIT } \\
\text { IIT } \\
\text { IIT } \\
\text { TIT }\end{array}$ & 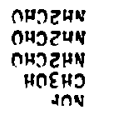 & 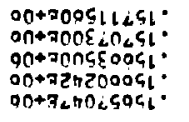 & $\begin{array}{l}\text { II } \\
\text { III } \\
\text { ITI } \\
\text { II } \\
\text { ITI }\end{array}$ & 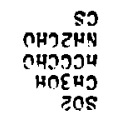 & 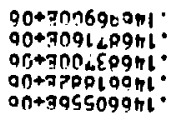 \\
\hline $\begin{array}{l}\text { TIT } \\
\text { III } \\
\text { TIT } \\
\text { IT } \\
\text { TI }\end{array}$ & 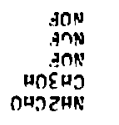 & 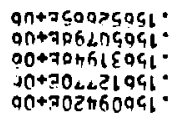 & $\begin{array}{l}\text { TII } \\
\text { ITI } \\
\text { ITI I I } \\
\text { II }\end{array}$ & 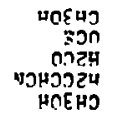 & 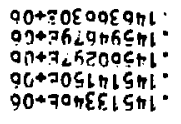 \\
\hline 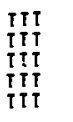 & 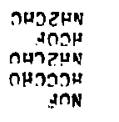 & 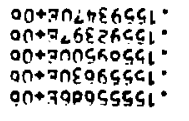 & $\begin{array}{l}\text { II } \\
\text { IT } \\
\text { IT } \\
\text { II } \\
\text { II }\end{array}$ & 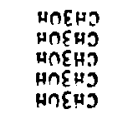 & 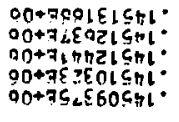 \\
\hline 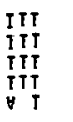 & 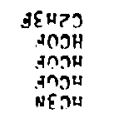 & 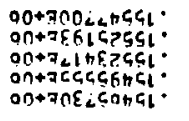 & $\begin{array}{l}\text { IT } \\
\text { III } \\
\text { III } \\
\text { II IT }\end{array}$ & 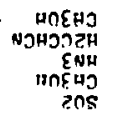 & 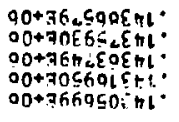 \\
\hline จาดท. 1 & จากวรากผ & nN & गา7४ J. & צר & ח \\
\hline
\end{tabular}


NU

$.10341030 t+00$ - 10 16 10 $.163^{17} 5340 \mathrm{c}+00$ - $103092^{2}$ OE+OO $.16500114 \mathrm{E}+06$

$.16509931 \mathrm{k+06}$

$.105177102+06$

$.16519053 c+06$

$.16536543 \mathrm{k}+06$

$.16536444 t=00$

$.16541293 t+06$

$.16545434 \mathrm{E}+06$

$.16546939 \mathrm{t}+06$

$.165517932+06$

$.16534031 \mathrm{a}+06$

$.16555610 E+00$

$.165565^{7} 1 t+06$

$.16556 d 95 E-06$

$.1656^{7} 0^{7} ? \mathrm{E}+06$

$.16500033 \mathrm{t}+00$

$.16616721 E+06$

$.10641670 \mathrm{~B}+06$

$.16641070 \mathrm{~b}+06$

$.16652279 \mathrm{E}+06$

$.16652279 E+06$

$.10660245 E+00$

$.160002 d 5 E+C 6$

- $16664700 \mathrm{t}+06$

$.10665766 E+06$

.16606205E+06

$.10609640 E+06$

$.16685180 E+06$

$.16732090 E+06$

$16757507 E+0 b$

16874170E+06

$.10874170 E+06$

$.16876280 \mathrm{E}+06$

$-16876200 \mathrm{E}+06$

$.16929950 \mathrm{E}+06$

.169299508+06

$.16906360 \varepsilon+06$

$.16978650 \mathrm{e}+06$

$.169706505+06$

$.16979130 \mathrm{E}+06$

$.16979130 \mathrm{~s}+06$

$.16961130 t+06$

$.16981130 t+06$

$16966200 E+06$

$.16995620 \mathrm{E}+06$

$170045902+06$

$.170039508+06$

$.17006063 t+06$

17014014E+06

-17020749E+06

17061160t+06

.17074605E+06

170788295406

$170824138+06$

$.17085350 \mathrm{~b}+06$

170476276006

$.17089259 E+06$

-17090237E\$06

- 17090s

. $17127490 E+06$

$.17162110 E+06$

171670705406

17167070 i 06

$171607906+65$
$171711006+06$

\begin{tabular}{|c|c|c|c|c|}
\hline MULECCULE & LAULE & $\mathrm{Bd}$ & MLLblint. & JhELE \\
\hline $\begin{array}{l}\text { C2n3r } \\
\text { HCBN } \\
\text { NAZCHU } \\
\text { CH3OA } \\
\text { Cn3Un }\end{array}$ & $\begin{array}{l}111 \\
11 A \\
111 \\
11 \\
11\end{array}$ & $\begin{array}{l}17171100 t+00 \\
17172020 E+06 \\
17177000 t+06 \\
17177940 \mathrm{c}+60 \\
17210140 t+00\end{array}$ & 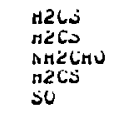 & $\begin{array}{ll}1 & 1 \\
1 & 1 \\
1 & 1 \\
1 & 1 \\
1 & 1 \\
1 & 1 \\
1 & C\end{array}$ \\
\hline $\begin{array}{l}\text { Cnzun } \\
\text { ntícina } \\
\text { Cn3Un } \\
\text { Cn } 3 \mathrm{CN} \\
\mathrm{CH} 3 \mathrm{OH}\end{array}$ & $\begin{array}{l}\text { II } \\
\text { III } \\
\text { II } \\
\text { II } \\
\text { II }\end{array}$ & $\begin{array}{l}17220^{7} 10 t+00 \\
17230120 \mathrm{t}+00 \\
17244595 \mathrm{E}+00 \\
17240060 \mathrm{t}+00 \\
17270340 \mathrm{O}+00\end{array}$ & 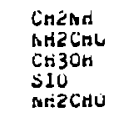 & $\begin{array}{l}111 \\
111 \\
11 \\
11 \\
111\end{array}$ \\
\hline $\begin{array}{l}\mathrm{CH} 3 \mathrm{CH} \\
\mathrm{CH} 3 \mathrm{CN} \\
\mathrm{CH} 3 \mathrm{CN} \\
\mathrm{CH} 3 \mathrm{CN} \\
\mathrm{CH} 3 \mathrm{CN}\end{array}$ & $\begin{array}{l}11 \\
I I \\
11 \\
I I \\
I I\end{array}$ & $\begin{array}{l}17310330 E+00 \\
17310400 E+00 \\
17340100 \mathrm{~d}+06 \\
17300000 E+00 \\
173^{\circ} 2500<+00\end{array}$ & $\begin{array}{l}\text { MHACHO } \\
\text { MA2CHU } \\
\text { n2CU } \\
\text { SIU } \\
\text { nCCCHO }\end{array}$ & 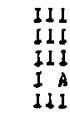 \\
\hline $\begin{array}{l}\text { CnjCN } \\
\text { CHjCN } \\
\text { Ca3CH } \\
\text { Cr:SUN } \\
\text { HNJ }\end{array}$ & $\begin{array}{l}11 \\
1 I \\
I I \\
1 I \\
I I J\end{array}$ & $\begin{array}{l}173^{77} 200 E+00 \\
17434400 E+00 \\
174402<0 E+00 \\
17400400 E+00 \\
17500900 E+00\end{array}$ & 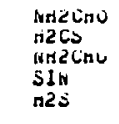 & $\begin{array}{ll}I & 1 \\
1 & 1 \\
1 & 1 \\
I & I \\
1 & I \\
1 & A \\
1 & 1\end{array}$ \\
\hline $\begin{array}{l}\text { CH3On } \\
\text { HN3 } \\
\text { hN3 } \\
\text { HN3 } \\
\text { SN3 }\end{array}$ & $\begin{array}{l}\text { II } \\
\text { III } \\
\text { III } \\
\text { III } \\
\text { III }\end{array}$ & $\begin{array}{l}17511901 k+00 \\
17510570 E+00 \\
17579020 E+00 \\
17504360 E+00 \\
1750 b 502 E+U 0\end{array}$ & 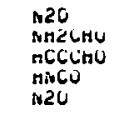 & 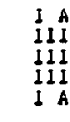 \\
\hline $\begin{array}{l}\text { HN3 } \\
\text { HN3 } \\
\text { HCCCHO } \\
\text { HN3 } \\
\text { HN3 }\end{array}$ & $\begin{array}{l}\text { III } \\
\text { IIII } \\
\text { III } \\
\text { IIII } \\
\text { III }\end{array}$ & $\begin{array}{l}175690301+06 \\
17592559 E+06 \\
17023417 \mathrm{0}+00 \\
17625796 \mathrm{0}+00 \\
17632350 E+00\end{array}$ & $\begin{array}{l}\text { Nn2CHU } \\
\$ 20 \\
120 \\
N 20 \\
N 20\end{array}$ & $\begin{array}{ll}1 & 1 \\
1 & A \\
I & h \\
I & A \\
I & A\end{array}$ \\
\hline $\begin{array}{l}\text { HN3 } \\
\text { CH2NH } \\
\text { NH2CHO } \\
\text { HW3 } \\
\text { WH2CHO }\end{array}$ & $\begin{array}{l}111 \\
\text { III } \\
\text { III } \\
\text { III } \\
\text { III }\end{array}$ & $\begin{array}{l}17 \text { C } 32500 E+00 \\
176920005+00 \\
17090^{7} 00 E+06 \\
17720040 E+00 \\
177230 ? 0 E+06\end{array}$ & $\begin{array}{l}\text { N2O } \\
\text { HCCCHU } \\
\text { NH2CHO } \\
\text { NH2CNU } \\
\text { HCN }\end{array}$ & $\begin{array}{ll}I & A \\
1 & 1 \\
1 & 1 \\
1 & 1 \\
I & 1\end{array}$ \\
\hline $\begin{array}{l}\text { NH2CHO } \\
\text { H2S } \\
\text { H2CS } \\
\text { MH2CHO } \\
\text { MHZCHO }\end{array}$ & $\begin{array}{l}\text { III } \\
\text { IIII } \\
\text { IIII } \\
\text { III } \\
\text { III }\end{array}$ & $\begin{array}{l}17720110 E+00 \\
17732750 t+00 \\
17732850 \mathrm{t}+06 \\
17734230 E+06 \\
17734270 \mathrm{1}+00\end{array}$ & $\begin{array}{l}\text { nCN } \\
\text { HCCCnO } \\
\text { HCCLiHO } \\
\text { HCCCHO } \\
\text { HCCCHO }\end{array}$ & 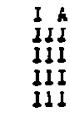 \\
\hline $\begin{array}{l}\text { Wh2CHO } \\
\text { WH2CHO } \\
\text { MH2CHO } \\
\text { MH2CHO } \\
\text { Mn2CHO }\end{array}$ & $\begin{array}{l}111 \\
11 I \\
111 \\
11 I \\
\text { III }\end{array}$ & $\begin{array}{l}.17730490 t+06 \\
17740300 \mathrm{0}+00 \\
17744020 t+00 \\
17745999 t+00 \\
.17746300 t+00\end{array}$ & $\begin{array}{l}\text { nCCCHO } \\
\text { nCCCHO } \\
\text { HCCCHU } \\
\text { nuz } \\
\text { nuf }\end{array}$ & $\begin{array}{l}1 \pm ! \\
1 !\rfloor \\
11 ! \\
11 ! \\
11 !\end{array}$ \\
\hline $\begin{array}{l}\text { NH2CHO } \\
\text { SH2CHO } \\
\text { NH2CHO } \\
\text { HH2CHO } \\
\text { MHZCHO }\end{array}$ & $\begin{array}{l}\text { III } \\
\text { III } \\
\text { III } \\
\text { IIII } \\
\text { III }\end{array}$ & 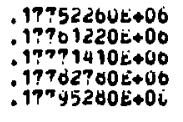 & $\begin{array}{l}\text { HCCCHO } \\
\text { MCCCHO } \\
\text { MCLCHO } \\
\text { HCCCHO } \\
\text { MCCCHO }\end{array}$ & $\begin{array}{l}\text { III } \\
\text { III } \\
\text { III } \\
\text { III } \\
\text { III }\end{array}$ \\
\hline $\begin{array}{l}\text { CH2N⿴ } \\
\text { WH2CHO } \\
\text { CH3OH } \\
\text { WOF } \\
\text { OCS }\end{array}$ & $\begin{array}{l}\text { III } \\
\text { III } \\
\text { II } \\
\text { III } \\
I A\end{array}$ & $\begin{array}{l}.17795260 \mathrm{~b}+06 \\
.17797420 \mathrm{E}+06 \\
.17007340 \mathrm{E}+06 \\
.17008660 \mathrm{0}+06 \\
.1700060 \mathrm{E}+06\end{array}$ & $\begin{array}{l}\text { НCCCHO } \\
\text { MCCCHO } \\
\text { CHZNH } \\
\text { HCCCHO } \\
\text { HCCCHO }\end{array}$ & $\begin{array}{l}111 \\
11 ! \\
111 \\
111 \\
111\end{array}$ \\
\hline $\begin{array}{l}\text { HCCCHO } \\
\text { CH3C2A } \\
\text { CH3C2H } \\
\text { CH3C2H } \\
\text { CH3C2H }\end{array}$ & $\begin{array}{l}I I I \\
I I I \\
I I \\
\text { II } \\
\text { II }\end{array}$ & $\begin{array}{l}.17813650 E+00 \\
.17823540 E+06 \\
.17024540 E+06 \\
.173340108+06 \\
.17839310 \mathrm{~B}+06\end{array}$ & $\begin{array}{l}\text { ACH } \\
\text { HCCCHO } \\
H C C C H O \\
\text { H2CS } \\
\text { НCCCHO }\end{array}$ & $\begin{array}{l}\text { II } \\
\text { III } \\
\text { IIII } \\
\text { III } \\
\text { III }\end{array}$ \\
\hline 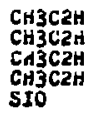 & $\begin{array}{l}I I \\
I I \\
I I \\
I I \\
I A\end{array}$ & $\begin{array}{l}17050040 \mathrm{O}+06 \\
.17863780 \mathrm{0}+06 \\
.17860290 \mathrm{0}+06 \\
.17005365 \mathrm{06} \\
.17005820 \mathrm{0}+06\end{array}$ & $\begin{array}{l}\text { MOF } \\
\operatorname{MH} 2 \mathrm{CHO} \\
\mathrm{NH2CHO} \\
\mathrm{NOF} \\
\text { NOF }\end{array}$ & $\begin{array}{l}\text { III } \\
\text { III } \\
\text { III } \\
\text { III } \\
\text { III }\end{array}$ \\
\hline $\begin{array}{l}\text { MH2CHO } \\
\text { H2CS } \\
\text { H2CS } \\
42 C S \\
\text { H2CS }\end{array}$ & $\begin{array}{l}\text { III } \\
\text { III } \\
\text { III } \\
\text { III } \\
\text { III }\end{array}$ & 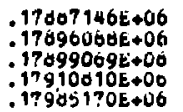 & $\begin{array}{l}\text { NOF } \\
\text { NOF } \\
\text { NOF } \\
\text { MHZCho } \\
\text { nCCCnO }\end{array}$ & $\begin{array}{l}I 1 I \\
\text { III } \\
I I I \\
I I I \\
I I I\end{array}$ \\
\hline
\end{tabular}




\begin{tabular}{|c|c|c|c|c|c|}
\hline mo & MLELULE & JAELE & $: u$ & MuLELULE & $I A E L E$ \\
\hline 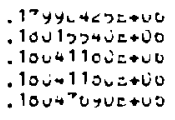 & 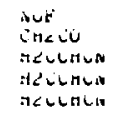 & 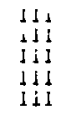 & 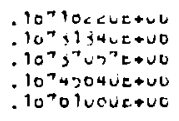 & 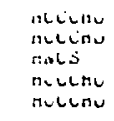 & $\begin{array}{l}111 \\
111 \\
111 \\
11 \\
11\end{array}$ \\
\hline 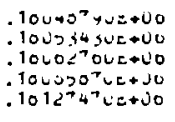 & 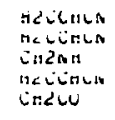 & 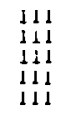 & 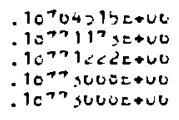 & 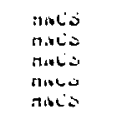 & $\begin{array}{lll}1 & 1 & 1 \\
1 & 1 & 1 \\
1 & 1 & 1 \\
1 & 1 & 1 \\
1 & 1 & 1\end{array}$ \\
\hline 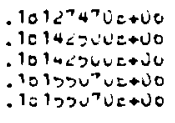 & 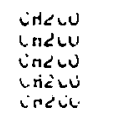 & $\begin{array}{l}111 \\
111 \\
111 \\
111 \\
11\end{array}$ & 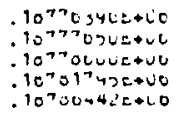 & 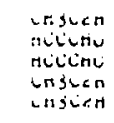 & $\begin{array}{ll}1 & 1 \\
1 & 1 \\
j & 1 \\
\vdots \\
i\end{array}$ \\
\hline 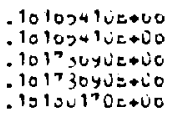 & 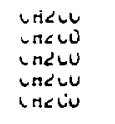 & $\begin{array}{l}11 j \\
11 j \\
112 \\
1+1 \\
1+1\end{array}$ & 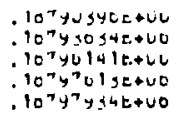 & 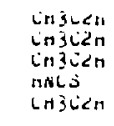 & $\begin{array}{l}11 \\
11 \\
11 \\
1 \text { iI } \\
1 i\end{array}$ \\
\hline 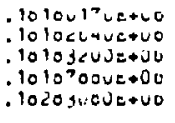 & $\begin{array}{l}\text { lnawu } \\
\text { li:Ll } \\
\text { in:2w } \\
\text { inewu } \\
\text { nucinu }\end{array}$ & $\begin{array}{lll}1 & 1 & 1 \\
1 & 1 & 1 \\
1 & 1 & 1 \\
1 & 1 & 1 \\
1 & 1 & 1 \\
1 & 1 & 1\end{array}$ & 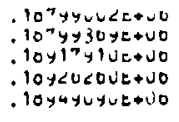 & 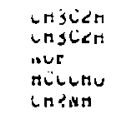 & $\begin{array}{l}1: \\
11 \\
111 \\
111 \\
111\end{array}$ \\
\hline $\begin{array}{l}10<0 y y+U E+00 \\
.10312904 E+U 0 \\
1033100 y=+00 \\
10334306=+00 \\
.1034 y 000=+00\end{array}$ & 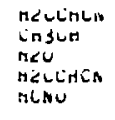 & $\begin{array}{l}111 \\
11 \\
11 \\
11 \\
11 \\
1\end{array}$ & 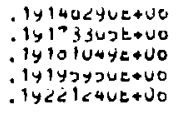 & 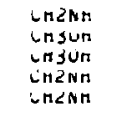 & $\begin{array}{l}111 \\
11 \\
11 \\
111 \\
111\end{array}$ \\
\hline 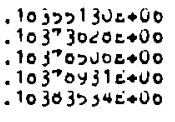 & $\begin{array}{l}\text { CnLCO } \\
\text { CnjCN } \\
\text { NUF } \\
\text { CHJCN } \\
\text { injicN }\end{array}$ & $\begin{array}{l}111 \\
11 \\
\vdots 11 \\
11 \\
11\end{array}$ & 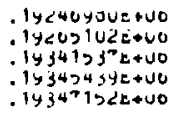 & $\begin{array}{l}\operatorname{cin} 2 n n \\
\text { juz } \\
\text { cnsun } \\
\text { Lnjun } \\
\text { Lnjun }\end{array}$ & $\begin{array}{lll}1 & 1 & 1 \\
1 & 1 & 1 \\
1 & 1 \\
1 & 1 \\
1 & 1\end{array}$ \\
\hline 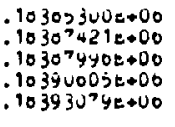 & $\begin{array}{l}\text { Cnjur } \\
\text { SnjCa } \\
\text { Lnjun } \\
\text { Cnjun } \\
\text { Lnjina }\end{array}$ & $\begin{array}{l}11 \\
11 \\
11 \\
11 \\
11\end{array}$ & $\begin{array}{l}19347493 E+U 0 \\
19340003 E+U 0 \\
1935 U 00 U E+U 0 \\
19321121 t+U 0 \\
119450044 E+U 0\end{array}$ & $\begin{array}{l}\text { Cnjon } \\
\text { injun } \\
\text { Cnjun } \\
\text { Lnjun } \\
\text { uns }\end{array}$ & $\begin{array}{l}\text { II } \\
\text { il } \\
\text { II } \\
\text { il } \\
\text { I A }\end{array}$ \\
\hline $\begin{array}{l}103940 u v e+00 \\
103 y 404 y e+00 \\
10342300 E+00 \\
103 y 300 \cup E+00 \\
10345 y 0 E+00\end{array}$ & $\begin{array}{l}\text { nCnO } \\
\text { CH3Lin } \\
\text { nino } \\
\text { LnEma } \\
\text { LnSLn }\end{array}$ & $\begin{array}{ll}1 & b \\
11 & \\
1 & 0 \\
1 & 1 \\
11 & 1\end{array}$ & $\begin{array}{l}194001200+40 \\
1950000+00 \\
195140700+40 \\
195520200+40 \\
1950010200+00\end{array}$ & $\begin{array}{l}\text { mur } \\
\text { inizan } \\
\text { Cnjün } \\
\text { lj } \\
\text { Ln2mn }\end{array}$ & $\begin{array}{lll}1 & 1 & 1 \\
1 & 1 & 1 \\
1 & 1 \\
1 & 11 \\
1 & 1 & 1\end{array}$ \\
\hline 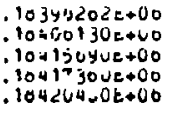 & $\begin{array}{l}\text { Lnjun } \\
\text { nLWu } \\
\text { hine } \\
\text { netu } \\
\text { ninu }\end{array}$ & $\begin{array}{ll}1 & 1 \\
1 & 0 \\
1 & 0 \\
1 & 0 \\
1 & 0\end{array}$ & 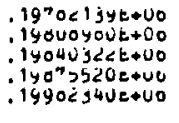 & $\begin{array}{l}\text { GNCU } \\
\text { neCS } \\
\text { UnJün } \\
\text { nCOUn } \\
\text { CHinn }\end{array}$ & $\begin{array}{l}11 \\
111 \\
111 \\
1 \\
1 \\
111\end{array}$ \\
\hline $\begin{array}{l}10424200 c+00 \\
1042 y 100 E+00 \\
10401140 e+00 \\
10407900=+06 \\
104927 y 0 E+00\end{array}$ & $\begin{array}{l}\text { nCNo } \\
\text { ninu } \\
\text { ninu } \\
\text { nCCCnO } \\
\text { ninu }\end{array}$ & $\begin{array}{ll}1 & 0 \\
1 & 0 \\
1 & 0 \\
1 & 1 \\
1 & 1 \\
1 & 0\end{array}$ & $\begin{array}{l}.2000324^{7} E+00 \\
.200 y^{7} 520 e+00 \\
.2012 J^{7} 30 E+00 \\
.20121502 E+00 \\
.20123471 E+00\end{array}$ & $\begin{array}{l}\text { NUF } \\
\text { N2O } \\
\text { nUF } \\
\text { nUE } \\
\text { Nuf }\end{array}$ & 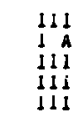 \\
\hline $\begin{array}{l}10509 y y u E+00 \\
105000^{\circ} 0 \mathrm{Je}+06 \\
10020010 \mathrm{0}+00 \\
10000520 \mathrm{0}+06 \\
100000^{7} 0 \mathrm{c}+00\end{array}$ & 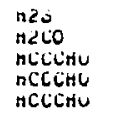 & $\begin{array}{l}111 \\
111 \\
111 \\
111 \\
111\end{array}$ & $\begin{array}{l}.2012^{7} 000 E+00 \\
.20130000 E+U 0 \\
.201414 \angle A E+00 \\
.2010^{7} 3^{7} 0 t+00 \\
.20140^{7} 02 E+00\end{array}$ & $\begin{array}{l}\text { Nut } \\
\text { nuF } \\
\text { nuF } \\
\text { ACUUn } \\
\text { CnjSCN }\end{array}$ & $\begin{array}{l}111 \\
111 \\
111 \\
\text { III } \\
11\end{array}$ \\
\hline 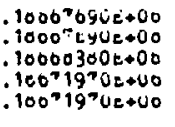 & 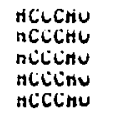 & $\begin{array}{l}111 \\
111 \\
111 \\
111 \\
111\end{array}$ & 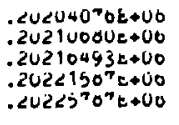 & 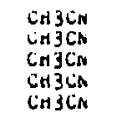 & $\begin{array}{l}11 \\
11 \\
11 \\
11 \\
11\end{array}$ \\
\hline $\begin{array}{l}100 ? 0<00 E+U R \\
100^{7} 0<9 U E+00 \\
1000024 U E+30 \\
100 y 3020 E+00 \\
1000310 E+00\end{array}$ & 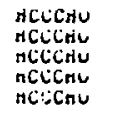 & 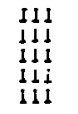 & $\begin{array}{l}.2 U<29370 E+00 \\
.20232154 c+00 \\
. \angle U<34 \cup 1 U E+00 \\
.20232145 E+00 \\
.20235501 E+00\end{array}$ & $\begin{array}{l}\mathrm{Cn} 3 \mathrm{CN} \\
\mathrm{CH} 3 \mathrm{Cn} \\
\mathrm{CH} 3 \mathrm{Cl} \\
\mathrm{CH} 3 \mathrm{CN} \\
\mathrm{CH} 3 \mathrm{CH}\end{array}$ & $\begin{array}{l}11 \\
\text { II } \\
\text { 1: } \\
\text { il } \\
\text { II }\end{array}$ \\
\hline
\end{tabular}


EnewenCt LJUI ya

nu

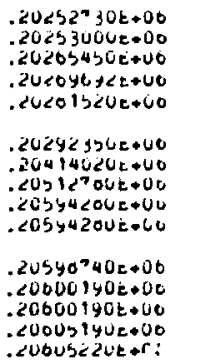

200042 '

$20615010 t+06$

JU4 $3044 E+00$

$\angle 40092356+06$

2004420000

$.20700600 k+0 t$

$.20^{7} 17400 t+00$

. $20^{n} 14060 \mathrm{e}+0$

$.20^{7} 223 y 0 E+06$

$.20732500 \mathrm{E}+00$

$.20>30030 \mathrm{c}+00$

$.20^{\circ} 00500 \mathrm{~L}+00$

.20004 14UE +06

$20420010 t+06$

.$<10407600+00$

$.21121150 c+00$

.213173130000

$.213700906<00$
$.21370090 t \rightarrow 00$

$.213 y 5972=000$

$.213959^{7} 2 t+06$

. $214095936+00$

$2140959 g t+00$

$.21419059 t+00$

$21419059 t-06$

$21422290 \mathrm{E}+00$

$.21426^{77} 2 \mathrm{e}+06$
$.2142^{77} 22 \mathrm{0}+00$

$.214310092+00$

$.214920508+00$

$.21544000 \mathrm{t}+06$

$.21619000 \mathrm{c}+06$

$<10500^{\circ} 0 E+00$

$10400 \mathrm{~b}+06$

$.21 ? 10460 t+06$
$.21822220 t+06$

$.21822220 \mathrm{k}+66$
$.21647560 \mathrm{E}+06$

$.21647560 E+06$

$.210303500 * 00$

$.2189032 ? E+06$

$.22040396 \mathrm{E}+0 \mathrm{~h}$

$.22047604 E+06$

$.22053930 E+06$

$.22059450 E+06$

$.22064112 E+06$

$.22067932 t+06$
$.2207090 \$ E+06$

$.2207302 ? E+06$

220?4299E+06

$.220 ? 4724 E+06$

$.2209123 ? \varepsilon+06$

$.22304995 E+00$

$.22355474 E+06$

$.22355909 \mathrm{E}+06$
MULÉCULE

Howe

ncuur

nLUUA

nCuvin

not

nLCuIr

$n<L$

जिए

nluun

$n<4$

netio

n2कs

racs

nels

$n<2=$

ideus

H2LS

HCNU

nut

HCAU

nciso

TCAO

HCh

HChu

HCAO

HCHu

Linzmn

HCKO

nces

HeLS

Cnenn
newo

nelo

Hing

तn

HN 3

I:N 3

nh 3

HA

Cat2un

nat

nin 3

inzan

Ha 3

Nor

H2LO

Hद.

SIU

i2 20

haco

H2Cs

OCs

$\mathrm{CH} 3 \mathrm{CH}$

$\mathrm{CH} 3 \mathrm{CH}$

$\mathrm{CN3CH}$

CH3CN

$\mathrm{CH} 3 \mathrm{CN}$

CHJCN

CH 3 CN

Cn 3 CN

Ca 3 CN

HUE

noF

NOF
hU

\begin{tabular}{|c|c|}
\hline KubEciUate & I AEL \\
\hline nur & 11 \\
\hline nur & \\
\hline Atut & 11 \\
\hline aur & !l \\
\hline inur & id \\
\hline hut & 11 \\
\hline vnchn & \\
\hline sur & 11 \\
\hline $\begin{array}{l}n \in 6 \\
v \pi<6)\end{array}$ & I1 \\
\hline neiv & II \\
\hline nZs & 11 \\
\hline $\operatorname{MCm}$ & 1 \\
\hline aubi & I \\
\hline MlNu & $i$ \\
\hline riglid & 1 \\
\hline runu & \rfloor \\
\hline risu & 1 \\
\hline BCNU & 1 \\
\hline ninu & 1 \\
\hline & 1 \\
\hline nETH & I \\
\hline inzins & 1 \\
\hline
\end{tabular}

$.25013054 c+00$

$.25072000 \mathrm{~T}+00$

. 24U1700UE+U6

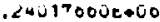

$\angle 4 v \angle 01+4 v_{e}+06$

C4u20 4 J Je+ve

$.24 u<00 j u=+00$

C4U9S1 4 UEषUO

$2403314 \mathrm{U} c+00$

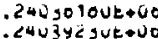

- 40 jy 3ute+00

$.240340306+60$

$.24321009 E+00$

$24404700 \varepsilon+00$

$.244935^{7} 0 t+06$

$.24512000 k+00$

- $25016190 k+00$

25044000e+00
$. \angle 5121150 \mathrm{0}+00$

-251511j4cewo

$.25142140 \mathrm{t}+00$

$.251740122+00$

.251? OONOE+vo

$2510^{7}$ y5OE+UE

- $25100000 \mathrm{e}+00$ $25241400 \mathrm{e}+\mathrm{UO}$ $.25307400 \mathrm{~T}+00$ $.25,20050 \mathrm{~L}+00$ .et $24090 \mathrm{t}+00$

.2万 $325220 t+00$

$.25320390 \mathrm{~b}+00$

$.25332350 \mathrm{t} \bullet 06$

$.2533690 \mathrm{k}+06$

$.253029^{\circ} 0 \mathrm{E}+00$

$.25420000 E+00$

$25460520 E+06$

$25501600 \mathrm{E}+00$

$.2550^{\circ} 2405+00$

$25010540 \mathrm{E}+06$

$.25617700 e+00$

$.25711<00 t+00$

$25^{\circ} 02500=06$

$20051700 t+06$

$.20390010 k+00$ nटu 11

M2CS

nंटिय

mel II

neci 11

H2C: 11

it

$\begin{array}{ll}\mathrm{r} 2 \mathrm{CS} & 111 \\ \mathrm{ri2s} & 111\end{array}$

खर्

Crent 111

$\mathrm{C} 20 \mathrm{dat}$

Cnenn II

$x<U$ iो

inzun ild

$\lim _{\rightarrow 2 u}$

a 20 \&

MLNU I

"Cliu 1 o

nenv 10

nunu Io

nCNio 10

HCNO

ACNOS I

Cnatro III

Calinn III

CenzhH III

Carat

CHEAr

Silo

Siv
HCNu IO 
w

$.204 \angle^{7} 02 U E+00$

- 20507010

$.57052000 t+00$

$.274 j^{7} 150 \mathrm{~s}+00$

$.20451500 * 00$

$.2^{7} 440220 \mathrm{~s}+00$

$.20445220 \mathrm{C}+40$

$.27452000=000$

$.27457750 \mathrm{c} \leftrightarrow 06$

$.27401040 c+00$

$.27405 y 10 \mathrm{E}+00$

27405910 c\$0

$.274702200+06$

$.27473160 \mathrm{0}+00$

. 27473320 +00

. $274952005+00$

$.27500^{7}$ DOE +00
$.275 \mathrm{~N}+\mathrm{OOE}+00$

. 270ट<410E $\$ 06$

. $7625000 \mathrm{c}+06$

.27620130t+00

$.27620130 \mathrm{E}+00$

.276340305006

$.276420005+00$

$.27609990 k+06$

27692920E+06

.27692920E+06

270042?0E $\$ 06$

.27000650E+06

.27910210E०06

$.20152090 \mathrm{Q}+00$

$.20183000 t+00$

$.28425410=006$

.2847?4205 +06

$247470706+06$

$29031410 \mathrm{E}+06$

$.29062340 E+06$

$29123770 \mathrm{0} \bullet 06$

$29130030 \mathrm{0}+00$

$.29136030 E+06$
$.291364205+06$

$.29194000 E+06$

$.29242190 \mathrm{E} \bullet 06$

$.292421906+00$

. 9242 1906006

$.292421908+06$

$.29243610 E+06$

$.292436105+06$

$.29244440 E+06$ $292444406+06$ $29246170 \mathrm{t}+06$ $29246170 \pm 406$ 292496105406

.292496 toE४06 $292501208+06$ 29250120E+06 .292623001406
.292624005406

.292064505406 $.29290260 E+06$ 203027 OE +06 $293912004+06$ .294043204+06

.30050560E+06 3 Uod $36608+06$ 3014 270E+06 $.30225620 E+06$

MOLECuLE IAE

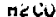

$\operatorname{neco}$

Cninn

Crist

niels

$1+1$

II

ए।

$n<i s$

n2LS

necs

Hels

Heto

H2LS

h2 is

H2CS

nacs

rizis

H2 L'S

$\mathrm{HCNO}$

HCNO

nCNo

HCWU

HCNO

HCNO

bicino

inctu

HCHO

HCUUt

HLinu

CH2hin

H2CS

HCOUH

$\mathrm{H} 2 \mathrm{CO}$

HCoOn

Chema

Chann

Crand

HCCO

$\mathrm{H} 2 \mathrm{CO}$

$\mathrm{H} 2 \mathrm{CO}$
$\mathrm{H} 2 \mathrm{CO}$

H2CO

H26O

acoOA

acoos

HCOH

HCOOH

$\mathrm{HCOOA}$

HCOOH

HCOOH

$\mathrm{HCOOH}$

HCOOH

ACOOA

$\mathrm{HCOOH}$

$\mathrm{HCOOH}$

$\mathrm{HCOOH}$

ACOOH

HCOUH

HCOOH

$\mathrm{HCOOH}$

HCAC:

CS

HCOOH

111
111
111
151
111

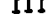

111

111

ItI

III

$1 \mathrm{~B}$

I $\mathrm{B}$

I

I

I 8

111

III

III

III

III

$42 \mathrm{~s}$

$42 \mathrm{CO}$

420

120

III

III

III

III

III

III

III

III

III nu

. $303+<040 \mathrm{~s}+00$ . $31443^{7} 00=+40$ . $321<<204 E+00$ - gej أ .3ayounuUe+uv

. 30y1ultuz+ue

- joy $1 \angle 0 y 0 z+00$

. 3ouly $y^{7} \mathrm{z}$ +ud

. 3y<01700e+vo

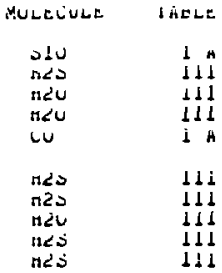

\& U.S. GOVERnMENT PAINTING OFFICE 1977-777-018/20 